\title{
Natural Products Containing the Oxetane and Related Moieties Present Additional Challenge for Structure Elucidation: a DU8+ Computational Case Study.
}

Andrei G. Kutateladze, ${ }^{*}$ Tina Holt, and D. Sai Reddy.

\section{Supporting Information}

\section{Contents}

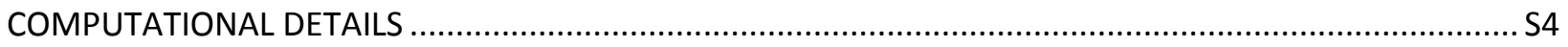

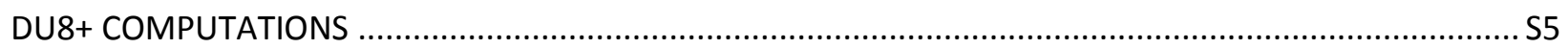

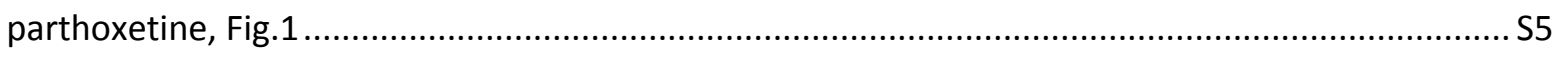

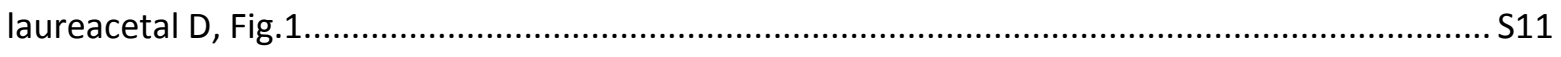

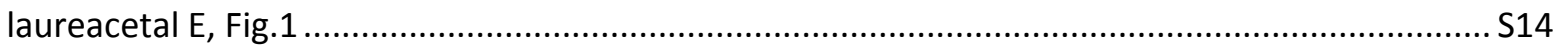

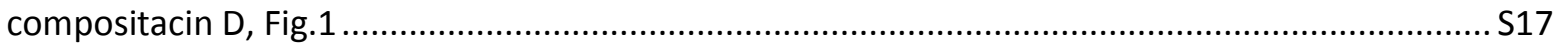

dictyoxetane, Fig.1

dichrocephone B, Fig.1

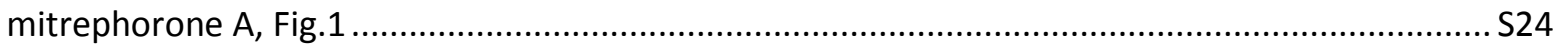

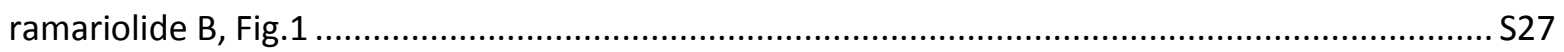

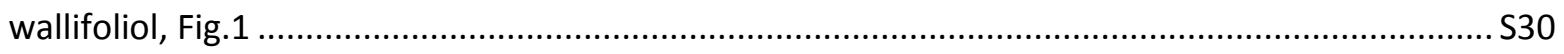

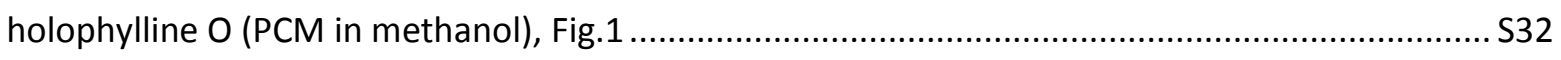

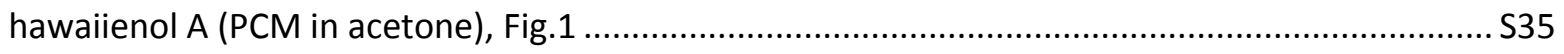

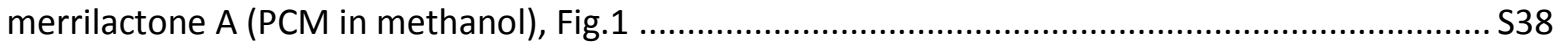

vibralactone, Fig.1

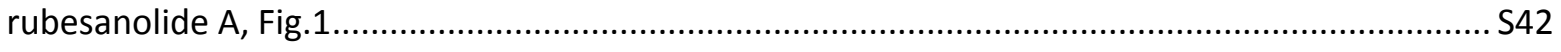

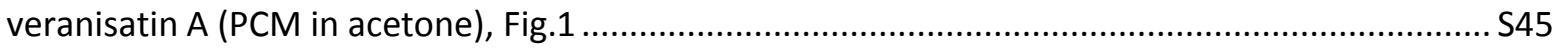

2-oxatetracyclo[4.4.0.0 $0^{1,4} \cdot 0^{6,8}$ ]decane (cmpd 47), Fig.2 .......................................................... 447

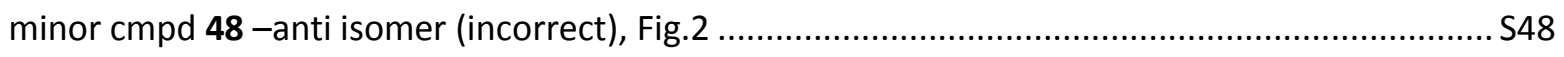

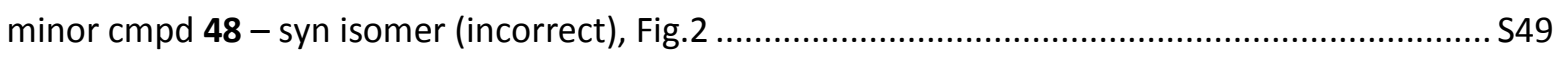

revised cmpd 48 - the oxametathesis product, Fig.2 ................................................................ S50

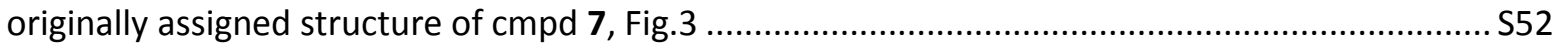

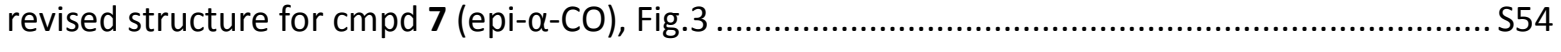

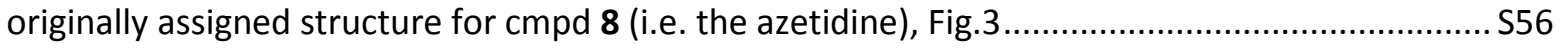

revised structure for compound $\mathbf{8}$ is the same as the originally assigned for 7 , Fig.3 ...................S58 
originally assigned structure of cleroindicin A (PCM pyridine), Fig.4A ....................................... S60

revised structure of cleroindicin A - syn open triol (PCM in pyridine), Fig.4A............................... S63

originally assigned structure of $2 \alpha, 6 \alpha$-epoxy-3-himachalene, Fig.4B .......................................... S65

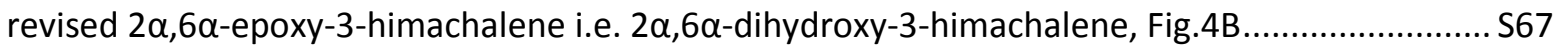

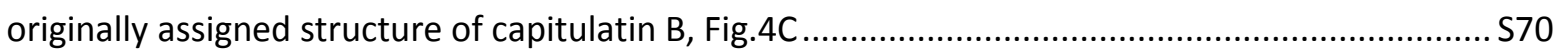

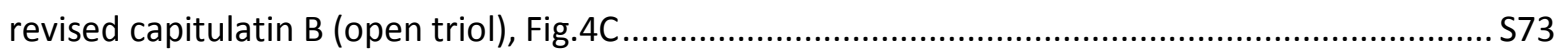

originally assigned structure of 11-hydroxycapitulatin B (PCM methanol), Fig.4D ........................S77

revised structure of 11-hydroxycapitulatin B (PCM methanol), Fig.4D ........................................ S80

originally assigned structure of cephaloziellin B, Fig.4E ............................................................. S82

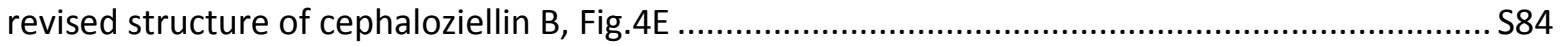

correctly assigned cephaloziellin A (accuracy test for DU8+) ...................................................... S87

originally assigned structure of 13,17-epoxy-ent-kaurenal, Fig.4F............................................... S90

revised structure of 13,17-epoxy-ent-kaurenal, Fig.4F...........................................................S93

originally assigned structure of 1,4-epoxy-6-deoxypseudoanisatin (cmpd 1), Fig.4G.................... S99

revised structure for cmpd 1 (1 $\alpha$-hydroxy-6-deoxypseudoanisatin), Fig.4G ................................ S101

originally assigned structure for 8-hydroxy-1,7-epoxy-2-menthene, Fig.5 ….............................. S103

revised "8-hydroxy-1,7-epoxy-2-menthene" (7-hydroxy-1,8-epoxy), Fig.5 ...................................S105

originally assigned structure of triptergulide A, Fig.5 ................................................................... S106

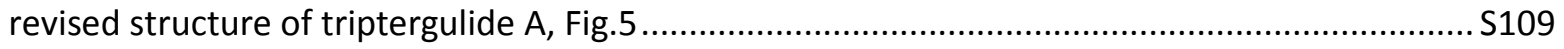

originally assigned structure of myrotheciumone B, Fig.5 ….................................................... S113

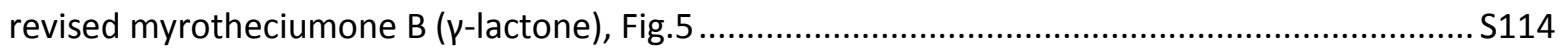

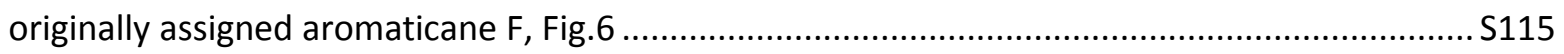

revised structure of aromaticane F, Fig.6 ….......................................................................... S119

originally assigned structure of moroccolide A (cmpd 2, PCM methanol), Fig.7 ........................... S121

revised structure of moroccolide A (cmpd 2, PCM methanol), Fig.7 ........................................... S123

originally assigned structure of saharanolide A ( $\mathrm{cmpd} \mathrm{5),} \mathrm{Fig.7} \mathrm{.................................................} \mathrm{S125}$

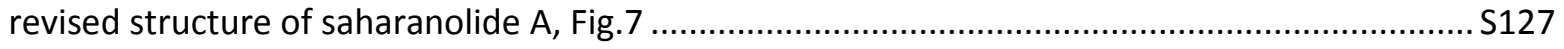

originally assigned structure of saharanolide B (cmpd 6, PCM methanol), Fig.7 ......................... S130

revised structure of saharanolide B (cmpd 6, PCM methanol), Fig.7 …..................................... S132

originally assigned structure of 6,11 -epoxy-eudesmane, Fig. 8 .................................................. S134

revised structure of 6,11-epoxy-eudesmane, Fig. 8 ................................................................. S136

originally assigned structure of okamuragenin (first possible diastereomer), Fig.8 ….................S138 
originally assigned structure of okamuragenin (second possible diastereomer, i.e. C10-epimer), Fig.8 S140

revised structure of okamuragenin, Fig. 8 S142

3-phenyloxete (test for the oxete structural type), Fig.9A S144

4-ethoxycarbonyl-4-trifluoromethyl-2-(p-methoxyphenyl)oxete (cmpd 4n) (Fig.9A).... S145

2-(o-methoxyphenyl)-3-phenyl-4-methyleneoxete - incorrect, Fig.9A..... S146

originally assigned structure of fusariumin D, Fig.9B S148

revised structure of fusariumin $D$, Fig.9B S150

revised structure of cheliensisamine (cmpd IV), Fig.9B. S154

2-acetyl-2-methyl-6-methoxybenzoxete (cmpd 4d) Fig.10 S155

originally assigned structure of phomopsidone A, Fig.10. S157

revised phomopsidone $A$ - excelsione ( $\mathrm{cmpd}$ 1), Fig.10 S159

originally assigned structure of amentotaxone, Fig.10..... S161

revised structure of amentotaxone (PCM methanol), Fig.10 S162

originally assigned structure of buxifoliadine (PCM DMSO), Fig.11 . S164

revised buxifoliadine (PCM DMSO), Fig 11. S166

originally assigned structure of neohupehenolide B (PCM methanol), Fig.13 S168

revised neohupehenolide $B$, Fig. 13 . S170

originally assigned structure of isoflavon cmpd 3, Fig.14. S172 revised structure of isoflavon $\mathrm{cmpd} 3$, Fig. 14 . S175

originally assigned structure of isoflavon cmpd 4, Fig. 14 S178

revised structure of isoflavon cmpd 4, Fig.14 S182

originally assigned structure for the sesquiterpene dimer from Xylopia aromatica, Fig.15 S185

revised sesquiterpene dimer from xylopia aromatica, Fig.15 S189

$\beta$-lactol from Caesalpinia decapetala (cmpd 1), Fig.16. S193

2,2,3,3-tetramethyl-4-methoxy-4-(p-cyanophenyl)oxetane, Fig.16. S194 truncated bidenphytin B (hydroxydioxetane moiety), Fig.17. S196 sphaerococcenol A. S197

bromosphaerol. S200

originally assigned structure of sphaeroxetane (cmpd 3), Fig.18 S203

A candidate structure for sphaeroxetane (ignoring multiplicities and fitting to 13C), Fig.18 ........S206 originally assigned structure of stereumone A, Fig.18 S209 


\section{COMPUTATIONAL DETAILS}

The structures of the oxetane-containing organic molecules and natural products were pre-optimized with the force field MMFF94 as implemented in OpenBabel. (O'Boyle, N. M.; Banck, M.; James, C. A.; Morley, C.; Vandermeersch, T.; Hutchison, G. R. J. Cheminform, 2011, 3, 8). For structures with freely rotatable groups, conformers were generated using OpenBabel's confab, whereas the conformers resulting from conformational changes in cyclic cores were generated manually, using Chem3D. As the empirical corrections for DU8+ were developed on a training set of ${ }^{1} \mathrm{H}$ and ${ }^{13} \mathrm{C}$ NMR spectra recorded in $\mathrm{CDCl}_{3}$, fast gas phase computations were used for such cases. For the experimental data obtained in DMSO- $d_{6}$ or methanol- $d_{4}$, a PCM model with additional linear scaling of chemical shifts was utilized (in these cases, corrected rmsd's, i.e. crmsd, were reported). For details on DU8+ method see: Kutateladze, A. G.; Reddy, D. S. J. Org. Chem. 2017, 82, 3368

All DFT calculations were performed with Gaussian 2009, Revision A.02, M. J. Frisch, G. W. Trucks, H. B. Schlegel, G. E. Scuseria, M. A. Robb, J. R. Cheeseman, G. Scalmani, V. Barone, B. Mennucci, G. A. Petersson, H. Nakatsuji, M. Caricato, X. Li, H. P. Hratchian, A. F. Izmaylov, J. Bloino, G. Zheng, J. L. Sonnenberg, M. Hada, M. Ehara, K. Toyota, R. Fukuda, J. Hasegawa, M. Ishida, T. Nakajima, Y. Honda, O. Kitao, H. Nakai, T. Vreven, J. A. Montgomery, Jr., J. E. Peralta, F. Ogliaro, M. Bearpark, J. J. Heyd, E. Brothers, K. N. Kudin, V. N. Staroverov, R. Kobayashi, J. Normand, K. Raghavachari, A. Rendell, J. C. Burant, S. S. Iyengar, J. Tomasi, M. Cossi, N. Rega, J. M. Millam, M. Klene, J. E. Knox, J. B. Cross, V. Bakken, C. Adamo, J. Jaramillo, R. Gomperts, R. E. Stratmann, O. Yazyev, A. J. Austin, R. Cammi, C. Pomelli, J. W. Ochterski, R. L. Martin, K. Morokuma, V. G. Zakrzewski, G. A. Voth, P. Salvador, J. J. Dannenberg, S. Dapprich, A. D. Daniels, O. Farkas, J. B. Foresman, J. V. Ortiz, J. Cioslowski, and D. J. Fox, Gaussian, Inc., Wallingford CT, 2009.

HOW TO READ THE TABLES BELOW:

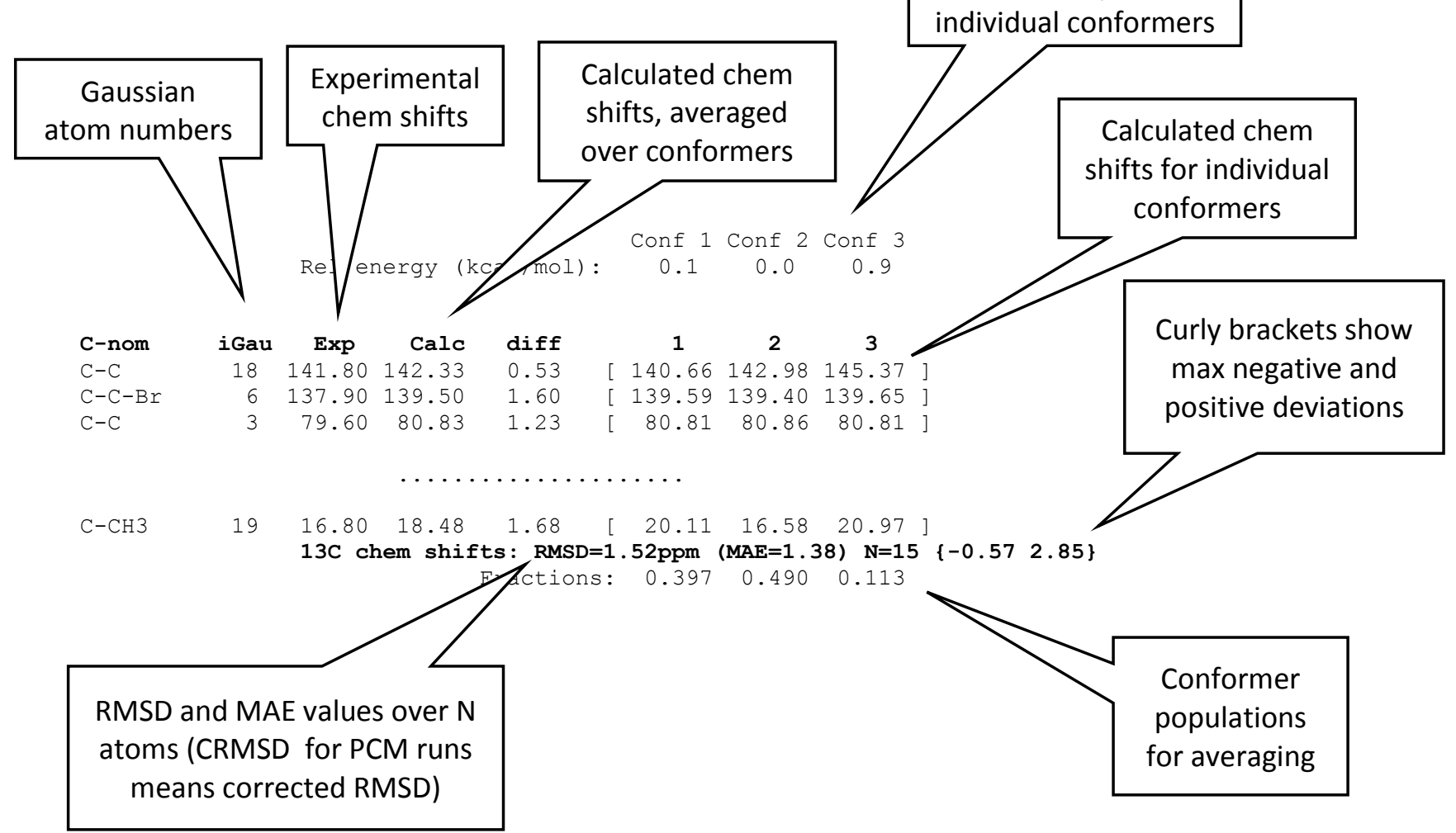




\section{DU8+ COMPUTATIONS}

parthoxetine, Fig.1

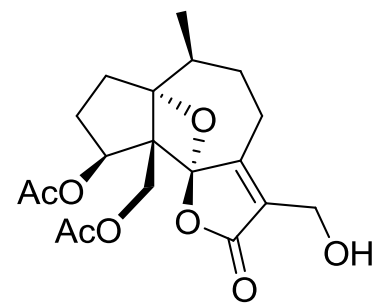

Conf 1 Conf 2 Conf 3 Conf 4 Conf 5 Conf 6

Rel energy (kcal/mol): $\begin{array}{cccccc}2.9 & 3.1 & 0.0 & 1.0 & 0.0 & 0.6\end{array}$

\begin{tabular}{|c|c|c|c|c|c|c|c|c|c|c|}
\hline C-nom & iGau & $\operatorname{Exp}$ & Calc & $\operatorname{diff}$ & 1 & 2 & 3 & 4 & 5 & 6 \\
\hline $\mathrm{C} 1-\mathrm{C}$ & 1 & 94.33 & 92.23 & -2.10 & 90.68 & 91.06 & 92.25 & 92.67 & 92.24 & 91.95 \\
\hline $\mathrm{C} 2-\mathrm{CH} 2$ & 5 & 34.94 & 35.01 & 0.07 & 31.70 & 34.57 & 35.21 & 32.94 & 35.21 & 35.06 \\
\hline $\mathrm{C} 3-\mathrm{CH} 2$ & 4 & 31.55 & 32.28 & 0.73 & 32.35 & 31.32 & 32.25 & 32.96 & 32.25 & 32.09 \\
\hline $\mathrm{C} 4-\mathrm{CH}$ & 3 & 77.46 & 78.98 & 1.52 & 78.90 & 80.11 & 79.08 & 77.95 & 79.08 & 78.98 \\
\hline C5-C & 2 & 61.01 & 61.99 & 0.98 & 64.50 & 64.85 & 61.82 & 61.70 & 61.83 & 62.94 \\
\hline C6-C & 6 & 105.67 & 105.17 & -0.50 & [ 105.85 & 106.26 & 105.08 & 105.85 & 105.08 & 105.28 \\
\hline $\mathrm{C} 7-\mathrm{C}$ & 7 & 161.64 & 160.96 & -0.68 & [ 168.82 & 165.24 & 160.33 & 165.13 & 160.33 & 162.05 \\
\hline $\mathrm{C} 8-\mathrm{CH} 2$ & 8 & 23.30 & 22.35 & -0.95 & {$\left[\begin{array}{ll}25.38 \\
{[}\end{array}\right.$} & 22.90 & 22.20 & 24.65 & 22.20 & 21.90 \\
\hline $\mathrm{C} 9-\mathrm{CH} 2$ & 9 & 29.07 & 27.57 & -1.50 & 29.68 & 26.64 & 27.34 & 31.11 & 27.35 & 26.97 \\
\hline $\mathrm{C} 10-\mathrm{CH}$ & 10 & 36.21 & 39.22 & 3.01 & 38.49 & 40.03 & 39.25 & 38.69 & 39.25 & 39.31 \\
\hline C11-C & 13 & 127.13 & 129.62 & 2.49 & {$[128.68$} & 129.66 & 129.73 & 129.58 & 129.73 & 129.03 \\
\hline $\mathrm{C} 12-\mathrm{C}$ & 12 & 169.66 & 169.28 & -0.38 & {$[170.10$} & 170.16 & 169.15 & 168.93 & 169.15 & 170.12 \\
\hline $\mathrm{C} 13-\mathrm{CH} 2$ & 16 & 54.66 & 53. & -1.14 & {$\left[\begin{array}{ll}{[} & 55.87\end{array}\right.$} & 57.68 & 53.48 & 54.00 & 53.49 & 53.37 \\
\hline $\mathrm{C} 14-\mathrm{CH} 3$ & 15 & 15.68 & 14.39 & -1.29 & 19.12 & 13.08 & 14.22 & 17.88 & 14.22 & 13.45 \\
\hline $\mathrm{C} 15-\mathrm{CH} 2$ & 14 & 59.63 & 60.29 & 0.66 & 62.50 & 63.35 & 60.90 & 61.17 & 60.89 & 56.45 \\
\hline C16-C & 22 & 170.64 & 170.54 & -0.10 & [ 172.57 & 173.70 & 170.24 & 170.19 & 170.25 & 172.25 \\
\hline C17-C & 26 & 169.09 & 170.22 & 1.13 & [ 169.63 & 169.55 & 170.20 & 170.31 & 170.20 & 170.34 \\
\hline $\mathrm{C} 18-\mathrm{CH} 3$ & 23 & 21.00 & 20.11 & -0.89 & {$\left[\begin{array}{ll}19.19 \\
{[}\end{array}\right.$} & 18.66 & 20.16 & 20.32 & 20.16 & 19.78 \\
\hline \multirow[t]{3}{*}{$\mathrm{C} 19-\mathrm{CH} 3$} & 27 & 20.44 & 20.2 & -0.24 & 20.26 & 20.12 & 20.18 & 20.19 & 20.18 & 20.33 \\
\hline & & & & & & & & $\{-2.10$ & $3.01\}$ & \\
\hline & & & & Fracti & $: 0.003$ & 0.002 & 0.389 & 0.073 & 0.389 & 0.143 \\
\hline
\end{tabular}

\section{Conformer 1}

Energy: -1340.22358 Hartree (Rel: $2.9 \mathrm{kcal} / \mathrm{mol}$ ) XYZ coordinates for conf 1 :

$\begin{array}{lrrr}C & -0.62440 & 2.12093 & -0.43519 \\ C & -0.69938 & 0.65780 & 0.14036 \\ C & -2.13476 & 0.24587 & -0.32642 \\ C & -2.98123 & 1.50914 & -0.15433 \\ C & -2.06568 & 2.61465 & -0.70962 \\ C & 0.32295 & 0.34739 & -0.99218 \\ C & 1.80821 & 0.36189 & -0.73430 \\ C & 2.49702 & 1.62041 & -0.30175 \\ C & 1.72005 & 2.55466 & 0.64177 \\ C & 0.37459 & 3.14841 & 0.14601 \\ C & 0.06566 & -0.85165 & -1.69174 \\ O & 1.23812 & -1.60712 & -1.74323 \\ C & 2.32850 & -0.80874 & -1.14144 \\ C & -0.42886 & 0.30152 & 1.61812 \\ C & -0.21056 & 4.02818 & 1.27005 \\ C & 3.73445 & -1.33820 & -1.01980 \\ C & 4.38438 & -0.90860 & 0.16367 \\ O & 1.25341 & -2.71300 & -2.21917 \\ O & -0.06221 & 1.54823 & -1.67836 \\ O & 0.89554 & -0.22225 & 1.83742 \\ O & 2.32997 & -1.88712 & 1.99889 \\ O & 1.16606 & -1.54473 & 1.97330 \\ \mathrm{O} & 0.02191 & -2.52549 & 2.10149\end{array}$

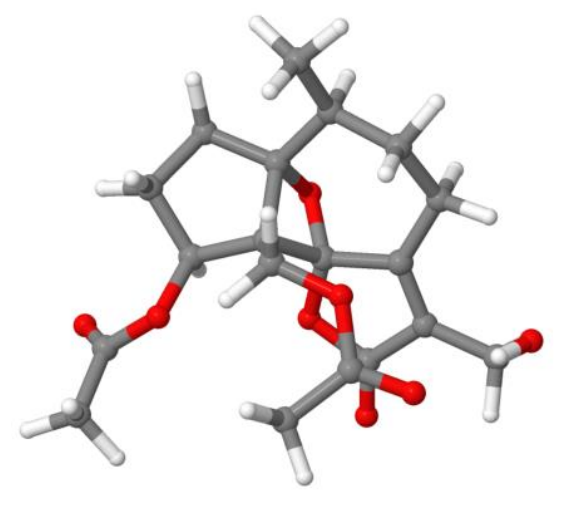




$\begin{array}{rrr}-2.61705 & -0.89177 & 0.41632 \\ -4.21608 & -1.23522 & -1.15455 \\ -3.65938 & -1.57673 & -0.13808 \\ -3.99169 & -2.80336 & 0.67751 \\ -2.11311 & -0.03529 & -1.37899 \\ -3.93884 & 1.42842 & -0.67345 \\ -3.18544 & 1.67236 & 0.91297 \\ -2.28697 & 3.58640 & -0.26568 \\ -2.17985 & 2.71304 & -1.79376 \\ 2.72716 & 2.17260 & -1.22630 \\ 3.45730 & 1.35093 & 0.14675 \\ 1.57597 & 2.05045 & 1.60227 \\ 2.37614 & 3.40761 & 0.85583 \\ 0.60050 & 3.80634 & -0.70652 \\ -0.48454 & 1.19333 & 2.24500 \\ -1.17646 & -0.40377 & 1.97460 \\ -1.02198 & 4.67329 & 0.92246 \\ 0.57137 & 4.68136 & 1.67235 \\ -0.59346 & 3.43203 & 2.10760 \\ 3.69260 & -2.43544 & -1.09518 \\ 4.35374 & -0.98802 & -1.85484 \\ 3.82797 & -1.19586 & 0.91423 \\ -0.64508 & -2.47586 & 1.23514 \\ 0.44872 & -3.52530 & 2.17973 \\ -0.57751 & -2.31762 & 2.99517 \\ -3.23517 & -3.57633 & 0.49832 \\ -3.99157 & -2.57641 & 1.74745 \\ -4.96593 & -3.18621 & 0.37122\end{array}$

\section{Conformer 2}

Energy: -1340.22319 Hartree (Rel: $3.1 \mathrm{kcal} / \mathrm{mol}$ ) XYZ coordinates for conf 2 :

\begin{tabular}{|c|c|c|c|}
\hline C & -0.56986 & 2.07787 & -0.46366 \\
\hline C & -0.74295 & 0.64815 & 0.17960 \\
\hline C & -2.22037 & 0.34647 & -0.25765 \\
\hline C & -2.96949 & 1.67080 & -0.09023 \\
\hline C & -1.96829 & 2.71256 & -0.62139 \\
\hline C & 0.21698 & 0.22043 & -0.96073 \\
\hline C & 1.71741 & 0.16835 & -0.72585 \\
\hline C & 2.49116 & 1.33226 & -0.16949 \\
\hline $\mathrm{C}$ & 1.93652 & 2.71345 & -0.56501 \\
\hline $\mathrm{C}$ & 0.52816 & 3.08444 & -0.04363 \\
\hline 0 & -0.11606 & -0.98940 & -1.60760 \\
\hline C & 1.01778 & -1.78804 & -1.69792 \\
\hline $\mathrm{C}$ & 2.16286 & -1.03016 & -1.14430 \\
\hline C & -0.54092 & 0.28294 & 1.65920 \\
\hline $\mathrm{C}$ & 0.51697 & 3.41347 & 1.45980 \\
\hline $\mathrm{C}$ & 3.53863 & -1.64472 & -1.09333 \\
\hline 0 & 4.38512 & -1.08081 & -0.11085 \\
\hline 0 & 0.97483 & -2.90372 & -2.14989 \\
\hline 0 & -0.15699 & 1.39050 & -1.70881 \\
\hline 0 & 0.82985 & 0.07115 & 2.03586 \\
\hline 0 & 2.58334 & -1.24340 & 2.26396 \\
\hline C & 1.38072 & -1.16743 & 2.12514 \\
\hline $\mathrm{C}$ & 0.47830 & -2.38309 & 2.07376 \\
\hline 0 & -2.77674 & -0.73897 & 0.50980 \\
\hline 0 & -4.37990 & -1.03300 & -1.06617 \\
\hline $\mathrm{C}$ & -3.85060 & -1.37832 & -0.03627 \\
\hline C & -4.25885 & -2.55649 & 0.81550 \\
\hline $\mathrm{H}$ & -2.23616 & 0.05070 & -1.30573 \\
\hline $\mathrm{H}$ & -3.92239 & 1.65951 & -0.62408 \\
\hline $\mathrm{H}$ & -3.18087 & 1.84002 & 0.97397 \\
\hline $\mathrm{H}$ & -2.03952 & 3.66111 & -0.08009 \\
\hline $\mathrm{H}$ & -2.12509 & 2.92674 & -1.68400 \\
\hline $\mathrm{H}$ & 3.52681 & 1.24280 & -0.50646 \\
\hline $\mathrm{H}$ & 2.53088 & 1.24140 & 0.92141 \\
\hline $\mathrm{H}$ & 2.63722 & 3.47211 & -0.19358 \\
\hline $\mathrm{H}$ & 1.93372 & 2.79602 & -1.65594 \\
\hline $\mathrm{H}$ & 0.26178 & 4.01211 & -0.57193 \\
\hline $\mathrm{H}$ & -0.89514 & 1.09664 & 2.29629 \\
\hline $\mathrm{H}$ & -1.13294 & -0.59775 & 1.89499 \\
\hline
\end{tabular}

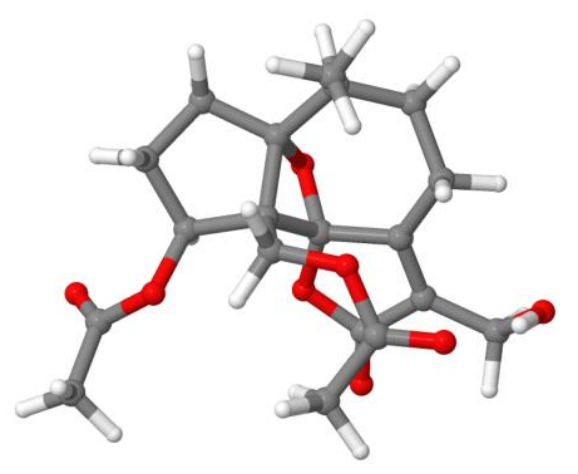




$\begin{array}{rrrr}\text { H } & -0.49351 & 3.64207 & 1.82135 \\ \mathrm{H} & 1.13616 & 4.29652 & 1.65202 \\ \mathrm{H} & 0.91809 & 2.59436 & 2.06372 \\ \mathrm{H} & 3.41816 & -2.73254 & -0.96667 \\ \mathrm{H} & 4.04896 & -1.50402 & -2.05437 \\ \mathrm{H} & 3.95256 & -1.18706 & 0.75662 \\ \mathrm{H} & -0.08767 & -2.43286 & 1.13775 \\ \mathrm{H} & 1.10903 & -3.26786 & 2.16008 \\ \mathrm{H} & -0.24228 & -2.37396 & 2.89930 \\ \mathrm{H} & -3.48655 & -3.33230 & 0.76600 \\ \mathrm{H} & -4.36289 & -2.25860 & 1.86344 \\ \mathrm{H} & -5.20078 & -2.96156 & 0.44443\end{array}$

\section{Conformer 3}

Energy: -1340.22812 Hartree (Rel: $0.0 \mathrm{kcal} / \mathrm{mol}$ ) XYZ coordinates for conf 3 :

\begin{tabular}{|c|c|c|c|}
\hline $\mathrm{C}$ & 0.01753 & -1.04296 & -1.58794 \\
\hline C & 0.33631 & -0.12487 & -0.34797 \\
\hline C & 1.28066 & 0.93490 & -1.00007 \\
\hline C & 2.12487 & 0.15998 & -2.01757 \\
\hline C & 1.13170 & -0.84280 & -2.63598 \\
\hline C & -1.13794 & 0.30508 & -0.51285 \\
\hline $\mathrm{C}$ & -2.21115 & -0.39922 & 0.29935 \\
\hline C & -2.29288 & -1.89906 & 0.36712 \\
\hline $\mathrm{C}$ & -1.95249 & -2.60886 & -0.96189 \\
\hline C & -0.49226 & -2.49802 & -1.46971 \\
\hline 0 & -1.41288 & 1.68067 & -0.39760 \\
\hline C & -2.52567 & 1.87241 & 0.41130 \\
\hline C & -3.02968 & 0.53243 & 0.81708 \\
\hline C & 0.80857 & -0.58631 & 1.01946 \\
\hline C & 0.47001 & -3.43615 & -0.72037 \\
\hline C & -4.30447 & 0.38984 & 1.59787 \\
\hline 0 & -5.45481 & 0.66137 & 0.80424 \\
\hline 0 & -2.92129 & 2.96707 & 0.70662 \\
\hline 0 & -1.13552 & -0.15665 & -1.87353 \\
\hline 0 & 2.11199 & -1.18761 & 0.88044 \\
\hline 0 & 2.22689 & -1.34682 & 3.13177 \\
\hline C & 2.73365 & -1.50630 & 2.04423 \\
\hline C & 4.11150 & -2.06724 & 1.78342 \\
\hline 0 & 2.05282 & 1.59733 & 0.01698 \\
\hline 0 & 2.49779 & 3.29337 & -1.41932 \\
\hline C & 2.58029 & 2.80595 & -0.31504 \\
\hline C & 3.25642 & 3.43780 & 0.87859 \\
\hline $\mathrm{H}$ & 0.69018 & 1.69314 & -1.51492 \\
\hline $\mathrm{H}$ & 2.58255 & 0.83129 & -2.74784 \\
\hline $\mathrm{H}$ & 2.92104 & -0.36960 & -1.48432 \\
\hline $\mathrm{H}$ & 1.61715 & -1.79003 & -2.89236 \\
\hline $\mathrm{H}$ & 0.66756 & -0.45307 & -3.54843 \\
\hline $\mathrm{H}$ & -3.30504 & -2.18733 & 0.67405 \\
\hline $\mathrm{H}$ & -1.63324 & -2.27305 & 1.16213 \\
\hline $\mathrm{H}$ & -2.18464 & -3.67449 & -0.84190 \\
\hline $\mathrm{H}$ & -2.62096 & -2.22435 & -1.73773 \\
\hline $\mathrm{H}$ & -0.52909 & -2.85375 & -2.51017 \\
\hline $\mathrm{H}$ & 0.88345 & 0.27117 & 1.69227 \\
\hline $\mathrm{H}$ & 0.13625 & -1.31078 & 1.48055 \\
\hline $\mathrm{H}$ & 1.50752 & -3.29012 & -1.03749 \\
\hline $\mathrm{H}$ & 0.20220 & -4.47994 & -0.91830 \\
\hline $\mathrm{H}$ & 0.43902 & -3.28829 & 0.36301 \\
\hline $\mathrm{H}$ & -4.35697 & -0.60683 & 2.06120 \\
\hline $\mathrm{H}$ & -4.32538 & 1.13399 & 2.39887 \\
\hline $\mathrm{H}$ & -5.43543 & 0.06427 & 0.03986 \\
\hline $\mathrm{H}$ & 4.74428 & -1.30004 & 1.32396 \\
\hline $\mathrm{H}$ & 4.55492 & -2.39181 & 2.72531 \\
\hline $\mathrm{H}$ & 4.05722 & -2.90815 & 1.08475 \\
\hline $\mathrm{H}$ & 2.49914 & 3.74093 & 1.61036 \\
\hline $\mathrm{H}$ & 3.92133 & 2.72223 & 1.37149 \\
\hline $\mathrm{H}$ & 3.81718 & 4.31532 & 0.55490 \\
\hline
\end{tabular}

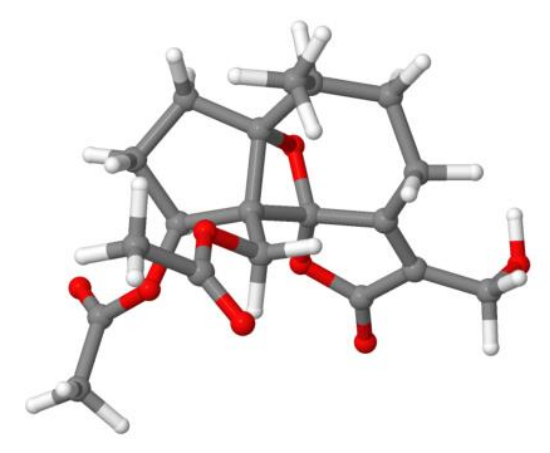




\section{Conformer 4}

Energy: -1340.22654 Hartree (Rel: $1.0 \mathrm{kcal} / \mathrm{mol}$ ) XYZ coordinates for conf 4 :

\begin{tabular}{|c|c|c|c|}
\hline C & 0.06450 & -1.19629 & -1.51638 \\
\hline C & 0.29573 & -0.15593 & -0.35819 \\
\hline C & 1.24086 & 0.87564 & -1.05370 \\
\hline C & 2.13034 & 0.04467 & -1.98252 \\
\hline C & 1.16228 & -0.98928 & -2.58550 \\
\hline C & -1.18903 & 0.20404 & -0.61871 \\
\hline C & -2.28863 & -0.43258 & 0.20210 \\
\hline C & -2.54336 & -1.90939 & 0.09552 \\
\hline C & -1.31928 & -2.83811 & -0.01647 \\
\hline C & -0.35958 & -2.65148 & -1.22235 \\
\hline O & -1.46920 & 1.58787 & -0.61722 \\
\hline C & -2.51761 & 1.84627 & 0.25846 \\
\hline C & -3.03986 & 0.54024 & 0.74587 \\
\hline C & 0.71141 & -0.47333 & 1.06861 \\
\hline $\mathrm{C}$ & 0.82716 & -3.61773 & -1.03809 \\
\hline C & -4.29831 & 0.46482 & 1.56240 \\
\hline O & -5.47120 & 0.67015 & 0.78063 \\
\hline O & -2.87336 & 2.96349 & 0.51902 \\
\hline O & -1.13854 & -0.41440 & -1.90793 \\
\hline O & 1.98505 & -1.15212 & 1.04297 \\
\hline O & 2.24169 & -0.68901 & 3.24268 \\
\hline C & 2.67189 & -1.16332 & 2.21656 \\
\hline C & 4.01383 & -1.83550 & 2.04753 \\
\hline O & 1.97203 & 1.62051 & -0.06397 \\
\hline O & 2.42476 & 3.22791 & -1.59666 \\
\hline C & 2.48447 & 2.81703 & -0.46040 \\
\hline C & 3.10897 & 3.54177 & 0.70797 \\
\hline $\mathrm{H}$ & 0.65741 & 1.58598 & -1.64020 \\
\hline $\mathrm{H}$ & 2.62312 & 0.67036 & -2.73059 \\
\hline $\mathrm{H}$ & 2.90022 & -0.45423 & -1.38351 \\
\hline $\mathrm{H}$ & 1.67617 & -1.91164 & -2.86207 \\
\hline $\mathrm{H}$ & 0.67376 & -0.60083 & -3.48486 \\
\hline $\mathrm{H}$ & -3.15057 & -2.04470 & -0.81225 \\
\hline $\mathrm{H}$ & -3.16666 & -2.23995 & 0.93494 \\
\hline $\mathrm{H}$ & -0.74392 & -2.80822 & 0.91526 \\
\hline $\mathrm{H}$ & -1.70588 & -3.86288 & -0.07925 \\
\hline $\mathrm{H}$ & -0.91031 & -2.95489 & -2.12565 \\
\hline $\mathrm{H}$ & 0.81582 & 0.45311 & 1.63577 \\
\hline $\mathrm{H}$ & -0.01182 & -1.10013 & 1.59190 \\
\hline $\mathrm{H}$ & 1.41450 & -3.73708 & -1.95250 \\
\hline $\mathrm{H}$ & 0.45938 & -4.61180 & -0.75930 \\
\hline $\mathrm{H}$ & 1.49728 & -3.27089 & -0.24477 \\
\hline $\mathrm{H}$ & -4.33774 & -0.49067 & 2.10743 \\
\hline $\mathrm{H}$ & -4.29943 & 1.27351 & 2.29817 \\
\hline $\mathrm{H}$ & -5.48352 & -0.00153 & 0.08095 \\
\hline $\mathrm{H}$ & 4.62376 & -1.27733 & 1.32910 \\
\hline $\mathrm{H}$ & 4.52283 & -1.87385 & 3.01112 \\
\hline $\mathrm{H}$ & 3.88768 & -2.84870 & 1.65219 \\
\hline $\mathrm{H}$ & 2.32040 & 3.89195 & 1.38364 \\
\hline $\mathrm{H}$ & 3.75716 & 2.87154 & 1.28013 \\
\hline $\mathrm{H}$ & 3.67603 & 4.39841 & 0.34193 \\
\hline
\end{tabular}

\section{Conformer 5}

Energy: -1340.22812 Hartree (Rel: XYZ coordinates for conf 5:

$\begin{array}{lrrr}\text { C } & 0.01630 & -1.04475 & -1.58695 \\ \text { C } & 0.33595 & -0.12586 & -0.34774 \\ \text { C } & 1.28060 & 0.93295 & -1.00102 \\ \text { C } & 2.12410 & 0.15682 & -2.01815 \\ \text { C } & 1.13014 & -0.84580 & -2.63556 \\ \text { C } & -1.13815 & 0.30459 & -0.51240 \\ \text { C } & -2.21158 & -0.39866 & 0.30047 \\ \text { C } & -2.29372 & -1.89840 & 0.36951 \\ \text { C } & -1.95408 & -2.60942 & -0.95903 \\ \text { C } & -0.49400 & -2.49952 & -1.46746 \\ \text { O } & -1.41246 & 1.68039 & -0.39808 \\ C & -2.52533 & 1.87318 & 0.41046 \\ \text { C } & -3.02987 & 0.53372 & 0.81727\end{array}$

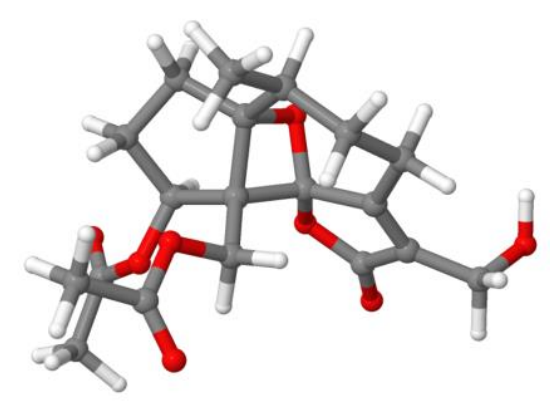




$\begin{array}{rrr}0.80852 & -0.58637 & 1.01991 \\ 0.46821 & -3.43741 & -0.71776 \\ -4.30494 & 0.39222 & 1.59782 \\ -5.45487 & 0.66371 & 0.80366 \\ -2.92063 & 2.96821 & 0.70483 \\ -1.13645 & -0.15817 & -1.87274 \\ 2.11159 & -1.18841 & 0.88092 \\ 2.23090 & -1.33848 & 3.13268 \\ 2.73553 & -1.50233 & 2.04482 \\ 4.11287 & -2.06430 & 1.78358 \\ 2.05338 & 1.59585 & 0.01525 \\ 2.49884 & 3.29038 & -1.42264 \\ 2.58138 & 2.80389 & -0.31794 \\ 3.25780 & 3.43670 & 0.87502 \\ 0.69030 & 1.69105 & -1.51629 \\ 2.58202 & 0.82728 & -2.74905 \\ 2.92007 & -0.37289 & -1.48474 \\ 1.61502 & -1.79340 & -2.89163 \\ 0.66585 & -0.45630 & -3.54803 \\ -3.30582 & -2.18616 & 0.67714 \\ -1.63382 & -2.27189 & 1.16453 \\ -2.18658 & -3.67487 & -0.83807 \\ -2.62270 & -2.22532 & -1.73496 \\ -0.53135 & -2.85606 & -2.50763 \\ 0.88398 & 0.27159 & 1.69203 \\ 0.13605 & -1.31023 & 1.48180 \\ 1.50563 & -3.29217 & -1.03557 \\ 0.19980 & -4.48126 & -0.91455 \\ 0.43792 & -3.28849 & 0.36549 \\ -4.35800 & -0.60412 & 2.06180 \\ -4.32576 & 1.13688 & 2.39836 \\ -5.43518 & 0.06652 & 0.03936 \\ 4.74476 & -1.29897 & 1.31980 \\ 4.55815 & -2.38506 & 2.72591 \\ 4.05722 & -2.90805 & 1.08846 \\ 2.50024 & 3.74437 & 1.60463 \\ 3.91960 & 2.72045 & 1.37104 \\ 3.82174 & 4.31165 & 0.54985\end{array}$

\section{Conformer 6}

Energy: -1340.22718 Hartree (Rel: $0.6 \mathrm{kcal} / \mathrm{mol}$ ) XYZ coordinates for conf 6 :

\begin{tabular}{|c|c|c|c|}
\hline C & -0.52678 & 2.14257 & -0.42715 \\
\hline C & -0.75511 & 0.67352 & 0.11107 \\
\hline C & -2.11958 & 0.32067 & -0.56071 \\
\hline C & -2.92267 & 1.62642 & -0.54477 \\
\hline C & -1.88661 & 2.70981 & -0.88477 \\
\hline C & 0.42556 & 0.34283 & -0.82702 \\
\hline C & 1.83096 & 0.31178 & -0.25403 \\
\hline C & 2.35383 & 1.42382 & 0.60987 \\
\hline C & 1.90036 & 2.83448 & 0.17140 \\
\hline C & 0.39062 & 3.17450 & 0.26739 \\
\hline 0 & 0.30399 & -0.81617 & -1.61458 \\
\hline C & 1.46844 & -1.56473 & -1.52316 \\
\hline C & 2.44068 & -0.80125 & -0.69662 \\
\hline C & -0.68008 & 0.25290 & 1.57610 \\
\hline C & -0.05522 & 3.54819 & 1.69315 \\
\hline C & 3.85610 & -1.27335 & -0.52865 \\
\hline O & 4.63230 & -1.07507 & -1.70658 \\
\hline O & 1.57059 & -2.65403 & -2.02167 \\
\hline 0 & 0.19957 & 1.55199 & -1.57056 \\
\hline 0 & -0.40161 & -1.16449 & 1.63211 \\
\hline 0 & 1.36365 & -0.82489 & 3.02405 \\
\hline C & 0.65214 & -1.57046 & 2.38381 \\
\hline C & 0.80879 & -3.06963 & 2.29865 \\
\hline 0 & -2.77232 & -0.73678 & 0.16024 \\
\hline 0 & -4.04717 & -1.20222 & -1.65515 \\
\hline C & -3.70289 & -1.45401 & -0.52273 \\
\hline & -4.22444 & -2.58807 & 0.32721 \\
\hline & -1.96452 & -0.01161 & -1.58746 \\
\hline & -3.76794 & 1.58452 & -1.23586 \\
\hline
\end{tabular}

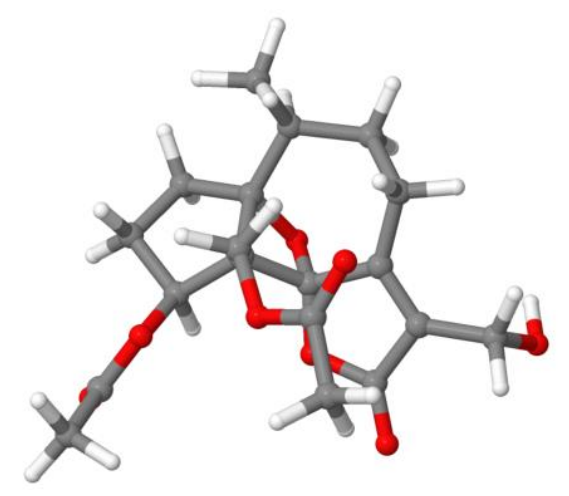




$\begin{array}{rrrr}\text { H } & -3.32307 & 1.78297 & 0.46598 \\ \mathrm{H} & -2.11601 & 3.66393 & -0.39959 \\ \mathrm{H} & -1.82604 & 2.89400 & -1.96258 \\ \mathrm{H} & 3.44980 & 1.39353 & 0.60096 \\ \mathrm{H} & 2.07206 & 1.22777 & 1.65262 \\ \mathrm{H} & 2.44610 & 3.56592 & 0.78053 \\ \mathrm{H} & 2.21958 & 2.98716 & -0.86403 \\ \mathrm{H} & 0.26953 & 4.08335 & -0.34070 \\ \mathrm{H} & 0.09554 & 0.77127 & 2.13170 \\ \mathrm{H} & -1.63766 & 0.41049 & 2.07868 \\ \mathrm{H} & -1.13851 & 3.70471 & 1.75450 \\ \mathrm{H} & 0.42674 & 4.48217 & 2.00178 \\ \mathrm{H} & 0.21308 & 2.78887 & 2.43328 \\ \mathrm{H} & 4.31616 & -0.79256 & 0.34707 \\ \mathrm{H} & 3.86204 & -2.35393 & -0.36063 \\ \mathrm{H} & 4.59738 & -0.13102 & -1.92723 \\ \mathrm{H} & 1.00025 & -3.36702 & 1.26182 \\ \mathrm{H} & 1.63543 & -3.38632 & 2.93542 \\ \mathrm{H} & -0.11637 & -3.56362 & 2.61232 \\ \mathrm{H} & -3.41080 & -3.28710 & 0.54823 \\ \mathrm{H} & -4.59818 & -2.20801 & 1.28342 \\ \mathrm{H} & -5.02121 & -3.10624 & -0.20733\end{array}$


laureacetal D, Fig.1<smiles>C=C(C)[C@@H]1C[C@@]23O[C@]2(C)C(Br)=CC(Br)=C3C1(C)C</smiles>

Rel energy (kcal/mol): $\begin{array}{cccc}\text { Conf } 1 & \text { Conf } 2 & \text { Conf } 3 \\ 0.1 & 0.0 & 0.9\end{array}$

\begin{tabular}{|c|c|c|c|c|c|c|c|c|}
\hline C-nom & iGau & $\operatorname{Exp}$ & Calc & $\operatorname{diff}$ & 1 & 2 & 3 & \\
\hline $\mathrm{C}-\mathrm{C}$ & 18 & 141.80 & 142.33 & 0.53 & 140.66 & 142.98 & 145.37 ] & ] \\
\hline $\mathrm{C}-\mathrm{C}-\mathrm{Br}$ & 6 & 137.90 & 139.50 & 1.60 & 139.59 & 139.40 & $139.65]$ & ] \\
\hline $\mathrm{C}-\mathrm{C}$ & 3 & 79.60 & 80.83 & 1.23 & 80.81 & 80.86 & $80.81]$ & ] \\
\hline $\mathrm{C}-\mathrm{C}$ & 4 & 62.50 & 63.75 & 1.25 & 63.81 & 63.65 & $64.00]$ & ] \\
\hline $\mathrm{C}-\mathrm{C}$ & 5 & 39.50 & 41.49 & 1.99 & 41.35 & 41.60 & $41.53]$ & ] \\
\hline $\mathrm{C}-\mathrm{CH}$ & 1 & 128.60 & 129.96 & 1.36 & [ 129.90 & 130.02 & $129.93]$ & ] \\
\hline $\mathrm{C}-\mathrm{CH}$ & 8 & 104.80 & 105.93 & 1.13 & {$[105.78$} & 106.23 & $105.16]$ & ] \\
\hline $\mathrm{C}-\mathrm{CH}$ & 10 & 83.80 & 84.47 & 0.67 & 83.09 & 85.91 & $83.06]$ & ] \\
\hline $\mathrm{C}-\mathrm{CH}-\mathrm{Br}$ & 2 & 51.50 & 54.35 & 2.85 & 54.30 & 54.37 & $54.47]$ & ] \\
\hline $\mathrm{C}-\mathrm{CH} 2$ & 20 & 111.20 & 112.61 & 1.41 & 111.02 & 114.40 & $110.41]$ & ] \\
\hline $\mathrm{C}-\mathrm{CH} 2$ & 11 & 31.90 & 34.37 & 2.47 & 35.58 & 33.87 & $32.28]$ & ] \\
\hline $\mathrm{C}-\mathrm{CH} 3$ & 16 & 24.90 & 25.60 & 0.70 & 25.67 & 25.56 & $25.56]$ & ] \\
\hline $\mathrm{C}-\mathrm{CH} 3$ & 13 & 23.60 & 23.03 & -0.57 & 22.91 & 23.16 & $22.86]$ & ] \\
\hline $\mathrm{C}-\mathrm{CH} 3$ & 12 & 21.10 & 22.32 & 1.22 & 22.23 & 22.41 & $22.27]$ & ] \\
\hline $\mathrm{C}-\mathrm{CH} 3$ & 19 & $\begin{array}{l}16.80 \\
13 \mathrm{C} \mathrm{C}\end{array}$ & $\begin{array}{l}18.48 \\
\text { shi }\end{array}$ & $\begin{array}{l}1.68 \\
.\end{array}$ & $\begin{array}{r}20.11 \\
1.52 \mathrm{ppm}\end{array}$ & $\begin{array}{r}16.58 \\
\operatorname{MAE}=1\end{array}$ & 38) $\mathrm{N}=15$ & $\begin{cases}-0.57 \quad 2.85 \\
0\end{cases}$ \\
\hline
\end{tabular}

\section{Conformer 1}

Energy: -5876.82326 Hartree (Rel: $0.1 \mathrm{kcal} / \mathrm{mol}$ ) XYZ coordinates for conf 1 :

\begin{tabular}{|c|c|c|c|}
\hline C & -1.92944 & 0.00083 & -0.88614 \\
\hline C & -1.29320 & -1.33959 & -0.90892 \\
\hline C & 0.22006 & -1.36069 & -0.63616 \\
\hline C & 0.79414 & -0.05972 & 0.04611 \\
\hline C & -0.19076 & 0.95516 & 0.70861 \\
\hline C & -1.44740 & 1.01370 & -0.15812 \\
\hline O & 0.83312 & -0.96740 & -1.90505 \\
\hline C & 1.29326 & 0.29475 & -1.37692 \\
\hline O & 2.66998 & 0.45544 & -1.36896 \\
\hline C & 3.22012 & -0.29947 & -0.28016 \\
\hline C & 2.11251 & -0.29127 & 0.81571 \\
\hline C & -0.60288 & 0.49430 & 2.12936 \\
\hline $\mathrm{C}$ & 0.48758 & 2.34166 & 0.80649 \\
\hline $\mathrm{Br}$ & -2.37284 & -2.50416 & 0.38606 \\
\hline $\mathrm{Br}$ & -2.51204 & 2.61922 & -0.11746 \\
\hline $\mathrm{C}$ & 0.73321 & -2.72941 & -0.20867 \\
\hline $\mathrm{H}$ & 0.86256 & 1.13056 & -1.93271 \\
\hline C & 4.53906 & 0.28238 & 0.17475 \\
\hline C & 5.29274 & -0.56036 & 1.17448 \\
\hline C & 4.99296 & 1.45268 & -0.28004 \\
\hline $\mathrm{H}$ & -2.86047 & 0.11163 & -1.43140 \\
\hline $\mathrm{H}$ & -1.46974 & -1.85747 & -1.85132 \\
\hline $\mathrm{H}$ & 3.39086 & -1.33057 & -0.62430 \\
\hline $\mathrm{H}$ & 2.30754 & 0.52909 & 1.50883 \\
\hline $\mathrm{H}$ & 2.10704 & -1.21497 & 1.39883 \\
\hline $\mathrm{H}$ & -1.31872 & 1.20166 & 2.56142 \\
\hline $\mathrm{H}$ & 0.27117 & 0.45572 & 2.78914 \\
\hline $\mathrm{H}$ & -1.07335 & -0.49305 & 2.11092 \\
\hline $\mathrm{H}$ & -0.18168 & 3.05731 & 1.28835 \\
\hline $\mathrm{H}$ & 0.75241 & 2.74112 & -0.17739 \\
\hline $\mathrm{H}$ & 1.39904 & 2.29099 & 1.40664 \\
\hline $\mathrm{H}$ & 0.31894 & -3.50030 & -0.86699 \\
\hline $\mathrm{H}$ & 0.44119 & -2.97330 & 0.81562 \\
\hline $\mathrm{H}$ & 1.82114 & -2.77059 & -0.29211 \\
\hline
\end{tabular}

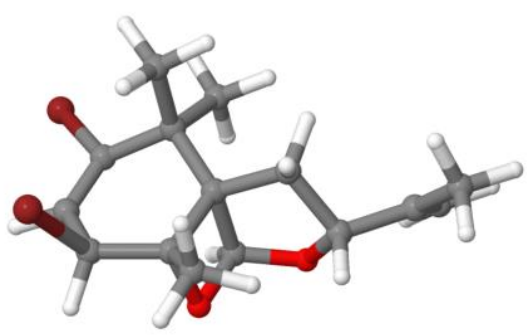




$\begin{array}{rrrr}\mathrm{H} & 5.51466 & -1.55665 & 0.76745 \\ \mathrm{H} & 4.71232 & -0.71904 & 2.09380 \\ \mathrm{H} & 6.24002 & -0.08996 & 1.45319 \\ \mathrm{H} & 5.94235 & 1.85753 & 0.06102 \\ \mathrm{H} & 4.43665 & 2.02945 & -1.01023\end{array}$

\section{Conformer 2}

Energy: -5876.82346 Hartree (Rel: $0.0 \mathrm{kcal} / \mathrm{mol}$ ) XYZ coordinates for conf 2 :

\begin{tabular}{|c|c|c|c|}
\hline C & -1.90697 & 0.00204 & -0.90189 \\
\hline C & -1.30236 & -1.35281 & -0.87501 \\
\hline C & 0.20455 & -1.40220 & -0.57015 \\
\hline C & 0.79906 & -0.09482 & 0.08226 \\
\hline C & -0.17628 & 0.95638 & 0.70095 \\
\hline C & -1.41424 & 1.02308 & -0.19278 \\
\hline 0 & 0.84861 & -1.06352 & -1.83947 \\
\hline C & 1.32938 & 0.20405 & -1.34416 \\
\hline 0 & 2.70967 & 0.32975 & -1.31909 \\
\hline C & 3.21889 & -0.42941 & -0.20289 \\
\hline C & 2.10284 & -0.33254 & 0.87613 \\
\hline C & -0.62797 & 0.53448 & 2.12198 \\
\hline C & 0.52867 & 2.32990 & 0.78707 \\
\hline $\mathrm{Br}$ & -2.43989 & -2.45687 & 0.42332 \\
\hline $\mathrm{Br}$ & -2.44151 & 2.65343 & -0.21727 \\
\hline $\mathrm{C}$ & 0.67210 & -2.76941 & -0.08886 \\
\hline $\mathrm{H}$ & 0.92769 & 1.03262 & -1.93173 \\
\hline C & 4.56450 & 0.11747 & 0.21733 \\
\hline C & 4.70542 & 1.61552 & 0.31731 \\
\hline C & 5.56568 & -0.72307 & 0.49646 \\
\hline $\mathrm{H}$ & -2.82461 & 0.11914 & -1.46810 \\
\hline $\mathrm{H}$ & -1.47015 & -1.89131 & -1.80738 \\
\hline $\mathrm{H}$ & 3.34148 & -1.47224 & -0.51797 \\
\hline $\mathrm{H}$ & 2.30898 & 0.50674 & 1.54236 \\
\hline $\mathrm{H}$ & 2.07322 & -1.23194 & 1.49540 \\
\hline $\mathrm{H}$ & -1.33460 & 1.26751 & 2.52558 \\
\hline $\mathrm{H}$ & 0.23193 & 0.48742 & 2.79961 \\
\hline $\mathrm{H}$ & -1.12201 & -0.44134 & 2.11320 \\
\hline $\mathrm{H}$ & -0.14595 & 3.07644 & 1.21067 \\
\hline $\mathrm{H}$ & 0.85377 & 2.68975 & -0.19418 \\
\hline $\mathrm{H}$ & 1.40471 & 2.27809 & 1.43776 \\
\hline $\mathrm{H}$ & 0.24987 & -3.55058 & -0.72978 \\
\hline $\mathrm{H}$ & 0.35263 & -2.97046 & 0.93659 \\
\hline $\mathrm{H}$ & 1.75971 & -2.84347 & -0.14679 \\
\hline $\mathrm{H}$ & 3.97380 & 2.05141 & 1.01024 \\
\hline $\mathrm{H}$ & 4.52865 & 2.08254 & -0.65775 \\
\hline $\mathrm{H}$ & 5.70506 & 1.89358 & 0.66365 \\
\hline $\mathrm{H}$ & 6.53126 & -0.36551 & 0.84437 \\
\hline $\mathrm{H}$ & 5.45784 & -1.79977 & 0.38831 \\
\hline
\end{tabular}

\section{Conformer 3}

Energy: -5876.82207 Hartree (Rel: $0.9 \mathrm{kcal} / \mathrm{mol}$ ) XYZ coordinates for conf 3 :

$\begin{array}{lrrr}\text { C } & -1.89220 & -0.00202 & -0.93371 \\ \text { C } & -1.26803 & -1.34769 & -0.89553 \\ \text { C } & 0.22887 & -1.37718 & -0.54188 \\ \text { C } & 0.78137 & -0.06249 & 0.13049 \\ \text { C } & -0.22560 & 0.97228 & 0.72470 \\ \text { C } & -1.43811 & 1.02252 & -0.20394 \\ \text { O } & 0.91139 & -1.02680 & -1.78856 \\ \text { C } & 1.34612 & 0.25245 & -1.27770 \\ \text { O } & 2.71951 & 0.41528 & -1.20800 \\ \text { C } & 3.20609 & -0.35082 & -0.08071 \\ \text { C } & 2.06635 & -0.27883 & 0.96191 \\ \text { C } & -0.71144 & 0.54056 & 2.13129 \\ \text { C } & 0.45741 & 2.35595 & 0.83146 \\ \text { Br } & -2.43065 & -2.47447 & 0.36169 \\ \text { Br } & -2.49207 & 2.63501 & -0.24807 \\ \text { C } & 0.69934 & -2.73830 & -0.04652 \\ \text { H } & 0.94091 & 1.07179 & -1.87549 \\ \text { C } & 4.56877 & 0.17383 & 0.31757 \\ \text { C } & 5.51804 & 0.42894 & -0.82724\end{array}$
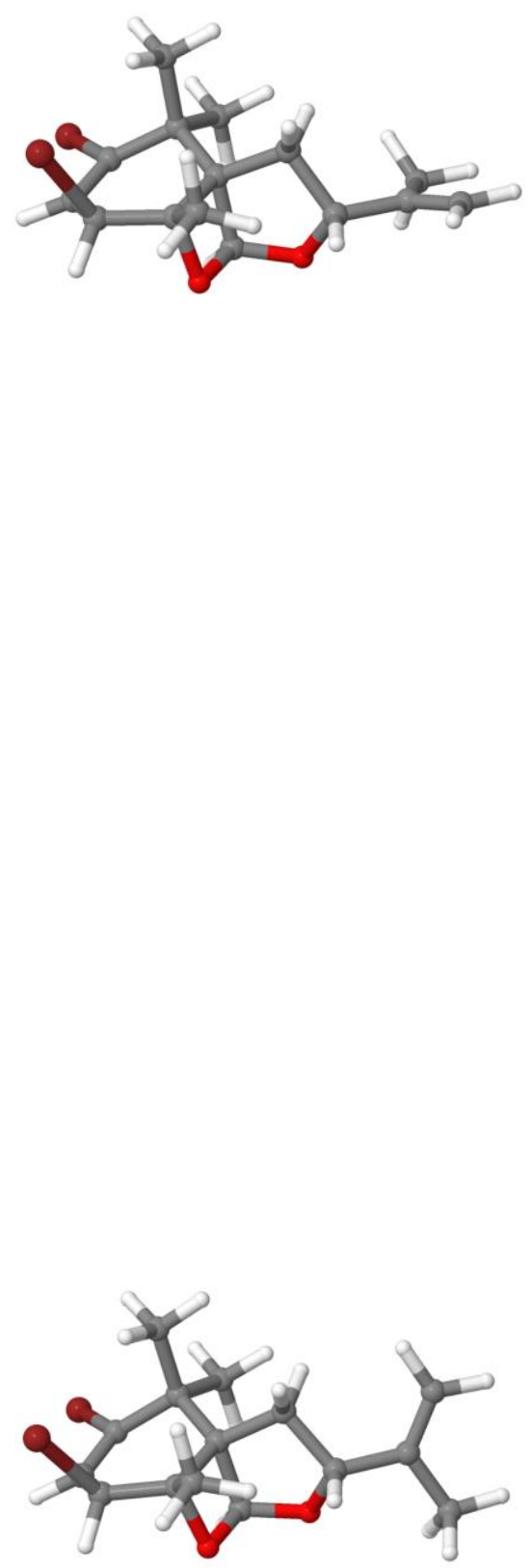


$\begin{array}{rrr}4.93085 & 0.33131 & 1.59458 \\ -2.79472 & 0.10368 & -1.52587 \\ -1.39862 & -1.88400 & -1.83506 \\ 3.33426 & -1.38903 & -0.42285 \\ 2.23924 & 0.57173 & 1.62213 \\ 2.02465 & -1.17477 & 1.58565 \\ -1.44486 & 1.25884 & 2.51293 \\ 0.12801 & 0.51132 & 2.83502 \\ -1.18507 & -0.44521 & 2.10734 \\ -0.23707 & 3.09020 & 1.24443 \\ 0.79642 & 2.72481 & -0.14162 \\ 1.32233 & 2.31541 & 1.49759 \\ 0.30698 & -3.52524 & -0.69921 \\ 0.35180 & -2.94333 & 0.96896 \\ 1.78915 & -2.79778 & -0.07132 \\ 5.12187 & 1.20124 & -1.49516 \\ 5.64946 & -0.47438 & -1.43819 \\ 6.50042 & 0.74473 & -0.46371 \\ 5.93482 & 0.65584 & 1.85546 \\ 4.26119 & 0.13414 & 2.42637\end{array}$


laureacetal E, Fig.1<smiles>C=C(C)[C@@H]1C[C@@]23[C@@H](O[C@H]2C(Br)=C[C@H]1Cl)OC3(C)C</smiles>

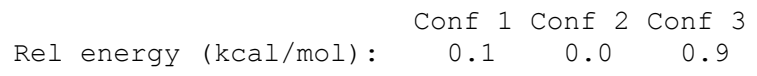

\begin{tabular}{|c|c|c|c|c|c|c|c|c|}
\hline C-nom & iGau & Exp & Calc & $\operatorname{diff}$ & 1 & 2 & 3 & \\
\hline$C-C$ & 18 & 141.80 & 142.34 & 0.54 & {$[140.69$} & 143.07 & 145.14 & ] \\
\hline $\mathrm{C}-\mathrm{C}-\mathrm{Br}$ & 6 & 138.70 & 140.78 & 2.08 & [ 140.83 & 140.70 & 140.91 & ] \\
\hline $\mathrm{C}-\mathrm{C}$ & 3 & 80.10 & 80.74 & 0.64 & {$\left[\begin{array}{ll}{[} & 80.71\end{array}\right.$} & 80.77 & 80.72 & ] \\
\hline$C-C$ & 4 & 62.40 & 63.56 & 1.16 & 63.65 & 63.42 & 63.80 & ] \\
\hline $\mathrm{C}-\mathrm{C}$ & 5 & 39.50 & 41.50 & 2.00 & 41.33 & 41.63 & 41.53 & ] \\
\hline $\mathrm{C}-\mathrm{CH}$ & 1 & 127.80 & 128.82 & 1.02 & [ 128.74 & 128.91 & 128.76 & ] \\
\hline $\mathrm{C}-\mathrm{CH}$ & 8 & 104.80 & 105.87 & 1.07 & [ 105.68 & 106.20 & 105.10 & ] \\
\hline $\mathrm{C}-\mathrm{CH}$ & 10 & 83.80 & 84.46 & 0.66 & {$\left[\begin{array}{ll}{[} & 83.00\end{array}\right.$} & 85.98 & 83.12 & ] \\
\hline $\mathrm{C}-\mathrm{CH}-\mathrm{Cl}$ & 2 & 59.40 & 61.27 & 1.87 & 61.25 & 61.27 & 61.34 & ] \\
\hline $\mathrm{C}-\mathrm{CH} 2$ & 20 & 111.20 & 112.65 & 1.45 & [ 111.08 & 114.46 & 110.46 & ] \\
\hline $\mathrm{C}-\mathrm{CH} 2$ & 11 & 31.90 & 34.36 & 2.46 & {$\left[\begin{array}{ll}35.55 \\
\end{array}\right.$} & 33.85 & 32.31 & \\
\hline $\mathrm{C}-\mathrm{CH} 3$ & 16 & 23.40 & 24.01 & 0.61 & 24.08 & 23.97 & 23.95 & \\
\hline $\mathrm{C}-\mathrm{CH} 3$ & 12 & 22.80 & 23.38 & 0.58 & 23.29 & 23.48 & 23.30 & \\
\hline $\mathrm{C}-\mathrm{CH} 3$ & 13 & 22.50 & 23.03 & 0.53 & 22.89 & 23.19 & 22.85 & ] \\
\hline $\mathrm{C}-\mathrm{CH} 3$ & 19 & 16.80 & 18.44 & 1.64 & 19.94 & 16.58 & 21.05 & \\
\hline
\end{tabular}

\section{Conformer 1} Energy: -3765.61091 Hartree (Rel: $0.1 \mathrm{kcal} / \mathrm{mol}$ )
XYZ coordinates for conf 1:

$\begin{array}{lrrr}\text { C } & 2.10184 & 0.74364 & -0.74686 \\ \text { C } & 1.21729 & 1.94165 & -0.68199 \\ \text { C } & -0.27780 & 1.63807 & -0.46544 \\ \text { C } & -0.59828 & 0.20132 & 0.10060 \\ \text { C } & 0.55555 & -0.65064 & 0.71743 \\ \text { C } & 1.81463 & -0.39458 & -0.10945 \\ \text { O } & -0.77724 & 1.23513 & -1.77788 \\ \text { C } & -0.99333 & -0.12940 & -1.36045 \\ \text { O } & -2.31252 & -0.55155 & -1.40971 \\ \text { C } & -3.01826 & -0.00693 & -0.28598 \\ \text { C } & -1.94968 & 0.11210 & 0.84207 \\ \text { C } & 0.84311 & -0.23341 & 2.18131 \\ \text { C } & 0.16223 & -2.14633 & 0.68900 \\ \text { Cl } & 1.93597 & 3.07423 & 0.61970 \\ \text { Br } & 3.16989 & -1.76610 & -0.15449 \\ \text { C } & -1.05193 & 2.84516 & 0.04855 \\ \text { H } & -0.39901 & -0.82024 & -1.96226 \\ \text { C } & -4.20547 & -0.86827 & 0.07894 \\ \text { C } & -5.12773 & -0.27293 & 1.11497 \\ \text { C } & -4.41467 & -2.06245 & -0.48030 \\ \text { H } & 3.04532 & 0.85931 & -1.26923 \\ \text { H } & 1.29445 & 2.54003 & -1.59123 \\ \text { H } & -3.38165 & 0.99588 & -0.55708 \\ \text { H } & -1.99343 & -0.78182 & 1.46698 \\ \text { H } & -2.13466 & 0.97101 & 1.49109 \\ \text { H } & 1.69144 & -0.80319 & 2.57515 \\ \text { H } & -0.02464 & -0.44086 & 2.81739 \\ \text { H } & 1.08694 & 0.83017 & 2.25555 \\ \text { H } & 0.94337 & -2.75280 & 1.15200 \\ \text { H } & 0.01421 & -2.51406 & -0.33101 \\ \text { H } & -0.76092 & -2.31934 & 1.24698 \\ \text { H } & -0.78922 & 3.72982 & -0.54123 \\ & & & \end{array}$

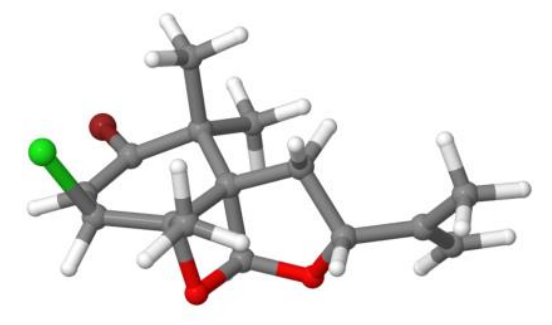




$\begin{array}{rrrr}\mathrm{H} & -0.82299 & 3.06116 & 1.09522 \\ \mathrm{H} & -2.12656 & 2.68319 & -0.05741 \\ \mathrm{H} & -5.53130 & 0.69268 & 0.77988 \\ \mathrm{H} & -4.60779 & -0.08350 & 2.06420 \\ \mathrm{H} & -5.97070 & -0.93843 & 1.32171 \\ \mathrm{H} & -5.27302 & -2.66919 & -0.20354 \\ \mathrm{H} & -3.74451 & -2.45873 & -1.23474\end{array}$

\section{Conformer 2}

Energy: -3765.61108 Hartree (Rel: $0.0 \mathrm{kcal} / \mathrm{mol}$ ) XYZ coordinates for conf 2 :

\begin{tabular}{|c|c|c|c|}
\hline $\mathrm{C}$ & 2.08252 & 0.75534 & -0.75572 \\
\hline C & 1.21389 & 1.96103 & -0.64218 \\
\hline C & -0.27945 & 1.66885 & -0.39790 \\
\hline C & -0.60472 & 0.22260 & 0.14111 \\
\hline $\mathrm{C}$ & 0.55515 & -0.65029 & 0.71683 \\
\hline C & 1.79714 & -0.39325 & -0.13649 \\
\hline 0 & -0.81019 & 1.30249 & -1.70898 \\
\hline $\mathrm{C}$ & -1.03286 & -0.06948 & -1.32062 \\
\hline 0 & -2.35850 & -0.47362 & -1.35318 \\
\hline C & -3.03504 & 0.07909 & -0.20513 \\
\hline $\mathrm{C}$ & -1.94503 & 0.12933 & 0.90339 \\
\hline C & 0.88128 & -0.25822 & 2.17971 \\
\hline C & 0.14847 & -2.14167 & 0.67876 \\
\hline $\mathrm{Cl}$ & 1.97758 & 3.05667 & 0.66553 \\
\hline $\mathrm{Br}$ & 3.13502 & -1.77820 & -0.24635 \\
\hline $\mathrm{C}$ & -1.02542 & 2.87340 & 0.16200 \\
\hline $\mathrm{H}$ & -0.45876 & -0.75287 & -1.95018 \\
\hline C & -4.24810 & -0.76030 & 0.12715 \\
\hline C & -4.08018 & -2.25889 & 0.11560 \\
\hline C & -5.40644 & -0.16506 & 0.42833 \\
\hline $\mathrm{H}$ & 3.01396 & 0.87173 & -1.29908 \\
\hline $\mathrm{H}$ & 1.27573 & 2.57882 & -1.53951 \\
\hline $\mathrm{H}$ & -3.36259 & 1.09624 & -0.45035 \\
\hline $\mathrm{H}$ & -1.98899 & -0.78139 & 1.50306 \\
\hline $\mathrm{H}$ & -2.11292 & 0.96728 & 1.58374 \\
\hline $\mathrm{H}$ & 1.72918 & -0.84631 & 2.54685 \\
\hline $\mathrm{H}$ & 0.02394 & -0.46131 & 2.83127 \\
\hline $\mathrm{H}$ & 1.14310 & 0.80017 & 2.26386 \\
\hline $\mathrm{H}$ & 0.94814 & -2.76357 & 1.08601 \\
\hline $\mathrm{H}$ & -0.05988 & -2.48761 & -0.33847 \\
\hline $\mathrm{H}$ & -0.74184 & -2.31792 & 1.28690 \\
\hline $\mathrm{H}$ & -0.76531 & 3.76799 & -0.41379 \\
\hline $\mathrm{H}$ & -0.76777 & 3.06219 & 1.20721 \\
\hline $\mathrm{H}$ & -2.10432 & 2.72855 & 0.08050 \\
\hline $\mathrm{H}$ & -3.28920 & -2.58901 & 0.80178 \\
\hline $\mathrm{H}$ & -3.79113 & -2.60379 & -0.88318 \\
\hline $\mathrm{H}$ & -5.00840 & -2.76018 & 0.40486 \\
\hline $\mathrm{H}$ & -6.28490 & -0.73736 & 0.71470 \\
\hline & -5.52047 & 0.91607 & 0.40019 \\
\hline
\end{tabular}

\section{Conformer 3}

Energy: -3765.60970 Hartree (Rel: $0.9 \mathrm{kcal} / \mathrm{mol}$ ) XYZ coordinates for conf 3 :

$\begin{array}{lrrr}\text { C } & 2.07733 & 0.73103 & -0.79804 \\ \text { C } & 1.20936 & 1.93564 & -0.66856 \\ \text { C } & -0.27484 & 1.64405 & -0.37245 \\ \text { C } & -0.57892 & 0.19934 & 0.18084 \\ \text { C } & 0.59663 & -0.67255 & 0.72449 \\ \text { C } & 1.81439 & -0.41362 & -0.16168 \\ \text { O } & -0.85360 & 1.27334 & -1.66250 \\ \text { C } & -1.05160 & -0.10058 & -1.26404 \\ \text { O } & -2.37159 & -0.51958 & -1.24915 \\ \text { C } & -3.01078 & 0.04815 & -0.08138 \\ \text { C } & -1.89411 & 0.10418 & 0.98699 \\ \text { C } & 0.96111 & -0.28180 & 2.17853 \\ \text { C } & 0.18987 & -2.16462 & 0.69195 \\ \text { C1 } & 2.01351 & 3.04234 & 0.60566 \\ \text { Br } & 3.15632 & -1.79249 & -0.29388 \\ \text { C } & -1.00134 & 2.84952 & 0.21068 \\ \text { H } & -0.49183 & -0.78047 & -1.90996\end{array}$

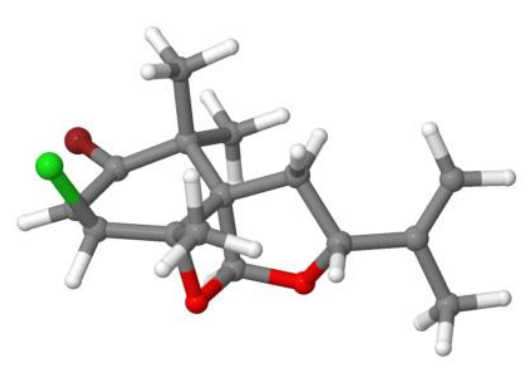




$\begin{array}{lrrr}\text { C } & -4.25762 & -0.75057 & 0.23168 \\ \text { C } & -5.12672 & -1.07449 & -0.95860 \\ \text { C } & -4.60182 & -1.08419 & 1.47941 \\ \text { H } & 2.99356 & 0.84689 & -1.36684 \\ \text { H } & 1.24184 & 2.54637 & -1.57227 \\ \text { H } & -3.32613 & 1.06907 & -0.34579 \\ \text { H } & -1.91435 & -0.81373 & 1.57574 \\ \text { H } & -2.02982 & 0.93921 & 1.67822 \\ \text { H } & 1.82090 & -0.86709 & 2.52156 \\ \text { H } & 0.12272 & -0.48915 & 2.85294 \\ \text { H } & 1.22123 & 0.77764 & 2.25681 \\ \text { H } & 0.99497 & -2.78568 & 1.08976 \\ \text { H } & -0.02882 & -2.51011 & -0.32317 \\ \text { H } & -0.69477 & -2.34293 & 1.30759 \\ \text { H } & -0.76396 & 3.74275 & -0.37687 \\ \text { H } & -0.70612 & 3.04216 & 1.24517 \\ \text { H } & -2.08227 & 2.70254 & 0.16956 \\ \text { H } & -4.58380 & -1.69779 & -1.67704 \\ \text { H } & -5.41683 & -0.16072 & -1.49481 \\ \text { H } & -6.03765 & -1.59762 & -0.65282 \\ \text { H } & -5.53141 & -1.60992 & 1.68145 \\ \text { H } & -3.99236 & -0.84124 & 2.34469\end{array}$


compositacin D, Fig.1<smiles>C[C@H]1C(Br)=C[C@@H](O)[C@]2(C)C[C@H]3CC(C)(O3)C12C</smiles>

Rel energy (kcal/mol): 0.0

\begin{tabular}{|c|c|c|c|c|c|}
\hline C-nom & iGau & $\operatorname{Exp}$ & Calc & $\operatorname{diff}$ & 1 \\
\hline $\mathrm{C} 1-\mathrm{CH} 2$ & 32 & 39.10 & 39.85 & 0.75 & 39.85 \\
\hline $\mathrm{C} 2-\mathrm{CH}-\mathrm{Br}$ & 29 & 58.50 & 60.12 & 1.62 & 60.12 \\
\hline $\mathrm{C} 3-\mathrm{C}$ & 24 & 85.20 & 85.12 & -0.08 & 85.12 \\
\hline $\mathrm{C} 4-\mathrm{CH} 2$ & 23 & 40.80 & 42.86 & 2.06 & 42.86 \\
\hline $\mathrm{C} 5-\mathrm{CH}$ & 14 & 80.90 & 80.05 & -0.85 & 80.05 \\
\hline C6-C & 7 & 51.20 & 53.02 & 1.82 & 53.02 \\
\hline $\mathrm{C} 7-\mathrm{C}$ & 6 & 59.50 & 57.93 & -1.57 & 57.93 \\
\hline $\mathrm{C} 8-\mathrm{CH}$ & 5 & 56.30 & 54.85 & -1.45 & {$[\quad 54.85$} \\
\hline $\mathrm{C} 9-\mathrm{CH}$ & 4 & 125.00 & 125.47 & 0.47 & {$[125.47$} \\
\hline $\mathrm{C} 10-\mathrm{C}-\mathrm{Br}$ & 2 & 144.20 & 143.70 & -0.50 & 143.70 \\
\hline $\mathrm{C} 11-\mathrm{C}$ & 8 & 45.60 & 47.11 & 1.51 & 47.11 \\
\hline $\mathrm{C} 12-\mathrm{CH} 3$ & 15 & 24.10 & 22.97 & -1.13 & 22.97 \\
\hline $\mathrm{C} 13-\mathrm{CH} 3$ & 16 & 24.40 & 23.93 & -0.47 & 23.93 \\
\hline $\mathrm{C} 14-\mathrm{CH} 3$ & 10 & 25.70 & 25.51 & -0.19 & 25.51 \\
\hline $\mathrm{C} 15-\mathrm{CH} 3$ & 27 & 26.80 & 24.50 & -2.30 & 24.50 \\
\hline
\end{tabular}

13C chem shifts: RMSD=1.31ppm (MAE=1.12) $\mathrm{N}=15 \quad\left\{\begin{array}{l}-2.30 \\ 2.06\end{array}\right\}$

\section{Conformer 1}

Energy: -5876.78962 Hartree (Rel: $0.0 \mathrm{kcal} / \mathrm{mol}$ ) XYZ coordinates for conf 1 :

\begin{tabular}{|c|c|c|c|}
\hline $\mathrm{Br}$ & -4.04970 & -1.17241 & 0.26134 \\
\hline $\mathrm{C}$ & -2.61736 & 0.06886 & -0.10730 \\
\hline 0 & -0.90248 & 2.73091 & 0.68943 \\
\hline $\mathrm{C}$ & -2.89972 & 1.36350 & 0.05071 \\
\hline $\mathrm{C}$ & -1.88711 & 2.38165 & -0.30454 \\
\hline C & -0.48143 & 1.98130 & -0.48112 \\
\hline C & -0.10257 & 0.49310 & -0.18815 \\
\hline $\mathrm{C}$ & -1.28822 & -0.48000 & -0.65039 \\
\hline $\mathrm{H}$ & -3.87078 & 1.69303 & 0.40607 \\
\hline C & 0.35791 & 2.81120 & -1.43599 \\
\hline $\mathrm{H}$ & -2.26042 & 3.24094 & -0.86560 \\
\hline $\mathrm{H}$ & 0.30706 & 2.42714 & -2.46079 \\
\hline $\mathrm{H}$ & -0.01472 & 3.83963 & -1.43682 \\
\hline C & 0.16983 & 0.32965 & 1.33573 \\
\hline C & -1.05946 & -1.93120 & -0.16632 \\
\hline C & -1.41161 & -0.49824 & -2.19667 \\
\hline $\mathrm{H}$ & -0.53678 & -0.96857 & -2.65704 \\
\hline $\mathrm{H}$ & -1.81970 & -2.58644 & -0.59819 \\
\hline $\mathrm{H}$ & -1.11436 & -2.02259 & 0.92043 \\
\hline $\mathrm{H}$ & -0.08351 & -2.30675 & -0.47644 \\
\hline $\mathrm{H}$ & 1.41168 & 2.83966 & -1.13877 \\
\hline $\mathrm{H}$ & -0.74379 & 0.24233 & 1.92489 \\
\hline C & 1.27481 & 1.26238 & 1.85367 \\
\hline $\mathrm{C}$ & 2.20084 & 0.09355 & 1.43986 \\
\hline $\mathrm{H}$ & 1.23329 & 1.36758 & 2.94085 \\
\hline $\mathrm{H}$ & 1.41077 & 2.24290 & 1.39494 \\
\hline C & 3.34820 & -0.29849 & 2.34147 \\
\hline 0 & 1.06003 & -0.81562 & 1.54049 \\
\hline $\mathrm{C}$ & 2.50366 & 0.30691 & -0.05831 \\
\hline $\mathrm{H}$ & 2.93508 & 1.29770 & -0.20806 \\
\hline $\mathrm{Br}$ & 3.94189 & -0.88784 & -0.78870 \\
\hline $\mathrm{C}$ & 1.23129 & 0.06688 & -0.89719 \\
\hline $\mathrm{H}$ & 1.34383 & 0.53212 & -1.87766 \\
\hline $\mathrm{H}$ & 1.18763 & -1.00537 & -1.07145 \\
\hline $\mathrm{H}$ & 4.16593 & 0.42725 & 2.26499 \\
\hline $\mathrm{H}$ & 3.00246 & -0.33406 & 3.37898 \\
\hline
\end{tabular}

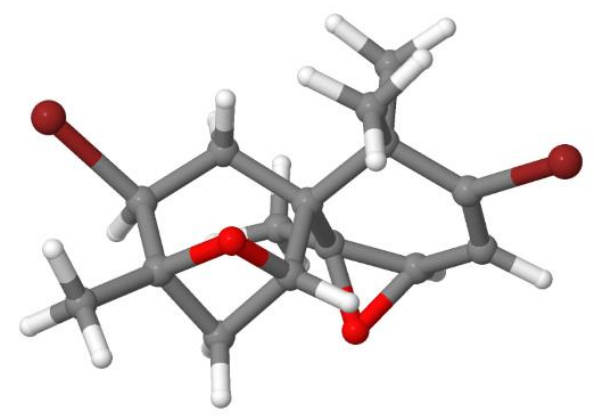




$\begin{array}{rrrr}\mathrm{H} & 3.74010 & -1.28106 & 2.06553 \\ \mathrm{H} & -2.29294 & -1.07646 & -2.49332 \\ \mathrm{H} & -1.52241 & 0.51103 & -2.60700\end{array}$


dictyoxetane, Fig.1

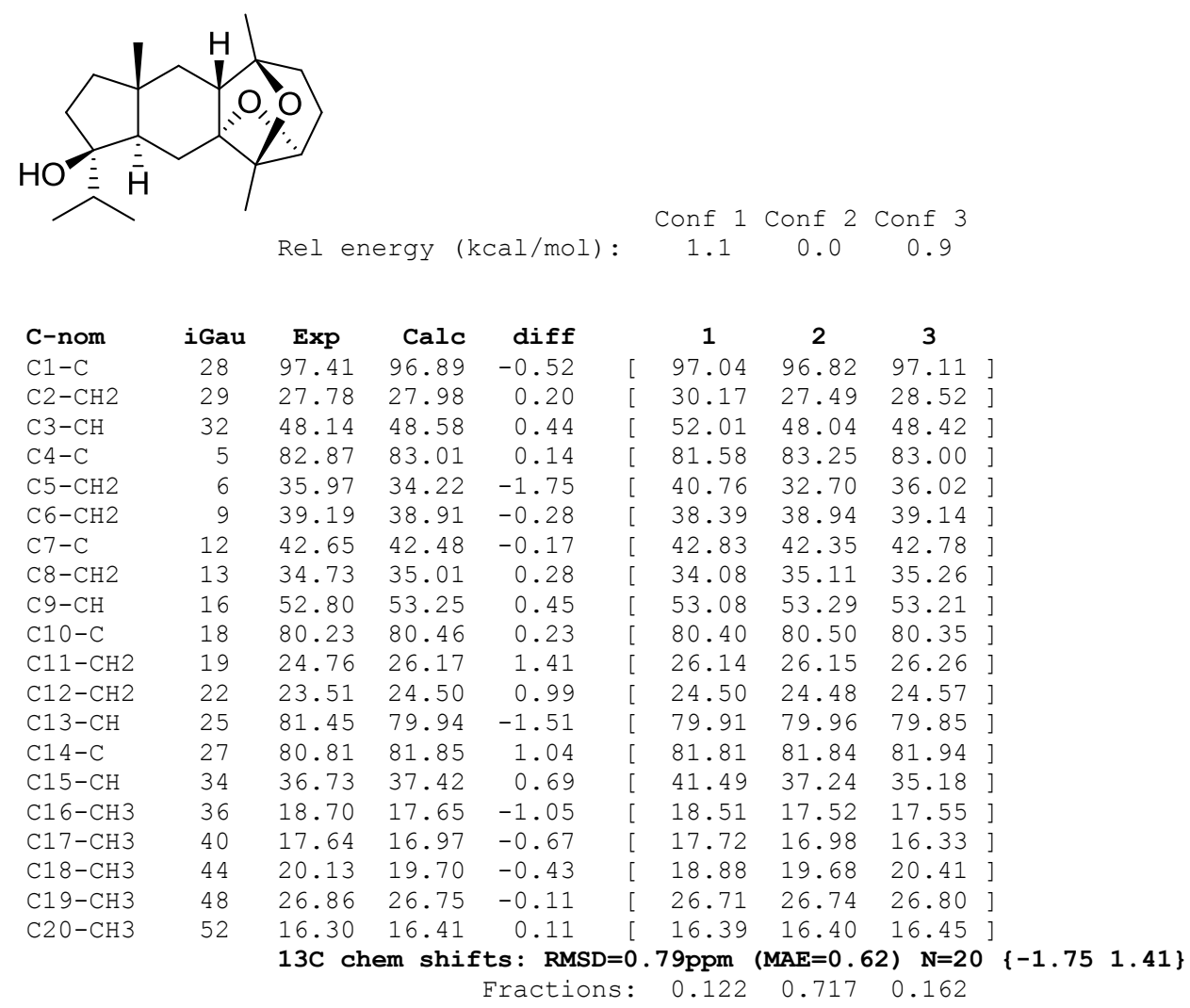

\section{Conformer 1}

Energy: -1007.03214 Hartree (Rel: $1.1 \mathrm{kcal} / \mathrm{mol}$ ) XYZ coordinates for conf 1 :

\begin{tabular}{|c|c|c|c|}
\hline & & & \\
\hline O & -3.26369 & 0.11327 & 1.47853 \\
\hline $\mathrm{H}$ & -2.93548 & -0.70405 & 1.88660 \\
\hline O & 3.31505 & -0.28218 & 0.90179 \\
\hline O & 1.02877 & -1.16352 & -1.03917 \\
\hline C & -2.86077 & 0.10060 & 0.09613 \\
\hline C & -3.30930 & 1.46923 & -0.51616 \\
\hline $\mathrm{H}$ & -4.01426 & 1.33473 & -1.34193 \\
\hline $\mathrm{H}$ & -3.83342 & 2.03408 & 0.26016 \\
\hline C & -2.02951 & 2.20313 & -0.98513 \\
\hline $\mathrm{H}$ & -1.80388 & 1.94187 & -2.02801 \\
\hline $\mathrm{H}$ & -2.13231 & 3.29371 & -0.93935 \\
\hline C & -0.92067 & 1.65852 & -0.06425 \\
\hline C & 0.52601 & 1.79769 & -0.61118 \\
\hline $\mathrm{H}$ & 0.84456 & 2.84922 & -0.59828 \\
\hline $\mathrm{H}$ & 0.54958 & 1.46049 & -1.65486 \\
\hline C & 1.45984 & 0.92952 & 0.24209 \\
\hline $\mathrm{H}$ & 1.39513 & 1.28022 & 1.28005 \\
\hline C & 2.99015 & 0.81539 & 0.00050 \\
\hline C & 3.39448 & 0.40267 & -1.44227 \\
\hline $\mathrm{H}$ & 2.70072 & 0.84958 & -2.16155 \\
\hline $\mathrm{H}$ & 4.38787 & 0.80527 & -1.67154 \\
\hline C & 3.41928 & -1.13275 & -1.61167 \\
\hline $\mathrm{H}$ & 3.31093 & -1.40605 & -2.66781 \\
\hline $\mathrm{H}$ & 4.38089 & -1.53483 & -1.26876 \\
\hline C & 2.31826 & -1.77756 & -0.78470 \\
\hline $\mathrm{H}$ & 2.26919 & -2.86531 & -0.92461 \\
\hline C & 2.31695 & -1.27757 & 0.68728 \\
\hline C & 1.00375 & -0.53423 & 0.28434 \\
\hline C & -0.39419 & -0.68411 & 0.87983 \\
\hline $\mathrm{H}$ & -0.67420 & -1.74153 & 0.90189 \\
\hline $\mathrm{H}$ & -0.42121 & -0.30884 & 1.90979 \\
\hline
\end{tabular}

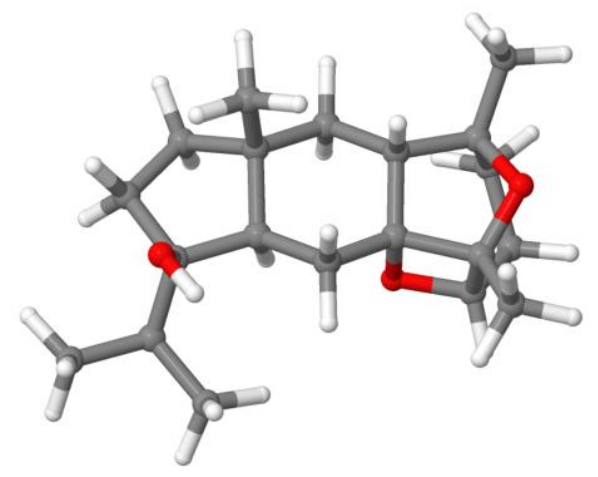




$\begin{array}{rrr}-1.31093 & 0.14584 & -0.04722 \\ -1.10655 & -0.21600 & -1.06455 \\ -3.53895 & -1.08732 & -0.65592 \\ -3.35988 & -0.90682 & -1.72626 \\ -5.05927 & -1.10278 & -0.42069 \\ -5.53063 & -1.88992 & -1.02066 \\ -5.52979 & -0.15127 & -0.68876 \\ -5.28632 & -1.29319 & 0.63320 \\ -2.95172 & -2.47233 & -0.33164 \\ -3.52772 & -3.25319 & -0.84165 \\ -3.00119 & -2.69627 & 0.74326 \\ -1.91099 & -2.56699 & -0.65501 \\ -1.04407 & 2.35587 & 1.31187 \\ -0.35296 & 1.95805 & 2.06087 \\ -2.04809 & 2.25538 & 1.72839 \\ -0.82057 & 3.42461 & 1.20013 \\ 3.76658 & 2.03605 & 0.47467 \\ 3.48448 & 2.30838 & 1.49715 \\ 3.57198 & 2.89255 & -0.18126 \\ 4.84244 & 1.83139 & 0.45732 \\ 2.35677 & -2.29176 & 1.80614 \\ 1.57946 & -3.05152 & 1.67040 \\ 3.32973 & -2.79576 & 1.82974 \\ 2.20551 & -1.80319 & 2.77458\end{array}$

Conformer 2

Energy: -1007.03382 Hartree (Rel: $0.0 \mathrm{kcal} / \mathrm{mol}$ ) XYZ coordinates for conf 2:

$\begin{array}{lrrr}\mathrm{O} & -3.18969 & 0.38100 & 1.60818 \\ \mathrm{H} & -2.84277 & -0.32408 & 2.17906 \\ \mathrm{O} & 3.33448 & -0.19062 & 0.91501 \\ \mathrm{O} & 1.03303 & -1.26894 & -0.90724 \\ \mathrm{C} & -2.83480 & 0.04626 & 0.24896 \\ \mathrm{C} & -3.33727 & 1.23311 & -0.63147 \\ \mathrm{H} & -3.94511 & 0.88656 & -1.47256 \\ \mathrm{H} & -3.98228 & 1.86276 & -0.01233 \\ \mathrm{C} & -2.08195 & 2.00444 & -1.13011 \\ \mathrm{H} & -1.82221 & 1.68084 & -2.14660 \\ \mathrm{H} & -2.24873 & 3.08702 & -1.16952 \\ \mathrm{C} & -0.94822 & 1.60711 & -0.15614 \\ \mathrm{C} & 0.48866 & 1.69620 & -0.74055 \\ \mathrm{H} & 0.79941 & 2.74478 & -0.84600 \\ \mathrm{H} & 0.49993 & 1.24749 & -1.74155 \\ \mathrm{C} & 1.44396 & 0.92942 & 0.18466 \\ \mathrm{H} & 1.37753 & 1.37483 & 1.18580 \\ \mathrm{C} & 2.97410 & 0.82397 & -0.06601 \\ \mathrm{C} & 3.36769 & 0.30389 & -1.47693 \\ \mathrm{H} & 2.66033 & 0.68482 & -2.22050 \\ \mathrm{H} & 4.35274 & 0.70055 & -1.74859 \\ \mathrm{C} & 3.41324 & -1.23959 & -1.52600 \\ \mathrm{H} & 3.28938 & -1.59654 & -2.55512 \\ \mathrm{H} & 4.38765 & -1.59954 & -1.17256 \\ \mathrm{C} & 2.33965 & -1.83555 & -0.62931 \\ \mathrm{H} & 2.31028 & -2.93177 & -0.68077 \\ \mathrm{C} & 2.35375 & -1.21876 & 0.79774 \\ \mathrm{C} & 1.02086 & -0.53455 & 0.36088 \\ \mathrm{C} & -0.36093 & -0.65578 & 0.99703 \\ \mathrm{H} & -0.64230 & -1.71360 & 1.07907 \\ \mathrm{H} & -0.36474 & -0.22318 & 2.00562 \\ \mathrm{C} & -1.30366 & 0.10205 & 0.04332 \\ \mathrm{H} & -1.12771 & -0.34435 & -0.94239 \\ \mathrm{C} & -3.47390 & -1.33473 & -0.08511 \\ \mathrm{H} & -3.06987 & -2.03910 & 0.66223 \\ \mathrm{C} & -3.10855 & -1.89481 & -1.47013 \\ \mathrm{H} & -3.65220 & -2.82982 & -1.65012 \\ \mathrm{H} & -2.04047 & -2.11231 & -1.56827 \\ \mathrm{H} & -3.38733 & -1.19932 & -2.27185 \\ \mathrm{C} & -5.00119 & -1.30691 & 0.10003 \\ \mathrm{H} & -5.41360 & -2.32109 & 0.04363 \\ \mathrm{H} & -5.48594 & -0.71243 & -0.68414 \\ \mathrm{H} & -5.27184 & -0.87365 & 1.06642 \\ & & & \end{array}$

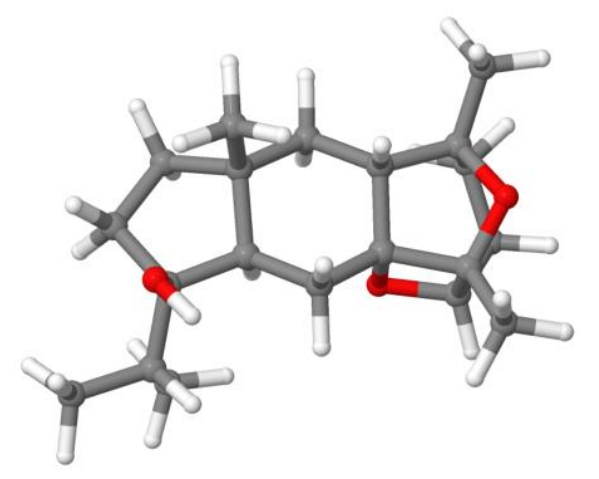




$\begin{array}{rrrr}\mathrm{C} & -1.05987 & 2.47576 & 1.11821 \\ \mathrm{H} & -0.41210 & 2.13115 & 1.93118 \\ \mathrm{H} & -2.07781 & 2.48398 & 1.51011 \\ \mathrm{H} & -0.76673 & 3.50712 & 0.88243 \\ \mathrm{C} & 3.73102 & 2.09436 & 0.29595 \\ \mathrm{H} & 3.45866 & 2.44139 & 1.29822 \\ \mathrm{H} & 3.50610 & 2.89098 & -0.42264 \\ \mathrm{H} & 4.81087 & 1.91202 & 0.27593 \\ \mathrm{C} & 2.43014 & -2.13988 & 1.99272 \\ \mathrm{H} & 1.66315 & -2.91940 & 1.93137 \\ \mathrm{H} & 3.41158 & -2.62553 & 2.03825 \\ \mathrm{H} & 2.28727 & -1.57816 & 2.92196\end{array}$

\section{Conformer 3}

Energy: -1007.03241 Hartree (Rel: $0.9 \mathrm{kcal} / \mathrm{mol}$ ) XYZ coordinates for conf 3 :

\begin{tabular}{|c|c|c|c|}
\hline 0 & -3.06092 & 0.31218 & 1.65197 \\
\hline $\mathrm{H}$ & -4.01659 & 0.41299 & 1.77890 \\
\hline 0 & 3.33461 & -0.19321 & 0.91442 \\
\hline 0 & 1.02870 & -1.26948 & -0.90267 \\
\hline C & -2.82701 & 0.04666 & 0.25580 \\
\hline $\mathrm{C}$ & -3.33378 & 1.23452 & -0.63748 \\
\hline $\mathrm{H}$ & -3.93737 & 0.88806 & -1.48245 \\
\hline $\mathrm{H}$ & -3.98157 & 1.88041 & -0.03441 \\
\hline $\mathrm{C}$ & -2.07684 & 2.00630 & -1.13328 \\
\hline $\mathrm{H}$ & -1.81682 & 1.67931 & -2.14845 \\
\hline $\mathrm{H}$ & -2.24383 & 3.08880 & -1.17664 \\
\hline $\mathrm{C}$ & -0.94541 & 1.61082 & -0.15642 \\
\hline C & 0.49115 & 1.69352 & -0.74294 \\
\hline $\mathrm{H}$ & 0.80391 & 2.74089 & -0.85510 \\
\hline $\mathrm{H}$ & 0.49987 & 1.23903 & -1.74142 \\
\hline C & 1.44554 & 0.92881 & 0.18475 \\
\hline $\mathrm{H}$ & 1.38098 & 1.37836 & 1.18408 \\
\hline C & 2.97497 & 0.81911 & -0.06830 \\
\hline $\mathrm{C}$ & 3.36542 & 0.29407 & -1.47850 \\
\hline $\mathrm{H}$ & 2.65781 & 0.67397 & -2.22242 \\
\hline $\mathrm{H}$ & 4.35095 & 0.68791 & -1.75286 \\
\hline $\mathrm{C}$ & 3.40773 & -1.24965 & -1.52256 \\
\hline $\mathrm{H}$ & 3.28230 & -1.60954 & -2.55057 \\
\hline $\mathrm{H}$ & 4.38171 & -1.61046 & -1.16875 \\
\hline $\mathrm{C}$ & 2.33270 & -1.83959 & -0.62334 \\
\hline $\mathrm{H}$ & 2.30028 & -2.93601 & -0.67164 \\
\hline $\mathrm{C}$ & 2.34996 & -1.21944 & 0.80209 \\
\hline C & 1.01787 & -0.53333 & 0.36684 \\
\hline C & -0.36209 & -0.65081 & 1.00520 \\
\hline $\mathrm{H}$ & -0.65101 & -1.70534 & 1.08766 \\
\hline $\mathrm{H}$ & -0.37855 & -0.22024 & 2.01200 \\
\hline $\mathrm{C}$ & -1.30160 & 0.10608 & 0.05009 \\
\hline $\mathrm{H}$ & -1.12951 & -0.33932 & -0.93644 \\
\hline C & -3.46010 & -1.33692 & -0.08655 \\
\hline $\mathrm{H}$ & -3.03909 & -2.03735 & 0.64807 \\
\hline $\mathrm{C}$ & -3.11737 & -1.86974 & -1.48857 \\
\hline $\mathrm{H}$ & -3.64353 & -2.81475 & -1.66869 \\
\hline $\mathrm{H}$ & -2.04758 & -2.06454 & -1.60927 \\
\hline $\mathrm{H}$ & -3.42567 & -1.17306 & -2.27838 \\
\hline $\mathrm{C}$ & -4.98804 & -1.33979 & 0.11273 \\
\hline $\mathrm{H}$ & -5.39044 & -2.34533 & -0.05371 \\
\hline $\mathrm{H}$ & -5.49209 & -0.66568 & -0.59092 \\
\hline $\mathrm{H}$ & -5.27899 & -1.05061 & 1.12976 \\
\hline C & -1.05265 & 2.48631 & 1.11302 \\
\hline $\mathrm{H}$ & -0.41224 & 2.13808 & 1.92929 \\
\hline $\mathrm{H}$ & -2.07113 & 2.50294 & 1.50340 \\
\hline $\mathrm{H}$ & -0.75262 & 3.51486 & 0.87323 \\
\hline $\mathrm{C}$ & 3.73569 & 2.08883 & 0.28854 \\
\hline $\mathrm{H}$ & 3.46613 & 2.43894 & 1.29050 \\
\hline $\mathrm{H}$ & 3.51105 & 2.88417 & -0.43168 \\
\hline $\mathrm{H}$ & 4.81515 & 1.90413 & 0.26671 \\
\hline $\mathrm{C}$ & 2.42593 & -2.13682 & 1.99980 \\
\hline $\mathrm{H}$ & 1.65483 & -2.91245 & 1.94310 \\
\hline $\mathrm{H}$ & 3.40548 & -2.62653 & 2.04479 \\
\hline $\mathrm{H}$ & 2.28660 & -1.57123 & 2.92712 \\
\hline
\end{tabular}

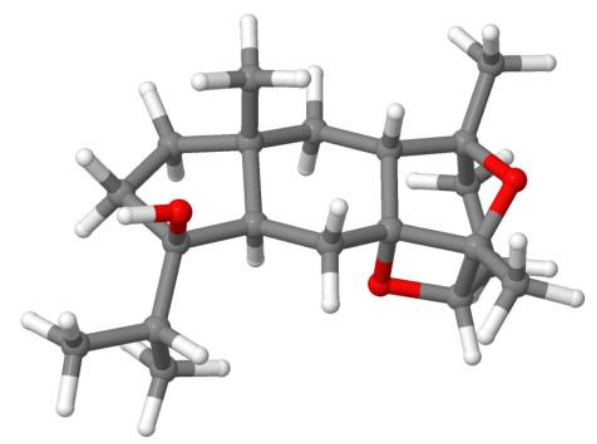


dichrocephone B, Fig.1

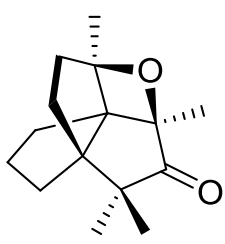

$$
\text { Rel energy }(\mathrm{kcal} / \mathrm{mol}): \begin{array}{cc}
\text { Conf } 1 & \text { Conf } 2 \\
0.0 & 0.0
\end{array}
$$

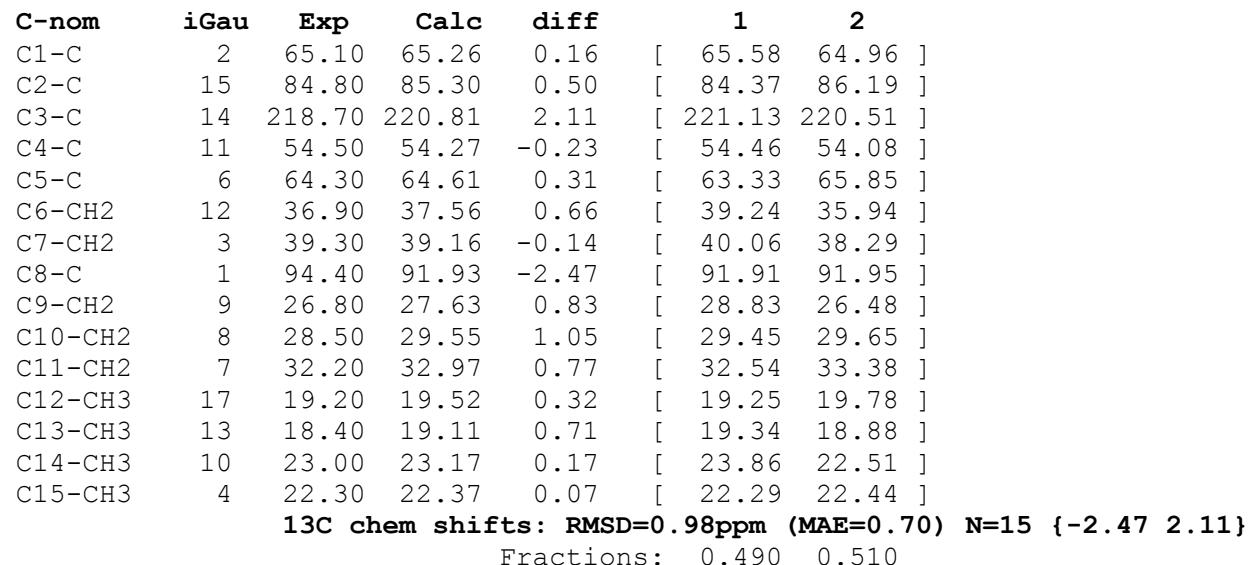

\section{Conformer 1}

Energy: -735.26067 Hartree (Rel: $0.0 \mathrm{kcal} / \mathrm{mol}$ ) XYZ coordinates for conf 1 :

$\begin{array}{rrrr}\mathrm{C} & 1.71886 & -0.40400 & 0.60562 \\ \mathrm{C} & 0.68923 & 0.07218 & -0.45636 \\ \mathrm{C} & 1.57355 & 0.51367 & 1.81739 \\ \mathrm{C} & 3.15034 & -0.70939 & 0.19917 \\ \mathrm{O} & 0.96000 & -1.65231 & 0.77335 \\ \mathrm{C} & -0.34533 & 0.98769 & 0.31557 \\ \mathrm{C} & -0.15162 & 2.40338 & -0.30542 \\ \mathrm{C} & 1.08780 & 2.31258 & -1.23047 \\ \mathrm{C} & 1.13094 & 0.84260 & -1.69702 \\ \mathrm{C} & -2.34886 & 0.53280 & -1.31056 \\ \mathrm{C} & -1.73897 & 0.29529 & 0.09856 \\ \mathrm{C} & 0.08916 & 0.90243 & 1.81438 \\ \mathrm{C} & -2.82597 & 0.61696 & 1.13237 \\ \mathrm{C} & -1.35453 & -1.19973 & 0.09075 \\ \mathrm{C} & 0.11104 & -1.35285 & -0.38199 \\ \mathrm{O} & -2.08434 & -2.12304 & 0.37786 \\ \mathrm{C} & 0.27195 & -2.37570 & -1.48896 \\ \mathrm{H} & 1.88340 & 0.02150 & 2.74671 \\ \mathrm{H} & 2.22064 & 1.39036 & 1.67605 \\ \mathrm{H} & 3.65149 & -1.26261 & 1.00183 \\ \mathrm{H} & 3.71642 & 0.21189 & 0.01670 \\ \mathrm{H} & 3.18959 & -1.32475 & -0.70522 \\ \mathrm{H} & -0.02463 & 3.17071 & 0.46585 \\ \mathrm{H} & -1.02667 & 2.70018 & -0.89153 \\ \mathrm{H} & 1.04863 & 3.02399 & -2.06267 \\ \mathrm{H} & 1.99799 & 2.53890 & -0.66029 \\ \mathrm{H} & 2.12052 & 0.54217 & -2.05902 \\ \mathrm{H} & -3.43069 & 0.68617 & -2.52742 \\ \mathrm{H} & -1.64729 & -0.12289 & -1.44332 \\ \mathrm{H} & -2.69486 & 0.32696 & -2.12491 \\ \mathrm{H} & -0.11313 & 1.56616 & -1.41841 \\ \mathrm{H} & -0.46500 & 1.83193 & 2.35709 \\ \mathrm{H} & 0.10919 & 2.32413 \\ \mathrm{H} & -0.02062 & 0.95877 \\ & & & \end{array}$

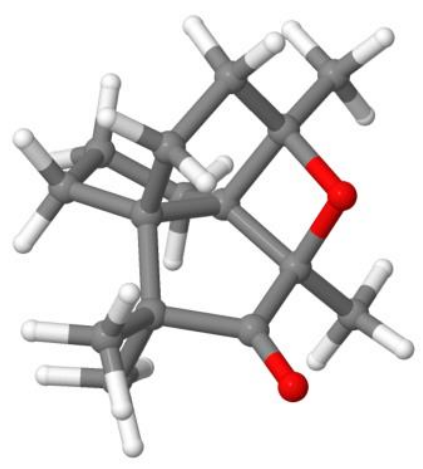




$\begin{array}{rrrr}\text { H } & -3.14208 & 1.66363 & 1.04822 \\ \mathrm{H} & -2.49720 & 0.44236 & 2.15956 \\ \mathrm{H} & -0.11631 & -3.33946 & -1.14318 \\ \mathrm{H} & 1.32411 & -2.49639 & -1.76383 \\ \mathrm{H} & -0.29240 & -2.08397 & -2.38301\end{array}$

\section{Conformer 2}

Energy: -735.26071 Hartree (Rel: $0.0 \mathrm{kcal} / \mathrm{mol}$ ) XYZ coordinates for conf 2:

$\begin{array}{lrrr}\text { C } & 1.82329 & -0.17560 & 0.55750 \\ \mathrm{C} & 0.69589 & 0.22780 & -0.43240 \\ \mathrm{C} & 1.58820 & 0.61320 & 1.84440 \\ \mathrm{C} & 3.26859 & -0.23320 & 0.09310 \\ \mathrm{O} & 1.26149 & -1.53120 & 0.64280 \\ \mathrm{C} & -0.42920 & 0.89911 & 0.44550 \\ \mathrm{C} & -0.45750 & 2.36591 & -0.04140 \\ \mathrm{C} & 0.03460 & 2.36711 & -1.50690 \\ \mathrm{C} & 0.95650 & 1.12370 & -1.65290 \\ \mathrm{C} & -2.38510 & 0.30401 & -1.18320 \\ \mathrm{C} & -1.71361 & 0.03741 & 0.19170 \\ \mathrm{C} & 0.06220 & 0.75840 & 1.92020 \\ \mathrm{C} & -2.80441 & 0.12281 & 1.26650 \\ \mathrm{C} & -1.12111 & -1.37919 & 0.05440 \\ \mathrm{C} & 0.33469 & -1.27350 & -0.45970 \\ \mathrm{O} & -1.70071 & -2.41949 & 0.27760 \\ \mathrm{C} & 0.60549 & -2.17200 & -1.64990 \\ \mathrm{H} & 2.01409 & 0.11220 & 2.72150 \\ \mathrm{H} & 2.08090 & 1.59110 & 1.75200 \\ \mathrm{H} & 3.86739 & -0.78700 & 0.82530 \\ \mathrm{H} & 3.69610 & 0.77180 & -0.00330 \\ \mathrm{H} & 3.36229 & -0.74320 & -0.87080 \\ \mathrm{H} & 0.23430 & 2.95300 & 0.57680 \\ \mathrm{H} & -1.44280 & 2.83401 & 0.07210 \\ \mathrm{H} & -0.81050 & 2.29721 & -2.19770 \\ \mathrm{H} & 0.56010 & 3.29570 & -1.75510 \\ \mathrm{H} & 2.01030 & 1.42420 & -1.67800 \\ \mathrm{H} & 0.77180 & 0.59520 & -2.59570 \\ \mathrm{H} & -3.21961 & -0.39219 & -1.31810 \\ \mathrm{H} & -1.70331 & 0.16941 & -2.02920 \\ \mathrm{H} & -2.78480 & 1.32281 & -1.22770 \\ \mathrm{H} & -0.25970 & 1.60000 & 2.54340 \\ \mathrm{H} & -0.34151 & -0.15039 & 2.37540 \\ \mathrm{H} & -3.57471 & -0.62939 & 1.06850 \\ \mathrm{H} & -3.27940 & 1.11121 & 1.26000 \\ \mathrm{H} & -2.42031 & -0.06279 & 2.27230 \\ \mathrm{H} & 0.39109 & -3.20810 & -1.36770 \\ \mathrm{H} & 1.64979 & -2.10160 & -1.96910 \\ \mathrm{H} & -0.03881 & -1.91349 & -2.49900 \\ & & & \end{array}$

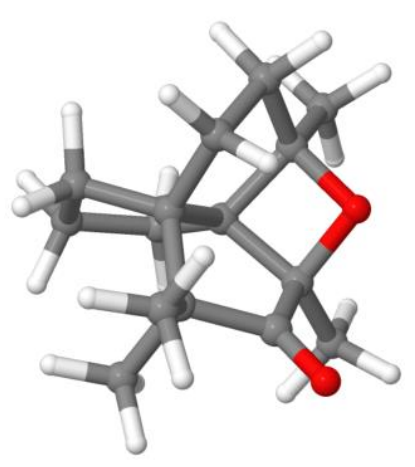


mitrephorone A, Fig.1

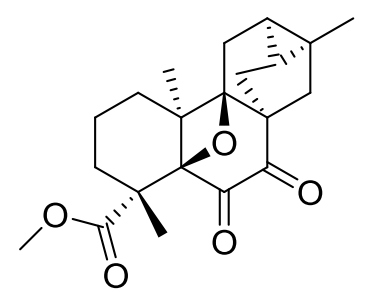

$$
\text { Rel energy (kcal/mol): } \begin{array}{ccc}
\text { Conf } 1 & \text { Conf 2 } \\
0.0 & 1.2
\end{array}
$$

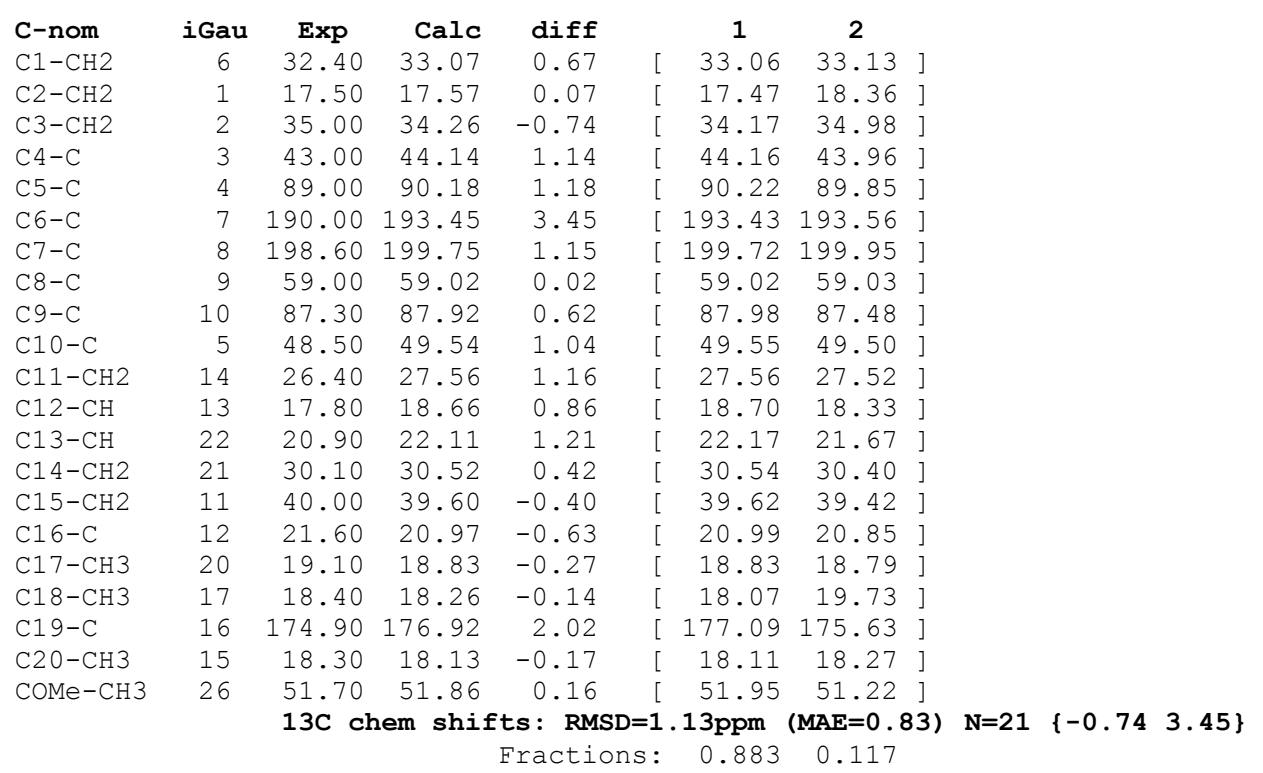

\section{Conformer 1}

Energy: -1191.94008 Hartree (Rel: $0.0 \mathrm{kcal} / \mathrm{mol})$ XYZ coordinates for conf 1 :

\begin{tabular}{|c|c|c|c|}
\hline C & -2.04624 & 2.61519 & -0.47281 \\
\hline $\mathrm{C}$ & -2.42478 & 1.95266 & 0.85475 \\
\hline $\mathrm{C}$ & -2.17740 & 0.41497 & 0.87239 \\
\hline $\mathrm{C}$ & -0.79357 & 0.08112 & 0.26953 \\
\hline $\mathrm{C}$ & -0.12526 & 0.93909 & -0.85905 \\
\hline $\mathrm{C}$ & -0.54778 & 2.42718 & -0.73553 \\
\hline C & -0.59935 & -1.42095 & 0.00758 \\
\hline $\mathrm{C}$ & 0.83691 & -1.79299 & -0.46310 \\
\hline C & 1.87692 & -0.69830 & -0.31956 \\
\hline $\mathrm{C}$ & 1.17989 & 0.65182 & -0.02229 \\
\hline C & 2.81391 & -1.02476 & 0.88945 \\
\hline C & 4.02862 & -0.13842 & 0.64813 \\
\hline C & 3.66978 & 1.24138 & 0.14046 \\
\hline C & 2.23486 & 1.75283 & 0.19011 \\
\hline C & -0.24612 & 0.47922 & -2.31362 \\
\hline C & -3.24587 & -0.20475 & -0.03517 \\
\hline C & -2.26247 & -0.09794 & 2.32338 \\
\hline 0 & -1.43819 & -2.27650 & 0.18131 \\
\hline 0 & 1.06610 & -2.89872 & -0.90513 \\
\hline C & 5.27177 & -0.36895 & 1.47629 \\
\hline $\mathrm{C}$ & 2.88457 & -0.57091 & -1.49032 \\
\hline C & 4.05609 & 0.15133 & -0.84210 \\
\hline 0 & 0.32374 & 0.41594 & 1.15791 \\
\hline 0 & -4.32073 & -0.63612 & 0.64765 \\
\hline 0 & -3.18118 & -0.22815 & -1.25041 \\
\hline $\mathrm{C}$ & -5.37037 & -1.20445 & -0.15339 \\
\hline $\mathrm{H}$ & -2.27158 & 3.68778 & -0.42279 \\
\hline
\end{tabular}

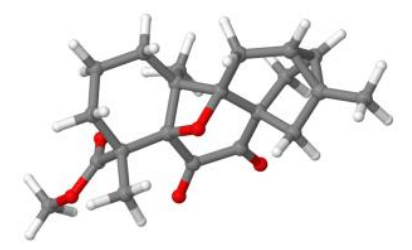




$\begin{array}{rrrr}\text { H } & -2.64451 & 2.20023 & -1.29085 \\ \mathrm{H} & -3.47679 & 2.13678 & 1.10537 \\ \mathrm{H} & -1.82699 & 2.39448 & 1.66267 \\ \mathrm{H} & -0.24803 & 2.95466 & -1.64968 \\ \mathrm{H} & -0.01157 & 2.90473 & 0.09061 \\ \mathrm{H} & 3.10148 & -2.08304 & 0.84555 \\ \mathrm{H} & 2.31190 & -0.84484 & 1.84564 \\ \mathrm{H} & 4.42093 & 2.01163 & 0.30738 \\ \mathrm{H} & 2.03543 & 2.23113 & 1.15698 \\ \mathrm{H} & 2.10958 & 2.53190 & -0.57217 \\ \mathrm{H} & -0.00988 & -0.57840 & -2.46734 \\ \mathrm{H} & -1.27295 & 0.62465 & -2.65861 \\ \mathrm{H} & 0.42230 & 1.06642 & -2.95421 \\ \mathrm{H} & -1.42493 & 0.30464 & 2.90021 \\ \mathrm{H} & -2.22911 & -1.18857 & 2.36685 \\ \mathrm{H} & -3.19664 & 0.23209 & 2.78588 \\ \mathrm{H} & 5.08335 & -0.17921 & 2.54089 \\ \mathrm{H} & 6.08680 & 0.29310 & 1.16140 \\ \mathrm{H} & 5.62535 & -1.40372 & 1.38064 \\ \mathrm{H} & 3.17382 & -1.57216 & -1.82911 \\ \mathrm{H} & 2.46233 & -0.03667 & -2.34859 \\ \mathrm{H} & 4.99542 & 0.23464 & -1.38321 \\ \mathrm{H} & -6.14379 & -1.50678 & 0.55319 \\ \mathrm{H} & -5.75921 & -0.46570 & -0.85939 \\ \mathrm{H} & -4.99531 & -2.06757 & -0.70875\end{array}$

\section{Conformer 2}

Energy: -1191.93817 Hartree (Rel: $1.2 \mathrm{kcal} / \mathrm{mol}$ ) XYZ coordinates for conf 2:

\begin{tabular}{|c|c|c|c|}
\hline C & -2.17964 & 2.46896 & -0.72596 \\
\hline C & -2.47517 & 2.05442 & 0.71804 \\
\hline $\mathrm{C}$ & -2.18307 & 0.55210 & 1.00832 \\
\hline $\mathrm{C}$ & -0.83874 & 0.12191 & 0.37939 \\
\hline $\mathrm{C}$ & -0.24406 & 0.78253 & -0.91552 \\
\hline $\mathrm{C}$ & -0.69063 & 2.26356 & -1.02654 \\
\hline C & -0.62684 & -1.40048 & 0.36439 \\
\hline C & 0.78771 & -1.83145 & -0.12545 \\
\hline $\mathrm{C}$ & 1.81523 & -0.72096 & -0.22176 \\
\hline $\mathrm{C}$ & 1.10858 & 0.65292 & -0.11729 \\
\hline C & 2.81729 & -0.82886 & 0.97459 \\
\hline C & 4.00115 & 0.01705 & 0.52455 \\
\hline $\mathrm{C}$ & 3.59139 & 1.28747 & -0.18847 \\
\hline C & 2.15171 & 1.78546 & -0.15237 \\
\hline C & -0.42054 & 0.08820 & -2.26869 \\
\hline C & -3.36170 & -0.25160 & 0.43760 \\
\hline C & -2.14865 & 0.32942 & 2.53386 \\
\hline 0 & -1.43614 & -2.22575 & 0.72397 \\
\hline 0 & 1.00686 & -2.99127 & -0.40423 \\
\hline C & 5.28812 & -0.05896 & 1.31338 \\
\hline $\mathrm{C}$ & 2.76138 & -0.78184 & -1.44827 \\
\hline C & 3.94932 & 0.05127 & -0.99253 \\
\hline 0 & 0.32409 & 0.61221 & 1.13036 \\
\hline 0 & -3.20445 & -0.54681 & -0.87669 \\
\hline 0 & -4.36587 & -0.52379 & 1.05517 \\
\hline $\mathrm{C}$ & -4.25423 & -1.34333 & -1.45376 \\
\hline $\mathrm{H}$ & -2.43316 & 3.52757 & -0.86189 \\
\hline $\mathrm{H}$ & -2.80442 & 1.90189 & -1.42535 \\
\hline $\mathrm{H}$ & -3.51824 & 2.25879 & 0.98661 \\
\hline $\mathrm{H}$ & -1.85163 & 2.65293 & 1.39482 \\
\hline $\mathrm{H}$ & -0.44399 & 2.63180 & -2.03029 \\
\hline $\mathrm{H}$ & -0.12902 & 2.88267 & -0.31998 \\
\hline $\mathrm{H}$ & 3.12308 & -1.87619 & 1.09380 \\
\hline $\mathrm{H}$ & 2.36014 & -0.49585 & 1.91189 \\
\hline $\mathrm{H}$ & 4.33541 & 2.08219 & -0.19324 \\
\hline $\mathrm{H}$ & 1.99070 & 2.41997 & 0.72767 \\
\hline $\mathrm{H}$ & 1.97376 & 2.42053 & -1.02914 \\
\hline $\mathrm{H}$ & -0.21027 & -0.98568 & -2.25315 \\
\hline $\mathrm{H}$ & -1.45097 & 0.20739 & -2.61475 \\
\hline $\mathrm{H}$ & 0.23878 & 0.54551 & -3.01559 \\
\hline $\mathrm{H}$ & -1.31681 & 0.88786 & 2.97066 \\
\hline $\mathrm{H}$ & -2.03468 & -0.72917 & 2.77899 \\
\hline
\end{tabular}

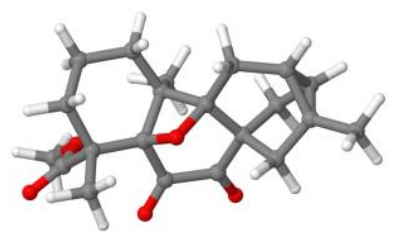




$\begin{array}{rrrr}\mathrm{H} & -3.08658 & 0.67562 & 2.97529 \\ \mathrm{H} & 5.15015 & 0.30607 & 2.33928 \\ \mathrm{H} & 6.07465 & 0.54741 & 0.84931 \\ \mathrm{H} & 5.65428 & -1.09195 & 1.37426 \\ \mathrm{H} & 3.05189 & -1.82282 & -1.62982 \\ \mathrm{H} & 2.28783 & -0.40337 & -2.36086 \\ \mathrm{H} & 4.85886 & 0.05216 & -1.58817 \\ \mathrm{H} & -3.97916 & -1.47724 & -2.50052 \\ \mathrm{H} & -4.31166 & -2.30890 & -0.94536 \\ \mathrm{H} & -5.21648 & -0.83272 & -1.36815\end{array}$


ramariolide $B$, Fig.1

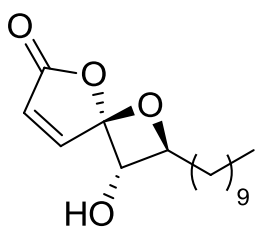

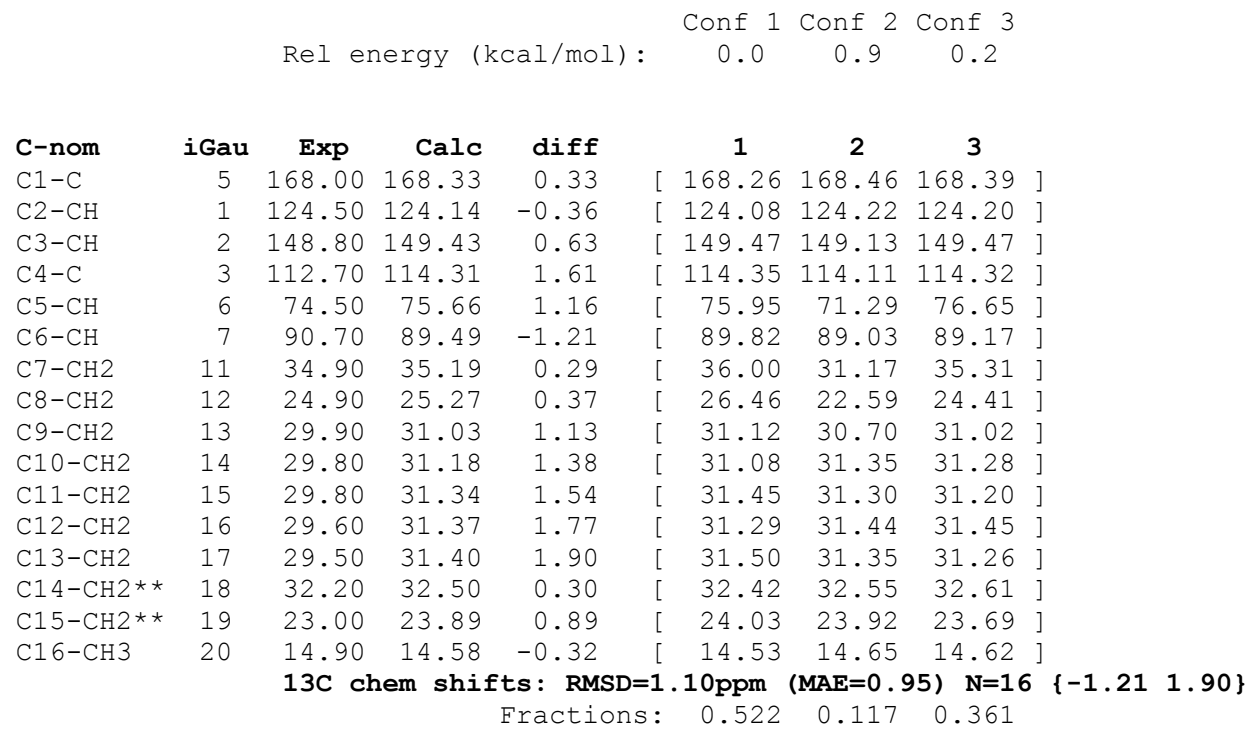

\section{Conformer 1}

Energy: -926.21689 Hartree (Rel: $0.0 \mathrm{kcal} / \mathrm{mol}$ ) XYZ coordinates for conf 1:

$\begin{array}{lrrr}\mathrm{C} & 6.06265 & -1.58977 & 1.04560 \\ \mathrm{C} & 4.90711 & -1.03742 & 1.41856 \\ \mathrm{C} & 4.34364 & -0.22986 & 0.28777 \\ \mathrm{O} & 5.27624 & -0.42632 & -0.79252 \\ \mathrm{C} & 6.33517 & -1.21049 & -0.36507 \\ \mathrm{C} & 3.99844 & 1.28784 & 0.39208 \\ \mathrm{C} & 2.68757 & 0.87604 & -0.32857 \\ \mathrm{O} & 3.02022 & -0.53784 & -0.08875 \\ \mathrm{O} & 7.26895 & -1.47877 & -1.07247 \\ \mathrm{O} & 4.87793 & 2.20027 & -0.17795 \\ \mathrm{C} & 1.35155 & 1.26964 & 0.26690 \\ \mathrm{C} & 0.15895 & 0.55575 & -0.38622 \\ \mathrm{C} & -1.18366 & 0.91083 & 0.26610 \\ \mathrm{C} & -2.38198 & 0.20346 & -0.38054 \\ \mathrm{C} & -3.72054 & 0.52901 & 0.29560 \\ \mathrm{C} & -4.92120 & -0.17581 & -0.34966 \\ \mathrm{C} & -6.25637 & 0.13350 & 0.34067 \\ \mathrm{C} & -7.45810 & -0.57014 & -0.30351 \\ \mathrm{C} & -8.79179 & -0.26904 & 0.39317 \\ \mathrm{C} & -9.98674 & -0.97435 & -0.25720 \\ \mathrm{H} & 6.73620 & -2.21952 & 1.61170 \\ \mathrm{H} & 4.39936 & -1.11713 & 2.37260 \\ \mathrm{H} & 3.81044 & 1.60499 & 1.42277 \\ \mathrm{H} & 2.72681 & 1.08758 & -1.40570 \\ \mathrm{H} & 5.27560 & 1.76660 & -0.95365 \\ \mathrm{H} & 1.24359 & 2.35931 & 0.16287 \\ \mathrm{H} & 1.36910 & 1.05341 & 1.34445 \\ \mathrm{H} & 0.32030 & -0.52876 & -0.32994 \\ \mathrm{H} & -1.33586 & 0.80842 & -1.45600 \\ \mathrm{H} & -1.14479 & 1.99961 & 0.22134 \\ \mathrm{H} & -2.21734 & -0.88386 & -0.35487 \\ \mathrm{H} & & & \end{array}$

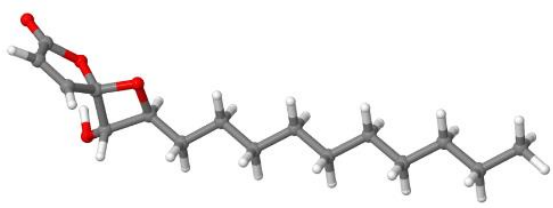




$\begin{array}{rrrr}\text { H } & -2.43483 & 0.47718 & -1.44468 \\ \mathrm{H} & -3.88391 & 1.61686 & 0.27496 \\ \mathrm{H} & -3.66562 & 0.25109 & 1.35876 \\ \mathrm{H} & -4.75142 & -1.26278 & -0.34028 \\ \mathrm{H} & -4.98459 & 0.11170 & -1.40969 \\ \mathrm{H} & -6.42593 & 1.22061 & 0.33379 \\ \mathrm{H} & -6.19189 & -0.15616 & 1.40019 \\ \mathrm{H} & -7.28652 & -1.65704 & -0.30219 \\ \mathrm{H} & -7.52778 & -0.27609 & -1.36154 \\ \mathrm{H} & -8.96309 & 0.81695 & 0.39291 \\ \mathrm{H} & -8.72261 & -0.56498 & 1.44974 \\ \mathrm{H} & -10.92251 & -0.74015 & 0.26336 \\ \mathrm{H} & -9.86037 & -2.06406 & -0.24133 \\ \mathrm{H} & -10.10360 & -0.67120 & -1.30510\end{array}$

\section{Conformer 2}

Energy: -926.21548 Hartree (Rel: $0.9 \mathrm{kcal} / \mathrm{mol}$ ) XYZ coordinates for conf 2 :

\begin{tabular}{|c|c|c|c|}
\hline $\mathrm{C}$ & -5.12106 & 2.34285 & 0.10217 \\
\hline C & -3.90437 & 1.79671 & 0.13561 \\
\hline C & -4.00839 & 0.31702 & -0.08113 \\
\hline 0 & -5.41278 & 0.08435 & -0.30202 \\
\hline C & -6.11433 & 1.27104 & -0.16797 \\
\hline C & -3.46945 & -0.74736 & 0.92365 \\
\hline C & -2.92228 & -1.43777 & -0.35503 \\
\hline 0 & -3.21346 & -0.21379 & -1.11833 \\
\hline 0 & -7.31119 & 1.32962 & -0.26051 \\
\hline O & -4.38316 & -1.39685 & 1.74590 \\
\hline C & -1.47551 & -1.88964 & -0.46885 \\
\hline C & -0.41520 & -0.83106 & -0.13584 \\
\hline C & 1.01668 & -1.34308 & -0.34430 \\
\hline C & 2.09035 & -0.29073 & -0.03756 \\
\hline C & 3.52335 & -0.80544 & -0.22868 \\
\hline C & 4.59756 & 0.25102 & 0.06238 \\
\hline C & 6.03105 & -0.26734 & -0.11477 \\
\hline C & 7.10520 & 0.79158 & 0.16648 \\
\hline $\mathrm{C}$ & 8.53917 & 0.27298 & -0.00573 \\
\hline $\mathrm{C}$ & 9.60469 & 1.33833 & 0.27382 \\
\hline $\mathrm{H}$ & -5.41249 & 3.37651 & 0.23385 \\
\hline $\mathrm{H}$ & -2.95068 & 2.28604 & 0.29464 \\
\hline $\mathrm{H}$ & -2.67439 & -0.35246 & 1.56109 \\
\hline $\mathrm{H}$ & -3.59679 & -2.24091 & -0.68127 \\
\hline $\mathrm{H}$ & -5.21156 & -1.48056 & 1.24140 \\
\hline $\mathrm{H}$ & -1.31497 & -2.24862 & -1.49522 \\
\hline $\mathrm{H}$ & -1.35544 & -2.76738 & 0.18289 \\
\hline $\mathrm{H}$ & -0.52396 & -0.50158 & 0.90735 \\
\hline $\mathrm{H}$ & -0.58532 & 0.05326 & -0.76296 \\
\hline $\mathrm{H}$ & 1.13111 & -1.68615 & -1.38308 \\
\hline $\mathrm{H}$ & 1.18290 & -2.22788 & 0.28798 \\
\hline $\mathrm{H}$ & 1.96753 & 0.06358 & 0.99688 \\
\hline $\mathrm{H}$ & 1.93029 & 0.58744 & -0.68055 \\
\hline $\mathrm{H}$ & 3.64360 & -1.17068 & -1.25942 \\
\hline $\mathrm{H}$ & 3.68511 & -1.67715 & 0.42269 \\
\hline $\mathrm{H}$ & 4.47111 & 0.62416 & 1.08969 \\
\hline $\mathrm{H}$ & 4.44096 & 1.11813 & -0.59642 \\
\hline $\mathrm{H}$ & 6.15573 & -0.64712 & -1.13983 \\
\hline $\mathrm{H}$ & 6.18927 & -1.13018 & 0.54929 \\
\hline $\mathrm{H}$ & 6.97937 & 1.17457 & 1.19038 \\
\hline $\mathrm{H}$ & 6.94961 & 1.65307 & -0.50015 \\
\hline $\mathrm{H}$ & 8.66451 & -0.11105 & -1.02823 \\
\hline $\mathrm{H}$ & 8.69603 & -0.58590 & 0.66234 \\
\hline $\mathrm{H}$ & 10.61649 & 0.93766 & 0.14280 \\
\hline $\mathrm{H}$ & 9.52659 & 1.71685 & 1.30061 \\
\hline $\mathrm{H}$ & 9.49531 & 2.19507 & -0.40267 \\
\hline
\end{tabular}

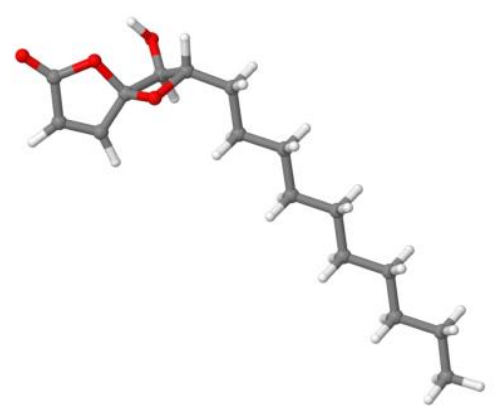

Conformer 3

Energy: -926.21654 Hartree (Rel: $0.2 \mathrm{kcal} / \mathrm{mol}$ ) XYZ coordinates for conf 3 :

$\begin{array}{llll}\mathrm{C} & 6.67544 & -0.48567 & 1.10069 \\ \mathrm{C} & 5.42721 & -0.94003 & 1.22339 \\ \mathrm{C} & 4.55431 & -0.30982 & 0.18014\end{array}$

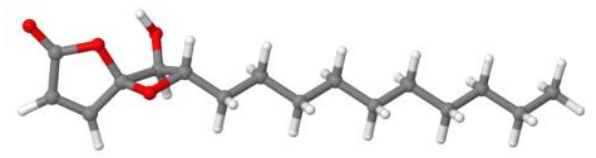




$\begin{array}{lrrr} & & & \\ \mathrm{O} & 5.45268 & 0.51267 & -0.58995 \\ \mathrm{C} & 6.72586 & 0.45634 & -0.04752 \\ \mathrm{C} & 3.26746 & 0.51034 & 0.50044 \\ \mathrm{C} & 2.65083 & -0.31077 & -0.66422 \\ \mathrm{O} & 3.82064 & -1.20046 & -0.63032 \\ \mathrm{O} & 7.64507 & 1.09502 & -0.48532 \\ \mathrm{O} & 3.34127 & 1.89703 & 0.45172 \\ \mathrm{C} & 1.34433 & -1.04865 & -0.45449 \\ \mathrm{C} & 0.13056 & -0.10705 & -0.48341 \\ \mathrm{C} & -1.20083 & -0.83487 & -0.25393 \\ \mathrm{C} & -2.41761 & 0.09911 & -0.29367 \\ \mathrm{C} & -3.75182 & -0.62432 & -0.06730 \\ \mathrm{C} & -4.96840 & 0.31011 & -0.10904 \\ \mathrm{C} & -6.30395 & -0.41168 & 0.11460 \\ \mathrm{C} & -7.52005 & 0.52314 & 0.07390 \\ \mathrm{C} & -8.85641 & -0.19772 & 0.29559 \\ \mathrm{C} & -10.06481 & 0.74389 & 0.25582 \\ \mathrm{H} & 7.55155 & -0.71958 & 1.69098 \\ \mathrm{H} & 5.03696 & -1.65114 & 1.94198 \\ \mathrm{H} & 2.82698 & 0.23332 & 1.46372 \\ \mathrm{H} & 2.64281 & 0.25773 & -1.60414 \\ \mathrm{H} & 3.98315 & 2.12394 & -0.24426 \\ \mathrm{H} & 1.38880 & -1.58494 & 0.50379 \\ \mathrm{H} & 1.24470 & -1.81411 & -1.23619 \\ \mathrm{H} & 0.09826 & 0.41334 & -1.45143 \\ \mathrm{H} & 0.25435 & 0.67712 & 0.27721 \\ \mathrm{H} & -1.16987 & -1.35199 & 0.71647 \\ \mathrm{H} & -1.31979 & -1.62135 & -1.01365 \\ \mathrm{H} & -2.44557 & 0.61693 & -1.26386 \\ \mathrm{H} & -2.29653 & 0.88541 & 0.46606 \\ \mathrm{H} & -3.72436 & -1.14187 & 0.90321 \\ \mathrm{H} & -3.87191 & -1.41104 & -0.82693 \\ \mathrm{H} & -4.99427 & 0.82876 & -1.07896 \\ \mathrm{H} & -4.84839 & 1.09610 & 0.65136 \\ \mathrm{H} & -6.27790 & -0.93124 & 1.08414 \\ \mathrm{H} & -6.42420 & -1.19699 & -0.64657 \\ \mathrm{H} & -7.54629 & 1.04391 & -0.89508 \\ \mathrm{H} & -7.40111 & 1.30800 & 0.83589 \\ \mathrm{H} & -8.83010 & -0.71903 & 1.26320 \\ \mathrm{H} & -8.97626 & -0.98020 & -0.46734 \\ \mathrm{H} & -11.00266 & 0.19981 & 0.41665 \\ \mathrm{H} & -10.13781 & 1.25448 & -0.71251 \\ \mathrm{H} & -9.99051 & 1.51648 & 1.03119 \\ & & & \end{array}$


wallifoliol, Fig.1

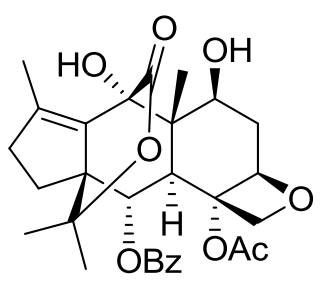

Rel energy (kcal/mol): $\begin{array}{cc}\text { Conf } 1 \\ 0.0\end{array}$

\begin{tabular}{|c|c|c|c|c|c|c|c|}
\hline C-nom & iGau & Exp & Calc & $\operatorname{diff}$ & 1 & & \\
\hline $\mathrm{C} 1-\mathrm{C}$ & 2 & 59.90 & 60.54 & 0.64 & 60.54 & ] & \\
\hline $\mathrm{C} 2-\mathrm{CH}$ & 3 & 68.20 & 70.66 & 2.46 & 70.66 & ] & \\
\hline $\mathrm{C} 3-\mathrm{CH}$ & 4 & 43.20 & 44.89 & 1.69 & 44.89 & ] & \\
\hline $\mathrm{C} 4-\mathrm{C}$ & 7 & 80.50 & 82.29 & 1.79 & 82.29 & ] & \\
\hline $\mathrm{C} 5-\mathrm{CH}$ & 8 & 84.20 & 83.18 & -1.02 & 83.18 & ] & \\
\hline $\mathrm{C} 6-\mathrm{CH} 2$ & 9 & 37.30 & 37.32 & 0.02 & 37.32 & ] & \\
\hline $\mathrm{C} 7-\mathrm{CH}$ & 10 & 71.00 & 72.53 & 1.53 & 72.53 & ] & \\
\hline $\mathrm{C} 8-\mathrm{C}$ & 5 & 48.30 & 49.98 & 1.68 & 49.98 & ] & \\
\hline $\mathrm{C} 9-\mathrm{C}$ & 6 & 84.90 & 85.26 & 0.36 & {$[\quad 85.26$} & ] & \\
\hline $\mathrm{C} 10-\mathrm{C}-\mathrm{HBnd}$ & 15 & 174.60 & 175.27 & 0.67 & {$[175.27$} & ] & \\
\hline $\mathrm{C} 11-\mathrm{C}$ & 1 & 131.00 & 133.49 & 2.49 & {$[133.49$} & ] & \\
\hline $\mathrm{C} 12-\mathrm{C}$ & 11 & 139.90 & 141.88 & 1.98 & [ 141.88 & ] & \\
\hline $\mathrm{Cl} 3-\mathrm{CH}$ & 12 & 79.60 & 79.96 & 0.36 & {$[\quad 79.96$} & ] & \\
\hline $\mathrm{C} 14-\mathrm{CH} 2$ & 13 & 37.10 & 39.41 & 2.31 & 39.41 & ] & \\
\hline C15-C & 19 & 90.30 & 91.42 & 1.12 & 91.42 & ] & \\
\hline $\mathrm{C} 16-\mathrm{CH} 3$ & 23 & 24.80 & 24.33 & -0.47 & 24.33 & ] & \\
\hline $\mathrm{C} 17-\mathrm{CH} 3$ & 24 & 22.40 & 22.31 & -0.09 & 22.31 & ] & \\
\hline $\mathrm{C} 18-\mathrm{CH} 3$ & 14 & 10.90 & 11.64 & 0.74 & 11.64 & ] & \\
\hline $\mathrm{C} 19-\mathrm{CH} 3$ & 16 & 10.10 & 10.87 & 0.77 & 10.87 & ] & \\
\hline $\mathrm{C} 20-\mathrm{CH} 2$ & 18 & 74.20 & 72.88 & -1.32 & {$[\quad 72.88$} & ] & \\
\hline $\mathrm{COAC} 4-\mathrm{C}$ & 31 & 170.20 & 170.55 & 0.35 & {$[170.55$} & ] & \\
\hline $\mathrm{COAC} 4-\mathrm{CH} 3$ & 32 & 21.30 & 20.87 & -0.43 & {$[\quad 20.87$} & ] & \\
\hline $\mathrm{CPhCO}-\mathrm{C}$ & 34 & 164.70 & 167.33 & 2.63 & {$[167.33$} & ] & \\
\hline CPhi-C & 38 & 129.70 & 129.89 & 0.19 & [ 129.89 & ] & \\
\hline $\mathrm{CPho}-\mathrm{CH}$ & 37 & 129.50 & 130.77 & 1.27 & {$[130.77$} & ] & \\
\hline $\mathrm{CPho-CH}$ & 39 & 129.50 & 130.77 & 1.27 & {$[130.77$} & ] & \\
\hline $\mathrm{CPhm}-\mathrm{CH}$ & 36 & 128.60 & 127.24 & -1.36 & {$[127.24$} & ] & \\
\hline $\mathrm{CPhm}-\mathrm{CH}$ & 40 & 128.60 & 127.24 & -1.36 & [ 127.24 & ] & \\
\hline $\mathrm{CPhp}-\mathrm{CH}$ & 35 & 133.60 & 132.47 & -1.13 & {$[132.47$} & ] & \\
\hline
\end{tabular}

\section{Conformer 1}

Energy: -1877.71441 Hartree (Rel: $0.0 \mathrm{kcal} / \mathrm{mol}$ ) XYZ coordinates for conf 1 :

$\begin{array}{rrrr}\text { C } & 1.97880 & -1.16492 & 0.64601 \\ \text { C } & 0.65953 & -1.63574 & 0.04486 \\ \text { C } & -0.11082 & -0.40986 & -0.56889 \\ \text { C } & 0.49969 & 0.90074 & -0.03792 \\ \text { C } & 1.96844 & 1.07147 & -0.59191 \\ \text { C } & 2.76222 & -0.32904 & -0.31620 \\ \text { C } & -0.38348 & 2.15512 & -0.18759 \\ \text { C } & 0.35621 & 3.51538 & -0.11889 \\ \text { C } & 1.84888 & 3.55551 & 0.18822 \\ \text { C } & 2.60296 & 2.20939 & 0.28404 \\ \text { C } & 2.21108 & -1.62224 & 1.88514 \\ \text { C } & 1.07166 & -2.52135 & 2.32718 \\ \text { C } & -0.03664 & -2.27446 & 1.27697 \\ \text { C } & 3.37821 & -1.40855 & 2.79994 \\ \text { C } & 2.90144 & -1.16861 & -1.62797 \\ \text { C } & 2.09022 & 1.44828 & -2.08186 \\ \text { O } & 0.04431 & 3.76924 & -1.52132 \\ \text { C } & -0.83979 & 2.62963 & -1.58012\end{array}$

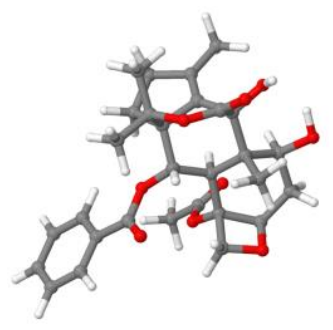




\begin{tabular}{|c|c|c|}
\hline 0.99460 & -2.64445 & -1.09768 \\
\hline 1.89884 & -1.95473 & -2.04829 \\
\hline 3.92394 & -1.08245 & -2.27854 \\
\hline 0.61753 & 0.78502 & 1.04495 \\
\hline 1.75133 & -3.89358 & -0.62762 \\
\hline-0.20406 & -3.06834 & -1.94947 \\
\hline 4.10542 & -0.10947 & 0.10379 \\
\hline 3.93563 & 2.52955 & -0.07280 \\
\hline 4.44109 & 1.72245 & 0.12706 \\
\hline 1.59313 & 0.73041 & -2.74313 \\
\hline-1.53569 & 2.06993 & 0.68766 \\
\hline-0.27668 & 2.50514 & 2.52306 \\
\hline-1.35446 & 2.28117 & 2.01268 \\
\hline-2.67334 & 2.23446 & 2.74799 \\
\hline-1.51071 & -0.55057 & -0.22558 \\
\hline-2.44338 & -0.37184 & -1.18965 \\
\hline-6.47289 & -0.92319 & 0.15859 \\
\hline-6.20448 & -0.53691 & -1.15739 \\
\hline-4.88775 & -0.36310 & -1.57755 \\
\hline-3.82978 & -0.57168 & -0.68160 \\
\hline-4.10195 & -0.96180 & 0.63809 \\
\hline-5.42122 & -1.13663 & 1.05401 \\
\hline-2.17912 & -0.08951 & -2.34494 \\
\hline-0.04961 & -0.41699 & -1.65506 \\
\hline-0.18864 & 4.23153 & 0.50515 \\
\hline 2.01776 & 4.09385 & 1.12535 \\
\hline 2.32745 & 4.13929 & -0.60529 \\
\hline 2.56463 & 1.86059 & 1.32788 \\
\hline 0.67273 & -2.18156 & 3.65485 \\
\hline 1.41495 & -3.57007 & 2.31531 \\
\hline-0.61911 & -3.16807 & 1.03062 \\
\hline-0.72469 & -1.55016 & 1.71765 \\
\hline 3.84506 & -2.37085 & 3.05391 \\
\hline 4.13338 & -0.76155 & 2.35558 \\
\hline 3.02992 & -0.97500 & 3.74433 \\
\hline 3.14668 & 1.48218 & -2.35775 \\
\hline 1.67151 & 2.43624 & -2.27759 \\
\hline-1.89673 & 2.92071 & -1.59322 \\
\hline-0.62975 & 1.98090 & -2.43294 \\
\hline 2.63898 & -3.63750 & -0.04334 \\
\hline 2.06840 & -4.47059 & -1.50082 \\
\hline 1.10422 & -4.53107 & -0.01680 \\
\hline-0.70205 & -2.22359 & -2.42859 \\
\hline 0.13143 & -3.75021 & -2.73686 \\
\hline-0.93440 & -3.59765 & -1.32892 \\
\hline 4.64271 & -0.29681 & -0.69660 \\
\hline-3.28718 & 1.40037 & 2.39744 \\
\hline-2.48730 & 2.15393 & 3.81988 \\
\hline-3.23073 & 3.15760 & 2.55014 \\
\hline-7.50010 & -1.05937 & 0.48603 \\
\hline-7.02121 & -0.37194 & -1.85440 \\
\hline-4.65669 & -0.06530 & -2.59486 \\
\hline-3.28215 & -1.13181 & 1.32671 \\
\hline-5.62986 & -1.44118 & 2.07590 \\
\hline 0.15461 & -2.91912 & 4.01059 \\
\hline
\end{tabular}


holophylline O (PCM in methanol), Fig.1

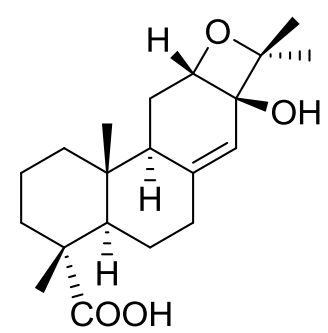

Rel energy (kcal/mol): $\begin{array}{ccc}\text { Conf } 1 & \text { Conf } 2 \\ 0.3 & 0.0\end{array}$

\begin{tabular}{|c|c|c|c|c|c|c|c|}
\hline C-nom & iGau & Exp & Calc & $\operatorname{diff}$ & 1 & 2 & \\
\hline $\mathrm{C} 1-\mathrm{CH} 2$ & 6 & 40.30 & 39.43 & -0.87 & 38.43 & $38.44]$ & \\
\hline $\mathrm{C} 2-\mathrm{CH} 2$ & 1 & 19.40 & 19.85 & 0.45 & 18.61 & $18.64]$ & \\
\hline $\mathrm{C} 3-\mathrm{CH} 2$ & 2 & 38.30 & 36.47 & -1.83 & 36.49 & $34.80]$ & \\
\hline $\mathrm{C} 4-\mathrm{C}$ & 3 & 48.50 & 47.97 & -0.53 & 46.63 & $47.33]$ & \\
\hline $\mathrm{C} 5-\mathrm{CH}$ & 4 & 49.90 & 48.50 & -1.40 & 46.41 & $48.32]$ & \\
\hline $\mathrm{C} 6-\mathrm{CH} 2$ & 7 & 25.10 & 25.08 & -0.02 & 24.50 & $23.56]$ & \\
\hline $\mathrm{C} 7-\mathrm{CH} 2$ & 8 & 35.80 & 35.73 & -0.07 & {$\left[\begin{array}{l}34.93 \\
{[}\end{array}\right.$} & $34.54]$ & \\
\hline $\mathrm{C} 8-\mathrm{C}$ & 9 & 142.80 & 146.06 & 3.26 & [ 146.63 & 146.09 ] & \\
\hline $\mathrm{C} 9-\mathrm{CH}$ & 10 & 47.30 & 47.32 & 0.02 & {$\left[\begin{array}{l}46.52 \\
0\end{array}\right.$} & $46.35]$ & \\
\hline $\mathrm{C} 10-\mathrm{C}$ & 5 & 37.80 & 38.21 & 0.41 & 37.19 & $37.21]$ & \\
\hline $\mathrm{C} 11-\mathrm{CH} 2$ & 14 & 24.80 & 25.66 & 0.86 & 24.63 & $24.42]$ & \\
\hline $\mathrm{C} 12-\mathrm{CH}$ & 13 & 83.70 & 81.91 & -1.79 & 81.51 & $81.34]$ & \\
\hline C13-C & 12 & 72.10 & 73.37 & 1.27 & 72.84 & $72.72]$ & \\
\hline $\mathrm{C} 14-\mathrm{CH}$ & 11 & 123.40 & 123.99 & 0.59 & {$[123.81$} & $124.07]$ & \\
\hline C15-C & 15 & 93.00 & 90.19 & -2.81 & [ 89.84 & 89.75 ] & \\
\hline $\mathrm{C} 16-\mathrm{CH} 3$ & 24 & 24.50 & 24.59 & 0.09 & 23.52 & $23.36]$ & \\
\hline $\mathrm{C} 17-\mathrm{CH} 3$ & 23 & 23.10 & 23.32 & 0.22 & 22.22 & $22.08]$ & \\
\hline $\mathrm{C} 18-\mathrm{C}$ & 25 & 182.90 & 181.75 & -1.15 & [ 182.15 & $182.54]$ & \\
\hline C19-СH3 & 17 & 17.90 & 19.26 & 1.36 & {$\left[\begin{array}{l}17.78 \\
0\end{array}\right.$} & $18.18]$ & \\
\hline \multirow[t]{3}{*}{$\mathrm{C} 20-\mathrm{CH} 3$} & 20 & 16.00 & 18.34 & 2.34 & {$\left[\begin{array}{ll}17.37 \\
{[}\end{array}\right.$} & $16.94]$ & \\
\hline & & $13 \mathrm{C} c$ & nem shif & ts: CRN & $=1.41 \mathrm{ppm}$ & $(\mathrm{CMAE}=1.07)$ & $\mathrm{N}=20 \quad\{-2.81 \quad 3.26\}$ \\
\hline & & & & Fractic & $: \quad 0.375$ & 0.625 & \\
\hline
\end{tabular}

Conformer 1

Energy: -1081.05983 Hartree (Rel: $0.3 \mathrm{kcal} / \mathrm{mol}$ ) XYZ coordinates for conf 1 :

\begin{tabular}{|c|c|c|c|}
\hline C & -2.85268 & 2.34478 & -0.79331 \\
\hline C & -3.70475 & 1.09010 & -1.00097 \\
\hline C & -3.38276 & -0.02916 & 0.03925 \\
\hline C & -1.83665 & -0.28356 & 0.09119 \\
\hline C & -0.89834 & 0.97093 & 0.16577 \\
\hline C & -1.35974 & 2.01195 & -0.88469 \\
\hline C & -1.42962 & -1.31723 & 1.15439 \\
\hline C & -0.00030 & -1.80521 & 0.88906 \\
\hline C & 1.01110 & -0.70378 & 0.63632 \\
\hline C & 0.55426 & 0.47487 & -0.21542 \\
\hline C & 2.28470 & -0.87201 & 1.03063 \\
\hline C & 3.44451 & -0.02204 & 0.61994 \\
\hline C & 3.03286 & 1.18018 & -0.25459 \\
\hline C & 1.58581 & 1.62348 & -0.21466 \\
\hline C & 4.18544 & -0.51399 & -0.68119 \\
\hline 0 & 3.43054 & 0.47416 & -1.46103 \\
\hline C & -4.04368 & 0.30551 & 1.39663 \\
\hline $\mathrm{H}$ & 0.48105 & 0.09905 & -1.24875 \\
\hline $\mathrm{H}$ & -1.61759 & -0.74233 & -0.88244 \\
\hline C & -0.86432 & 1.62155 & 1.57157 \\
\hline $\mathrm{H}$ & 3.69391 & 2.04094 & -0.07987 \\
\hline 0 & 4.33627 & 0.21710 & 1.70046 \\
\hline $\mathrm{C}$ & 5.67671 & -0.17952 & -0.70095 \\
\hline & 3.92370 & -1.92309 & -1.19102 \\
\hline & -4.03755 & -1.29594 & -0.54115 \\
\hline
\end{tabular}

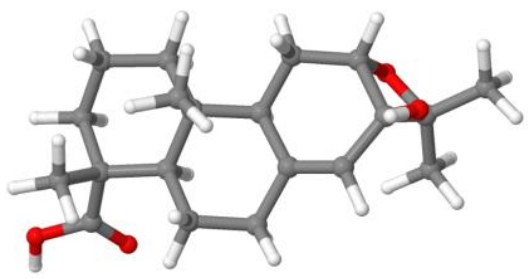




$\begin{array}{rrr}-3.47145 & -2.17955 & -1.15817 \\ -5.37900 & -1.31854 & -0.36598 \\ -3.11322 & 3.08526 & -1.55988 \\ -3.08319 & 2.81102 & 0.17288 \\ -3.50860 & 0.70410 & -2.01129 \\ -4.77362 & 1.32809 & -0.95432 \\ -0.77148 & 2.93103 & -0.78263 \\ -1.15342 & 1.61753 & -1.89119 \\ -2.10616 & -2.17857 & 1.12899 \\ -1.50278 & -0.88985 & 2.16070 \\ 0.35069 & -2.45734 & 1.69796 \\ -0.03055 & -2.43123 & -0.01753 \\ 2.54873 & -1.75808 & 1.61019 \\ 1.39609 & 2.30411 & -1.05213 \\ 1.48294 & 2.21882 & 0.70019 \\ -3.79889 & -0.43670 & 2.16261 \\ -5.13046 & 0.33202 & 1.29103 \\ -3.72813 & 1.28325 & 1.76455 \\ -0.40890 & 2.61632 & 1.52967 \\ -1.85928 & 1.75016 & 1.99877 \\ -0.27961 & 1.02602 & 2.28059 \\ 3.79591 & 0.39501 & 2.48733 \\ 6.06023 & -0.28970 & -1.72189 \\ 6.23601 & -0.85722 & -0.04721 \\ 5.86480 & 0.84448 & -0.36752 \\ 2.85372 & -2.14401 & -1.21385 \\ 4.41999 & -2.66336 & -0.55260 \\ 4.32409 & -2.03074 & -2.20625 \\ -5.70536 & -2.12025 & -0.81919\end{array}$

\section{Conformer 2}

Energy: -1081.06031 Hartree (Rel: $0.0 \mathrm{kcal} / \mathrm{mol}$ ) XYZ coordinates for conf 2 :

$\begin{array}{lrrr}\text { C } & -2.86220 & 2.37915 & -0.78373 \\ \text { C } & -3.71926 & 1.12910 & -1.00609 \\ \text { C } & -3.41522 & 0.01119 & 0.03328 \\ \text { C } & -1.86115 & -0.26539 & 0.07100 \\ \text { C } & -0.91713 & 0.98156 & 0.17518 \\ \text { C } & -1.36895 & 2.04108 & -0.86218 \\ \text { C } & -1.46412 & -1.33043 & 1.10582 \\ \text { C } & -0.03586 & -1.81864 & 0.83526 \\ \mathrm{C} & 0.98126 & -0.71475 & 0.61829 \\ \mathrm{C} & 0.53557 & 0.48627 & -0.20768 \\ \mathrm{C} & 2.25115 & -0.89848 & 1.01734 \\ \mathrm{C} & 3.41740 & -0.04342 & 0.63642 \\ \mathrm{C} & 3.01703 & 1.18156 & -0.21110 \\ \mathrm{C} & 1.57159 & 1.63027 & -0.17091 \\ \mathrm{C} & 4.16524 & -0.50665 & -0.67100 \\ \mathrm{O} & 3.41989 & 0.50357 & -1.43162 \\ \mathrm{C} & -4.06243 & 0.36112 & 1.38846 \\ \mathrm{H} & 0.46918 & 0.13597 & -1.25038 \\ \mathrm{H} & -1.64495 & -0.69265 & -0.91797 \\ \mathrm{C} & -0.89017 & 1.60184 & 1.59422 \\ \mathrm{H} & 3.68030 & 2.03491 & -0.01064 \\ \mathrm{O} & 4.30222 & 0.16448 & 1.72886 \\ \mathrm{C} & 5.65798 & -0.17793 & -0.67173 \\ \mathrm{C} & 3.90216 & -1.90174 & -1.21759 \\ \mathrm{C} & -4.08283 & -1.28494 & -0.45459 \\ \mathrm{O} & -4.90967 & -1.93361 & 0.15845 \\ \mathrm{O} & -3.66213 & -1.66957 & -1.68298 \\ \mathrm{H} & -3.10924 & 3.12408 & -1.55049 \\ \mathrm{H} & -3.09863 & 2.84534 & 0.18101 \\ \mathrm{H} & -3.52222 & 0.74661 & -2.01410 \\ \mathrm{H} & -4.78733 & 1.37908 & -0.96353 \\ \mathrm{H} & -0.77874 & 2.95651 & -0.74032 \\ \mathrm{H} & -1.15313 & 1.66138 & -1.87233 \\ \mathrm{H} & -2.14682 & -2.18846 & 1.06299 \\ \mathrm{H} & -1.54018 & -0.92850 & 2.12212 \\ \mathrm{H} & 0.30621 & -2.49252 & 1.62977 \\ \mathrm{H} & -0.06199 & -2.42157 & -0.08703 \\ \mathrm{H} & 2.50715 & -1.79993 & 1.57635 \\ & & & \end{array}$

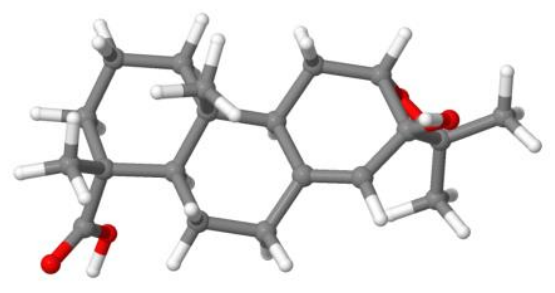




$\begin{array}{rrr}1.39142 & 2.33197 & -0.99296 \\ 1.46369 & 2.20347 & 0.75734 \\ -3.77624 & -0.34173 & 2.17528 \\ -5.15164 & 0.32166 & 1.29602 \\ -3.79641 & 1.36755 & 1.71512 \\ -0.44318 & 2.60101 & 1.57397 \\ -1.88645 & 1.71097 & 2.02301 \\ -0.30005 & 0.99574 & 2.28988 \\ 3.75731 & 0.32472 & 2.51637 \\ 6.04835 & -0.26369 & -1.69241 \\ 6.20985 & -0.87427 & -0.03141 \\ 5.84801 & 0.83651 & -0.31116 \\ 2.83153 & -2.11718 & -1.25726 \\ 4.38849 & -2.65972 & -0.59246 \\ 4.31255 & -1.98644 & -2.23098 \\ -4.13963 & -2.49422 & -1.89754\end{array}$


hawaiienol A (PCM in acetone), Fig.1<smiles>COc1ccc(C23CC4(O)OC(O2)C(C(O)(O)c2ccc(O)cc2)C4O3)cc1</smiles>

Rel energy (kcal/mol): $\begin{array}{cccc}\text { Conf } 1 & \text { Conf } 2 & \text { Conf } 3 \\ 0.5 & 0.0 & 0.5\end{array}$

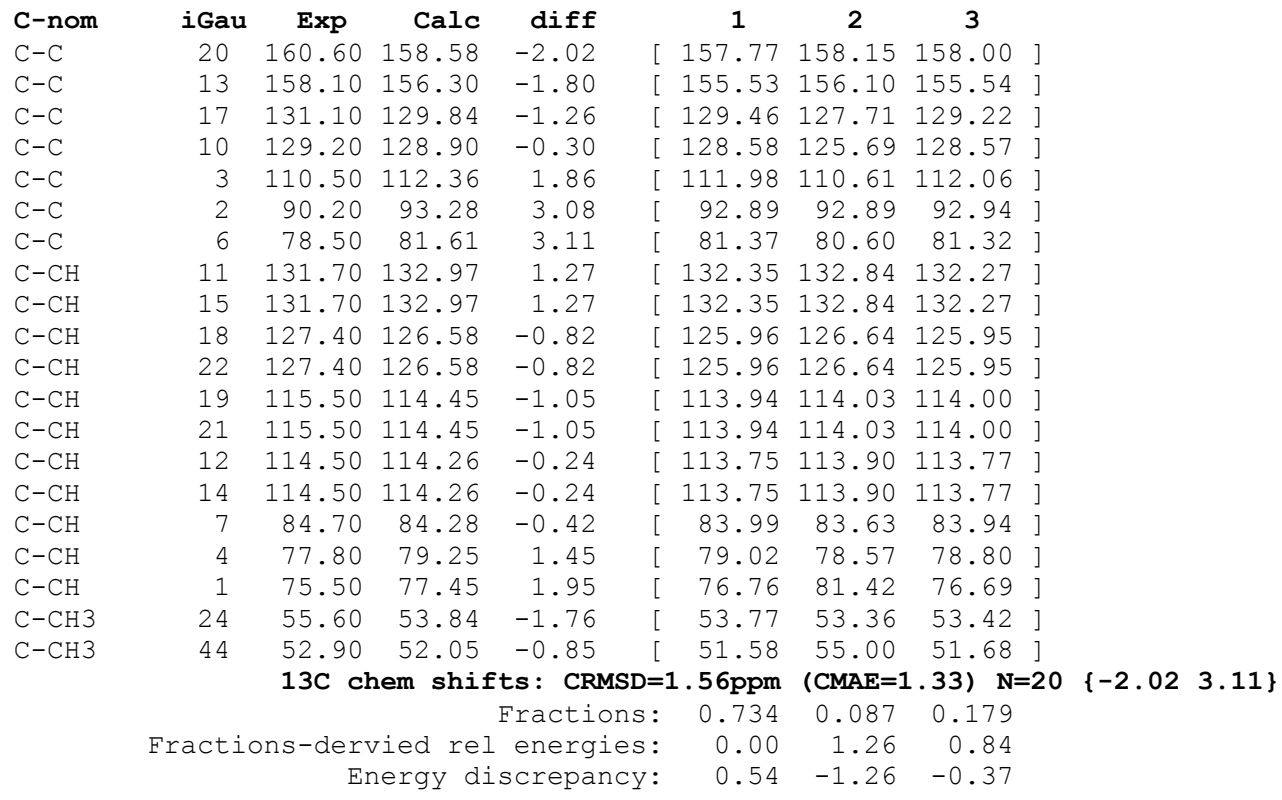

Conformer 1

Energy: -1300.62850 Hartree (Rel: $0.5 \mathrm{kcal} / \mathrm{mol})$ XYZ coordinates for conf 1 :

$\begin{array}{lrrr}\text { C } & -0.12171 & 0.81188 & -0.78066 \\ C & -0.89238 & 0.24603 & 0.44726 \\ C & 0.94417 & 1.70026 & -0.04600 \\ C & -0.46624 & 1.28050 & 1.53575 \\ O & 0.07693 & 2.40629 & 0.87610 \\ C & 1.82640 & 0.84570 & 0.93543 \\ C & 0.67811 & 0.26002 & 1.78777 \\ C & 2.53884 & 1.73250 & 1.80093 \\ O & -0.02407 & -0.77097 & 1.03989 \\ O & 2.77760 & -0.18375 & 0.33927 \\ C & 3.20575 & -1.26189 & 1.13454 \\ C & 4.14720 & -2.17753 & 0.67735 \\ C & 4.69848 & -2.02820 & -0.60200 \\ C & 4.29587 & -0.95732 & -1.40579 \\ C & 3.34784 & -0.04741 & -0.93493 \\ C & 5.61760 & -2.95403 & -1.00483 \\ \text { C } & -2.30426 & -0.20813 & 0.24135 \\ O & -2.70333 & -1.50671 & 0.59219 \\ C & -4.01765 & -1.92213 & 0.40992 \\ C & -4.97315 & -1.04131 & -0.11948 \\ C & -4.59033 & 0.26190 & -0.46582 \\ C & -3.26460 & 0.66272 & -0.28652 \\ C & -6.23488 & -1.54096 & -0.25501 \\ C & -7.24682 & -0.68717 & -0.78143 \\ O & 0.34895 & -0.18315 & -1.65131\end{array}$

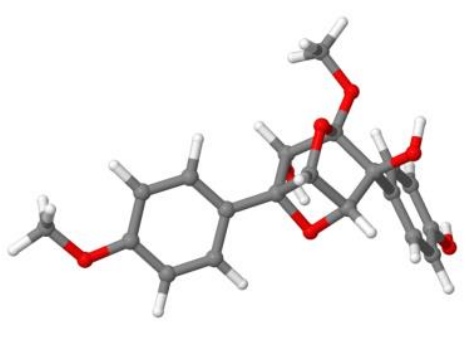




$\begin{array}{rrr}-0.76794 & 1.47152 & -1.36517 \\ 1.72842 & 2.57415 & -0.76666 \\ -1.15550 & 1.56007 & 2.32865 \\ 0.96137 & -0.05655 & 2.79191 \\ 2.94448 & 2.40777 & 1.22871 \\ 2.79971 & -1.39241 & 2.13341 \\ 4.46521 & -3.01005 & 1.29755 \\ 4.72126 & -0.83234 & -2.39906 \\ 3.05063 & 0.77662 & -1.57197 \\ 5.91679 & -2.74056 & -1.90416 \\ -1.97417 & -2.19117 & 1.01198 \\ -4.32710 & -2.92830 & 0.67650 \\ -5.30611 & 0.96637 & -0.87299 \\ -2.98899 & 1.67870 & -0.55795 \\ -8.16105 & -1.28235 & -0.79692 \\ -7.00662 & -0.36271 & -1.80133 \\ -7.39726 & 0.19430 & -0.14625 \\ 0.73958 & -0.87746 & -1.08930 \\ 1.05273 & 3.49914 & -1.63102 \\ 1.81513 & 4.20390 & -1.96592 \\ 0.26677 & 4.03596 & -1.09219 \\ 0.62743 & 2.98828 & -2.50156\end{array}$

\section{Conformer 2} XYZ coordinates for conf 2

\begin{tabular}{|c|c|c|c|}
\hline $\mathrm{C}$ & -0.18698 & 0.70523 & -0.91504 \\
\hline $\mathrm{C}$ & -0.97625 & 0.26662 & 0.34464 \\
\hline $\mathrm{C}$ & 0.91254 & 1.61690 & -0.27478 \\
\hline $\mathrm{C}$ & -0.49703 & 1.34609 & 1.36443 \\
\hline O & 0.08414 & 2.41643 & 0.63015 \\
\hline C & 1.78084 & 0.83754 & 0.79246 \\
\hline $\mathrm{C}$ & 0.61355 & 0.30074 & 1.66207 \\
\hline 0 & 2.51108 & 1.77042 & 1.60168 \\
\hline O & -0.13497 & -0.74590 & 0.98790 \\
\hline C & 2.76602 & -0.20416 & 0.30797 \\
\hline C & 3.29008 & -1.12771 & 1.22913 \\
\hline $\mathrm{C}$ & 4.24976 & -2.06187 & 0.85520 \\
\hline C & 4.71662 & -2.08790 & -0.46577 \\
\hline C & 4.21300 & -1.17261 & -1.39561 \\
\hline C & 3.25114 & -0.23941 & -1.00644 \\
\hline O & 5.65776 & -3.02409 & -0.78238 \\
\hline C & -2.40735 & -0.14912 & 0.19143 \\
\hline C & -2.88513 & -1.30837 & 0.82320 \\
\hline C & -4.21797 & -1.68542 & 0.71039 \\
\hline C & -5.11605 & -0.90231 & -0.03131 \\
\hline C & -4.65571 & 0.26275 & -0.65954 \\
\hline C & -3.31137 & 0.62458 & -0.54584 \\
\hline 0 & -6.40150 & -1.35364 & -0.07959 \\
\hline C & -7.35637 & -0.59659 & -0.81840 \\
\hline 0 & 0.24535 & -0.38335 & -1.68732 \\
\hline $\mathrm{H}$ & -0.77628 & 1.35672 & -1.56532 \\
\hline 0 & 1.50062 & 2.38333 & -1.24932 \\
\hline $\mathrm{H}$ & -1.16289 & 1.70373 & 2.14557 \\
\hline $\mathrm{H}$ & 0.89956 & 0.03128 & 2.67911 \\
\hline $\mathrm{H}$ & 1.89111 & 2.49502 & 1.80854 \\
\hline $\mathrm{H}$ & 2.94814 & -1.11613 & 2.25990 \\
\hline $\mathrm{H}$ & 4.64707 & -2.77423 & 1.57169 \\
\hline $\mathrm{H}$ & 4.57153 & -1.18643 & -2.42232 \\
\hline $\mathrm{H}$ & 2.87081 & 0.46096 & -1.74001 \\
\hline $\mathrm{H}$ & 5.89919 & -2.93492 & -1.71920 \\
\hline $\mathrm{H}$ & -2.19983 & -1.91667 & 1.40330 \\
\hline $\mathrm{H}$ & -4.58633 & -2.58568 & 1.19307 \\
\hline $\mathrm{H}$ & -5.32516 & 0.88891 & -1.23756 \\
\hline $\mathrm{H}$ & -2.97898 & 1.53356 & -1.03967 \\
\hline $\mathrm{H}$ & -8.30138 & -1.13316 & -0.72216 \\
\hline $\mathrm{H}$ & -7.08138 & -0.53063 & -1.87809 \\
\hline $\mathrm{H}$ & -7.46864 & 0.41470 & -0.40899 \\
\hline $\mathrm{H}$ & 0.54747 & -1.05950 & -1.05171 \\
\hline C & 2.45532 & 3.38954 & -0.87416 \\
\hline $\mathrm{H}$ & 2.67862 & 3.92966 & -1.79606 \\
\hline
\end{tabular}

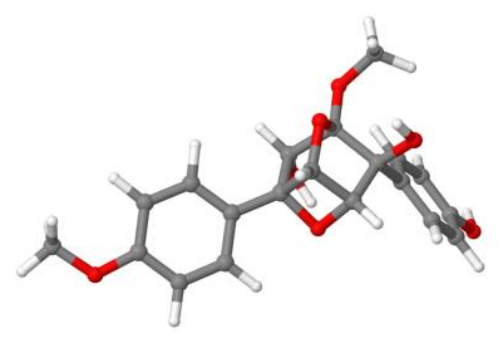




$\begin{array}{llll}\mathrm{H} & 3.36810 & 2.94702 & -0.47003 \\ \mathrm{H} & 2.02754 & 4.08311 & -0.14341\end{array}$

\section{Conformer 3}

Energy: -1300.62861 Hartree (Rel: $0.5 \mathrm{kcal} / \mathrm{mol}$ ) XYZ coordinates for conf 3 :

$\begin{array}{lrrr}\mathrm{C} & -0.06200 & 0.91072 & -0.81856 \\ \mathrm{C} & -0.89948 & 0.40206 & 0.39164 \\ \mathrm{C} & 1.04448 & 1.72497 & -0.05878 \\ \mathrm{C} & -0.42926 & 1.40743 & 1.48930 \\ \mathrm{O} & 0.20621 & 2.49141 & 0.84212 \\ \mathrm{C} & 1.84143 & 0.81357 & 0.94459 \\ \mathrm{C} & 0.63559 & 0.31150 & 1.76947 \\ \mathrm{O} & 2.59322 & 1.65155 & 1.82557 \\ \mathrm{O} & -0.11729 & -0.67071 & 1.00601 \\ \mathrm{C} & 2.73172 & -0.28184 & 0.37210 \\ \mathrm{C} & 3.05288 & -1.39297 & 1.17213 \\ \mathrm{C} & 3.93630 & -2.37509 & 0.73744 \\ \mathrm{C} & 4.53627 & -2.26179 & -0.52345 \\ \mathrm{C} & 4.24061 & -1.15956 & -1.33137 \\ \mathrm{C} & 3.34975 & -0.18293 & -0.88330 \\ \mathrm{O} & 5.39479 & -3.25246 & -0.90503 \\ \mathrm{C} & -2.33329 & 0.04579 & 0.15149 \\ \mathrm{C} & -2.82457 & -1.22185 & 0.47161 \\ \mathrm{C} & -4.16514 & -1.55787 & 0.25666 \\ \mathrm{C} & -5.04129 & -0.60704 & -0.27948 \\ \mathrm{C} & -4.55931 & 0.67436 & -0.59649 \\ \mathrm{C} & -3.22410 & 0.99171 & -0.38612 \\ \mathrm{O} & -6.36533 & -0.82060 & -0.52685 \\ \mathrm{C} & -6.91146 & -2.09948 & -0.21640 \\ \mathrm{O} & 0.36036 & -0.11696 & -1.67572 \\ \mathrm{H} & -0.64637 & 1.61184 & -1.41993 \\ \mathrm{O} & 1.90519 & 2.54135 & -0.75928 \\ \mathrm{H} & -1.11614 & 1.73575 & 2.26539 \\ \mathrm{H} & 0.87166 & -0.02059 & 2.78081 \\ \mathrm{H} & 3.04996 & 2.30197 & 1.26315 \\ \mathrm{H} & 2.60714 & -1.49739 & 2.15702 \\ \mathrm{H} & 4.17116 & -3.23218 & 1.36120 \\ \mathrm{H} & 4.70467 & -1.06273 & -2.31041 \\ \mathrm{H} & 3.13560 & 0.66422 & -1.52341 \\ \mathrm{H} & 5.73674 & -3.05766 & -1.79328 \\ \mathrm{H} & -2.15248 & -1.95926 & 0.89707 \\ \mathrm{H} & -4.50877 & -2.55338 & 0.51263 \\ \mathrm{H} & -5.24961 & 1.40491 & -1.00713 \\ \mathrm{H} & -2.87557 & 1.99108 & -0.63430 \\ \mathrm{H} & -7.96702 & -2.04709 & -0.48722 \\ \mathrm{H} & -6.82058 & -2.32409 & 0.85334 \\ \mathrm{H} & -6.42580 & -2.89384 & -0.79621 \\ \mathrm{H} & 0.68769 & -0.83477 & -1.10296 \\ \mathrm{C} & 1.31633 & 3.50852 & -1.64097 \\ \mathrm{H} & 2.12926 & 4.17110 & -1.94139 \\ \mathrm{H} & 0.54203 & 4.08521 & -1.12736 \\ \mathrm{H} & 0.89641 & 3.02711 & -2.53064\end{array}$

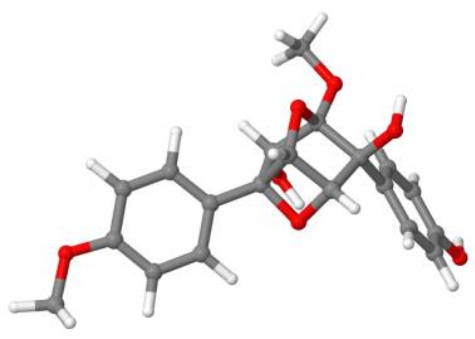


merrilactone A (PCM in methanol), Fig.1

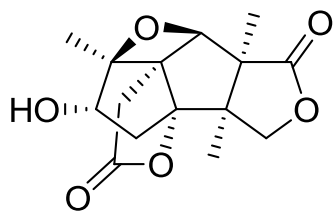

Conf 1 Conf 2

Rel energy (kcal/mol): $0.1 \quad 0.0$

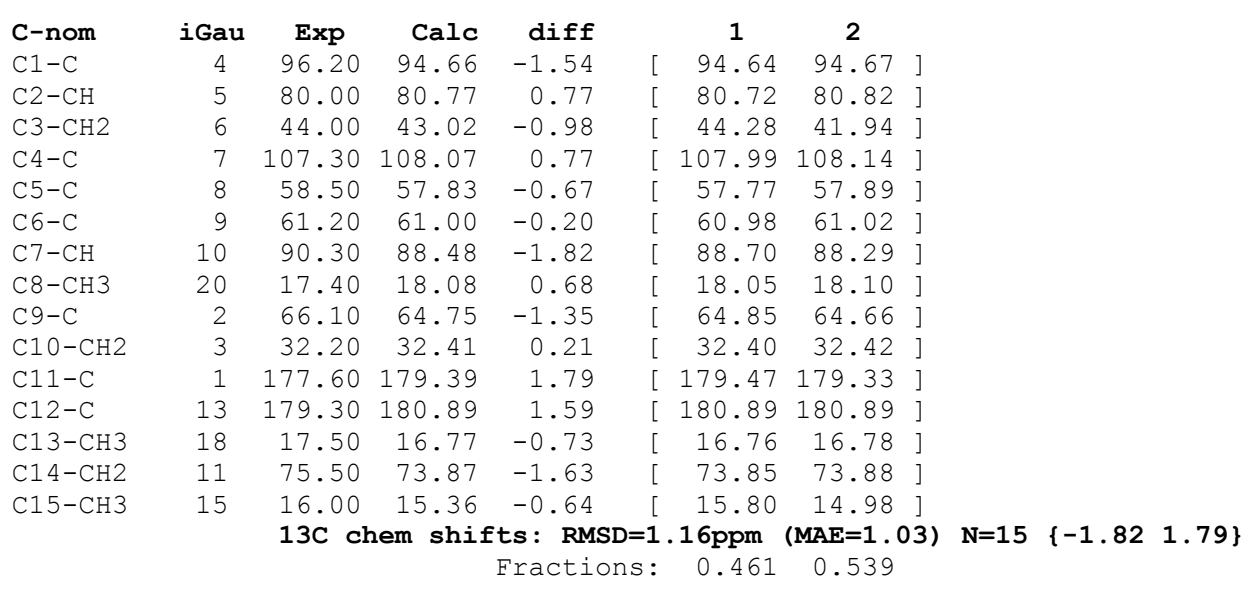

\section{Conformer 1}

Energy: -1033.76067 Hartree (Rel: $0.1 \mathrm{kcal} / \mathrm{mol}$ ) XYZ coordinates for conf 1 :

$\begin{array}{lrrr}\text { C } & 2.21186 & 1.69295 & -0.33882 \\ \mathrm{C} & 0.78166 & -0.18588 & -0.73874 \\ \mathrm{C} & 1.77046 & 0.72693 & -1.43409 \\ \mathrm{C} & 1.09161 & -1.64082 & -0.31548 \\ \mathrm{C} & 1.41935 & -1.59979 & 1.18268 \\ \mathrm{C} & 0.52425 & -0.47453 & 1.72552 \\ \mathrm{C} & 0.39253 & 0.55491 & 0.57693 \\ \mathrm{C} & -1.04474 & 1.15766 & 0.33325 \\ \mathrm{C} & -1.70603 & 0.15072 & -0.68254 \\ \mathrm{C} & -0.55537 & -0.72451 & -1.24957 \\ \mathrm{C} & -1.98618 & 1.06653 & 1.55458 \\ \mathrm{O} & -2.70460 & -0.18610 & 1.44988 \\ \mathrm{C} & -2.65054 & -0.68103 & 0.18836 \\ \mathrm{O} & -0.32129 & -1.97778 & -0.53771 \\ \mathrm{C} & 2.00049 & -2.49981 & -1.17051 \\ \mathrm{O} & 2.80674 & -1.26948 & 1.25743 \\ \mathrm{O} & 1.39627 & 1.58286 & 0.74782 \\ \mathrm{C} & -0.96377 & 2.61328 & -0.14985 \\ \mathrm{O} & 3.12679 & 2.48005 & -0.36196 \\ \mathrm{C} & -2.52839 & 0.77112 & -1.82792 \\ \mathrm{O} & -3.30660 & -1.63895 & -0.14776 \\ \mathrm{H} & 1.32180 & 1.30752 & -2.24926 \\ \mathrm{H} & 2.64144 & 0.21194 & -1.84754 \\ \mathrm{H} & 1.21478 & -2.56217 & 1.67264 \\ \mathrm{H} & -0.44735 & -0.89148 & 1.99431 \\ \mathrm{H} & 0.94327 & -0.00736 & 2.62228 \\ \mathrm{H} & -0.66028 & -0.90475 & -2.32405 \\ \mathrm{H} & -2.72599 & 1.87224 & 1.54710 \\ \mathrm{H} & -1.47252 & 1.07255 & 2.51713 \\ \mathrm{H} & 1.73437 & -2.41675 & -2.22939 \\ \mathrm{H} & 1.89938 & -3.55008 & -0.87416 \\ \mathrm{H} & 3.04593 & -2.20749 & -1.04236 \\ \mathrm{H} & 3.02947 & -1.13336 & 2.19218 \\ \mathrm{H} & -1.96123 & 3.00014 & -0.37385 \\ \mathrm{H} & -0.35562 & 2.72448 & -1.05254 \\ & & & \\ & & & \end{array}$

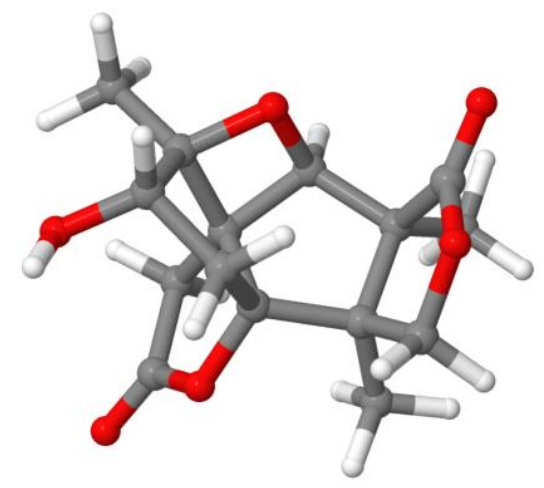




$\begin{array}{rrrr}\mathrm{H} & -0.52223 & 3.24479 & 0.62647 \\ \mathrm{H} & -3.32015 & 1.42968 & -1.45821 \\ \mathrm{H} & -1.88839 & 1.35031 & -2.50055 \\ \mathrm{H} & -2.99830 & -0.03151 & -2.40448\end{array}$

\section{Conformer 2}

Energy: -1033.76081 Hartree (Rel: $0.0 \mathrm{kcal} / \mathrm{mol}$ ) XYZ coordinates for conf 2 :

$\begin{array}{lrrr}\mathrm{C} & 2.19757 & 1.71856 & -0.32899 \\ \mathrm{C} & 0.78458 & -0.17297 & -0.73765 \\ \mathrm{C} & 1.76721 & 0.75110 & -1.42747 \\ \mathrm{C} & 1.10016 & -1.62936 & -0.32500 \\ \mathrm{C} & 1.41398 & -1.60279 & 1.18197 \\ \mathrm{C} & 0.52910 & -0.47643 & 1.72543 \\ \mathrm{C} & 0.39003 & 0.55727 & 0.58157 \\ \mathrm{C} & -1.05131 & 1.15198 & 0.34006 \\ \mathrm{C} & -1.70515 & 0.14721 & -0.68271 \\ \mathrm{C} & -0.54778 & -0.71415 & -1.25839 \\ \mathrm{C} & -1.99322 & 1.04625 & 1.55978 \\ \mathrm{O} & -2.69920 & -0.21286 & 1.44805 \\ \mathrm{C} & -2.64173 & -0.69878 & 0.18308 \\ \mathrm{O} & -0.30917 & -1.97620 & -0.56514 \\ \mathrm{C} & 2.02017 & -2.47192 & -1.18631 \\ \mathrm{O} & 2.77577 & -1.22234 & 1.38983 \\ \mathrm{O} & 1.38587 & 1.59251 & 0.75827 \\ \mathrm{C} & -0.98024 & 2.61121 & -0.13358 \\ \mathrm{O} & 3.10250 & 2.51727 & -0.35087 \\ \mathrm{C} & -2.53447 & 0.76953 & -1.82207 \\ \mathrm{O} & -3.29011 & -1.65970 & -0.15918 \\ \mathrm{H} & 1.31486 & 1.32955 & -2.24212 \\ \mathrm{H} & 2.64414 & 0.24650 & -1.84123 \\ \mathrm{H} & 1.19468 & -2.56662 & 1.66081 \\ \mathrm{H} & -0.44087 & -0.89027 & 2.00167 \\ \mathrm{H} & 0.96935 & -0.02008 & 2.61570 \\ \mathrm{H} & -0.64840 & -0.87859 & -2.33587 \\ \mathrm{H} & -2.74071 & 1.84484 & 1.55475 \\ \mathrm{H} & -1.48109 & 1.05300 & 2.52312 \\ \mathrm{H} & 1.73846 & -2.40315 & -2.24183 \\ \mathrm{H} & 1.95499 & -3.52384 & -0.88492 \\ \mathrm{H} & 3.06163 & -2.14839 & -1.09085 \\ \mathrm{H} & 3.34029 & -1.98342 & 1.18029 \\ \mathrm{H} & -1.98022 & 2.99248 & -0.35599 \\ \mathrm{H} & -0.37224 & 2.73243 & -1.03508 \\ \mathrm{H} & -0.54375 & 3.24082 & 0.64710 \\ \mathrm{H} & -3.33086 & 1.41905 & -1.44633 \\ \mathrm{H} & -1.90012 & 1.35855 & -2.49146 \\ \mathrm{H} & -2.99888 & -0.03259 & -2.40380 \\ & & & \end{array}$

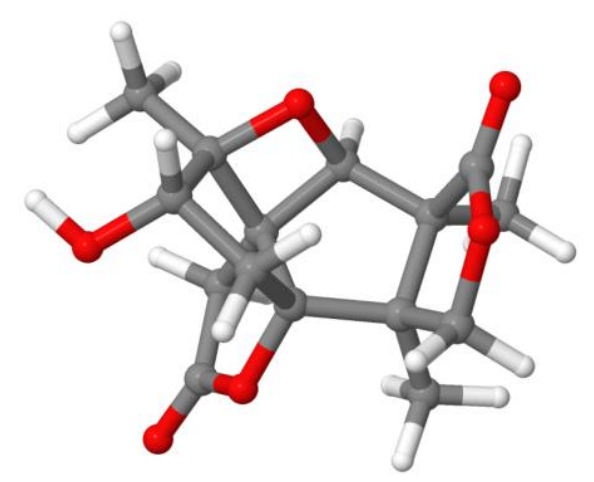


vibralactone, Fig.1<smiles>CC(C)=CC[C@]12C=C(CO)C[C@H]1OC2=O</smiles>

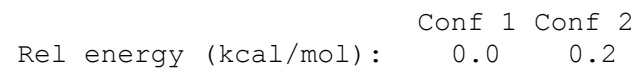

\begin{tabular}{|c|c|c|c|c|c|c|c|}
\hline C-nom & iGau & $\operatorname{Exp}$ & $\mathrm{Calc}$ & diff & 1 & 2 & \\
\hline $\mathrm{C} 1$ & 4 & 75.10 & 76.38 & 1.28 & 75.82 & $77.13]$ & \\
\hline C2 & 7 & 122.40 & 123.18 & 0.78 & {$[123.0$} & $123.35]$ & \\
\hline C3 & 6 & 146.60 & 148.21 & 1.61 & {$[148.3$} & $148.00]$ & \\
\hline C4 & 5 & 37.30 & 36.89 & -0.41 & {$[36.8]$} & $36.99]$ & \\
\hline C5 & 3 & 78.50 & 75.81 & -2.69 & 76.15 & $75.36]$ & \\
\hline $\mathrm{C} 7$ & 1 & 173.00 & 171.99 & -1.01 & {$[171.8]$} & $172.21]$ & \\
\hline $\mathrm{C} 8$ & 9 & 27.60 & 28.91 & 1.31 & {$\left[\begin{array}{ll}29.2 \\
2\end{array}\right.$} & $28.41]$ & \\
\hline C9 & 13 & 117.20 & 119.06 & 1.86 & {$[120.5$} & $117.03]$ & \\
\hline C10 & 14 & 136.00 & 136.64 & 0.64 & {$[134.4$} & $139.51]$ & \\
\hline C11 & 16 & 18.00 & 17.12 & -0.88 & {$[\quad 16.7$} & 17.67 ] & \\
\hline $\mathrm{C} 12$ & 15 & 25.80 & 25.26 & -0.54 & 25.1 & $25.43]$ & \\
\hline C13 & 11 & 61.30 & 61.46 & 0.16 & {$\left[\begin{array}{ll}61.4\end{array}\right.$} & $61.42]$ & \\
\hline & & & & $\begin{array}{l}\text { ts: RM } \\
\text { Fracti }\end{array}$ & $\begin{array}{l}1.29 \mathrm{ppm} \\
: \quad 0.57\end{array}$ & $\begin{array}{c}(\mathrm{MAE}=1.10) \\
0.429\end{array}$ & D) $\mathrm{N}=12 \quad\left\{\begin{array}{lll}-2.69 & 1.86\end{array}\right\}$ \\
\hline
\end{tabular}

\section{Conformer 1}

Energy: -692.51617 Hartree (Rel: $0.0 \mathrm{kcal} / \mathrm{mol}$ ) XYZ coordinates for conf 1 :

$\begin{array}{lrrr}\mathrm{C} & -0.11379 & 1.82101 & -0.19770 \\ \mathrm{O} & -0.26219 & 1.78591 & 1.16970 \\ \mathrm{C} & -0.27699 & 0.31251 & 1.18630 \\ \mathrm{C} & -0.21959 & 0.29091 & -0.36640 \\ \mathrm{C} & -1.62910 & -0.25558 & 1.62230 \\ \mathrm{C} & -2.38510 & -0.41698 & 0.31590 \\ \mathrm{C} & -1.62020 & -0.12938 & -0.74680 \\ \mathrm{H} & 0.59470 & -0.04999 & 1.73260 \\ \mathrm{C} & 0.92040 & -0.40329 & -1.13060 \\ \mathrm{O} & 0.04792 & 2.78031 & -0.89390 \\ \mathrm{C} & -3.82500 & -0.84797 & 0.28510 \\ \mathrm{O} & -4.31150 & -1.14977 & -1.01120 \\ \mathrm{C} & 2.29470 & 0.04320 & -0.70380 \\ \mathrm{C} & 3.26540 & -0.68471 & -0.12820 \\ \mathrm{C} & 4.59220 & -0.05242 & 0.22300 \\ \mathrm{C} & 3.16679 & -2.15291 & 0.21080 \\ \mathrm{H} & -1.51740 & -1.21398 & 2.14920 \\ \mathrm{H} & -2.12489 & 0.43282 & 2.32000 \\ \mathrm{H} & -1.96020 & -0.14938 & -1.77720 \\ \mathrm{H} & 0.79150 & -1.48629 & -1.03200 \\ \mathrm{H} & 0.78040 & -0.17119 & -2.19670 \\ \mathrm{H} & -3.96861 & -1.69577 & 0.97930 \\ \mathrm{H} & -4.46330 & -0.03716 & 0.66000 \\ \mathrm{H} & -3.77451 & -1.87927 & -1.35910 \\ \mathrm{H} & 2.50251 & 1.09600 & -0.89790 \\ \mathrm{H} & 4.80230 & -0.14542 & 1.29780 \\ \mathrm{H} & 4.62061 & 1.00968 & -0.03830 \\ \mathrm{H} & 5.41860 & -0.55482 & -0.29920 \\ \mathrm{H} & 3.34419 & -2.31401 & 1.28330 \\ \mathrm{H} & 3.94229 & -2.72371 & -0.31850 \\ \mathrm{H} & 2.19949 & -2.59560 & -0.03910\end{array}$

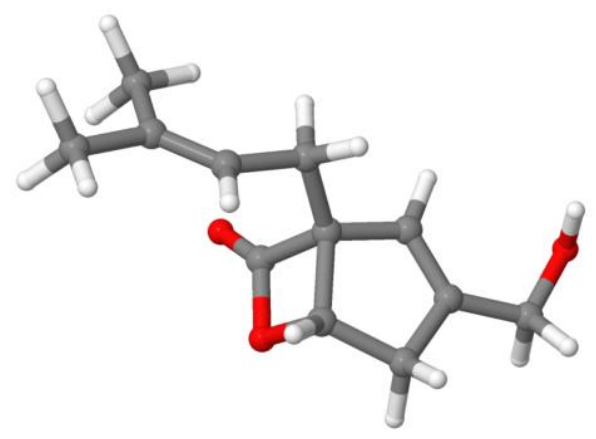




\begin{tabular}{|c|c|c|c|}
\hline $0.2 \mathrm{kcal} / \mathrm{mol}$ ) & $\begin{array}{l}692.51590 \\
\text { inates for }\end{array}$ & $\begin{array}{l}\text { ee }(\operatorname{Rel}: \\
2:\end{array}$ & $\mathrm{cal} / \mathrm{mol}$ ) \\
\hline C & -0.26060 & 0.52201 & 1.22050 \\
\hline 0 & 0.12330 & 1.79201 & 0.85490 \\
\hline $\mathrm{C}$ & 0.53610 & 1.34320 & -0.48500 \\
\hline C & 0.21629 & -0.12880 & -0.09390 \\
\hline $\mathrm{C}$ & 2.04580 & 1.46090 & -0.70540 \\
\hline $\mathrm{C}$ & 2.57769 & 0.11159 & -0.25900 \\
\hline $\mathrm{C}$ & 1.59089 & -0.73820 & 0.05870 \\
\hline $\mathrm{H}$ & -0.09050 & 1.82711 & -1.23530 \\
\hline $\mathrm{C}$ & -0.81761 & -0.98169 & -0.84950 \\
\hline 0 & -0.77631 & 0.17891 & 2.24400 \\
\hline $\mathrm{C}$ & 4.05249 & -0.17301 & -0.19680 \\
\hline 0 & 4.37059 & -1.53351 & 0.04000 \\
\hline $\mathrm{C}$ & -2.12401 & -0.27609 & -1.11910 \\
\hline $\mathrm{C}$ & -3.27511 & -0.38768 & -0.43590 \\
\hline $\mathrm{C}$ & -4.48911 & 0.41323 & -0.84390 \\
\hline $\mathrm{C}$ & -3.48601 & -1.27568 & 0.76540 \\
\hline H & 2.28860 & 1.66630 & -1.75780 \\
\hline $\mathrm{H}$ & 2.45230 & 2.29539 & -0.11820 \\
\hline H & 1.74029 & -1.74730 & 0.42910 \\
\hline H & -0.36531 & -1.29469 & -1.80070 \\
\hline $\mathrm{H}$ & -0.97352 & -1.89749 & -0.26860 \\
\hline H & 4.53749 & 0.20198 & -1.11620 \\
\hline H & 4.50340 & 0.38578 & 0.63370 \\
\hline $\mathrm{H}$ & 4.00318 & -2.04891 & -0.69550 \\
\hline H & -2.10880 & 0.41271 & -1.96490 \\
\hline $\mathrm{H}$ & -5.33271 & -0.24777 & -1.08750 \\
\hline H & -4.29050 & 1.04682 & -1.71420 \\
\hline $\mathrm{H}$ & -4.82710 & 1.05833 & -0.02130 \\
\hline $\mathrm{H}$ & -4.33102 & -1.95697 & 0.59570 \\
\hline H & -3.73781 & -0.66898 & 1.64480 \\
\hline $\mathrm{H}$ & -2.60952 & -1.87018 & 1.02910 \\
\hline
\end{tabular}

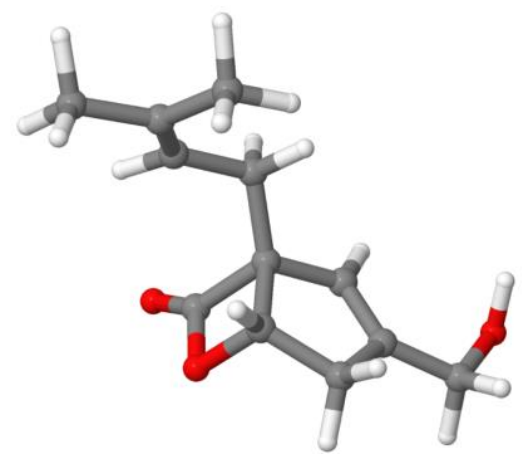


rubesanolide A, Fig.1

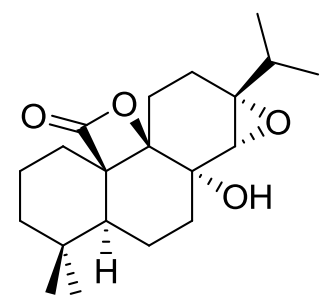

Rel energy (kcal/mol): $\quad \begin{array}{llll}0.0 & 0.7 & 1.1\end{array}$

\begin{tabular}{|c|c|c|c|c|c|c|c|c|c|}
\hline C-nom & iGau & $\operatorname{Exp}$ & Calc & $\operatorname{diff}$ & & 1 & 2 & 3 & \\
\hline $\mathrm{C} 1-\mathrm{CH} 2$ & 6 & 28.20 & 28.55 & 0.35 & [ & 28.58 & 28.46 & 28.56 & ] \\
\hline $\mathrm{C} 2-\mathrm{CH} 2$ & 1 & 19.10 & 19.58 & 0.48 & [ & 19.60 & 19.52 & 19.58 & ] \\
\hline $\mathrm{C} 3-\mathrm{CH} 2$ & 2 & 41.20 & 40.00 & -1.20 & [ & 40.00 & 39.94 & 40.11 & ] \\
\hline C4-C & 3 & 33.40 & 33.22 & -0.18 & [ & 33.23 & 33.25 & 33.12 & ] \\
\hline $\mathrm{C} 5-\mathrm{CH}$ & 4 & 42.20 & 42.34 & 0.14 & [ & 42.29 & 42.50 & 42.39 & ] \\
\hline $\mathrm{C} 6-\mathrm{CH} 2$ & 7 & 18.09 & 19.22 & 1.13 & [ & 19.21 & 19.23 & 19.30 & ] \\
\hline $\mathrm{C} 7-\mathrm{CH} 2$ & 8 & 29.20 & 30.88 & 1.68 & [ & 30.88 & 30.88 & 30.91 & ] \\
\hline $\mathrm{C} 8-\mathrm{C}$ & 9 & 67.80 & 69.06 & 1.26 & [ & 69.09 & 68.88 & 69.18 & ] \\
\hline $\mathrm{C} 9-\mathrm{C}$ & 10 & 80.40 & 80.94 & 0.54 & [ & 80.93 & 81.07 & 80.72 & \\
\hline $\mathrm{C} 10-\mathrm{C}$ & 5 & 61.20 & 62.04 & 0.84 & [ & 62.02 & 62.12 & 62.02 & \\
\hline $\mathrm{C} 11-\mathrm{CH} 2$ & 14 & 19.50 & 22.56 & 3.06 & [ & 22.42 & 22.89 & 22.84 & ] \\
\hline $\mathrm{C} 12-\mathrm{CH} 2$ & 13 & 19.50 & 21.24 & 1.74 & [ & 19.38 & 25.64 & 25.49 & ] \\
\hline C13-C & 12 & 68.10 & 68.00 & -0.10 & [ & 68.19 & 67.53 & 67.60 & \\
\hline $\mathrm{Cl} 4-\mathrm{CH}$ & 11 & 63.30 & 65.16 & 1.86 & [ & 66.70 & 60.54 & 63.57 & \\
\hline $\mathrm{C} 15-\mathrm{CH}$ & 20 & 34.30 & 35.60 & 1.30 & [ & 36.99 & 32.61 & 31.85 & \\
\hline $\mathrm{C} 16-\mathrm{CH} 3$ & 21 & 17.60 & 17.38 & -0.22 & & 16.87 & 19.36 & 17.01 & ] \\
\hline $\mathrm{C} 17-\mathrm{CH} 3$ & 22 & 18.13 & 17.59 & -0.54 & ] & 18.17 & 15.37 & 17.93 & ] \\
\hline $\mathrm{C} 18-\mathrm{CH} 3$ & 25 & 31.40 & 31.25 & -0.15 & ] & 31.26 & 31.23 & 31.20 & ] \\
\hline $\mathrm{C} 19-\mathrm{CH} 3$ & 24 & 20.20 & 20.76 & 0.56 & & 20.80 & 20.67 & 20.62 & \\
\hline C20-C & 16 & 174.10 & 175.42 & 1.32 & & 175.42 & 175.41 & 175.47 & \\
\hline
\end{tabular}

\section{Conformer 1}

Energy: -1081.05226 Hartree (Rel: $0.0 \mathrm{kcal} / \mathrm{mol}$ ) XYZ coordinates for conf 1:

$\begin{array}{llrr}\text { C } & 3.33538 & -1.69562 & -1.26472 \\ \mathrm{C} & 4.10102 & -0.37647 & -1.14291 \\ \mathrm{C} & 3.65532 & 0.49526 & 0.05789 \\ \mathrm{C} & 2.09978 & 0.69690 & -0.00824 \\ \mathrm{C} & 1.27277 & -0.60579 & -0.25142 \\ \mathrm{C} & 1.83377 & -1.43300 & -1.41914 \\ \mathrm{C} & 1.52722 & 1.46454 & 1.20261 \\ \mathrm{C} & 0.01866 & 1.80622 & 1.08316 \\ \mathrm{C} & -0.70478 & 1.12324 & -0.08400 \\ \mathrm{C} & -0.28034 & -0.35154 & -0.23517 \\ \mathrm{C} & -2.22614 & 1.25259 & 0.09201 \\ \mathrm{C} & -3.16982 & 0.16758 & -0.24516 \\ \mathrm{C} & -2.61761 & -1.15960 & -0.73511 \\ \mathrm{C} & -1.16743 & -1.07764 & -1.24172 \\ \mathrm{O} & -0.36726 & -1.07270 & 1.06524 \\ \mathrm{C} & 0.96129 & -1.41335 & 1.01685 \\ \mathrm{O} & 1.53539 & -2.14502 & 1.77635 \\ \mathrm{O} & -2.95413 & 1.28250 & -1.15398 \\ \mathrm{O} & -0.33953 & 1.79677 & -1.29597 \\ \mathrm{C} & -4.55604 & 0.18015 & 0.40013 \\ \mathrm{C} & -5.64537 & -0.29095 & -0.57694 \\ \mathrm{C} & -4.58361 & -0.61633 & 1.71519 \\ \mathrm{H} & 1.91514 & 1.30429 & -0.90284 \\ \mathrm{C} & 4.13805 & -0.14384 & 1.37867 \\ \mathrm{C} & 4.34962 & 1.86715 & -0.09255 \\ \mathrm{H} & 3.69655 & -2.26153 & -2.13272 \\ \mathrm{H} & 3.50805 & -2.32629 & -0.38457 \\ \mathrm{H} & 3.95630 & 0.19787 & -2.07070\end{array}$

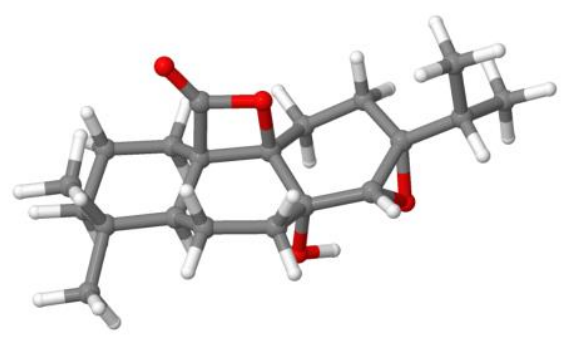




$\begin{array}{rrr}5.17948 & -0.56701 & -1.06261 \\ 1.65420 & -0.87212 & -2.34727 \\ 1.29952 & -2.38477 & -1.51225 \\ 2.08961 & 2.39123 & 1.34512 \\ 1.68934 & 0.87126 & 2.10956 \\ -0.12457 & 2.88242 & 0.93839 \\ -0.49291 & 1.53652 & 2.01389 \\ -2.54777 & 2.01918 & 0.79921 \\ -2.65820 & -1.87412 & 0.09415 \\ -3.26251 & -1.55252 & -1.53003 \\ -0.80257 & -2.09403 & -1.41242 \\ -1.11877 & -0.55401 & -2.20197 \\ -1.13155 & 1.75824 & -1.86415 \\ -4.76061 & 1.23303 & 0.63702 \\ -6.63911 & -0.14450 & -0.13889 \\ -5.60185 & 0.27429 & -1.51428 \\ -5.54558 & -1.35704 & -0.81315 \\ -5.54536 & -0.47804 & 2.22182 \\ -3.79094 & -0.29242 & 2.39934 \\ -4.45630 & -1.69108 & 1.54170 \\ 3.93579 & 0.50982 & 2.23444 \\ 5.22425 & -0.29402 & 1.33889 \\ 3.66842 & -1.10823 & 1.58569 \\ 4.01513 & 2.38892 & -0.99776 \\ 4.16512 & 2.52369 & 0.76439 \\ 5.43556 & 1.73157 & -0.16774\end{array}$

\section{Conformer 2}

Energy: -1081.05107 Hartree (Rel: $0.7 \mathrm{kcal} / \mathrm{mol}$ ) XYZ coordinates for conf 2:

\begin{tabular}{|c|c|c|c|}
\hline C & 3.47883 & -1.55261 & -1.18196 \\
\hline C & 4.14255 & -0.18171 & -1.03836 \\
\hline C & 3.59026 & 0.65922 & 0.14017 \\
\hline C & 2.02836 & 0.75082 & 0.00968 \\
\hline C & 1.30622 & -0.60966 & -0.25311 \\
\hline $\mathrm{C}$ & 1.96849 & -1.40087 & -1.39217 \\
\hline C & 1.35297 & 1.48593 & 1.18663 \\
\hline C & -0.16768 & 1.72601 & 0.99577 \\
\hline C & -0.79389 & 0.97722 & -0.18742 \\
\hline C & -0.26041 & -0.46592 & -0.29579 \\
\hline $\mathrm{C}$ & -2.32690 & 1.00862 & -0.07584 \\
\hline C & -3.17691 & -0.15011 & -0.41285 \\
\hline C & -2.51396 & -1.44200 & -0.86010 \\
\hline C & -1.05646 & -1.27151 & -1.31842 \\
\hline 0 & -0.34332 & -1.17246 & 1.01291 \\
\hline C & 1.00529 & -1.42696 & 1.01149 \\
\hline 0 & 1.59932 & -2.11512 & 1.79590 \\
\hline 0 & -3.00428 & 0.94848 & -1.34868 \\
\hline 0 & -0.42719 & 1.65732 & -1.39542 \\
\hline C & -4.57504 & -0.30583 & 0.20014 \\
\hline C & -4.47338 & -0.79658 & 1.65629 \\
\hline C & -5.43037 & 0.96560 & 0.10487 \\
\hline $\mathrm{H}$ & 1.83959 & 1.33615 & -0.89873 \\
\hline C & 4.06266 & 0.05707 & 1.48184 \\
\hline $\mathrm{C}$ & 4.19203 & 2.07609 & 0.01171 \\
\hline $\mathrm{H}$ & 3.91170 & -2.09240 & -2.03361 \\
\hline $\mathrm{H}$ & 3.66548 & -2.16712 & -0.29317 \\
\hline $\mathrm{H}$ & 3.99357 & 0.37875 & -1.97394 \\
\hline $\mathrm{H}$ & 5.22789 & -0.29512 & -0.91575 \\
\hline $\mathrm{H}$ & 1.78177 & -0.86038 & -2.33091 \\
\hline $\mathrm{H}$ & 1.50865 & -2.38964 & -1.49616 \\
\hline $\mathrm{H}$ & 1.84585 & 2.44893 & 1.34532 \\
\hline $\mathrm{H}$ & 1.51271 & 0.91075 & 2.10557 \\
\hline $\mathrm{H}$ & -0.37342 & 2.78795 & 0.82378 \\
\hline $\mathrm{H}$ & -0.70077 & 1.43869 & 1.90902 \\
\hline $\mathrm{H}$ & -2.72171 & 1.78065 & 0.58539 \\
\hline $\mathrm{H}$ & -2.54077 & -2.15182 & -0.02631 \\
\hline $\mathrm{H}$ & -3.11178 & -1.87907 & -1.67051 \\
\hline $\mathrm{H}$ & -0.61543 & -2.26280 & -1.45384 \\
\hline $\mathrm{H}$ & -1.00960 & -0.76481 & -2.28784 \\
\hline $\mathrm{H}$ & -1.18606 & 1.54726 & -1.99818 \\
\hline
\end{tabular}

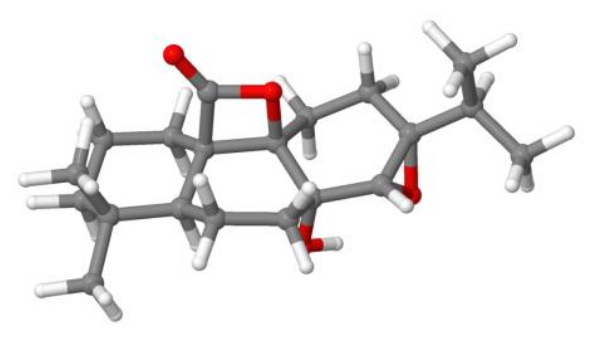




$\begin{array}{rrr}-5.08274 & -1.08842 & -0.38396 \\ -5.47161 & -0.99475 & 2.06227 \\ -3.88842 & -1.71774 & 1.74567 \\ -3.99832 & -0.03882 & 2.29091 \\ -6.43906 & 0.76056 & 0.48048 \\ -5.50808 & 1.32049 & -0.92574 \\ -5.01665 & 1.78005 & 0.71167 \\ 3.77677 & 0.69337 & 2.32688 \\ 5.15775 & -0.01219 & 1.48782 \\ 3.65684 & -0.93942 & 1.67071 \\ 3.85615 & 2.57347 & -0.90660 \\ 3.93063 & 2.71820 & 0.85954 \\ 5.28682 & 2.01643 & -0.02280\end{array}$

Conformer 3

Energy: -1081.05043 Hartree (Rel: $1.1 \mathrm{kcal} / \mathrm{mol}$ ) XYZ coordinates for conf 3 :

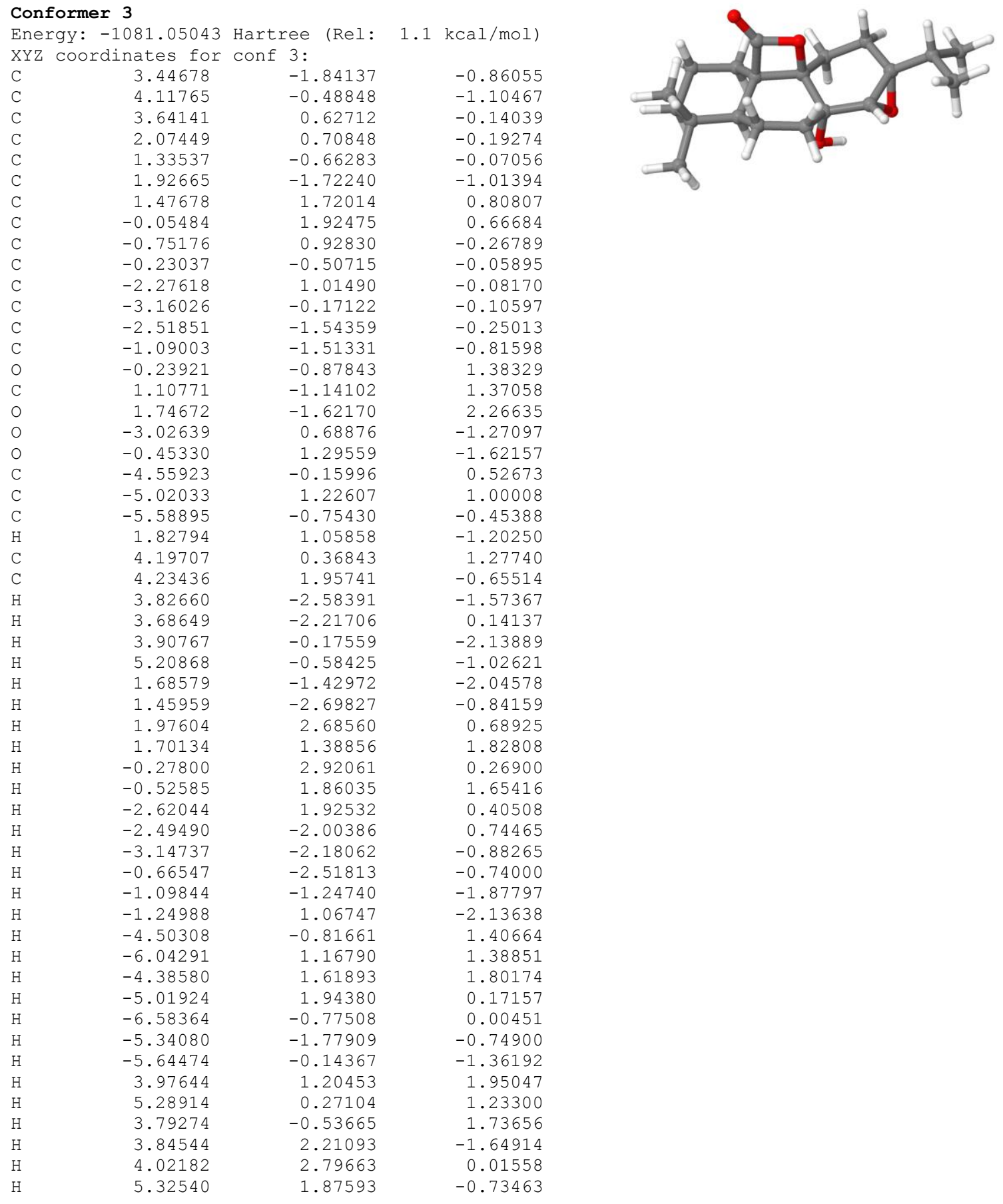


veranisatin A (PCM in acetone), Fig.1

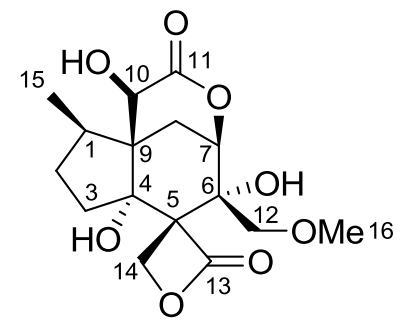

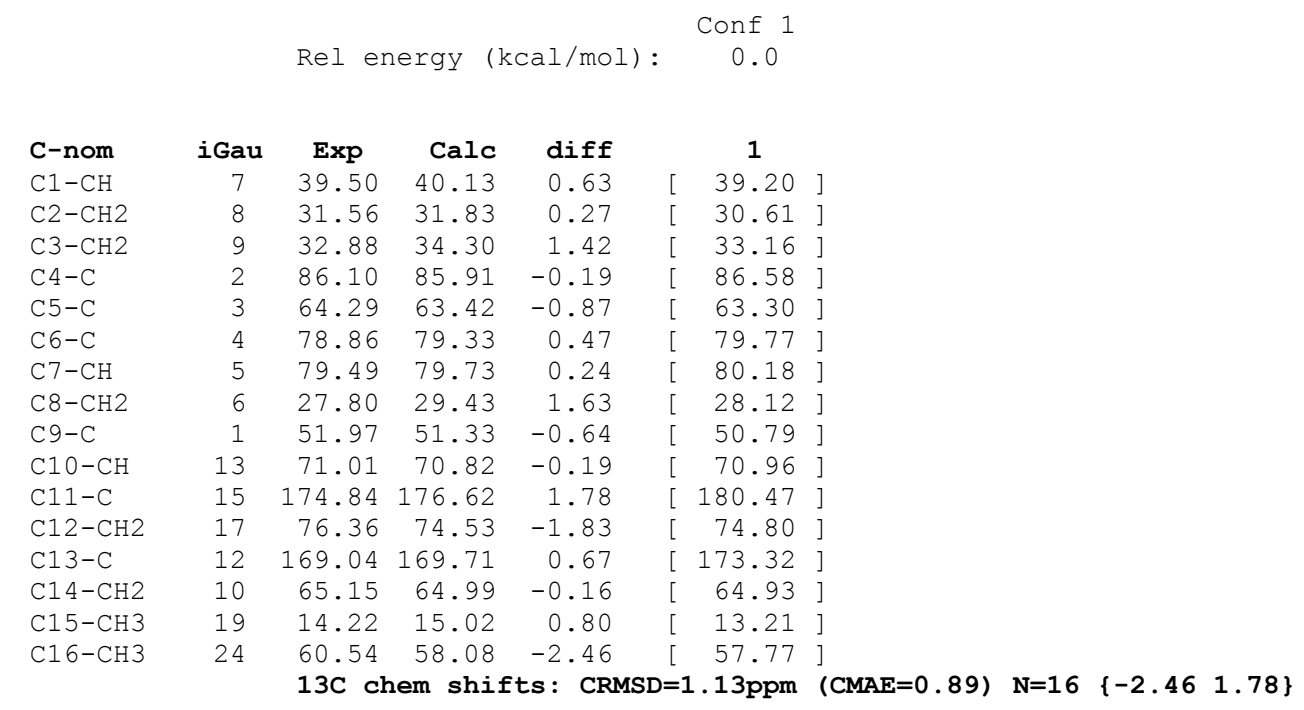

\section{Conformer 1}

Energy: -1224.68149 Hartree (Rel: $0.0 \mathrm{kcal} / \mathrm{mol}$ ) XYZ coordinates for conf 1 :

\begin{tabular}{|c|c|c|c|}
\hline C & -1.69482 & 0.06967 & -0.54856 \\
\hline C & -0.70773 & -1.06646 & -0.09061 \\
\hline C & 0.70253 & -0.51712 & 0.30131 \\
\hline C & 1.28334 & 0.34689 & -0.88409 \\
\hline C & 0.24206 & 1.35856 & -1.43195 \\
\hline C & -1.10812 & 0.74230 & -1.79870 \\
\hline C & -2.99926 & -0.75943 & -0.75148 \\
\hline C & -2.97563 & -1.81160 & 0.39974 \\
\hline C & -1.51801 & -1.87180 & 0.94023 \\
\hline C & 0.87203 & -0.02750 & 1.76590 \\
\hline 0 & 1.64866 & -1.23524 & 2.08677 \\
\hline C & 1.61188 & -1.65893 & 0.79070 \\
\hline C & -1.92022 & 1.24148 & 0.43588 \\
\hline 0 & 0.09785 & 2.40855 & -0.43200 \\
\hline C & -0.76091 & 2.23231 & 0.58677 \\
\hline 0 & -0.66541 & 2.92033 & 1.58462 \\
\hline C & 2.59088 & 1.07776 & -0.54359 \\
\hline 0 & -0.53797 & -1.98463 & -1.17887 \\
\hline C & -4.31169 & 0.02333 & -0.84195 \\
\hline 0 & 1.56722 & -0.52669 & -1.98995 \\
\hline 0 & 2.12228 & -2.64432 & 0.33257 \\
\hline 0 & -2.34380 & 0.84163 & 1.72378 \\
\hline 0 & 3.53934 & 0.07480 & -0.23432 \\
\hline C & 4.84661 & 0.58333 & 0.00080 \\
\hline $\mathrm{H}$ & 0.68528 & 1.86429 & -2.29130 \\
\hline $\mathrm{H}$ & -0.96766 & 0.01521 & -2.60160 \\
\hline $\mathrm{H}$ & -1.76107 & 1.53103 & -2.19002 \\
\hline $\mathrm{H}$ & -2.86048 & -1.29496 & -1.69585 \\
\hline $\mathrm{H}$ & -3.30959 & -2.78632 & 0.03091 \\
\hline
\end{tabular}

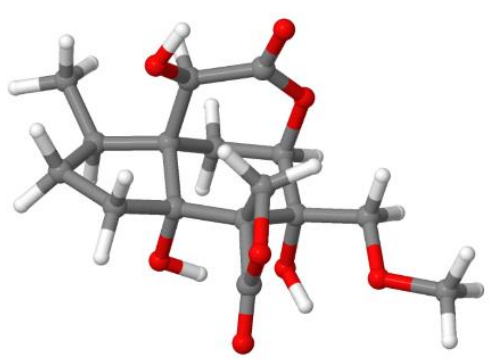




$\begin{array}{rrrr}\text { H } & -3.66058 & -1.51397 & 1.19928 \\ \mathrm{H} & -1.47043 & -1.42776 & 1.93394 \\ \mathrm{H} & -1.12644 & -2.89127 & 0.99353 \\ \mathrm{H} & -0.03126 & 0.02595 & 2.37005 \\ \mathrm{H} & 1.48877 & 0.85669 & 1.91939 \\ \mathrm{H} & -2.70573 & 1.86020 & -0.03356 \\ \mathrm{H} & 2.90598 & 1.64796 & -1.43081 \\ \mathrm{H} & 2.47123 & 1.78538 & 0.28485 \\ \mathrm{H} & 0.20203 & -1.66704 & -1.73490 \\ \mathrm{H} & -5.13955 & -0.66407 & -1.05174 \\ \mathrm{H} & -4.29243 & 0.76494 & -1.65032 \\ \mathrm{H} & -4.54435 & 0.53982 & 0.09610 \\ \mathrm{H} & 2.40209 & -0.98147 & -1.76349 \\ \mathrm{H} & -2.19857 & 1.61610 & 2.29993 \\ \mathrm{H} & 5.48434 & -0.27382 & 0.22525 \\ \mathrm{H} & 5.23267 & 1.10473 & -0.88601 \\ \mathrm{H} & 4.85277 & 1.27631 & 0.85310\end{array}$


2-oxatetracyclo[4.4.0.0 $\left.0^{1,4} \cdot 0^{6,8}\right]$ decane (cmpd 47), Fig.2

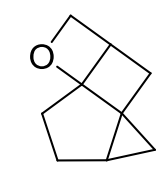

$$
\text { Rel energy (kcal/mol): } \begin{gathered}
\text { Conf } 1 \\
0.0
\end{gathered}
$$

\begin{tabular}{|c|c|c|c|c|c|}
\hline C-nom & iGau & Exp & Calc & diff & 1 \\
\hline $\mathrm{C}$ & 5 & 106.30 & 104.96 & $-1 \cdot 34$ & [ 104.96$]$ \\
\hline C & 8 & 75.80 & 75.82 & 0.02 & {$\left[\begin{array}{lll}75.82 & ]\end{array}\right.$} \\
\hline $\mathrm{C}$ & 7 & 40.80 & 40.84 & 0.04 & $40.84]$ \\
\hline C & 4 & 39.30 & 39.25 & -0.05 & $39.25]$ \\
\hline C & 6 & 32.80 & 32.78 & -0.02 & $32.78]$ \\
\hline $\mathrm{C}$ & 1 & 31.80 & 31.77 & -0.03 & $31.77]$ \\
\hline C & 2 & 26.30 & 26.22 & -0.08 & $26.22]$ \\
\hline $\mathrm{C}$ & 3 & 25.80 & 25.79 & -0.01 & $25.79]$ \\
\hline $\mathrm{C}$ & 10 & 13.70 & 14.44 & 0.74 & $14.44]$ \\
\hline
\end{tabular}

\section{Conformer 1}

Energy: -425.29362 Hartree (Rel: $0.0 \mathrm{kcal} / \mathrm{mol})$ XYZ coordinates for conf 1 :

$\begin{array}{lrrr}\mathrm{C} & -0.64162 & -1.63463 & -0.11504 \\ \mathrm{C} & -1.96892 & -0.96275 & -0.55920 \\ \mathrm{C} & -1.81452 & 0.53341 & -0.25810 \\ \mathrm{C} & -0.39470 & 0.81913 & 0.16371 \\ \mathrm{C} & 0.36009 & -0.49751 & 0.05228 \\ \mathrm{C} & 0.68509 & 1.59114 & -0.60502 \\ \mathrm{C} & 1.37303 & 0.21816 & -0.91149 \\ \mathrm{C} & 2.42341 & -0.20387 & 0.12460 \\ \mathrm{O} & 1.41746 & -0.75552 & 1.00936 \\ \mathrm{C} & -1.50433 & 1.04172 & 1.13982 \\ \mathrm{H} & -0.74169 & -2.11151 & 0.86854 \\ \mathrm{H} & -0.30576 & -2.41391 & -0.80783 \\ \mathrm{H} & -2.11565 & -1.08702 & -1.63920 \\ \mathrm{H} & -2.84238 & -1.41161 & -0.06984 \\ \mathrm{H} & -2.33426 & 1.23123 & -0.91222 \\ \mathrm{H} & 1.30863 & 2.25981 & -0.00020 \\ \mathrm{H} & 0.32755 & 2.14444 & -1.47990 \\ \mathrm{H} & 1.52832 & -0.05337 & -1.95752 \\ \mathrm{H} & 3.11947 & -0.98338 & -0.21477 \\ \mathrm{H} & 2.99436 & 0.60639 & 0.59662 \\ \mathrm{H} & -1.80875 & 2.06351 & 1.35708 \\ \mathrm{H} & -1.57467 & 0.37076 & 1.99512 \\ & & & \end{array}$

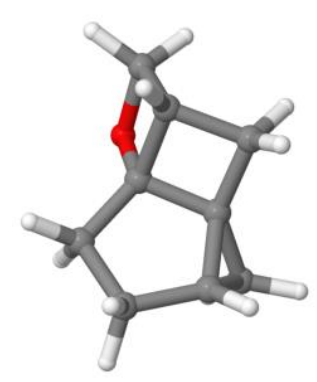


minor cmpd 48 -anti isomer (incorrect), Fig.2

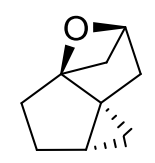

$$
\text { Rel energy (kcal/mol): } \begin{gathered}
\text { Conf } 1 \\
0.0
\end{gathered}
$$

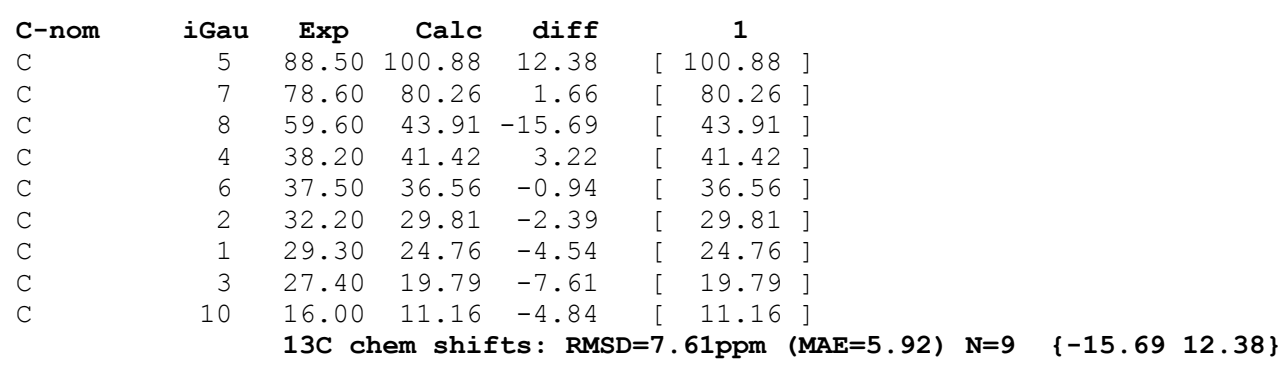

\section{Conformer 1}

Energy: -425.31030 Hartree (Rel: $0.0 \mathrm{kcal} / \mathrm{mol}$ ) XYZ coordinates for conf 1 :

$\begin{array}{rrrr}\mathrm{C} & -1.00014 & -1.48666 & 0.38040 \\ \mathrm{C} & -2.01831 & -0.71267 & -0.51285 \\ \mathrm{C} & -1.56318 & 0.75204 & -0.49606 \\ \mathrm{C} & -0.15515 & 0.79520 & 0.03408 \\ \mathrm{C} & 0.26207 & -0.65951 & 0.26747 \\ \mathrm{C} & 1.18191 & 1.38898 & -0.42843 \\ \mathrm{C} & 1.98394 & 0.06165 & -0.30488 \\ \mathrm{C} & 1.57705 & -0.54888 & 1.06347 \\ \mathrm{O} & 1.07658 & -0.89201 & -0.94469 \\ \mathrm{C} & -1.26757 & 1.38080 & 0.85444 \\ \mathrm{H} & -1.33181 & -1.51374 & 1.42508 \\ \mathrm{H} & -0.84960 & -2.52040 & 0.05651 \\ \mathrm{H} & -1.96570 & -1.09024 & -1.54069 \\ \mathrm{H} & -3.05109 & -0.84446 & -0.16737 \\ \mathrm{H} & -1.89873 & 1.40390 & -1.29933 \\ \mathrm{H} & 1.55612 & 2.18654 & 0.22366 \\ \mathrm{H} & 1.17699 & 1.74721 & -1.46274 \\ \mathrm{H} & 3.00510 & 0.02553 & -0.68956 \\ \mathrm{H} & 2.06287 & -1.51315 & 1.23011 \\ \mathrm{H} & 1.59700 & 0.07638 & 1.96318 \\ \mathrm{H} & -1.32166 & 2.46634 & 0.90426 \\ \mathrm{H} & -1.59580 & 0.88651 & 1.76859\end{array}$

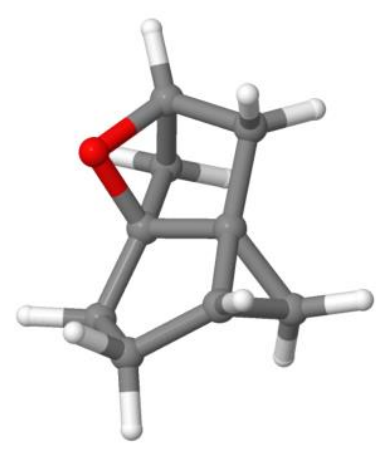


minor cmpd 48 - syn isomer (incorrect), Fig.2<smiles>CC1(C)C2CCCC1(O)CC2</smiles>

Rel energy (kcal/mol): $\begin{gathered}\text { Conf } 1 \\ 0.0\end{gathered}$

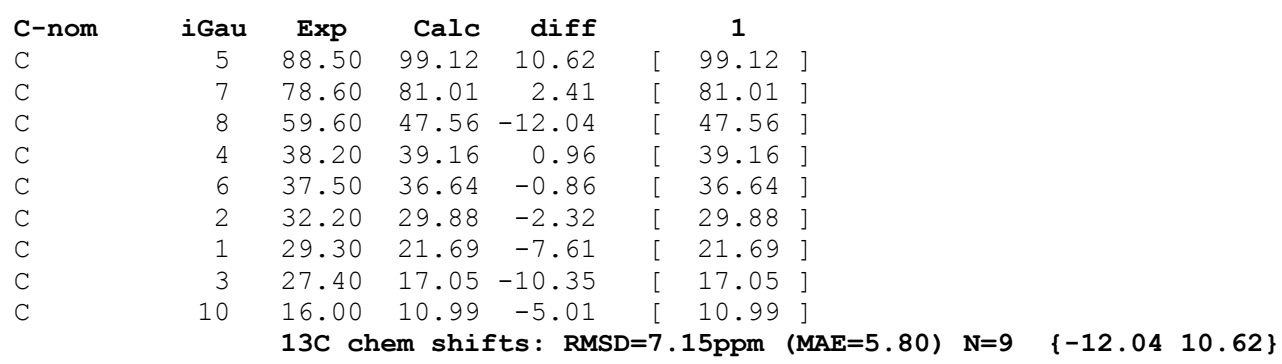

\section{Conformer 1}

Energy: -425.30243 Hartree (Rel: $0.0 \mathrm{kcal} / \mathrm{mol}$ ) XYZ coordinates for conf 1 :

$\begin{array}{lrrr}\text { C } & 1.03120 & -1.42175 & -0.50148 \\ \mathrm{C} & 2.03173 & -0.71894 & 0.47487 \\ \mathrm{C} & 1.56323 & 0.74463 & 0.58695 \\ \mathrm{C} & 0.14738 & 0.78960 & 0.06389 \\ \mathrm{C} & -0.24297 & -0.65661 & -0.22346 \\ \mathrm{C} & -1.23703 & 1.37788 & 0.37473 \\ \mathrm{C} & -2.02499 & 0.05906 & 0.09725 \\ \mathrm{C} & -1.24034 & -0.97834 & 0.91604 \\ \mathrm{O} & -1.39862 & -0.49578 & -1.11898 \\ \mathrm{C} & 1.25254 & 1.41443 & -0.73558 \\ \mathrm{H} & 1.33639 & -1.28512 & -1.54478 \\ \mathrm{H} & 0.95161 & -2.49735 & -0.31890 \\ \mathrm{H} & 1.98198 & -1.19822 & 1.46103 \\ \mathrm{H} & 3.06877 & -0.80191 & 0.12770 \\ \mathrm{H} & 1.92918 & 1.34451 & 1.41719 \\ \mathrm{H} & -1.51049 & 2.17802 & -0.31939 \\ \mathrm{H} & -1.38329 & 1.73266 & 1.40135 \\ \mathrm{H} & -3.11450 & 0.09498 & 0.04609 \\ \mathrm{H} & -1.65217 & -1.98562 & 0.81785 \\ \mathrm{H} & -0.96683 & -0.74920 & 1.95220 \\ \mathrm{H} & 1.26674 & 2.50253 & -0.73896 \\ \mathrm{H} & 1.59703 & 0.97123 & -1.66881\end{array}$

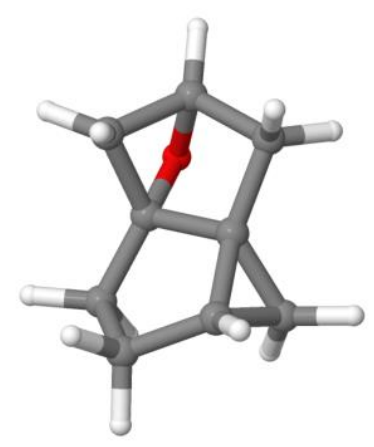


revised cmpd 48 - the oxametathesis product, Fig.2

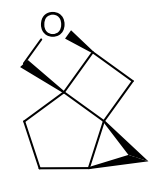

Rel energy (kcal/mol): $\begin{gathered}\text { Conf } 1 \\ 0.0\end{gathered}$

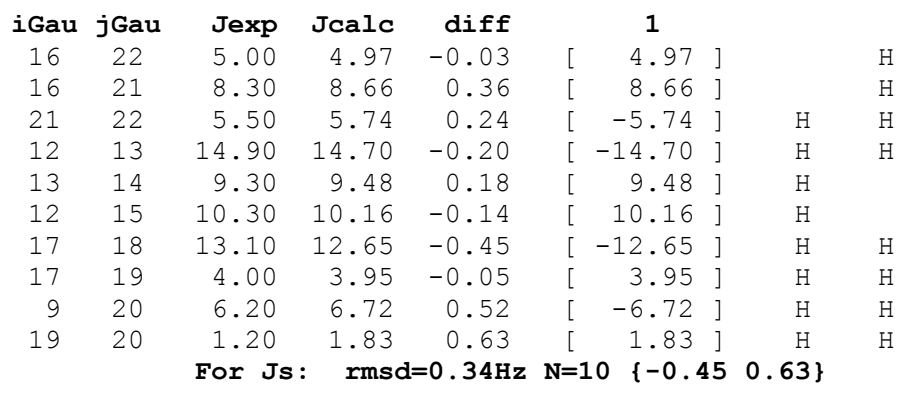

$\begin{array}{lcccccc}\text { H-nom } & \text { iGau } & \text { Exp } & \text { Calc } & \text { diff } & 1 \\ \mathrm{H} & 22 & 0.52 & 0.84 & 0.32 & {[} & 0.84] \\ \mathrm{H} & 21 & 0.97 & 1.13 & 0.16 & {[} & 1.13] \\ \mathrm{H} & 13 & 1.34 & 1.28 & -0.06 & {[} & 1.28] \\ \mathrm{H} & 12 & 2.29 & 2.24 & -0.05 & {[} & 2.24] \\ \mathrm{H} & 17 & 2.57 & 2.45 & -0.12 & {[} & 2.45 \\ \mathrm{H} & 18 & 2.75 & 2.83 & 0.08 & {[} & 2.83] \\ \mathrm{H} & 9 & 4.77 & 4.64 & -0.13 & {[} & 4.64] \\ \mathrm{H} & 20 & 4.97 & 4.97 & 0.00 & {[} & 4.97] \\ \mathrm{H} & 19 & 5.31 & 5.13 & -0.18 & {[} & 5.13]\end{array}$

$1 \mathrm{H}$ chem shifts: $\mathrm{RMSD}=0.15 \mathrm{ppm}(\mathrm{MAE}=0.12) \quad \mathrm{N}=9 \quad\left\{\begin{array}{lll}-0.18 & 0.32\end{array}\right\}$

$\mathrm{m}=1.000 \quad \mathrm{~b}=0.00$

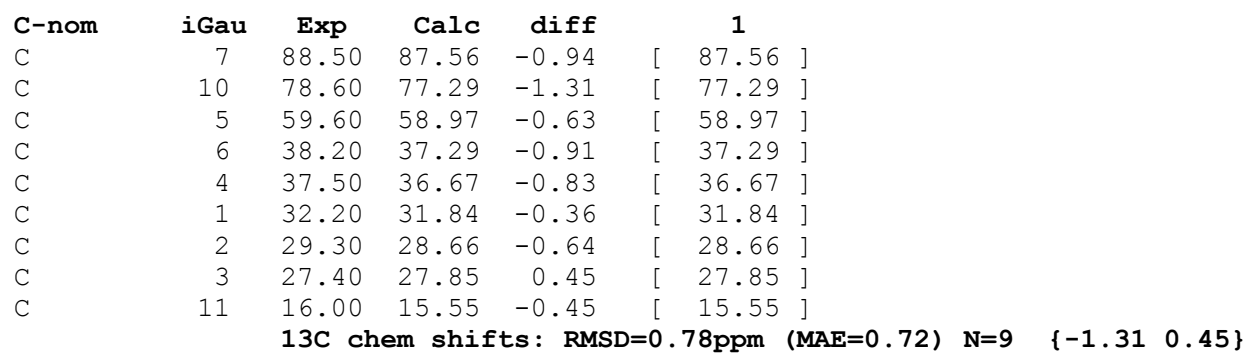

\section{Conformer 1}

Energy: -425.28973 Hartree (Rel: $0.0 \mathrm{kcal} / \mathrm{mol}$ ) XYZ coordinates for conf 1 :

$\begin{array}{lrrr}\mathrm{C} & -0.68683 & 1.64160 & -0.15053 \\ \mathrm{C} & -2.00958 & 0.92759 & -0.55700 \\ \mathrm{C} & -1.81686 & -0.55549 & -0.23331 \\ \mathrm{C} & -0.38860 & -0.80094 & 0.18197 \\ \mathrm{C} & 0.34494 & 0.53387 & 0.07032 \\ \mathrm{C} & 0.68507 & -1.56457 & -0.60601 \\ \mathrm{C} & 1.36069 & -0.18547 & -0.88668 \\ \mathrm{O} & 2.45814 & 0.08102 & 0.00748 \\ \mathrm{H} & 1.87986 & 1.81156 & 1.06446 \\ \mathrm{C} & 1.58953 & 0.75765 & 0.95059 \\ \mathrm{C} & -1.48830 & -1.04694 & 1.16798 \\ \mathrm{H} & -0.36947 & 2.38303 & -0.89326 \\ \mathrm{H} & -0.81620 & 2.18947 & 0.79289 \\ \mathrm{H} & -2.88172 & 1.37062 & -0.06008 \\ \mathrm{H} & -2.18020 & 1.02729 & -1.63602 \\ \mathrm{H} & -2.31616 & -1.27551 & -0.87923\end{array}$

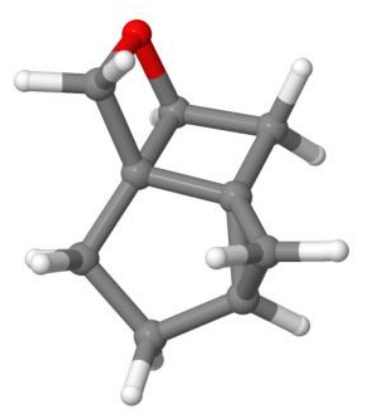




$\begin{array}{rrrr}\mathrm{H} & 0.31698 & -2.11441 & -1.47953 \\ \mathrm{H} & 1.34554 & -2.21323 & -0.02200 \\ \mathrm{H} & 1.57900 & 0.09467 & -1.92271 \\ \mathrm{H} & 1.58552 & 0.27856 & 1.93924 \\ \mathrm{H} & -1.76873 & -2.07338 & 1.39482 \\ \mathrm{H} & -1.57991 & -0.37068 & 2.01768\end{array}$


originally assigned structure of cmpd 7, Fig.3<smiles>CC(C)(C)OC(=O)N1CC2C3C(=O)CCCC23C1</smiles>

Rel energy (kcal/mol): $\begin{array}{ccc}\text { Conf } 1 & \text { Conf } 2 \\ 0.0 & 0.9\end{array}$

\begin{tabular}{|c|c|c|c|c|c|c|}
\hline C-nom & iGau & Exp & Calc & $\operatorname{diff}$ & 1 & 2 \\
\hline C & 2 & 206.82 & 209.58 & 2.76 & [ 209.62 & 209.37 \\
\hline $\mathrm{C}$ & 3 & 156.43 & 158.27 & 1.84 & [ 158.27 & 158.27 \\
\hline $\mathrm{C}$ & 27 & 79.47 & 79.10 & -0.37 & {$\left[\begin{array}{l}79.05 \\
0\end{array}\right.$} & 79.34 \\
\hline $\mathrm{C}$ & 5 & 77.43 & 74.91 & -2.52 & 74.97 & 74.61 \\
\hline $\mathrm{C}$ & 4 & 58.86 & 60.49 & 1.63 & 60.35 & 61.18 \\
\hline $\mathrm{C}$ & 13 & 47.90 & 51.96 & 4.06 & 51.98 & 51.86 \\
\hline $\mathrm{C}$ & 8 & 43.50 & 39.42 & -4.08 & 39.49 & 39.11 \\
\hline $\mathrm{C}$ & 10 & 39.97 & 38.87 & -1.10 & 38.88 & 38.81 \\
\hline C & 9 & 38.24 & 37.43 & -0.81 & 37.49 & 37.17 \\
\hline $\mathrm{C}$ & 30 & 28.33 & 26.74 & -1.59 & 26.73 & 26.80 \\
\hline C & 31 & 28.33 & 26.74 & -1.59 & 26.73 & 26.80 \\
\hline $\mathrm{C}$ & 32 & 28.33 & 26.74 & -1.59 & 26.73 & 26.80 \\
\hline C & 6 & 28.15 & 26.50 & -1.65 & 26.35 & 27.22 \\
\hline C & 7 & 25.36 & 24.29 & -1.07 & 24.31 & 24.18 \\
\hline
\end{tabular}

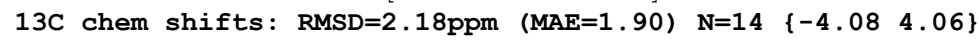

Fractions: $0.826 \quad 0.174$

Conformer 1 (enantiomer is computed)

Energy: -826.53460 Hartree (Rel: $0.0 \mathrm{kcal} / \mathrm{mol}$ ) XYZ coordinates for conf 1 :

$\begin{array}{lrrr}\mathrm{O} & -4.48837 & -1.24830 & -0.15309 \\ \mathrm{C} & -3.63972 & -0.42551 & -0.44076 \\ \mathrm{C} & 1.26180 & 0.37569 & -0.01214 \\ \mathrm{C} & -2.16888 & -0.76583 & -0.60661 \\ \mathrm{C} & -1.25124 & 0.21344 & 0.20036 \\ \mathrm{C} & -1.55937 & 1.69357 & 0.12492 \\ \mathrm{C} & -3.07378 & 1.93104 & 0.29782 \\ \mathrm{C} & -3.93731 & 1.05524 & -0.64365 \\ \mathrm{C} & -1.53285 & -1.81826 & 0.33734 \\ \mathrm{C} & -1.54410 & -0.70746 & 1.43334 \\ \mathrm{~N} & 0.08316 & -0.28984 & -0.21608 \\ \mathrm{O} & 1.35832 & 1.56699 & 0.24859 \\ \mathrm{C} & -0.06429 & -1.76859 & -0.12702 \\ \mathrm{O} & 2.30149 & -0.48113 & -0.18935 \\ \mathrm{H} & -1.93607 & -0.84027 & -1.67869 \\ \mathrm{H} & -0.99059 & 2.25381 & 0.87065 \\ \mathrm{H} & -1.23409 & 2.06629 & -0.85489 \\ \mathrm{H} & -3.29856 & 2.98728 & 0.11189 \\ \mathrm{H} & -3.36710 & 1.73286 & 1.33707 \\ \mathrm{H} & -5.00617 & 1.22552 & -0.48509 \\ \mathrm{H} & -3.70127 & 1.31669 & -1.68659 \\ \mathrm{H} & -1.97752 & -2.80152 & 0.49291 \\ \mathrm{H} & -0.78077 & -0.74107 & 2.21783 \\ \mathrm{H} & -2.53442 & -0.56501 & 1.87169 \\ \mathrm{H} & 0.10415 & -2.24744 & -1.09784 \\ \mathrm{H} & 0.63176 & -2.19581 & 0.60047 \\ \mathrm{C} & 3.69200 & -0.01361 & -0.10105 \\ \mathrm{H} & 5.56402 & -1.07985 & -0.34134 \\ \mathrm{H} & 4.24615 & -1.69592 & -1.36238 \\ \mathrm{C} & 4.49014 & -1.29206 & -0.37436 \\ \mathrm{C} & 3.96610 & 1.03688 & -1.18335 \\ \mathrm{C} & 3.98446 & 0.51472 & 1.30832 \\ \mathrm{H} & 4.26512 & -2.05716 & 0.37610 \\ \mathrm{H} & 5.03071 & 1.29732 & -1.18248 \\ \mathrm{H} & 3.71496 & 0.63794 & -2.17256 \\ \mathrm{H} & 3.38161 & 1.94111 & -1.00754 \\ & & & \end{array}$

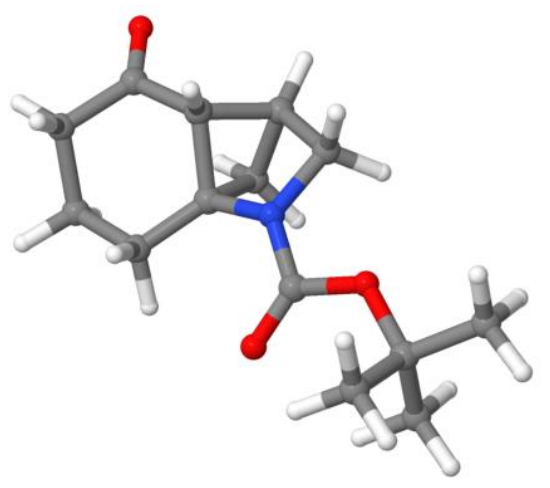




$\begin{array}{rrrr}\mathrm{H} & 5.05118 & 0.74927 & 1.39995 \\ \mathrm{H} & 3.73959 & -0.24671 & 2.05757 \\ \mathrm{H} & 3.40578 & 1.41549 & 1.51768\end{array}$

\section{Conformer 2}

Energy: -826.53313 Hartree (Rel: $0.9 \mathrm{kcal} / \mathrm{mol}$ ) XYZ coordinates for conf 2:

\begin{tabular}{lrrr} 
O & -4.58817 & -0.15575 & -0.00043 \\
$\mathrm{C}$ & -3.51156 & 0.28991 & -0.34947 \\
$\mathrm{C}$ & 1.36624 & -0.96145 & -0.22129 \\
$\mathrm{C}$ & -2.28511 & -0.57459 & -0.58207 \\
$\mathrm{C}$ & -1.02002 & -0.01316 & 0.15346 \\
$\mathrm{C}$ & -0.76731 & 1.47795 & 0.07073 \\
$\mathrm{C}$ & -2.07438 & 2.26013 & 0.31563 \\
$\mathrm{C}$ & -3.24768 & 1.77433 & -0.56921 \\
$\mathrm{C}$ & -2.03824 & -1.78972 & 0.34760 \\
$\mathrm{C}$ & -1.56372 & -0.75933 & 1.41867 \\
$\mathrm{~N}$ & 0.00011 & -0.98588 & -0.33101 \\
$\mathrm{O}$ & 2.05029 & -1.96936 & -0.33056 \\
$\mathrm{C}$ & -0.69242 & -2.29648 & -0.20168 \\
$\mathrm{O}$ & 1.83703 & 0.29531 & -0.02620 \\
$\mathrm{H}$ & -2.15794 & -0.73193 & -1.66292 \\
$\mathrm{H}$ & -0.00163 & 1.78954 & 0.78575 \\
$\mathrm{H}$ & -0.37691 & 1.71054 & -0.92828 \\
$\mathrm{H}$ & -1.89857 & 3.32553 & 0.12864 \\
$\mathrm{H}$ & -2.36592 & 2.17686 & 1.37070 \\
$\mathrm{H}$ & -4.16578 & 2.33197 & -0.36284 \\
$\mathrm{H}$ & -2.98395 & 1.92871 & -1.62660 \\
$\mathrm{H}$ & -2.81207 & -2.53053 & 0.54941 \\
$\mathrm{H}$ & -0.82378 & -1.08186 & 2.15886 \\
$\mathrm{H}$ & -2.39859 & -0.25388 & 1.90997 \\
$\mathrm{H}$ & -0.77702 & -2.80001 & -1.17082 \\
$\mathrm{H}$ & -0.15443 & -2.95410 & 0.48716 \\
$\mathrm{C}$ & 3.28525 & 0.55410 & 0.01882 \\
$\mathrm{H}$ & 4.39219 & 2.39990 & 0.26976 \\
$\mathrm{H}$ & 2.85884 & 2.35566 & 1.16364 \\
$\mathrm{C}$ & 3.34876 & 2.07042 & 0.22648 \\
$\mathrm{C}$ & 3.91687 & -0.17970 & 1.20754 \\
$\mathrm{C}$ & 3.93225 & 0.16464 & -1.31511 \\
$\mathrm{H}$ & 2.85403 & 2.59596 & -0.59698 \\
$\mathrm{H}$ & 4.96676 & 0.11846 & 1.30862 \\
$\mathrm{H}$ & 3.39853 & 0.08554 & 2.13601 \\
$\mathrm{H}$ & 3.86777 & -1.26078 & 1.07119 \\
$\mathrm{H}$ & 4.98610 & 0.46584 & -1.31466 \\
$\mathrm{H}$ & 3.43240 & 0.67810 & -2.14416 \\
$\mathrm{H}$ & 3.87255 & -0.91226 & -1.47844 \\
& & & \\
& & & \\
\hline
\end{tabular}

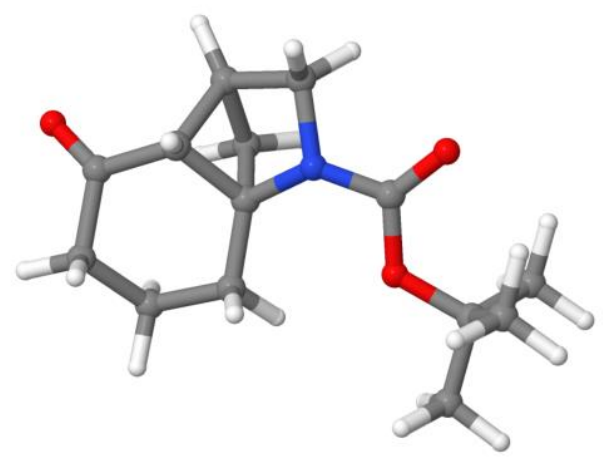


revised structure for $\mathrm{cmpd} 7$ (epi- $\alpha-\mathrm{CO})$, Fig.3<smiles>CC(C)(C)OC(=O)N1CC2CC13CCCC(=O)C23</smiles>

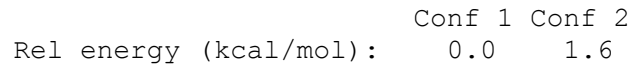

\begin{tabular}{|c|c|c|c|c|c|c|c|}
\hline C-nom & iGau & Exp & Calc & diff & 1 & 2 & \\
\hline $\mathrm{C}$ & 2 & 206.82 & 206.58 & -0.24 & {$[206.63$} & $205.87]$ & \\
\hline C & 3 & 156.43 & 158.40 & 1.97 & [ 158.35 & 159.17 ] & \\
\hline C & 27 & 79.47 & 79.36 & -0.11 & {$\left[\begin{array}{l}79.34 \\
0\end{array}\right.$} & $79.61]$ & \\
\hline C & 5 & 77.43 & 77.49 & 0.06 & 77.51 & $77.25]$ & \\
\hline C & 4 & 58.86 & 58.33 & -0.53 & 58.26 & $59.27]$ & \\
\hline C & 13 & 47.90 & 47.43 & -0.47 & 47.39 & $48.00]$ & \\
\hline C & 10 & 43.50 & 43.91 & 0.41 & 43.93 & $43.63]$ & \\
\hline C & 8 & 39.97 & 38.95 & -1.02 & 38.96 & $38.83]$ & \\
\hline C & 9 & 38.24 & 38.50 & 0.26 & 38.51 & $38.36]$ & \\
\hline C & 30 & 28.33 & 26.61 & -1.72 & 26.60 & $26.75]$ & \\
\hline C & 31 & 28.33 & 26.61 & -1.72 & 26.60 & $26.75]$ & \\
\hline C & 32 & 28.33 & 26.61 & -1.72 & 26.60 & $26.75]$ & \\
\hline C & 6 & 28.15 & 27.53 & -0.62 & 27.45 & $28.69]$ & \\
\hline C & 7 & 25.36 & 25.98 & 0.62 & 26.00 & $25.67]$ & \\
\hline & & $13 \mathrm{C} c$ & & $\begin{array}{l}\text { Es: RMs } \\
\text { ractic }\end{array}$ & $\begin{array}{l}=1.05 \mathrm{ppm} \\
: \quad 0.933\end{array}$ & $\begin{array}{c}(\mathrm{MAE}=0.82 \\
0.067\end{array}$ & $\mathrm{~N}=14 \quad\left\{\begin{array}{lll}-1.72 & 1.97\end{array}\right\}$ \\
\hline
\end{tabular}

\section{Conformer 1}

Energy: -826.53065 Hartree (Rel: $0.0 \mathrm{kcal} / \mathrm{mol}$ ) XYZ coordinates for conf 1 :

$\begin{array}{lrrr}\mathrm{O} & -2.53504 & -0.38170 & 2.36386 \\ \mathrm{C} & -2.68455 & 0.04970 & 1.23709 \\ \mathrm{C} & 0.91001 & 0.23166 & -0.40424 \\ \mathrm{C} & -2.59509 & -0.76134 & -0.03514 \\ \mathrm{C} & -1.53154 & -0.15679 & -0.99795 \\ \mathrm{C} & -1.82890 & 1.26473 & -1.42527 \\ \mathrm{C} & -2.00101 & 2.12801 & -0.15197 \\ \mathrm{C} & -2.95974 & 1.52288 & 0.91334 \\ \mathrm{C} & -1.80264 & -2.07099 & -0.29568 \\ \mathrm{C} & -1.60865 & -1.51760 & -1.75606 \\ \mathrm{~N} & -0.29850 & -0.36826 & -0.17183 \\ \mathrm{O} & 1.07871 & 1.23394 & -1.08726 \\ \mathrm{C} & -0.44277 & -1.75444 & 0.34135 \\ \mathrm{O} & 1.88190 & -0.42799 & 0.27576 \\ \mathrm{H} & -3.57147 & -0.77189 & -0.53396 \\ \mathrm{H} & -2.76187 & 1.24915 & -2.00516 \\ \mathrm{H} & -1.04139 & 1.68553 & -2.05206 \\ \mathrm{H} & -1.01365 & 2.27544 & 0.29674 \\ \mathrm{H} & -2.36916 & 3.12220 & -0.43100 \\ \mathrm{H} & -3.99343 & 1.58085 & 0.53755 \\ \mathrm{H} & -2.91798 & 2.09573 & 1.84474 \\ \mathrm{H} & -2.22206 & -3.05783 & -0.09612 \\ \mathrm{H} & -0.72098 & -1.83822 & -2.31167 \\ \mathrm{H} & -2.50752 & -1.61861 & -2.37044 \\ \mathrm{H} & 0.36114 & -2.40074 & -0.02198 \\ \mathrm{H} & -0.46314 & -1.77652 & 1.43369 \\ \mathrm{C} & 3.27133 & 0.05347 & 0.27383 \\ \mathrm{H} & 5.04109 & -0.72457 & 1.25415 \\ \mathrm{H} & 3.88326 & -1.97709 & 0.75648 \\ \mathrm{C} & 3.97652 & -0.96792 & 1.17128 \\ \mathrm{C} & 3.34627 & 1.45582 & 0.88808 \\ \mathrm{C} & 3.84198 & 0.01023 & -1.14855 \\ \mathrm{H} & 3.54047 & -0.96633 & 2.17551\end{array}$

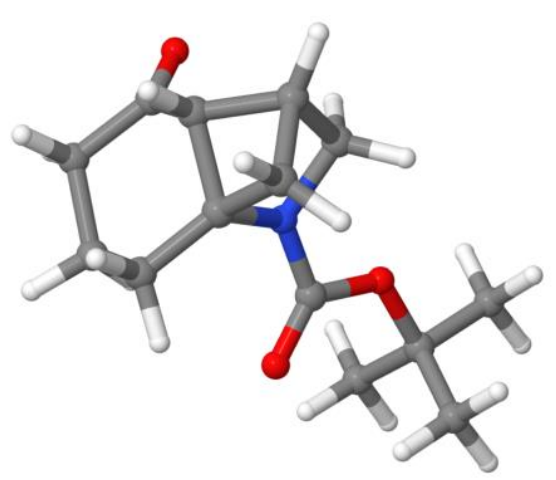




$\begin{array}{rrrr}\text { H } & 4.39540 & 1.75546 & 0.99228 \\ \mathrm{H} & 2.83149 & 2.18604 & 0.26202 \\ \mathrm{H} & 2.89159 & 1.45798 & 1.88499 \\ \mathrm{H} & 4.90948 & 0.25762 & -1.12506 \\ \mathrm{H} & 3.32909 & 0.72123 & -1.79787 \\ \mathrm{H} & 3.73705 & -0.99620 & -1.56951\end{array}$

\section{Conformer 2}

Energy: -826.52816 Hartree (Rel: $1.6 \mathrm{kcal} / \mathrm{mol}$ ) XYZ coordinates for conf 2 :

\begin{tabular}{|c|c|c|c|}
\hline 0 & 3.28020 & 0.24321 & -1.82055 \\
\hline C & 2.84267 & 0.64104 & -0.75899 \\
\hline C & -0.90392 & -0.82229 & -0.44741 \\
\hline C & 2.62276 & -0.20219 & 0.47599 \\
\hline C & 1.15015 & -0.09832 & 0.97274 \\
\hline C & 0.75285 & 1.31327 & 1.35207 \\
\hline C & 1.00090 & 2.23183 & 0.13120 \\
\hline C & 2.41445 & 2.09082 & -0.50044 \\
\hline C & 2.34567 & -1.72504 & 0.57316 \\
\hline C & 1.47113 & -1.36163 & 1.82979 \\
\hline N & 0.44461 & -0.72637 & -0.19845 \\
\hline 0 & -1.37622 & -1.68117 & -1.17730 \\
\hline C & 1.27667 & -1.91935 & -0.50886 \\
\hline 0 & -1.61051 & 0.15905 & 0.16753 \\
\hline $\mathrm{H}$ & 3.29519 & 0.13995 & 1.27227 \\
\hline $\mathrm{H}$ & 1.39020 & 1.62020 & 2.19245 \\
\hline $\mathrm{H}$ & -0.28568 & 1.39195 & 1.67636 \\
\hline $\mathrm{H}$ & 0.24907 & 2.00256 & -0.63056 \\
\hline $\mathrm{H}$ & 0.84792 & 3.27794 & 0.42184 \\
\hline $\mathrm{H}$ & 3.15717 & 2.52665 & 0.18618 \\
\hline $\mathrm{H}$ & 2.47701 & 2.64397 & -1.44244 \\
\hline $\mathrm{H}$ & 3.14734 & -2.46328 & 0.61462 \\
\hline $\mathrm{H}$ & 0.63832 & -2.02666 & 2.08202 \\
\hline $\mathrm{H}$ & 2.07246 & -1.14116 & 2.71638 \\
\hline $\mathrm{H}$ & 0.69589 & -2.83819 & -0.39211 \\
\hline $\mathrm{H}$ & 1.67755 & -1.87098 & -1.52418 \\
\hline C & -3.06603 & 0.26982 & -0.03001 \\
\hline $\mathrm{H}$ & -4.50472 & 1.68291 & 0.76253 \\
\hline $\mathrm{H}$ & -2.89646 & 2.37963 & 0.47599 \\
\hline C & -3.42881 & 1.48838 & 0.82447 \\
\hline C & -3.76730 & -0.98634 & 0.50002 \\
\hline C & -3.38023 & 0.53390 & -1.50663 \\
\hline $\mathrm{H}$ & -3.17017 & 1.31771 & 1.87500 \\
\hline $\mathrm{H}$ & -4.85339 & -0.84412 & 0.46261 \\
\hline $\mathrm{H}$ & -3.50478 & -1.86237 & -0.09432 \\
\hline $\mathrm{H}$ & -3.48535 & -1.16678 & 1.54365 \\
\hline $\mathrm{H}$ & -4.45273 & 0.72631 & -1.62513 \\
\hline H & -3.10432 & -0.32124 & -2.12492 \\
\hline $\mathrm{H}$ & -2.83629 & 1.41747 & -1.85926 \\
\hline
\end{tabular}

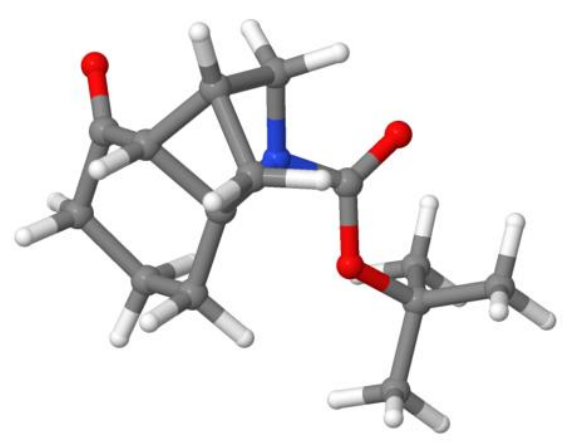


originally assigned structure for cmpd 8 (i.e. the azetidine), Fig.3<smiles>CC(C)(C)OC(=O)N1C[C@@H]2C[C@H]1C2=O</smiles>

$$
\text { Rel energy (kcal/mol): } \begin{array}{cc}
0.6 & 0.0
\end{array}
$$

\begin{tabular}{|c|c|c|c|c|c|c|}
\hline $\mathrm{C}$-nom & iGau & $\operatorname{Exp}$ & Calc & $\operatorname{diff}$ & 1 & 2 \\
\hline $\mathrm{C}$ & 2 & 206.82 & 213.18 & 6.36 & [ 213.10 & 213.21 \\
\hline C & 3 & 156.43 & 157.10 & 0.67 & [ 156.55 & 157.30 \\
\hline C & 27 & 79.47 & 79.13 & -0.34 & {$[\quad 79.07$} & 79.15 \\
\hline C & 5 & 77.43 & 76.22 & -1.21 & 75.81 & 76.37 \\
\hline C & 4 & 58.86 & 50.58 & -8.28 & 51.30 & 50.32 \\
\hline C & 13 & 47.90 & 55.40 & 7.50 & 54.45 & 55.75 \\
\hline C & 10 & 43.50 & 30.42 & -13.08 & 30.43 & 30.41 \\
\hline C & 8 & 39.97 & 39.29 & -0.68 & 39.46 & 39.23 \\
\hline C & 9 & 38.24 & 33.38 & -4.86 & 33.18 & 33.46 \\
\hline C & 30 & 28.33 & 26.67 & -1.66 & 26.87 & 26.60 \\
\hline C & 31 & 28.33 & 26.67 & -1.66 & 26.87 & 26.60 \\
\hline C & 32 & 28.33 & 26.67 & -1.66 & 26.87 & 26.60 \\
\hline $\mathrm{C}$ & 6 & 28.15 & 28.94 & 0.79 & 29.52 & 28.73 \\
\hline $\mathrm{C}$ & 7 & 25.36 & 21.44 & -3.92 & 21.16 & 21.55 \\
\hline
\end{tabular}

13C chem shifts: RMSD $=5.26 \mathrm{ppm} \quad(\mathrm{MAE}=3.76) \quad \mathrm{N}=14 \quad\left\{\begin{array}{lll}-13.08 & 7.50\end{array}\right.$

Fractions: $0.270 \quad 0.730$

\section{Conformer 1}

Energy: -826.52814 Hartree ( $\operatorname{Rel}: 0.6 \mathrm{kcal} / \mathrm{mol})$ XYZ coordinates for conf 1 :

$\begin{array}{lrrr}\mathrm{O} & -3.36097 & -1.33220 & -1.69013 \\ \mathrm{C} & -2.52628 & -1.17323 & -0.81940 \\ \mathrm{C} & 1.36957 & 1.11039 & 0.12847 \\ \mathrm{C} & -1.55412 & 0.00471 & -0.86083 \\ \mathrm{C} & -1.14433 & 0.59099 & 0.52878 \\ \mathrm{C} & -1.29596 & -0.27709 & 1.76902 \\ \mathrm{C} & -2.48624 & -1.24246 & 1.68123 \\ \mathrm{C} & -2.41748 & -2.08503 & 0.38995 \\ \mathrm{C} & -1.92975 & 1.91540 & 0.18272 \\ \mathrm{C} & -2.22948 & 1.35661 & -1.23618 \\ \mathrm{~N} & 0.08954 & 1.40652 & 0.50045 \\ \mathrm{O} & 2.20003 & 1.95926 & -0.15524 \\ \mathrm{C} & -0.59372 & 2.68840 & 0.28263 \\ \mathrm{O} & 1.55916 & -0.23705 & 0.13937 \\ \mathrm{H} & -0.69934 & -0.30075 & -1.47707 \\ \mathrm{H} & -1.37203 & 0.36245 & 2.65755 \\ \mathrm{H} & -0.37061 & -0.85811 & 1.87557 \\ \mathrm{H} & -2.49460 & -1.90378 & 2.55501 \\ \mathrm{H} & -3.43440 & -0.68720 & 1.70289 \\ \mathrm{H} & -3.21709 & -2.82926 & 0.33659 \\ \mathrm{H} & -1.45218 & -2.61245 & 0.35845 \\ \mathrm{H} & -2.77239 & 2.27513 & 0.77642 \\ \mathrm{H} & -3.28948 & 1.24283 & -1.47557 \\ \mathrm{H} & -1.75086 & 1.91038 & -2.04966 \\ \mathrm{H} & -0.50449 & 3.36516 & 1.14081 \\ \mathrm{H} & -0.27257 & 3.21783 & -0.62149 \\ \mathrm{C} & 2.87987 & -0.81248 & -0.16237 \\ \mathrm{H} & 3.55476 & -2.87169 & -0.21277 \\ \mathrm{H} & 1.86110 & -2.65727 & -0.70198 \\ \mathrm{C} & 2.63432 & -2.31590 & -0.00539 \\ \mathrm{C} & 3.91336 & -0.32296 & 0.85846 \\ \mathrm{C} & 3.28305 & -0.47557 & -1.60223 \\ \mathrm{H} & 2.31113 & -2.55160 & 1.01413 \\ \mathrm{H} & 4.86209 & -0.84910 & 0.70273 \\ \mathrm{H} & 4.08440 & 0.74990 & 0.75872 \\ \mathrm{H} & 3.57111 & -0.53449 & 1.87768\end{array}$

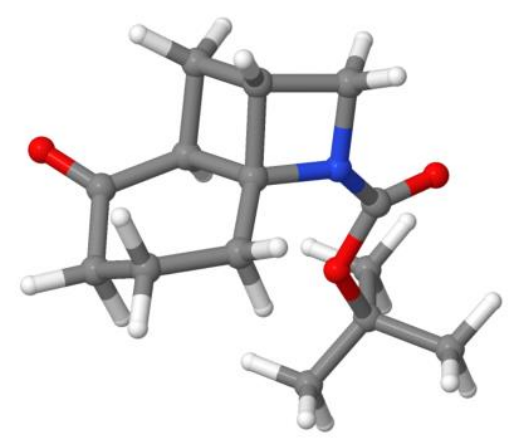




$\begin{array}{rrrr}\mathrm{H} & 4.21515 & -0.99359 & -1.85531 \\ \mathrm{H} & 3.43162 & 0.59832 & -1.72381 \\ \mathrm{H} & 2.50932 & -0.80911 & -2.30318\end{array}$

\section{Conformer 2}

Energy: -826.52908 Hartree (Rel: $0.0 \mathrm{kcal} / \mathrm{mol}$ ) XYZ coordinates for conf 2:

\begin{tabular}{|c|c|c|c|}
\hline 0 & 3.96592 & 0.15009 & 1.85484 \\
\hline $\mathrm{C}$ & 3.16623 & -0.32592 & 1.07073 \\
\hline C & -1.15401 & -0.27126 & -0.20824 \\
\hline C & 1.80925 & 0.32943 & 0.82459 \\
\hline $\mathrm{C}$ & 1.28594 & 0.25075 & -0.64514 \\
\hline C & 1.82500 & -0.83820 & -1.56113 \\
\hline $\mathrm{C}$ & 3.28353 & -1.21047 & -1.26014 \\
\hline C & 3.46584 & -1.55609 & 0.23407 \\
\hline C & 1.46396 & 1.81502 & -0.77476 \\
\hline C & 1.87496 & 1.88102 & 0.72306 \\
\hline $\mathrm{N}$ & -0.16525 & 0.48058 & -0.77181 \\
\hline 0 & -0.96671 & -1.39438 & 0.24413 \\
\hline C & -0.06136 & 1.92983 & -1.00628 \\
\hline 0 & -2.32883 & 0.40109 & -0.24660 \\
\hline $\mathrm{H}$ & 1.10700 & -0.09000 & 1.55588 \\
\hline $\mathrm{H}$ & 1.70375 & -0.53386 & -2.60867 \\
\hline $\mathrm{H}$ & 1.19153 & -1.72194 & -1.41028 \\
\hline $\mathrm{H}$ & 3.58351 & -2.06516 & -1.87690 \\
\hline $\mathrm{H}$ & 3.95645 & -0.38325 & -1.52639 \\
\hline $\mathrm{H}$ & 4.48137 & -1.89311 & 0.46027 \\
\hline $\mathrm{H}$ & 2.76341 & -2.35855 & 0.50332 \\
\hline $\mathrm{H}$ & 2.12702 & 2.27854 & -1.50776 \\
\hline $\mathrm{H}$ & 2.87884 & 2.26894 & 0.91167 \\
\hline $\mathrm{H}$ & 1.17088 & 2.42577 & 1.35992 \\
\hline $\mathrm{H}$ & -0.36411 & 2.21500 & -2.02108 \\
\hline $\mathrm{H}$ & -0.60962 & 2.54656 & -0.28522 \\
\hline C & -3.56952 & -0.21041 & 0.25472 \\
\hline $\mathrm{H}$ & -5.59600 & 0.55433 & 0.33566 \\
\hline $\mathrm{H}$ & -4.66558 & 1.12299 & -1.06777 \\
\hline C & -4.60782 & 0.88584 & -0.00042 \\
\hline C & -3.44381 & -0.50090 & 1.75469 \\
\hline $\mathrm{C}$ & -3.89788 & -1.46962 & -0.55507 \\
\hline $\mathrm{H}$ & -4.34632 & 1.80038 & 0.54208 \\
\hline $\mathrm{H}$ & -4.40807 & -0.84508 & 2.14565 \\
\hline $\mathrm{H}$ & -2.69240 & -1.26920 & 1.94339 \\
\hline $\mathrm{H}$ & -3.16420 & 0.41002 & 2.29600 \\
\hline $\mathrm{H}$ & -4.87688 & -1.85759 & -0.25150 \\
\hline $\mathrm{H}$ & -3.14634 & -2.24418 & -0.39457 \\
\hline $\mathrm{H}$ & -3.94230 & -1.23321 & -1.62406 \\
\hline
\end{tabular}

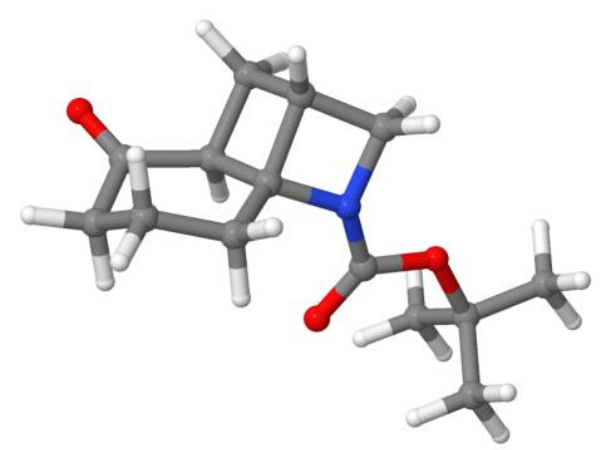


revised structure for compound $\mathbf{8}$ is the same as the originally assigned for 7, Fig.3<smiles>CC(C)(C)OC(=O)N1CC2C3C(=O)CCCC23C1</smiles>

Rel energy (kcal/mol): $\begin{array}{ccc}\text { Conf } 1 & \text { Conf } 2 \\ 0.0 & 0.9\end{array}$

\begin{tabular}{|c|c|c|c|c|c|c|c|c|}
\hline iGau & jGau & Jexp & Jcalc & $\operatorname{diff}$ & & 1 & 2 & \\
\hline 25 & 26 & 8.90 & 8.99 & 0.09 & [ & -8.94 & -9.25 & $\mathrm{H}$ \\
\hline 22 & 24 & 3.10 & 3.10 & -0.00 & [ & 3.09 & 3.12 & $\mathrm{H}$ \\
\hline 22 & 26 & 1.00 & 1.10 & 0.10 & {[} & 1.10 & 1.10 & $\mathrm{H}$ \\
\hline 22 & 25 & 1.00 & 0.93 & -0.07 & {[} & 0.92 & 0.95 & $\mathrm{H}$ \\
\hline 16 & 17 & 14.80 & 15.02 & 0.22 & {[} & -15.10 & -14.66 & $\mathrm{H}$ \\
\hline 23 & 24 & 8.00 & 8.45 & 0.45 & {[} & -8.46 & -8.40 & \\
\hline
\end{tabular}

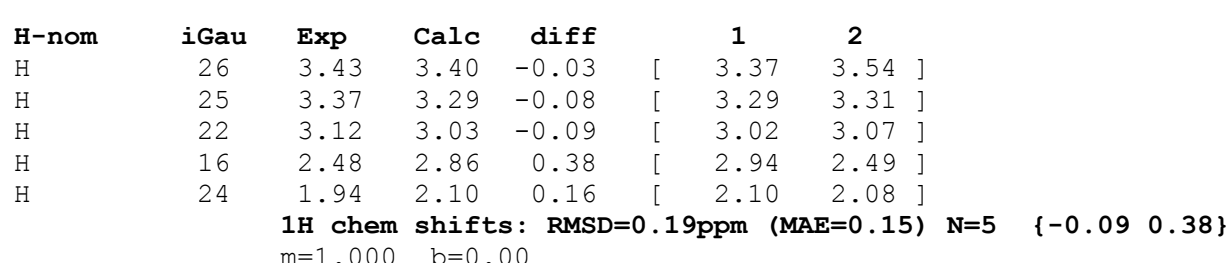

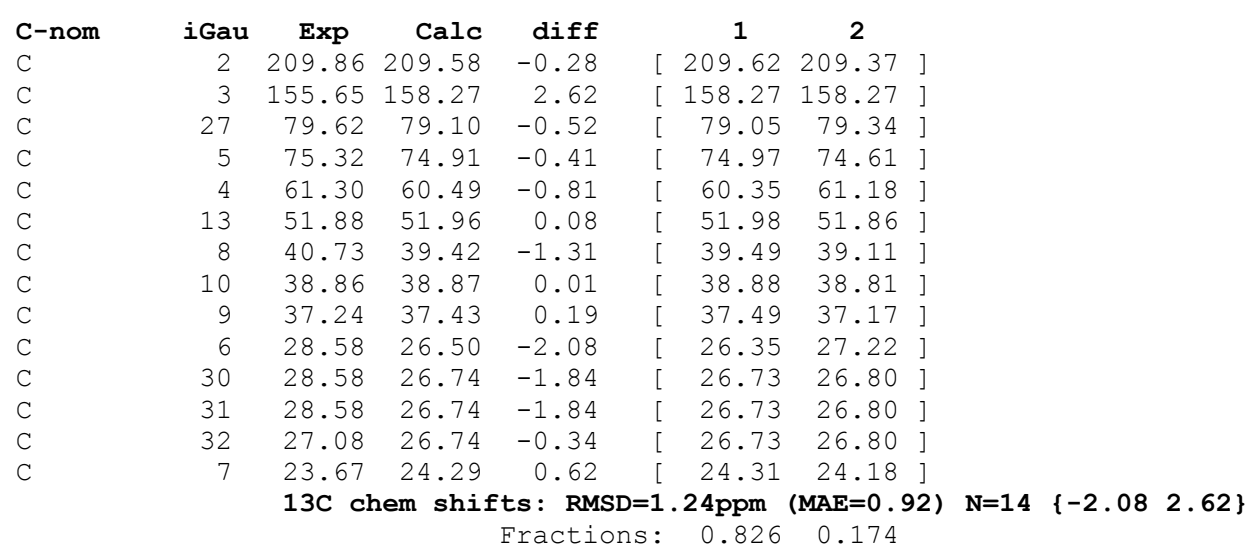

Conformer 1

Energy: -826.53460 Hartree (Rel: $0.0 \mathrm{kcal} / \mathrm{mol})$ XYZ coordinates for conf 1:

$\begin{array}{lrrr}\mathrm{O} & -4.48837 & -1.24830 & -0.15309 \\ \mathrm{C} & -3.63972 & -0.42551 & -0.44076 \\ \mathrm{C} & 1.26180 & 0.37569 & -0.01214 \\ \mathrm{C} & -2.16888 & -0.76583 & -0.60661 \\ \mathrm{C} & -1.25124 & 0.21344 & 0.20036 \\ \mathrm{C} & -1.55937 & 1.69357 & 0.12492 \\ \mathrm{C} & -3.07378 & 1.93104 & 0.29782 \\ \mathrm{C} & -3.93731 & 1.05524 & -0.64365 \\ \mathrm{C} & -1.53285 & -1.81826 & 0.33734 \\ \mathrm{C} & -1.54410 & -0.70746 & 1.43334 \\ \mathrm{~N} & 0.08316 & -0.28984 & -0.21608 \\ \mathrm{O} & 1.35832 & 1.56699 & 0.24859 \\ \mathrm{C} & -0.06429 & -1.76859 & -0.12702 \\ \mathrm{O} & 2.30149 & -0.48113 & -0.18935\end{array}$

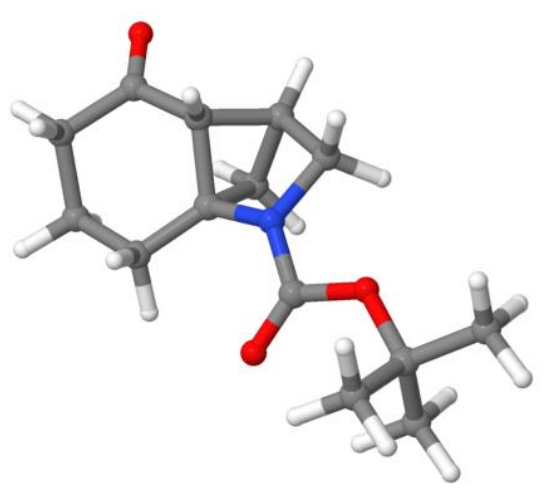




$\begin{array}{lrrr}\text { H } & -1.93607 & -0.84027 & -1.67869 \\ \mathrm{H} & -0.99059 & 2.25381 & 0.87065 \\ \mathrm{H} & -1.23409 & 2.06629 & -0.85489 \\ \mathrm{H} & -3.29856 & 2.98728 & 0.11189 \\ \mathrm{H} & -3.36710 & 1.73286 & 1.33707 \\ \mathrm{H} & -5.00617 & 1.22552 & -0.48509 \\ \mathrm{H} & -3.70127 & 1.31669 & -1.68659 \\ \mathrm{H} & -1.97752 & -2.80152 & 0.49291 \\ \mathrm{H} & -0.78077 & -0.74107 & 2.21783 \\ \mathrm{H} & -2.53442 & -0.56501 & 1.87169 \\ \mathrm{H} & 0.10415 & -2.24744 & -1.09784 \\ \mathrm{H} & 0.63176 & -2.19581 & 0.60047 \\ \mathrm{C} & 3.69200 & -0.01361 & -0.10105 \\ \mathrm{H} & 5.56402 & -1.07985 & -0.34134 \\ \mathrm{H} & 4.24615 & -1.69592 & -1.36238 \\ \mathrm{C} & 4.49014 & -1.29206 & -0.37436 \\ \mathrm{C} & 3.96610 & 1.03688 & -1.18335 \\ \mathrm{C} & 3.98446 & 0.51472 & 1.30832 \\ \mathrm{H} & 4.26512 & -2.05716 & 0.37610 \\ \mathrm{H} & 5.03071 & 1.29732 & -1.18248 \\ \mathrm{H} & 3.71496 & 0.63794 & -2.17256 \\ \mathrm{H} & 3.38161 & 1.94111 & -1.00754 \\ \mathrm{H} & 5.05118 & 0.74927 & 1.39995 \\ \mathrm{H} & 3.73959 & -0.24671 & 2.05757 \\ \mathrm{H} & 3.40578 & 1.41549 & 1.51768\end{array}$

\section{Conformer 2}

Energy: -826.53313 Hartree (Rel: $0.9 \mathrm{kcal} / \mathrm{mol}$ ) XYZ coordinates for conf 2:

\begin{tabular}{lrrr} 
O & -4.58817 & -0.15575 & -0.00043 \\
$\mathrm{C}$ & -3.51156 & 0.28991 & -0.34947 \\
$\mathrm{C}$ & 1.36624 & -0.96145 & -0.22129 \\
$\mathrm{C}$ & -2.28511 & -0.57459 & -0.58207 \\
$\mathrm{C}$ & -1.02002 & -0.01316 & 0.15346 \\
$\mathrm{C}$ & -0.76731 & 1.47795 & 0.07073 \\
$\mathrm{C}$ & -2.07438 & 2.26013 & 0.31563 \\
$\mathrm{C}$ & -3.24768 & 1.77433 & -0.56921 \\
$\mathrm{C}$ & -2.03824 & -1.78972 & 0.34760 \\
$\mathrm{C}$ & -1.56372 & -0.75933 & 1.41867 \\
$\mathrm{~N}$ & 0.00011 & -0.98588 & -0.33101 \\
$\mathrm{O}$ & 2.05029 & -1.96936 & -0.33056 \\
$\mathrm{C}$ & -0.69242 & -2.29648 & -0.20168 \\
$\mathrm{O}$ & 1.83703 & 0.29531 & -0.02620 \\
$\mathrm{H}$ & -2.15794 & -0.73193 & -1.66292 \\
$\mathrm{H}$ & -0.00163 & 1.78954 & 0.78575 \\
$\mathrm{H}$ & -0.37691 & 1.71054 & -0.92828 \\
$\mathrm{H}$ & -1.89857 & 3.32553 & 0.12864 \\
$\mathrm{H}$ & -2.36592 & 2.17686 & 1.37070 \\
$\mathrm{H}$ & -4.16578 & 2.33197 & -0.36284 \\
$\mathrm{H}$ & -2.98395 & 1.92871 & -1.62660 \\
$\mathrm{H}$ & -2.81207 & -2.53053 & 0.54941 \\
$\mathrm{H}$ & -0.82378 & -1.08186 & 2.15886 \\
$\mathrm{H}$ & -2.39859 & -0.25388 & 1.90997 \\
$\mathrm{H}$ & -0.77702 & -2.80001 & -1.17082 \\
$\mathrm{H}$ & -0.15443 & -2.95410 & 0.48716 \\
$\mathrm{C}$ & 3.28525 & 0.55410 & 0.01882 \\
$\mathrm{H}$ & 4.39219 & 2.39990 & 0.26976 \\
$\mathrm{H}$ & 2.85884 & 2.35566 & 1.16364 \\
$\mathrm{C}$ & 3.34876 & 2.07042 & 0.22648 \\
$\mathrm{C}$ & 3.91687 & -0.17970 & 1.20754 \\
$\mathrm{C}$ & 3.93225 & 0.16464 & -1.31511 \\
$\mathrm{H}$ & 2.85403 & 2.59596 & -0.59698 \\
$\mathrm{H}$ & 4.96676 & 0.11846 & 1.30862 \\
$\mathrm{H}$ & 3.39853 & 0.08554 & 2.13601 \\
$\mathrm{H}$ & 3.86777 & -1.26078 & 1.07119 \\
$\mathrm{H}$ & 4.98610 & 0.46584 & -1.31466 \\
$\mathrm{H}$ & 3.43240 & 0.67810 & -2.14416 \\
$\mathrm{H}$ & 3.87255 & -0.91226 & -1.47844 \\
& & & \\
& & & \\
\hline
\end{tabular}

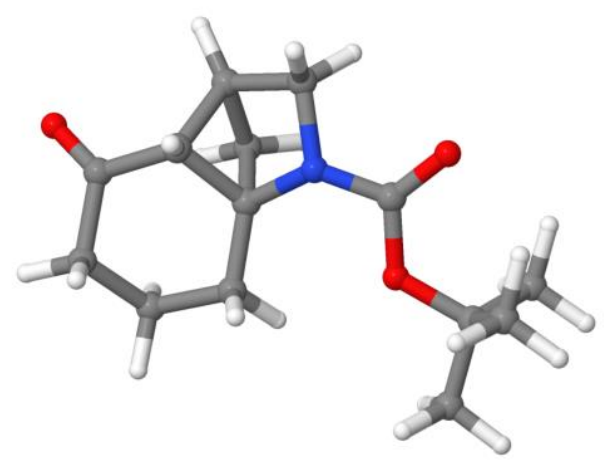


originally assigned structure of cleroindicin A (PCM pyridine), Fig.4A<smiles>OC1CCC2(CCO2)CC1</smiles>

Conf 1 Conf 2 Conf 3 Conf 4 Conf 5 Conf 6 Rel energy (kcal/mol): $\begin{array}{cccccc}0.3 & 0.3 & 0.0 & 0.7 & 0.7 & 1.4\end{array}$

\begin{tabular}{|c|c|c|c|c|c|c|c|c|c|c|}
\hline C-nom & iGau & Exp & Calc & $\operatorname{diff}$ & 1 & 2 & 3 & 4 & 5 & 6 \\
\hline $\mathrm{C} 4-\mathrm{C}$ & 6 & 70.30 & 81.69 & 11.39 & 83.41 & 83.41 & 83.38 & 84.70 & 84.70 & 83.81 \\
\hline $\mathrm{C} 7-\mathrm{CH}$ & 3 & 70.10 & 66.75 & -3.35 & 68.18 & 68.18 & 68.34 & 64.62 & 64.62 & 64.78 \\
\hline $\mathrm{C} 2-\mathrm{CH} 2$ & 8 & 59.10 & 62.87 & 3.77 & 63.22 & 63.22 & 63.26 & 63.05 & 63.05 & 63.14 \\
\hline $\mathrm{C} 3-\mathrm{CH} 2$ & 9 & 45.40 & 33.47 & -11.93 & 31.48 & 31.48 & 31.45 & 30.45 & 30.45 & 30.13 \\
\hline $\mathrm{C} 9-\mathrm{CH} 2$ & 5 & 36.40 & 37.25 & 0.85 & 35.68 & 35.88 & 36.42 & 32.90 & 33.09 & 32.46 \\
\hline $\mathrm{C} 5-\mathrm{CH} 2$ & 1 & 36.40 & 37.25 & 0.85 & 35.88 & 35.68 & 36.42 & 33.09 & 32.90 & 32.46 \\
\hline $\mathrm{C} 6-\mathrm{CH} 2$ & 2 & 31.90 & 32.35 & 0.45 & 28.30 & 31.30 & 30.96 & 27.64 & 30.48 & 28.62 \\
\hline \multirow[t]{2}{*}{$\mathrm{C} 8-\mathrm{CH} 2$} & 4 & 31.90 & 32.35 & 0.45 & 31.30 & 28.30 & 30.96 & 30.48 & 27.64 & 28.62 \\
\hline & & & & & & $(\mathrm{CMAE}=$ & 101 & $\{-1\}$ & 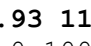 & 39\} \\
\hline
\end{tabular}

\section{Conformer 1}

Energy: -463.69224 Hartree (Rel: $0.3 \mathrm{kcal} / \mathrm{mol})$ XYZ coordinates for conf 1 :

$\begin{array}{lrrr}\mathrm{C} & 0.16771 & 1.26095 & 0.61345 \\ \mathrm{C} & -1.19857 & 1.26660 & -0.08973 \\ \mathrm{C} & -2.00408 & 0.01214 & 0.24766 \\ \mathrm{C} & -1.20411 & -1.25076 & -0.09461 \\ \mathrm{C} & 0.16059 & -1.26067 & 0.61281 \\ \mathrm{C} & 0.96843 & -0.00207 & 0.28869 \\ \mathrm{O} & 1.38315 & -0.00363 & -1.12379 \\ \mathrm{C} & 2.78375 & -0.00811 & -0.75336 \\ \mathrm{C} & 2.44658 & -0.00583 & 0.74732 \\ \mathrm{O} & -3.22968 & 0.08371 & -0.48576 \\ \mathrm{H} & 0.03193 & 1.31265 & 1.70360 \\ \mathrm{H} & 0.74835 & 2.14415 & 0.32171 \\ \mathrm{H} & -1.04984 & 1.29984 & -1.17685 \\ \mathrm{H} & -1.77089 & 2.15999 & 0.18696 \\ \mathrm{H} & -2.21945 & 0.01151 & 1.33086 \\ \mathrm{H} & -1.05410 & -1.28011 & -1.18151 \\ \mathrm{H} & -1.77817 & -2.14651 & 0.17902 \\ \mathrm{H} & 0.02122 & -1.31133 & 1.70259 \\ \mathrm{H} & 0.73536 & -2.14813 & 0.32236 \\ \mathrm{H} & 3.30546 & 0.88210 & -1.12613 \\ \mathrm{H} & 3.29941 & -0.90251 & -1.12461 \\ \mathrm{H} & 2.75470 & -0.89784 & 1.29935 \\ \mathrm{H} & 2.75926 & 0.88584 & 1.29735 \\ \mathrm{H} & -3.73279 & -0.72368 & -0.29163\end{array}$

\section{Conformer 2}

Energy: -463.69224 Hartree (Rel: $0.3 \mathrm{kcal} / \mathrm{mol}$ ) XYZ coordinates for conf 2 :

$\begin{array}{llrr}\text { C } & -0.16059 & -1.26067 & 0.61280 \\ \text { C } & 1.20411 & -1.25076 & -0.09461 \\ \text { C } & 2.00409 & 0.01214 & 0.24766 \\ \text { C } & 1.19857 & 1.26660 & -0.08973 \\ \text { C } & -0.16771 & 1.26094 & 0.61345 \\ \text { C } & -0.96843 & -0.00208 & 0.28869 \\ \text { O } & -1.38315 & -0.00363 & -1.12379 \\ \text { C } & -2.78375 & -0.00810 & -0.75336\end{array}$
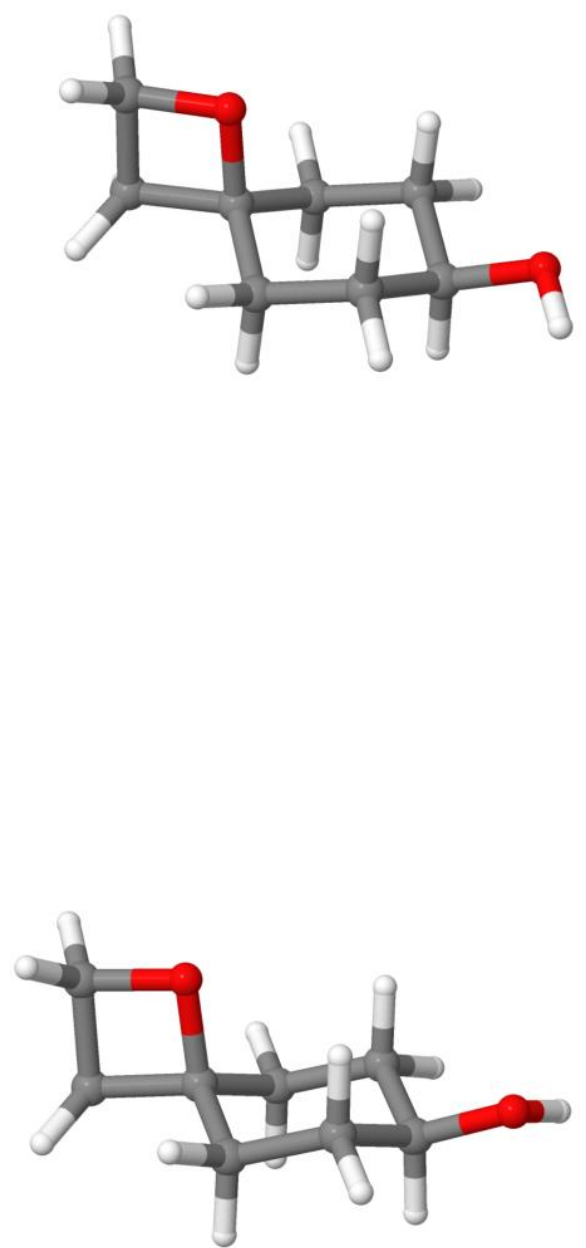


$\begin{array}{rrr}-2.44658 & -0.00584 & 0.74732 \\ 3.22968 & 0.08372 & -0.48576 \\ -0.02122 & -1.31134 & 1.70259 \\ -0.73535 & -2.14813 & 0.32235 \\ 1.05410 & -1.28011 & -1.18152 \\ 1.77817 & -2.14651 & 0.17902 \\ 2.21945 & 0.01150 & 1.33086 \\ 1.04983 & 1.29984 & -1.17685 \\ 1.77088 & 2.15999 & 0.18697 \\ -0.03193 & 1.31265 & 1.70361 \\ -0.74835 & 2.14415 & 0.32171 \\ -3.29942 & -0.90249 & -1.12462 \\ -3.30546 & 0.88212 & -1.12612 \\ -2.75926 & 0.88582 & 1.29736 \\ -2.75470 & -0.89785 & 1.29934 \\ 3.73280 & -0.72367 & -0.29163\end{array}$

\section{Conformer 3}

Energy: -463.69265 Hartree (Rel: $0.0 \mathrm{kcal} / \mathrm{mol}$ ) XYZ coordinates for conf 3 :

$\begin{array}{lrrr}\mathrm{C} & -0.16690 & 1.26063 & -0.62128 \\ \mathrm{C} & 1.20278 & 1.26168 & 0.07737 \\ \mathrm{C} & 2.00885 & 0.00001 & -0.25570 \\ \mathrm{C} & 1.20276 & -1.26167 & 0.07730 \\ \mathrm{C} & -0.16692 & -1.26057 & -0.62135 \\ \mathrm{C} & -0.96871 & 0.00003 & -0.29077 \\ \mathrm{O} & -1.37237 & -0.00001 & 1.12492 \\ \mathrm{C} & -2.77594 & -0.00006 & 0.76524 \\ \mathrm{C} & -2.45037 & 0.00003 & -0.73806 \\ \mathrm{O} & 3.28837 & -0.00002 & 0.38463 \\ \mathrm{H} & -0.03271 & 1.30870 & -1.71177 \\ \mathrm{H} & -0.74441 & 2.14646 & -0.33095 \\ \mathrm{H} & 1.04817 & 1.30265 & 1.16544 \\ \mathrm{H} & 1.77892 & 2.15191 & -0.20095 \\ \mathrm{H} & 2.24246 & 0.00003 & -1.32897 \\ \mathrm{H} & 1.04814 & -1.30271 & 1.16536 \\ \mathrm{H} & 1.77889 & -2.15190 & -0.20107 \\ \mathrm{H} & -0.03273 & -1.30858 & -1.71184 \\ \mathrm{H} & -0.74444 & -2.14641 & -0.33106 \\ \mathrm{H} & -3.29168 & 0.89220 & 1.14136 \\ \mathrm{H} & -3.29158 & -0.89243 & 1.14126 \\ \mathrm{H} & -2.76491 & -0.89182 & -1.28662 \\ \mathrm{H} & -2.76494 & 0.89195 & -1.28649 \\ \mathrm{H} & 3.12946 & -0.00025 & 1.34345\end{array}$

\section{Conformer 4}

Energy: -463.69151 Hartree (Rel: $0.7 \mathrm{kcal} / \mathrm{mol}$ ) XYZ coordinates for conf 4 :

$\begin{array}{lrrr}\text { C } & -0.00464 & 1.25765 & -0.36060 \\ \text { C } & -1.22438 & 1.27929 & 0.57616 \\ \text { C } & -2.08278 & 0.01821 & 0.43559 \\ \text { C } & -1.22778 & -1.24661 & 0.61216 \\ \text { C } & -0.01605 & -1.25758 & -0.33343 \\ \text { C } & 0.83984 & -0.00184 & -0.16271 \\ \text { O } & 1.97937 & -0.01707 & -1.08468 \\ \text { C } & 2.92531 & -0.01195 & 0.01274 \\ \mathrm{C} & 1.79905 & 0.00584 & 1.05941 \\ \mathrm{O} & -2.68711 & 0.06849 & -0.86391 \\ \mathrm{H} & 0.61638 & 2.14743 & -0.19982 \\ \mathrm{H} & -0.34189 & 1.28150 & -1.40414 \\ \mathrm{H} & -1.84145 & 2.16241 & 0.37329 \\ \mathrm{H} & -0.89442 & 1.35477 & 1.62091 \\ \mathrm{H} & -2.86972 & 0.03111 & 1.20613 \\ \mathrm{H} & -1.84604 & -2.13949 & 0.44692 \\ \mathrm{H} & -0.89116 & -1.29578 & 1.65721 \\ \mathrm{H} & 0.59746 & -2.15057 & -0.16281 \\ \mathrm{H} & -0.36265 & -1.29530 & -1.37388 \\ \mathrm{H} & 3.56965 & 0.87587 & -0.00960 \\ \mathrm{H} & 3.55799 & -0.90840 & 0.00883 \\ \mathrm{H} & 1.74241 & -0.87811 & 1.70026 \\ \mathrm{H} & 1.75192 & 0.90461 & 1.68007\end{array}$
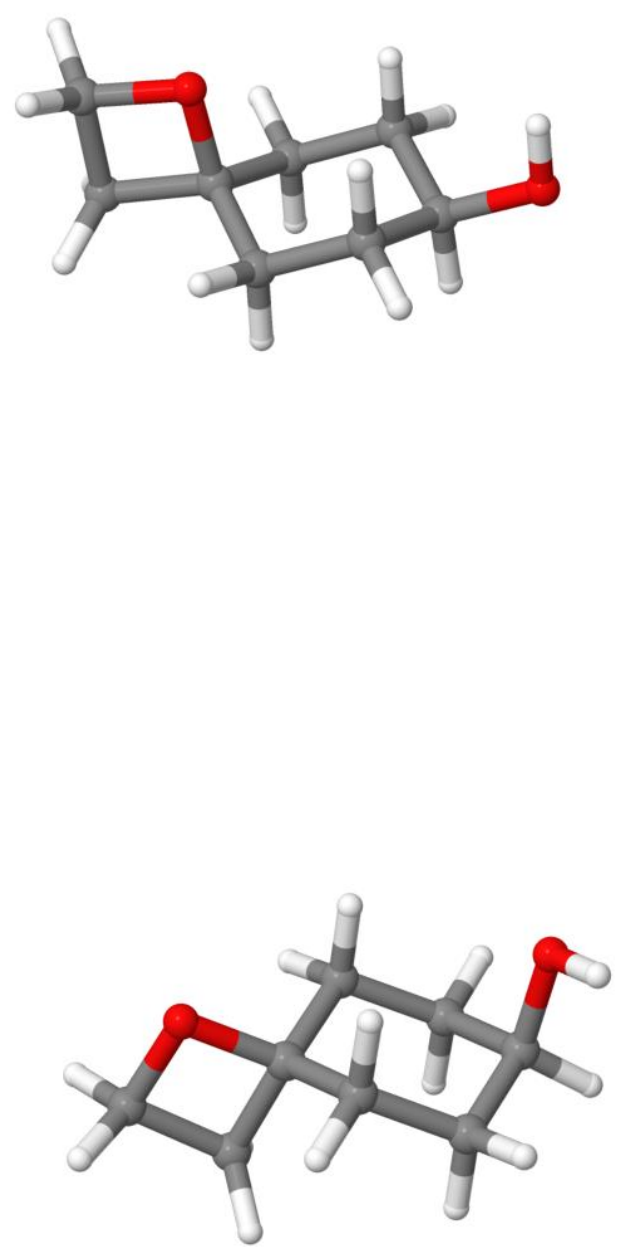


\section{Conformer 5}

Energy: -463.69151 Hartree (Rel: $0.7 \mathrm{kcal} / \mathrm{mol}$ ) XYZ coordinates for conf 5:

$\begin{array}{lrrr}\mathrm{C} & 0.01604 & -1.25757 & -0.33343 \\ \mathrm{C} & 1.22777 & -1.24662 & 0.61216 \\ \mathrm{C} & 2.08278 & 0.01820 & 0.43559 \\ \mathrm{C} & 1.22439 & 1.27929 & 0.57616 \\ \mathrm{C} & 0.00465 & 1.25766 & -0.36060 \\ \mathrm{C} & -0.83984 & -0.00183 & -0.16271 \\ \mathrm{O} & -1.97937 & -0.01705 & -1.08468 \\ \mathrm{C} & -2.92531 & -0.01197 & 0.01274 \\ \mathrm{C} & -1.79905 & 0.00586 & 1.05941 \\ \mathrm{O} & 2.68711 & 0.06848 & -0.86391 \\ \mathrm{H} & -0.59747 & -2.15055 & -0.16281 \\ \mathrm{H} & 0.36264 & -1.29530 & -1.37388 \\ \mathrm{H} & 1.84602 & -2.13950 & 0.44693 \\ \mathrm{H} & 0.89115 & -1.29577 & 1.65721 \\ \mathrm{H} & 2.86972 & 0.03110 & 1.20613 \\ \mathrm{H} & 1.84146 & 2.16241 & 0.37328 \\ \mathrm{H} & 0.89443 & 1.35477 & 1.62090 \\ \mathrm{H} & -0.61637 & 2.14744 & -0.19981 \\ \mathrm{H} & 0.34190 & 1.28150 & -1.40414 \\ \mathrm{H} & -3.55795 & -0.90846 & 0.00883 \\ \mathrm{H} & -3.56969 & 0.87580 & -0.00960 \\ \mathrm{H} & -1.75194 & 0.90465 & 1.68004 \\ \mathrm{H} & -1.74239 & -0.87807 & 1.70029 \\ \mathrm{H} & 3.17793 & -0.75953 & -0.99058\end{array}$

\section{Conformer 6}

Energy: -463.69035 Hartree (Rel: $1.4 \mathrm{kcal} / \mathrm{mol}$ ) XYZ coordinates for conf 6 :

$\begin{array}{lrrr}\mathrm{C} & 0.00797 & -1.25860 & -0.34635 \\ \mathrm{C} & 1.22842 & -1.26479 & 0.59094 \\ \mathrm{C} & 2.08950 & 0.00003 & 0.43359 \\ \mathrm{C} & 1.22837 & 1.26481 & 0.59097 \\ \mathrm{C} & 0.00793 & 1.25860 & -0.34633 \\ \mathrm{O} & -0.84260 & -0.00001 & -0.16201 \\ \mathrm{C} & -1.98249 & -0.00003 & -1.08007 \\ \mathrm{C} & -2.92656 & 0.00003 & 0.01987 \\ \mathrm{O} & -1.79699 & -0.00003 & 1.06367 \\ \mathrm{H} & 2.81673 & 0.00006 & -0.80300 \\ \mathrm{H} & -0.60756 & -2.15031 & -0.17821 \\ \mathrm{H} & 0.33162 & -1.30275 & -1.39680 \\ \mathrm{H} & 1.84644 & -2.15051 & 0.40439 \\ \mathrm{H} & 0.88775 & -1.32698 & 1.63356 \\ \mathrm{H} & 2.87174 & 0.00003 & 1.20047 \\ \mathrm{H} & 1.84637 & 2.15054 & 0.40444 \\ \mathrm{H} & 0.88770 & 1.32696 & 1.63358 \\ \mathrm{H} & -0.60762 & 2.15029 & -0.17818 \\ \mathrm{H} & 0.33162 & 1.30275 & -1.39677 \\ \mathrm{H} & -3.56458 & -0.89225 & 0.00788 \\ \mathrm{H} & -3.56446 & 0.89240 & 0.00787 \\ \mathrm{H} & -1.74263 & 0.89132 & 1.69398 \\ \mathrm{H} & -1.74269 & -0.89142 & 1.69390 \\ & 2.17617 & -0.00049 & -1.53173\end{array}$
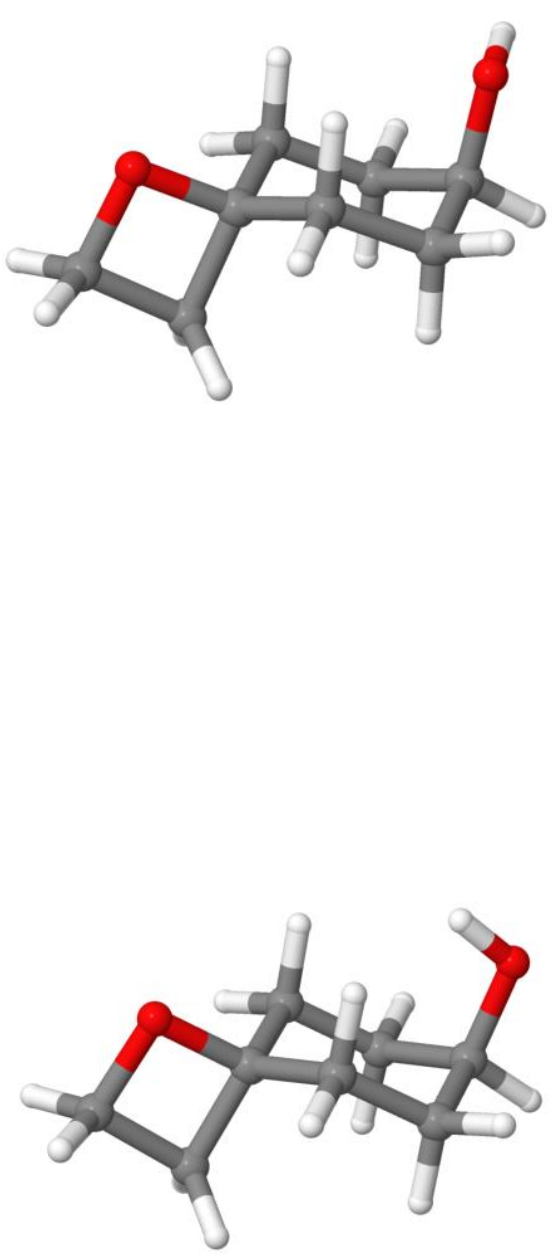
revised structure of cleroindicin A - syn open triol (PCM in pyridine), Fig.4A assuming the solvent (pyridine) disrupt intramolecular H-bonds<smiles>OCCC1(O)CCC(O)CC1</smiles>

$$
\text { Rel energy (kcal/mol): } \begin{array}{ccc}
\operatorname{Conf} 1 & \operatorname{Conf} 2 \\
0.0 & 0.0
\end{array}
$$

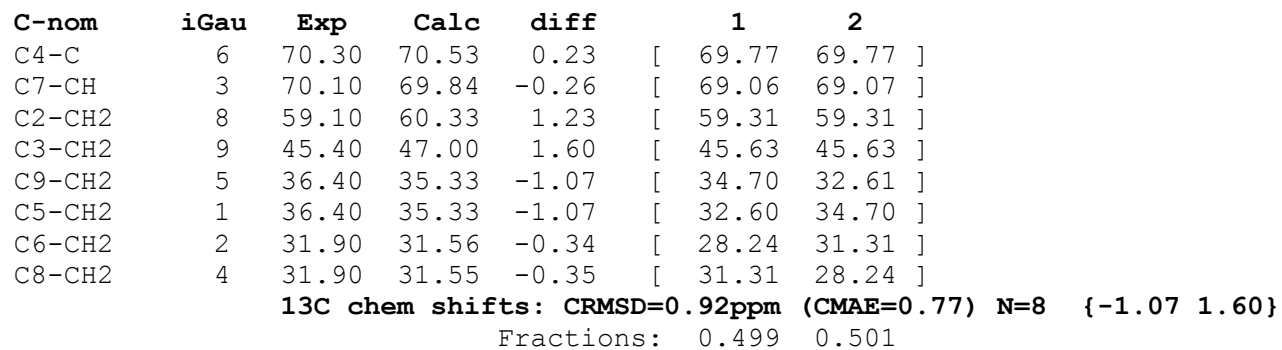

\section{Conformer 1}

Energy: -540.13243 Hartree (Rel: $0.0 \mathrm{kcal} / \mathrm{mol}$ ) XYZ coordinates for conf 1 :

$\begin{array}{lrrr}\mathrm{C} & -0.14780 & -0.13838 & -1.26186 \\ \mathrm{C} & -1.68427 & -0.10552 & -1.26361 \\ \mathrm{C} & -2.25432 & -0.68052 & 0.03212 \\ \mathrm{C} & -1.68283 & 0.05416 & 1.25042 \\ \mathrm{C} & -0.14720 & 0.00562 & 1.25874 \\ \mathrm{C} & 0.45784 & 0.57970 & -0.04131 \\ \mathrm{O} & 0.06477 & 1.95981 & -0.18840 \\ \mathrm{C} & 2.66830 & -0.83431 & 0.05534 \\ \mathrm{C} & 2.00142 & 0.53598 & -0.04119 \\ \mathrm{O} & -3.67859 & -0.56836 & -0.04068 \\ \mathrm{H} & 0.24030 & 0.32712 & -2.17584 \\ \mathrm{H} & 0.19325 & -1.18121 & -1.26459 \\ \mathrm{H} & -2.07310 & -0.67168 & -2.11868 \\ \mathrm{H} & -2.03468 & 0.92753 & -1.36909 \\ \mathrm{H} & -1.97050 & -1.74600 & 0.09887 \\ \mathrm{H} & -2.07618 & -0.39102 & 2.17469 \\ \mathrm{H} & -2.02321 & 1.09572 & 1.21720 \\ \mathrm{H} & 0.24696 & 0.56161 & 2.12083 \\ \mathrm{H} & 0.18378 & -1.03361 & 1.38467 \\ \mathrm{H} & 2.36996 & -1.47151 & -0.79012 \\ \mathrm{H} & 2.36928 & -1.35025 & 0.97961 \\ \mathrm{H} & 2.35229 & 1.02746 & -0.95715 \\ \mathrm{H} & 2.36319 & 1.14372 & 0.80101 \\ \mathrm{H} & -4.04039 & -0.92110 & 0.78841 \\ \mathrm{H} & 0.39145 & 2.43992 & 0.59075 \\ \mathrm{O} & 4.07999 & -0.61349 & 0.04112 \\ \mathrm{H} & 4.51143 & -1.48078 & 0.09122\end{array}$

\section{Conformer 2}

Energy: -540.13243 Hartree (Rel: $0.0 \mathrm{kcal} / \mathrm{mol}$ ) XYZ coordinates for conf 2 :

$\begin{array}{lrrr}\text { C } & 0.14732 & 0.00808 & 1.25878 \\ \mathrm{C} & 1.68294 & 0.05680 & 1.25025 \\ \mathrm{C} & 2.25427 & -0.68042 & 0.03342 \\ \mathrm{C} & 1.68414 & -0.10795 & -1.26343 \\ \mathrm{C} & 0.14769 & -0.14090 & -1.26146 \\ \mathrm{C} & -0.45785 & 0.57968 & -0.04228 \\ \mathrm{O} & -0.06483 & 1.95953 & -0.19211 \\ \mathrm{C} & -2.66819 & -0.83426 & 0.05680 \\ \mathrm{C} & -2.00138 & 0.53593 & -0.04200 \\ \mathrm{O} & 3.67856 & -0.56854 & -0.03973\end{array}$

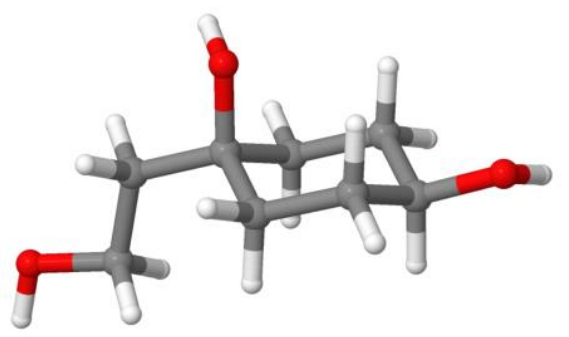




$\begin{array}{rrrr}\mathrm{H} & -0.24692 & 0.56551 & 2.11989 \\ \mathrm{H} & -0.18355 & -1.03098 & 1.38651 \\ \mathrm{H} & 2.07649 & -0.38632 & 2.17540 \\ \mathrm{H} & 2.02308 & 1.09836 & 1.21469 \\ \mathrm{H} & 1.97036 & -1.74572 & 0.10235 \\ \mathrm{H} & 2.07297 & -0.67576 & -2.11739 \\ \mathrm{H} & 2.03445 & 0.92493 & -1.37091 \\ \mathrm{H} & -0.24064 & 0.32259 & -2.17637 \\ \mathrm{H} & -0.19333 & -1.18376 & -1.26184 \\ \mathrm{H} & -2.36921 & -1.34860 & 0.98198 \\ \mathrm{H} & -2.36969 & -1.47292 & -0.78751 \\ \mathrm{H} & -2.36315 & 1.14496 & 0.79926 \\ \mathrm{H} & -2.35230 & 1.02586 & -0.95875 \\ \mathrm{H} & 4.04040 & -0.92014 & 0.78983 \\ \mathrm{H} & -0.39229 & 2.44127 & 0.58570 \\ \mathrm{O} & -4.07985 & -0.61354 & 0.04202 \\ \mathrm{H} & -4.51130 & -1.48063 & 0.09533\end{array}$


originally assigned structure of $2 \alpha, 6 \alpha$-epoxy-3-himachalene, Fig.4B

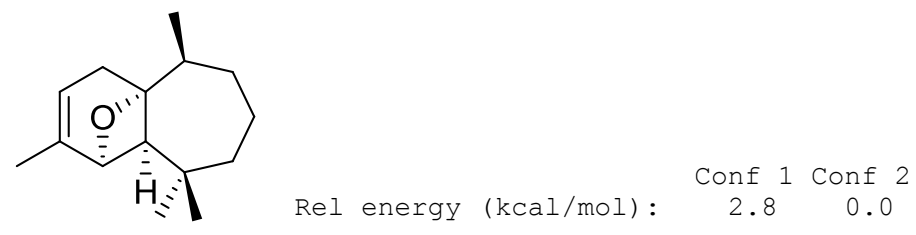

\begin{tabular}{|c|c|c|c|c|c|c|c|}
\hline C-nom & iGau & Exp & Calc & diff & & 1 & 2 \\
\hline $\mathrm{C} 1-\mathrm{d}$ & 4 & 60.90 & 57.32 & -3.58 & {[} & 61.81 & 57.28 \\
\hline$C 2-d$ & 3 & 72.10 & 80.83 & 8.73 & {[} & 80.65 & 80.83 \\
\hline C3-s & 2 & 136.70 & 141.08 & 4.38 & {[} & 139.46 & 141.09 \\
\hline$C 4-d$ & 1 & 119.60 & 124.39 & 4.79 & {[} & 123.98 & 124.39 \\
\hline$c 5-t$ & 6 & 30.50 & 28.69 & -1.81 & {[} & 31.08 & 28.67 \\
\hline $\mathrm{C} 6-\mathrm{s}$ & 5 & 78.00 & 90.33 & 12.33 & {[} & 90.58 & 90.33 \\
\hline$C 7-d$ & 11 & 40.80 & 46.02 & 5.22 & {[} & 42.87 & 46.05 \\
\hline$c 8-t$ & 10 & 31.90 & 34.11 & 2.21 & {[} & 31.32 & 34.13 \\
\hline C9-t & 9 & 19.70 & 23.14 & 3.44 & {[} & 22.40 & 23.15 \\
\hline C10-t & 8 & 40.30 & 42.26 & 1.96 & {[} & 42.65 & 42.26 \\
\hline C11-s & 7 & 35.10 & 31.52 & -3.58 & {[} & 33.13 & 31.51 \\
\hline $\mathrm{C} 12-\mathrm{q}$ & 14 & 34.20 & 31.54 & -2.66 & {[} & 32.08 & 31.54 \\
\hline C13-q & 13 & 22.50 & 28.14 & 5.64 & {[} & 22.34 & 28.19 \\
\hline $\mathrm{C} 14-\mathrm{q}$ & 12 & 16.80 & 14.60 & -2.20 & {[} & 14.53 & 14.60 \\
\hline C15-q & 15 & 21.00 & 20.26 & -0.74 & {[} & 19.96 & 20.26 \\
\hline
\end{tabular}

$13 \mathrm{C}$ chem shifts: RMSD=5.11ppm (MAE=4.22) $\mathrm{N}=15 \quad\left\{\begin{array}{l}-3.58 \quad 12.33\end{array}\right.$

Fractions: 0.0090 .991

\section{Conformer 1}

Energy: -661.20729 Hartree (Rel: $2.8 \mathrm{kcal} / \mathrm{mol})$ XYZ coordinates for conf 1 :

\begin{tabular}{|c|c|c|c|}
\hline C & 1.44817 & -1.34262 & 1.29413 \\
\hline C & 2.17626 & -1.02145 & 0.21883 \\
\hline C & 1.36133 & -0.70851 & -1.02544 \\
\hline $\mathrm{C}$ & 0.25358 & 0.35211 & -0.78661 \\
\hline C & -0.50216 & -0.87883 & -0.20967 \\
\hline $\mathrm{C}$ & -0.06261 & -1.34045 & 1.19715 \\
\hline C & 0.30018 & 1.76482 & -0.17705 \\
\hline C & -1.18332 & 2.22878 & -0.03756 \\
\hline C & -2.08679 & 1.40675 & 0.90907 \\
\hline C & -2.78949 & 0.13179 & 0.37005 \\
\hline C & -2.00025 & -0.89411 & -0.49565 \\
\hline C & -2.62595 & -2.29413 & -0.40160 \\
\hline C & 1.04055 & 1.91733 & 1.16645 \\
\hline C & 0.99717 & 2.69568 & -1.19522 \\
\hline C & 3.67528 & -0.96040 & 0.16574 \\
\hline $\mathrm{H}$ & 1.91118 & -1.61123 & 2.24269 \\
\hline $\mathrm{H}$ & -0.20497 & 0.48733 & -1.77482 \\
\hline $\mathrm{H}$ & 1.96098 & -0.65075 & -1.93977 \\
\hline $\mathrm{H}$ & -0.50343 & -0.72158 & 1.98881 \\
\hline $\mathrm{H}$ & -1.16714 & 3.26742 & 0.32112 \\
\hline $\mathrm{H}$ & -1.63877 & 2.26480 & -1.03829 \\
\hline $\mathrm{H}$ & -1.51490 & 1.15471 & 1.80933 \\
\hline $\mathrm{H}$ & -2.88997 & 2.06604 & 1.26375 \\
\hline $\mathrm{H}$ & -3.66540 & 0.43784 & -0.21739 \\
\hline $\mathrm{H}$ & -3.19700 & -0.39193 & 1.24607 \\
\hline $\mathrm{H}$ & -2.08344 & -0.58254 & -1.54539 \\
\hline $\mathrm{H}$ & -2.07792 & -3.00577 & -1.02791 \\
\hline $\mathrm{H}$ & -3.66779 & -2.27119 & -0.74305 \\
\hline $\mathrm{H}$ & -2.62774 & -2.67272 & 0.62752 \\
\hline $\mathrm{H}$ & 0.95924 & 2.95636 & 1.51109 \\
\hline $\mathrm{H}$ & 2.10468 & 1.68505 & 1.06212 \\
\hline $\mathrm{H}$ & 0.64681 & 1.27399 & 1.95638 \\
\hline $\mathrm{H}$ & 0.99719 & 3.73394 & -0.84116 \\
\hline $\mathrm{H}$ & 2.04178 & 2.39918 & -1.35179 \\
\hline $\mathrm{H}$ & 0.49330 & 2.67416 & -2.16937 \\
\hline $\mathrm{H}$ & 4.07566 & -1.65491 & -0.58606 \\
\hline
\end{tabular}

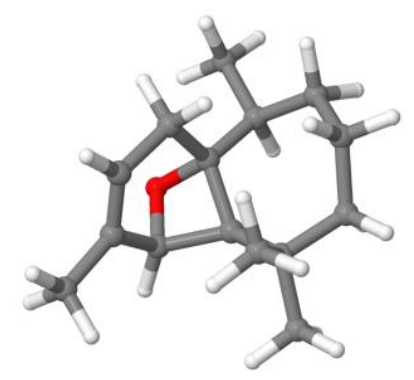




$\begin{array}{rrrr}\mathrm{H} & 4.12206 & -1.21253 & 1.13324 \\ \mathrm{H} & 4.02935 & 0.04182 & -0.11651 \\ \mathrm{O} & 0.29126 & -1.70628 & -1.14076 \\ \mathrm{H} & -0.44566 & -2.35779 & 1.35977\end{array}$

\section{Conformer 2}

Energy: -661.21174 Hartree (Rel: $0.0 \mathrm{kcal} / \mathrm{mol}$ ) XYZ coordinates for conf 2 :

$\begin{array}{lrrr}\text { C } & 1.62829 & -1.26559 & 1.20811 \\ \mathrm{C} & 2.27119 & -0.86848 & 0.10427 \\ \mathrm{C} & 1.36298 & -0.56093 & -1.07512 \\ \mathrm{C} & 0.18413 & 0.39658 & -0.72958 \\ \mathrm{C} & -0.43241 & -0.91930 & -0.17711 \\ \mathrm{C} & 0.11594 & -1.33896 & 1.20563 \\ \mathrm{C} & 0.18282 & 1.77491 & -0.02099 \\ \mathrm{C} & -1.15135 & 1.94726 & 0.76963 \\ \mathrm{C} & -2.45502 & 1.41876 & 0.12920 \\ \mathrm{C} & -2.77125 & -0.07205 & 0.37745 \\ \mathrm{C} & -1.93232 & -1.10265 & -0.40624 \\ \mathrm{C} & -2.41178 & -2.53891 & -0.14340 \\ \mathrm{C} & 1.34426 & 2.03129 & 0.95854 \\ \mathrm{C} & 0.26487 & 2.85552 & -1.12493 \\ \mathrm{C} & 3.76012 & -0.74614 & -0.04142 \\ \mathrm{H} & 2.16269 & -1.54888 & 2.11403 \\ \mathrm{H} & -0.33754 & 0.54108 & -1.68306 \\ \mathrm{H} & 1.90182 & -0.41709 & -2.01753 \\ \mathrm{H} & -0.30408 & -0.71400 & 2.00627 \\ \mathrm{H} & -1.04431 & 1.47613 & 1.75686 \\ \mathrm{H} & -1.26805 & 3.02102 & 0.97140 \\ \mathrm{H} & -3.28691 & 1.99795 & 0.55068 \\ \mathrm{H} & -2.46970 & 1.62738 & -0.94978 \\ \mathrm{H} & -3.82516 & -0.24771 & 0.12200 \\ \mathrm{H} & -2.69034 & -0.27499 & 1.45587 \\ \mathrm{H} & -2.07722 & -0.90178 & -1.47772 \\ \mathrm{H} & -1.77255 & -3.26208 & -0.66104 \\ \mathrm{H} & -3.43759 & -2.67175 & -0.50681 \\ \mathrm{H} & -2.40941 & -2.78380 & 0.92541 \\ \mathrm{H} & 1.24592 & 3.03836 & 1.38377 \\ \mathrm{H} & 2.31640 & 1.98076 & 0.45508 \\ \mathrm{H} & 1.36184 & 1.31819 & 1.78593 \\ \mathrm{H} & 0.30128 & 3.86089 & -0.68820 \\ \mathrm{H} & 1.16663 & 2.72406 & -1.73619 \\ \mathrm{H} & -0.59913 & 2.81398 & -1.79908 \\ \mathrm{H} & 4.13593 & -1.40318 & -0.83834 \\ \mathrm{H} & 4.27701 & -1.00956 & 0.88733 \\ \mathrm{H} & 4.05868 & 0.27635 & -0.31356 \\ \mathrm{O} & 0.37921 & -1.64272 & -1.17128 \\ \mathrm{H} & -0.20279 & -2.36747 & 1.42271 \\ & & & \end{array}$

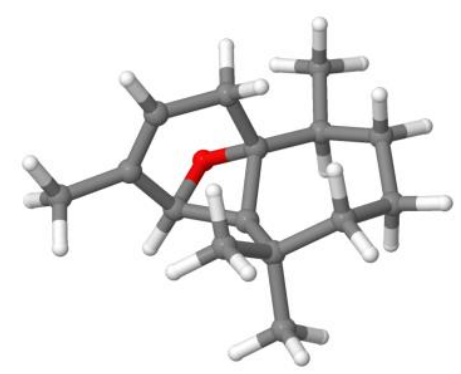




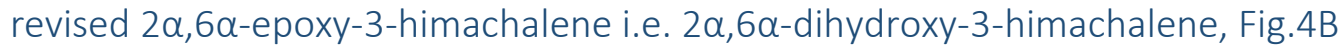<smiles>CC1=CC[C@]2(O)[C@@H](C)CCCC(C)(C)[C@H]2[C@H]1O</smiles>

$$
\text { Rel energy (kcal/mol): } \begin{array}{cc}
\operatorname{Conf} 1 & \operatorname{Conf} 2 \\
0.0 & 0.6
\end{array}
$$

\begin{tabular}{|c|c|c|c|c|c|c|c|}
\hline C-nom & iGau & $\operatorname{Exp}$ & Calc & $\operatorname{diff}$ & 1 & 2 & \\
\hline $\mathrm{C} 1-\mathrm{d}$ & 4 & 60.90 & 60.78 & -0.12 & 59.51 & $64.11]$ & \\
\hline$c 2-d$ & 3 & 72.10 & 71.63 & -0.47 & 71.58 & 71.75 ] & \\
\hline $\mathrm{C} 3-\mathrm{s}$ & 2 & 136.70 & 140.38 & 3.68 & [ 141.42 & $137.65]$ & \\
\hline$C 4-d$ & 1 & 119.60 & 117.90 & -1.70 & [ 116.94 & $120.39]$ & \\
\hline$c 5-t$ & 6 & 30.50 & 32.18 & 1.68 & {$\left[\begin{array}{ll}32.95 \\
{[}\end{array}\right.$} & $30.16]$ & \\
\hline $\mathrm{C} 6-\mathrm{s}$ & 5 & 78.00 & 79.68 & 1.68 & 79.79 & $79.38]$ & \\
\hline$C 7-d$ & 11 & 40.80 & 41.89 & 1.09 & 41.46 & $43.03]$ & \\
\hline$c 8-t$ & 10 & 31.90 & 31.12 & -0.78 & 30.88 & $31.76]$ & \\
\hline$c 9-t$ & 9 & 19.70 & 20.07 & 0.37 & 20.10 & 19.99 ] & \\
\hline $\mathrm{c} 10-\mathrm{t}$ & 8 & 40.30 & 39.13 & -1.17 & 39.06 & 39.30 ] & \\
\hline C11-s & 7 & 35.10 & 34.85 & -0.25 & 34.79 & $35.00]$ & \\
\hline $\mathrm{C} 12-\mathrm{q}$ & 14 & 34.20 & 33.71 & -0.49 & 33.70 & $33.75]$ & \\
\hline C13-q & 13 & 22.50 & 22.84 & 0.34 & 22.75 & 23.07 ] & \\
\hline C14-q & 12 & 16.80 & 17.08 & 0.28 & 16.78 & $17.86]$ & \\
\hline $\mathrm{C} 15-\mathrm{q}$ & 15 & 21.00 & 21.69 & 0.69 & [ 21.71 & $21.64]$ & \\
\hline & & $13 \mathrm{C} \mathrm{Cl}$ & sem shif & S : RM & 1. 33ppm & $(\mathrm{MAE}=0.99)$ & $N=15 \begin{cases}-1.70 & 3.68\}\end{cases}$ \\
\hline
\end{tabular}

Tweaking the ratio of the nearly energy-degenerate conformers, while keeping the energy discrepancy within $0.6 \mathrm{kcal} / \mathrm{mol}$ for each, produces even better match:

$$
\text { Rel energy (kcal/mol): } 0.00 .6
$$

\begin{tabular}{|c|c|c|c|c|c|c|c|}
\hline C-nom & iGau & $\operatorname{Exp}$ & Calc & $\operatorname{diff}$ & 1 & 2 & \\
\hline $\mathrm{C} 1-\mathrm{d}$ & 4 & 60.90 & 62.82 & 1.92 & 59.51 & 64.11 ] & \\
\hline$c 2-d$ & 3 & 72.10 & 71.70 & -0.40 & 71.58 & 71.75 ] & \\
\hline C3-s & 2 & 136.70 & 138.71 & 2.01 & [ 141.42 & 137.65 & \\
\hline$c 4-d$ & 1 & 119.60 & 119.42 & -0.18 & [ 116.94 & $120.39]$ & \\
\hline$c 5-t$ & 6 & 30.50 & 30.94 & 0.44 & [ 32.95 & 30.16 ] & \\
\hline C6-s & 5 & 78.00 & 79.49 & 1.49 & 79.79 & $79.38]$ & \\
\hline$C 7-d$ & 11 & 40.80 & 42.59 & 1.79 & 41.46 & $43.03]$ & \\
\hline$c 8-t$ & 10 & 31.90 & 31.51 & -0.39 & 30.88 & $31.76]$ & \\
\hline$c 9-t$ & 9 & 19.70 & 20.02 & 0.32 & 20.10 & 19.99 ] & \\
\hline C10-t & 8 & 40.30 & 39.23 & -1.07 & 39.06 & 39.30 ] & \\
\hline C11-s & 7 & 35.10 & 34.94 & -0.16 & 34.79 & $35.00]$ & \\
\hline $\mathrm{C} 12-\mathrm{q}$ & 14 & 34.20 & 33.74 & -0.46 & 33.70 & $33.75]$ & \\
\hline C13-q & 13 & 22.50 & 22.98 & 0.48 & 22.75 & $23.07]$ & \\
\hline $\mathrm{C} 14-\mathrm{q}$ & 12 & 16.80 & 17.56 & 0.76 & 16.78 & $17.86]$ & \\
\hline \multirow[t]{5}{*}{ C15-q } & 15 & 21.00 & 21.66 & 0.66 & {$\left[\begin{array}{ll}21.71 \\
\text { n }\end{array}\right.$} & $21.64]$ & \\
\hline & & $13 \mathrm{C} c$ & hem shif & ts: RMS & $=1.05 \mathrm{ppm}$ & $(\mathrm{MAE}=0.83)$ & \multirow[t]{4}{*}{$\mathrm{N}=15 \quad \begin{cases}-1.07 & 2.01\}\end{cases}$} \\
\hline & & & & Fractio & $\mathrm{s}: \quad 0.280$ & 0.720 & \\
\hline & Fracti & ons-der & vied rel & energi & 0.56 & 0.00 & \\
\hline & & & nergy di & screpan & -0.56 & 0.57 & \\
\hline
\end{tabular}




\section{Conformer 1}

Energy: -737.65669 Hartree (Rel: $0.0 \mathrm{kcal} / \mathrm{mol}$ ) XYZ coordinates for conf 1 :

\begin{tabular}{|c|c|c|c|}
\hline C & 1.43596 & -1.13027 & 1.50556 \\
\hline C & 2.26716 & -0.48789 & 0.67674 \\
\hline C & 1.78644 & 0.17747 & -0.60683 \\
\hline C & 0.23697 & 0.35169 & -0.70547 \\
\hline C & -0.44929 & -0.94983 & -0.17460 \\
\hline $\mathrm{C}$ & -0.04678 & -1.26603 & 1.27511 \\
\hline C & -0.22857 & 1.77713 & -0.21574 \\
\hline $\mathrm{C}$ & -1.77164 & 1.94664 & -0.11986 \\
\hline C & -2.49966 & 1.15933 & 0.97877 \\
\hline C & -2.88371 & -0.28286 & 0.59751 \\
\hline C & -1.98898 & -1.01764 & -0.42417 \\
\hline C & -2.50347 & -2.46654 & -0.54637 \\
\hline C & 0.41025 & 2.20997 & 1.12578 \\
\hline C & 0.22405 & 2.79513 & -1.29477 \\
\hline C & 3.75186 & -0.40855 & 0.91875 \\
\hline $\mathrm{H}$ & 1.83213 & -1.59641 & 2.40817 \\
\hline $\mathrm{H}$ & 0.02463 & 0.33584 & -1.78242 \\
\hline $\mathrm{H}$ & 2.25500 & 1.16538 & -0.65623 \\
\hline $\mathrm{H}$ & -0.60018 & -0.62407 & 1.97266 \\
\hline $\mathrm{H}$ & -1.95014 & 3.01679 & 0.05123 \\
\hline $\mathrm{H}$ & -2.22423 & 1.72735 & -1.09695 \\
\hline $\mathrm{H}$ & -1.89123 & 1.16412 & 1.88977 \\
\hline $\mathrm{H}$ & -3.41887 & 1.69487 & 1.24805 \\
\hline $\mathrm{H}$ & -3.89298 & -0.27390 & 0.16478 \\
\hline $\mathrm{H}$ & -2.96570 & -0.88984 & 1.51000 \\
\hline $\mathrm{H}$ & -2.14356 & -0.55312 & -1.40764 \\
\hline $\mathrm{H}$ & -2.03767 & -2.99437 & -1.38207 \\
\hline $\mathrm{H}$ & -3.58572 & -2.46191 & -0.71867 \\
\hline $\mathrm{H}$ & -2.33195 & -3.04264 & 0.37311 \\
\hline $\mathrm{H}$ & 0.01045 & 3.18689 & 1.42510 \\
\hline $\mathrm{H}$ & 1.49583 & 2.31845 & 1.04020 \\
\hline $\mathrm{H}$ & 0.22250 & 1.51052 & 1.94389 \\
\hline $\mathrm{H}$ & -0.01563 & 3.81731 & -0.97797 \\
\hline $\mathrm{H}$ & 1.30079 & 2.75572 & -1.48632 \\
\hline $\mathrm{H}$ & -0.28792 & 2.61119 & -2.24701 \\
\hline $\mathrm{H}$ & 4.29483 & -0.79621 & 0.04886 \\
\hline $\mathrm{H}$ & 4.05276 & -0.97686 & 1.80511 \\
\hline $\mathrm{H}$ & 4.07722 & 0.63286 & 1.05516 \\
\hline 0 & 2.32236 & -0.50511 & -1.74341 \\
\hline $\mathrm{H}$ & -0.36782 & -2.29040 & 1.52402 \\
\hline $\mathrm{H}$ & 1.80489 & -1.33087 & -1.78031 \\
\hline 0 & 0.15441 & -1.96173 & -1.04087 \\
\hline $\mathrm{H}$ & 0.08495 & -2.81847 & -0.59265 \\
\hline
\end{tabular}

Conformer 2

Energy: -737.65579 Hartree (Rel: $0.6 \mathrm{kcal} / \mathrm{mol}$ ) XYZ coordinates for conf 2 :

$\begin{array}{lrrr}\mathrm{C} & 1.41021 & -1.14297 & 1.50717 \\ \mathrm{C} & 2.25232 & -0.50686 & 0.68562 \\ \mathrm{C} & 1.78586 & 0.09950 & -0.62871 \\ \mathrm{C} & 0.24297 & 0.32661 & -0.71863 \\ \mathrm{C} & -0.48209 & -0.95487 & -0.17453 \\ \mathrm{C} & -0.06677 & -1.30110 & 1.26095 \\ \mathrm{C} & -0.16193 & 1.77784 & -0.24317 \\ \mathrm{C} & -1.69634 & 2.00240 & -0.13215 \\ \mathrm{C} & -2.43332 & 1.25294 & 0.98589 \\ \mathrm{C} & -2.88040 & -0.17463 & 0.62408 \\ \mathrm{C} & -2.02895 & -0.97027 & -0.39082 \\ \mathrm{C} & -2.60692 & -2.40012 & -0.45142 \\ \mathrm{C} & 0.50635 & 2.20173 & 1.08618 \\ \mathrm{C} & 0.31396 & 2.76733 & -1.33858 \\ \mathrm{C} & 3.73131 & -0.40417 & 0.95390 \\ \mathrm{H} & 1.79387 & -1.58837 & 2.42544 \\ \mathrm{H} & 0.02232 & 0.32028 & -1.79678 \\ \mathrm{H} & 2.28831 & 1.06268 & -0.75588 \\ \mathrm{H} & -0.63013 & -0.68357 & 1.97271 \\ \mathrm{H} & -1.83663 & 3.08018 & 0.02593 \\ \mathrm{H} & -2.16872 & 1.78508 & -1.10059 \\ & & & \end{array}$
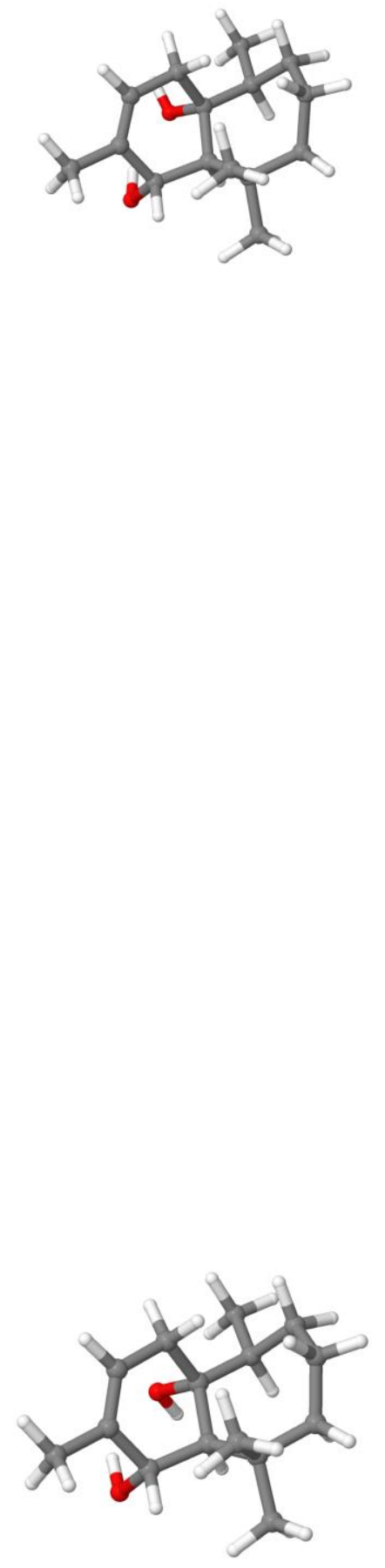


$\begin{array}{rrrr}\mathrm{H} & -1.80819 & 1.23914 & 1.88511 \\ \mathrm{H} & -3.32633 & 1.82483 & 1.26828 \\ \mathrm{H} & -3.89129 & -0.12840 & 0.19779 \\ \mathrm{H} & -2.98121 & -0.76560 & 1.54459 \\ \mathrm{H} & -2.19054 & -0.51796 & -1.38273 \\ \mathrm{H} & -2.16274 & -3.00537 & -1.24413 \\ \mathrm{H} & -3.68791 & -2.35111 & -0.62732 \\ \mathrm{H} & -2.45228 & -2.93371 & 0.49344 \\ \mathrm{H} & 0.14350 & 3.19485 & 1.37967 \\ \mathrm{H} & 1.59409 & 2.27147 & 0.98818 \\ \mathrm{H} & 0.30341 & 1.51553 & 1.91160 \\ \mathrm{H} & 0.10896 & 3.80011 & -1.03233 \\ \mathrm{H} & 1.38742 & 2.69375 & -1.53807 \\ \mathrm{H} & -0.21186 & 2.58962 & -2.28469 \\ \mathrm{H} & 4.29809 & -0.80736 & 0.10616 \\ \mathrm{H} & 4.02019 & -0.94804 & 1.85926 \\ \mathrm{H} & 4.04305 & 0.64367 & 1.07270 \\ \mathrm{O} & 2.28713 & -0.68346 & -1.71993 \\ \mathrm{H} & -0.36156 & -2.33872 & 1.46296 \\ \mathrm{H} & 1.86730 & -1.55289 & -1.58632 \\ \mathrm{O} & 0.06007 & -2.05629 & -0.97004 \\ \mathrm{H} & -0.25633 & -1.92222 & -1.87988\end{array}$


originally assigned structure of capitulatin B, Fig.4C

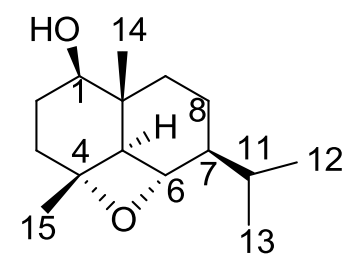
Rel energy (kcal/mol): $\begin{array}{cccc}\text { Conf } 1 & \text { Conf } 2 & \text { Conf } 3 \\ 0.0 & 0.9 & 1.7\end{array}$

\begin{tabular}{|c|c|c|c|c|c|c|c|c|}
\hline C-nom & iGau & $\operatorname{Exp}$ & Calc & $\operatorname{diff}$ & 1 & 2 & 3 & \\
\hline $\mathrm{C} 1-\mathrm{CH}$ & 6 & 78.80 & 81.75 & 2.95 & 81.75 & 81.71 & 81.86 & ] \\
\hline $\mathrm{C} 2-\mathrm{CH} 2$ & 1 & 28.00 & 35.76 & 7.76 & 35.78 & 35.68 & 35.80 & ] \\
\hline $\mathrm{C} 3-\mathrm{CH} 2$ & 2 & 40.20 & 41.77 & 1.57 & 41.75 & 41.87 & 41.83 & ] \\
\hline $\mathrm{C} 4-\mathrm{C}$ & 3 & 73.60 & 87.47 & 13.87 & 87.40 & 87.99 & 86.58 & ] \\
\hline $\mathrm{C} 5-\mathrm{CH}$ & 4 & 60.00 & 57.48 & -2.52 & 57.37 & 57.89 & 57.83 & ] \\
\hline $\mathrm{C} 6-\mathrm{CH}$ & 7 & 70.80 & 81.48 & 10.68 & 82.70 & 76.89 & 78.38 & ] \\
\hline $\mathrm{C} 7-\mathrm{CH}$ & 8 & 51.30 & 54.37 & 3.07 & 54.60 & 53.59 & 53.52 & ] \\
\hline $\mathrm{C} 8-\mathrm{CH} 2$ & 9 & 18.30 & 27.66 & 9.36 & 27.83 & 28.25 & 22.03 & ] \\
\hline $\mathrm{C} 9-\mathrm{CH} 2$ & 10 & 39.30 & 42.05 & 2.75 & 42.05 & 42.02 & 42.07 & ] \\
\hline C10-C & 5 & 40.30 & 36.37 & -3.93 & 36.34 & 36.44 & 36.62 & ] \\
\hline $\mathrm{Cl1}-\mathrm{CH}$ & 15 & 25.30 & 32.81 & 7.51 & 33.39 & 31.54 & 27.42 & \\
\hline $\mathrm{C} 12-\mathrm{CH} 3$ & 16 & 15.80 & 20.57 & 4.77 & 21.70 & 15.54 & 21.06 & ] \\
\hline $\mathrm{C} 13-\mathrm{CH} 3$ & 17 & 21.10 & 19.96 & -1.14 & 19.67 & 22.31 & 15.34 & ] \\
\hline $\mathrm{C} 14-\mathrm{CH} 3$ & 11 & 13.90 & 13.10 & -0.80 & 13.09 & 13.16 & 13.14 & ] \\
\hline $\mathrm{C} 15-\mathrm{CH} 3$ & 13 & 23.60 & 17.98 & -5.62 & 17.96 & 18.05 & 18.00 & ] \\
\hline & & & & & & & & \\
\hline
\end{tabular}

Conformer 1

Energy: -737.61748 Hartree (Rel: $0.0 \mathrm{kcal} / \mathrm{mol})$ XYZ coordinates for conf 1 :

$\begin{array}{lrrr}\mathrm{C} & 2.98311 & 0.59734 & -0.78493 \\ \mathrm{C} & 1.99249 & 1.78783 & -1.05607 \\ \mathrm{C} & 0.90107 & 1.66460 & 0.01850 \\ \mathrm{C} & 0.46211 & 0.22925 & -0.05324 \\ \mathrm{C} & 1.20692 & -1.02398 & 0.27274 \\ \mathrm{C} & 2.41119 & -0.86759 & -0.71869 \\ \mathrm{C} & -0.90918 & 0.64991 & 0.34347 \\ \mathrm{C} & -1.90628 & -0.35167 & -0.24184 \\ \mathrm{C} & -1.33012 & -1.73658 & 0.24384 \\ \mathrm{C} & 0.14581 & -2.10353 & -0.14385 \\ \mathrm{C} & 1.65877 & -1.19979 & 1.72681 \\ \mathrm{H} & 0.31363 & 0.12684 & -1.14124 \\ \mathrm{C} & 1.30342 & 2.31295 & 1.33924 \\ \mathrm{O} & -0.55870 & 1.99168 & -0.21889 \\ \mathrm{C} & -3.37579 & -0.11075 & 0.16828 \\ \mathrm{C} & -3.81786 & 1.30495 & -0.24164 \\ \mathrm{C} & -4.34350 & -1.15459 & -0.41200 \\ \mathrm{O} & 3.44367 & -1.77682 & -0.35575 \\ \mathrm{H} & 3.75780 & 0.60692 & -1.56506 \\ \mathrm{H} & 3.50905 & 0.77978 & 0.16066 \\ \mathrm{H} & 1.54444 & 1.69658 & -2.05250 \\ \mathrm{H} & 2.54868 & 2.73371 & -1.02777 \\ \mathrm{H} & 2.03152 & -1.12088 & -1.72328 \\ \mathrm{H} & -1.08676 & 0.76589 & 1.42224 \\ \mathrm{H} & -1.84016 & -0.30570 & -1.33980 \\ \mathrm{H} & -1.97089 & -2.54113 & -0.13255 \\ \mathrm{H} & -1.42652 & -1.76695 & 1.33968 \\ \mathrm{H} & 0.19133 & -2.23794 & -1.23339 \\ \mathrm{H} & 0.38261 & -3.07909 & 0.29845 \\ \mathrm{H} & 2.20768 & -2.13870 & 1.84397 \\ \mathrm{H} & 2.31883 & -0.38920 & 2.05240 \\ \mathrm{H} & 0.79595 & -1.21782 & 2.40309 \\ \mathrm{H} & 2.34095 & 2.08817 & 1.61001 \\ \mathrm{H} & 0.65967 & 1.99851 & 2.16592 \\ & & & \end{array}$

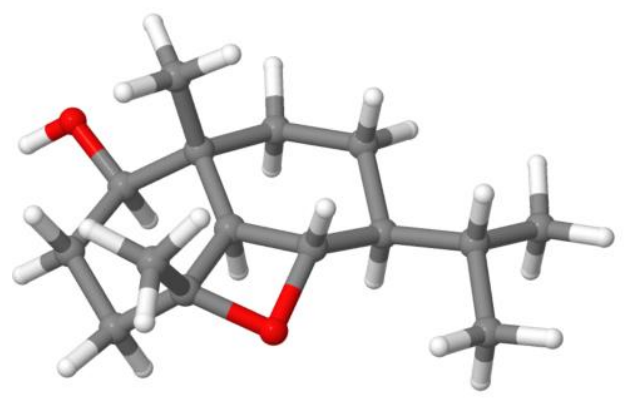




$\begin{array}{rrrr}\mathrm{H} & 1.20964 & 3.40038 & 1.23702 \\ \mathrm{H} & -3.42650 & -0.17643 & 1.26724 \\ \mathrm{H} & -4.83536 & 1.51296 & 0.11037 \\ \mathrm{H} & -3.15097 & 2.07450 & 0.16037 \\ \mathrm{H} & -3.81513 & 1.40986 & -1.33439 \\ \mathrm{H} & -5.37734 & -0.91836 & -0.13327 \\ \mathrm{H} & -4.13189 & -2.16811 & -0.05538 \\ \mathrm{H} & -4.29317 & -1.16663 & -1.50897 \\ \mathrm{H} & 4.17032 & -1.67605 & -0.99042\end{array}$

\section{Conformer 2}

Energy: -737.61609 Hartree (Rel: $0.9 \mathrm{kcal} / \mathrm{mol}$ ) XYZ coordinates for conf 2 :

\begin{tabular}{lrrr} 
CYZ Coordinates for & Conf & \multicolumn{1}{l}{ L } & \\
$\mathrm{C}$ & 2.96547 & 0.81286 & -0.56437 \\
$\mathrm{C}$ & 1.91679 & 1.95524 & -0.82578 \\
$\mathrm{C}$ & 0.74996 & 1.66589 & 0.13108 \\
$\mathrm{C}$ & 0.42487 & 0.21692 & -0.09967 \\
$\mathrm{C}$ & 1.22832 & -1.01085 & 0.18432 \\
$\mathrm{C}$ & 2.49802 & -0.68593 & -0.67681 \\
$\mathrm{C}$ & -1.00284 & 0.51111 & 0.20655 \\
$\mathrm{C}$ & -1.85722 & -0.50248 & -0.55618 \\
$\mathrm{C}$ & -1.23232 & -1.88362 & -0.12728 \\
$\mathrm{C}$ & 0.29106 & -2.11770 & -0.41859 \\
$\mathrm{H}$ & 1.56939 & -1.28575 & 1.65315 \\
$\mathrm{C}$ & 0.37782 & 0.20396 & -1.20106 \\
$\mathrm{O}$ & 0.99190 & 2.21905 & 1.53215 \\
$\mathrm{C}$ & -0.70429 & 1.91740 & -0.21032 \\
$\mathrm{C}$ & -3.39135 & -0.42640 & -0.37201 \\
$\mathrm{C}$ & -3.86929 & -0.58374 & 1.08162 \\
$\mathrm{O}$ & -3.93109 & 0.88169 & -0.97577 \\
$\mathrm{H}$ & 3.55922 & -1.55585 & -0.30087 \\
$\mathrm{H}$ & 3.80321 & 0.94373 & -1.26436 \\
$\mathrm{H}$ & 3.39047 & 0.94207 & 0.43899 \\
$\mathrm{H}$ & 1.56324 & 1.92300 & -1.86304 \\
$\mathrm{H}$ & 2.39773 & 2.92948 & -0.66910 \\
$\mathrm{H}$ & 2.22442 & -0.86922 & -1.72994 \\
$\mathrm{H}$ & -1.26885 & 0.52021 & 1.27114 \\
$\mathrm{H}$ & -1.65786 & -0.36293 & -1.62897 \\
$\mathrm{H}$ & -1.79683 & -2.68632 & -0.61932 \\
$\mathrm{H}$ & -1.39979 & -2.01842 & 0.94985 \\
$\mathrm{H}$ & 0.43589 & -2.14636 & -1.50732 \\
$\mathrm{H}$ & 0.56519 & -3.11128 & -0.04312 \\
$\mathrm{H}$ & 2.17162 & -2.19467 & 1.74126 \\
$\mathrm{H}$ & 2.14177 & -0.46612 & 2.09990 \\
$\mathrm{H}$ & 0.65783 & -1.41681 & 2.24816 \\
$\mathrm{H}$ & 2.01602 & 2.03649 & 1.87560 \\
$\mathrm{H}$ & 0.30487 & 1.79280 & 2.26907 \\
$\mathrm{H}$ & 0.83089 & 3.30322 & 1.51221 \\
$\mathrm{H}$ & -3.81851 & -1.26239 & -0.94763 \\
$\mathrm{H}$ & -4.96494 & -0.57949 & 1.12118 \\
$\mathrm{H}$ & -3.52926 & -1.52228 & 1.53312 \\
$\mathrm{H}$ & -3.51971 & 0.24174 & 1.71339 \\
$\mathrm{H}$ & -5.02330 & 0.93266 & -0.89105 \\
$\mathrm{H}$ & -3.67112 & 0.96731 & -2.03755 \\
$\mathrm{H}$ & -3.50986 & 1.75555 & -0.46445 \\
& 4.32952 & -1.34607 & -0.85189 \\
$\mathrm{H}$ & & &
\end{tabular}

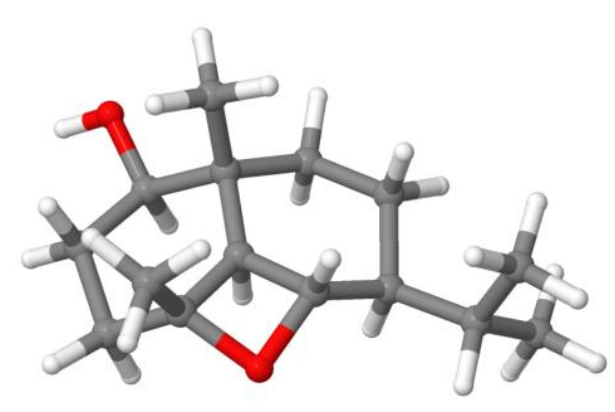

Conformer 3

Energy: -737.61471 Hartree (Rel: $1.7 \mathrm{kcal} / \mathrm{mol}$ ) XYZ coordinates for conf 3 :

$\begin{array}{lrrr}\text { C } & 3.07702 & 0.37661 & -0.69771 \\ \text { C } & 2.21883 & 1.63590 & -1.08762 \\ \text { C } & 1.05753 & 1.66675 & -0.08256 \\ \text { C } & 0.49410 & 0.27418 & -0.11583 \\ \text { C } & 1.09554 & -1.02518 & 0.31101 \\ \text { C } & 2.36804 & -1.02638 & -0.60584 \\ \text { C } & -0.85499 & 0.83700 & 0.16831 \\ \text { C } & -1.88917 & -0.10215 & -0.45137 \\ \text { C } & -1.48858 & -1.50476 & 0.14663 \\ \text { C } & -0.03363 & -2.02448 & -0.12962 \\ \text { C } & 1.44224 & -1.16992 & 1.79695\end{array}$

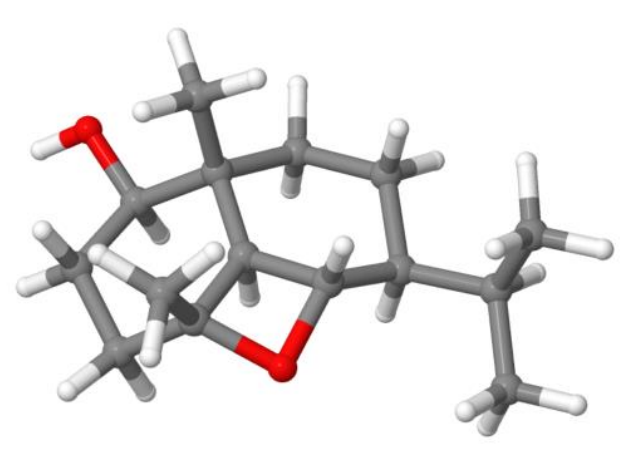




$\begin{array}{lrrr}\text { H } & 0.40657 & 0.13129 & -1.20551 \\ \mathrm{C} & 1.43529 & 2.34715 & 1.22926 \\ \mathrm{O} & -0.34804 & 2.10983 & -0.43285 \\ \mathrm{C} & -3.37683 & 0.28982 & -0.26284 \\ \mathrm{C} & -4.30451 & -0.53689 & -1.16889 \\ \mathrm{C} & -3.86864 & 0.24428 & 1.19369 \\ \mathrm{O} & 3.28793 & -2.00527 & -0.13701 \\ \mathrm{H} & 3.89762 & 0.27699 & -1.42276 \\ \mathrm{H} & 3.55525 & 0.55831 & 0.27312 \\ \mathrm{H} & 1.82547 & 1.53340 & -2.10588 \\ \mathrm{H} & 2.85749 & 2.52847 & -1.06689 \\ \mathrm{H} & 2.02919 & -1.29305 & -1.62147 \\ \mathrm{H} & -1.08175 & 1.02548 & 1.22577 \\ \mathrm{H} & -1.70059 & -0.12639 & -1.53464 \\ \mathrm{H} & -2.18513 & -2.26523 & -0.22596 \\ \mathrm{H} & -1.64242 & -1.46714 & 1.23388 \\ \mathrm{H} & 0.06644 & -2.21380 & -1.20725 \\ \mathrm{H} & 0.08494 & -2.99487 & 0.36817 \\ \mathrm{H} & 1.88826 & -2.14972 & 1.99059 \\ \mathrm{H} & 2.15928 & -0.41017 & 2.12521 \\ \mathrm{H} & 0.54492 & -1.07020 & 2.41877 \\ \mathrm{H} & 2.43049 & 2.04888 & 1.57694 \\ \mathrm{H} & 0.71781 & 2.13312 & 2.02686 \\ \mathrm{H} & 1.44466 & 3.43173 & 1.06999 \\ \mathrm{H} & -3.44599 & 1.33561 & -0.59782 \\ \mathrm{H} & -5.33641 & -0.17129 & -1.10737 \\ \mathrm{H} & -3.99212 & -0.47990 & -2.21868 \\ \mathrm{H} & -4.31631 & -1.59527 & -0.88024 \\ \mathrm{H} & -4.88521 & 0.64891 & 1.26333 \\ \mathrm{H} & -3.23511 & 0.83650 & 1.86340 \\ \mathrm{H} & -3.89987 & -0.78165 & 1.57997 \\ \mathrm{H} & 4.06009 & -1.99800 & -0.72412 \\ & & & \\ & & & \end{array}$


revised capitulatin $\mathrm{B}$ (open triol), Fig.4C

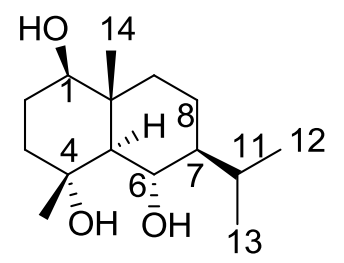

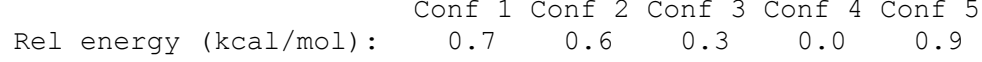

\begin{tabular}{|c|c|c|c|c|c|c|c|c|c|}
\hline C-nom & iGau & Exp & $\mathrm{Calc}$ & $\operatorname{diff}$ & 1 & 2 & 3 & 4 & 5 \\
\hline $\mathrm{Cl}-\mathrm{CH}$ & 6 & 78.80 & 78.90 & 0.10 & 78.70 & 78.31 & 78.42 & 79.59 & 78.35 \\
\hline $\mathrm{C} 2-\mathrm{CH} 2$ & 1 & 28.00 & 28.82 & 0.82 & 30.08 & 29.95 & 30.44 & 26.69 & 30.34 \\
\hline $\mathrm{C} 3-\mathrm{CH} 2$ & 2 & 40.20 & 41.24 & 1.04 & 38.34 & 40.70 & 41.49 & 42.06 & 41.54 \\
\hline $\mathrm{C} 4-\mathrm{C}$ & 3 & 73.60 & 74.93 & 1.33 & 70.94 & 74.88 & 75.50 & 75.60 & 75.61 \\
\hline $\mathrm{C} 5-\mathrm{CH}$ & 4 & 60.00 & 57.01 & -2.99 & 55.52 & 53.33 & 58.38 & 57.63 & 57.96 \\
\hline $\mathrm{C} 6-\mathrm{CH}$ & 7 & 70.80 & 68.77 & -2.03 & 72.65 & 68.24 & 68.09 & 68.02 & 69.92 \\
\hline $\mathrm{C} 7-\mathrm{CH}$ & 8 & 51.30 & 51.25 & -0.05 & 50.75 & 52.58 & 51.23 & 51.26 & 49.77 \\
\hline $\mathrm{C} 8-\mathrm{CH} 2$ & 9 & 18.30 & 19.21 & 0.91 & 18.71 & 18.83 & 18.33 & 18.48 & 26.98 \\
\hline $\mathrm{C} 9-\mathrm{CH} 2$ & 10 & 39.30 & 39.47 & 0.17 & 38.72 & 39.27 & 40.04 & 39.19 & 40.39 \\
\hline C10-C & 5 & 40.30 & 40.50 & 0.20 & 40.23 & 40.61 & 40.47 & 40.58 & 40.41 \\
\hline $\mathrm{Cl1}-\mathrm{CH}$ & 15 & 25.30 & 25.94 & 0.64 & 26.13 & 25.15 & 25.04 & 24.97 & 34.62 \\
\hline $\mathrm{C} 12-\mathrm{CH} 3$ & 16 & 21.10 & 20.74 & -0.36 & 21.06 & 21.21 & 21.19 & 21.12 & 16.17 \\
\hline $\mathrm{C} 13-\mathrm{CH} 3$ & 17 & 15.80 & 15.51 & -0.29 & 14.23 & 14.83 & 14.85 & 14.76 & 24.32 \\
\hline $\mathrm{Cl} 4-\mathrm{CH} 3$ & 11 & 13.90 & 15.09 & 1.19 & 14.28 & 13.92 & 15.36 & 15.47 & 15.53 \\
\hline \multirow[t]{3}{*}{$\mathrm{C} 15-\mathrm{CH} 3$} & 13 & 23.60 & 22.34 & -1.26 & 25.37 & 25.00 & 21.29 & 21.43 & 21.31 \\
\hline & & & & & $1.19 \mathrm{ppm}$ & $\mathrm{MAE}=0.89$ & ) $\mathrm{N}=15$ & $\{-2.99$ & $1.33\}$ \\
\hline & & & & Eract & $: \quad 0.117$ & 0.139 & 0.262 & 0.400 & 0.082 \\
\hline
\end{tabular}

\section{Conformer 1}

Energy: -814.10633 Hartree (Rel: $0.7 \mathrm{kcal} / \mathrm{mol}$ ) XYZ coordinates for conf 1 :

$\begin{array}{lrrr}\mathrm{C} & 3.35072 & -0.37724 & -0.07062 \\ \mathrm{C} & 2.98319 & 1.02516 & -0.55954 \\ \mathrm{C} & 1.60613 & 1.53228 & -0.08530 \\ \mathrm{C} & 0.52190 & 0.42758 & -0.35427 \\ \mathrm{C} & 0.87851 & -1.03280 & 0.10360 \\ \mathrm{C} & 2.27253 & -1.38740 & -0.47213 \\ \mathrm{C} & -0.90806 & 0.79233 & 0.11166 \\ \mathrm{C} & -1.96541 & -0.17290 & -0.47666 \\ \mathrm{C} & -1.61254 & -1.63416 & -0.13968 \\ \mathrm{C} & -0.17107 & -1.98066 & -0.53184 \\ \mathrm{C} & 0.88608 & -1.25849 & 1.63507 \\ \mathrm{H} & 0.47859 & 0.39205 & -1.45341 \\ \mathrm{C} & 1.66218 & 2.04857 & 1.36417 \\ \mathrm{O} & 1.35411 & 2.65153 & -0.94300 \\ \mathrm{C} & -3.42487 & 0.22553 & -0.11368 \\ \mathrm{C} & -4.45244 & -0.56540 & -0.94054 \\ \mathrm{C} & -3.75673 & 0.12747 & 1.38617 \\ \mathrm{O} & 2.59900 & -2.71258 & -0.04884 \\ \mathrm{H} & 4.31028 & -0.67959 & -0.51565 \\ \mathrm{H} & 3.49615 & -0.40301 & 1.01593 \\ \mathrm{H} & 2.95396 & 1.03043 & -1.65654 \\ \mathrm{H} & 3.74761 & 1.75549 & -0.26862 \\ \mathrm{H} & 2.18502 & -1.36659 & -1.57357 \\ \mathrm{H} & -0.94717 & 0.76664 & 1.20842 \\ \mathrm{H} & -1.88145 & -0.05593 & -1.56838 \\ \mathrm{H} & -2.29831 & -2.31014 & -0.66390 \\ \mathrm{H} & -1.76024 & -1.82351 & 0.93062 \\ \mathrm{H} & -0.07685 & -1.92367 & -1.62631 \\ \mathrm{H} & 0.06108 & -3.01379 & -0.25247 \\ \mathrm{H} & 1.04481 & -2.32061 & 1.83954 \\ \mathrm{H} & 1.68436 & -0.71114 & 2.14004 \\ \mathrm{H} & -0.05497 & -0.96293 & 2.10633 \\ \mathrm{H} & 2.36623 & 2.88630 & 1.40243\end{array}$

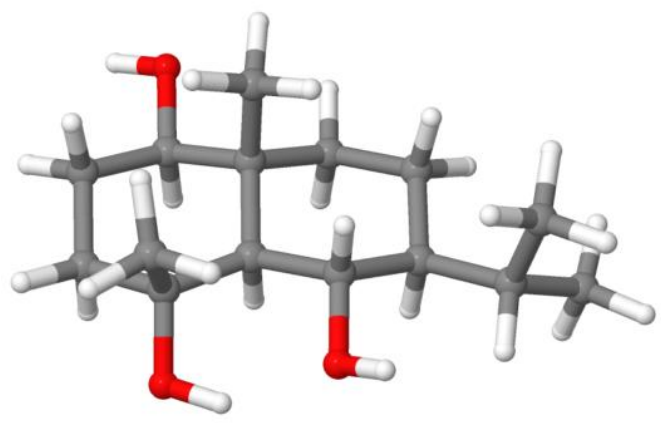




$\begin{array}{rrrr}\text { H } & 0.68458 & 2.42450 & 1.68483 \\ \mathrm{H} & 1.98801 & 1.29455 & 2.08623 \\ \mathrm{H} & -3.53927 & 1.27764 & -0.41319 \\ \mathrm{H} & -5.46171 & -0.16515 & -0.78797 \\ \mathrm{H} & -4.22891 & -0.50943 & -2.01264 \\ \mathrm{H} & -4.47478 & -1.62409 & -0.65684 \\ \mathrm{H} & -4.77363 & 0.49061 & 1.57613 \\ \mathrm{H} & -3.07701 & 0.72155 & 2.00956 \\ \mathrm{H} & -3.70856 & -0.90798 & 1.74210 \\ \mathrm{H} & 3.48158 & -2.91847 & -0.39322 \\ \mathrm{H} & 0.41972 & 2.88850 & -0.80007 \\ \mathrm{O} & -1.19052 & 2.14505 & -0.31310 \\ \mathrm{H} & -1.94051 & 2.47648 & 0.20157\end{array}$

\section{Conformer 2}

Energy: -814.10649 Hartree (Rel: $0.6 \mathrm{kcal} / \mathrm{mol}$ ) XYZ coordinates for conf 2 :

\begin{tabular}{|c|c|c|c|}
\hline & & & \\
\hline C & 3.35133 & -0.38482 & -0.06398 \\
\hline C & 2.97782 & 1.02072 & -0.54216 \\
\hline $\mathrm{C}$ & 1.59151 & 1.50835 & -0.07113 \\
\hline C & 0.50667 & 0.42027 & -0.34214 \\
\hline C & 0.87843 & -1.03949 & 0.10231 \\
\hline C & 2.27188 & -1.39024 & -0.47860 \\
\hline C & -0.92336 & 0.79683 & 0.13994 \\
\hline $\mathrm{C}$ & -1.96961 & -0.16788 & -0.46968 \\
\hline $\mathrm{C}$ & -1.61753 & -1.63239 & -0.14883 \\
\hline C & -0.17500 & -1.98089 & -0.53874 \\
\hline $\mathrm{C}$ & 0.89218 & -1.27652 & 1.63191 \\
\hline $\mathrm{H}$ & 0.45719 & 0.38782 & -1.44214 \\
\hline $\mathrm{C}$ & 1.61527 & 2.06438 & 1.35826 \\
\hline 0 & 1.22378 & 2.60353 & -0.96679 \\
\hline $\mathrm{C}$ & -3.42395 & 0.23836 & -0.10337 \\
\hline $\mathrm{C}$ & -4.45474 & -0.49362 & -0.97855 \\
\hline $\mathrm{C}$ & -3.76998 & 0.07252 & 1.38616 \\
\hline $\mathrm{O}$ & 2.60244 & -2.71667 & -0.06743 \\
\hline $\mathrm{H}$ & 4.31275 & -0.67734 & -0.51032 \\
\hline $\mathrm{H}$ & 3.49371 & -0.41927 & 1.02229 \\
\hline $\mathrm{H}$ & 2.95491 & 1.02901 & -1.63921 \\
\hline $\mathrm{H}$ & 3.74226 & 1.74984 & -0.23932 \\
\hline $\mathrm{H}$ & 2.18516 & -1.35786 & -1.57980 \\
\hline $\mathrm{H}$ & -0.95728 & 0.70681 & 1.23552 \\
\hline $\mathrm{H}$ & -1.88300 & -0.03658 & -1.56084 \\
\hline $\mathrm{H}$ & -2.30125 & -2.30577 & -0.67983 \\
\hline $\mathrm{H}$ & -1.76784 & -1.83251 & 0.91915 \\
\hline $\mathrm{H}$ & -0.07654 & -1.91809 & -1.63287 \\
\hline $\mathrm{H}$ & 0.05701 & -3.01555 & -0.26452 \\
\hline $\mathrm{H}$ & 1.07705 & -2.33619 & 1.82648 \\
\hline $\mathrm{H}$ & 1.67786 & -0.71574 & 2.14407 \\
\hline $\mathrm{H}$ & -0.05601 & -1.00681 & 2.10149 \\
\hline $\mathrm{H}$ & 2.34472 & 2.88395 & 1.42459 \\
\hline $\mathrm{H}$ & 0.63235 & 2.45767 & 1.63162 \\
\hline $\mathrm{H}$ & 1.90918 & 1.31425 & 2.09569 \\
\hline $\mathrm{H}$ & -3.49262 & 1.30661 & -0.33840 \\
\hline $\mathrm{H}$ & -5.46188 & -0.09515 & -0.80535 \\
\hline $\mathrm{H}$ & -4.22927 & -0.37456 & -2.04569 \\
\hline $\mathrm{H}$ & -4.48979 & -1.56877 & -0.76219 \\
\hline $\mathrm{H}$ & -4.77929 & 0.45317 & 1.58427 \\
\hline $\mathrm{H}$ & -3.08121 & 0.62821 & 2.03230 \\
\hline $\mathrm{H}$ & -3.75303 & -0.97927 & 1.69774 \\
\hline $\mathrm{H}$ & 3.45981 & -2.94087 & -0.46056 \\
\hline $\mathrm{H}$ & 1.74350 & 3.38306 & -0.71053 \\
\hline O & -1.28820 & 2.13719 & -0.17888 \\
\hline & -0.52822 & 2.55409 & -0.62717 \\
\hline
\end{tabular}

Conformer 3

Energy: -814.10710 Hartree (Rel: $0.3 \mathrm{kcal} / \mathrm{mol})$ XYZ coordinates for conf 3 :

$\begin{array}{llrl}\mathrm{C} & 3.35019 & -0.38223 & -0.11692 \\ \mathrm{C} & 2.97007 & 1.03337 & -0.56127 \\ \mathrm{C} & 1.60221 & 1.50769 & -0.02996 \\ \mathrm{C} & 0.51015 & 0.42974 & -0.34551\end{array}$

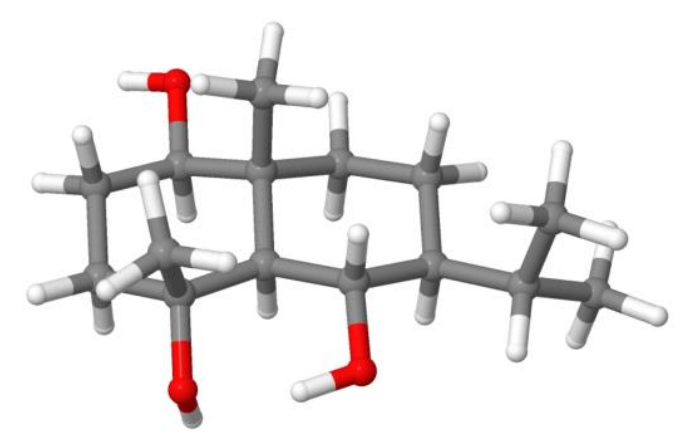




$\begin{array}{rrr}0.87375 & -1.03415 & 0.09354 \\ 2.26032 & -1.38898 & -0.50213 \\ -0.93217 & 0.82203 & 0.08537 \\ -1.97945 & -0.17100 & -0.46685 \\ -1.61921 & -1.62484 & -0.11588 \\ -0.18676 & -1.97334 & -0.53615 \\ 0.89238 & -1.27162 & 1.62387 \\ 0.48200 & 0.39728 & -1.44964 \\ 1.68453 & 1.96611 & 1.43006 \\ 1.27864 & 2.74215 & -0.73348 \\ -3.43028 & 0.24162 & -0.08839 \\ -4.47110 & -0.51918 & -0.92657 \\ -3.75066 & 0.11233 & 1.41044 \\ 2.59586 & -2.71026 & -0.08018 \\ 4.29767 & -0.67304 & -0.59314 \\ 3.52528 & -0.42521 & 0.96417 \\ 2.92349 & 1.05220 & -1.66123 \\ 3.73818 & 1.76040 & -0.27255 \\ 2.15626 & -1.36944 & -1.60267 \\ -0.98045 & 0.81950 & 1.18632 \\ -1.91068 & -0.07113 & -1.56224 \\ -2.31480 & -2.30940 & -0.61588 \\ -1.74219 & -1.80208 & 0.95945 \\ -0.11047 & -1.90547 & -1.63197 \\ 0.05281 & -3.00852 & -0.27073 \\ 0.96287 & -2.34493 & 1.81987 \\ 1.74612 & -0.80367 & 2.11816 \\ -0.01039 & -0.89713 & 2.11191 \\ 2.33680 & 2.84369 & 1.48322 \\ 0.70103 & 2.25422 & 1.81271 \\ 2.09347 & 1.19453 & 2.08356 \\ -3.51076 & 1.30242 & -0.35065 \\ -5.47678 & -0.11884 & -0.74992 \\ -4.26196 & -0.43027 & -1.99993 \\ -4.49924 & -1.58759 & -0.67839 \\ -4.75849 & 0.49289 & 1.61574 \\ -3.05445 & 0.68719 & 2.03146 \\ -3.72264 & -0.93127 & 1.74747 \\ 3.45487 & -2.93494 & -0.46964 \\ 1.20027 & 2.52947 & -1.67939 \\ -1.30622 & 2.10854 & -0.39664 \\ -0.50804 & 2.67039 & -0.38562 \\ & & \end{array}$

\section{Conformer 4}

Energy: -814.10750 Hartree (Rel: $0.0 \mathrm{kcal} / \mathrm{mol}$ ) XYZ coordinates for conf 4 :

$\begin{array}{lrrr}\text { C } & 3.35535 & -0.38651 & -0.11833 \\ \text { C } & 2.97128 & 1.02814 & -0.56059 \\ \text { C } & 1.60322 & 1.50618 & -0.03410 \\ \text { C } & 0.50928 & 0.42729 & -0.34256 \\ \text { C } & 0.87775 & -1.03296 & 0.10297 \\ \text { C } & 2.26942 & -1.38906 & -0.50306 \\ \text { C } & -0.93180 & 0.82258 & 0.08889 \\ \text { C } & -1.97908 & -0.16997 & -0.46407 \\ \text { C } & -1.62345 & -1.62164 & -0.10212 \\ \text { C } & -0.18897 & -1.97386 & -0.51482 \\ \text { C } & 0.90760 & -1.26595 & 1.63177 \\ \text { H } & 0.48004 & 0.38998 & -1.44671 \\ \text { C } & 1.68396 & 1.97807 & 1.42190 \\ \text { O } & 1.27673 & 2.73464 & -0.74791 \\ \text { C } & -3.43123 & 0.24444 & -0.09315 \\ \text { C } & -4.46948 & -0.52135 & -0.92996 \\ \text { C } & -3.75629 & 0.12592 & 1.40550 \\ \text { O } & 2.73817 & -2.67503 & -0.10421 \\ \text { H } & 4.29529 & -0.68906 & -0.59355 \\ \text { H } & 3.53129 & -0.42980 & 0.96270 \\ \text { H } & 2.92826 & 1.04783 & -1.66084 \\ \text { H } & 3.73876 & 1.75500 & -0.26971 \\ \text { H } & 2.16174 & -1.36210 & -1.60320 \\ \text { H } & -0.97919 & 0.81745 & 1.19008 \\ \text { H } & -1.90610 & -0.07605 & -1.55968\end{array}$

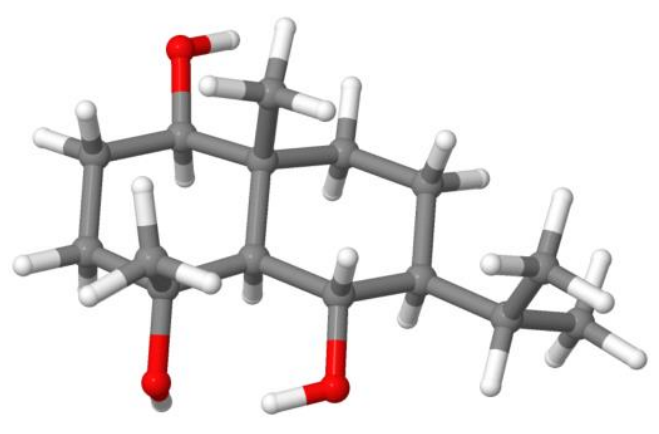




$\begin{array}{rrrr}\text { H } & -2.31769 & -2.30973 & -0.59891 \\ \mathrm{H} & -1.74780 & -1.79023 & 0.97412 \\ \mathrm{H} & -0.11194 & -1.92745 & -1.61191 \\ \mathrm{H} & 0.02362 & -3.01327 & -0.22598 \\ \mathrm{H} & 1.03259 & -2.33433 & 1.83134 \\ \mathrm{H} & 1.73941 & -0.75855 & 2.12303 \\ \mathrm{H} & -0.01129 & -0.93573 & 2.12180 \\ \mathrm{H} & 2.32761 & 2.86245 & 1.46414 \\ \mathrm{H} & 0.69913 & 2.25994 & 1.80587 \\ \mathrm{H} & 2.10482 & 1.21757 & 2.08078 \\ \mathrm{H} & -3.50938 & 1.30343 & -0.36291 \\ \mathrm{H} & -5.47516 & -0.11820 & -0.76042 \\ \mathrm{H} & -4.25611 & -0.44084 & -2.00314 \\ \mathrm{H} & -4.50093 & -1.58787 & -0.67371 \\ \mathrm{H} & -4.76478 & 0.50786 & 1.60448 \\ \mathrm{H} & -3.06222 & 0.70558 & 2.02427 \\ \mathrm{H} & -3.72993 & -0.91520 & 1.75065 \\ \mathrm{H} & 2.12353 & -3.33965 & -0.45082 \\ \mathrm{H} & 1.22963 & 2.52064 & -1.69554 \\ \mathrm{O} & -1.30579 & 2.10907 & -0.39024 \\ \mathrm{H} & -0.50147 & 2.66282 & -0.40680\end{array}$

\section{Conformer 5}

Energy: -814.10600 Hartree (Rel: $0.9 \mathrm{kcal} / \mathrm{mol}$ ) XYZ coordinates for conf 5:

\begin{tabular}{|c|c|c|c|}
\hline C & 3.35917 & 0.08865 & -0.12209 \\
\hline C & 2.73026 & 1.42837 & -0.51581 \\
\hline C & 1.30149 & 1.63161 & 0.02762 \\
\hline C & 0.41786 & 0.38947 & -0.33342 \\
\hline C & 1.03990 & -1.00165 & 0.05597 \\
\hline C & 2.46474 & -1.08099 & -0.54753 \\
\hline C & -1.06909 & 0.50030 & 0.10999 \\
\hline C & -1.92890 & -0.63355 & -0.48959 \\
\hline C & -1.30892 & -2.01110 & -0.19059 \\
\hline $\mathrm{C}$ & 0.16171 & -2.09094 & -0.61031 \\
\hline C & 1.10399 & -1.28909 & 1.57700 \\
\hline $\mathrm{H}$ & 0.39558 & 0.39305 & -1.43840 \\
\hline C & 1.30197 & 2.04495 & 1.50357 \\
\hline 0 & 0.76435 & 2.81530 & -0.63201 \\
\hline $\mathrm{C}$ & -3.43406 & -0.59281 & -0.07610 \\
\hline C & -3.65845 & -0.26217 & 1.40920 \\
\hline $\mathrm{C}$ & -4.27643 & 0.33572 & -0.96708 \\
\hline 0 & 3.03144 & -2.33641 & -0.17486 \\
\hline $\mathrm{H}$ & 4.34165 & -0.01075 & -0.60580 \\
\hline $\mathrm{H}$ & 3.54294 & 0.03911 & 0.95714 \\
\hline $\mathrm{H}$ & 2.67693 & 1.47800 & -1.61446 \\
\hline $\mathrm{H}$ & 3.35640 & 2.27055 & -0.19885 \\
\hline $\mathrm{H}$ & 2.35406 & -1.03889 & -1.64665 \\
\hline $\mathrm{H}$ & -1.10418 & 0.42454 & 1.20770 \\
\hline $\mathrm{H}$ & -1.88999 & -0.48938 & -1.58088 \\
\hline $\mathrm{H}$ & -1.88052 & -2.78122 & -0.72453 \\
\hline $\mathrm{H}$ & -1.41210 & -2.24761 & 0.87695 \\
\hline $\mathrm{H}$ & 0.22445 & -1.97301 & -1.70290 \\
\hline $\mathrm{H}$ & 0.57913 & -3.07648 & -0.37891 \\
\hline $\mathrm{H}$ & 1.34892 & -2.34321 & 1.73327 \\
\hline $\mathrm{H}$ & 1.87370 & -0.70854 & 2.08959 \\
\hline $\mathrm{H}$ & 0.15472 & -1.08509 & 2.07786 \\
\hline $\mathrm{H}$ & 1.82213 & 3.00371 & 1.59705 \\
\hline $\mathrm{H}$ & 0.28246 & 2.17879 & 1.87755 \\
\hline $\mathrm{H}$ & 1.80666 & 1.31597 & 2.13853 \\
\hline $\mathrm{H}$ & -3.81019 & -1.61491 & -0.23863 \\
\hline $\mathrm{H}$ & -4.71518 & -0.39250 & 1.67184 \\
\hline $\mathrm{H}$ & -3.07470 & -0.91238 & 2.07279 \\
\hline $\mathrm{H}$ & -3.38819 & 0.77772 & 1.62247 \\
\hline $\mathrm{H}$ & -5.33877 & 0.26631 & -0.69994 \\
\hline $\mathrm{H}$ & -4.18151 & 0.05830 & -2.02459 \\
\hline $\mathrm{H}$ & -3.95485 & 1.37433 & -0.86118 \\
\hline $\mathrm{H}$ & 3.91701 & -2.38833 & -0.56621 \\
\hline $\mathrm{H}$ & 0.72792 & 2.63002 & -1.58617 \\
\hline 0 & -1.66097 & 1.73290 & -0.28919 \\
\hline $\mathrm{H}$ & -0.96448 & 2.41709 & -0.27114 \\
\hline
\end{tabular}

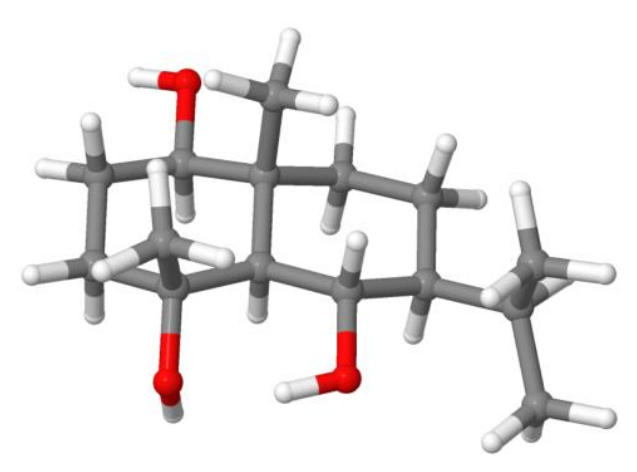


originally assigned structure of 11-hydroxycapitulatin B (PCM methanol), Fig.4D

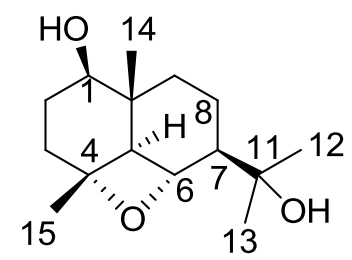

Rel energy (kcal/mol): Conf 1 Conf 2 Conf

\begin{tabular}{|c|c|c|c|c|c|c|c|c|}
\hline C-nom & iGau & $\operatorname{Exp}$ & Calc & $\operatorname{diff}$ & 1 & 2 & 3 & \\
\hline $\mathrm{C} 1-\mathrm{CH}$ & 6 & 79.20 & 81.46 & 2.26 & 81.54 & 81.29 & 81.43 & \\
\hline $\mathrm{C} 2-\mathrm{CH} 2$ & 1 & 28.30 & 34.94 & 6.64 & 34.95 & 34.97 & 34.89 & \\
\hline $\mathrm{C} 3-\mathrm{CH} 2$ & 2 & 40.70 & 41.52 & 0.82 & 41.47 & 41.69 & 41.47 & \\
\hline $\mathrm{C} 4-\mathrm{C}$ & 3 & 73.90 & 90.61 & 16.71 & 89.60 & 93.87 & 90.01 ] & \\
\hline $\mathrm{C} 5-\mathrm{CH}$ & 4 & 56.50 & 57.66 & 1.16 & 57.52 & 57.72 & $57.89]$ & \\
\hline $\mathrm{C} 6-\mathrm{CH}$ & 7 & 72.80 & 79.12 & 6.32 & 79.21 & 78.32 & $79.61]$ & \\
\hline $\mathrm{C} 7-\mathrm{CH}$ & 8 & 55.40 & 57.64 & 2.24 & 56.52 & 60.54 & 57.56 & \\
\hline $\mathrm{C} 8-\mathrm{CH} 2$ & 9 & 23.10 & 24.36 & 1.26 & 23.94 & 25.79 & $24.05]$ & \\
\hline $\mathrm{C} 9-\mathrm{CH} 2$ & 10 & 40.40 & 41.59 & 1.19 & 41.59 & 41.77 & $41.46]$ & \\
\hline C10-C & 5 & 40.90 & 36.50 & -4.40 & 36.34 & 36.68 & 36.69 ] & \\
\hline $\mathrm{C} 11-\mathrm{C}$ & 15 & 75.40 & 71.92 & -3.48 & 71.46 & 73.43 & $71.61]$ & \\
\hline $\mathrm{C} 12-\mathrm{CH} 3$ & 17 & 23.30 & 27.39 & 4.09 & 27.34 & 23.25 & 30.93 & \\
\hline $\mathrm{C} 13-\mathrm{CH} 3$ & 16 & 29.50 & 27.71 & -1.79 & 30.11 & 26.92 & 23.40 & \\
\hline $\mathrm{C} 14-\mathrm{CH} 3$ & 11 & 13.90 & 12.95 & -0.95 & 12.98 & 12.92 & 12.93 ] & \\
\hline $\mathrm{C} 15-\mathrm{CH} 3$ & 13 & 23.40 & 17.83 & -5.57 & [ 17.86 & 17.82 & $17.76]$ & \\
\hline & & $13 \mathrm{C} \mathrm{ch}$ & 。 & $\begin{array}{l}\text { ts: RMS } \\
\text { eractio }\end{array}$ & $\begin{array}{l}5.55 \mathrm{ppm} \\
: \quad 0.532\end{array}$ & $\begin{array}{c}\mathrm{MAE}=3.93 \\
0.212\end{array}$ & $\begin{array}{l}\mathrm{N}=15 \\
0.256\end{array}$ & $\left\{\begin{array}{lll}-5.57 & 16.71\end{array}\right\}$ \\
\hline
\end{tabular}

\section{Conformer 1}

Energy: -812.83920 Hartree (Rel: $0.0 \mathrm{kcal} / \mathrm{mol}$ ) XYZ coordinates for conf 1 :

$\begin{array}{lrrr}\text { C } & 3.23815 & 0.58363 & -0.68144 \\ \mathrm{C} & 2.27671 & 1.78587 & -1.00212 \\ \mathrm{C} & 1.12403 & 1.66446 & 0.00507 \\ \mathrm{C} & 0.67091 & 0.23644 & -0.10537 \\ \mathrm{C} & 1.38527 & -1.02644 & 0.25161 \\ \mathrm{C} & 2.64549 & -0.87286 & -0.66877 \\ \mathrm{C} & -0.71522 & 0.66465 & 0.22684 \\ \mathrm{C} & -1.68403 & -0.32429 & -0.42123 \\ \mathrm{C} & -1.15105 & -1.72001 & 0.08430 \\ \mathrm{C} & 0.33924 & -2.09171 & -0.23380 \\ \mathrm{C} & 1.74712 & -1.21167 & 1.72990 \\ \mathrm{H} & 0.58250 & 0.14371 & -1.20068 \\ \mathrm{C} & 1.45347 & 2.29557 & 1.35365 \\ \mathrm{O} & -0.32344 & 2.01246 & -0.31391 \\ \mathrm{C} & -3.18039 & -0.07114 & -0.11365 \\ \mathrm{C} & -3.56754 & 1.35054 & -0.55590 \\ \mathrm{C} & -4.09816 & -1.09642 & -0.79872 \\ \mathrm{O} & 3.64948 & -1.80177 & -0.26540 \\ \mathrm{H} & 4.05791 & 0.59249 & -1.41204 \\ \mathrm{H} & 3.70471 & 0.75357 & 0.29707 \\ \mathrm{H} & 1.89131 & 1.70292 & -2.02524 \\ \mathrm{H} & 2.83905 & 2.72546 & -0.93164 \\ \mathrm{H} & 2.32195 & -1.10783 & -1.69573 \\ \mathrm{H} & -0.95163 & 0.77924 & 1.28994 \\ \mathrm{H} & -1.56368 & -0.27177 & -1.51206 \\ \mathrm{H} & -1.77684 & -2.51333 & -0.33801 \\ \mathrm{H} & -1.30801 & -1.75767 & 1.16905 \\ \mathrm{H} & 0.44142 & -2.21155 & -1.32106 \\ \mathrm{H} & 0.54277 & -3.07478 & 0.20925 \\ & & & \end{array}$

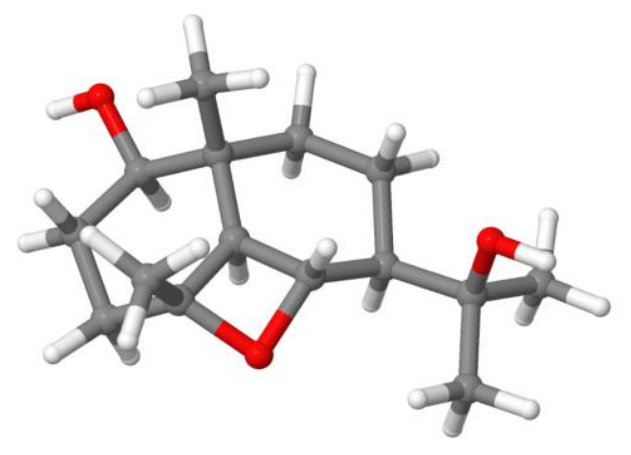




$\begin{array}{rrrr}\mathrm{H} & 2.24894 & -2.17202 & 1.88423 \\ \mathrm{H} & 2.41831 & -0.42442 & 2.08918 \\ \mathrm{H} & 0.84773 & -1.19330 & 2.35602 \\ \mathrm{H} & 2.46822 & 2.04887 & 1.68296 \\ \mathrm{H} & 0.75541 & 1.98525 & 2.13688 \\ \mathrm{H} & 1.39067 & 3.38605 & 1.25650 \\ \mathrm{O} & -3.31836 & -0.18267 & 1.31745 \\ \mathrm{H} & -4.61524 & 1.55492 & -0.30111 \\ \mathrm{H} & -2.93908 & 2.09914 & -0.06440 \\ \mathrm{H} & -3.45849 & 1.47043 & -1.63998 \\ \mathrm{H} & -5.14950 & -0.85104 & -0.60221 \\ \mathrm{H} & -3.91517 & -2.10759 & -0.42434 \\ \mathrm{H} & -3.95447 & -1.09594 & -1.88554 \\ \mathrm{H} & 4.39564 & -1.71699 & -0.88094 \\ \mathrm{H} & -4.23985 & 0.03224 & 1.53659\end{array}$

\section{Conformer 2}

Energy: -812.83834 Hartree (Rel: $0.5 \mathrm{kcal} / \mathrm{mol}$ ) XYZ coordinates for conf 2 :

\begin{tabular}{|c|c|c|c|}
\hline C & 3.21545 & 0.58221 & -0.71772 \\
\hline C & 2.24305 & 1.78009 & -1.02789 \\
\hline C & 1.11414 & 1.66195 & 0.00552 \\
\hline C & 0.66935 & 0.22866 & -0.08560 \\
\hline C & 1.39222 & -1.02913 & 0.27064 \\
\hline C & 2.63218 & -0.87913 & -0.67792 \\
\hline C & -0.71161 & 0.64740 & 0.26874 \\
\hline C & -1.67900 & -0.34812 & -0.37147 \\
\hline C & -1.14961 & -1.73172 & 0.16129 \\
\hline C & 0.33760 & -2.10452 & -0.18032 \\
\hline $\mathrm{C}$ & 1.78602 & -1.19855 & 1.74259 \\
\hline $\mathrm{H}$ & 0.56281 & 0.12687 & -1.17776 \\
\hline C & 1.46344 & 2.31090 & 1.33910 \\
\hline O & -0.35262 & 1.99356 & -0.28466 \\
\hline C & -3.18510 & -0.05267 & -0.14833 \\
\hline C & -4.06934 & -1.00893 & -0.95490 \\
\hline C & -3.60969 & -0.05852 & 1.32933 \\
\hline 0 & 3.64984 & -1.79630 & -0.28438 \\
\hline $\mathrm{H}$ & 4.01766 & 0.58873 & -1.46724 \\
\hline $\mathrm{H}$ & 3.70402 & 0.76333 & 0.24791 \\
\hline $\mathrm{H}$ & 1.83627 & 1.68778 & -2.04166 \\
\hline $\mathrm{H}$ & 2.80378 & 2.72156 & -0.97612 \\
\hline $\mathrm{H}$ & 2.28912 & -1.12749 & -1.69521 \\
\hline $\mathrm{H}$ & -0.91301 & 0.76241 & 1.34051 \\
\hline $\mathrm{H}$ & -1.52519 & -0.31240 & -1.45880 \\
\hline $\mathrm{H}$ & -1.78639 & -2.53282 & -0.23012 \\
\hline $\mathrm{H}$ & -1.27344 & -1.75203 & 1.25224 \\
\hline $\mathrm{H}$ & 0.41648 & -2.23908 & -1.26748 \\
\hline $\mathrm{H}$ & 0.55300 & -3.08002 & 0.27280 \\
\hline $\mathrm{H}$ & 2.29617 & -2.15479 & 1.89421 \\
\hline $\mathrm{H}$ & 2.46052 & -0.40483 & 2.08046 \\
\hline $\mathrm{H}$ & 0.90055 & -1.17963 & 2.38829 \\
\hline $\mathrm{H}$ & 2.48570 & 2.07259 & 1.65066 \\
\hline $\mathrm{H}$ & 0.78269 & 2.00668 & 2.13963 \\
\hline $\mathrm{H}$ & 1.39376 & 3.39967 & 1.22994 \\
\hline 0 & -3.45092 & 1.25313 & -0.71011 \\
\hline $\mathrm{H}$ & -5.11166 & -0.67482 & -0.90969 \\
\hline $\mathrm{H}$ & -3.76131 & -1.02772 & -2.00620 \\
\hline $\mathrm{H}$ & -4.02219 & -2.02802 & -0.55920 \\
\hline $\mathrm{H}$ & -4.67264 & 0.19503 & 1.40184 \\
\hline $\mathrm{H}$ & -3.05117 & 0.68192 & 1.91252 \\
\hline $\mathrm{H}$ & -3.46122 & -1.03949 & 1.79419 \\
\hline $\mathrm{H}$ & 4.38001 & -1.71979 & -0.91982 \\
\hline $\mathrm{H}$ & -2.76939 & 1.86370 & -0.38101 \\
\hline
\end{tabular}

Conformer 3

Energy: -812.83851 Hartree (Rel: $0.4 \mathrm{kcal} / \mathrm{mol})$ XYZ coordinates for conf 3 :

$\begin{array}{lllr}\text { C } & 3.22939 & 0.59466 & -0.68864 \\ \text { C } & 2.26551 & 1.80231 & -0.98060 \\ \text { C } & 1.12624 & 1.66928 & 0.04020 \\ \text { C } & 0.67118 & 0.24279 & -0.08265\end{array}$
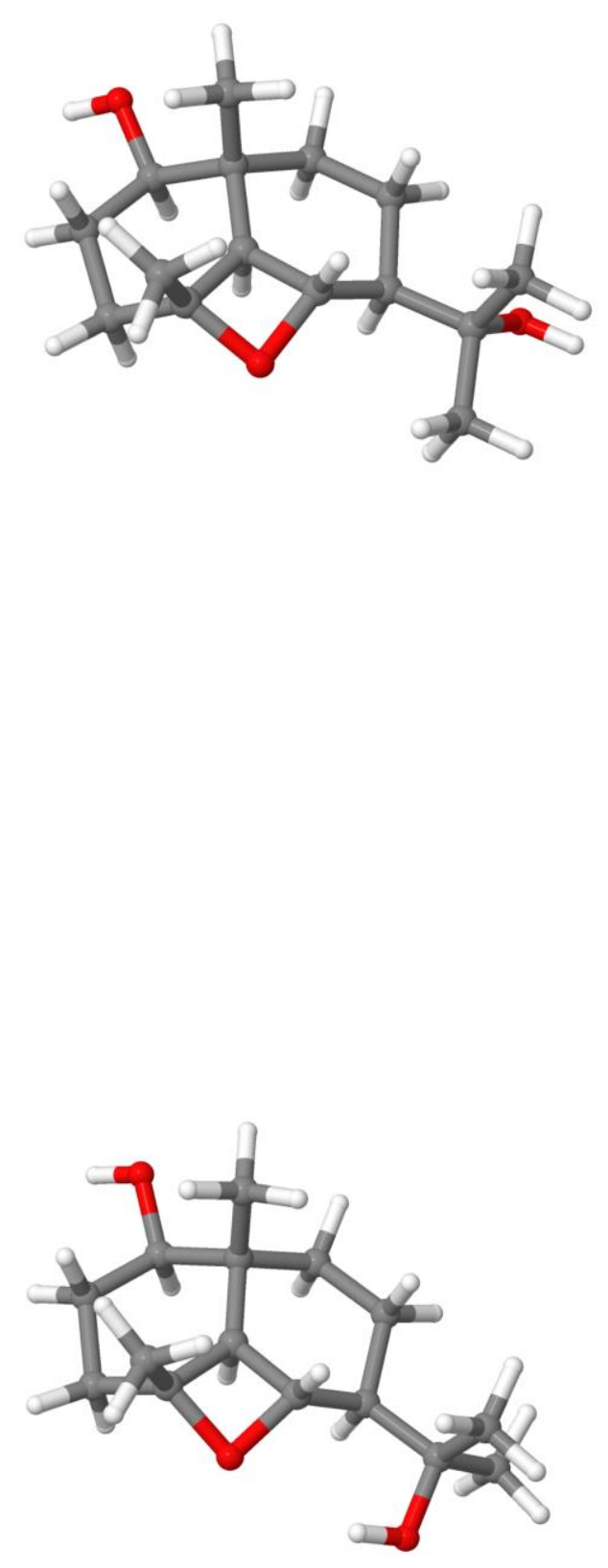


$\begin{array}{rrr}1.38794 & -1.02679 & 0.24814 \\ 2.63568 & -0.86164 & -0.68742 \\ -0.71260 & 0.66919 & 0.26714 \\ -1.67562 & -0.31891 & -0.38823 \\ -1.14695 & -1.71415 & 0.11545 \\ 0.33447 & -2.08729 & -0.23590 \\ 1.77143 & -1.23092 & 1.71853 \\ 0.57076 & 0.16462 & -1.17752 \\ 1.47214 & 2.28567 & 1.39111 \\ -0.32707 & 2.02161 & -0.25699 \\ -3.18700 & -0.08339 & -0.15370 \\ -3.62179 & -0.22916 & 1.31278 \\ -3.57830 & 1.30307 & -0.69138 \\ 3.64413 & -1.79630 & -0.30959 \\ 4.03896 & 0.61269 & -1.43028 \\ 3.70956 & 0.75123 & 0.28549 \\ 1.86637 & 1.73350 & -1.99944 \\ 2.82998 & 2.74014 & -0.90497 \\ 2.29825 & -1.08299 & -1.71287 \\ -0.92506 & 0.76990 & 1.33857 \\ -1.53057 & -0.27157 & -1.47639 \\ -1.80303 & -2.49223 & -0.28691 \\ -1.26099 & -1.75272 & 1.20692 \\ 0.41771 & -2.19271 & -1.32617 \\ 0.54618 & -3.07552 & 0.19126 \\ 2.27617 & -2.19264 & 1.85295 \\ 2.44732 & -0.44770 & 2.07775 \\ 0.88194 & -1.22138 & 2.35887 \\ 2.49098 & 2.03534 & 1.70475 \\ 0.78451 & 1.96729 & 2.18038 \\ 1.40836 & 3.37707 & 1.30643 \\ -3.83981 & -1.09860 & -0.94483 \\ -4.70033 & -0.04675 & 1.40004 \\ -3.41993 & -1.23692 & 1.68704 \\ -3.11556 & 0.49241 & 1.96316 \\ -4.65978 & 1.46002 & -0.59106 \\ -3.31637 & 1.38823 & -1.75164 \\ -3.06751 & 2.10453 & -0.14677 \\ 4.38101 & -1.70618 & -0.93541 \\ -4.79734 & -0.97969 & -0.83162\end{array}$


revised structure of 11-hydroxycapitulatin B (PCM methanol), Fig.4D

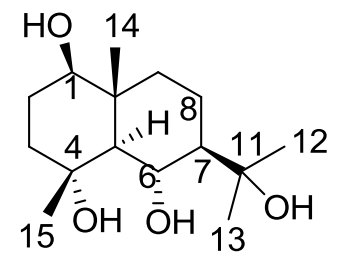

$$
\text { Rel energy (kcal/mol): } \begin{array}{ccc}
\text { Conf } 1 & \text { Conf } 2 \\
0.0 & 0.3
\end{array}
$$

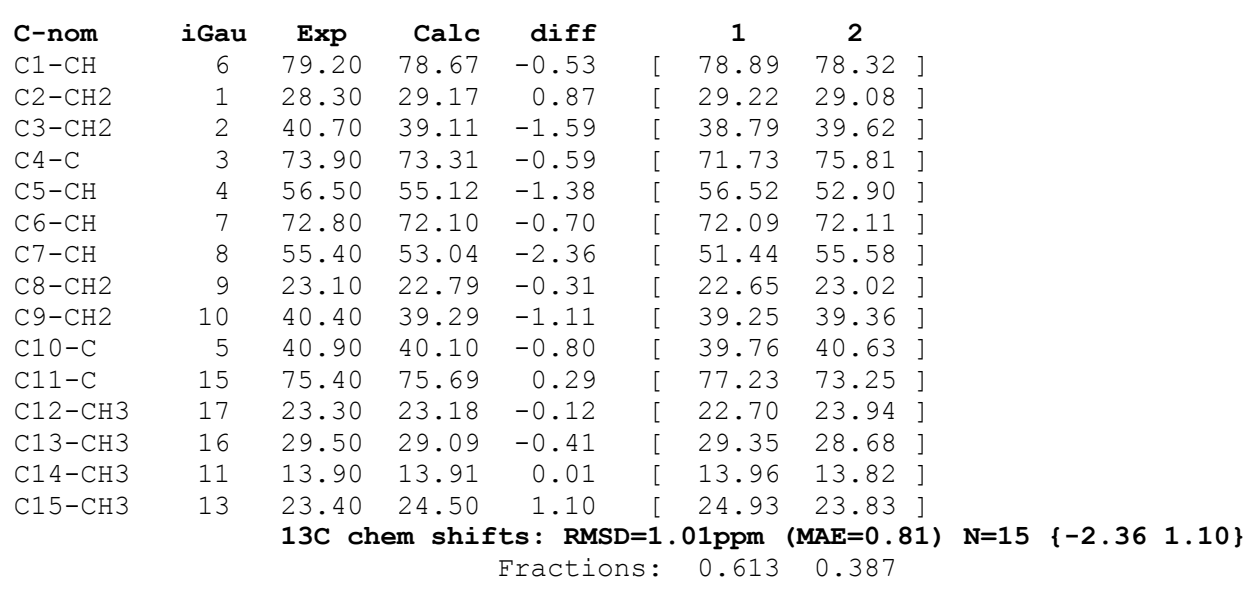

\section{Conformer 1}

Energy: -889.33781 Hartree (Rel: $0.0 \mathrm{kcal} / \mathrm{mol}$ ) XYZ coordinates for conf 1 :

\begin{tabular}{lrrr} 
XYZ Cordinates for Conf & \multicolumn{1}{l}{ I. } \\
$\mathrm{C}$ & 3.57295 & -0.13958 & -0.13476 \\
$\mathrm{C}$ & 3.06199 & 1.22728 & -0.59400 \\
$\mathrm{C}$ & 1.65927 & 1.59272 & -0.06683 \\
$\mathrm{C}$ & 0.67744 & 0.39472 & -0.31694 \\
$\mathrm{C}$ & 1.18263 & -1.03007 & 0.10872 \\
$\mathrm{C}$ & 2.58211 & -1.24169 & -0.51987 \\
$\mathrm{C}$ & -0.76671 & 0.63189 & 0.19480 \\
$\mathrm{C}$ & -1.74619 & -0.39782 & -0.42181 \\
$\mathrm{C}$ & -1.26609 & -1.82682 & -0.10104 \\
$\mathrm{C}$ & 0.19584 & -2.05151 & -0.51225 \\
$\mathrm{H}$ & 1.25666 & -1.27789 & 1.63528 \\
$\mathrm{C}$ & 0.60586 & 0.36441 & -1.41535 \\
$\mathrm{O}$ & 1.71470 & 2.09146 & 1.38844 \\
$\mathrm{C}$ & 1.24893 & 2.69588 & -0.89623 \\
$\mathrm{C}$ & -3.23897 & -0.14943 & -0.04568 \\
$\mathrm{C}$ & -4.16288 & -1.24146 & -0.60126 \\
$\mathrm{O}$ & -3.47380 & 0.04073 & 1.45911 \\
$\mathrm{H}$ & 3.05660 & -2.53711 & -0.13823 \\
$\mathrm{H}$ & 4.54163 & -0.34682 & -0.61038 \\
$\mathrm{H}$ & 3.74993 & -0.15929 & 0.94712 \\
$\mathrm{H}$ & 2.99993 & 1.23660 & -1.69006 \\
$\mathrm{H}$ & 3.76668 & 2.02068 & -0.31498 \\
$\mathrm{H}$ & 2.45526 & -1.21187 & -1.61621 \\
$\mathrm{H}$ & -0.78097 & 0.51686 & 1.28599 \\
$\mathrm{H}$ & -1.69989 & -0.26665 & -1.51393 \\
$\mathrm{H}$ & -1.89148 & -2.55412 & -0.62825 \\
$\mathrm{H}$ & -1.39112 & -2.03330 & 0.96953 \\
$\mathrm{H}$ & 0.26714 & -1.98559 & -1.60770 \\
$\mathrm{H}$ & 0.50448 & -3.06672 & -0.24012 \\
$\mathrm{H}$ & 1.51730 & -2.32467 & 1.81591 \\
$\mathrm{H}$ & 2.01520 & -0.66723 & 2.12907 \\
$\mathrm{H}$ & 0.30673 & -1.08113 & 2.13801 \\
$\mathrm{H}$ & 2.15356 & 1.36500 & 2.07782 \\
$\mathrm{H}$ & 0.71507 & 2.34727 & 1.75383 \\
& 2.32644 & 2.99981 & 1.42044
\end{tabular}

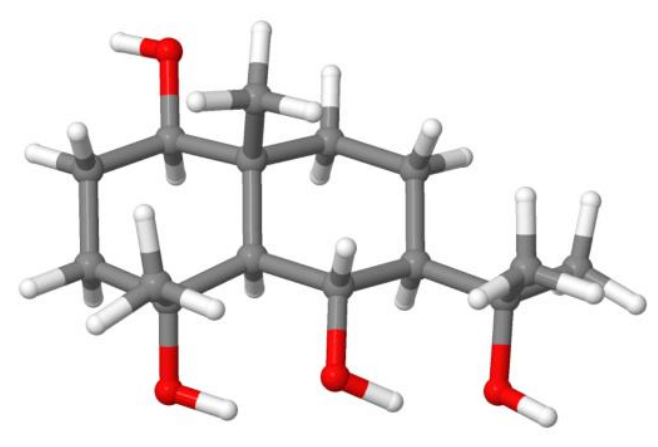




$\begin{array}{rrrr}\mathrm{O} & -3.57261 & 1.09373 & -0.74023 \\ \mathrm{H} & -5.20727 & -0.92399 & -0.49792 \\ \mathrm{H} & -3.96346 & -1.41807 & -1.66373 \\ \mathrm{H} & -4.04985 & -2.18614 & -0.06106 \\ \mathrm{H} & -4.54359 & 0.17776 & 1.65962 \\ \mathrm{H} & -2.94271 & 0.92006 & 1.83498 \\ \mathrm{H} & -3.14338 & -0.83534 & 2.02684 \\ \mathrm{H} & 3.92236 & -2.66099 & -0.55911 \\ \mathrm{H} & -4.41845 & 1.41997 & -0.38888 \\ \mathrm{H} & 0.28332 & 2.76931 & -0.74740 \\ \mathrm{O} & -1.16859 & 1.98441 & -0.09393 \\ \mathrm{H} & -2.10711 & 1.95366 & -0.38681\end{array}$

\section{Conformer 2}

Energy: -889.33738 Hartree (Rel: $0.3 \mathrm{kcal} / \mathrm{mol}$ ) XYZ coordinates for conf 2:

$\begin{array}{lrrr}\mathrm{C} & 3.56419 & -0.17498 & -0.14188 \\ \mathrm{C} & 3.05779 & 1.19661 & -0.59509 \\ \mathrm{C} & 1.65807 & 1.55878 & -0.05613 \\ \mathrm{C} & 0.66201 & 0.38445 & -0.31076 \\ \mathrm{C} & 1.16529 & -1.04298 & 0.11204 \\ \mathrm{C} & 2.56058 & -1.26679 & -0.52417 \\ \mathrm{C} & -0.78015 & 0.62722 & 0.21005 \\ \mathrm{C} & -1.76343 & -0.39298 & -0.41532 \\ \mathrm{C} & -1.29157 & -1.82245 & -0.08563 \\ \mathrm{C} & 0.16775 & -2.05980 & -0.50025 \\ \mathrm{C} & 1.24731 & -1.28584 & 1.63874 \\ \mathrm{H} & 0.58858 & 0.35333 & -1.40907 \\ \mathrm{C} & 1.70509 & 2.08707 & 1.38285 \\ \mathrm{O} & 1.14740 & 2.63338 & -0.91107 \\ \mathrm{C} & -3.26285 & -0.11186 & -0.06438 \\ \mathrm{C} & -4.18150 & -1.20083 & -0.63382 \\ \mathrm{C} & -3.50780 & 0.03852 & 1.44919 \\ \mathrm{O} & 3.02330 & -2.56484 & -0.14270 \\ \mathrm{H} & 4.52721 & -0.38458 & -0.62650 \\ \mathrm{H} & 3.74969 & -0.19792 & 0.93820 \\ \mathrm{H} & 2.98959 & 1.20747 & -1.69022 \\ \mathrm{H} & 3.76905 & 1.98458 & -0.31561 \\ \mathrm{H} & 2.42921 & -1.23557 & -1.61972 \\ \mathrm{H} & -0.79019 & 0.50017 & 1.29901 \\ \mathrm{H} & -1.70160 & -0.26221 & -1.50701 \\ \mathrm{H} & -1.92244 & -2.55073 & -0.60532 \\ \mathrm{H} & -1.41449 & -2.02231 & 0.98663 \\ \mathrm{H} & 0.23640 & -1.99920 & -1.59625 \\ \mathrm{H} & 0.47529 & -3.07421 & -0.22447 \\ \mathrm{H} & 1.51074 & -2.33182 & 1.81841 \\ \mathrm{H} & 2.00784 & -0.67489 & 2.12954 \\ \mathrm{H} & 0.29907 & -1.09087 & 2.14465 \\ \mathrm{H} & 2.13762 & 1.36401 & 2.07725 \\ \mathrm{H} & 0.70524 & 2.35401 & 1.73589 \\ \mathrm{H} & 2.33269 & 2.98677 & 1.42134 \\ \mathrm{O} & -3.67353 & 1.09754 & -0.72757 \\ \mathrm{H} & -5.21968 & -0.85756 & -0.56495 \\ \mathrm{H} & -3.95655 & -1.39149 & -1.68971 \\ \mathrm{H} & -4.09825 & -2.14231 & -0.08279 \\ \mathrm{H} & -4.57852 & 0.18134 & 1.63303 \\ \mathrm{H} & -2.98168 & 0.91223 & 1.84715 \\ \mathrm{H} & -3.18210 & -0.84563 & 2.01015 \\ \mathrm{H} & 3.87715 & -2.70710 & -0.58169 \\ \mathrm{H} & -2.92953 & 1.72045 & -0.58963 \\ \mathrm{H} & 1.60211 & 3.45669 & -0.66391 \\ \mathrm{O} & -1.24152 & 1.96937 & -0.04152 \\ \mathrm{H} & -0.51235 & 2.45898 & -0.48425 \\ & & & \end{array}$

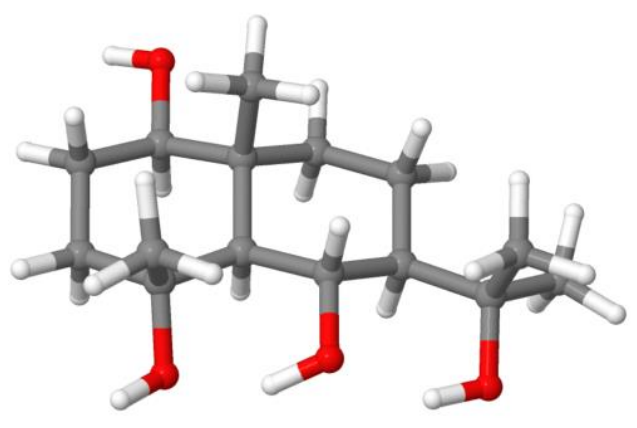


originally assigned structure of cephaloziellin B, Fig.4E

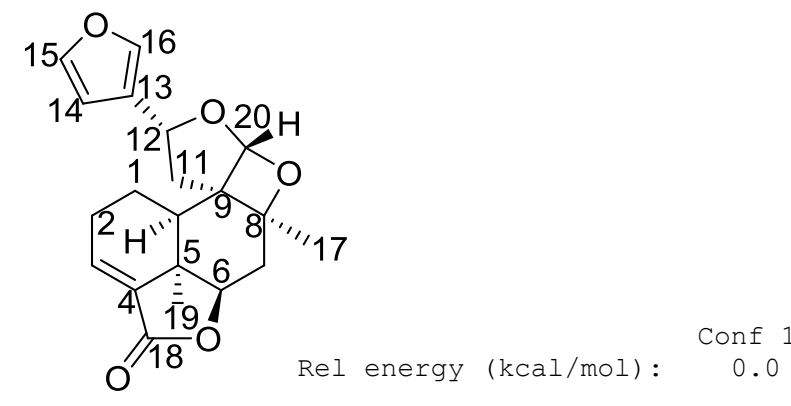

\begin{tabular}{|c|c|c|c|c|c|c|}
\hline iGau & jGau & Jexp & Jcalc & $\operatorname{diff}$ & 1 & \\
\hline 31 & 32 & 15.60 & 14.37 & -1.23 & -14.37 & H1alpha H1beta \\
\hline 28 & 32 & 7.20 & 6.67 & -0.53 & 6.67 & H2beta H1beta \\
\hline 33 & 35 & 11.40 & 12.11 & 0.71 & 12.11 & H6 H7beta \\
\hline 33 & 34 & 7.80 & 5.79 & -2.01 & 5.79 & H6 H7alpha \\
\hline 34 & 35 & 12.50 & 14.76 & 2.26 & -14.76 & H7alpha H7beta \\
\hline 40 & 41 & 13.20 & 14.27 & 1.07 & {$[-14.27$} & H11beta H11alpha \\
\hline 39 & 41 & 7.80 & 5.05 & -2.75 & {$[\quad 5.05$} & H12 H11alpha \\
\hline 39 & 40 & 9.00 & 10.08 & 1.08 & 10.08 & H12 H11beta \\
\hline & & For Js & & $1.63 \mathrm{~Hz}$ & $\{-2.7$ & $52.26\}$ \\
\hline
\end{tabular}

\begin{tabular}{|c|c|c|c|c|c|c|}
\hline H-nom & iGau & $\operatorname{Exp}$ & Calc & diff & & 1 \\
\hline Hlalpha & 31 & 2.11 & 1.99 & -0.12 & {[} & 1.99 \\
\hline H1beta & 32 & 1.86 & 1.87 & 0.01 & {[} & 1.87 \\
\hline H2alpha & 29 & 2.40 & 2.39 & -0.01 & {[} & 2.39 \\
\hline H2beta & 28 & 2.62 & 2.41 & -0.21 & {[} & 2.41 \\
\hline H3 & 30 & 6.95 & 7.03 & 0.08 & {[} & 7.03 \\
\hline H6 & 33 & 4.60 & 4.21 & -0.39 & {[} & 4.21 \\
\hline H7alpha & 34 & 2.30 & 2.56 & 0.26 & {[} & 2.56 \\
\hline H7beta & 35 & 1.78 & 1.93 & 0.15 & {[} & 1.93 \\
\hline $\mathrm{H} 10$ & 21 & 2.00 & 1.76 & -0.24 & {[} & 1.76 \\
\hline H11alpha & 41 & 3.00 & 2.64 & -0.36 & {[} & 2.64 \\
\hline H11beta & 40 & 1.98 & 2.15 & 0.17 & {[} & 2.15 \\
\hline $\mathrm{H} 12$ & 39 & 5.32 & 5.42 & 0.10 & {[} & 5.42 \\
\hline $\mathrm{H} 14$ & 47 & 6.50 & 7.06 & 0.56 & {[} & 7.06 \\
\hline H1 5 & 46 & 7.37 & 7.31 & -0.06 & {[} & 7.31 \\
\hline H1 6 & 45 & 7.44 & 7.19 & -0.25 & {[} & 7.19 \\
\hline $\mathrm{H} 2 \mathrm{O}$ & 27 & 5.49 & 5.55 & 0.06 & {[} & 5.55 \\
\hline
\end{tabular}

$1 \mathrm{H}$ chem shifts: RMSD $=0.24 \mathrm{ppm}(\mathrm{MAE}=0.19) \quad \mathrm{N}=16 \quad\{-0.390 .56\}$ $\mathrm{m}=1.000 \quad \mathrm{~b}=0.00$

\begin{tabular}{|c|c|c|c|c|c|}
\hline C-nom & iGau & $\operatorname{Exp}$ & Calc & $\operatorname{diff}$ & 1 \\
\hline $\mathrm{C} 1-\mathrm{CH} 2$ & 6 & 19.40 & 20.17 & 0.77 & 20.17 \\
\hline $\mathrm{C} 2-\mathrm{CH} 2$ & 1 & 23.80 & 23.42 & -0.38 & 23.42 \\
\hline $\mathrm{C} 3-\mathrm{CH}$ & 2 & 138.10 & 134.57 & -3.53 & 134.57 \\
\hline $\mathrm{C} 4-\mathrm{C}$ & 3 & 132.70 & 134.24 & 1.54 & [ 134.24 \\
\hline C5-C & 4 & 39.50 & 40.36 & 0.86 & {$[40.36$} \\
\hline $\mathrm{C} 6-\mathrm{CH}$ & 7 & 84.90 & 80.17 & -4.73 & 80.17 \\
\hline $\mathrm{C} 7-\mathrm{CH} 2$ & 8 & 42.10 & 44.30 & 2.2 & 44.30 \\
\hline $\mathrm{C} 8-\mathrm{C}$ & 9 & 72.80 & 75.61 & 2.8 & 75.61 \\
\hline $\mathrm{C} 9-\mathrm{C}$ & 10 & 60.30 & 60.08 & -0.2 & 60.08 \\
\hline $\mathrm{ClO}-\mathrm{CH}$ & 5 & 46.20 & 42.31 & -3.8 & 42.31 \\
\hline $\mathrm{C} 11-\mathrm{CH} 2$ & 19 & 37.80 & 44.68 & 6.8 & 44.68 \\
\hline $\mathrm{C} 12-\mathrm{CH}$ & 18 & 74.50 & 77.55 & 3. & 77.55 \\
\hline C13-C & 22 & 128.70 & 128.34 & -0.36 & 128.34 \\
\hline $\mathrm{Cl} 4-\mathrm{CH}$ & 26 & 109.50 & 110.26 & 0.7 & 110.26 \\
\hline $\mathrm{C} 15-\mathrm{CH}$ & 25 & 143.80 & 142.46 & $-1 \cdot 34$ & {$[142.46$} \\
\hline $\mathrm{Cl} 6-\mathrm{CH}$ & 23 & 140.10 & 138.34 & $-1 \cdot 7$ & [ 138.34 \\
\hline $\mathrm{C} 17-\mathrm{CH} 3$ & 20 & 24.40 & 22.95 & -1.4 & 22.95 \\
\hline $\mathrm{C} 18-\mathrm{C}$ & 11 & 169.50 & 169.21 & -0.29 & 169.21 \\
\hline $\mathrm{C} 19-\mathrm{CH} 3$ & 14 & 30.40 & 27.71 & -2.6 & 27.71 \\
\hline $\mathrm{C} 20-\mathrm{CH}$ & 16 & 100.70 & 106.33 & 5.63 & [ 106.33 \\
\hline
\end{tabular}




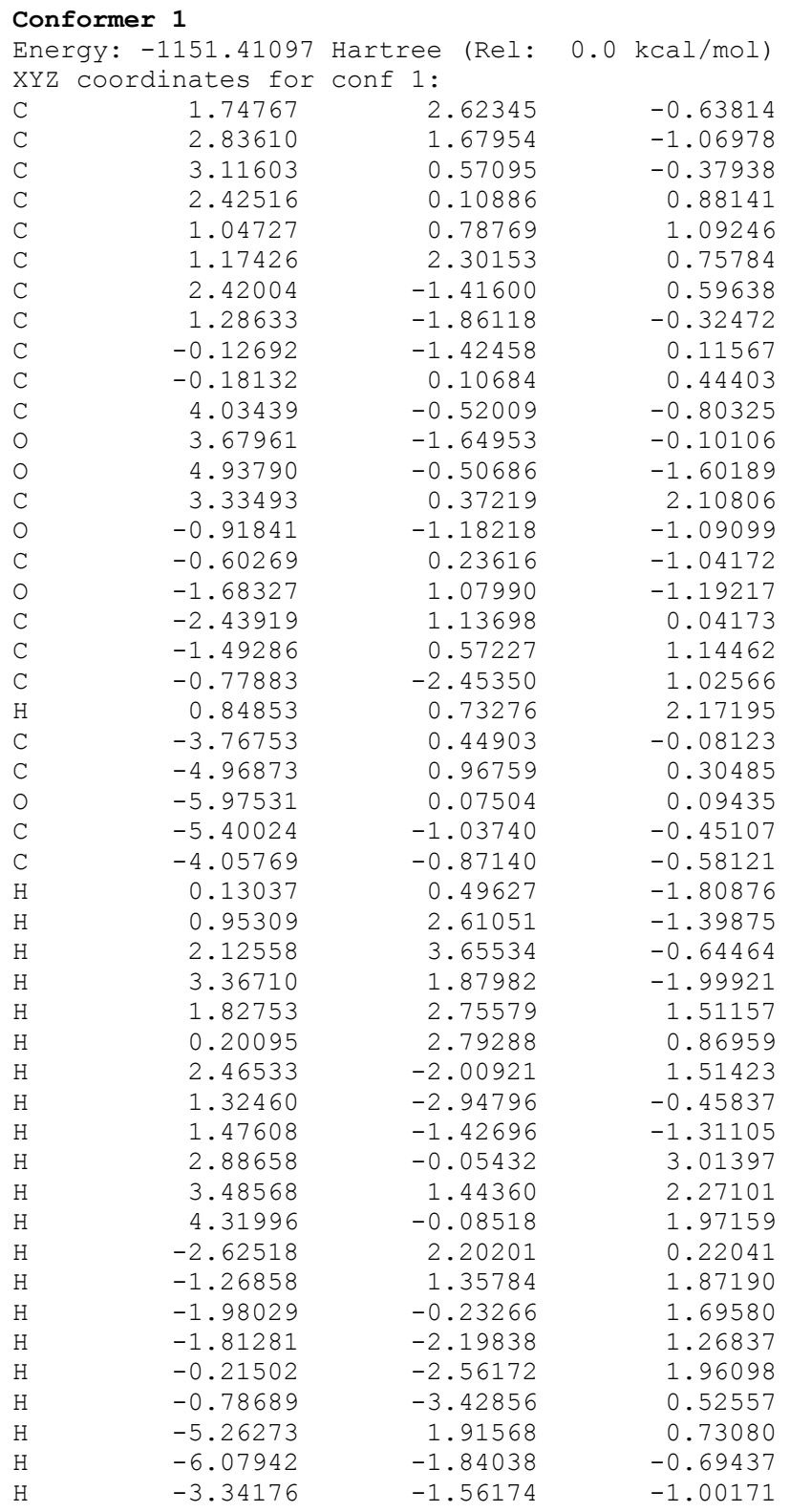


revised structure of cephaloziellin B, Fig.4E

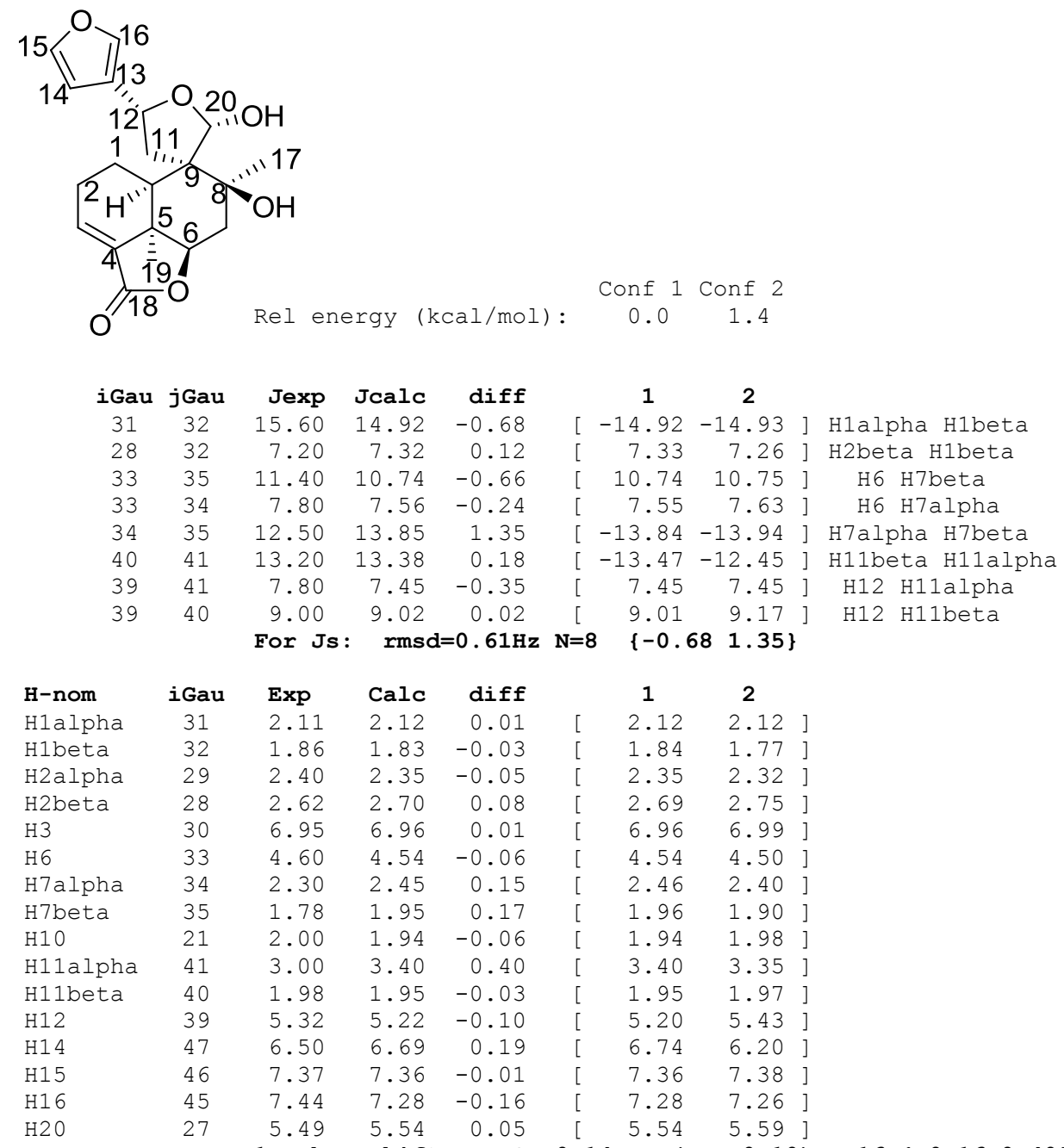

$1 \mathrm{H}$ chem shifts: RMSD $=0.14 \mathrm{ppm}(\mathrm{MAE}=0.10) \quad \mathrm{N}=16 \quad\left\{\begin{array}{lll}-0.16 & 0.40\end{array}\right.$ $\mathrm{m}=1.000 \quad \mathrm{~b}=0.00$

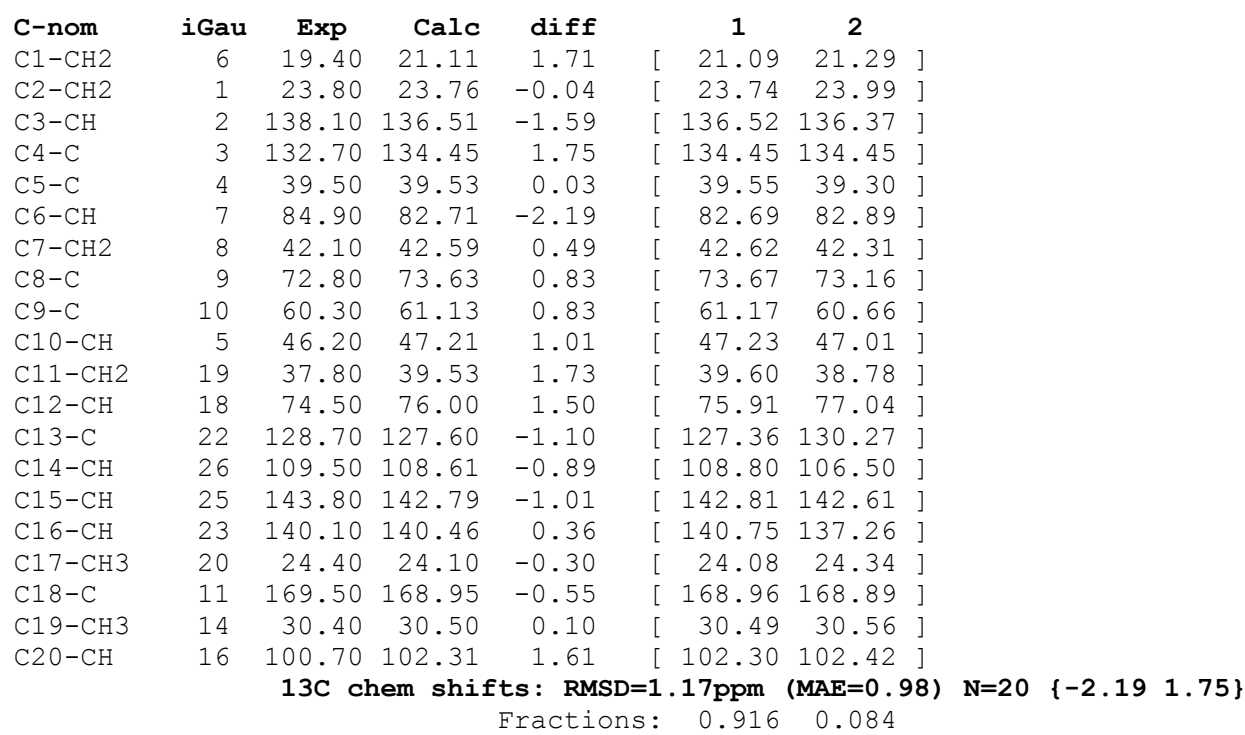




\section{Conformer 1}

Energy: -1227.85205 Hartree (Rel: $0.0 \mathrm{kcal} / \mathrm{mol}$ ) XYZ coordinates for conf 1 :

$\begin{array}{lrrr}\mathrm{C} & -1.21510 & -2.81384 & \\ \mathrm{C} & -2.33198 & -2.25348 & -0.59579 \\ \mathrm{C} & -2.83743 & -1.03811 & -0.36781 \\ \mathrm{C} & -2.45059 & -0.11686 & 0.76295 \\ \mathrm{C} & -0.98943 & -0.37380 & 1.25006 \\ \mathrm{C} & -0.80750 & -1.91308 & 1.42828 \\ \mathrm{C} & -2.76493 & 1.26524 & 0.11246 \\ \mathrm{C} & -1.57748 & 1.95355 & -0.55854 \\ \mathrm{C} & -0.30337 & 1.90791 & 0.31285 \\ \mathrm{C} & 0.14138 & 0.40060 & 0.47510 \\ \mathrm{C} & -3.80304 & -0.32527 & -1.24110 \\ \mathrm{O} & -3.80187 & 0.99499 & -0.87338 \\ \mathrm{O} & -4.49067 & -0.75177 & -2.13723 \\ \mathrm{C} & -3.44627 & -0.31247 & 1.93717 \\ \mathrm{O} & 0.71829 & 2.70559 & -0.27902 \\ \mathrm{C} & 0.50630 & -0.30958 & -0.86573 \\ \mathrm{O} & 1.45879 & -1.28750 & -0.55817 \\ \mathrm{C} & 2.29083 & -0.84983 & 0.55669 \\ \mathrm{C} & 1.50199 & 0.30183 & 1.22235 \\ \mathrm{C} & -0.53910 & 2.59840 & 1.66793 \\ \mathrm{H} & -0.92442 & 0.02982 & 2.26689 \\ \mathrm{C} & 3.67960 & -0.49705 & 0.11115 \\ \mathrm{C} & 4.80019 & -1.24499 & 0.32415 \\ \mathrm{O} & 5.89942 & -0.63980 & -0.20252 \\ \mathrm{C} & 5.46888 & 0.52611 & -0.76875 \\ \mathrm{C} & 4.12730 & 0.66961 & -0.60905 \\ \mathrm{H} & -0.33188 & -0.82509 & -1.33606 \\ \mathrm{H} & -0.35212 & -2.98835 & -0.41773 \\ \mathrm{H} & -1.49545 & -3.80797 & 0.61515 \\ \mathrm{H} & -2.68513 & -2.82468 & -1.45329 \\ \mathrm{H} & -1.41599 & -2.19870 & 2.29346 \\ \mathrm{H} & 0.22410 & -2.13922 & 1.71168 \\ \mathrm{H} & -3.22678 & 1.93846 & 0.83869 \\ \mathrm{H} & -1.82561 & 2.99859 & -0.77290 \\ \mathrm{H} & -1.37601 & 1.47413 & -1.52290 \\ \mathrm{H} & -3.22340 & 0.38778 & 2.75202 \\ \mathrm{H} & -3.40985 & -1.32990 & 2.33620 \\ \mathrm{H} & -4.47297 & -0.12560 & 1.60407 \\ \mathrm{H} & 2.35683 & -1.72032 & 1.21765 \\ \mathrm{H} & 1.37128 & 0.11687 & 2.29277 \\ \mathrm{H} & 2.03616 & 1.24692 & 1.10879 \\ \mathrm{H} & 0.37213 & 2.57488 & 2.27304 \\ \mathrm{H} & -1.35333 & 2.16103 & 2.25083 \\ \mathrm{H} & -0.78108 & 3.64878 & 1.47717 \\ \mathrm{H} & 4.97367 & -2.19052 & 0.81659 \\ \mathrm{H} & 6.23414 & 1.12937 & -1.23304 \\ \mathrm{H} & 3.51539 & 1.49391 & -0.94522 \\ \mathrm{H} & 0.95731 & 2.27361 & -1.11917 \\ \mathrm{O} & 1.04430 & 0.62009 & -1.81509 \\ \mathrm{H} & 1.50554 & 0.09412 & -2.48893 \\ & & & \end{array}$

\section{Conformer 2}

Energy: -1227.84979 Hartree (Rel: $1.4 \mathrm{kcal} / \mathrm{mol}$ ) XYZ coordinates for conf 2 :

\begin{tabular}{llrr} 
CYZ Coordinates for Conf & \multicolumn{1}{l}{ : } & \\
C & -1.21575 & -2.71131 & 0.76083 \\
C & -2.32236 & -2.31753 & -0.17538 \\
C & -2.82779 & -1.08107 & -0.18520 \\
C & -2.45126 & 0.03653 & 0.75631 \\
C & -0.99529 & -0.12448 & 1.30060 \\
C & -0.81945 & -1.60361 & 1.76537 \\
C & -2.75711 & 1.27278 & -0.14562 \\
C & -1.56037 & 1.83547 & -0.91128 \\
C & -0.30292 & 1.95194 & -0.02293 \\
C & 0.14098 & 0.49660 & 0.40631 \\
C & -3.77861 & -0.54523 & -1.19120 \\
O & -3.77742 & 0.82017 & -1.08055 \\
O & -4.45475 & -1.13339 & -2.00048 \\
C & -3.46068 & 0.06532 & 1.93469
\end{tabular}

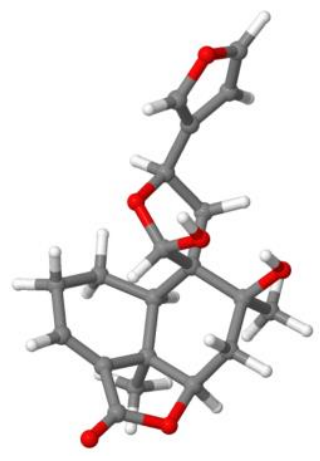




$\begin{array}{rrr}0.72788 & 2.64286 & -0.71863 \\ 0.51325 & -0.43958 & -0.78417 \\ 1.44711 & -1.36389 & -0.28458 \\ 2.27128 & -0.73162 & 0.72508 \\ 1.50141 & 0.53756 & 1.15990 \\ -0.56710 & 2.86651 & 1.18714 \\ -0.94111 & 0.46128 & 2.22510 \\ 3.66230 & -0.49197 & 0.20441 \\ 4.23701 & -1.06258 & -0.89250 \\ 5.52891 & -0.64746 & -1.03442 \\ 5.78526 & 0.19888 & 0.00071 \\ 4.68681 & 0.33164 & 0.79248 \\ -0.32228 & -1.02469 & -1.17115 \\ -0.34663 & -3.00532 & 0.15737 \\ -1.50136 & -3.61747 & 1.31414 \\ -2.66667 & -3.03923 & -0.91493 \\ -1.44142 & -1.72035 & 2.65973 \\ 0.20715 & -1.77366 & 2.10162 \\ -3.23315 & 2.06535 & 0.43695 \\ -1.80685 & 2.82472 & -1.31159 \\ -1.34024 & 1.19474 & -1.77232 \\ -3.24617 & 0.90775 & 2.60414 \\ -3.43046 & -0.85784 & 2.52016 \\ -4.48309 & 0.18444 & 1.55996 \\ 2.32264 & -1.45338 & 1.54991 \\ 1.36748 & 0.56829 & 2.24539 \\ 2.04203 & 1.43602 & 0.85383 \\ 0.32766 & 2.94033 & 1.81244 \\ -1.40310 & 2.54778 & 1.81464 \\ -0.78749 & 3.86999 & 0.80941 \\ 3.88045 & -1.76087 & -1.63269 \\ 6.77767 & 0.62266 & 0.02672 \\ 4.60484 & 0.94313 & 1.68065 \\ 1.03558 & 2.05447 & -1.43233 \\ 1.08038 & 0.31185 & -1.85691 \\ 1.53005 & -0.31472 & -2.44592\end{array}$


correctly assigned cephaloziellin A (accuracy test for DU8+)

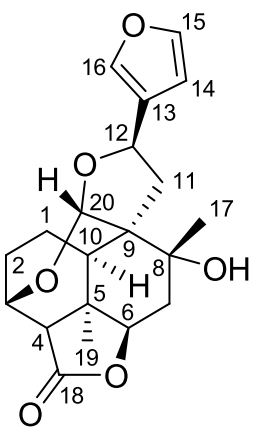

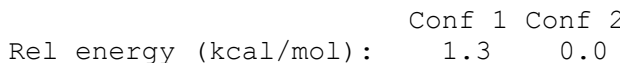

\begin{tabular}{|c|c|c|c|}
\hline C & -0.72696 & -2.55960 & 0.51422 \\
\hline C & -1.48068 & -1.89991 & -0.64593 \\
\hline C & -2.72760 & -1.20096 & -0.08470 \\
\hline C & -2.38632 & -0.03916 & 0.87993 \\
\hline C & -0.90156 & -0.11391 & 1.35627 \\
\hline $\mathrm{C}$ & -0.57443 & -1.58671 & 1.71346 \\
\hline C & -2.77276 & 1.21824 & 0.03859 \\
\hline C & -1.65138 & 1.92690 & -0.72068 \\
\hline $\mathrm{C}$ & -0.34620 & 2.01471 & 0.07548 \\
\hline C & 0.14549 & 0.55446 & 0.40132 \\
\hline $\mathrm{C}$ & -3.63437 & -0.58490 & -1.14420 \\
\hline 0 & -3.74588 & 0.75485 & -0.93249 \\
\hline 0 & -4.22319 & -1.15622 & -2.02612 \\
\hline C & -3.33042 & -0.07404 & 2.10092 \\
\hline o & -0.70188 & -0.97703 & -1.43422 \\
\hline C & 0.37923 & -0.25872 & -0.91134 \\
\hline 0 & 1.50191 & -1.10904 & -0.69442 \\
\hline C & 2.28149 & -0.70625 & 0.44022 \\
\hline & 1.55296 & 0.52112 & 1.05067 \\
\hline & 0.65354 & 2.89391 & -0.69682 \\
\hline 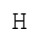 & -0.84023 & 0.46889 & 2.28055 \\
\hline c & 3.70309 & -0.43926 & 0.03860 \\
\hline
\end{tabular}

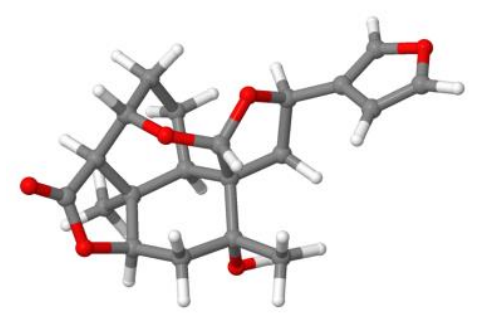




$\begin{array}{rrr}4.82231 & -0.95535 & \\ 5.94907 & -0.47576 & 0.02126 \\ 5.53686 & 0.36095 & -0.97175 \\ 4.17981 & 0.42549 & -1.01121 \\ 0.62735 & 0.41332 & -1.74061 \\ 0.23850 & -2.91676 & 0.15478 \\ -1.30505 & -3.44008 & 0.82014 \\ 0.13399 & 2.86219 & 1.78663 \\ -1.25588 & -1.88835 & 2.51623 \\ 0.42592 & -1.66225 & 2.14871 \\ -3.29540 & 1.94012 & 0.66895 \\ -1.98527 & 2.93631 & -0.98380 \\ -1.46811 & 1.39330 & -1.65666 \\ -3.14528 & 0.78491 & 2.75602 \\ -3.20464 & -0.98799 & 2.69111 \\ -4.37705 & -0.03183 & 1.77685 \\ 2.28305 & -1.54166 & 1.15229 \\ 1.50653 & 0.44434 & 2.14210 \\ 2.12605 & 1.42154 & 0.81961 \\ 1.55898 & 3.10092 & -0.11595 \\ 0.96101 & 2.45612 & -1.65162 \\ 0.17617 & 3.85571 & -0.90766 \\ 4.98008 & -1.64743 & 1.43511 \\ 6.32276 & 0.81543 & -1.55568 \\ 3.58250 & 0.99450 & -1.70994 \\ -0.68800 & 2.69816 & 1.29647 \\ -1.79465 & -2.65551 & -1.37205 \\ -3.32031 & -1.97743 & 0.41624\end{array}$

\section{Conformer 2}

Energy: -1227.85828 Hartree (Rel: $0.0 \mathrm{kcal} / \mathrm{mol}$ ) XYZ coordinates for conf 2 :

\begin{tabular}{|c|c|c|c|}
\hline C & -1.17721 & -2.48760 & 0.97994 \\
\hline C & -1.63437 & -1.92589 & -0.36861 \\
\hline C & -2.81099 & -0.97300 & -0.11746 \\
\hline C & -2.42362 & 0.26513 & 0.72686 \\
\hline C & -1.04206 & 0.07184 & 1.42610 \\
\hline C & -0.97563 & -1.35939 & 2.02579 \\
\hline C & -2.50143 & 1.41759 & -0.32372 \\
\hline C & -1.19948 & 1.82181 & -1.01416 \\
\hline C & -0.00198 & 1.86784 & -0.06327 \\
\hline C & 0.20761 & 0.43964 & 0.55769 \\
\hline C & -3.46554 & -0.41098 & -1.37457 \\
\hline 0 & -3.41590 & 0.94861 & -1.34904 \\
\hline 0 & -4.00494 & -1.02811 & -2.25740 \\
\hline C & -3.51188 & 0.53994 & 1.78639 \\
\hline 0 & -0.59885 & -1.27668 & -1.13775 \\
\hline C & 0.48518 & -0.62660 & -0.55084 \\
\hline 0 & 1.38561 & -1.57244 & 0.03484 \\
\hline C & 2.28512 & -0.87050 & 0.89287 \\
\hline C & 1.49054 & 0.35183 & 1.43612 \\
\hline C & 1.21146 & 2.44230 & -0.81426 \\
\hline $\mathrm{H}$ & -1.01387 & 0.77751 & 2.26295 \\
\hline $\mathrm{C}$ & 3.55094 & -0.50405 & 0.15889 \\
\hline C & 3.91998 & -0.93258 & -1.08184 \\
\hline 0 & 5.14244 & -0.43124 & -1.42319 \\
\hline $\mathrm{C}$ & 5.56539 & 0.32476 & -0.37341 \\
\hline C & 4.63860 & 0.31933 & 0.62313 \\
\hline $\mathrm{H}$ & 0.98416 & -0.16310 & -1.40930 \\
\hline $\mathrm{H}$ & -0.26585 & -3.06841 & 0.83820 \\
\hline $\mathrm{H}$ & -1.95650 & -3.18006 & 1.32156 \\
\hline $\mathrm{H}$ & 0.40424 & 2.93816 & 1.53875 \\
\hline $\mathrm{H}$ & -1.75105 & -1.43045 & 2.79634 \\
\hline $\mathrm{H}$ & -0.02899 & -1.50576 & 2.55402 \\
\hline $\mathrm{H}$ & -2.96922 & 2.29820 & 0.12023 \\
\hline $\mathrm{H}$ & -1.33449 & 2.80363 & -1.48042 \\
\hline $\mathrm{H}$ & -0.99199 & 1.10930 & -1.81708 \\
\hline $\mathrm{H}$ & -3.28651 & 1.45616 & 2.34418 \\
\hline $\mathrm{H}$ & -3.60097 & -0.28330 & 2.50350 \\
\hline $\mathrm{H}$ & -4.48973 & 0.66899 & 1.30741 \\
\hline $\mathrm{H}$ & 2.53002 & -1.56282 & 1.70807 \\
\hline
\end{tabular}

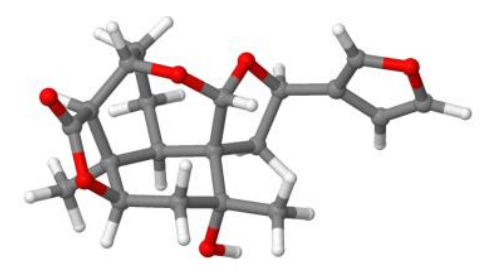




$\begin{array}{rrr}1.23498 & 0.20735 & 2.48925 \\ 2.10089 & 1.25619 & 1.38323 \\ 2.09051 & 2.55190 & -0.17027 \\ 1.50961 & 1.83315 & -1.67258 \\ 0.94585 & 3.43784 & -1.18335 \\ 3.44653 & -1.57356 & -1.80825 \\ 6.53100 & 0.79312 & -0.49013 \\ 4.71785 & 0.82860 & 1.57438 \\ -0.37315 & 2.79513 & 0.97523 \\ -1.96821 & -2.73352 & -1.02651 \\ -3.58384 & -1.56978 & 0.38439\end{array}$


originally assigned structure of 13,17-epoxy-ent-kaurenal, Fig.4F

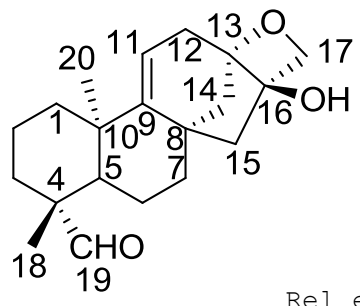

Rel energy (kcal/mol): $\begin{array}{cccc}\text { Conf } 1 & \text { Conf } 2 & \text { Conf } 3 \\ 0.0 & 0.6 & 0.7\end{array}$

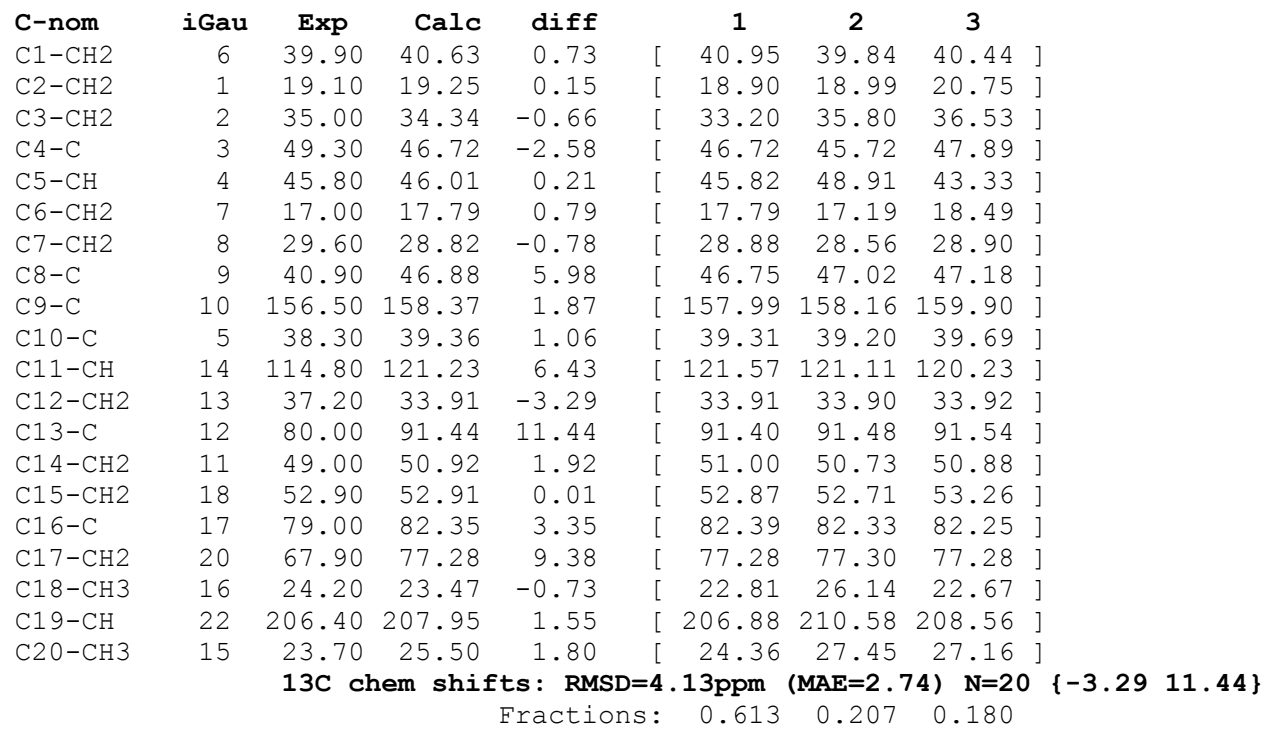

Conformer 1

Energy: -1004.56929 Hartree (Rel: $0.0 \mathrm{kcal} / \mathrm{mol}$ ) XYZ coordinates for conf 1 :

$\begin{array}{lrrr}\mathrm{C} & -2.84531 & 2.00340 & 0.77810 \\ \mathrm{C} & -3.34053 & 0.71182 & 1.43213 \\ \mathrm{C} & -3.04962 & -0.53466 & 0.57429 \\ \mathrm{C} & -1.52370 & -0.58797 & 0.20445 \\ \mathrm{C} & -0.95946 & 0.71647 & -0.46007 \\ \mathrm{C} & -1.35296 & 1.92620 & 0.43667 \\ \mathrm{C} & -1.11551 & -1.89144 & -0.53293 \\ \mathrm{C} & 0.25199 & -1.80979 & -1.24056 \\ \mathrm{C} & 1.21887 & -0.81079 & -0.60312 \\ \mathrm{C} & 0.58531 & 0.60062 & -0.54306 \\ \mathrm{C} & 2.55209 & -0.68830 & -1.37377 \\ \mathrm{C} & 3.31921 & 0.24466 & -0.42943 \\ \mathrm{C} & 2.89120 & 1.67302 & -0.68197 \\ \mathrm{C} & 1.37181 & 1.69472 & -0.62006 \\ \mathrm{C} & -1.50934 & 0.94575 & -1.89277 \\ \mathrm{C} & -3.43474 & -1.80354 & 1.38390 \\ \mathrm{C} & 2.98281 & -0.30357 & 0.98996 \\ \mathrm{C} & 1.76809 & -1.24162 & 0.80175 \\ \mathrm{O} & 4.72410 & -0.04451 & -0.26029 \\ \mathrm{C} & 4.45159 & -0.77462 & 0.96634 \\ \mathrm{O} & 2.81713 & 0.64422 & 2.02639 \\ \mathrm{C} & -3.98108 & -0.56795 & -0.63693 \\ \mathrm{O} & -4.96983 & 0.12251 & -0.77146 \\ \mathrm{H} & -3.01294 & 2.84894 & 1.45730 \\ \mathrm{H} & -3.44047 & 2.21103 & -0.11823 \\ \mathrm{H} & -4.41682 & 0.77299 & 1.62313 \\ \mathrm{H} & -2.84483 & 0.58074 & 2.40493 \\ \mathrm{H} & -1.03096 & -0.62647 & 1.18695 \\ \mathrm{H} & -1.04900 & 2.86090 & -0.04775\end{array}$

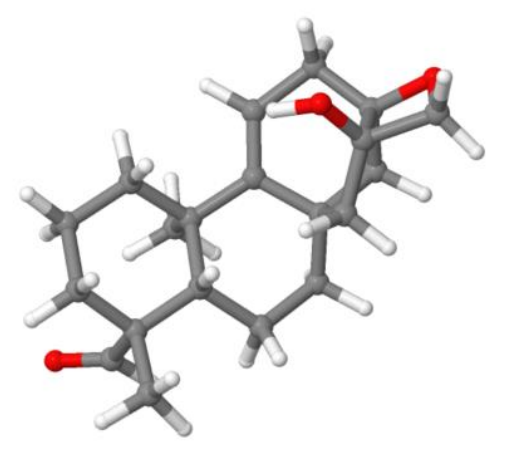




$\begin{array}{rrrr}\mathrm{H} & -0.77914 & 1.86202 & 1.37255 \\ \mathrm{H} & -1.09490 & -2.70288 & 0.20272 \\ \mathrm{H} & -1.86849 & -2.18278 & -1.27292 \\ \mathrm{H} & 0.72009 & -2.80236 & -1.26035 \\ \mathrm{H} & 0.11269 & -1.52479 & -2.29067 \\ \mathrm{H} & 3.04878 & -1.66324 & -1.44807 \\ \mathrm{H} & 2.42901 & -0.28454 & -2.38481 \\ \mathrm{H} & 3.30951 & 2.33560 & 0.08596 \\ \mathrm{H} & 3.25461 & 2.04158 & -1.65277 \\ \mathrm{H} & 0.92058 & 2.68434 & -0.61941 \\ \mathrm{H} & -1.28415 & 0.11128 & -2.56367 \\ \mathrm{H} & -1.05124 & 1.84223 & -2.32420 \\ \mathrm{H} & -2.59316 & 1.09578 & -1.90181 \\ \mathrm{H} & -3.38530 & -2.71961 & 0.78597 \\ \mathrm{H} & -2.75807 & -1.92145 & 2.23866 \\ \mathrm{H} & -4.45603 & -1.70933 & 1.76869 \\ \mathrm{H} & 2.06000 & -2.29980 & 0.76649 \\ \mathrm{H} & 1.05693 & -1.12694 & 1.62532 \\ \mathrm{H} & 4.60404 & -1.85494 & 0.84471 \\ \mathrm{H} & 5.04907 & -0.40151 & 1.80373 \\ \mathrm{H} & 1.98933 & 1.12117 & 1.84386 \\ \mathrm{H} & -3.74469 & -1.34014 & -1.40089\end{array}$

\section{Conformer 2}

Energy: -1004.56826 Hartree (Rel: $0.6 \mathrm{kcal} / \mathrm{mol}$ ) XYZ coordinates for conf 2 :

\begin{tabular}{lrrr} 
CYZ & Coordinates for ronf & \multicolumn{1}{l}{ 2. } & \\
C & -2.79888 & 2.30405 & 0.43446 \\
$\mathrm{C}$ & -3.34566 & 1.16274 & 1.29736 \\
$\mathrm{C}$ & -3.10138 & -0.23732 & 0.69049 \\
$\mathrm{C}$ & -1.57434 & -0.39608 & 0.32856 \\
$\mathrm{C}$ & -0.96727 & 0.75488 & -0.54567 \\
$\mathrm{C}$ & -1.30900 & 2.11326 & 0.12984 \\
$\mathrm{C}$ & -1.23181 & -1.81673 & -0.18954 \\
$\mathrm{C}$ & 0.11631 & -1.90523 & -0.93164 \\
$\mathrm{C}$ & 1.14066 & -0.87016 & -0.46557 \\
$\mathrm{C}$ & 0.57132 & 0.56292 & -0.60635 \\
$\mathrm{C}$ & 2.46459 & -0.92051 & -1.25956 \\
$\mathrm{C}$ & 3.28979 & 0.09938 & -0.46563 \\
$\mathrm{C}$ & 2.92070 & 1.49716 & -0.90972 \\
$\mathrm{C}$ & 1.40458 & 1.59821 & -0.84113 \\
$\mathrm{C}$ & -1.51439 & 0.76379 & -1.99770 \\
$\mathrm{C}$ & -3.49874 & -1.31281 & 1.73031 \\
$\mathrm{C}$ & 2.95668 & -0.22985 & 1.02030 \\
$\mathrm{C}$ & 1.69233 & -1.11802 & 0.98186 \\
$\mathrm{O}$ & 4.68396 & -0.22704 & -0.27523 \\
$\mathrm{C}$ & 4.39974 & -0.77541 & 1.04021 \\
$\mathrm{O}$ & 2.86398 & 0.85945 & 1.91814 \\
$\mathrm{C}$ & -4.02994 & -0.43084 & -0.51528 \\
$\mathrm{O}$ & -4.23102 & -1.48839 & -1.07477 \\
$\mathrm{H}$ & -2.94318 & 3.26006 & 0.95339 \\
$\mathrm{H}$ & -3.36970 & 2.38403 & -0.49967 \\
$\mathrm{H}$ & -4.42084 & 1.30068 & 1.47895 \\
$\mathrm{H}$ & -2.86338 & 1.19652 & 2.28423 \\
$\mathrm{H}$ & -1.08220 & -0.28251 & 1.30669 \\
$\mathrm{H}$ & -0.96678 & 2.94151 & -0.50066 \\
$\mathrm{H}$ & -0.74435 & 2.18463 & 1.07014 \\
$\mathrm{H}$ & -1.21285 & -2.49299 & 0.67229 \\
$\mathrm{H}$ & -2.02916 & -2.18329 & -0.84121 \\
$\mathrm{H}$ & 0.54036 & -2.91058 & -0.81191 \\
$\mathrm{H}$ & -0.03758 & -1.77408 & -2.00977 \\
$\mathrm{H}$ & 2.91478 & -1.91884 & -1.20093 \\
$\mathrm{H}$ & 2.34239 & -0.65786 & -2.31622 \\
$\mathrm{H}$ & 3.38042 & 2.23885 & -0.24468 \\
$\mathrm{H}$ & 3.28579 & 1.71025 & -1.92547 \\
$\mathrm{H}$ & 0.99928 & 2.59773 & -0.98108 \\
$\mathrm{H}$ & -1.36481 & -0.19069 & -2.50857 \\
$\mathrm{H}$ & -1.00151 & 1.53621 & -2.58077 \\
$\mathrm{H}$ & -2.58621 & 0.98385 & -2.03404 \\
$\mathrm{H}$ & -3.48872 & -2.31446 & 1.29583 \\
$\mathrm{H}$ & -2.81156 & -1.28864 & 2.58430 \\
$\mathrm{H}$ & -4.51021 & -1.12345 & 2.10925 \\
& & &
\end{tabular}

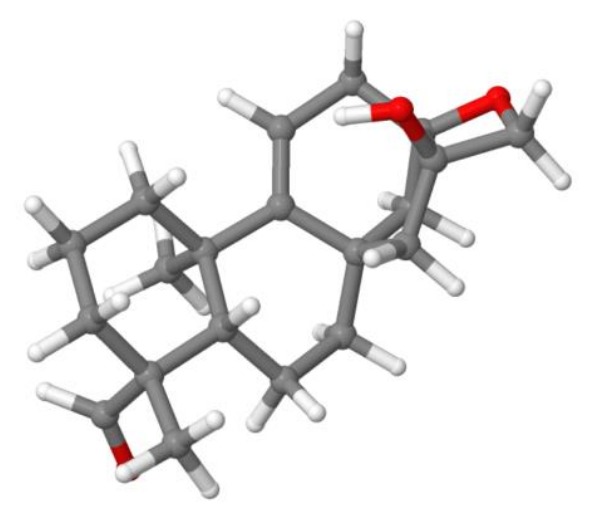




$\begin{array}{rrrr}\text { H } & 1.92736 & -2.18415 & 1.09979 \\ \mathrm{H} & 1.00111 & -0.84618 & 1.78527 \\ \mathrm{H} & 4.49470 & -1.86898 & 1.05915 \\ \mathrm{H} & 5.03238 & -0.32855 & 1.81320 \\ \mathrm{H} & 2.04982 & 1.34184 & 1.69477 \\ \mathrm{H} & -4.58237 & 0.47638 & -0.85091\end{array}$

\section{Conformer 3}

Energy: -1004.56813 Hartree (Rel: $0.7 \mathrm{kcal} / \mathrm{mol}$ ) XYZ coordinates for conf 3 :

\begin{tabular}{|c|c|c|c|}
\hline C & -2.86012 & 2.19456 & 0.53080 \\
\hline $\mathrm{C}$ & -3.32095 & 1.02435 & 1.40089 \\
\hline C & -3.06636 & -0.34817 & 0.73062 \\
\hline C & -1.57045 & -0.46038 & 0.28912 \\
\hline C & -0.99275 & 0.74852 & -0.53426 \\
\hline C & -1.37050 & 2.07086 & 0.19206 \\
\hline $\mathrm{C}$ & -1.21873 & -1.84391 & -0.31907 \\
\hline C & 0.13788 & -1.86812 & -1.05281 \\
\hline C & 1.14145 & -0.84371 & -0.52021 \\
\hline $\mathrm{C}$ & 0.54963 & 0.58706 & -0.58169 \\
\hline C & 2.47145 & -0.82458 & -1.30631 \\
\hline $\mathrm{C}$ & 3.27484 & 0.15872 & -0.44599 \\
\hline C & 2.88668 & 1.57505 & -0.80718 \\
\hline C & 1.36853 & 1.64787 & -0.74220 \\
\hline C & -1.51480 & 0.82738 & -1.99453 \\
\hline C & -3.40547 & -1.45151 & 1.77294 \\
\hline C & 2.93762 & -0.26319 & 1.01473 \\
\hline C & 1.69150 & -1.17114 & 0.91243 \\
\hline 0 & 4.67331 & -0.15677 & -0.26480 \\
\hline C & 4.39035 & -0.78198 & 1.01613 \\
\hline 0 & 2.81413 & 0.76991 & 1.97356 \\
\hline C & -4.12549 & -0.54153 & -0.35397 \\
\hline 0 & -3.98203 & -0.98353 & -1.47263 \\
\hline $\mathrm{H}$ & -3.03598 & 3.14114 & 1.05724 \\
\hline $\mathrm{H}$ & -3.46176 & 2.24161 & -0.38701 \\
\hline $\mathrm{H}$ & -4.38648 & 1.12147 & 1.65148 \\
\hline $\mathrm{H}$ & -2.77740 & 1.04462 & 2.35592 \\
\hline $\mathrm{H}$ & -1.03317 & -0.39754 & 1.24744 \\
\hline $\mathrm{H}$ & -1.07058 & 2.92839 & -0.42073 \\
\hline $\mathrm{H}$ & -0.79465 & 2.13936 & 1.12589 \\
\hline $\mathrm{H}$ & -1.19944 & -2.57826 & 0.49450 \\
\hline $\mathrm{H}$ & -2.00429 & -2.15854 & -1.00889 \\
\hline $\mathrm{H}$ & 0.57964 & -2.87093 & -0.98726 \\
\hline $\mathrm{H}$ & -0.01247 & -1.67711 & -2.12192 \\
\hline $\mathrm{H}$ & 2.93807 & -1.81710 & -1.30455 \\
\hline $\mathrm{H}$ & 2.35135 & -0.50051 & -2.34592 \\
\hline $\mathrm{H}$ & 3.33239 & 2.28202 & -0.09637 \\
\hline $\mathrm{H}$ & 3.25304 & 1.85438 & -1.80632 \\
\hline $\mathrm{H}$ & 0.94722 & 2.64716 & -0.82592 \\
\hline $\mathrm{H}$ & -1.35819 & -0.10122 & -2.54708 \\
\hline $\mathrm{H}$ & -0.98414 & 1.62731 & -2.52269 \\
\hline $\mathrm{H}$ & -2.58450 & 1.04150 & -2.03926 \\
\hline $\mathrm{H}$ & -3.32566 & -2.45810 & 1.35289 \\
\hline $\mathrm{H}$ & -2.72043 & -1.37805 & 2.62555 \\
\hline $\mathrm{H}$ & -4.42572 & -1.32598 & 2.15505 \\
\hline $\mathrm{H}$ & 1.94794 & -2.23782 & 0.96229 \\
\hline $\mathrm{H}$ & 0.99306 & -0.96521 & 1.72883 \\
\hline $\mathrm{H}$ & 4.50596 & -1.87300 & 0.97577 \\
\hline $\mathrm{H}$ & 5.00892 & -0.36682 & 1.81774 \\
\hline $\mathrm{H}$ & 1.99695 & 1.25304 & 1.76234 \\
\hline $\mathrm{H}$ & -5.14879 & -0.26937 & 0.00298 \\
\hline
\end{tabular}

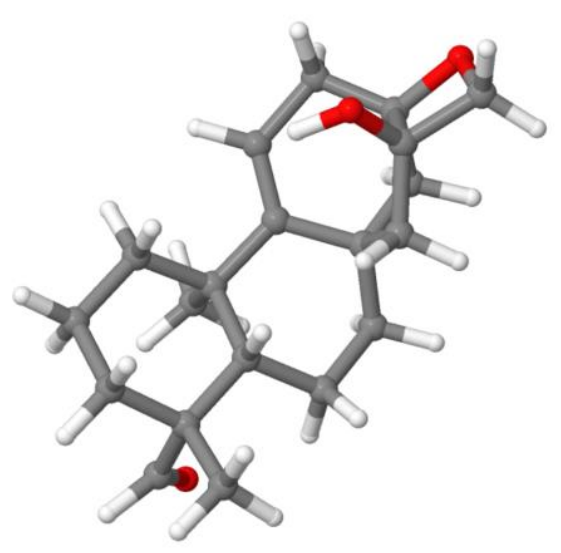


revised structure of 13,17-epoxy-ent-kaurenal, Fig.4F

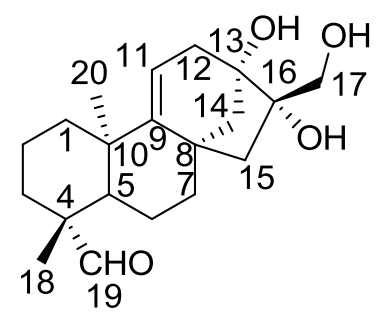

Rel energy (kcal/mol): $\begin{array}{cccccc} & \text { Conf } 1 & \text { Conf } 2 & \text { Conf } 3 & \text { Conf } 4 & \text { Conf } 5 \\ 0.8 & 0.1 & 0.7 & 0.6 & 0.0 & 0.7\end{array}$

\begin{tabular}{|c|c|c|c|c|c|c|c|c|c|c|}
\hline C-nom & iGau & $\operatorname{Exp}$ & Calc & $\operatorname{diff}$ & 1 & 2 & 3 & 4 & 5 & 6 \\
\hline $\mathrm{C} 1-\mathrm{CH} 2$ & 6 & 39.90 & 39.21 & -0.69 & 39.34 & 39.40 & 38.71 & 38.59 & 39.44 & 39.10 \\
\hline $\mathrm{C} 2-\mathrm{CH} 2$ & 1 & 19.10 & 19.06 & -0.04 & 20.64 & 18.67 & 18.84 & 18.79 & 18.66 & 20.56 \\
\hline $\mathrm{C} 3-\mathrm{CH} 2$ & 2 & 35.00 & 34.44 & -0.56 & 36.71 & 33.22 & 35.82 & 35.81 & 33.24 & 36.65 \\
\hline C4-C & 3 & 49.30 & 46.79 & -2.51 & 48.03 & 46.77 & 45.86 & 45.81 & 46.74 & 47.96 \\
\hline $\mathrm{C} 5-\mathrm{CH}$ & 4 & 45.80 & 46.28 & 0.48 & 43.54 & 46.04 & 49.15 & 49.22 & 46.10 & 43.73 \\
\hline $\mathrm{C} 6-\mathrm{CH} 2$ & 7 & 17.00 & 17.74 & 0.74 & 18.61 & 17.83 & 17.00 & 16.94 & 17.76 & 18.35 \\
\hline $\mathrm{C} 7-\mathrm{CH} 2$ & 8 & 29.60 & 29.98 & 0.38 & 30.01 & 30.08 & 29.59 & 29.66 & 30.10 & 30.04 \\
\hline C8-C & 9 & 40.90 & 40.62 & -0.28 & 41.48 & 41.14 & 41.34 & 40.18 & 39.98 & 40.34 \\
\hline C9-C & 10 & 156.50 & 158.64 & 2.14 & 160.10 & 158.39 & 158.50 & 158.29 & 158.14 & 160.19 \\
\hline $\mathrm{C} 10-\mathrm{C}$ & 5 & 38.30 & 38.87 & 0.57 & {$\left[\begin{array}{ll}39.19 \\
{[}\end{array}\right.$} & 38.85 & 38.73 & 38.73 & 38.83 & 39.10 \\
\hline $\mathrm{C} 11-\mathrm{CH}$ & 14 & 114.80 & 116.37 & 1.57 & [ 115.39 & 116.56 & 116.38 & 116.52 & 116.73 & 115.39 \\
\hline $\mathrm{C} 12-\mathrm{CH} 2$ & 13 & 37.20 & 36.90 & -0.30 & {$\left[\begin{array}{ll}{[} & 37.81\end{array}\right.$} & 37.76 & 37.87 & 36.23 & 36.15 & 36.10 \\
\hline C13-C & 12 & 80.00 & 79.79 & -0.21 & 80.41 & 80.55 & 80.41 & 79.08 & 79.22 & 79.34 \\
\hline $\mathrm{C} 14-\mathrm{CH} 2$ & 11 & 49.00 & 47.82 & -1.18 & 46.87 & 46.90 & 46.77 & 48.50 & 48.66 & 48.57 \\
\hline $\mathrm{C} 15-\mathrm{CH} 2$ & 18 & 52.90 & 51. & -1.24 & 52.35 & 52.03 & 52.06 & 51.17 & 51.25 & 51.58 \\
\hline C16-C & 17 & 79.00 & 80.42 & 1.42 & 80.35 & 80.58 & 80.39 & 80.19 & 80.41 & 80.37 \\
\hline $\mathrm{C} 17-\mathrm{CH} 2$ & 20 & 67.90 & 67.02 & -0.88 & 67.02 & 66.84 & 67.06 & 67.22 & 67.05 & 67.13 \\
\hline $\mathrm{C} 18-\mathrm{CH} 3$ & 16 & 24.20 & 23.42 & -0.78 & 22.55 & 22.73 & 26.06 & 26.12 & 22.74 & 22.57 \\
\hline $\mathrm{C} 19-\mathrm{CH}$ & 22 & 206.40 & 208.14 & 1.74 & 208.67 & 207.13 & 210.57 & 210.57 & 207.08 & 208.67 \\
\hline \multirow[t]{3}{*}{$\mathrm{C} 20-\mathrm{CH} 3$} & 15 & 23.70 & 25.06 & 1.36 & 26.74 & 23.93 & 26.91 & 26.89 & 23.91 & 26.41 \\
\hline & & & & & & & (35) $N=20$ & & & \\
\hline & & & & racti & 0.087 & 0.269 & 0.099 & 0.116 & 0.327 & 0.102 \\
\hline
\end{tabular}

\section{Conformer 1}

Energy: -1081.02525 Hartree (Rel: $0.8 \mathrm{kcal} / \mathrm{mol}$ ) XYZ coordinates for conf 1 :

$\begin{array}{lrrr}\text { C } & -3.18685 & 2.23144 & 0.48438 \\ \mathrm{C} & -3.60154 & 1.10516 & 1.43276 \\ \mathrm{C} & -3.34507 & -0.29952 & 0.83349 \\ \mathrm{C} & -1.86251 & -0.41332 & 0.35182 \\ \mathrm{C} & -1.33613 & 0.75374 & -0.56156 \\ \mathrm{C} & -1.70761 & 2.10975 & 0.10166 \\ \mathrm{C} & -1.50209 & -1.82746 & -0.17782 \\ \mathrm{C} & -0.20169 & -1.87122 & -1.00481 \\ \mathrm{C} & 0.82059 & -0.80200 & -0.60887 \\ \mathrm{C} & 0.20055 & 0.60787 & -0.65172 \\ \mathrm{C} & 2.07256 & -0.82697 & -1.51877 \\ \mathrm{C} & 3.01894 & 0.09224 & -0.74707 \\ \mathrm{C} & 2.52439 & 1.53633 & -0.91077 \\ \mathrm{C} & 1.02579 & 1.65970 & -0.76962 \\ \mathrm{C} & -1.90958 & 0.74958 & -2.00497 \\ \mathrm{C} & -3.63368 & -1.34718 & 1.94574 \\ \mathrm{C} & 2.90341 & -0.48641 & 0.71431 \\ \mathrm{C} & 1.45833 & -1.03563 & 0.80311 \\ \mathrm{O} & 4.36798 & -0.02747 & -1.21249 \\ \mathrm{C} & 3.27869 & 0.50191 & 1.81825 \\ \mathrm{O} & 3.84100 & -1.57300 & 0.82274 \\ \mathrm{C} & -4.43066 & -0.56927 & -0.20760 \\ \mathrm{O} & -4.31106 & -1.07482 & -1.30191 \\ \mathrm{H} & -3.35995 & 3.20306 & 0.96421 \\ \mathrm{H} & -3.82012 & 2.21986 & -0.41318 \\ \mathrm{H} & -4.66034 & 1.20071 & 1.71136\end{array}$

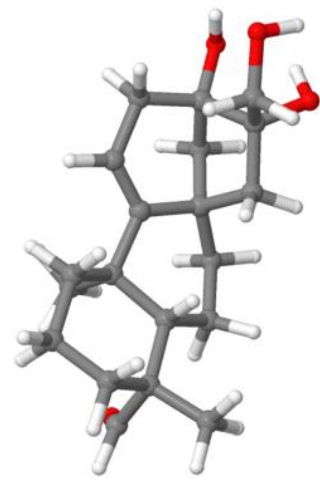




$\begin{array}{rrrr}\text { H } & -3.02876 & 1.18722 & 2.36717 \\ \mathrm{H} & -1.29466 & -0.27917 & 1.28550 \\ \mathrm{H} & -1.44310 & 2.93356 & -0.57136 \\ \mathrm{H} & -1.09964 & 2.23900 & 1.00761 \\ \mathrm{H} & -1.40103 & -2.49715 & 0.68425 \\ \mathrm{H} & -2.32002 & -2.21911 & -0.78576 \\ \mathrm{H} & 0.26227 & -2.86160 & -0.91410 \\ \mathrm{H} & -0.43400 & -1.74653 & -2.06887 \\ \mathrm{H} & 2.48844 & -1.84046 & -1.57298 \\ \mathrm{H} & 1.87093 & -0.47625 & -2.53610 \\ \mathrm{H} & 3.03995 & 2.20523 & -0.20813 \\ \mathrm{H} & 2.82974 & 1.86984 & -1.91423 \\ \mathrm{H} & 0.63678 & 2.67432 & -0.79713 \\ \mathrm{H} & -1.77467 & -0.20694 & -2.51322 \\ \mathrm{H} & -1.39788 & 1.51938 & -2.59336 \\ \mathrm{H} & -2.98005 & 0.96452 & -2.02313 \\ \mathrm{H} & -3.54030 & -2.37436 & 1.58203 \\ \mathrm{H} & -2.92993 & -1.20962 & 2.77488 \\ \mathrm{H} & -4.64711 & -1.22192 & 2.34593 \\ \mathrm{H} & 1.52168 & -2.10491 & 1.03201 \\ \mathrm{H} & 0.88443 & -0.55651 & 1.60355 \\ \mathrm{H} & 2.66841 & 1.40779 & 1.78120 \\ \mathrm{H} & 3.12125 & 0.02656 & 2.79836 \\ \mathrm{H} & 4.41088 & -1.48961 & 0.03135 \\ \mathrm{H} & -5.44790 & -0.28911 & 0.16071 \\ \mathrm{H} & 4.88187 & 0.59943 & -0.66870 \\ \mathrm{O} & 4.64114 & 0.90561 & 1.66673 \\ \mathrm{H} & 5.13523 & 0.06776 & 1.71797\end{array}$

\section{Conformer 2}

Energy: -1081.02632 Hartree (Rel: $0.1 \mathrm{kcal} / \mathrm{mol})$ XYZ coordinates for conf 2 :

$\begin{array}{lrrr}\mathrm{C} & -3.19516 & 2.01059 & 0.82421 \\ \mathrm{C} & -3.63879 & 0.71533 & 1.50813 \\ \mathrm{C} & -3.33904 & -0.53449 & 0.65800 \\ \mathrm{C} & -1.82258 & -0.55554 & 0.24971 \\ \mathrm{C} & -1.30954 & 0.75567 & -0.44295 \\ \mathrm{C} & -1.71046 & 1.96793 & 0.44466 \\ \mathrm{C} & -1.40099 & -1.85849 & -0.48553 \\ \mathrm{C} & -0.09497 & -1.73728 & -1.29570 \\ \mathrm{C} & 0.89135 & -0.71230 & -0.73094 \\ \mathrm{C} & 0.22931 & 0.66737 & -0.55663 \\ \mathrm{C} & 2.14272 & -0.55784 & -1.62849 \\ \mathrm{C} & 3.06397 & 0.25105 & -0.71578 \\ \mathrm{C} & 2.52772 & 1.68822 & -0.64727 \\ \mathrm{C} & 1.02619 & 1.74578 & -0.50026 \\ \mathrm{C} & -1.90027 & 0.96026 & -1.86375 \\ \mathrm{C} & -3.67227 & -1.80303 & 1.49025 \\ \mathrm{C} & 2.96717 & -0.56102 & 0.63064 \\ \mathrm{C} & 1.53221 & -1.14364 & 0.63322 \\ \mathrm{O} & 4.41500 & 0.24485 & -1.19026 \\ \mathrm{C} & 3.33504 & 0.23915 & 1.88011 \\ \mathrm{O} & 3.92101 & -1.63521 & 0.55132 \\ \mathrm{C} & -4.29881 & -0.60336 & -0.52960 \\ \mathrm{O} & -5.31210 & 0.05483 & -0.64249 \\ \mathrm{H} & -3.36766 & 2.86055 & 1.49675 \\ \mathrm{H} & -3.82009 & 2.18974 & -0.05787 \\ \mathrm{H} & -4.71087 & 0.75128 & 1.72759 \\ \mathrm{H} & -3.11367 & 0.60997 & 2.46851 \\ \mathrm{H} & -1.30237 & -0.56938 & 1.21918 \\ \mathrm{H} & -1.44579 & 2.90108 & -0.06637 \\ \mathrm{H} & -1.10900 & 1.93542 & 1.36359 \\ \mathrm{H} & -1.28725 & -2.64752 & 0.26611 \\ \mathrm{H} & -2.18911 & -2.20815 & -1.16092 \\ \mathrm{H} & 0.39630 & -2.71685 & -1.35133 \\ \mathrm{H} & -0.32313 & -1.45964 & -2.33172 \\ \mathrm{H} & 2.58598 & -1.53767 & -1.84341 \\ \mathrm{H} & 1.92994 & -0.05405 & -2.57697 \\ \mathrm{H} & 3.01976 & 2.24993 & 0.15839 \\ \mathrm{H} & 2.82713 & 2.18851 & -1.58065 \\ \mathrm{H} & 0.60948 & 2.74036 & -0.36564 \\ & & & \end{array}$

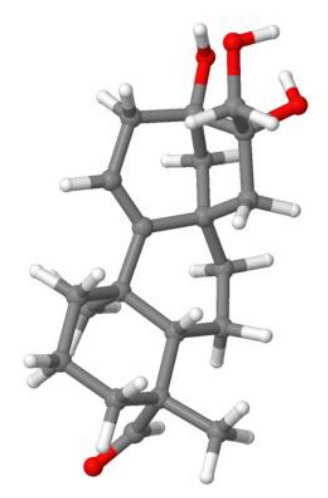




$\begin{array}{rrr}-1.68416 & 0.12470 & -2.53538 \\ -1.46764 & 1.86059 & -2.31342 \\ -2.98607 & 1.09422 & -1.84240 \\ -3.60534 & -2.72478 & 0.90277 \\ -2.97725 & -1.88994 & 2.33401 \\ -4.68903 & -1.73438 & 1.89224 \\ 1.61544 & -2.23367 & 0.70318 \\ 0.94718 & -0.79707 & 1.49168 \\ 2.70755 & 1.12602 & 1.99800 \\ 3.19818 & -0.39414 & 2.76954 \\ 4.49191 & -1.40205 & -0.20876 \\ -4.05735 & -1.37120 & -1.29633 \\ 4.91365 & 0.78216 & -0.54535 \\ 4.68773 & 0.69188 & 1.78669 \\ 5.20319 & -0.13090 & 1.71082\end{array}$

\section{Conformer 3}

Energy: -1081.02537 Hartree (Rel: $0.7 \mathrm{kcal} / \mathrm{mol})$ XYZ coordinates for conf 3 :

\begin{tabular}{|c|c|c|c|}
\hline C & -3.12779 & 2.33189 & 0.40917 \\
\hline C & -3.62032 & 1.22043 & 1.34135 \\
\hline $\mathrm{C}$ & -3.37618 & -0.20130 & 0.78659 \\
\hline C & -1.86204 & -0.35102 & 0.37114 \\
\hline C & -1.30947 & 0.77088 & -0.57409 \\
\hline C & -1.64800 & 2.15216 & 0.05292 \\
\hline C & -1.51431 & -1.78788 & -0.09838 \\
\hline C & -0.21758 & -1.88678 & -0.92537 \\
\hline $\mathrm{C}$ & 0.82236 & -0.81968 & -0.57499 \\
\hline C & 0.22382 & 0.59559 & -0.67643 \\
\hline C & 2.07311 & -0.90373 & -1.48257 \\
\hline C & 3.03287 & 0.03461 & -0.75209 \\
\hline C & 2.55950 & 1.47658 & -0.98330 \\
\hline $\mathrm{C}$ & 1.06330 & 1.62882 & -0.84682 \\
\hline C & -1.90724 & 0.71501 & -2.00563 \\
\hline $\mathrm{C}$ & -3.71259 & -1.23684 & 1.88618 \\
\hline C & 2.90856 & -0.47694 & 0.73380 \\
\hline C & 1.45548 & -1.00111 & 0.84646 \\
\hline 0 & 4.38012 & -0.12461 & -1.20990 \\
\hline C & 3.29930 & 0.55455 & 1.79179 \\
\hline 0 & 3.82904 & -1.57180 & 0.89089 \\
\hline $\mathrm{C}$ & -4.34455 & -0.46000 & -0.37423 \\
\hline 0 & -4.56079 & -1.54602 & -0.87027 \\
\hline $\mathrm{H}$ & -3.26781 & 3.30653 & 0.89370 \\
\hline $\mathrm{H}$ & -3.73794 & 2.36442 & -0.50284 \\
\hline $\mathrm{H}$ & -4.68964 & 1.34945 & 1.56064 \\
\hline $\mathrm{H}$ & -3.09903 & 1.30484 & 2.30512 \\
\hline $\mathrm{H}$ & -1.33316 & -0.18630 & 1.32269 \\
\hline $\mathrm{H}$ & -1.34757 & 2.95448 & -0.63092 \\
\hline $\mathrm{H}$ & -1.04591 & 2.27629 & 0.96328 \\
\hline $\mathrm{H}$ & -1.42214 & -2.41957 & 0.79219 \\
\hline $\mathrm{H}$ & -2.34161 & -2.20552 & -0.67832 \\
\hline $\mathrm{H}$ & 0.22911 & -2.88035 & -0.79358 \\
\hline $\mathrm{H}$ & -0.44748 & -1.80398 & -1.99460 \\
\hline $\mathrm{H}$ & 2.47319 & -1.92490 & -1.49155 \\
\hline $\mathrm{H}$ & 1.87650 & -0.59557 & -2.51464 \\
\hline $\mathrm{H}$ & 3.08441 & 2.17066 & -0.31288 \\
\hline $\mathrm{H}$ & 2.86923 & 1.75822 & -2.00118 \\
\hline $\mathrm{H}$ & 0.68986 & 2.64714 & -0.91639 \\
\hline $\mathrm{H}$ & -1.77319 & -0.25834 & -2.48353 \\
\hline $\mathrm{H}$ & -1.41754 & 1.46515 & -2.63602 \\
\hline $\mathrm{H}$ & -2.98050 & 0.93161 & -2.01303 \\
\hline $\mathrm{H}$ & -3.69866 & -2.25615 & 1.49509 \\
\hline $\mathrm{H}$ & -2.99365 & -1.16232 & 2.71049 \\
\hline $\mathrm{H}$ & -4.71258 & -1.04983 & 2.29564 \\
\hline $\mathrm{H}$ & 1.50347 & -2.06004 & 1.12206 \\
\hline $\mathrm{H}$ & 0.88743 & -0.47908 & 1.62399 \\
\hline $\mathrm{H}$ & 2.70138 & 1.46613 & 1.71463 \\
\hline $\mathrm{H}$ & 3.13777 & 0.12623 & 2.79256 \\
\hline $\mathrm{H}$ & 4.40146 & -1.53201 & 0.09803 \\
\hline $\mathrm{H}$ & -4.90980 & 0.42743 & -0.73978 \\
\hline $\mathrm{H}$ & 4.90217 & 0.51650 & -0.69081 \\
\hline
\end{tabular}

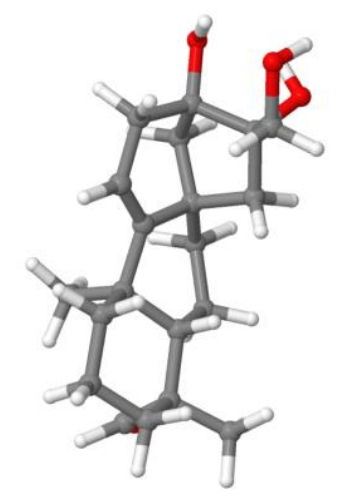




$\begin{array}{llll}\mathrm{O} & 4.66683 & 0.93295 & 1.61967 \\ \mathrm{H} & 5.15134 & 0.09330 & 1.71372\end{array}$

\section{Conformer 4}

Energy: -1081.02553 Hartree (Rel: $0.6 \mathrm{kcal} / \mathrm{mol}$ ) XYZ coordinates for conf 4 :

\begin{tabular}{|c|c|c|c|}
\hline C & -3.14531 & 2.30356 & 0.51949 \\
\hline C & -3.62661 & 1.14812 & 1.40286 \\
\hline C & -3.37618 & -0.24541 & 0.78325 \\
\hline C & -1.86276 & -0.36716 & 0.35653 \\
\hline C & -1.31907 & 0.80088 & -0.53690 \\
\hline C & -1.66539 & 2.15038 & 0.15153 \\
\hline C & -1.50897 & -1.77884 & -0.18128 \\
\hline C & -0.21734 & -1.83084 & -1.02032 \\
\hline C & 0.81979 & -0.77696 & -0.62468 \\
\hline C & 0.21471 & 0.63831 & -0.64851 \\
\hline$C$ & 2.06643 & -0.80306 & -1.54013 \\
\hline C & 3.03081 & 0.10011 & -0.75842 \\
\hline C & 2.54666 & 1.54707 & -0.90526 \\
\hline C & 1.05045 & 1.68229 & -0.76285 \\
\hline C & -1.91936 & 0.80669 & -1.96862 \\
\hline C & -3.70270 & -1.33181 & 1.83583 \\
\hline C & 2.91328 & -0.50890 & 0.69251 \\
\hline C & 1.46345 & -1.03543 & 0.78059 \\
\hline 0 & 4.39033 & 0.08182 & -1.18317 \\
\hline C & 3.33396 & 0.44769 & 1.80798 \\
\hline 0 & 3.81500 & -1.63498 & 0.74256 \\
\hline C & -4.34738 & -0.45756 & -0.38468 \\
\hline 0 & -4.55804 & -1.52136 & -0.92894 \\
\hline $\mathrm{H}$ & -3.29061 & 3.25473 & 1.04716 \\
\hline $\mathrm{H}$ & -3.75872 & 2.37172 & -0.38842 \\
\hline $\mathrm{H}$ & -4.69566 & 1.26018 & 1.63252 \\
\hline $\mathrm{H}$ & -3.10131 & 1.19202 & 2.36713 \\
\hline $\mathrm{H}$ & -1.33146 & -0.24450 & 1.31323 \\
\hline $\mathrm{H}$ & -1.37266 & 2.98380 & -0.49756 \\
\hline $\mathrm{H}$ & -1.06167 & 2.23892 & 1.06491 \\
\hline $\mathrm{H}$ & -1.40713 & -2.45031 & 0.67865 \\
\hline $\mathrm{H}$ & -2.33776 & -2.17456 & -0.77419 \\
\hline $\mathrm{H}$ & 0.23460 & -2.82761 & -0.94073 \\
\hline $\mathrm{H}$ & -0.45462 & -1.69690 & -2.08250 \\
\hline $\mathrm{H}$ & 2.46931 & -1.82204 & -1.60626 \\
\hline $\mathrm{H}$ & 1.86517 & -0.43894 & -2.55301 \\
\hline $\mathrm{H}$ & 3.06404 & 2.20953 & -0.19721 \\
\hline $\mathrm{H}$ & 2.86091 & 1.89201 & -1.90173 \\
\hline $\mathrm{H}$ & 0.67268 & 2.70127 & -0.77600 \\
\hline $\mathrm{H}$ & -1.77934 & -0.14202 & -2.49198 \\
\hline $\mathrm{H}$ & -1.43653 & 1.58890 & -2.56440 \\
\hline $\mathrm{H}$ & -2.99421 & 1.01540 & -1.96331 \\
\hline $\mathrm{H}$ & -3.68388 & -2.33236 & 1.39908 \\
\hline H & -2.98154 & -1.29021 & 2.66053 \\
\hline $\mathrm{H}$ & -4.70241 & -1.16977 & 2.25637 \\
\hline $\mathrm{H}$ & 1.51250 & -2.10850 & 0.99538 \\
\hline $\mathrm{H}$ & 0.90249 & -0.55898 & 1.59146 \\
\hline $\mathrm{H}$ & 2.74709 & 1.37506 & 1.77755 \\
\hline $\mathrm{H}$ & 3.17042 & -0.02138 & 2.78444 \\
\hline $\mathrm{H}$ & 4.60540 & -1.29823 & 1.20864 \\
\hline $\mathrm{H}$ & -4.92021 & 0.44147 & -0.70739 \\
\hline $\mathrm{H}$ & 4.69487 & -0.81810 & -0.95792 \\
\hline 0 & 4.73892 & 0.69939 & 1.72407 \\
\hline H & 4.92968 & 0.93567 & 0.79584 \\
\hline
\end{tabular}

\section{Conformer 5}

Energy: -1081.02650 Hartree (Rel: $0.0 \mathrm{kcal} / \mathrm{mol}$ ) XYZ coordinates for conf 5:

$\begin{array}{llrr}\text { C } & -3.19373 & 1.97175 & 0.91573 \\ \text { C } & -3.63457 & 0.64786 & 1.54455 \\ \text { C } & -3.33773 & -0.56435 & 0.64039 \\ \text { C } & -1.82283 & -0.56651 & 0.22614 \\ \text { C } & -1.31476 & 0.77281 & -0.41448 \\ \text { C } & -1.71135 & 1.94638 & 0.52567 \\ C & -1.40269 & -1.83756 & -0.56341\end{array}$
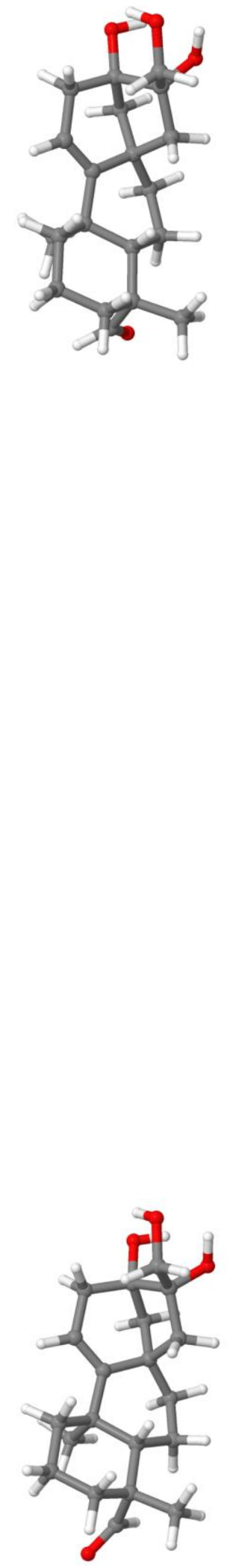


\begin{tabular}{|c|c|c|c|}
\hline $\mathrm{C}$ & -0.09647 & -1.68271 & -1.36801 \\
\hline C & 0.88869 & -0.67705 & -0.76698 \\
\hline C & 0.22379 & 0.69241 & -0.54006 \\
\hline $\mathrm{C}$ & 2.13909 & -0.48547 & -1.65736 \\
\hline C & 3.06222 & 0.29619 & -0.71269 \\
\hline $\mathrm{C}$ & 2.51855 & 1.72454 & -0.59679 \\
\hline $\mathrm{C}$ & 1.01788 & 1.77063 & -0.45026 \\
\hline $\mathrm{C}$ & -1.91380 & 1.03387 & -1.82223 \\
\hline $\mathrm{C}$ & -3.66760 & -1.86750 & 1.41879 \\
\hline $\mathrm{C}$ & 2.96618 & -0.57462 & 0.59901 \\
\hline $\mathrm{C}$ & 1.53594 & -1.16017 & 0.57683 \\
\hline O & 4.42218 & 0.41163 & -1.12110 \\
\hline $\mathrm{C}$ & 3.35267 & 0.17359 & 1.87559 \\
\hline O & 3.90680 & -1.65815 & 0.44738 \\
\hline $\mathrm{C}$ & -4.30186 & -0.58202 & -0.54545 \\
\hline O & -5.31713 & 0.07805 & -0.62417 \\
\hline $\mathrm{H}$ & -3.36219 & 2.79128 & 1.62603 \\
\hline $\mathrm{H}$ & -3.82341 & 2.19016 & 0.04596 \\
\hline $\mathrm{H}$ & -4.70588 & 0.67417 & 1.76902 \\
\hline $\mathrm{H}$ & -3.10642 & 0.50093 & 2.49778 \\
\hline $\mathrm{H}$ & -1.29891 & -0.61953 & 1.19241 \\
\hline $\mathrm{H}$ & -1.45075 & 2.90048 & 0.05261 \\
\hline $\mathrm{H}$ & -1.10400 & 1.87593 & 1.43858 \\
\hline $\mathrm{H}$ & -1.29066 & -2.65821 & 0.15384 \\
\hline $\mathrm{H}$ & -2.19120 & -2.15644 & -1.25341 \\
\hline $\mathrm{H}$ & 0.39681 & -2.65837 & -1.46234 \\
\hline $\mathrm{H}$ & -0.32549 & -1.36502 & -2.39231 \\
\hline $\mathrm{H}$ & 2.58402 & -1.45824 & -1.90418 \\
\hline $\mathrm{H}$ & 1.92652 & 0.05005 & -2.58850 \\
\hline $\mathrm{H}$ & 3.00453 & 2.26567 & 0.22692 \\
\hline $\mathrm{H}$ & 2.82253 & 2.25947 & -1.50894 \\
\hline $\mathrm{H}$ & 0.59773 & 2.75813 & -0.27932 \\
\hline $\mathrm{H}$ & -1.70497 & 0.22235 & -2.52477 \\
\hline $\mathrm{H}$ & -1.48006 & 1.94829 & -2.24131 \\
\hline $\mathrm{H}$ & -2.99889 & 1.17140 & -1.78934 \\
\hline $\mathrm{H}$ & -3.60324 & -2.76315 & 0.79202 \\
\hline $\mathrm{H}$ & -2.96884 & -1.99009 & 2.25496 \\
\hline $\mathrm{H}$ & -4.68266 & -1.81635 & 1.82763 \\
\hline $\mathrm{H}$ & 1.62444 & -2.25197 & 0.60010 \\
\hline $\mathrm{H}$ & 0.95375 & -0.85373 & 1.45200 \\
\hline $\mathrm{H}$ & 2.73565 & 1.07116 & 2.01267 \\
\hline $\mathrm{H}$ & 3.20370 & -0.47269 & 2.74754 \\
\hline $\mathrm{H}$ & 4.68475 & -1.38408 & 0.97213 \\
\hline $\mathrm{H}$ & -4.06194 & -1.31377 & -1.34723 \\
\hline $\mathrm{H}$ & 4.76335 & -0.50187 & -1.07365 \\
\hline O & 4.74867 & 0.48079 & 1.84917 \\
\hline $\mathrm{H}$ & 4.93179 & 0.89299 & 0.98288 \\
\hline
\end{tabular}

\section{Conformer 6}

Energy: -1081.02540 Hartree (Rel: $0.7 \mathrm{kcal} / \mathrm{mol}$ ) XYZ coordinates for conf 6 :

$\begin{array}{lrrr}\text { C } & -3.21373 & 2.16614 & 0.68523 \\ C & -3.60724 & 0.95759 & 1.53629 \\ C & -3.34369 & -0.38604 & 0.81298 \\ C & -1.86370 & -0.44370 & 0.31281 \\ C & -1.35110 & 0.80662 & -0.49197 \\ C & -1.73568 & 2.09579 & 0.28584 \\ C & -1.49975 & -1.79804 & -0.35282 \\ C & -0.19660 & -1.75860 & -1.17690 \\ C & 0.81751 & -0.72051 & -0.68789 \\ C & 0.18638 & 0.68130 & -0.58970 \\ C & 2.06381 & -0.63925 & -1.60124 \\ C & 3.01446 & 0.20106 & -0.73679 \\ C & 2.50542 & 1.64753 & -0.73973 \\ C & 1.00666 & 1.74355 & -0.59304 \\ C & -1.92414 & 0.92014 & -1.93142 \\ C & -3.61591 & -1.53174 & 1.82807 \\ C & 2.91318 & -0.54849 & 0.64584 \\ C & 1.46763 & -1.09073 & 0.69072 \\ \text { O } & 4.37319 & 0.24822 & -1.16521 \\ C & 3.33647 & 0.29594 & 1.84794\end{array}$

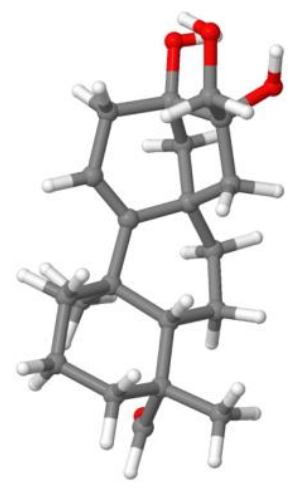




$\begin{array}{rrr}3.82341 & -1.66706 & 0.57750 \\ -4.43597 & -0.56824 & -0.24031 \\ -4.32235 & -0.96792 & -1.37817 \\ -3.39532 & 3.09115 & 1.24700 \\ -3.85330 & 2.22235 & -0.20627 \\ -4.66390 & 1.01730 & 1.83247 \\ -3.02547 & 0.96400 & 2.46879 \\ -1.28951 & -0.39506 & 1.25095 \\ -1.48395 & 2.97665 & -0.31630 \\ -1.12438 & 2.15536 & 1.19675 \\ -1.40063 & -2.55019 & 0.43855 \\ -2.31639 & -2.12683 & -0.99860 \\ 0.27500 & -2.74950 & -1.17274 \\ -0.42767 & -1.54423 & -2.22657 \\ 2.48388 & -1.64022 & -1.76454 \\ 1.85426 & -0.18394 & -2.57464 \\ 3.01137 & 2.24453 & 0.03177 \\ 2.81565 & 2.09460 & -1.69603 \\ 0.61145 & 2.75318 & -0.51598 \\ -1.78232 & 0.01004 & -2.51710 \\ -1.41809 & 1.74023 & -2.45299 \\ -2.99625 & 1.12769 & -1.93169 \\ -3.51814 & -2.52105 & 1.37222 \\ -2.90648 & -1.46407 & 2.66097 \\ -4.62686 & -1.45117 & 2.24550 \\ 1.52407 & -2.17751 & 0.81706 \\ 0.90558 & -0.68571 & 1.53860 \\ 2.74214 & 1.21657 & 1.91482 \\ 3.18503 & -0.26938 & 2.77415 \\ 4.61592 & -1.37038 & 1.06665 \\ -5.45199 & -0.33232 & 0.16085 \\ 4.69183 & -0.66601 & -1.04006 \\ 4.73855 & 0.56650 & 1.77805 \\ 4.91799 & 0.89421 & 0.87556\end{array}$


originally assigned structure of 1,4-epoxy-6-deoxypseudoanisatin (cmpd 1), Fig.4G

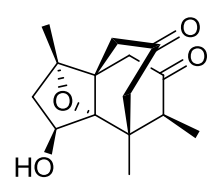

Rel energy (kcal/mol): $\begin{gathered}\operatorname{Conf} 1 \\ 0.0\end{gathered}$

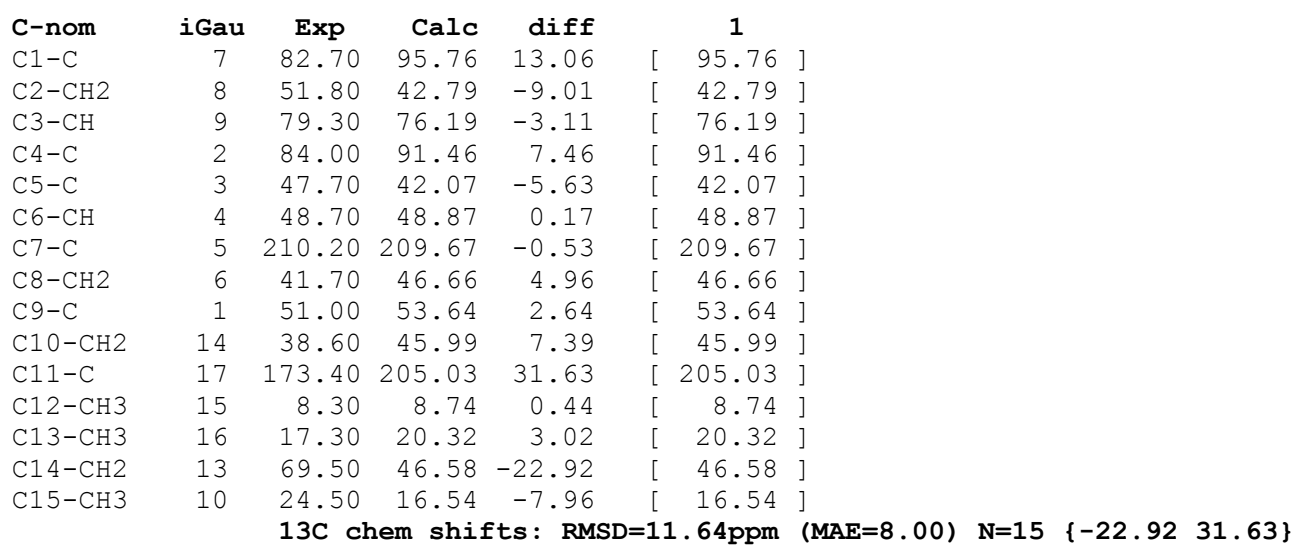

\section{Conformer 1}

Energy: -884.45380 Hartree (Rel: $0.0 \mathrm{kcal} / \mathrm{mol}$ ) XYZ coordinates for conf 1 :

$\begin{array}{lrrr}\mathrm{C} & -0.76387 & 0.66618 & 0.58068 \\ \mathrm{C} & -0.59598 & -0.28355 & -0.61445 \\ \mathrm{C} & 0.83496 & -0.75407 & -0.83698 \\ \mathrm{C} & 1.73450 & 0.50295 & -1.11272 \\ \mathrm{C} & 1.64231 & 1.46842 & 0.07090 \\ \mathrm{C} & 0.22850 & 1.85437 & 0.48989 \\ \mathrm{C} & -2.08099 & 0.97267 & -0.21925 \\ \mathrm{C} & -2.90209 & -0.32660 & -0.16339 \\ \mathrm{C} & -1.77042 & -1.31111 & -0.57200 \\ \mathrm{C} & -2.82513 & 2.28233 & -0.19709 \\ \mathrm{O} & -1.55641 & -2.40648 & 0.31069 \\ \mathrm{O} & -1.27528 & 0.72604 & -1.45739 \\ \mathrm{C} & 1.22386 & -1.43548 & 0.52542 \\ \mathrm{C} & -0.58596 & -0.05605 & 1.91507 \\ \mathrm{C} & 3.19273 & 0.20605 & -1.47352 \\ \mathrm{C} & 0.95647 & -1.77416 & -1.98147 \\ \mathrm{C} & 0.76256 & -0.80468 & 1.86101 \\ \mathrm{O} & 2.62299 & 1.93901 & 0.61501 \\ \mathrm{O} & 1.42906 & -0.96329 & 2.86317 \\ \mathrm{H} & 1.25855 & 1.02384 & -1.95782 \\ \mathrm{H} & 0.28558 & 2.39235 & 1.44092 \\ \mathrm{H} & -0.10865 & 2.56093 & -0.27646 \\ \mathrm{H} & -3.30191 & -0.54665 & 0.83265 \\ \mathrm{H} & -3.73026 & -0.30025 & -0.87819 \\ \mathrm{H} & -1.91877 & -1.67678 & -1.59498 \\ \mathrm{H} & -2.15449 & 3.14382 & -0.25989 \\ \mathrm{H} & -3.40723 & 2.36571 & 0.72821 \\ \mathrm{H} & -3.52157 & 2.33114 & -1.04168 \\ \mathrm{H} & -2.31108 & -3.00932 & 0.22623 \\ \mathrm{H} & 0.78638 & -2.44077 & 0.53528 \\ \mathrm{H} & 2.30794 & -1.56002 & 0.60003 \\ \mathrm{H} & -0.57073 & 0.60876 & 2.78410 \\ \mathrm{H} & -1.35644 & -0.81719 & 2.07578 \\ \mathrm{H} & 3.74864 & 1.14347 & -1.55714 \\ \mathrm{H} & 3.26795 & -0.32445 & -2.42698\end{array}$

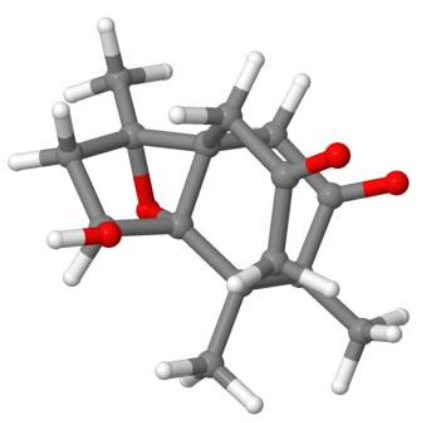




$\begin{array}{llll}\mathrm{H} & 3.69268 & -0.38985 & -0.70317 \\ \mathrm{H} & 0.27947 & -2.61873 & -1.81808 \\ \mathrm{H} & 0.71045 & -1.31498 & -2.94646 \\ \mathrm{H} & 1.97182 & -2.17680 & -2.04684\end{array}$


revised structure for cmpd 1 (1 1 -hydroxy-6-deoxypseudoanisatin), Fig.4G

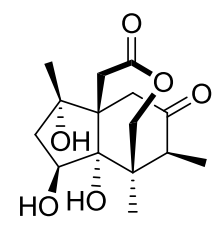

Rel energy (kcal/mol): $\begin{array}{cc}\text { Conf } 1 \text { Conf } 2 \\ 0.9 & 0.0\end{array}$

\begin{tabular}{|c|c|c|c|c|c|c|c|}
\hline C-nom & iGau & Exp & Calc & $\operatorname{diff}$ & 1 & 2 & \\
\hline $\mathrm{C} 1-\mathrm{C}$ & 7 & 82.70 & 84.88 & 2.18 & 83.06 & $85.26]$ & \\
\hline $\mathrm{C} 2-\mathrm{CH} 2$ & 8 & 51.80 & 52.81 & 1.01 & 53.01 & $52.77]$ & \\
\hline $\mathrm{C} 3-\mathrm{CH}$ & 9 & 79.30 & 81.70 & 2.40 & 81.62 & $81.72]$ & \\
\hline $\mathrm{C} 4-\mathrm{C}$ & 2 & 84.00 & 86.41 & 2.41 & 89.13 & $85.84]$ & \\
\hline $\mathrm{C} 5-\mathrm{C}$ & 3 & 47.70 & 47.25 & -0.45 & 46.47 & $47.41]$ & \\
\hline $\mathrm{C} 6-\mathrm{CH}$ & 4 & 48.70 & 48.84 & 0.14 & 49.20 & $48.76]$ & \\
\hline $\mathrm{C} 7-\mathrm{C}$ & 5 & 210.20 & 207.82 & -2.38 & [ 207.22 & $207.94 \quad]$ & \\
\hline $\mathrm{C} 8-\mathrm{CH} 2$ & 6 & 41.70 & 41.11 & -0.59 & {$[\quad 41.61$} & $41.01]$ & \\
\hline $\mathrm{C} 9-\mathrm{C}$ & 1 & 51.00 & 51.71 & 0.71 & 51.47 & $51.76]$ & \\
\hline $\mathrm{C} 10-\mathrm{CH} 2$ & 14 & 38.60 & 38.91 & 0.31 & 38.65 & $38.97]$ & \\
\hline $\mathrm{C} 11-\mathrm{C}$ & 17 & 173.40 & 170.24 & -3.16 & {$[170.46$} & 170.19 ] & \\
\hline C12-CH3 & 15 & 8.30 & 9.25 & 0.95 & 9.23 & $9.25]$ & \\
\hline $\mathrm{C} 13-\mathrm{CH} 3$ & 16 & 17.30 & 18.09 & 0.79 & 18.02 & $18.10]$ & \\
\hline $\mathrm{C} 14-\mathrm{CH} 2$ & 13 & 69.50 & 68.47 & -1.03 & 67.73 & $68.63]$ & \\
\hline \multirow[t]{3}{*}{$\mathrm{C} 15-\mathrm{CH} 3$} & 10 & 24.50 & 25.37 & 0.87 & {$\left[\begin{array}{ll}{[} & 24.17\end{array}\right.$} & $25.62]$ & \\
\hline & & $13 \mathrm{C} \mathrm{cl}$ & em shi & Es: RMS & $=1.58 \mathrm{ppm}$ & $(\mathrm{MAE}=1,29)$ & $\mathrm{N}=15 \quad \begin{cases}-3.16 & 2.41\}\end{cases}$ \\
\hline & & & & Fracti & $: \quad 0.173$ & 0.827 & \\
\hline
\end{tabular}

\section{Conformer 1}

Energy: -1036.13469 Hartree (Rel: $0.9 \mathrm{kcal} / \mathrm{mol}$ ) XYZ coordinates for conf 1 :

\begin{tabular}{|c|c|c|c|}
\hline & & & \\
\hline C & 0.80738 & -0.56004 & -0.18122 \\
\hline C & 0.42252 & 0.94376 & -0.24110 \\
\hline C & -1.08054 & 1.22075 & 0.07132 \\
\hline C & -1.92148 & 0.49666 & -1.04168 \\
\hline C & -1.48644 & -0.96261 & -1.31684 \\
\hline C & -0.00176 & -1.34149 & -1.23046 \\
\hline C & 2.37444 & -0.50491 & -0.45478 \\
\hline C & 2.80881 & 0.79695 & 0.29850 \\
\hline C & 1.55236 & 1.67935 & 0.53888 \\
\hline C & 3.18257 & -1.72894 & -0.01765 \\
\hline O & 1.22961 & 1.82487 & 1.92401 \\
\hline 0 & 2.65448 & -0.39839 & -1.84821 \\
\hline C & -1.51890 & 0.73807 & 1.47555 \\
\hline C & 0.57089 & -1.16637 & 1.23776 \\
\hline C & -3.44374 & 0.56737 & -0.85941 \\
\hline C & -1.33833 & 2.74632 & 0.05380 \\
\hline C & -0.84843 & -1.60180 & 1.56812 \\
\hline O & -2.29503 & -1.78603 & -1.69752 \\
\hline O & -1.14101 & -2.74723 & 1.81196 \\
\hline $\mathrm{H}$ & -1.66891 & 1.01266 & -1.97876 \\
\hline $\mathrm{H}$ & 0.04601 & -2.42140 & -1.05824 \\
\hline $\mathrm{H}$ & 0.42936 & -1.16397 & -2.22196 \\
\hline $\mathrm{H}$ & 3.25601 & 0.55428 & 1.26955 \\
\hline $\mathrm{H}$ & 3.57161 & 1.30949 & -0.29474 \\
\hline $\mathrm{H}$ & 1.68117 & 2.68266 & 0.10678 \\
\hline $\mathrm{H}$ & 2.77726 & -2.64272 & -0.46506 \\
\hline $\mathrm{H}$ & 3.22056 & -1.85156 & 1.06744 \\
\hline $\mathrm{H}$ & 4.20699 & -1.60773 & -0.38353 \\
\hline $\mathrm{H}$ & 2.00313 & 2.20625 & 2.36835 \\
\hline $\mathrm{H}$ & -2.46797 & 1.21777 & 1.72258 \\
\hline $\mathrm{H}$ & 1.14197 & -2.08996 & 1.31591 \\
\hline $\mathrm{H}$ & 0.93317 & -0.48355 & 2.01325 \\
\hline $\mathrm{H}$ & -3.93097 & 0.09622 & -1.71556 \\
\hline $\mathrm{H}$ & -3.79531 & 1.60190 & -0.79262 \\
\hline $\mathrm{H}$ & -3.76474 & 0.02288 & 0.03275 \\
\hline
\end{tabular}

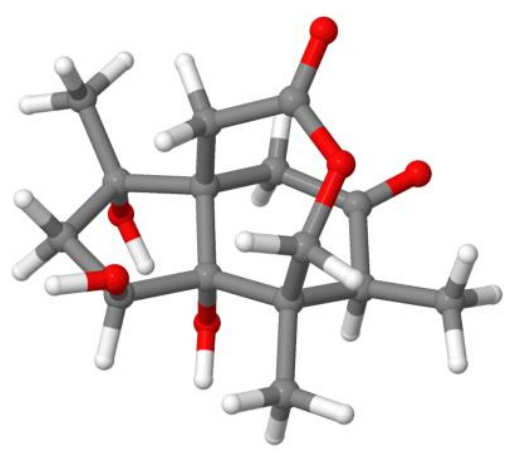




$\begin{array}{rrrr}\mathrm{H} & -0.76254 & 3.24561 & 0.84032 \\ \mathrm{H} & -1.07639 & 3.20241 & -0.90925 \\ \mathrm{H} & -2.39553 & 2.96840 & 0.22233 \\ \mathrm{H} & 2.12439 & 0.35127 & -2.17472 \\ \mathrm{O} & 0.66263 & 1.33379 & -1.62628 \\ \mathrm{H} & 0.52262 & 2.29103 & -1.69705 \\ \mathrm{O} & -1.82793 & -0.66001 & 1.57265 \\ \mathrm{H} & -0.78988 & 1.02358 & 2.23459\end{array}$

\section{Conformer 2}

Energy: -1036.13617 Hartree (Rel: $0.0 \mathrm{kcal} / \mathrm{mol}$ ) XYZ coordinates for conf 2 :

$\begin{array}{lrrr}\mathrm{C} & 0.80114 & -0.55856 & -0.17716 \\ \mathrm{C} & 0.42223 & 0.95231 & -0.27113 \\ \mathrm{C} & -1.07945 & 1.22654 & 0.04671 \\ \mathrm{C} & -1.91943 & 0.48479 & -1.05646 \\ \mathrm{C} & -1.49208 & -0.97658 & -1.29890 \\ \mathrm{C} & -0.00179 & -1.35329 & -1.22288 \\ \mathrm{C} & 2.36116 & -0.51114 & -0.41137 \\ \mathrm{C} & 2.79872 & 0.78701 & 0.33108 \\ \mathrm{C} & 1.54063 & 1.68556 & 0.53386 \\ \mathrm{C} & 3.17249 & -1.74371 & -0.00598 \\ \mathrm{O} & 1.22248 & 1.83662 & 1.92260 \\ \mathrm{O} & 2.54289 & -0.34138 & -1.84486 \\ \mathrm{C} & -1.52590 & 0.77238 & 1.45387 \\ \mathrm{C} & 0.55962 & -1.14708 & 1.24863 \\ \mathrm{C} & -3.44246 & 0.56446 & -0.88534 \\ \mathrm{C} & -1.32018 & 2.75196 & -0.01494 \\ \mathrm{C} & -0.86479 & -1.57107 & 1.58055 \\ \mathrm{O} & -2.29602 & -1.81727 & -1.65228 \\ \mathrm{O} & -1.15392 & -2.71607 & 1.83639 \\ \mathrm{H} & -1.64908 & 0.98691 & -1.99507 \\ \mathrm{H} & 0.04903 & -2.43278 & -1.04847 \\ \mathrm{H} & 0.40807 & -1.17174 & -2.22183 \\ \mathrm{H} & 3.21509 & 0.54206 & 1.31397 \\ \mathrm{H} & 3.58749 & 1.29704 & -0.23470 \\ \mathrm{H} & 1.67169 & 2.67429 & 0.08345 \\ \mathrm{H} & 2.78029 & -2.64522 & -0.48763 \\ \mathrm{H} & 3.19349 & -1.90048 & 1.07501 \\ \mathrm{H} & 4.21414 & -1.61648 & -0.33005 \\ \mathrm{H} & 1.90705 & 2.39442 & 2.32384 \\ \mathrm{H} & -2.47879 & 1.25285 & 1.68374 \\ \mathrm{H} & 1.12256 & -2.07402 & 1.34891 \\ \mathrm{H} & 0.91854 & -0.45143 & 2.01466 \\ \mathrm{H} & -3.92878 & 0.09301 & -1.74224 \\ \mathrm{H} & -3.78647 & 1.60160 & -0.82588 \\ \mathrm{H} & -3.77422 & 0.02721 & 0.00767 \\ \mathrm{H} & -0.78888 & 3.25488 & 0.80092 \\ \mathrm{H} & -0.96183 & 3.15806 & -0.96369 \\ \mathrm{H} & -2.38311 & 2.99032 & 0.08532 \\ \mathrm{H} & 3.49326 & -0.23877 & -2.01514 \\ \mathrm{O} & 0.59173 & 1.42177 & -1.61857 \\ \mathrm{H} & 1.36458 & 0.96305 & -2.00445 \\ \mathrm{O} & -1.83571 & -0.62847 & 1.58338 \\ \mathrm{H} & -0.80517 & 1.07199 & 2.21513\end{array}$

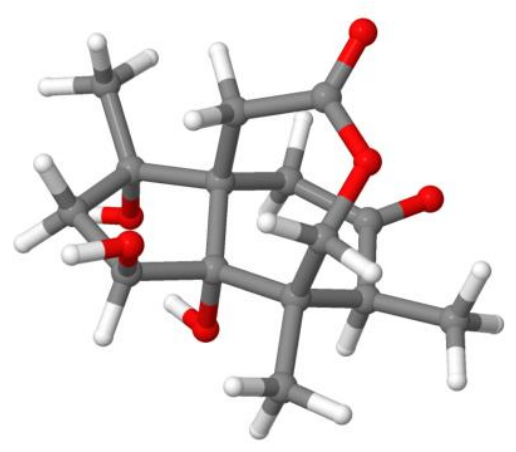


originally assigned structure for 8-hydroxy-1,7-epoxy-2-menthene, Fig.5

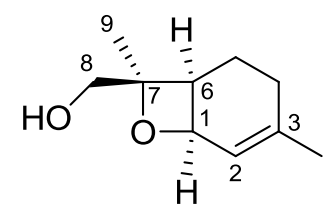

$$
\text { Rel energy (kcal/mol): } \begin{array}{ccc}
\text { Conf } 1 \text { Conf 2 } \\
0.9 & 0.0
\end{array}
$$

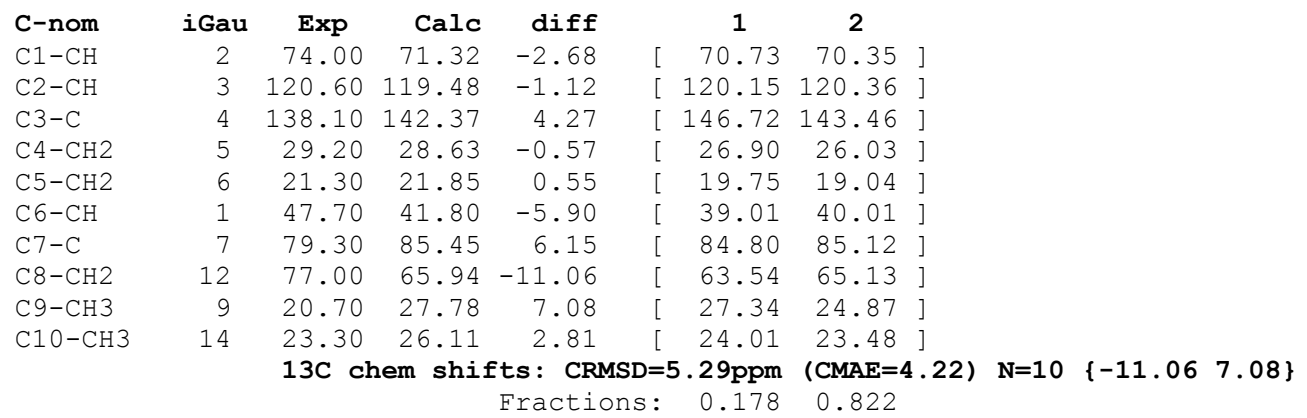

\section{Conformer 1}

Energy: -541.09379 Hartree (Rel: $0.9 \mathrm{kcal} / \mathrm{mol})$ XYZ coordinates for conf 1 :

$\begin{array}{lrrr}\mathrm{C} & 0.62292 & -0.79607 & 0.88721 \\ \mathrm{C} & 0.05538 & -1.38422 & -0.42266 \\ \mathrm{C} & -1.36039 & -1.04879 & -0.76384 \\ \mathrm{C} & -2.10002 & -0.15205 & -0.09000 \\ \mathrm{C} & -1.54728 & 0.62149 & 1.08683 \\ \mathrm{C} & -0.37813 & -0.08102 & 1.80065 \\ \mathrm{C} & 1.65965 & -0.06030 & -0.02029 \\ \mathrm{O} & 1.04590 & -0.62948 & -1.22624 \\ \mathrm{C} & 3.08711 & -0.56519 & 0.15934 \\ \mathrm{H} & 1.13777 & -1.55618 & 1.48269 \\ \mathrm{H} & 0.24382 & -2.45531 & -0.56060 \\ \mathrm{C} & 1.65067 & 1.47453 & -0.14100 \\ \mathrm{O} & 0.50268 & 1.95641 & -0.82011 \\ \mathrm{C} & -3.51508 & 0.17189 & -0.49212 \\ \mathrm{H} & -1.78944 & -1.56172 & -1.62446 \\ \mathrm{H} & -1.22791 & 1.60177 & 0.71430 \\ \mathrm{H} & -2.35053 & 0.80915 & 1.81189 \\ \mathrm{H} & 0.14505 & 0.62833 & 2.45427 \\ \mathrm{H} & -0.79894 & -0.84736 & 2.46454 \\ \mathrm{H} & 3.71281 & -0.26788 & -0.68958 \\ \mathrm{H} & 3.52842 & -0.14677 & 1.07302 \\ \mathrm{H} & 3.10860 & -1.65682 & 0.23957 \\ \mathrm{H} & 2.56266 & 1.78681 & -0.67559 \\ \mathrm{H} & 1.68206 & 1.93278 & 0.85428 \\ \mathrm{H} & 0.27463 & 1.23391 & -1.43814 \\ \mathrm{H} & -3.82230 & -0.37524 & -1.38916 \\ \mathrm{H} & -3.62733 & 1.24703 & -0.68899 \\ \mathrm{H} & -4.21686 & -0.06954 & 0.31800\end{array}$

Conformer 2

Energy: -541.09524 Hartree (Rel: $0.0 \mathrm{kcal} / \mathrm{mol}$ ) XYZ coordinates for conf 2 :

$\begin{array}{lrrr}\text { C } & 0.44616 & -0.58268 & 0.87726 \\ \text { C } & -0.06444 & -1.18160 & -0.45172 \\ \text { C } & -1.39924 & -0.72367 & -0.93604 \\ \text { C } & -2.29421 & -0.14461 & -0.11993 \\ \text { C } & -1.93179 & 0.16903 & 1.32063 \\ C & -0.44069 & 0.52627 & 1.46051 \\ C & 1.72392 & -0.20805 & 0.06201 \\ \text { O } & 1.08532 & -0.63782 & -1.19234 \\ \text { C } & 2.93825 & -1.06932 & 0.38600\end{array}$

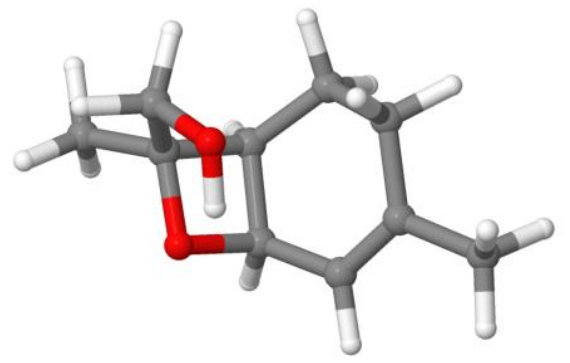




$\begin{array}{rrrr}\text { H } & 0.64472 & -1.34319 & 1.63879 \\ \mathrm{H} & -0.00386 & -2.27856 & -0.48474 \\ \mathrm{C} & 2.12415 & 1.26877 & -0.08842 \\ \mathrm{O} & 1.16159 & 2.00948 & -0.82389 \\ \mathrm{C} & -3.68416 & 0.21622 & -0.57191 \\ \mathrm{H} & -1.65378 & -0.94673 & -1.97215 \\ \mathrm{H} & -2.54607 & 1.00427 & 1.68005 \\ \mathrm{H} & -2.18559 & -0.68781 & 1.96353 \\ \mathrm{H} & -0.26771 & 1.46213 & 0.92339 \\ \mathrm{H} & -0.18813 & 0.69760 & 2.51331 \\ \mathrm{H} & 3.69180 & -0.98828 & -0.40520 \\ \mathrm{H} & 3.39392 & -0.74473 & 1.32988 \\ \mathrm{H} & 2.65705 & -2.12253 & 0.48925 \\ \mathrm{H} & 3.10494 & 1.31745 & -0.58911 \\ \mathrm{H} & 2.23351 & 1.73154 & 0.89911 \\ \mathrm{H} & 0.79882 & 1.35645 & -1.45445 \\ \mathrm{H} & -3.85996 & -0.06127 & -1.61623 \\ \mathrm{H} & -3.86302 & 1.29486 & -0.46376 \\ \mathrm{H} & -4.43964 & -0.28663 & 0.04781\end{array}$


revised "8-hydroxy-1,7-epoxy-2-menthene" (7-hydroxy-1,8-epoxy), Fig.5

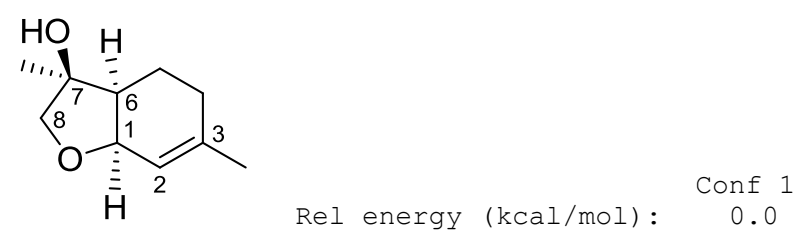

\begin{tabular}{|c|c|c|c|c|c|}
\hline $\mathrm{C}$-nom & iGau & $\operatorname{Exp}$ & Calc & $\operatorname{diff}$ & 1 \\
\hline $\mathrm{Cl}-\mathrm{CH}$ & 2 & 74.00 & 75.35 & 1.35 & 75.35 \\
\hline $\mathrm{C} 2-\mathrm{CH}$ & 3 & 120.60 & 120.48 & -0.12 & 121.77 \\
\hline $\mathrm{C} 3-\mathrm{C}$ & 4 & 138.10 & 138.73 & 0.63 & 140.55 \\
\hline $\mathrm{C} 4-\mathrm{CH} 2$ & 5 & 29.20 & 29.01 & -0.19 & 27.67 \\
\hline $\mathrm{C} 5-\mathrm{CH} 2$ & 6 & 21.30 & 20.24 & -1.06 & 18.65 \\
\hline $\mathrm{C} 6-\mathrm{CH}$ & 1 & 47.70 & 46.28 & -1.42 & 45.44 \\
\hline C $7-C$ & 7 & 79.30 & 78.84 & -0.46 & 78.94 \\
\hline $\mathrm{C} 8-\mathrm{CH} 2$ & 12 & 77.00 & 77.76 & 0.76 & 77.83 \\
\hline $\mathrm{C} 9-\mathrm{CH} 3$ & 9 & 20.70 & 21.15 & 0.45 & 19.59 \\
\hline $\mathrm{C} 10-\mathrm{CH} 3$ & 14 & 23.30 & 24.49 & 1.19 & 23.03 \\
\hline
\end{tabular}

13C chem shifts: CRMSD $=0.89 \mathrm{ppm}(\mathrm{CMAE}=0.77) \quad \mathrm{N}=10 \quad\{-1.42 \quad 1.35\}$

\section{Conformer 1}

Energy: -541.11696 Hartree (Rel: $0.0 \mathrm{kcal} / \mathrm{mol}$ ) XYZ coordinates for conf 1 :

$\begin{array}{rrrr}\mathrm{C} & 0.47858 & -0.45221 & -0.61771 \\ \mathrm{C} & -0.16052 & 0.97543 & -0.63781 \\ \mathrm{C} & -1.55780 & 1.07957 & -0.11059 \\ \mathrm{C} & -2.38667 & 0.02917 & -0.02274 \\ \mathrm{C} & -1.88524 & -1.36496 & -0.34370 \\ \mathrm{C} & -0.40563 & -1.52233 & 0.03721 \\ \mathrm{C} & 1.85498 & -0.20651 & 0.09348 \\ \mathrm{O} & 1.76694 & -0.42165 & 1.50910 \\ \mathrm{C} & 2.99283 & -1.07963 & -0.41377 \\ \mathrm{H} & 0.67922 & -0.74867 & -1.65370 \\ \mathrm{H} & -0.14228 & 1.35196 & -1.67534 \\ \mathrm{C} & 2.03381 & 1.30005 & -0.14307 \\ \mathrm{O} & 0.73273 & 1.82340 & 0.12542 \\ \mathrm{C} & -3.82817 & 0.16842 & 0.38948 \\ \mathrm{H} & -1.90908 & 2.08492 & 0.12004 \\ \mathrm{H} & -2.48599 & -2.10821 & 0.19603 \\ \mathrm{H} & -2.03365 & -1.57897 & -1.41369 \\ \mathrm{H} & -0.31771 & -1.44936 & 1.12544 \\ \mathrm{H} & -0.04503 & -2.51970 & -0.24216 \\ \mathrm{H} & 3.92389 & -0.83581 & 0.10964 \\ \mathrm{H} & 3.14701 & -0.93320 & -1.48759 \\ \mathrm{H} & 2.77299 & -2.13846 & -0.23886 \\ \mathrm{H} & 2.33849 & 1.51577 & -1.18040 \\ \mathrm{H} & 2.74424 & 1.75780 & 0.54995 \\ \mathrm{H} & -4.09807 & 1.21081 & 0.58780 \\ \mathrm{H} & -4.03774 & -0.42167 & 1.29231 \\ \mathrm{H} & -4.49800 & -0.21491 & -0.39304 \\ \mathrm{H} & 1.14728 & 0.25162 & 1.84269\end{array}$

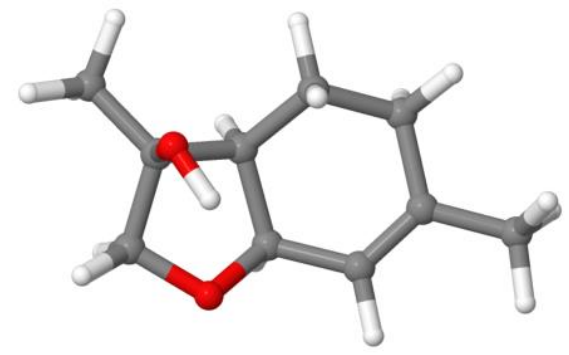


originally assigned structure of triptergulide A, Fig.5

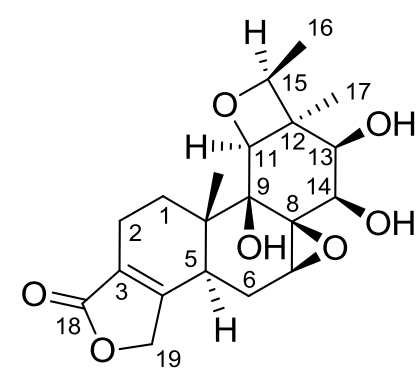

Rel energy (kcal/mol): $\begin{array}{ccc}\text { Conf } 1 \text { Conf } 2 \\ 1.1 & 0.0\end{array}$

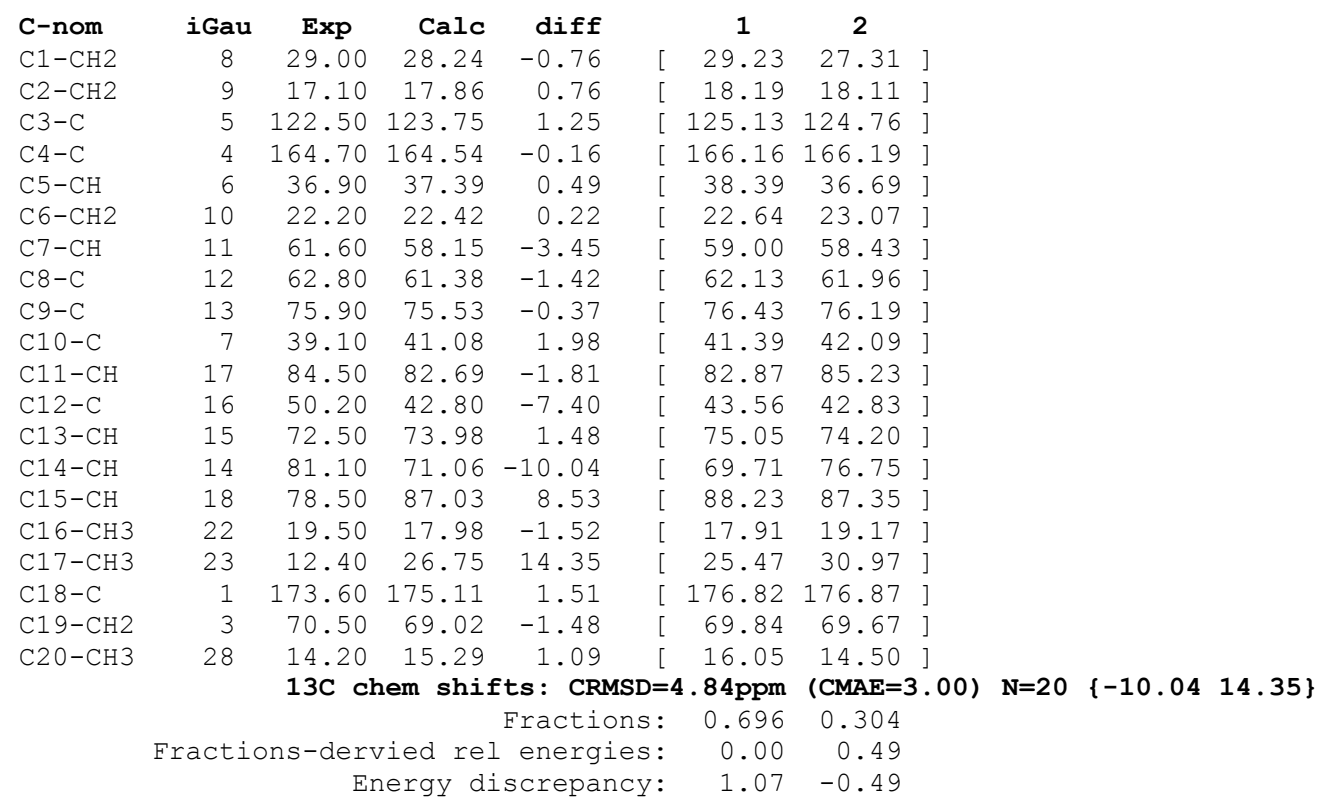

Conformer 1

Energy: -1304.25760 Hartree (Rel: $1.1 \mathrm{kcal} / \mathrm{mol}$ ) XYZ coordinates for conf 1 :

$\begin{array}{lrrr}C & -5.00547 & -0.69384 & 0.25810 \\ \mathrm{O} & -5.28996 & 0.45894 & 0.94808 \\ \mathrm{C} & -4.08230 & 1.22225 & 1.12415 \\ \mathrm{C} & -3.00915 & 0.40529 & 0.46089 \\ \mathrm{C} & -3.56297 & -0.71147 & -0.03730 \\ \mathrm{C} & -1.54727 & 0.74254 & 0.38792 \\ \mathrm{C} & -0.82797 & -0.13043 & -0.69001 \\ \mathrm{C} & -1.30053 & -1.60781 & -0.53278 \\ \mathrm{C} & -2.81562 & -1.78507 & -0.76085 \\ \mathrm{C} & -1.27199 & 2.24707 & 0.19749 \\ \mathrm{C} & 0.21774 & 2.51414 & 0.10654 \\ \mathrm{C} & 1.19920 & 1.43851 & -0.14710 \\ \mathrm{C} & 0.73650 & -0.00235 & -0.46587 \\ \mathrm{C} & 2.60532 & 1.64277 & 0.51115 \\ \mathrm{C} & 3.50427 & 0.39696 & 0.41027 \\ \mathrm{C} & 2.76636 & -0.78677 & 1.08604 \\ \mathrm{C} & 1.28082 & -0.94696 & 0.64163 \\ \mathrm{C} & 2.92508 & -2.25979 & 0.58733 \\ \mathrm{O} & 1.53971 & -2.26914 & 0.09570 \\ \mathrm{O} & 0.86622 & 2.39185 & -1.17051 \\ \mathrm{H} & 2.98463 & -2.93313 & 1.45178\end{array}$

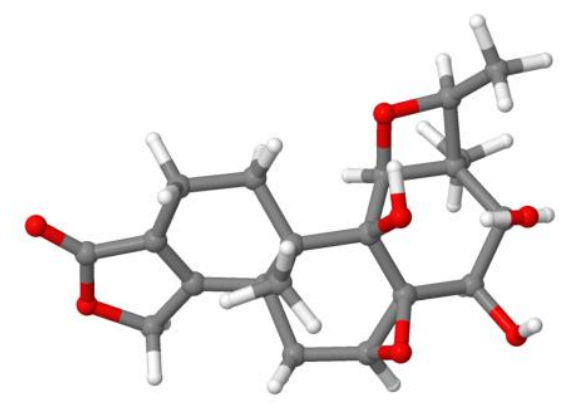




$\begin{array}{lrrr}\mathrm{C} & 3.92923 & -2.72296 & -0.44921 \\ \mathrm{C} & 2.95164 & -0.68578 & 2.60804 \\ \mathrm{H} & 0.58841 & -0.99502 & 1.48865 \\ \mathrm{O} & 3.92388 & 0.25232 & -0.94495 \\ \mathrm{O} & 3.26965 & 2.77018 & -0.01993 \\ \mathrm{O} & 1.40985 & -0.38438 & -1.68554 \\ \mathrm{C} & -1.19897 & 0.33753 & -2.11985 \\ \mathrm{H} & -1.12231 & 0.47981 & 1.37095 \\ \mathrm{O} & -5.86832 & -1.50493 & -0.01247 \\ \mathrm{H} & -3.91307 & 1.36547 & 2.19792 \\ \mathrm{H} & -4.22567 & 2.20651 & 0.66419 \\ \mathrm{H} & -0.75202 & -2.24879 & -1.22993 \\ \mathrm{H} & -1.07021 & -1.97071 & 0.47456 \\ \mathrm{H} & -3.13220 & -2.77352 & -0.40598 \\ \mathrm{H} & -3.06333 & -1.75872 & -1.83062 \\ \mathrm{H} & -1.66858 & 2.80429 & 1.05347 \\ \mathrm{H} & -1.77555 & 2.63535 & -0.69473 \\ \mathrm{H} & 0.58510 & 3.36919 & 0.67580 \\ \mathrm{H} & 2.43316 & 1.87347 & 1.56669 \\ \mathrm{H} & 4.42504 & 0.60240 & 0.96592 \\ \mathrm{H} & 4.94036 & -2.66950 & -0.02934 \\ \mathrm{H} & 3.90916 & -2.12256 & -1.35839 \\ \mathrm{H} & 3.72564 & -3.77011 & -0.70199 \\ \mathrm{H} & 2.65206 & 0.29090 & 3.00208 \\ \mathrm{H} & 2.35579 & -1.44670 & 3.12319 \\ \mathrm{H} & 4.00375 & -0.84471 & 2.87229 \\ \mathrm{H} & 3.11270 & 0.05657 & -1.46769 \\ \mathrm{H} & 3.63065 & 2.47042 & -0.87473 \\ \mathrm{H} & -2.48931 & -1.36031 & -1.61480 \\ \mathrm{H} & -0.88688 & 0.46835 & -2.21071 \\ \mathrm{H} & -0.71586 & -0.40995 & -2.85399 \\ \mathrm{H} & & 1.27924 & -2.37695\end{array}$

\section{Conformer 2}

Energy: -1304.25930 Hartree (Rel: $0.0 \mathrm{kcal} / \mathrm{mol})$ XYZ coordinates for conf 2:

\begin{tabular}{|c|c|c|c|}
\hline $\mathrm{C}$ & -5.01250 & -0.65528 & 0.29291 \\
\hline 0 & -5.22749 & 0.39764 & 1.14802 \\
\hline $\mathrm{C}$ & -3.98901 & 1.09742 & 1.36695 \\
\hline C & -2.97618 & 0.35756 & 0.53929 \\
\hline $\mathrm{C}$ & -3.58719 & -0.66762 & -0.07662 \\
\hline $\mathrm{C}$ & -1.50632 & 0.65344 & 0.44282 \\
\hline C & -0.88948 & -0.05339 & -0.80885 \\
\hline C & -1.38364 & -1.53247 & -0.84386 \\
\hline $\mathrm{C}$ & -2.91620 & -1.65078 & -0.98294 \\
\hline $\mathrm{C}$ & -1.16155 & 2.15452 & 0.53870 \\
\hline $\mathrm{C}$ & 0.31029 & 2.43343 & 0.27695 \\
\hline $\mathrm{C}$ & 1.20396 & 1.42001 & -0.33220 \\
\hline $\mathrm{C}$ & 0.67925 & 0.01233 & -0.66016 \\
\hline $\mathrm{C}$ & 2.71045 & 1.52023 & -0.14091 \\
\hline C & 3.22360 & 0.66209 & 1.03322 \\
\hline C & 2.53941 & -0.72153 & 1.20825 \\
\hline $\mathrm{C}$ & 1.19573 & -0.96691 & 0.46087 \\
\hline $\mathrm{C}$ & 3.03510 & -1.99887 & 0.45122 \\
\hline 0 & 1.70044 & -2.18455 & -0.14595 \\
\hline 0 & 0.69378 & 2.52363 & -1.10538 \\
\hline $\mathrm{H}$ & 3.21471 & -2.81938 & 1.15495 \\
\hline C & 4.13656 & -2.00608 & -0.58975 \\
\hline $\mathrm{C}$ & 2.41101 & -1.01088 & 2.71183 \\
\hline $\mathrm{H}$ & 0.38743 & -1.19445 & 1.15778 \\
\hline 0 & 4.64023 & 0.57186 & 0.96932 \\
\hline 0 & 3.41707 & 1.20536 & -1.34677 \\
\hline 0 & 1.30059 & -0.38119 & -1.89879 \\
\hline $\mathrm{C}$ & -1.34231 & 0.61155 & -2.13217 \\
\hline $\mathrm{H}$ & -1.05700 & 0.19205 & 1.33624 \\
\hline 0 & -5.91223 & -1.39999 & -0.04089 \\
\hline $\mathrm{H}$ & -3.76307 & 1.07916 & 2.43978 \\
\hline $\mathrm{H}$ & -4.12200 & 2.14135 & 1.06132 \\
\hline $\mathrm{H}$ & -0.89912 & -2.05529 & -1.67537 \\
\hline $\mathrm{H}$ & -1.09380 & -2.05785 & 0.07198 \\
\hline $\mathrm{H}$ & -3.23485 & -2.67122 & -0.73758 \\
\hline
\end{tabular}

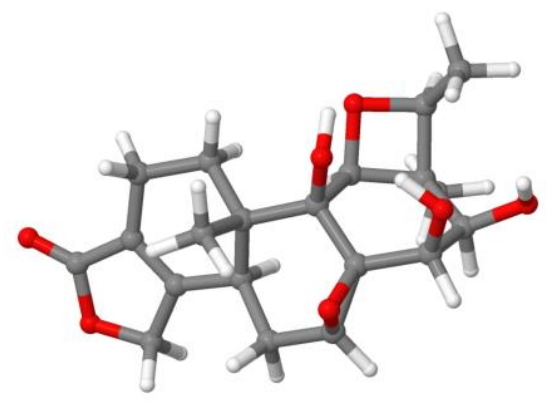




$\begin{array}{rrrr}\text { H } & -3.23621 & -1.47976 & -2.01948 \\ \mathrm{H} & -1.41397 & 2.52022 & 1.54023 \\ \mathrm{H} & -1.75116 & 2.74506 & -0.17225 \\ \mathrm{H} & 0.76924 & 3.20062 & 0.90111 \\ \mathrm{H} & 2.95500 & 2.56388 & 0.08410 \\ \mathrm{H} & 2.99710 & 1.23133 & 1.94280 \\ \mathrm{H} & 4.10054 & -2.95598 & -1.13567 \\ \mathrm{H} & 4.04396 & -1.18939 & -1.30820 \\ \mathrm{H} & 5.11259 & -1.93166 & -0.10067 \\ \mathrm{H} & 1.80600 & -0.24206 & 3.20686 \\ \mathrm{H} & 1.94112 & -1.98330 & 2.89653 \\ \mathrm{H} & 3.40239 & -1.01667 & 3.18023 \\ \mathrm{H} & 4.84447 & 0.54791 & 0.01522 \\ \mathrm{H} & 2.84238 & 0.58469 & -1.84536 \\ \mathrm{H} & 1.54614 & -1.32378 & -1.75839 \\ \mathrm{H} & -2.43261 & 0.69293 & -2.16711 \\ \mathrm{H} & -1.02129 & -0.00059 & -2.97952 \\ \mathrm{H} & -0.92308 & 1.60746 & -2.25915\end{array}$


revised structure of triptergulide A, Fig.5

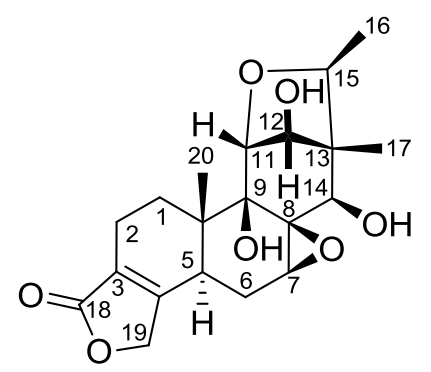

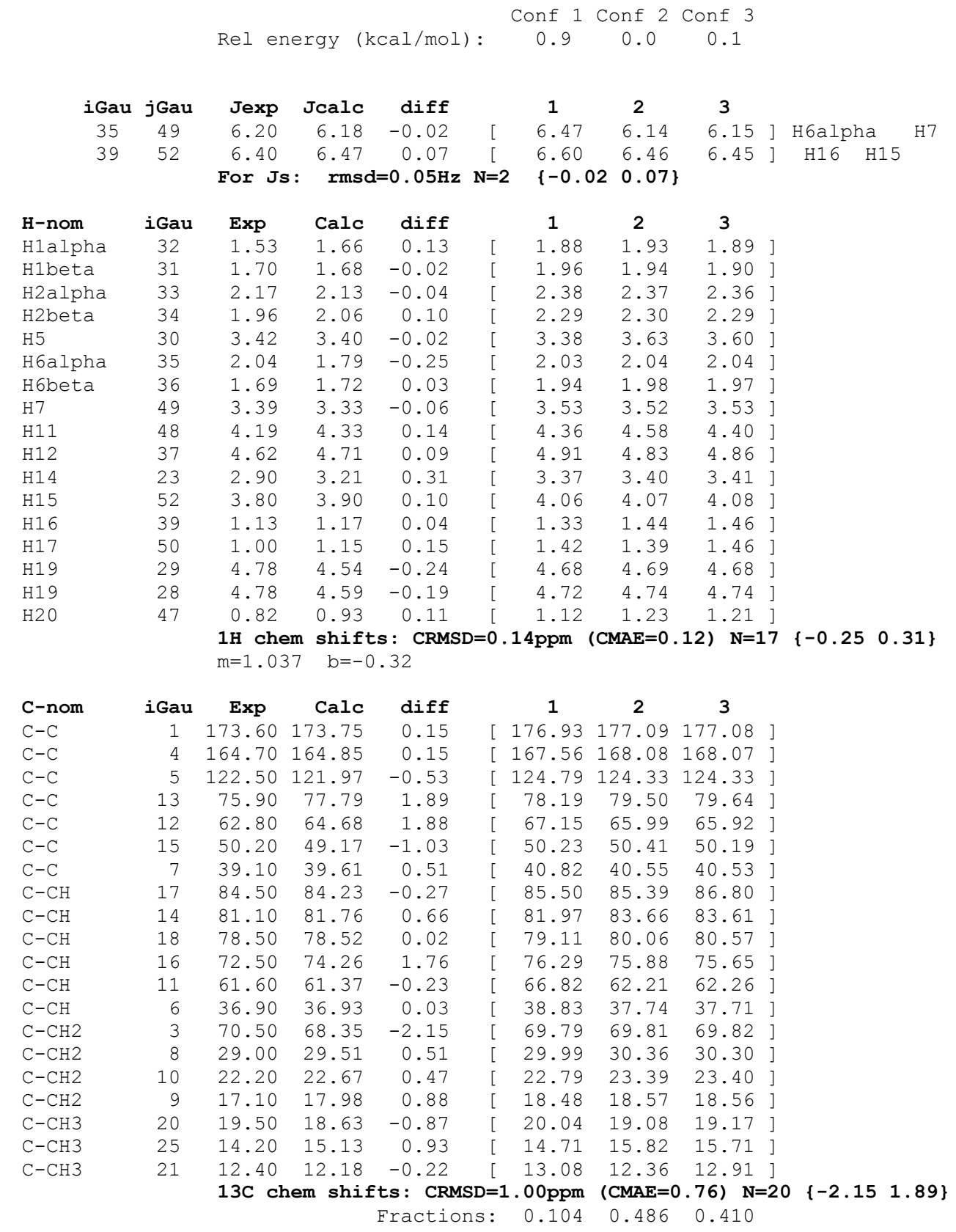




\section{Conformer 1}

Energy: -1304.27895 Hartree (Rel: $0.9 \mathrm{kcal} / \mathrm{mol}$ ) XYZ coordinates for conf 1 :

\begin{tabular}{|c|c|c|c|}
\hline $\mathrm{C}$ & 4.93566 & -0.74784 & -0.02772 \\
\hline 0 & 5.01835 & -0.54737 & -1.38415 \\
\hline $\mathrm{C}$ & 3.73603 & -0.12573 & -1.88417 \\
\hline $\mathrm{C}$ & 2.84262 & -0.08728 & -0.67612 \\
\hline C & 3.55759 & -0.45720 & 0.39932 \\
\hline C & 1.38046 & 0.25496 & -0.65432 \\
\hline $\mathrm{C}$ & 0.90691 & 0.55863 & 0.80289 \\
\hline C & 1.47335 & -0.54671 & 1.73845 \\
\hline $\mathrm{C}$ & 3.01479 & -0.56134 & 1.78963 \\
\hline C & 0.99278 & 1.37397 & -1.63981 \\
\hline C & -0.49026 & 1.67695 & -1.55221 \\
\hline $\mathrm{C}$ & -1.31155 & 1.25914 & -0.39615 \\
\hline C & -0.68180 & 0.58393 & 0.84496 \\
\hline $\mathrm{C}$ & -2.81286 & 1.03252 & -0.63744 \\
\hline C & -3.23846 & -0.43083 & -0.36547 \\
\hline C & -2.86795 & -0.73092 & 1.10638 \\
\hline $\mathrm{C}$ & -1.34513 & -0.81530 & 0.98029 \\
\hline $\mathrm{C}$ & -2.25009 & -1.36390 & -1.13562 \\
\hline 0 & -0.94588 & 2.64742 & -0.57863 \\
\hline $\mathrm{C}$ & -2.78300 & -2.70473 & -1.62732 \\
\hline $\mathrm{C}$ & -4.71837 & -0.62738 & -0.69898 \\
\hline 0 & -3.58804 & 1.90172 & 0.18205 \\
\hline $\mathrm{H}$ & -3.01441 & 1.25765 & -1.69666 \\
\hline 0 & -1.11408 & 1.26772 & 2.02816 \\
\hline $\mathrm{C}$ & 1.45985 & 1.92656 & 1.28249 \\
\hline 0 & 5.89612 & -1.10970 & 0.62255 \\
\hline 0 & -1.15938 & -1.61718 & -0.19798 \\
\hline $\mathrm{H}$ & 3.40316 & -0.84437 & -2.64248 \\
\hline $\mathrm{H}$ & 3.85766 & 0.85083 & -2.36644 \\
\hline $\mathrm{H}$ & 0.84103 & -0.64606 & -0.98043 \\
\hline $\mathrm{H}$ & 1.06865 & -0.41547 & 2.74826 \\
\hline $\mathrm{H}$ & 1.14755 & -1.52980 & 1.38054 \\
\hline $\mathrm{H}$ & 3.36403 & -1.48592 & 2.26545 \\
\hline $\mathrm{H}$ & 3.40254 & 0.25891 & 2.40865 \\
\hline $\mathrm{H}$ & 1.21375 & 1.05628 & -2.66487 \\
\hline $\mathrm{H}$ & 1.56910 & 2.28874 & -1.46232 \\
\hline $\mathrm{H}$ & -3.19972 & 0.05844 & 1.77673 \\
\hline 0 & -3.41231 & -1.94688 & 1.61167 \\
\hline $\mathrm{H}$ & -3.51546 & -2.55781 & -2.42769 \\
\hline $\mathrm{H}$ & -1.95497 & -3.29661 & -2.03103 \\
\hline $\mathrm{H}$ & -3.26089 & -3.28319 & -0.83071 \\
\hline $\mathrm{H}$ & -5.32306 & 0.10519 & -0.15705 \\
\hline $\mathrm{H}$ & -5.05754 & -1.62641 & -0.41598 \\
\hline $\mathrm{H}$ & -0.93334 & 2.21313 & 1.90025 \\
\hline $\mathrm{H}$ & 2.52917 & 2.00826 & 1.06824 \\
\hline $\mathrm{H}$ & 1.33050 & 2.03377 & 2.36362 \\
\hline $\mathrm{H}$ & 0.96474 & 2.76733 & 0.79288 \\
\hline $\mathrm{H}$ & -0.91772 & -1.35621 & 1.82508 \\
\hline $\mathrm{H}$ & -1.00463 & 1.78407 & -2.50694 \\
\hline $\mathrm{H}$ & -4.90289 & -0.48853 & -1.77080 \\
\hline $\mathrm{H}$ & -3.21693 & 2.79185 & 0.05561 \\
\hline $\mathrm{H}$ & -1.82886 & -0.83407 & -1.99979 \\
\hline $\mathrm{H}$ & -2.92331 & -2.68074 & 1.20403 \\
\hline
\end{tabular}

\section{Conformer 2}

Energy: -1304.28040 Hartree (Rel: $0.0 \mathrm{kcal} / \mathrm{mol}$ ) XYZ coordinates for conf 2:

$\begin{array}{llrr}\text { C } & 4.96446 & -0.72343 & 0.06814 \\ \text { O } & 5.04491 & -0.68437 & -1.30292 \\ C & 3.75481 & -0.35071 & -1.84730 \\ C & 2.85999 & -0.18385 & -0.65134 \\ C & 3.58152 & -0.40940 & 0.45949 \\ C & 1.39229 & 0.13241 & -0.66951 \\ C & 0.91970 & 0.61336 & 0.74186 \\ C & 1.49995 & -0.36588 & 1.80331 \\ C & 3.04136 & -0.35158 & 1.85349 \\ C & 0.98600 & 1.09825 & -1.80162 \\ C & -0.49165 & 1.44006 & -1.73712\end{array}$

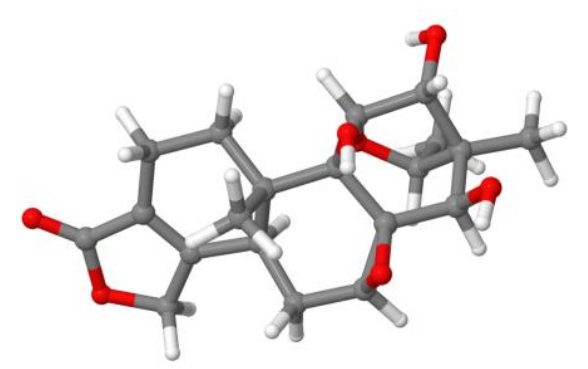




$\begin{array}{lrrr}\text { C } & -1.28240 & 1.21115 & -0.51185 \\ \mathrm{C} & -0.65954 & 0.62368 & 0.77277 \\ \mathrm{C} & -2.79897 & 1.04222 & -0.61569 \\ \mathrm{C} & -3.27810 & -0.39614 & -0.29261 \\ \mathrm{C} & -2.82091 & -0.69838 & 1.15446 \\ \mathrm{C} & -1.31306 & -0.79394 & 0.92922 \\ \mathrm{C} & -2.38334 & -1.38412 & -1.10040 \\ \mathrm{O} & -0.88000 & 2.54443 & -0.89104 \\ \mathrm{C} & -2.96830 & -2.75798 & -1.40791 \\ \mathrm{C} & -4.78308 & -0.52567 & -0.53302 \\ \mathrm{O} & -3.41438 & 1.95330 & 0.31718 \\ \mathrm{H} & -3.11396 & 1.30364 & -1.63489 \\ \mathrm{O} & -1.04282 & 1.41608 & 1.90570 \\ \mathrm{C} & 1.45567 & 2.03521 & 1.05179 \\ \mathrm{O} & 5.93147 & -0.98699 & 0.75535 \\ \mathrm{O} & -1.19023 & -1.55616 & -0.28431 \\ \mathrm{H} & 3.43657 & -1.16226 & -2.51253 \\ \mathrm{H} & 3.85706 & 0.56261 & -2.44434 \\ \mathrm{H} & 0.86711 & -0.81103 & -0.87442 \\ \mathrm{H} & 1.09142 & -0.12272 & 2.79047 \\ \mathrm{H} & 1.19307 & -1.39115 & 1.56827 \\ \mathrm{H} & 3.40510 & -1.20536 & 2.43836 \\ \mathrm{H} & 3.41586 & 0.54488 & 2.36573 \\ \mathrm{H} & 1.18977 & 0.63104 & -2.77176 \\ \mathrm{H} & 1.56872 & 2.02597 & -1.76865 \\ \mathrm{H} & -3.09589 & 0.09445 & 1.85131 \\ \mathrm{O} & -3.27114 & -1.95476 & 1.65501 \\ \mathrm{H} & -3.80690 & -2.67767 & -2.10813 \\ \mathrm{H} & -2.19722 & -3.37800 & -1.87822 \\ \mathrm{H} & -3.30816 & -3.25099 & -0.49478 \\ \mathrm{H} & -5.32422 & 0.23277 & 0.04072 \\ \mathrm{H} & -5.15116 & -1.51243 & -0.23848 \\ \mathrm{H} & -1.92015 & 1.79478 & 1.70620 \\ \mathrm{H} & 2.52009 & 2.10693 & 0.80673 \\ \mathrm{H} & 1.33532 & 2.26181 & 2.11405 \\ \mathrm{H} & 0.92467 & 2.79979 & 0.48577 \\ \mathrm{H} & -0.84257 & -1.35595 & 1.73590 \\ \mathrm{H} & -1.02441 & 1.43312 & -2.68843 \\ \mathrm{H} & -5.02656 & -0.38192 & -1.59228 \\ \mathrm{H} & -3.20025 & 2.84961 & 0.00401 \\ \mathrm{H} & -2.07193 & -0.92096 & -2.04604 \\ \mathrm{H} & -4.20212 & -1.85476 & 1.90867 \\ & & & \end{array}$

\section{Conformer 3}

Energy: -1304.28024 Hartree (Rel: $0.1 \mathrm{kcal} / \mathrm{mol}$ ) XYZ coordinates for conf 3 :

$\begin{array}{llrr}\text { C } & 4.96548 & -0.72293 & 0.06362 \\ \text { O } & 5.04509 & -0.68019 & -1.30735 \\ \text { C } & 3.75464 & -0.34495 & -1.85002 \\ \text { C } & 2.86042 & -0.18189 & -0.65309 \\ \text { C } & 3.58263 & -0.41060 & 0.45663 \\ \text { C } & 1.39274 & 0.13460 & -0.66948 \\ \text { C } & 0.92069 & 0.61109 & 0.74355 \\ \text { C } & 1.50175 & -0.37128 & 1.80164 \\ \text { C } & 3.04320 & -0.35710 & 1.85109 \\ \text { C } & 0.98619 & 1.10427 & -1.79815 \\ \text { C } & -0.49182 & 1.44426 & -1.73250 \\ \text { C } & -1.28178 & 1.21082 & -0.50761 \\ \text { C } & -0.65838 & 0.61998 & 0.77457 \\ \text { C } & -2.79856 & 1.04203 & -0.61044 \\ \text { C } & -3.28245 & -0.39609 & -0.28999 \\ \text { C } & -2.83176 & -0.69832 & 1.15041 \\ \text { C } & -1.31535 & -0.79568 & 0.92735 \\ \text { C } & -2.38625 & -1.38171 & -1.10165 \\ \text { O } & -0.88121 & 2.54590 & -0.88338 \\ \text { C } & -2.97055 & -2.75348 & -1.41905 \\ \text { C } & -4.78716 & -0.52178 & -0.53475 \\ \text { O } & -3.41120 & 1.95530 & 0.32155 \\ \text { H } & -3.11429 & 1.30217 & -1.62962 \\ \text { O } & -1.04308 & 1.40794 & 1.91115 \\ \text { C } & 1.45588 & 2.03213 & 1.05803\end{array}$

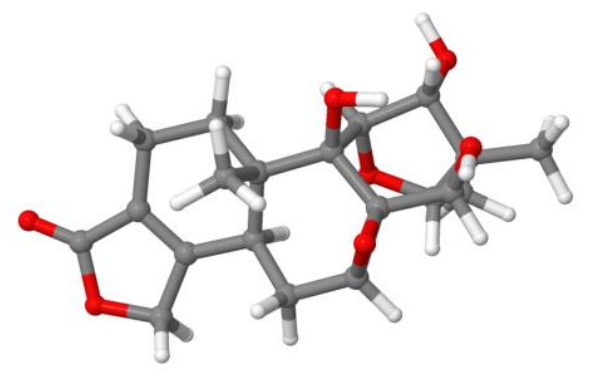




$\begin{array}{rrrr}\text { O } & 5.93268 & -0.98916 & 0.74954 \\ \mathrm{O} & -1.19422 & -1.55714 & -0.28544 \\ \mathrm{H} & 3.43618 & -1.15444 & -2.51764 \\ \mathrm{H} & 3.85658 & 0.57025 & -2.44422 \\ \mathrm{H} & 0.86704 & -0.80803 & -0.87707 \\ \mathrm{H} & 1.09395 & -0.13113 & 2.78987 \\ \mathrm{H} & 1.19487 & -1.39593 & 1.56363 \\ \mathrm{H} & 3.40732 & -1.21263 & 2.43317 \\ \mathrm{H} & 3.41794 & 0.53783 & 2.36585 \\ \mathrm{H} & 1.19059 & 0.64084 & -2.76995 \\ \mathrm{H} & 1.56806 & 2.03240 & -1.76139 \\ \mathrm{H} & -3.10892 & 0.09361 & 1.84783 \\ \mathrm{O} & -3.39082 & -1.93551 & 1.58031 \\ \mathrm{H} & -3.80943 & -2.66751 & -2.11826 \\ \mathrm{H} & -2.19926 & -3.36890 & -1.89510 \\ \mathrm{H} & -3.31011 & -3.25453 & -0.51038 \\ \mathrm{H} & -5.32417 & 0.24458 & 0.03195 \\ \mathrm{H} & -5.15558 & -1.50131 & -0.22366 \\ \mathrm{H} & -1.91989 & 1.78793 & 1.71161 \\ \mathrm{H} & 2.52053 & 2.10514 & 0.81428 \\ \mathrm{H} & 1.33445 & 2.25540 & 2.12088 \\ \mathrm{H} & 0.92500 & 2.79818 & 0.49384 \\ \mathrm{H} & -0.83894 & -1.35762 & 1.73214 \\ \mathrm{H} & -1.02488 & 1.43914 & -2.68365 \\ \mathrm{H} & -5.02376 & -0.38346 & -1.59651 \\ \mathrm{H} & -3.19228 & 2.85052 & 0.00837 \\ \mathrm{H} & -2.07481 & -0.91278 & -2.04450 \\ \mathrm{H} & -3.13157 & -2.06709 & 2.50653\end{array}$


originally assigned structure of myrotheciumone B, Fig.5<smiles>[3H][C@@H]1[C@@H](O)C[C@H]2OC(=O)[C@H]12</smiles>

Rel energy (kcal/mol): $\begin{gathered}\operatorname{Conf} 1 \\ 0.0\end{gathered}$

\begin{tabular}{|c|c|c|c|c|c|}
\hline C-nom & iGau & $\operatorname{Exp}$ & Calc & $\operatorname{diff}$ & 1 \\
\hline $\mathrm{C} 1-\mathrm{C}$ & 2 & 175.50 & 169.71 & -5.79 & [ 169.71 \\
\hline $\mathrm{C} 2-\mathrm{CH}$ & 3 & 58.00 & 61.24 & 3.24 & [ 61.24 \\
\hline $\mathrm{C} 3-\mathrm{CH}$ & 5 & 48.00 & 47.19 & -0.81 & 47.19 \\
\hline $\mathrm{C} 4-\mathrm{C}$ & 6 & 90.30 & 79.72 & -10.58 & 79.72 \\
\hline $\mathrm{C} 5-\mathrm{CH} 2$ & 7 & 43.90 & 43.69 & -0.21 & 43.69 \\
\hline $\mathrm{C} 6-\mathrm{CH}$ & 4 & 68.60 & 75.03 & 6.43 & 75.03 \\
\hline $\mathrm{C} 7-\mathrm{CH} 3$ & 11 & 9.30 & 9.96 & 0.66 & 9.96 \\
\hline $\mathrm{C} 8-\mathrm{CH} 3$ & 13 & 16.20 & 24.77 & 8.57 & 24.77 \\
\hline
\end{tabular}

13C chem shifts: RMSD=5.83ppm (MAE=4.54) $\mathrm{N}=8 \quad\{-10.58 \quad 8.57\}$

\section{Conformer 1}

Energy: -537.72697 Hartree (Rel: $0.0 \mathrm{kcal} / \mathrm{mol}$ ) XYZ coordinates for conf 1 :

$\begin{array}{rrrr}\mathrm{O} & -1.58059 & -1.18693 & 0.45823 \\ \mathrm{C} & -1.83013 & 0.13254 & 0.10563 \\ \mathrm{C} & -0.85242 & 0.12678 & -1.06575 \\ \mathrm{C} & -0.68966 & -1.37140 & -0.71584 \\ \mathrm{C} & 0.52507 & 0.78763 & -0.79076 \\ \mathrm{C} & 1.26193 & -0.20989 & 0.16972 \\ \mathrm{C} & 0.76070 & -1.59318 & -0.31740 \\ \mathrm{H} & -1.12601 & -2.11868 & -1.38063 \\ \mathrm{H} & -1.29438 & 0.41045 & -2.02368 \\ \mathrm{O} & -2.58484 & 0.88916 & 0.63959 \\ \mathrm{C} & 0.45452 & 2.23224 & -0.29570 \\ \mathrm{O} & 0.89460 & 0.00863 & 1.53648 \\ \mathrm{C} & 2.78074 & -0.06534 & 0.12410 \\ \mathrm{H} & 1.06596 & 0.76489 & -1.74797 \\ \mathrm{H} & 1.31976 & -1.90647 & -1.20870 \\ \mathrm{H} & 0.88470 & -2.36940 & 0.44520 \\ \mathrm{H} & 1.45972 & 2.65248 & -0.18263 \\ \mathrm{H} & -0.09370 & 2.86241 & -1.00502 \\ \mathrm{H} & -0.04608 & 2.28905 & 0.67364 \\ \mathrm{H} & 0.04267 & -0.42488 & 1.70532 \\ \mathrm{H} & 3.24927 & -0.82556 & 0.75730 \\ \mathrm{H} & 3.16080 & -0.17349 & -0.89765 \\ \mathrm{H} & 3.07943 & 0.91604 & 0.50647\end{array}$

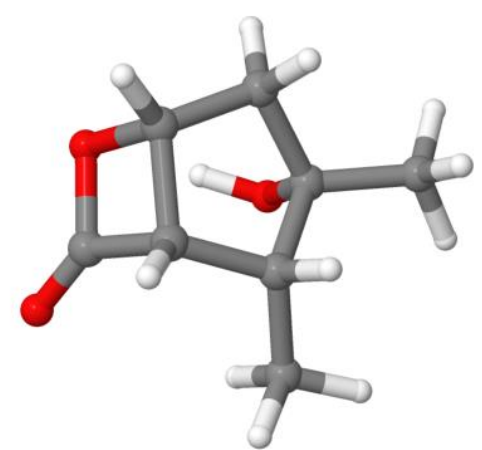


revised myrotheciumone $B$ ( $\gamma$-lactone), Fig.5

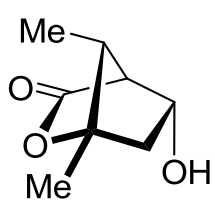

$$
\text { Rel energy (kcal/mol): } \begin{gathered}
\text { Conf } 1 \\
0.0
\end{gathered}
$$

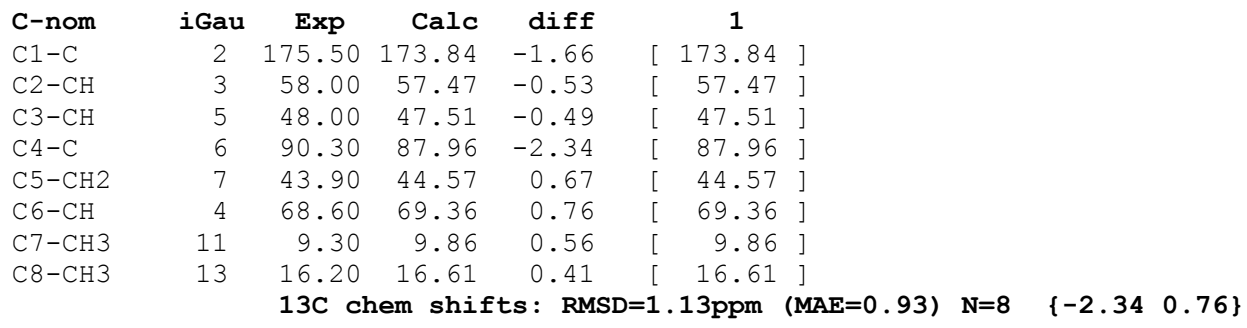

\section{Conformer 1}

Energy: -537.73921 Hartree (Rel: $0.0 \mathrm{kcal} / \mathrm{mol})$ XYZ coordinates for conf 1 :

$\begin{array}{lrrr}\mathrm{O} & 2.59602 & -0.80984 & 0.31498 \\ \mathrm{C} & 0.60091 & 1.06791 & -0.57428 \\ \mathrm{C} & 0.57405 & 0.57325 & 0.86607 \\ \mathrm{C} & 1.27110 & -0.82570 & 0.79644 \\ \mathrm{C} & -0.91032 & 0.17785 & 1.01251 \\ \mathrm{C} & -0.89292 & -0.64062 & -0.30744 \\ \mathrm{C} & 0.24504 & -1.65172 & -0.05205 \\ \mathrm{H} & 1.34407 & -1.23202 & 1.80972 \\ \mathrm{H} & 1.01749 & 1.27021 & 1.57697 \\ \mathrm{O} & 1.33537 & 1.86465 & -1.10559 \\ \mathrm{C} & -1.91239 & 1.32774 & 1.09804 \\ \mathrm{O} & -0.32996 & 0.33848 & -1.25751 \\ \mathrm{C} & -2.18786 & -1.17277 & -0.87634 \\ \mathrm{H} & -1.05236 & -0.49702 & 1.86669 \\ \mathrm{H} & -0.11836 & -2.53110 & 0.49015 \\ \mathrm{H} & 0.69237 & -1.99141 & -0.99093 \\ \mathrm{H} & -2.93958 & 0.94931 & 1.14614 \\ \mathrm{H} & -1.73624 & 1.92215 & 2.00132 \\ \mathrm{H} & -1.84108 & 1.99833 & 0.23512 \\ \mathrm{H} & -2.00784 & -1.69672 & -1.82068 \\ \mathrm{H} & -2.64500 & -1.87825 & -0.17366 \\ \mathrm{H} & -2.89640 & -0.36076 & -1.06624 \\ \mathrm{H} & 2.64593 & -0.23454 & -0.46728\end{array}$

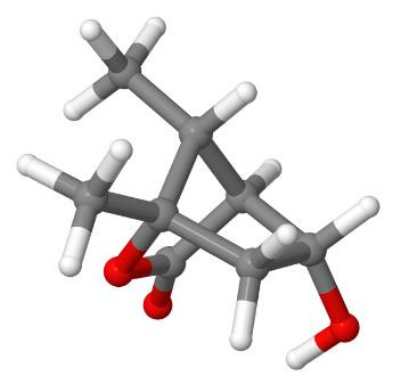


originally assigned aromaticane F, Fig.6

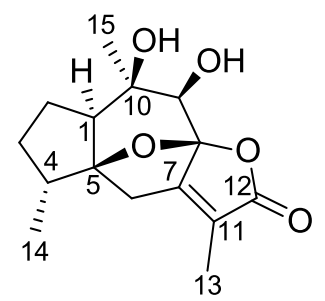

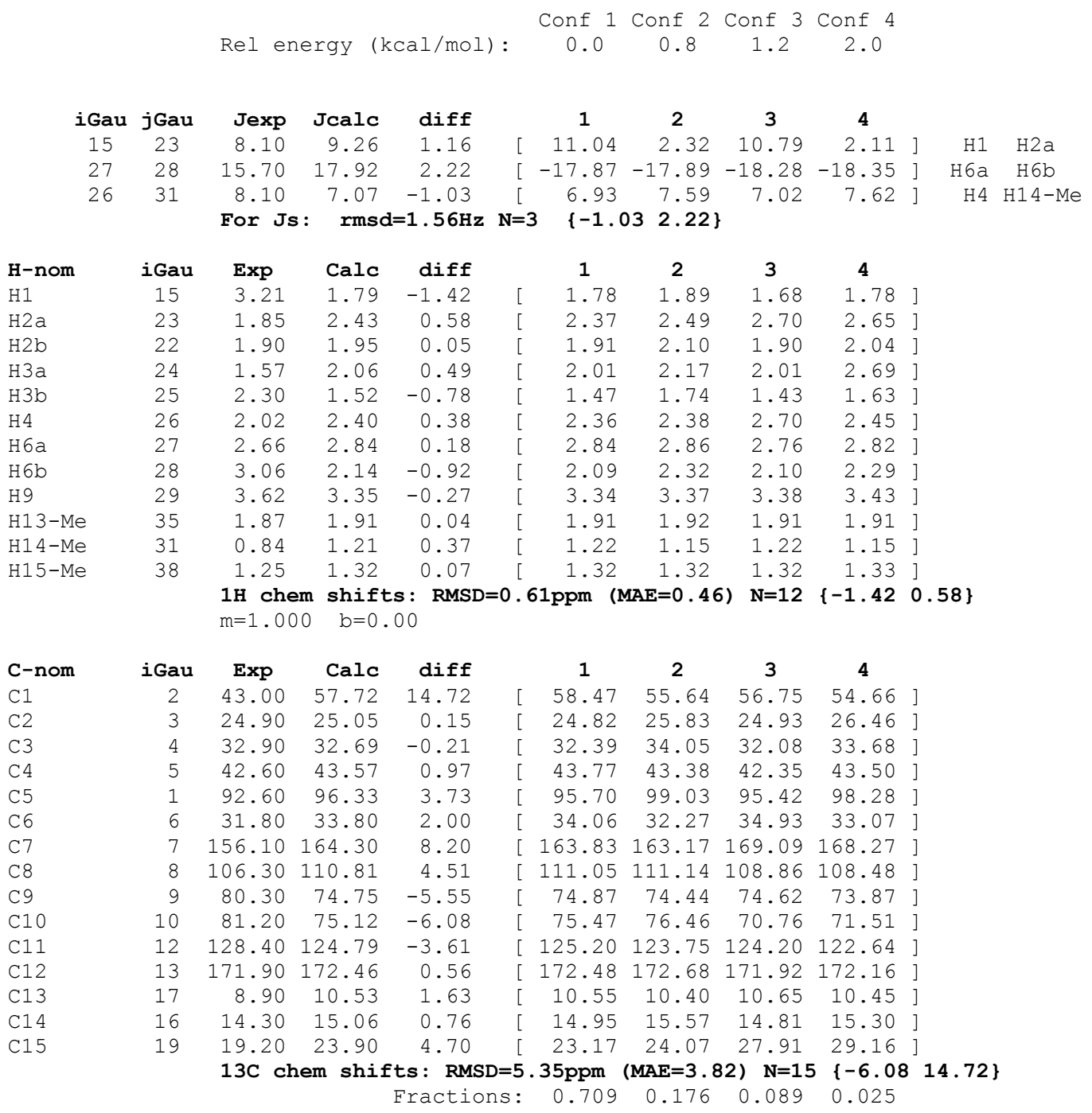




\section{Conformer 1}

Energy: -959.72077 Hartree (Rel: $0.0 \mathrm{kcal} / \mathrm{mol}$ ) XYZ coordinates for conf 1 :

\begin{tabular}{lrrr}
$\mathrm{C}$ & -0.90941 & 0.90960 & 0.00402 \\
$\mathrm{C}$ & -1.82034 & -0.19279 & 0.65674 \\
$\mathrm{C}$ & -3.26626 & 0.16881 & 0.25019 \\
$\mathrm{C}$ & -3.21665 & 1.68838 & 0.03151 \\
$\mathrm{C}$ & -1.87216 & 1.90688 & -0.68756 \\
$\mathrm{C}$ & 0.14273 & 1.47056 & 1.03087 \\
$\mathrm{C}$ & 1.33640 & 0.63397 & 0.68681 \\
$\mathrm{C}$ & 0.88291 & -0.45191 & -0.25310 \\
$\mathrm{C}$ & 0.20456 & -1.71342 & 0.41701 \\
$\mathrm{C}$ & -1.34585 & -1.61393 & 0.26967 \\
$\mathrm{O}$ & -0.08678 & 0.27347 & -1.03282 \\
$\mathrm{C}$ & 2.66425 & 0.76721 & 0.60688 \\
$\mathrm{C}$ & 3.12599 & -0.18493 & -0.45128 \\
$\mathrm{O}$ & 2.01204 & -0.82214 & -0.99345 \\
$\mathrm{H}$ & -1.73608 & -0.11200 & 1.74790 \\
$\mathrm{C}$ & -1.41415 & 3.36211 & -0.78917 \\
$\mathrm{C}$ & 3.61321 & 1.73278 & 1.23841 \\
$\mathrm{O}$ & 4.23650 & -0.36983 & -0.87080 \\
$\mathrm{C}$ & -2.04008 & -2.69356 & 1.09780 \\
$\mathrm{O}$ & 0.66152 & -2.92842 & -0.11713 \\
$\mathrm{O}$ & -1.64752 & -1.92040 & -1.10369 \\
$\mathrm{H}$ & -3.99989 & -0.13459 & 1.00357 \\
$\mathrm{H}$ & -3.52300 & -0.34098 & -0.68489 \\
$\mathrm{H}$ & -4.06384 & 2.07052 & -0.54834 \\
$\mathrm{H}$ & -3.21804 & 2.21681 & 0.99604 \\
$\mathrm{H}$ & -1.98266 & 1.51796 & -1.70968 \\
$\mathrm{H}$ & 0.36457 & 2.52854 & 0.86610 \\
$\mathrm{H}$ & -0.20639 & 1.36625 & 2.06358 \\
$\mathrm{H}$ & 0.48365 & -1.72220 & 1.47742 \\
$\mathrm{H}$ & -0.42867 & 3.43975 & -1.26259 \\
$\mathrm{H}$ & -2.11976 & 3.93677 & -1.39948 \\
$\mathrm{H}$ & -1.36444 & 3.84672 & 0.19334 \\
$\mathrm{H}$ & 4.21419 & 2.24002 & 0.47542 \\
$\mathrm{H}$ & 3.08456 & 2.48297 & 1.83397 \\
$\mathrm{H}$ & 4.31817 & 1.20841 & 1.89543 \\
$\mathrm{H}$ & -1.63661 & -3.67577 & 0.83886 \\
$\mathrm{H}$ & -1.88454 & -2.52648 & 2.16914 \\
$\mathrm{H}$ & -3.11510 & -2.69248 & 0.89379 \\
$\mathrm{H}$ & 0.14968 & -3.05802 & -0.93798 \\
$\mathrm{H}$ & -1.25284 & -1.19209 & -1.62120 \\
& & & \\
\hline & & & \\
\hline
\end{tabular}

\section{Conformer 2}

Energy: -959.71946 Hartree (Rel: $0.8 \mathrm{kcal} / \mathrm{mol}$ ) XYZ coordinates for conf 2 :

$\begin{array}{lrrr}\text { C } & 0.88237 & -0.96165 & 0.00604 \\ \text { C } & 1.82064 & 0.11443 & 0.65761 \\ \text { C } & 3.22658 & -0.12894 & 0.03740 \\ \text { C } & 2.98697 & -1.06763 & -1.16231 \\ \text { C } & 1.80678 & -1.95904 & -0.72153 \\ \text { C } & -0.19816 & -1.55263 & 0.98477 \\ \text { C } & -1.39462 & -0.73482 & 0.61706 \\ \text { C } & -0.91071 & 0.40481 & -0.23418 \\ \text { C } & -0.26621 & 1.60451 & 0.56566 \\ \text { C } & 1.28252 & 1.55308 & 0.40884 \\ \text { O } & 0.09142 & -0.26432 & -1.02181 \\ \text { C } & -2.71555 & -0.87429 & 0.46894 \\ \text { C } & -3.13874 & 0.14033 & -0.54754 \\ \mathrm{O} & -2.00773 & 0.82102 & -0.99626 \\ \text { H } & 1.85320 & -0.03743 & 1.74290 \\ \text { C } & 2.28007 & -3.11713 & 0.17324 \\ \text { C } & -3.68527 & -1.87788 & 1.00215 \\ \mathrm{O} & -4.23113 & 0.34530 & -1.00321 \\ \mathrm{C} & 1.95006 & 2.57042 & 1.33246 \\ \mathrm{O} & -0.75479 & 2.85824 & 0.16591 \\ \mathrm{O} & 1.56602 & 1.99611 & -0.93146 \\ \mathrm{H} & 3.88198 & -0.60934 & 0.77217 \\ \mathrm{H} & 3.70408 & 0.80488 & -0.26726 \\ \mathrm{H} & 2.70271 & -0.48281 & -2.04439\end{array}$
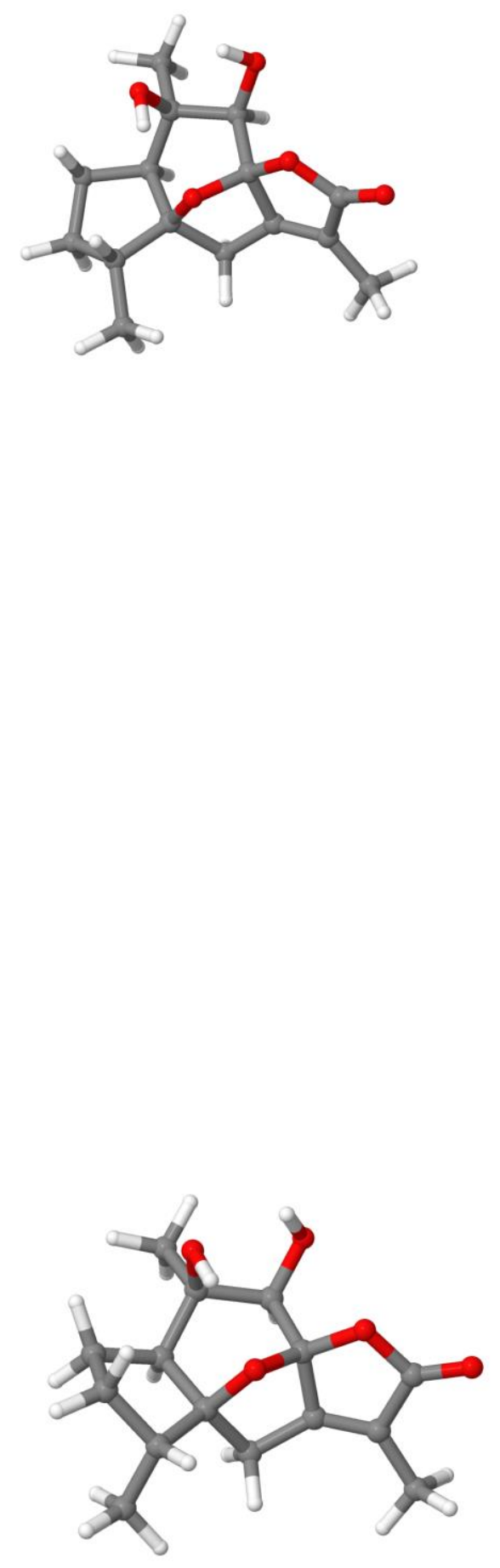


$\begin{array}{rrrr}\text { H } & 3.87513 & -1.64993 & -1.43118 \\ \mathrm{H} & 1.26558 & -2.37356 & -1.58001 \\ \mathrm{H} & -0.38642 & -2.61368 & 0.79429 \\ \mathrm{H} & 0.11195 & -1.45880 & 2.03148 \\ \mathrm{H} & -0.54328 & 1.49515 & 1.62103 \\ \mathrm{H} & 1.45473 & -3.76600 & 0.48411 \\ \mathrm{H} & 2.99455 & -3.73939 & -0.37655 \\ \mathrm{H} & 2.78442 & -2.76109 & 1.07930 \\ \mathrm{H} & -4.25552 & -2.33542 & 0.18612 \\ \mathrm{H} & -3.17739 & -2.66464 & 1.56792 \\ \mathrm{H} & -4.41576 & -1.39675 & 1.66435 \\ \mathrm{H} & 1.54319 & 3.56650 & 1.14148 \\ \mathrm{H} & 1.77157 & 2.31933 & 2.38360 \\ \mathrm{H} & 3.02999 & 2.59475 & 1.15722 \\ \mathrm{H} & -0.26827 & 3.07568 & -0.65161 \\ \mathrm{H} & 1.20280 & 1.30052 & -1.51190\end{array}$

\section{Conformer 3}

Energy: -959.71881 Hartree (Rel: $1.2 \mathrm{kcal} / \mathrm{mol}$ ) XYZ coordinates for conf 3 :

$\begin{array}{lrrr}\mathrm{C} & -0.92568 & 0.90311 & -0.03760 \\ \mathrm{C} & -1.77607 & -0.20921 & 0.68405 \\ \mathrm{C} & -3.25052 & 0.11582 & 0.34782 \\ \mathrm{C} & -3.24205 & 1.62715 & 0.07137 \\ \mathrm{C} & -1.94355 & 1.83753 & -0.72750 \\ \mathrm{C} & 0.11311 & 1.52672 & 0.96742 \\ \mathrm{C} & 1.30703 & 0.66984 & 0.67149 \\ \mathrm{C} & 0.86903 & -0.39844 & -0.30332 \\ \mathrm{C} & 0.24433 & -1.70104 & 0.29197 \\ \mathrm{C} & -1.31755 & -1.63718 & 0.31748 \\ \mathrm{O} & -0.08186 & 0.30860 & -1.07128 \\ \mathrm{C} & 2.63899 & 0.79815 & 0.64261 \\ \mathrm{C} & 3.14484 & -0.14523 & -0.39366 \\ \mathrm{O} & 2.04328 & -0.75609 & -1.01301 \\ \mathrm{H} & -1.63734 & -0.09369 & 1.76635 \\ \mathrm{C} & -1.52173 & 3.29305 & -0.92871 \\ \mathrm{C} & 3.56148 & 1.76267 & 1.31578 \\ \mathrm{O} & 4.26880 & -0.36292 & -0.75567 \\ \mathrm{C} & -1.85808 & -2.67822 & 1.31307 \\ \mathrm{O} & 0.58599 & -2.83822 & -0.49135 \\ \mathrm{O} & -1.80661 & -1.93833 & -0.98133 \\ \mathrm{H} & -3.93449 & -0.16926 & 1.15381 \\ \mathrm{H} & -3.54637 & -0.42805 & -0.55380 \\ \mathrm{H} & -4.12639 & 1.96942 & -0.47730 \\ \mathrm{H} & -3.20409 & 2.19510 & 1.01303 \\ \mathrm{H} & -2.09157 & 1.38250 & -1.71607 \\ \mathrm{H} & 0.34369 & 2.57043 & 0.73313 \\ \mathrm{H} & -0.23621 & 1.49111 & 2.00404 \\ \mathrm{H} & 0.62534 & -1.83241 & 1.31602 \\ \mathrm{H} & -0.56077 & 3.36059 & -1.45187 \\ \mathrm{H} & -2.26549 & 3.82174 & -1.53546 \\ \mathrm{H} & -1.43553 & 3.83271 & 0.02238 \\ \mathrm{H} & 4.17003 & 2.29877 & 0.57870 \\ \mathrm{H} & 3.00729 & 2.48982 & 1.91607 \\ \mathrm{H} & 4.26019 & 1.23394 & 1.97578 \\ \mathrm{H} & -1.49466 & -3.67467 & 1.04094 \\ \mathrm{H} & -1.54293 & -2.46543 & 2.34208 \\ \mathrm{H} & -2.95180 & -2.69316 & 1.27757 \\ \mathrm{H} & 1.42961 & -2.63561 & -0.93355 \\ \mathrm{H} & -1.14685 & -2.54654 & -1.36437 \\ & & & \end{array}$

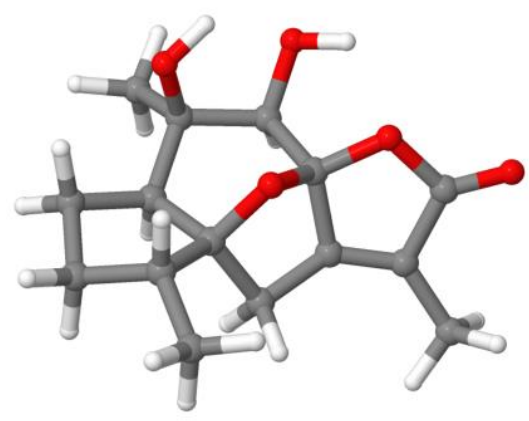




\section{Conformer 4}

Energy: -959.71762 Hartree (Rel: $2.0 \mathrm{kcal} / \mathrm{mol}$ ) XYZ coordinates for conf 4 :

\begin{tabular}{|c|c|c|c|}
\hline C & -0.88784 & 0.96575 & -0.03960 \\
\hline C & -1.78882 & -0.10527 & 0.67689 \\
\hline C & -3.22043 & 0.11316 & 0.10623 \\
\hline C & -3.01642 & 0.97386 & -1.15366 \\
\hline $\mathrm{C}$ & -1.85017 & 1.91310 & -0.78401 \\
\hline C & 0.18240 & 1.60169 & 0.92263 \\
\hline C & 1.37320 & 0.75529 & 0.60138 \\
\hline C & 0.89256 & -0.37072 & -0.27964 \\
\hline C & 0.28634 & -1.60720 & 0.45544 \\
\hline C & -1.27431 & -1.55588 & 0.46790 \\
\hline O & -0.08168 & 0.28927 & -1.05866 \\
\hline C & 2.70039 & 0.88751 & 0.49525 \\
\hline C & 3.15900 & -0.12490 & -0.49752 \\
\hline O & 2.03155 & -0.78620 & -1.01199 \\
\hline $\mathrm{H}$ & -1.78254 & 0.08922 & 1.75557 \\
\hline C & -2.31822 & 3.10751 & 0.06414 \\
\hline C & 3.65179 & 1.89325 & 1.05902 \\
\hline O & 4.26433 & -0.36483 & -0.89987 \\
\hline C & -1.80638 & -2.49305 & 1.56612 \\
\hline O & 0.64839 & -2.82411 & -0.18766 \\
\hline O & -1.74348 & -2.01051 & -0.79453 \\
\hline $\mathrm{H}$ & -3.83757 & 0.64429 & 0.84002 \\
\hline $\mathrm{H}$ & -3.71873 & -0.83021 & -0.12358 \\
\hline $\mathrm{H}$ & -2.71620 & 0.32797 & -1.98421 \\
\hline $\mathrm{H}$ & -3.91891 & 1.52013 & -1.45089 \\
\hline $\mathrm{H}$ & -1.33694 & 2.29404 & -1.67458 \\
\hline $\mathrm{H}$ & 0.38756 & 2.64803 & 0.67425 \\
\hline $\mathrm{H}$ & -0.13299 & 1.56939 & 1.97087 \\
\hline $\mathrm{H}$ & 0.66457 & -1.61828 & 1.48853 \\
\hline $\mathrm{H}$ & -1.49461 & 3.78377 & 0.31794 \\
\hline $\mathrm{H}$ & -3.05723 & 3.69084 & -0.49616 \\
\hline $\mathrm{H}$ & -2.79225 & 2.79358 & 1.00173 \\
\hline $\mathrm{H}$ & 4.23126 & 2.37018 & 0.26055 \\
\hline $\mathrm{H}$ & 3.12401 & 2.66581 & 1.62556 \\
\hline $\mathrm{H}$ & 4.37502 & 1.41092 & 1.72807 \\
\hline $\mathrm{H}$ & -1.44554 & -3.51204 & 1.39146 \\
\hline $\mathrm{H}$ & -1.48247 & -2.17891 & 2.56605 \\
\hline $\mathrm{H}$ & -2.90068 & -2.51016 & 1.54358 \\
\hline $\mathrm{H}$ & 1.46370 & -2.64745 & -0.69035 \\
\hline $\mathrm{H}$ & -1.08082 & -2.66482 & -1.08622 \\
\hline
\end{tabular}

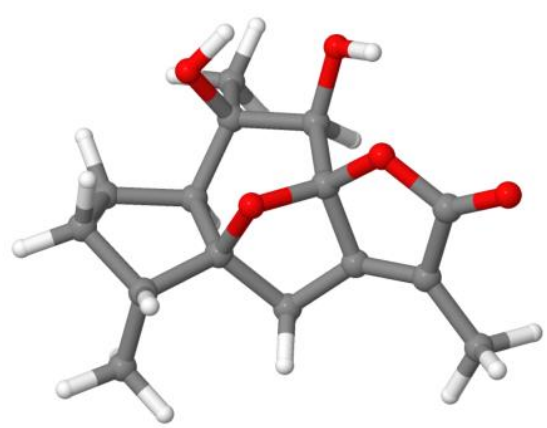


revised structure of aromaticane F, Fig. 6

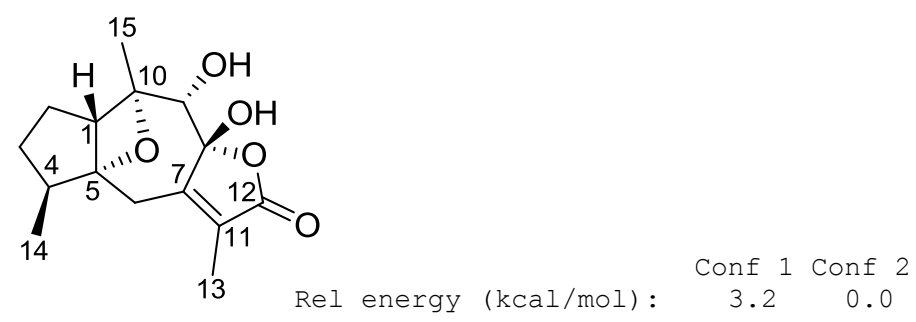

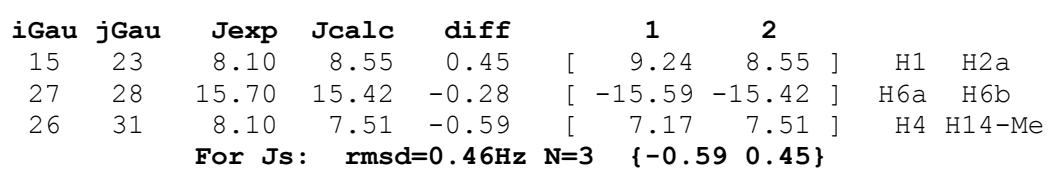

$\begin{array}{lccccccc}\text { H-nom } & \text { iGau } & \text { Exp } & \text { Calc } & \text { diff } & \mathbf{1} & \mathbf{2} & \\ \text { H1 } & 15 & 3.21 & 3.50 & 0.29 & {[} & 3.65 & 3.50] \\ \text { H2a } & 23 & 1.85 & 2.03 & 0.18 & {[} & 1.91 & 2.03] \\ \text { H2b } & 22 & 1.90 & 2.13 & 0.23 & {[} & 2.36 & 2.13] \\ \text { H3a } & 24 & 1.57 & 1.73 & 0.16 & {[} & 1.53 & 1.73] \\ \text { H3b } & 25 & 2.30 & 2.71 & 0.41 & {[} & 2.16 & 2.71] \\ \text { H4 } & 26 & 2.02 & 2.23 & 0.21 & {[} & 2.64 & 2.23] \\ \text { H6a } & 27 & 2.66 & 2.45 & -0.21 & {[} & 2.20 & 2.45] \\ \text { H6b } & 28 & 3.06 & 3.05 & -0.01 & {[} & 3.07 & 3.05] \\ \text { H9 } & 29 & 3.62 & 3.91 & 0.29 & {[} & 3.95 & 3.91] \\ \text { H13-Me } & 35 & 1.87 & 1.88 & 0.01 & {[} & 1.89 & 1.88] \\ \text { H14-Me } & 31 & 0.84 & 1.02 & 0.18 & {[} & 1.18 & 1.02] \\ \text { H15-Me } & 38 & 1.25 & 1.47 & 0.22 & {[} & 1.49 & 1.47]\end{array}$

\begin{tabular}{|c|c|c|c|c|c|c|c|}
\hline C-nom & iGau & $\operatorname{Exp}$ & Calc & diff & 1 & 2 & \\
\hline $\mathrm{C} 1$ & 2 & 43.00 & 45.57 & 2.57 & 48.28 & $45.56]$ & \\
\hline $\mathrm{C} 2$ & 3 & 24.90 & 25.97 & 1.07 & 25.41 & $25.97]$ & \\
\hline $\mathrm{C} 3$ & 4 & 32.90 & 33.47 & 0.57 & 36.76 & $33.45]$ & \\
\hline $\mathrm{C} 4$ & 5 & 42.60 & 43.14 & 0.54 & 46.11 & $43.13]$ & \\
\hline C5 & 1 & 92.60 & 90.65 & -1.95 & 88.46 & $90.66]$ & \\
\hline $\mathrm{C} 6$ & 6 & 31.80 & 33.06 & 1.26 & {$[\quad 32.59$} & $33.06]$ & \\
\hline C7 & 7 & 156.10 & 158.78 & 2.68 & {$[158.64$} & $158.78]$ & \\
\hline $\mathrm{C} 8$ & 8 & 106.30 & 108.05 & 1.75 & [ 107.92 & $108.05]$ & \\
\hline $\mathrm{C} 9$ & 9 & 80.30 & 81.00 & 0.70 & {$[\quad 80.94$} & $81.00]$ & \\
\hline C10 & 10 & 81.20 & 82.42 & 1.22 & {$[\quad 80.72$} & $82.43]$ & \\
\hline C11 & 12 & 128.40 & 128.55 & 0.15 & [ 128.99 & $128.55]$ & \\
\hline C12 & 13 & 171.90 & 171.17 & -0.73 & [ 171.08 & 171.17 ] & \\
\hline C13 & 17 & 8.90 & 9.88 & 0.98 & {$\left[\begin{array}{l}\quad 9.99 \\
\quad\end{array}\right.$} & $9.88]$ & \\
\hline C14 & 16 & 14.30 & 14.46 & 0.16 & 15.18 & $14.46]$ & \\
\hline \multirow[t]{3}{*}{ C15 } & 19 & 19.20 & 18.90 & -0.30 & {$\left[\begin{array}{ll}20.17\end{array}\right.$} & $18.89]$ & \\
\hline & & $13 \mathrm{C} \mathrm{ch}$ & lem shif & ts: RMS & $1.36 \mathrm{ppm}$ & $(\mathrm{MAE}=1.11)$ & 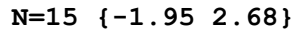 \\
\hline & & & & Eractic & $: \quad 0.005$ & 0.995 & \\
\hline
\end{tabular}

\section{Conformer 1}

Energy: -959.71125 Hartree (Rel: $3.2 \mathrm{kcal} / \mathrm{mol}$ ) XYZ coordinates for conf 1 :

$\begin{array}{lrrr}\text { C } & 1.42874 & 0.54126 & -0.12936 \\ \text { C } & 1.71141 & -0.75723 & 0.67813 \\ \text { C } & 3.24591 & -0.90156 & 0.71701 \\ \text { C } & 3.74645 & 0.55101 & 0.61203 \\ \text { C } & 2.80418 & 1.21471 & -0.41650 \\ \text { C } & 0.30732 & 1.45707 & 0.38472 \\ \text { C } & -1.04429 & 0.80530 & 0.31050 \\ \text { C } & -1.28282 & -0.61865 & 0.79077 \\ \text { C } & -0.57187 & -1.75364 & -0.01941 \\ \text { C } & 0.88537 & -1.45315 & -0.44101 \\ \text { O } & 0.92971 & -0.24175 & -1.25676 \\ \text { C } & -2.20196 & 1.32214 & -0.13322 \\ \text { C } & -3.26241 & 0.29767 & 0.02904\end{array}$

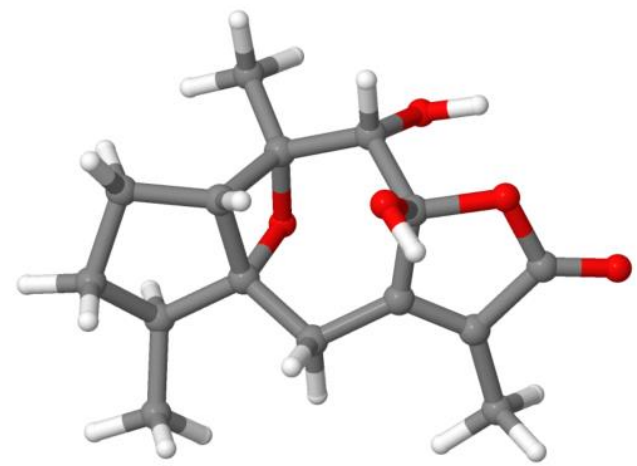




$\begin{array}{rrr}-2.70449 & -0.81720 & 0.62767 \\ 1.27883 & -0.81634 & 1.67720 \\ 2.86876 & 2.74443 & -0.41226 \\ -2.53564 & 2.65835 & -0.71453 \\ -4.42826 & 0.34960 & -0.27056 \\ 1.46288 & -2.64406 & -1.19139 \\ -1.29009 & -2.03227 & -1.19992 \\ -0.95337 & -0.81209 & 2.14167 \\ 3.62029 & -1.47863 & -0.13448 \\ 3.59227 & -1.40604 & 1.62488 \\ 3.64192 & 1.05682 & 1.58340 \\ 4.80144 & 0.62383 & 0.32412 \\ 3.08463 & 0.86106 & -1.41717 \\ 0.52860 & 1.75019 & 1.42236 \\ 0.27755 & 2.38237 & -0.19894 \\ -0.56020 & -2.62136 & 0.66142 \\ 2.22162 & 3.18515 & -1.17927 \\ 2.57987 & 3.16455 & 0.55902 \\ 3.89202 & 3.07808 & -0.61996 \\ -1.64044 & 3.25107 & -0.92038 \\ -3.09664 & 2.53245 & -1.64702 \\ -3.17936 & 3.23149 & -0.03571 \\ 0.80702 & -2.90203 & -2.02616 \\ 2.45312 & -2.41010 & -1.58917 \\ 1.54554 & -3.51613 & -0.53151 \\ -2.21501 & -2.17261 & -0.93925 \\ -1.43345 & -0.14597 & 2.66266\end{array}$

\section{Conformer 2}

Energy: -959.71629 Hartree (Rel: $0.0 \mathrm{kcal} / \mathrm{mol}$ ) XYZ coordinates for conf 2:

\begin{tabular}{lrrr}
$\mathrm{C}$ & 1.37579 & 0.65580 & -0.14497 \\
$\mathrm{C}$ & 1.73443 & -0.56585 & 0.75633 \\
$\mathrm{C}$ & 3.27194 & -0.66390 & 0.71596 \\
$\mathrm{C}$ & 3.71851 & 0.20985 & -0.47876 \\
$\mathrm{C}$ & 2.68663 & 1.36166 & -0.53146 \\
$\mathrm{C}$ & 0.20687 & 1.56043 & 0.26697 \\
$\mathrm{C}$ & -1.11020 & 0.84144 & 0.22439 \\
$\mathrm{C}$ & -1.28275 & -0.54175 & 0.83408 \\
$\mathrm{C}$ & -0.50720 & -1.71140 & 0.14136 \\
$\mathrm{C}$ & 0.93921 & -1.38295 & -0.29709 \\
$\mathrm{O}$ & 0.93681 & -0.24140 & -1.20947 \\
$\mathrm{C}$ & -2.28458 & 1.25383 & -0.27949 \\
$\mathrm{C}$ & -3.29456 & 0.19619 & -0.03059 \\
$\mathrm{O}$ & -2.69103 & -0.82622 & 0.67933 \\
$\mathrm{H}$ & 1.33390 & -0.59691 & 1.76976 \\
$\mathrm{C}$ & 3.05900 & 2.51636 & 0.41237 \\
$\mathrm{C}$ & -2.67465 & 2.51083 & -0.98824 \\
$\mathrm{O}$ & -4.45682 & 0.15935 & -0.34586 \\
$\mathrm{C}$ & 1.57312 & -2.60833 & -0.93913 \\
$\mathrm{O}$ & -1.19568 & -2.13732 & -1.01267 \\
$\mathrm{O}$ & -0.95631 & -0.59054 & 2.19823 \\
$\mathrm{H}$ & 3.63471 & -1.69476 & 0.64588 \\
$\mathrm{H}$ & 3.67318 & -0.25624 & 1.65171 \\
$\mathrm{H}$ & 4.74656 & 0.57367 & -0.37141 \\
$\mathrm{H}$ & 3.66823 & -0.36400 & -1.40913 \\
$\mathrm{H}$ & 2.58615 & 1.75402 & -1.55178 \\
$\mathrm{H}$ & 0.38709 & 1.95054 & 1.27911 \\
$\mathrm{H}$ & 0.15860 & 2.42951 & -0.39959 \\
$\mathrm{H}$ & -0.46204 & -2.50852 & 0.90238 \\
$\mathrm{H}$ & 2.32373 & 3.32790 & 0.37997 \\
$\mathrm{H}$ & 3.14190 & 2.18311 & 1.45433 \\
$\mathrm{H}$ & 4.02792 & 2.93987 & 0.12549 \\
$\mathrm{H}$ & -1.80608 & 3.12542 & -1.23925 \\
$\mathrm{H}$ & -3.21355 & 2.26979 & -1.91111 \\
$\mathrm{H}$ & -3.35648 & 3.11222 & -0.37418 \\
$\mathrm{H}$ & 0.96238 & -2.93404 & -1.78429 \\
$\mathrm{H}$ & 2.58027 & -2.38628 & -1.29954 \\
$\mathrm{H}$ & 1.63417 & -3.43374 & -0.21957 \\
$\mathrm{H}$ & -2.11654 & -2.29555 & -0.74773 \\
$\mathrm{H}$ & -1.46910 & 0.09981 & 2.65215 \\
& & & \\
& & & \\
\hline
\end{tabular}

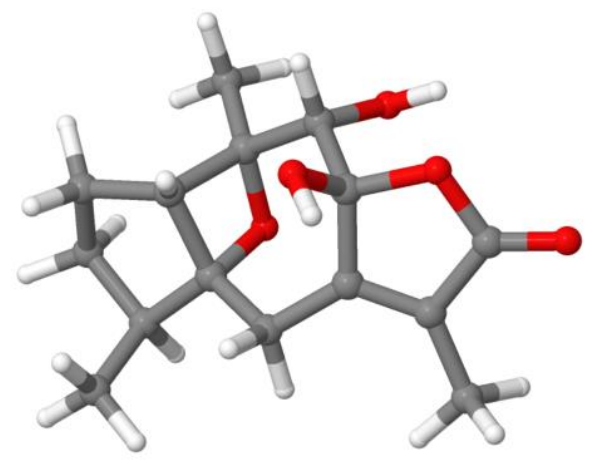


originally assigned structure of moroccolide A (cmpd 2, PCM methanol), Fig.7

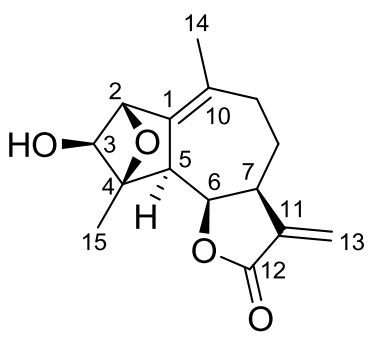

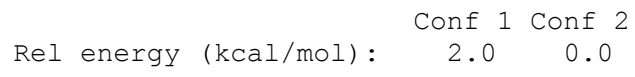

\begin{tabular}{|c|c|c|c|c|c|c|c|}
\hline C-nom & iGau & Exp & Calc & $\operatorname{diff}$ & & 1 & 2 \\
\hline $\mathrm{C} 1-\mathrm{C}$ & 3 & 136.90 & 127.90 & -9.00 & {[} & 133.26 & 131.27 \\
\hline $\mathrm{C} 2-\mathrm{CH}$ & 8 & 68.40 & 85.93 & 17.53 & {[} & 86.62 & 87.19 \\
\hline $\mathrm{C} 3-\mathrm{CH}$ & 9 & 73.20 & 82.88 & 9.68 & {[} & 84.34 & 83.94 \\
\hline $\mathrm{C} 4-\mathrm{C}$ & 10 & 65.40 & 92.64 & 27.24 & {[} & 95.03 & 94.20 \\
\hline $\mathrm{C} 5-\mathrm{CH}$ & 4 & 53.50 & 48.23 & -5.27 & {[} & 48.99 & 47.44 \\
\hline $\mathrm{C} 6-\mathrm{CH}$ & 5 & 83.00 & 79.17 & -3.83 & {[} & 75.48 & 80.21 \\
\hline $\mathrm{C} 7-\mathrm{CH}$ & 6 & 55.30 & 42.38 & -12.92 & {[} & 46.73 & 41.15 \\
\hline $\mathrm{C} 8-\mathrm{CH} 2$ & 7 & 26.20 & 30.45 & 4.25 & {[} & 30.54 & 28.72 \\
\hline $\mathrm{C} 9-\mathrm{CH} 2$ & 1 & 35.80 & 32.71 & -3.09 & {[} & 31.49 & 31.15 \\
\hline C10-C & 2 & 142.30 & 126.91 & -15.39 & {[} & 134.61 & 130.15 \\
\hline $\mathrm{C} 11-\mathrm{C}$ & 18 & 141.20 & 133.45 & -7.75 & {[} & 141.42 & 137.03 \\
\hline C12-C & 17 & 167.00 & 168.52 & 1.52 & {[} & 174.86 & 174.06 \\
\hline $\mathrm{C} 13-\mathrm{CH} 2$ & 21 & 118.20 & 122.94 & 4.74 & {[} & 122.91 & 126.23 \\
\hline $\mathrm{C} 14-\mathrm{CH} 3$ & 13 & 22.30 & 21.88 & -0.42 & {[} & 20.34 & 19.74 \\
\hline $\mathrm{C} 15-\mathrm{CH} 3$ & 11 & 19.80 & 14.43 & -5.37 & {[} & 11.82 & 11.92 \\
\hline
\end{tabular}

$13 \mathrm{C}$ chem shifts: CRMSD $=10.99 \mathrm{ppm}(\mathrm{CMAE}=8.53) \mathrm{N}=15 \quad\left\{\begin{array}{lll}-15.39 & 27.24\end{array}\right.$

Fractions: 0.0330 .967

\section{Conformer 1}

Energy: -883.24176 Hartree (Rel: $2.0 \mathrm{kcal} / \mathrm{mol}$ ) XYZ coordinates for conf 1 :

$\begin{array}{lrrr}\mathrm{C} & 0.62279 & 2.61921 & -0.66832 \\ \mathrm{C} & -0.62291 & 2.26209 & 0.12802 \\ \mathrm{C} & -1.11785 & 1.02195 & 0.06592 \\ \mathrm{C} & -0.63909 & -0.13545 & -0.81411 \\ \mathrm{C} & 0.79968 & -0.62557 & -0.70534 \\ \mathrm{C} & 1.96224 & 0.37055 & -0.94641 \\ \mathrm{C} & 1.85200 & 1.77024 & -0.28843 \\ \mathrm{C} & -2.32096 & 0.38723 & 0.71480 \\ \mathrm{C} & -3.04255 & -0.30819 & -0.47195 \\ \mathrm{C} & -1.73496 & -1.15892 & -0.38773 \\ \mathrm{H} & -1.66545 & -2.59543 & -0.82377 \\ \mathrm{C} & 0.86772 & 3.67319 & -0.49521 \\ \mathrm{H} & -1.21419 & 3.35900 & 0.97657 \\ \mathrm{H} & -1.52997 & 4.20421 & 0.34940 \\ \mathrm{O} & 1.88383 & 1.66613 & 0.80315 \\ \mathrm{C} & 1.09359 & -1.21235 & 0.60218 \\ \mathrm{C} & 2.44058 & -1.22699 & 0.79108 \\ \mathrm{H} & 3.07896 & -0.39619 & -0.27365 \\ \mathrm{O} & -0.76818 & 0.11302 & -1.87905 \\ \mathrm{C} & 2.95822 & -1.83216 & 1.70634 \\ \mathrm{H} & 4.39449 & -0.39182 & -0.49575 \\ \mathrm{H} & -2.08016 & 3.02923 & 1.55677 \\ \mathrm{O} & -0.46396 & 3.75449 & 1.67505 \\ \mathrm{H} & -4.21310 & -1.00549 & -0.12860 \\ \mathrm{H} & -4.00734 & -1.47765 & 0.69908 \\ \mathrm{H} & 0.91620 & -1.44243 & -1.42926 \\ \mathrm{H} & 2.12370 & 0.49216 & -2.02253 \\ \mathrm{H} & 2.76565 & 2.30726 & -0.57142 \\ & -2.86233 & 0.88775 & 1.51703\end{array}$

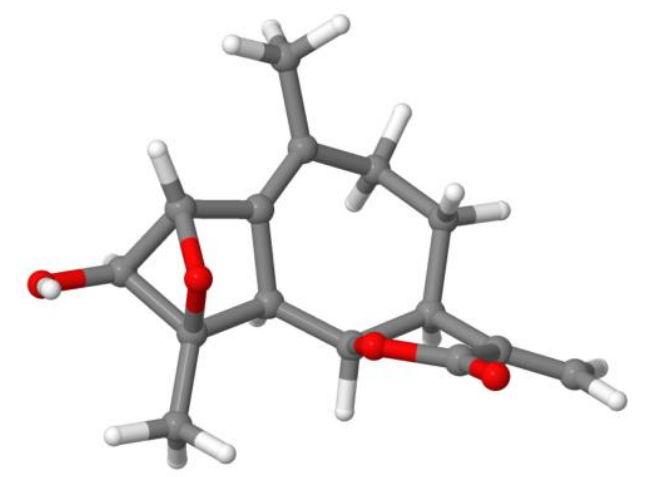




$\begin{array}{rrrr}\mathrm{O} & -1.80517 & -0.95413 & 1.08271 \\ \mathrm{H} & -3.24942 & 0.26470 & -1.38003 \\ \mathrm{H} & -1.55270 & -2.65790 & -1.91203 \\ \mathrm{H} & -2.58258 & -3.12307 & -0.54348 \\ \mathrm{H} & -0.81400 & -3.10152 & -0.35752 \\ \mathrm{H} & 4.84505 & 0.20890 & -1.28060 \\ \mathrm{H} & 5.05625 & -1.00381 & 0.11123 \\ \mathrm{H} & 0.42730 & 2.52815 & -1.74708\end{array}$

\section{Conformer 2}

Energy: -883.24494 Hartree (Rel: $0.0 \mathrm{kcal} / \mathrm{mol}$ ) XYZ coordinates for conf 2:

\begin{tabular}{lrrr}
$\mathrm{C}$ & 1.42923 & 2.11095 & -0.53031 \\
$\mathrm{C}$ & 0.09311 & 2.02960 & 0.18299 \\
$\mathrm{C}$ & -0.78293 & 1.02878 & 0.02748 \\
$\mathrm{C}$ & -0.70418 & -0.24139 & -0.83476 \\
$\mathrm{C}$ & 0.57159 & -1.06817 & -0.72252 \\
$\mathrm{C}$ & 1.85713 & -0.37755 & -1.28049 \\
$\mathrm{C}$ & 1.72091 & 1.11558 & -1.66259 \\
$\mathrm{C}$ & -2.10689 & 0.78804 & 0.71437 \\
$\mathrm{C}$ & -3.03517 & 0.27906 & -0.42022 \\
$\mathrm{C}$ & -2.01696 & -0.90134 & -0.32215 \\
$\mathrm{C}$ & -2.36331 & -2.31663 & -0.69083 \\
$\mathrm{H}$ & 2.22567 & 2.04466 & 0.22685 \\
$\mathrm{C}$ & -0.15413 & 3.19472 & 1.11445 \\
$\mathrm{H}$ & -0.22413 & 4.13382 & 0.54853 \\
$\mathrm{H}$ & 2.67143 & 1.40973 & -2.12432 \\
$\mathrm{O}$ & 0.84830 & -1.40469 & 0.66725 \\
$\mathrm{C}$ & 2.15025 & -1.21759 & 0.98686 \\
$\mathrm{C}$ & 2.86383 & -0.63753 & -0.18399 \\
$\mathrm{H}$ & -0.82588 & -0.02032 & -1.90412 \\
$\mathrm{O}$ & 2.58989 & -1.50379 & 2.08211 \\
$\mathrm{C}$ & 4.18143 & -0.42183 & -0.16470 \\
$\mathrm{H}$ & -1.06821 & 3.08986 & 1.70310 \\
$\mathrm{H}$ & 0.68652 & 3.31359 & 1.81132 \\
$\mathrm{O}$ & -4.34229 & -0.04223 & -0.01662 \\
$\mathrm{H}$ & -4.24846 & -0.51867 & 0.82866 \\
$\mathrm{H}$ & 0.42196 & -2.02509 & -1.23346 \\
$\mathrm{H}$ & 2.15513 & -0.90644 & -2.19264 \\
$\mathrm{H}$ & 0.96462 & 1.21328 & -2.45001 \\
$\mathrm{H}$ & -2.46221 & 1.44883 & 1.50370 \\
$\mathrm{O}$ & -1.97823 & -0.62944 & 1.13621 \\
$\mathrm{H}$ & -3.10375 & 0.84884 & -1.35098 \\
$\mathrm{H}$ & -2.30540 & -2.45407 & -1.77648 \\
$\mathrm{H}$ & -3.38134 & -2.55617 & -0.36799 \\
$\mathrm{H}$ & -1.67319 & -3.02222 & -0.21610 \\
$\mathrm{H}$ & 4.71465 & -0.00571 & -1.01469 \\
$\mathrm{H}$ & 4.76073 & -0.66580 & 0.72151 \\
$\mathrm{H}$ & 1.52690 & 3.12482 & -0.94593 \\
& & & \\
& & & \\
\hline
\end{tabular}

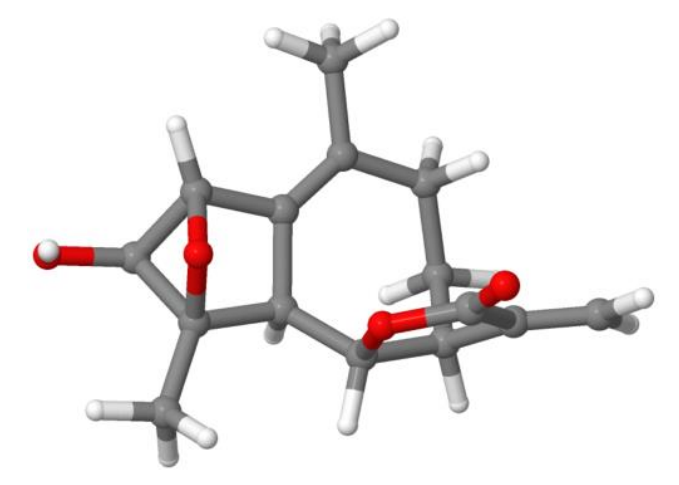


revised structure of moroccolide $A$ (cmpd 2, PCM methanol), Fig.7

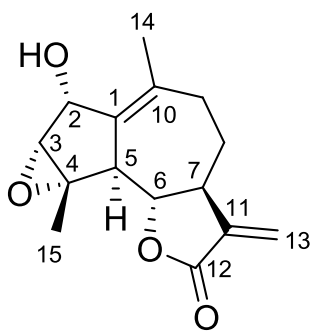

Rel energy (kcal/mol): $\begin{gathered}\text { Conf } 1 \\ 0.0\end{gathered}$

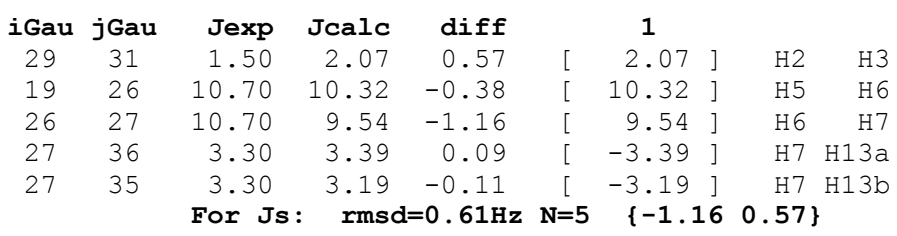

$\begin{array}{lcccccc}\text { H-nom } & \text { iGau } & \text { Exp } & \text { Calc } & \text { diff } & 1 \\ \text { H2 } & 29 & 4.70 & 4.57 & -0.13 & {[} & 4.58] \\ \text { H3 } & 31 & 3.45 & 3.44 & -0.01 & {[} & 3.44] \\ \text { H5 } & 19 & 3.28 & 3.13 & -0.15 & {[} & 3.12] \\ \text { H6 } & 26 & 3.69 & 3.53 & -0.16 & {[} & 3.53] \\ \text { H7 } & 27 & 2.88 & 2.64 & -0.24 & {[} & 2.63] \\ \text { H8a } & 15 & 2.13 & 2.01 & -0.12 & {[} & 1.99] \\ \text { H8b } & 28 & 1.35 & 1.45 & 0.10 & {[} & 1.42] \\ \text { H9a } & 37 & 2.26 & 2.31 & 0.05 & {[} & 2.29] \\ \text { H9b } & 12 & 2.11 & 2.11 & 0.00 & {[} & 2.09] \\ \text { H13a } & 36 & 6.05 & 6.37 & 0.32 & {[} & 6.40] \\ \text { H13b } & 35 & 5.50 & 5.51 & 0.01 & {[} & 5.53] \\ \text { H14-Me } & 23 & 1.89 & 2.06 & 0.17 & {[} & 2.04] \\ \text { H15-Me } & 34 & 1.60 & 1.77 & 0.17 & {[} & 1.74]\end{array}$

H15-Me $\quad 34 \quad 1.60 \quad 1.77 \quad 0.17 \quad\left[\begin{array}{ll}1.74\end{array}\right]$

$1 \mathrm{H}$ chem shifts: CRMSD $=0.15 \mathrm{ppm}$ ( $\mathrm{CMAE}=0.12) \quad \mathrm{N}=13 \quad\{-0.240 .32\}$ $\mathrm{m}=0.987 \mathrm{~b}=0.05$

C-nom igau Exp Calc diff 1

$\left.\begin{array}{cccccc}\mathrm{C} 1-\mathrm{C} & 3 & 136.90 & 140.01 & 3.11 & {[140.72}\end{array}\right]$

$\begin{array}{llllll}\mathrm{C} 2-\mathrm{CH}^{*} * & 8 & 73.20 & 74.01 & 0.81 & {\left[\begin{array}{l}73.48\end{array}\right]}\end{array}$

$\mathrm{C} 3-\mathrm{CH}^{*} * \quad 968.40 \quad 67.34 \quad-1.06 \quad\left[\begin{array}{lll}66.69\end{array}\right]$

$\begin{array}{llllll}\mathrm{C} 4-\mathrm{C} & 10 & 65.40 & 65.58 & 0.18 & {\left[\begin{array}{l}64.90\end{array}\right]}\end{array}$

$\left.\begin{array}{lllll}\mathrm{C} 5-\mathrm{CH} & 4 & 53.50 & 54.53 & 1.03\end{array}\right]\left[\begin{array}{l}53.64\end{array}\right]$

$\begin{array}{llllll}\mathrm{C} 6-\mathrm{CH} & 5 & 83.00 & 79.65 & -3.35 & {\left[\begin{array}{l}79.23\end{array}\right]}\end{array}$

$\left.\begin{array}{llllll}\mathrm{C} 7-\mathrm{CH} & 6 & 55.30 & 54.28 & -1.02 & {\left[\begin{array}{ll}53.39\end{array}\right]}\end{array}\right]$

$\begin{array}{lllll}\mathrm{C} 8-\mathrm{CH} 2 & 7 & 26.20 & 27.26 & 1.06\end{array}\left[\begin{array}{ll}25.86\end{array}\right]$

$\begin{array}{lllll}\mathrm{C} 9-\mathrm{CH} 2 & 1 & 35.80 & 35.98 & 0.18\end{array}\left[\begin{array}{l}34.74\end{array}\right]$

$\begin{array}{lllll}\mathrm{C} 10-\mathrm{C} & 2 & 142.30 & 142.35 & 0.05\end{array}\left[\begin{array}{l}143.10\end{array}\right]$

$\begin{array}{lllll}\mathrm{C} 11-\mathrm{C} & 18 & 141.20 & 137.87 & -3.33\end{array}\left[\begin{array}{l}138.54\end{array}\right]$

$\begin{array}{lllll}\mathrm{C} 12-\mathrm{C} & 17 & 167.00 & 168.23 & 1.23\end{array}\left[\begin{array}{l}169.47\end{array}\right]$

$\begin{array}{lllll}\mathrm{C} 13-\mathrm{CH} 2 & 21 & 118.20 & 118.47 & 0.27\end{array}\left[\begin{array}{l}118.78\end{array}\right]$

$\begin{array}{lllll}\mathrm{C} 14-\mathrm{CH} 3 & 13 & 22.30 & 22.74 & 0.44\end{array}\left[\begin{array}{r}21.25\end{array}\right]$

$\left.\begin{array}{lllll}\mathrm{C} 15-\mathrm{CH} 3 & 11 & 19.80 & 20.60 & 0.80\end{array}\right]\left[\begin{array}{ll}19.08\end{array}\right]$

13C chem shifts: $C R M S D=1.62 \mathrm{ppm}(\mathrm{CMAE}=1.19) \quad \mathrm{N}=15 \quad\{-3.353 .11\}$

\section{Conformer 1}

Energy: -883.24617 Hartree (Rel: $0.0 \mathrm{kcal} / \mathrm{mol}$ )

XYZ coordinates for conf 1 :

$\begin{array}{lrrr}\text { C } & 0.22616 & 2.54630 & 0.82092 \\ \text { C } & 1.38637 & 1.86755 & 0.10408 \\ \text { C } & 1.49774 & 0.53216 & -0.01024 \\ \text { C } & 0.46846 & -0.49886 & 0.48868 \\ C & -0.87584 & -0.23485 & -0.20744 \\ C & -1.68674 & 0.92220 & 0.41727\end{array}$

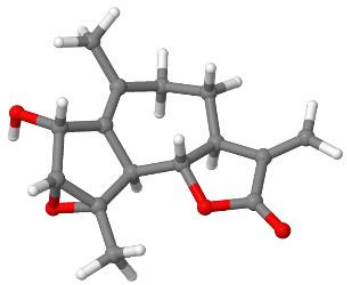




$\begin{array}{lrrr}\text { C } & -1.15623 & 2.33050 & 0.16824 \\ \text { C } & 2.64573 & -0.16881 & -0.75745 \\ \text { C } & 2.39194 & -1.64065 & -0.51902 \\ \text { C } & 1.13725 & -1.85875 & 0.21387 \\ \text { C } & 0.40054 & -3.17452 & 0.21329 \\ \text { H } & 0.42620 & 3.62254 & 0.86730 \\ \text { C } & 2.39133 & 2.82846 & -0.48905 \\ \text { H } & 2.79379 & 3.48502 & 0.29490 \\ \text { H } & -1.86526 & 3.05098 & 0.59671 \\ \text { O } & -1.79124 & -1.36285 & -0.13013 \\ \mathrm{C} & -3.08778 & -0.91955 & -0.20193 \\ \mathrm{C} & -3.07732 & 0.56803 & -0.04271 \\ \mathrm{H} & 0.31105 & -0.42083 & 1.57472 \\ \mathrm{O} & -4.02120 & -1.66620 & -0.35600 \\ \mathrm{C} & -4.14549 & 1.32529 & -0.29242 \\ \mathrm{H} & 3.23495 & 2.32981 & -0.96466 \\ \mathrm{H} & 1.89878 & 3.48764 & -1.21864 \\ \mathrm{O} & 2.41754 & -1.90066 & 0.89994 \\ \mathrm{H} & 4.00073 & -0.08432 & 0.60774 \\ \mathrm{H} & -0.70124 & -0.04223 & -1.27421 \\ \mathrm{H} & -1.66194 & 0.74735 & 1.50711 \\ \mathrm{H} & -1.10608 & 2.53755 & -0.90859 \\ \mathrm{H} & 2.62755 & 0.06566 & -1.82892 \\ \mathrm{O} & 3.95214 & 0.19632 & -0.32302 \\ \mathrm{H} & 2.86255 & -2.39704 & -1.14443 \\ \mathrm{H} & -0.15878 & -3.30819 & 1.14516 \\ \mathrm{H} & 1.12819 & -3.98710 & 0.12712 \\ \mathrm{H} & -0.31482 & -3.23780 & -0.60915 \\ \mathrm{H} & -4.13187 & 2.40611 & -0.18414 \\ \mathrm{H} & -5.07332 & 0.86184 & -0.61593 \\ \mathrm{H} & 0.17488 & 2.20319 & 1.86504 \\ & & & \end{array}$


originally assigned structure of saharanolide A (cmpd 5), Fig.7

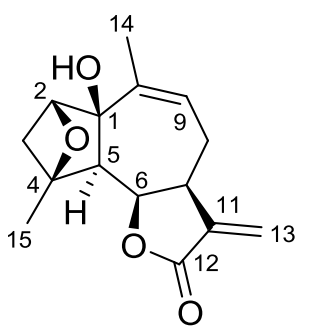

$$
\text { Rel energy (kcal/mol): } \begin{gathered}
\text { Conf } 1 \\
0.0
\end{gathered}
$$

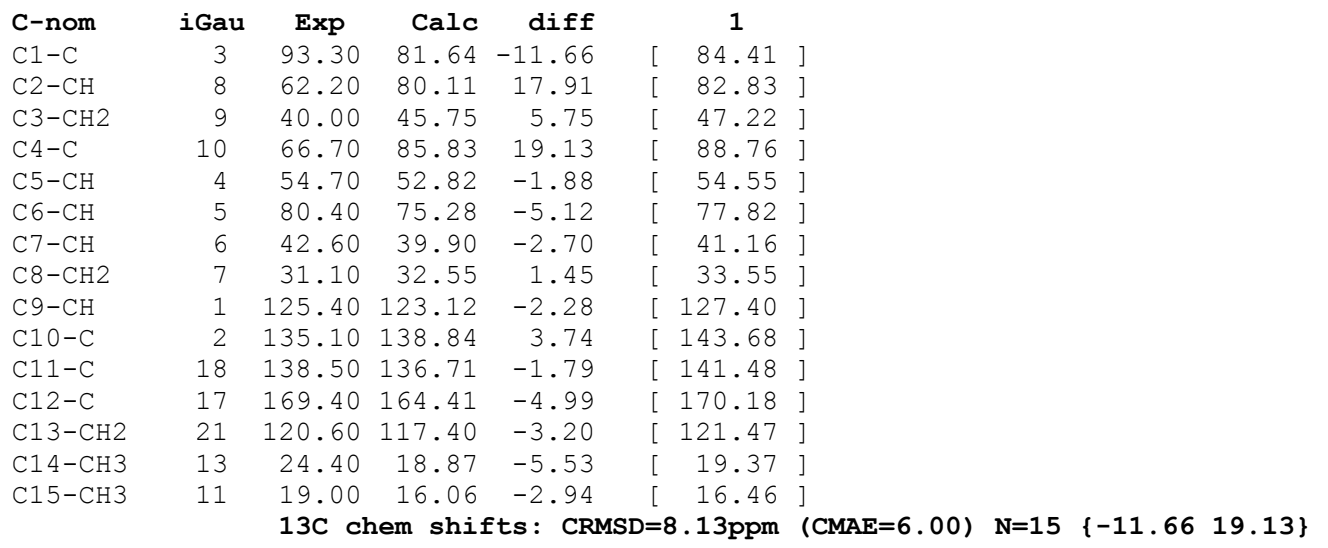

\section{Conformer 1}

Energy: -883.22098 Hartree (Rel: $0.0 \mathrm{kcal} / \mathrm{mol}$ ) XYZ coordinates for conf 1 :

\begin{tabular}{|c|c|c|c|}
\hline C & -0.19503 & 2.60749 & -0.25608 \\
\hline C & -1.34673 & 1.99244 & 0.05250 \\
\hline C & -1.27491 & 0.53775 & 0.48376 \\
\hline C & -0.72738 & -0.35618 & -0.69046 \\
\hline C & 0.77129 & -0.53613 & -0.78412 \\
\hline C & 1.61765 & 0.76747 & -0.91584 \\
\hline C & 1.20025 & 2.02543 & -0.08833 \\
\hline C & -2.54721 & -0.31693 & 0.75726 \\
\hline C & -3.05950 & -0.85941 & -0.58724 \\
\hline C & -1.68287 & -1.57269 & -0.49932 \\
\hline C & -1.36647 & -2.90538 & -1.12159 \\
\hline $\mathrm{H}$ & -0.24103 & 3.64893 & -0.57471 \\
\hline C & -2.68528 & 2.67827 & -0.02169 \\
\hline $\mathrm{H}$ & -3.37768 & 2.16966 & -0.70644 \\
\hline $\mathrm{H}$ & 1.34644 & 1.79902 & 0.97128 \\
\hline 0 & 1.33125 & -1.26531 & 0.33788 \\
\hline C & 2.64326 & -0.91216 & 0.50742 \\
\hline C & 2.93808 & 0.25146 & -0.38945 \\
\hline $\mathrm{H}$ & -0.99063 & 0.12284 & -1.64198 \\
\hline 0 & 3.37978 & -1.48889 & 1.26805 \\
\hline C & 4.18300 & 0.67953 & -0.60267 \\
\hline $\mathrm{H}$ & -3.17328 & 2.69344 & 0.96261 \\
\hline $\mathrm{H}$ & -2.58078 & 3.71405 & -0.35994 \\
\hline O & -0.48108 & 0.48283 & 1.66188 \\
\hline $\mathrm{H}$ & -0.41223 & -0.46725 & 1.87598 \\
\hline $\mathrm{H}$ & -3.25362 & -0.17299 & -1.41787 \\
\hline $\mathrm{H}$ & 0.98270 & -1.14772 & -1.67362 \\
\hline $\mathrm{H}$ & 1.67159 & 1.04500 & -1.97452 \\
\hline $\mathrm{H}$ & 1.93493 & 2.79681 & -0.34731 \\
\hline $\mathrm{H}$ & -3.19039 & -0.01802 & 1.58583 \\
\hline 0 & -1.88063 & -1.61405 & 0.96891 \\
\hline
\end{tabular}

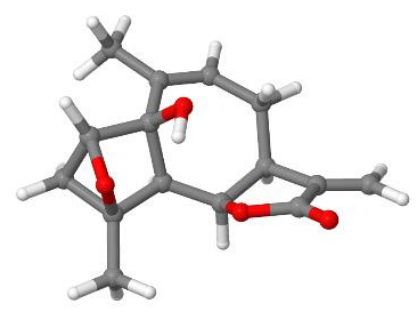




$\begin{array}{rrrr}\mathrm{H} & -3.88913 & -1.55643 & -0.44762 \\ \mathrm{H} & -1.28465 & -2.81186 & -2.21112 \\ \mathrm{H} & -2.15195 & -3.63272 & -0.89441 \\ \mathrm{H} & -0.41608 & -3.28839 & -0.73439 \\ \mathrm{H} & 4.40524 & 1.51527 & -1.26078 \\ \mathrm{H} & 5.01714 & 0.18795 & -0.10951\end{array}$


revised structure of saharanolide A, Fig.7
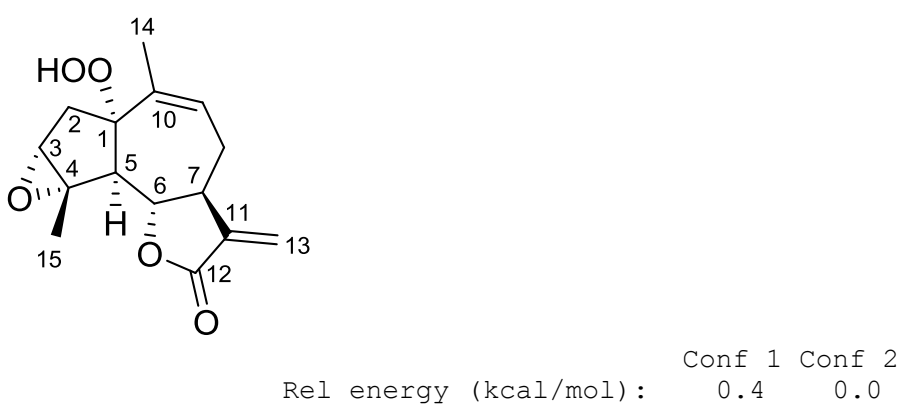

\begin{tabular}{|c|c|c|c|c|c|c|c|c|c|c|}
\hline iGau & jGau & Jexp & Jcalc & $\operatorname{diff}$ & & 1 & 2 & & & \\
\hline 28 & 29 & 15.80 & 15.11 & -0.69 & {[} & -15.08 & -15.12 & ] & $\mathrm{H} 2 \mathrm{a}$ & $\mathrm{H} 2 \mathrm{~b}$ \\
\hline 29 & 30 & 1.70 & 1.56 & -0.14 & {[} & 1.63 & 1.52 & ] & $\mathrm{H} 2 \mathrm{~b}$ & H3 \\
\hline 23 & 24 & 11.55 & 11.81 & 0.26 & {[} & 12.06 & 11.69 & ] & H5 & H6 \\
\hline 24 & 25 & 8.80 & 8.15 & -0.65 & {[} & 7.99 & 8.23 & ] & H6 & $\mathrm{H} 7$ \\
\hline 26 & 27 & 18.20 & 18.27 & 0.07 & {[} & -18.58 & -18.11 & ] & $\mathrm{H} 8 \mathrm{~b}$ & $\mathrm{H} 8 \mathrm{a}$ \\
\hline 22 & 27 & 5.80 & 6.61 & 0.81 & {[} & 6.17 & 6.83 & ] & $\mathrm{H} 9$ & $\mathrm{H} 8 \mathrm{a}$ \\
\hline 25 & 27 & 4.30 & 3.76 & -0.54 & {[} & 4.02 & 3.63 & ] & H7 & $\mathrm{H} 8 \mathrm{a}$ \\
\hline 25 & 38 & 3.50 & 3.62 & 0.12 & [ & -3.62 & -3.62 & ] & $\mathrm{H} 7$ & $\mathrm{H} 13 \mathrm{a}$ \\
\hline \multirow[t]{2}{*}{25} & 37 & 3.20 & 3.23 & 0.03 & [ & -3.22 & -3.23 & ] & $\mathrm{H} 7$ & $\mathrm{H} 13 \mathrm{~b}$ \\
\hline & & For Js & rmsc & $=0.47 \mathrm{~Hz}$ & $\mathbf{N}=\mathbf{y}$ & $\{-0.6$ & 90.81 & & & \\
\hline
\end{tabular}

\begin{tabular}{|c|c|c|c|c|c|c|c|}
\hline $\mathrm{H}-$ nom & iGau & Exp & Calc & $\operatorname{diff}$ & & 1 & 2 \\
\hline $\mathrm{H} 2 \mathrm{a}$ & 28 & 2.38 & 2.30 & -0.08 & {[} & 2.25 & 2.33 \\
\hline $\mathrm{H} 2 \mathrm{~b}$ & 29 & 2.21 & 1.81 & -0.40 & {[} & 1.85 & 1.79 \\
\hline H3 & 30 & 3.32 & 3.17 & -0.15 & {[} & 3.18 & 3.16 \\
\hline H5 & 23 & 2.99 & 2.93 & -0.06 & {[} & 3.20 & 2.80 \\
\hline H6 & 24 & 4.05 & 4.05 & 0.00 & {[} & 4.06 & 4.05 \\
\hline H7 & 25 & 3.25 & 3.20 & -0.05 & {[} & 3.24 & 3.18 \\
\hline $\mathrm{H} 8 \mathrm{a}$ & 27 & 2.72 & 2.52 & -0.20 & {[} & 2.51 & 2.53 \\
\hline $\mathrm{H} 8 \mathrm{~b}$ & 26 & 2.05 & 2.09 & 0.04 & {[} & 2.11 & 2.08 \\
\hline H9 & 22 & 5.69 & 5.59 & -0.10 & {[} & 5.68 & 5.54 \\
\hline $\mathrm{H} 13 \mathrm{a}$ & 38 & 6.27 & 6.50 & 0.23 & {[} & 6.50 & 6.50 \\
\hline $\mathrm{H} 13 \mathrm{~b}$ & 37 & 5.52 & 5.63 & 0.11 & {[} & 5.60 & 5.64 \\
\hline $\mathrm{H} 14-\mathrm{Me}$ & 35 & 1.88 & 1.94 & 0.06 & {[} & 1.87 & 1.98 \\
\hline $\mathrm{H} 15-\mathrm{Me}$ & 33 & 1.70 & 1.68 & -0.02 & L & 1.69 & 1.68 \\
\hline
\end{tabular}

$1 \mathrm{H}$ chem shifts: $\mathrm{RMSD}=0.16 \mathrm{ppm}(\mathrm{MAE}=0.12) \quad \mathrm{N}=13 \quad\{-0.40 \quad 0.23\}$

$\mathrm{m}=1.000 \mathrm{~b}=0.00$

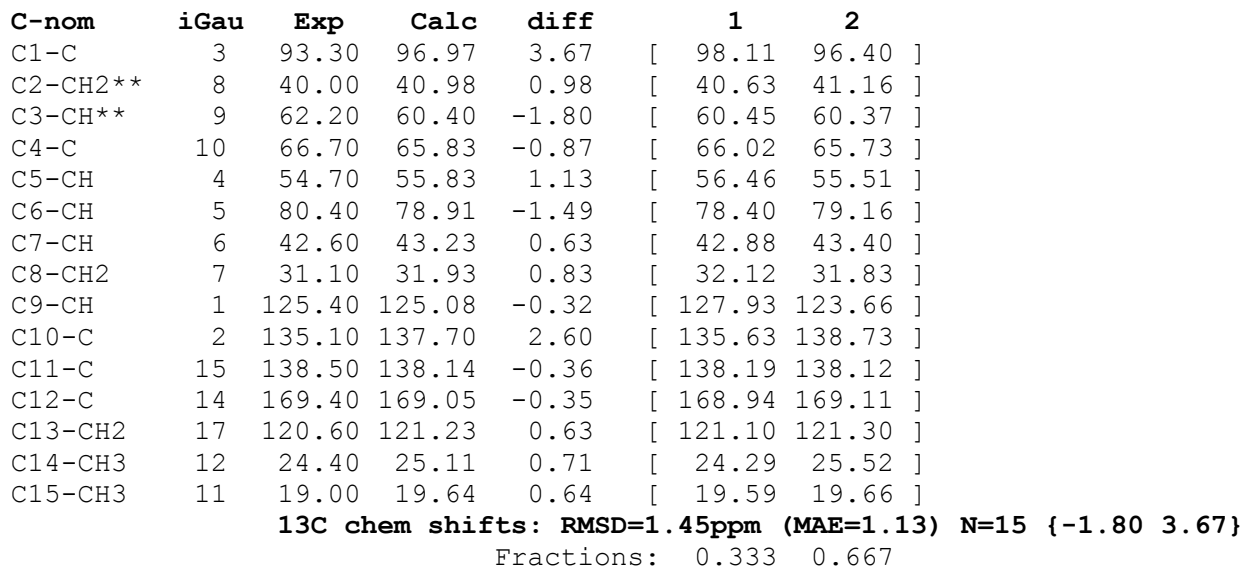




\section{Conformer 1}

Energy: -958.36256 Hartree (Rel: $0.4 \mathrm{kcal} / \mathrm{mol}$ ) XYZ coordinates for conf 1 :

\begin{tabular}{|c|c|c|c|}
\hline $\mathrm{C}$ & -0.40429 & -2.49670 & -0.80059 \\
\hline C & -1.48502 & -1.75026 & -0.50980 \\
\hline C & -1.62068 & -0.31903 & 0.05572 \\
\hline $\mathrm{C}$ & -0.40368 & 0.67975 & 0.24955 \\
\hline $\mathrm{C}$ & 0.89491 & 0.27722 & -0.44507 \\
\hline C & 1.48932 & -1.03772 & 0.10453 \\
\hline C & 1.07549 & -2.25042 & -0.71402 \\
\hline C & -2.67415 & 0.46146 & -0.79699 \\
\hline C & -2.30275 & 1.91497 & -0.70399 \\
\hline C & -0.95407 & 2.06827 & -0.14224 \\
\hline $\mathrm{C}$ & -0.08346 & 3.26824 & -0.42840 \\
\hline C & -2.84614 & -2.41262 & -0.71214 \\
\hline O & 1.90646 & 1.28998 & -0.19869 \\
\hline C & 3.13127 & 0.73581 & 0.04716 \\
\hline $\mathrm{C}$ & 2.96708 & -0.74453 & 0.14116 \\
\hline 0 & 4.13161 & 1.39944 & 0.15930 \\
\hline C & 4.01623 & -1.56266 & 0.23353 \\
\hline O & -2.28294 & -0.45724 & 1.34384 \\
\hline O & -1.37489 & -1.11573 & 2.26285 \\
\hline O & -2.13297 & 2.37090 & 0.64092 \\
\hline $\mathrm{H}$ & -1.55991 & -2.05511 & 2.07027 \\
\hline $\mathrm{H}$ & -0.62667 & -3.48981 & -1.19401 \\
\hline $\mathrm{H}$ & -0.18615 & 0.69657 & 1.32175 \\
\hline $\mathrm{H}$ & 0.76940 & 0.21764 & -1.53518 \\
\hline $\mathrm{H}$ & 1.11941 & -1.16668 & 1.13181 \\
\hline $\mathrm{H}$ & 1.48887 & -2.16561 & -1.73154 \\
\hline $\mathrm{H}$ & 1.54102 & -3.15202 & -0.29136 \\
\hline $\mathrm{H}$ & -3.68480 & 0.25990 & -0.42840 \\
\hline $\mathrm{H}$ & -2.62375 & 0.13462 & -1.84250 \\
\hline $\mathrm{H}$ & -2.73549 & 2.63359 & -1.40019 \\
\hline $\mathrm{H}$ & 0.56152 & 3.50049 & 0.42380 \\
\hline $\mathrm{H}$ & -0.73137 & 4.12937 & -0.61913 \\
\hline $\mathrm{H}$ & 0.56410 & 3.10987 & -1.29499 \\
\hline $\mathrm{H}$ & -3.48545 & -2.27746 & 0.16648 \\
\hline $\mathrm{H}$ & -3.38977 & -1.98932 & -1.56536 \\
\hline $\mathrm{H}$ & -2.73424 & -3.48475 & -0.89528 \\
\hline $\mathrm{H}$ & 3.91438 & -2.64246 & 0.29751 \\
\hline $\mathrm{H}$ & 5.02048 & -1.14844 & 0.25012 \\
\hline
\end{tabular}

\section{Conformer 2}

Energy: -958.36321 Hartree (Rel: $0.0 \mathrm{kcal} / \mathrm{mol}$ ) XYZ coordinates for conf 2 :

$\begin{array}{lrrr}\mathrm{C} & -0.38984 & -2.51330 & -0.79797 \\ \mathrm{C} & -1.46902 & -1.79269 & -0.44934 \\ \mathrm{C} & -1.61535 & -0.33467 & 0.03579 \\ \mathrm{C} & -0.40179 & 0.66625 & 0.24135 \\ \mathrm{C} & 0.91314 & 0.27687 & -0.42893 \\ \mathrm{C} & 1.50188 & -1.05218 & 0.08534 \\ \mathrm{C} & 1.08705 & -2.23849 & -0.76985 \\ \mathrm{C} & -2.60715 & 0.41066 & -0.91956 \\ \mathrm{C} & -2.25253 & 1.86967 & -0.83366 \\ \mathrm{C} & -0.94385 & 2.04682 & -0.19012 \\ \mathrm{C} & -0.06889 & 3.25195 & -0.44099 \\ \mathrm{C} & -2.81900 & -2.49551 & -0.53177 \\ \mathrm{O} & 1.91550 & 1.28498 & -0.12348 \\ \mathrm{C} & 3.14041 & 0.72446 & 0.11059 \\ \mathrm{C} & 2.97955 & -0.75848 & 0.13809 \\ \mathrm{O} & 4.13598 & 1.38728 & 0.26430 \\ \mathrm{C} & 4.02983 & -1.57829 & 0.19664 \\ \mathrm{O} & -2.36498 & -0.37209 & 1.27372 \\ \mathrm{O} & -1.54462 & -1.04760 & 2.27660 \\ \mathrm{O} & -2.17165 & 2.35261 & 0.51294 \\ \mathrm{H} & -1.58926 & -0.37439 & 2.97955 \\ \mathrm{H} & -0.60932 & -3.51915 & -1.15698 \\ \mathrm{H} & -0.19412 & 0.69217 & 1.31594 \\ \mathrm{H} & 0.81413 & 0.25295 & -1.52316 \\ \mathrm{H} & 1.12864 & -1.21427 & 1.10724 \\ \mathrm{H} & 1.46688 & -2.10359 & -1.79523\end{array}$
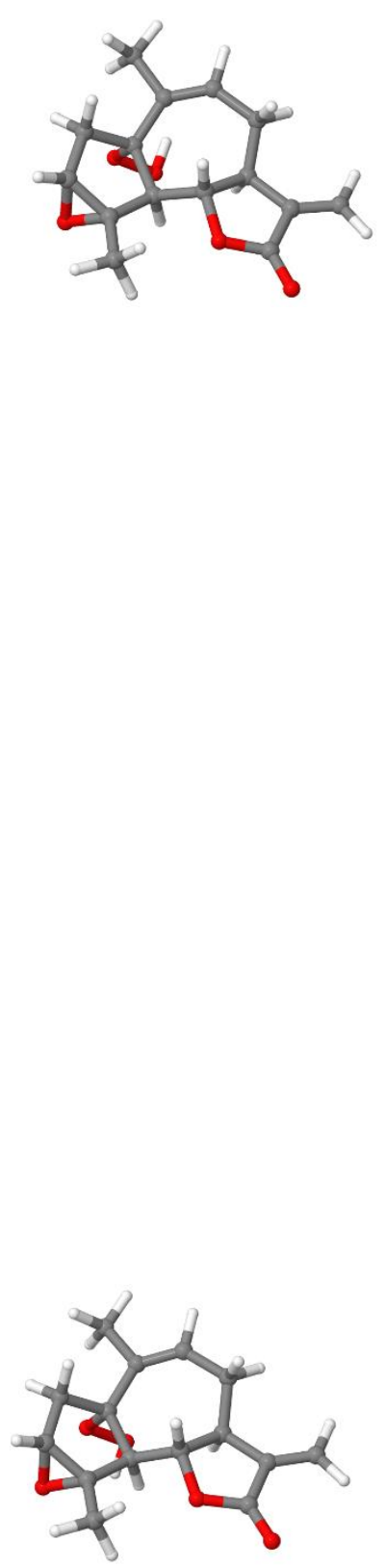


$\begin{array}{rrrr}\text { H } & 1.58448 & -3.14422 & -0.39613 \\ \mathrm{H} & -3.64033 & 0.21160 & -0.62045 \\ \mathrm{H} & -2.47509 & 0.04963 & -1.94670 \\ \mathrm{H} & -2.64632 & 2.57337 & -1.56692 \\ \mathrm{H} & 0.52879 & 3.49774 & 0.44159 \\ \mathrm{H} & -0.71354 & 4.10522 & -0.67327 \\ \mathrm{H} & 0.62498 & 3.09250 & -1.27041 \\ \mathrm{H} & -3.33441 & -2.45043 & 0.43210 \\ \mathrm{H} & -3.48533 & -2.03887 & -1.27367 \\ \mathrm{H} & -2.69079 & -3.54601 & -0.80548 \\ \mathrm{H} & 3.92844 & -2.65984 & 0.21144 \\ \mathrm{H} & 5.03362 & -1.16428 & 0.23412\end{array}$


originally assigned structure of saharanolide B (cmpd 6, PCM methanol), Fig.7

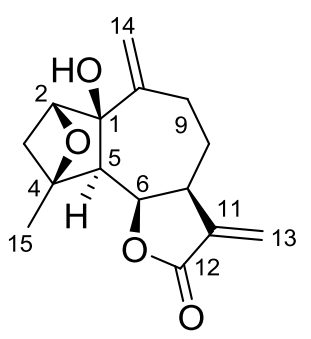

Rel energy (kcal/mol): $\begin{array}{ccc}\text { Conf } 1 & \text { Conf } 2 \\ 2.2 & 0.0\end{array}$

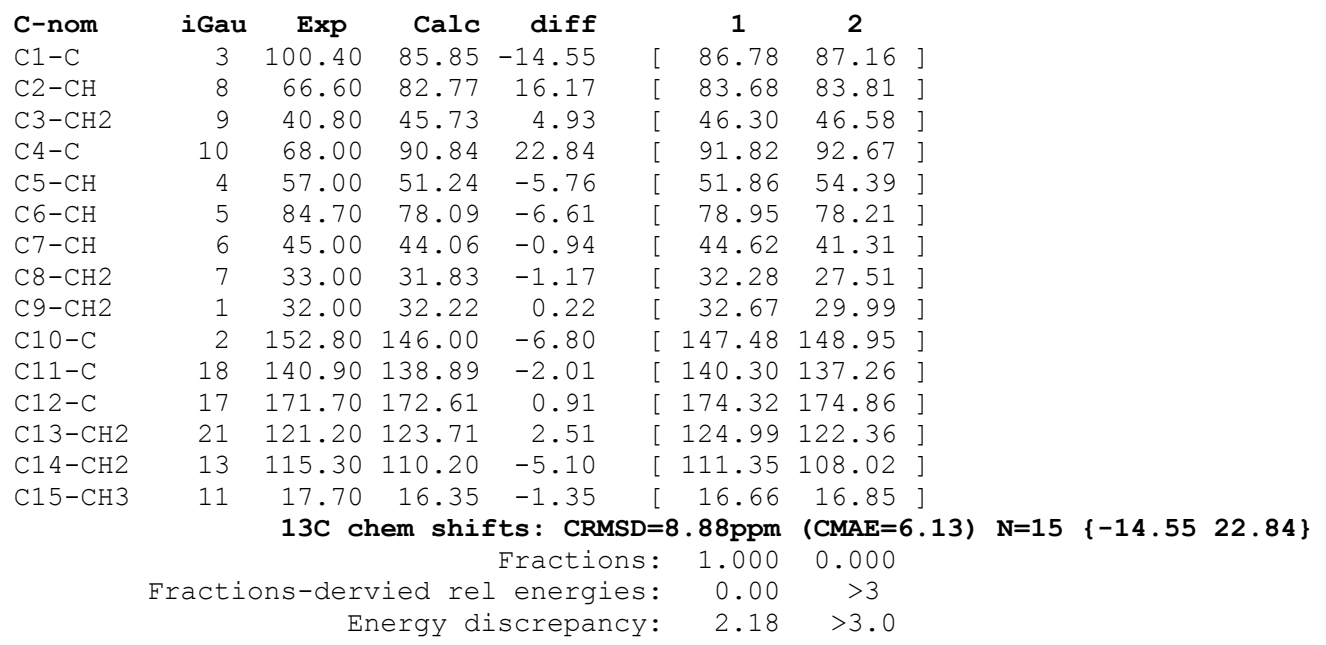

\section{Conformer 1}

Energy: -883.23501 Hartree (Rel: $2.2 \mathrm{kcal} / \mathrm{mol}$ ) XYZ coordinates for conf 1 :

$\begin{array}{lrrr}\mathrm{C} & -0.15400 & 2.58101 & -0.67628 \\ \mathrm{C} & -1.33969 & 2.04134 & 0.11320 \\ \mathrm{C} & -1.25799 & 0.57925 & 0.48548 \\ \mathrm{C} & -0.76516 & -0.30881 & -0.71787 \\ \mathrm{C} & 0.72179 & -0.58455 & -0.82069 \\ \mathrm{C} & 1.65046 & 0.65925 & -0.96083 \\ \mathrm{C} & 1.22942 & 2.00188 & -0.29236 \\ \mathrm{C} & -2.53629 & -0.24489 & 0.79836 \\ \mathrm{C} & -3.11853 & -0.70901 & -0.54227 \\ \mathrm{C} & -1.77326 & -1.48226 & -0.53937 \\ \mathrm{C} & -1.54895 & -2.79932 & -1.23051 \\ \mathrm{H} & -0.12414 & 3.66919 & -0.55064 \\ \mathrm{C} & -2.37664 & 2.80884 & 0.46034 \\ \mathrm{H} & -3.23130 & 2.41670 & 1.00519 \\ \mathrm{H} & 1.29639 & 1.90854 & 0.79339 \\ \mathrm{O} & 1.23144 & -1.34722 & 0.31720 \\ \mathrm{C} & 2.52546 & -1.01462 & 0.56574 \\ \mathrm{C} & 2.90912 & 0.12933 & -0.31187 \\ \mathrm{H} & -1.00863 & 0.18873 & -1.66458 \\ \mathrm{O} & 3.19362 & -1.60576 & 1.38788 \\ \mathrm{C} & 4.17407 & 0.54148 & -0.42136 \\ \mathrm{H} & -2.41124 & 3.86598 & 0.20638 \\ \mathrm{O} & -0.40750 & 0.49342 & 1.63326 \\ \mathrm{H} & -0.34150 & -0.46320 & 1.81671 \\ \mathrm{H} & -3.30862 & 0.02639 & -1.32907 \\ \mathrm{H} & 0.88914 & -1.22492 & -1.69503 \\ \mathrm{H} & 1.79912 & 0.85009 & -2.02934 \\ \mathrm{H} & -3.139556 & 2.72563 & -0.59584 \\ \mathrm{H} & -1.90665 & -1.57227 & 1.66240 \\ \mathrm{O} & & & 0.94072\end{array}$

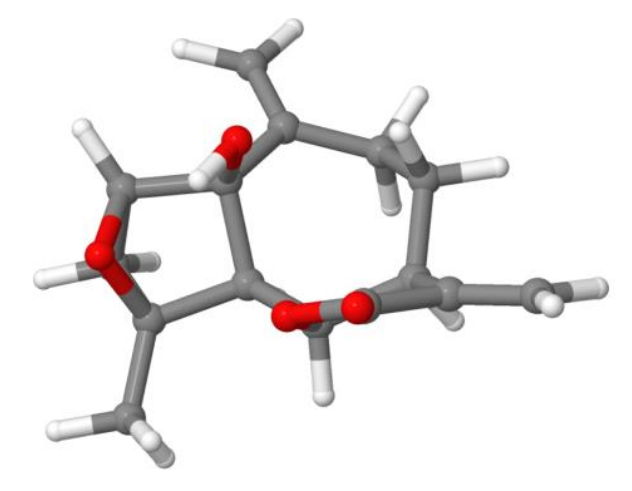




$\begin{array}{rrrr}\mathrm{H} & -3.97221 & -1.37653 & -0.40534 \\ \mathrm{H} & -1.51352 & -2.65636 & -2.31646 \\ \mathrm{H} & -2.35938 & -3.49774 & -1.00009 \\ \mathrm{H} & -0.60142 & -3.24740 & -0.91186 \\ \mathrm{H} & 4.46083 & 1.36390 & -1.07030 \\ \mathrm{H} & 4.96086 & 0.05003 & 0.14446 \\ \mathrm{H} & -0.32354 & 2.41243 & -1.75066\end{array}$

\section{Conformer 2}

Energy: -883.23848 Hartree (Rel: $0.0 \mathrm{kcal} / \mathrm{mol}$ ) XYZ coordinates for conf 2:

\begin{tabular}{|c|c|c|c|}
\hline $\mathrm{C}$ & -0.76797 & 2.47307 & 0.09166 \\
\hline $\mathrm{C}$ & 0.65927 & 2.14851 & -0.29839 \\
\hline C & 0.90669 & 0.68865 & -0.57370 \\
\hline $\mathrm{C}$ & 0.84493 & -0.18085 & 0.74182 \\
\hline $\mathrm{C}$ & -0.49857 & -0.79064 & 1.11326 \\
\hline $\mathrm{C}$ & -1.67014 & 0.24052 & 1.26685 \\
\hline $\mathrm{C}$ & -1.31946 & 1.74942 & 1.34556 \\
\hline C & 2.29067 & 0.17787 & -1.05692 \\
\hline $\mathrm{C}$ & 3.16810 & -0.02521 & 0.18580 \\
\hline C & 2.07206 & -1.09329 & 0.44137 \\
\hline C & 2.29241 & -2.36632 & 1.21140 \\
\hline $\mathrm{H}$ & -1.42619 & 2.24911 & -0.75667 \\
\hline C & 1.61596 & 3.07813 & -0.38051 \\
\hline $\mathrm{H}$ & 2.63351 & 2.84316 & -0.68102 \\
\hline $\mathrm{H}$ & -2.24942 & 2.26045 & 1.62354 \\
\hline 0 & -0.94354 & -1.77880 & 0.13929 \\
\hline C & -2.11618 & -1.42631 & -0.44744 \\
\hline $\mathrm{C}$ & -2.61708 & -0.16548 & 0.16275 \\
\hline $\mathrm{H}$ & 1.10849 & 0.44608 & 1.60144 \\
\hline 0 & -2.61777 & -2.10407 & -1.32023 \\
\hline C & -3.74918 & 0.40722 & -0.25195 \\
\hline $\mathrm{H}$ & 1.41158 & 4.12005 & -0.14577 \\
\hline 0 & -0.06055 & 0.28232 & -1.54586 \\
\hline $\mathrm{H}$ & 0.17697 & -0.64508 & -1.74118 \\
\hline $\mathrm{H}$ & 3.28896 & 0.79290 & 0.90034 \\
\hline $\mathrm{H}$ & -0.37233 & -1.34786 & 2.04583 \\
\hline $\mathrm{H}$ & -2.15864 & 0.01117 & 2.22294 \\
\hline $\mathrm{H}$ & -0.63789 & 1.90456 & 2.19129 \\
\hline $\mathrm{H}$ & 2.65443 & 0.54005 & -2.01913 \\
\hline 0 & 2.00027 & -1.26839 & -1.03771 \\
\hline $\mathrm{H}$ & 4.12819 & -0.47952 & -0.06904 \\
\hline $\mathrm{H}$ & 2.38670 & -2.14983 & 2.28153 \\
\hline $\mathrm{H}$ & 3.20809 & -2.86392 & 0.87737 \\
\hline $\mathrm{H}$ & 1.45083 & -3.05363 & 1.07251 \\
\hline $\mathrm{H}$ & -4.13337 & 1.32418 & 0.18516 \\
\hline $\mathrm{H}$ & -4.32333 & -0.04321 & -1.05678 \\
\hline $\mathrm{H}$ & -0.84306 & 3.55105 & 0.27432 \\
\hline
\end{tabular}

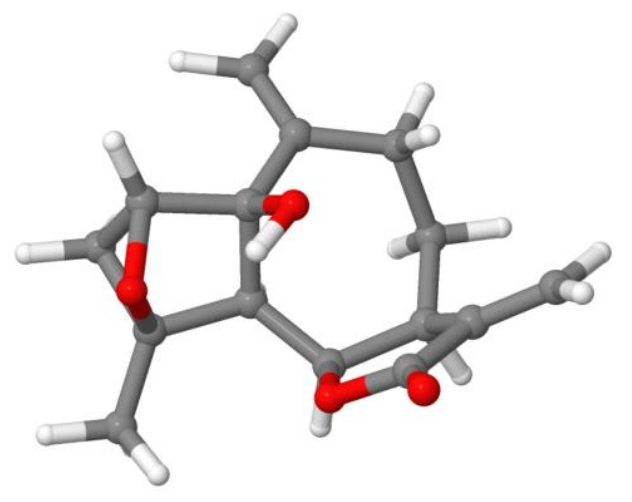


revised structure of saharanolide B (cmpd 6, PCM methanol), Fig.7

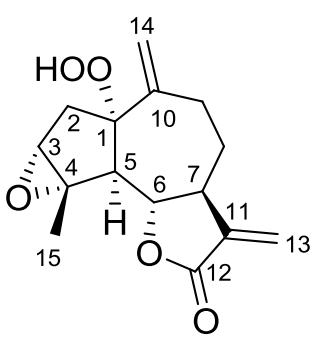

$$
\text { Rel energy (kcal/mol): } 1.1 \quad 0.0
$$

\begin{tabular}{|c|c|c|c|c|c|c|c|}
\hline $\mathrm{C}$-nom & iGau & $\operatorname{Exp}$ & Calc & $\operatorname{diff}$ & 1 & 2 & \\
\hline $\mathrm{C} 1-\mathrm{C}$ & 3 & 100.40 & 103.60 & 3.20 & [ 103.42 & $103.74]$ & \\
\hline $\mathrm{C} 2-\mathrm{CH} 2 * *$ & 8 & 40.80 & 41.36 & 0.56 & {$[\quad 36.78$} & 41.10 ] & \\
\hline $\mathrm{C} 3-\mathrm{CH}^{\star} \star$ & 9 & 66.60 & 65.73 & -0.87 & 63.94 & $65.45]$ & \\
\hline $\mathrm{C} 4-\mathrm{C}$ & 10 & 68.00 & 67.87 & -0.13 & 66.50 & $67.55]$ & \\
\hline $\mathrm{C} 5-\mathrm{CH}$ & 4 & 57.00 & 57.75 & 0.75 & 52.89 & $57.84]$ & \\
\hline $\mathrm{C} 6-\mathrm{CH}$ & 5 & 84.70 & 83.12 & -1.58 & 83.56 & $82.78]$ & \\
\hline $\mathrm{C} 7-\mathrm{CH}$ & 6 & 45.00 & 44.05 & -0.95 & 41.89 & $43.43]$ & \\
\hline $\mathrm{C} 8-\mathrm{CH} 2$ & 7 & 33.00 & 32.99 & -0.01 & 31.91 & $31.98]$ & \\
\hline $\mathrm{C} 9-\mathrm{CH} 2$ & 1 & 32.00 & 32.59 & 0.59 & {$[\quad 30.27$} & $31.79]$ & \\
\hline $\mathrm{C} 10-\mathrm{C}$ & 2 & 152.80 & 151.41 & -1.39 & [ 150.12 & $152.61]$ & \\
\hline $\mathrm{C} 11-\mathrm{C}$ & 15 & 140.90 & 137.28 & -3.62 & [ 138.12 & $137.86]$ & \\
\hline C12-C & 14 & 171.70 & 172.71 & 1.01 & {$[174.04$} & $173.86]$ & \\
\hline $\mathrm{C} 13-\mathrm{CH} 2$ & 17 & 121.20 & 123.89 & 2.69 & [ 124.35 & $124.29]$ & \\
\hline $\mathrm{C} 14-\mathrm{CH} 2$ & 12 & 115.30 & 115.60 & 0.30 & [ 115.87 & $115.88]$ & \\
\hline \multirow[t]{3}{*}{$\mathrm{C} 15-\mathrm{CH} 3$} & 11 & 17.70 & 17.91 & 0.21 & {$[\quad 16.75$} & $16.64]$ & \\
\hline & & $13 \mathrm{C} \mathrm{ch}$ & em shif & s: CRM & $=1 \cdot 61 \mathrm{ppm}$ & $(\mathrm{CMAE}=1.19)$ & $\mathrm{N}=15\{-3.623 .20\}$ \\
\hline & & & & ractic & $: \quad 0.145$ & 0.855 & \\
\hline
\end{tabular}

\section{Conformer 1}

Energy: -958.37863 Hartree (Rel: $1.1 \mathrm{kcal} / \mathrm{mol}$ ) XYZ coordinates for conf 1:

$\begin{array}{rrrr}\mathrm{C} & -0.01341 & -2.66071 & -0.71722 \\ \mathrm{C} & -1.14639 & -1.65965 & -0.89412 \\ \mathrm{C} & -1.48581 & -0.73372 & 0.28927 \\ \mathrm{C} & -0.59500 & 0.57142 & 0.29097 \\ \mathrm{C} & 0.77706 & 0.39741 & -0.35338 \\ \mathrm{C} & 1.60027 & -0.82789 & 0.11988 \\ \mathrm{C} & 1.40510 & -2.05286 & -0.78265 \\ \mathrm{C} & -2.95485 & -0.22835 & 0.27061 \\ \mathrm{C} & -2.89080 & 1.17068 & -0.30739 \\ \mathrm{C} & -1.49711 & 1.65433 & -0.33737 \\ \mathrm{C} & -0.98949 & 2.67934 & -1.32028 \\ \mathrm{C} & -1.78371 & -1.55782 & -2.06665 \\ \mathrm{O} & 1.58687 & 1.56832 & -0.02277 \\ \mathrm{C} & 2.88436 & 1.22417 & 0.18656 \\ \mathrm{C} & 2.99932 & -0.26095 & 0.16686 \\ \mathrm{O} & 3.74829 & 2.05782 & 0.36006 \\ \mathrm{C} & 4.18444 & -0.87411 & 0.19130 \\ \mathrm{O} & -1.21664 & -1.56750 & 1.43901 \\ \mathrm{O} & -1.42325 & -0.80521 & 2.65744 \\ \mathrm{O} & -2.43809 & 2.15457 & 0.64381 \\ \mathrm{H} & -2.22247 & -1.24368 & 3.00485 \\ \mathrm{H} & -0.13106 & -3.18156 & 0.23754 \\ \mathrm{H} & -0.43109 & 0.84511 & 1.33758 \\ \mathrm{H} & 0.69841 & 0.37926 & -1.44561 \\ \mathrm{H} & 1.29216 & -1.08229 & 1.14315 \\ \mathrm{H} & 1.63496 & -1.76634 & -1.81697 \\ \mathrm{H} & -3.33745 & -2.83107 & -0.50249 \\ \mathrm{H} & -3.60437 & -0.89150 & -0.30163 \\ \mathrm{H} & -3.64627 & 1.51914 & -1.00986 \\ \mathrm{H} & -0.20307 & 3.29630 & -0.87500 \\ \mathrm{H} & & & \end{array}$

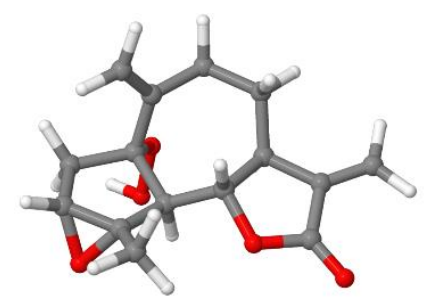




$\begin{array}{rrrr}\mathrm{H} & -1.81412 & 3.32936 & -1.62743 \\ \mathrm{H} & -0.57829 & 2.20226 & -2.21651 \\ \mathrm{H} & -2.58763 & -0.85318 & -2.25349 \\ \mathrm{H} & -1.49973 & -2.18936 & -2.90464 \\ \mathrm{H} & 4.28251 & -1.95534 & 0.17048 \\ \mathrm{H} & 5.09864 & -0.28865 & 0.23369 \\ \mathrm{H} & -0.09719 & -3.41602 & -1.50588\end{array}$

\section{Conformer 2}

Energy: -958.38031 Hartree (Rel: $0.0 \mathrm{kcal} / \mathrm{mol}$ ) XYZ coordinates for conf 2:

$\begin{array}{lrrr}\mathrm{C} & 0.25354 & -2.53617 & 0.74479 \\ \mathrm{C} & 1.26670 & -1.41640 & 0.92299 \\ \mathrm{C} & 1.53223 & -0.50033 & -0.28372 \\ \mathrm{C} & 0.49244 & 0.68669 & -0.37677 \\ \mathrm{C} & -0.85591 & 0.40186 & 0.28070 \\ \mathrm{C} & -1.54978 & -0.92073 & -0.13183 \\ \mathrm{C} & -1.22137 & -2.08487 & 0.80959 \\ \mathrm{C} & 2.93499 & 0.17403 & -0.25795 \\ \mathrm{C} & 2.69340 & 1.60048 & 0.18967 \\ \mathrm{C} & 1.25475 & 1.91823 & 0.16200 \\ \mathrm{C} & 0.61334 & 2.96428 & 1.03888 \\ \mathrm{C} & 1.85092 & -1.21112 & 2.10973 \\ \mathrm{O} & -1.77771 & 1.46588 & -0.10722 \\ \mathrm{C} & -3.03576 & 0.98293 & -0.28809 \\ \mathrm{C} & -3.00011 & -0.50234 & -0.18594 \\ \mathrm{O} & -3.97793 & 1.71615 & -0.50050 \\ \mathrm{C} & -4.11612 & -1.23338 & -0.15198 \\ \mathrm{O} & 1.31959 & -1.21342 & -1.51722 \\ \mathrm{O} & 2.27538 & -2.31367 & -1.57822 \\ \mathrm{O} & 2.14702 & 2.42973 & -0.85628 \\ \mathrm{H} & 2.87281 & -1.98549 & -2.27601 \\ \mathrm{H} & 0.42924 & -3.03921 & -0.20997 \\ \mathrm{H} & 0.29987 & 0.86014 & -1.44243 \\ \mathrm{H} & -0.77444 & 0.44529 & 1.37185 \\ \mathrm{H} & -1.22490 & -1.18054 & -1.14922 \\ \mathrm{H} & -1.47431 & -1.79167 & 1.83667 \\ \mathrm{H} & -1.85553 & -2.94372 & 0.55979 \\ \mathrm{H} & 3.35690 & 0.17548 & -1.26793 \\ \mathrm{H} & 3.63249 & -0.35580 & 0.39303 \\ \mathrm{H} & 3.39034 & 2.10120 & 0.85975 \\ \mathrm{H} & -0.21971 & 3.45355 & 0.52582 \\ \mathrm{H} & 1.35811 & 3.72133 & 1.30126 \\ \mathrm{H} & 0.22939 & 2.52614 & 1.96653 \\ \mathrm{H} & 2.56379 & -0.41376 & 2.29607 \\ \mathrm{H} & 1.61750 & -1.84625 & 2.96072 \\ \mathrm{H} & -4.10111 & -2.31581 & -0.06760 \\ \mathrm{H} & -5.08667 & -0.74845 & -0.20884 \\ \mathrm{H} & 0.41595 & -3.27861 & 1.53362 \\ & & & \end{array}$

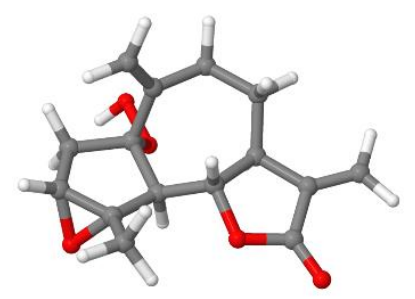


originally assigned structure of 6,11-epoxy-eudesmane, Fig.8

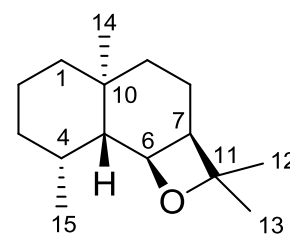

Rel energy (kcal/mol): $\begin{array}{cc}\text { Conf } 1 \text { Conf } 2 \\ 1.0 & 0.0\end{array}$

\begin{tabular}{|c|c|c|c|c|c|c|c|}
\hline C-nom & iGau & $\operatorname{Exp}$ & Calc & diff & 1 & 2 & \\
\hline $\mathrm{C} 1-\mathrm{CH} 2$ & 6 & 41.80 & 40.70 & $-1 \cdot 10$ & 42.52 & $40.39]$ & \\
\hline $\mathrm{C} 2-\mathrm{CH} 2$ & 1 & 22.20 & 17.98 & -4.22 & 18.94 & $17.81]$ & \\
\hline $\mathrm{C} 3-\mathrm{CH} 2$ & 2 & 36.60 & 32.50 & -4.10 & 31.78 & $32.62]$ & \\
\hline $\mathrm{C} 4-\mathrm{CH}$ & 3 & 33.80 & 27.91 & -5.89 & 26.90 & $28.08]$ & \\
\hline $\mathrm{C} 5-\mathrm{CH}$ & 4 & 55.50 & 52.07 & -3.43 & 47.15 & $52.92]$ & \\
\hline $\mathrm{C} 6-\mathrm{CH}$ & 7 & 78.00 & 73.02 & -4.98 & 71.83 & $73.23]$ & \\
\hline $\mathrm{C} 7-\mathrm{CH}$ & 8 & 40.10 & 39.96 & -0.14 & 41.92 & $39.62]$ & \\
\hline $\mathrm{C} 8-\mathrm{CH} 2$ & 9 & 18.90 & 19.25 & 0.35 & 19.31 & $19.24]$ & \\
\hline $\mathrm{C} 9-\mathrm{CH} 2$ & 10 & 39.90 & 42.24 & 2.34 & 38.83 & $42.82]$ & \\
\hline $\mathrm{C} 10-\mathrm{C}$ & 5 & 33.10 & 31.78 & $-1 \cdot 32$ & 31.34 & 31.85 ] & \\
\hline C11-C & 15 & 83.50 & 83.14 & -0.36 & 82.50 & $83.25]$ & \\
\hline $\mathrm{C} 12-\mathrm{CH} 3$ & 17 & 31.90 & 31.11 & -0.79 & 29.92 & $31.31]$ & \\
\hline $\mathrm{C} 13-\mathrm{CH} 3$ & 16 & 25.10 & 24.82 & -0.28 & 25.12 & $24.77]$ & \\
\hline $\mathrm{C} 14-\mathrm{CH} 3$ & 13 & 18.20 & 14.76 & -3.44 & 15.08 & $14.70]$ & \\
\hline $\mathrm{C} 15-\mathrm{CH} 3$ & 11 & 20.70 & 20.98 & 0.28 & {$[25.64$} & $20.18]$ & \\
\hline & & $13 \mathrm{C} \mathrm{ch}$ & m shif & s: RMS & 2. 91ppm & $\triangle A E=2.20)$ & $N=15 \quad\left\{\begin{array}{lll}-5.89 & 2.34\end{array}\right.$ \\
\hline
\end{tabular}

\section{Conformer 1}

Energy: -662.45253 Hartree (Rel: $1.0 \mathrm{kcal} / \mathrm{mol}$ ) XYZ coordinates for conf 1 :

$\begin{array}{lrrr}\mathrm{C} & 3.39184 & 0.04711 & -0.70639 \\ \mathrm{C} & 2.63976 & -1.20926 & -1.17589 \\ \mathrm{C} & 1.41267 & -1.53843 & -0.29660 \\ \mathrm{C} & 0.52535 & -0.26806 & -0.18223 \\ \mathrm{C} & 1.22644 & 1.05110 & 0.28484 \\ \mathrm{C} & 2.45901 & 1.26686 & -0.62978 \\ \mathrm{C} & -0.78507 & -0.46846 & 0.57042 \\ \mathrm{C} & -1.75003 & 0.73480 & 0.46525 \\ \mathrm{C} & -1.18520 & 1.87799 & -0.38252 \\ \mathrm{C} & 0.22220 & 2.25889 & 0.12081 \\ \mathrm{C} & 1.67673 & 1.02876 & 1.76420 \\ \mathrm{H} & 0.22798 & -0.07427 & -1.22292 \\ \mathrm{C} & 1.81185 & -2.24901 & 1.01008 \\ \mathrm{O} & -1.71959 & -1.34435 & -0.11085 \\ \mathrm{C} & -2.75146 & -0.30384 & -0.13736 \\ \mathrm{C} & -3.26582 & -0.09960 & -1.55767 \\ \mathrm{C} & -3.88813 & -0.67465 & 0.81424 \\ \mathrm{H} & 4.21873 & 0.26210 & -1.39570 \\ \mathrm{H} & 3.85586 & -0.13462 & 0.27181 \\ \mathrm{H} & 2.29925 & -1.04378 & -2.20869 \\ \mathrm{H} & 3.32047 & -2.07041 & -1.20860 \\ \mathrm{H} & 0.79377 & -2.25881 & -0.84909 \\ \mathrm{H} & 3.01592 & 2.15214 & -0.29123 \\ \mathrm{H} & 2.10155 & 1.49667 & -1.64502 \\ \mathrm{H} & -0.61389 & -0.82173 & 1.59584 \\ \mathrm{H} & -2.07975 & 1.12582 & 1.43474 \\ \mathrm{H} & -1.84155 & 2.75622 & -0.33752 \\ \mathrm{H} & -1.13582 & 1.59159 & -1.43814 \\ \mathrm{H} & 0.11004 & 2.76112 & 1.09040 \\ \mathrm{H} & 0.65378 & 3.00837 & -0.55501 \\ \mathrm{H} & 0.83674 & 0.83865 & 2.44235 \\ \mathrm{H} & 2.44349 & 0.28009 & 1.97273 \\ \mathrm{H} & 0.95056 & -2.40979 & 2.03331 \\ \mathrm{H} & & -3.23582 & 1.66720 \\ \mathrm{H} & 2.22789 & & \end{array}$

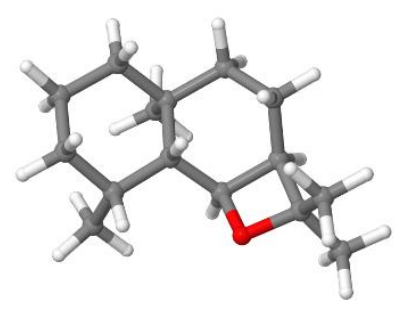




$\begin{array}{rrrr}\mathrm{H} & 2.57187 & -1.71000 & 1.58312 \\ \mathrm{H} & -3.97406 & 0.73643 & -1.60556 \\ \mathrm{H} & -3.78476 & -1.00515 & -1.89305 \\ \mathrm{H} & -2.44914 & 0.09343 & -2.25846 \\ \mathrm{H} & -3.50740 & -0.84790 & 1.82664 \\ \mathrm{H} & -4.63830 & 0.12477 & 0.86011 \\ \mathrm{H} & -4.38233 & -1.59250 & 0.47592\end{array}$

\section{Conformer 2}

Energy: -662.45420 Hartree (Rel: $0.0 \mathrm{kcal} / \mathrm{mol})$ XYZ coordinates for conf 2:

$\begin{array}{lrrr}\text { C } & 3.36631 & 0.20442 & -0.71209 \\ \mathrm{C} & 2.74978 & -1.15281 & -1.08514 \\ \mathrm{C} & 1.56473 & -1.53218 & -0.17101 \\ \mathrm{C} & 0.52780 & -0.37023 & -0.17821 \\ \mathrm{C} & 1.07323 & 1.05952 & 0.13194 \\ \mathrm{C} & 2.31362 & 1.32211 & -0.75899 \\ \mathrm{C} & -0.75316 & -0.69055 & 0.60575 \\ \mathrm{C} & -1.78803 & 0.45054 & 0.77212 \\ \mathrm{C} & -1.34950 & 1.90330 & 0.51801 \\ \mathrm{C} & -0.04215 & 2.05335 & -0.28347 \\ \mathrm{C} & 1.43497 & 1.29092 & 1.61653 \\ \mathrm{H} & 0.20438 & -0.31465 & -1.22792 \\ \mathrm{C} & 2.05807 & -2.03196 & 1.20049 \\ \mathrm{O} & -1.68876 & -1.47295 & -0.18646 \\ \mathrm{C} & -2.65778 & -0.37687 & -0.22750 \\ \mathrm{C} & -2.81341 & 0.15793 & -1.65020 \\ \mathrm{C} & -4.00088 & -0.84274 & 0.32803 \\ \mathrm{H} & 4.18548 & 0.44446 & -1.40217 \\ \mathrm{H} & 3.81974 & 0.15036 & 0.28635 \\ \mathrm{H} & 2.39639 & -1.10327 & -2.12550 \\ \mathrm{H} & 3.51373 & -1.94106 & -1.05393 \\ \mathrm{H} & 1.04480 & -2.38234 & -0.63552 \\ \mathrm{H} & 2.76464 & 2.28484 & -0.47971 \\ \mathrm{H} & 1.97566 & 1.43147 & -1.80053 \\ \mathrm{H} & -0.52274 & -1.21454 & 1.54042 \\ \mathrm{H} & -2.25393 & 0.39649 & 1.76195 \\ \mathrm{H} & -1.21711 & 2.39186 & 1.49112 \\ \mathrm{H} & -2.15443 & 2.45954 & 0.01931 \\ \mathrm{H} & 0.32635 & 3.08298 & -0.17376 \\ \mathrm{H} & -0.24053 & 1.91404 & -1.35450 \\ \mathrm{H} & 0.63355 & 0.98276 & 2.29657 \\ \mathrm{H} & 2.33702 & 0.75052 & 1.91427 \\ \mathrm{H} & 1.62280 & 2.35793 & 1.79356 \\ \mathrm{H} & 1.23253 & -2.29361 & 1.87047 \\ \mathrm{H} & 2.65779 & -2.93967 & 1.06083 \\ \mathrm{H} & 2.68874 & -1.30575 & 1.72177 \\ \mathrm{H} & -3.51528 & 1.00020 & -1.68183 \\ \mathrm{H} & -3.20778 & -0.63506 & -2.29606 \\ \mathrm{H} & -1.86011 & 0.48785 & -2.07114 \\ \mathrm{H} & -3.87582 & -1.27635 & 1.32560 \\ \mathrm{H} & -4.70729 & -0.00584 & 0.39708 \\ \mathrm{H} & -4.44010 & -1.60802 & -0.32266\end{array}$

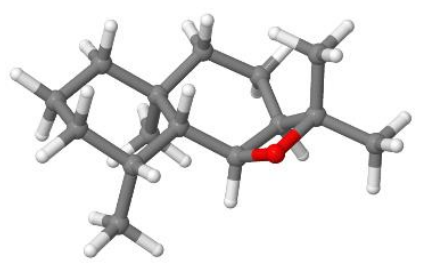


revised structure of 6,11-epoxy-eudesmane, Fig.8

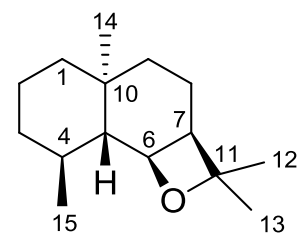

$$
\text { Rel energy (kcal/mol): } \begin{array}{ccc}
\text { Conf } 1 & \text { Conf } 2 \\
1.0 & 0.0
\end{array}
$$

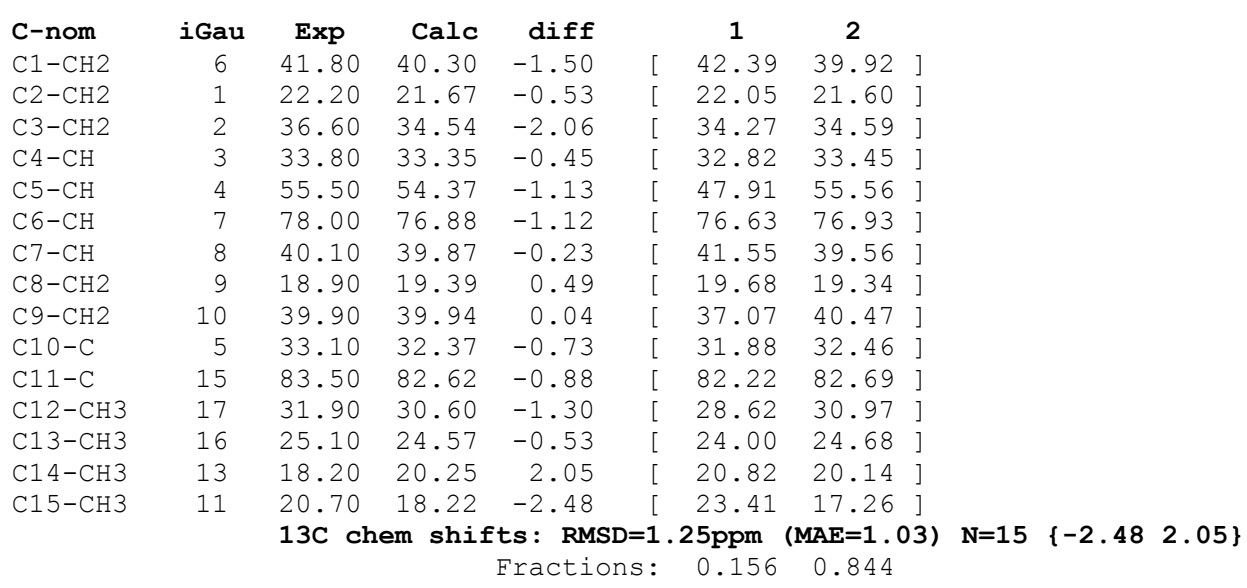

\section{Conformer 1}

Energy: -662.45977 Hartree (Rel: $1.0 \mathrm{kcal} / \mathrm{mol}$ ) XYZ coordinates for conf 1 :

$\begin{array}{lrrr}\mathrm{C} & -3.57790 & -0.01085 & -0.22148 \\ \mathrm{C} & -2.90809 & 1.34637 & -0.46924 \\ \mathrm{C} & -1.55000 & 1.48596 & 0.24586 \\ \mathrm{C} & -0.62293 & 0.29895 & -0.11398 \\ \mathrm{C} & -1.27119 & -1.10479 & 0.14732 \\ \mathrm{C} & -2.63268 & -1.16288 & -0.58723 \\ \mathrm{C} & 0.73915 & 0.35035 & 0.58306 \\ \mathrm{C} & 1.65520 & -0.87139 & 0.33284 \\ \mathrm{C} & 1.09428 & -1.78356 & -0.75739 \\ \mathrm{C} & -0.33742 & -2.24066 & -0.40830 \\ \mathrm{C} & -1.48722 & -1.35783 & 1.65968 \\ \mathrm{H} & -0.42760 & 0.36912 & -1.19454 \\ \mathrm{C} & -0.91062 & 2.84527 & -0.07962 \\ \mathrm{O} & 1.71168 & 1.24678 & -0.01679 \\ \mathrm{C} & 2.73220 & 0.19596 & -0.03560 \\ \mathrm{C} & 3.42087 & 0.13930 & -1.39335 \\ \mathrm{C} & 3.74579 & 0.42906 & 1.08608 \\ \mathrm{H} & -4.50094 & -0.08849 & -0.81065 \\ \mathrm{H} & -3.88073 & -0.08867 & 0.83139 \\ \mathrm{H} & -2.74851 & 1.47638 & -1.55105 \\ \mathrm{H} & -3.57259 & 2.16319 & -0.15684 \\ \mathrm{H} & -1.73461 & 1.45817 & 1.33099 \\ \mathrm{H} & -3.11219 & -2.13186 & -0.38869 \\ \mathrm{H} & -2.44390 & -1.12802 & -1.67074 \\ \mathrm{H} & 0.61203 & 0.59489 & 1.64778 \\ \mathrm{H} & 1.90136 & -1.45677 & 1.22678 \\ \mathrm{H} & 1.73911 & -2.65806 & -0.91012 \\ \mathrm{H} & 1.07365 & -1.24927 & -1.71302 \\ \mathrm{H} & -0.28549 & -3.05045 & 0.33108 \\ \mathrm{H} & -0.78695 & -2.68473 & -1.30554 \\ \mathrm{H} & -0.53518 & -1.43441 & 2.19634 \\ \mathrm{H} & -2.07704 & -0.57573 & 2.14684 \\ \mathrm{H} & -2.01773 & -2.30728 & 1.80427 \\ \mathrm{H} & -0.70457 & 2.93199 & -1.15389 \\ \mathrm{H} & -1.58801 & 3.66225 & 0.19869\end{array}$

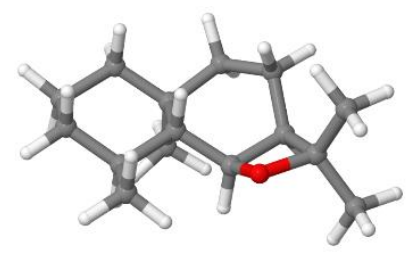




$\begin{array}{rrrr}\mathrm{H} & 0.03892 & 2.99087 & 0.44287 \\ \mathrm{H} & 4.12578 & -0.69943 & -1.44206 \\ \mathrm{H} & 3.98407 & 1.06552 & -1.55703 \\ \mathrm{H} & 2.70069 & 0.03916 & -2.20937 \\ \mathrm{H} & 3.24629 & 0.49575 & 2.05884 \\ \mathrm{H} & 4.47464 & -0.38978 & 1.13115 \\ \mathrm{H} & 4.28944 & 1.36570 & 0.91882\end{array}$

\section{Conformer 2}

Energy: -662.46137 Hartree (Rel: $0.0 \mathrm{kcal} / \mathrm{mol}$ ) XYZ coordinates for conf 2:

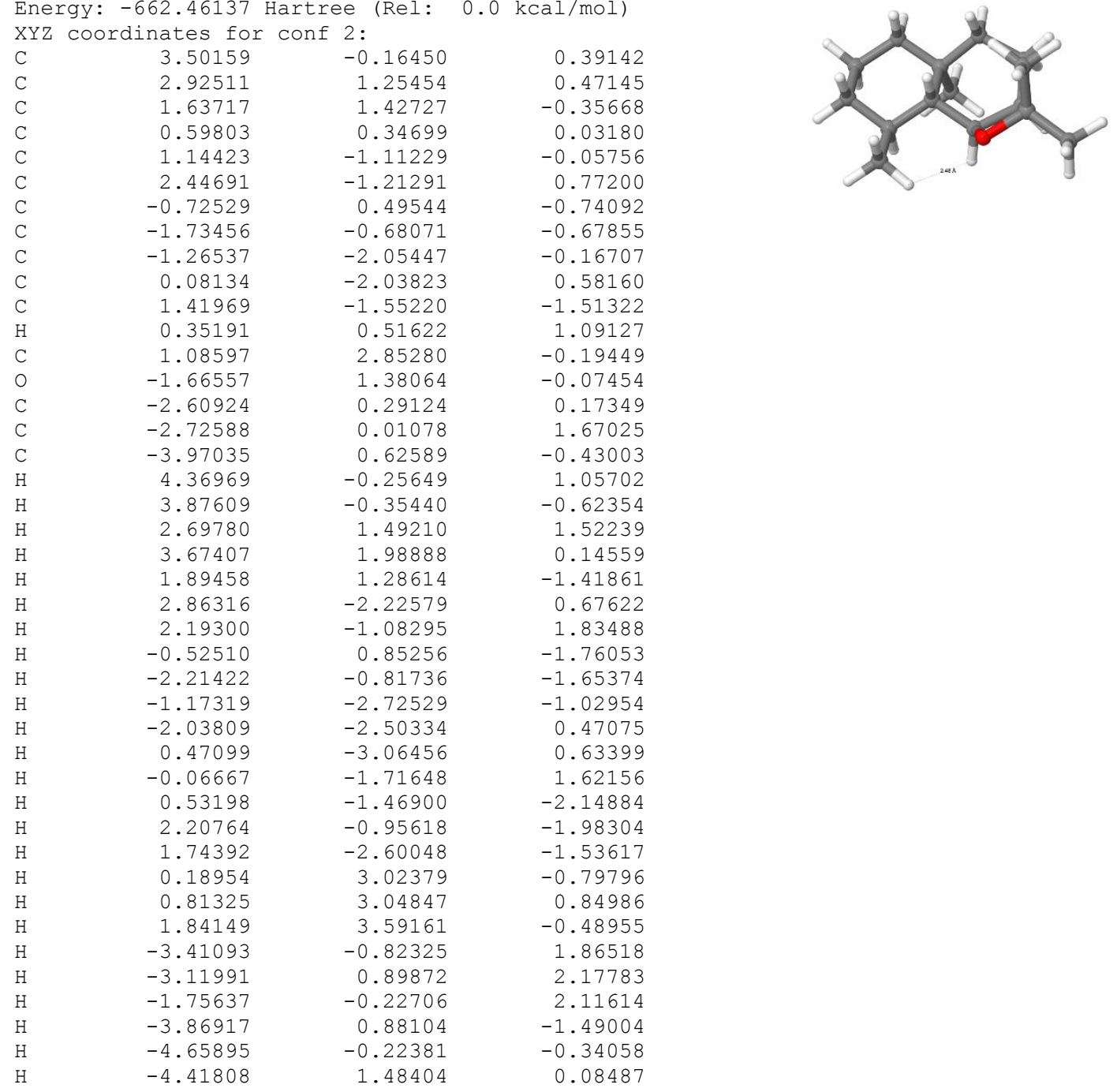


originally assigned structure of okamuragenin (first possible diastereomer), Fig.8

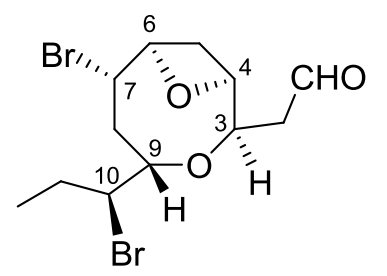

$$
\text { Rel energy (kcal/mol): } \begin{array}{ccc}
\text { Conf } 1 \text { Conf } 2 \\
2.1 & 0.0
\end{array}
$$

\begin{tabular}{|c|c|c|c|c|c|c|c|}
\hline iGau & jGau & Jexp & Jcalc & $\operatorname{diff}$ & & 1 & 2 \\
\hline 23 & 25 & 5.00 & 5.16 & 0.16 & {[} & 5.21 & 5.16 \\
\hline 23 & 24 & 8.70 & 9.17 & 0.47 & {[} & 9.15 & 9.17 \\
\hline 20 & 21 & 5.80 & 5.39 & -0.41 & {[} & 5.03 & 5.40 \\
\hline 21 & 22 & 14.10 & 14.31 & 0.21 & {[} & $-15.10-$ & -14.29 \\
\hline 16 & 22 & 7.40 & 7.44 & 0.04 & {[} & 6.74 & 7.46 \\
\hline \multirow[t]{2}{*}{16} & 29 & 3.50 & 3.94 & 0.44 & {[} & 1.74 & 4.00 \\
\hline & & For Js: & rmsd & $0.33 \mathrm{~Hz}$ & $\mathrm{~N}=6$ & $\{-0.41$ & $0.47\}$ \\
\hline
\end{tabular}

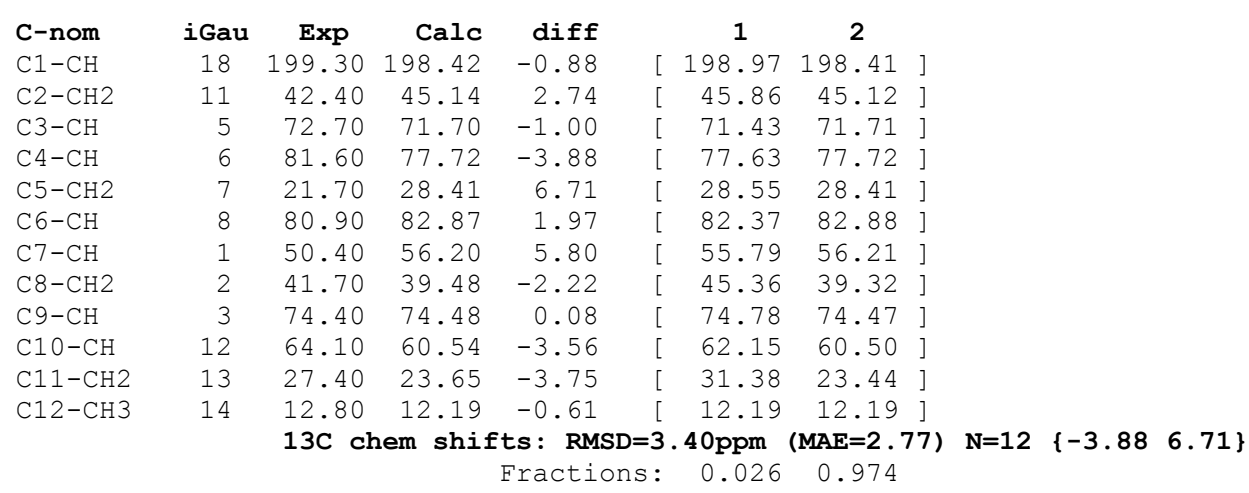

\section{Conformer 1}

Energy: -5836.52366 Hartree (Rel: $2.1 \mathrm{kcal} / \mathrm{mol}$ ) XYZ coordinates for conf 1 :

$\begin{array}{lrrr}\mathrm{C} & -2.02537 & -0.19313 & 0.66398 \\ \mathrm{C} & -0.76238 & -0.89602 & 0.15642 \\ \mathrm{C} & 0.58600 & -0.16844 & 0.40170 \\ \mathrm{O} & 0.98632 & 0.59624 & -0.73173 \\ \mathrm{C} & 0.69779 & 1.97893 & -0.86676 \\ \mathrm{C} & -0.76884 & 2.39611 & -0.66848 \\ \mathrm{C} & -1.42116 & 2.33461 & 0.72825 \\ \mathrm{C} & -2.28606 & 1.20968 & 0.11356 \\ \mathrm{O} & -1.70378 & 1.42879 & -1.19445 \\ \mathrm{H} & 0.93264 & 2.16209 & -1.92277 \\ \mathrm{C} & 1.64478 & 2.85325 & -0.00394 \\ \mathrm{C} & 1.72119 & -1.15909 & 0.71673 \\ \mathrm{C} & 3.06266 & -0.49192 & 1.01474 \\ \mathrm{C} & 4.13780 & -1.43782 & 1.55626 \\ \mathrm{Br} & 1.93749 & -2.47440 & -0.77760 \\ \mathrm{H} & 0.52766 & 0.46508 & 1.30021 \\ \mathrm{Br} & -3.58335 & -1.38385 & 0.22840 \\ \mathrm{C} & 1.53578 & 4.32603 & -0.33426 \\ \mathrm{O} & 1.11159 & 5.16903 & 0.42553 \\ \mathrm{H} & -2.05396 & -0.15278 & 1.75638 \\ \mathrm{H} & -0.72747 & -1.88275 & 0.62789 \\ \mathrm{H} & -0.84700 & -1.05769 & -0.91934 \\ \mathrm{H} & -0.89770 & 3.36944 & -1.16162 \\ \mathrm{H} & -1.98670 & 3.23278 & 0.98480 \\ \mathrm{H} & -0.78972 & 2.07117 & 1.57897 \\ \mathrm{H} & -3.35924 & 1.41864 & 0.10599\end{array}$

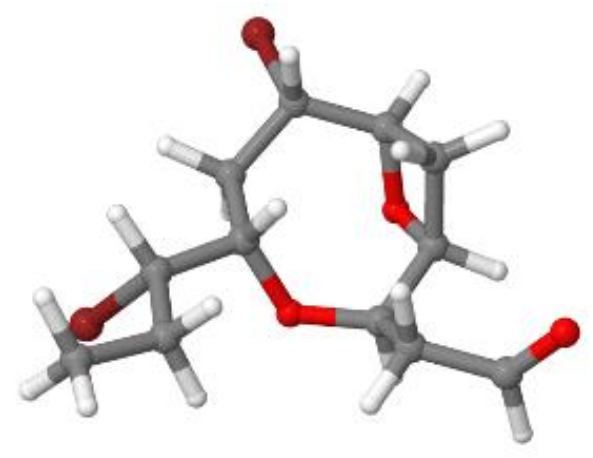




$\begin{array}{rrrr}\mathrm{H} & 1.44495 & 2.72578 & 1.06450 \\ \mathrm{H} & 2.66970 & 2.52266 & -0.21551 \\ \mathrm{H} & 1.40857 & -1.78247 & 1.55854 \\ \mathrm{H} & 3.41436 & 0.01322 & 0.10992 \\ \mathrm{H} & 2.86712 & 0.29474 & 1.76029 \\ \mathrm{H} & 5.05586 & -0.88434 & 1.78105 \\ \mathrm{H} & 3.80836 & -1.92846 & 2.48038 \\ \mathrm{H} & 4.37918 & -2.21734 & 0.82802 \\ \mathrm{H} & 1.87219 & 4.60312 & -1.35950\end{array}$

\section{Conformer 2}

Energy: -5836.52707 Hartree (Rel: $0.0 \mathrm{kcal} / \mathrm{mol}$ ) XYZ coordinates for conf 2 :

\begin{tabular}{|c|c|c|c|}
\hline $\mathrm{C}$ & -0.32699 & 1.81957 & -0.43347 \\
\hline C & 0.54482 & 0.85303 & 0.37390 \\
\hline C & 0.29244 & -0.65671 & 0.14527 \\
\hline 0 & -0.60860 & -1.17489 & 1.13323 \\
\hline C & -1.99075 & -1.35146 & 0.86330 \\
\hline C & -2.73379 & -0.09970 & 0.36253 \\
\hline C & -2.46364 & 0.46570 & -1.04793 \\
\hline $\mathrm{C}$ & -1.84188 & 1.66275 & -0.29100 \\
\hline 0 & -2.25249 & 1.11240 & 0.98534 \\
\hline $\mathrm{H}$ & -2.39485 & -1.57689 & 1.85837 \\
\hline C & -2.25442 & -2.57320 & -0.05292 \\
\hline C & 1.56375 & -1.51600 & 0.32399 \\
\hline C & 2.28671 & -1.34663 & 1.65619 \\
\hline C & 3.39733 & -2.37111 & 1.90328 \\
\hline $\mathrm{Br}$ & 2.79360 & -1.14384 & -1.21716 \\
\hline $\mathrm{H}$ & -0.07867 & -0.85410 & -0.86516 \\
\hline $\mathrm{Br}$ & 0.20211 & 3.68239 & 0.10298 \\
\hline $\mathrm{C}$ & -3.71749 & -2.96076 & -0.08600 \\
\hline 0 & -4.42677 & -2.87725 & -1.06407 \\
\hline $\mathrm{H}$ & -0.06972 & 1.79205 & -1.49563 \\
\hline $\mathrm{H}$ & 1.58494 & 1.08290 & 0.12833 \\
\hline $\mathrm{H}$ & 0.39777 & 1.04731 & 1.43879 \\
\hline $\mathrm{H}$ & -3.80217 & -0.24883 & 0.57082 \\
\hline $\mathrm{H}$ & -3.37402 & 0.72044 & -1.59450 \\
\hline $\mathrm{H}$ & -1.80948 & -0.10005 & -1.71369 \\
\hline $\mathrm{H}$ & -2.33587 & 2.61950 & -0.48125 \\
\hline $\mathrm{H}$ & -1.92527 & -2.38640 & -1.07959 \\
\hline $\mathrm{H}$ & -1.67988 & -3.41340 & 0.35889 \\
\hline $\mathrm{H}$ & 1.28417 & -2.56022 & 0.17220 \\
\hline $\mathrm{H}$ & 2.68870 & -0.33045 & 1.73530 \\
\hline $\mathrm{H}$ & 1.51373 & -1.44386 & 2.43015 \\
\hline $\mathrm{H}$ & 3.83905 & -2.22451 & 2.89464 \\
\hline $\mathrm{H}$ & 3.00892 & -3.39597 & 1.85866 \\
\hline $\mathrm{H}$ & 4.19455 & -2.27968 & 1.15905 \\
\hline $\mathrm{H}$ & -4.12561 & -3.33206 & 0.88195 \\
\hline
\end{tabular}

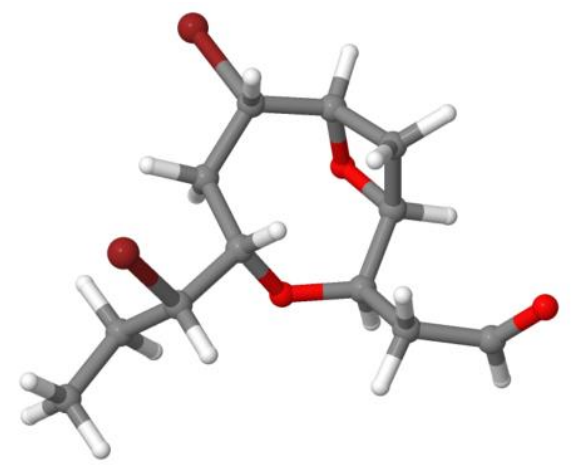


originally assigned structure of okamuragenin (second possible diastereomer, i.e. C10-epimer), Fig.8

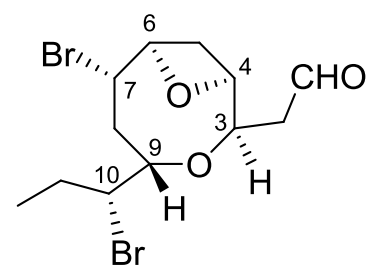

Rel energy (kcal/mol): $\begin{array}{ccc}\text { Conf } 1 & \text { Conf } 2 \\ 0.0 & 2.1\end{array}$

\begin{tabular}{|c|c|c|c|c|c|c|c|}
\hline iGau & jGau & Jexp & Jcalc & $\operatorname{diff}$ & & 1 & 2 \\
\hline 23 & 25 & 5.00 & 5.19 & 0.19 & {[} & 5.19 & 5.10 \\
\hline 23 & 24 & 8.70 & 9.20 & 0.50 & {[} & 9.20 & 9.15 \\
\hline 20 & 21 & 5.80 & 5.09 & -0.71 & {[} & 5.09 & 5.11 \\
\hline 21 & 22 & 14.10 & 14.29 & 0.19 & {[} & $-14.26-$ & -15.21 \\
\hline 16 & 22 & 7.40 & 6.89 & -0.51 & {[} & 6.90 & $6.65]$ \\
\hline \multirow[t]{2}{*}{16} & 29 & 3.50 & 1.88 & -1.62 & {[} & 1.87 & $2.35]$ \\
\hline & & For Js: & rmsc & $=0.78 \mathrm{~Hz}$ & $\mathrm{~N}=6$ & $\{-1.62$ & $0.50\}$ \\
\hline
\end{tabular}

\begin{tabular}{|c|c|c|c|c|c|c|}
\hline C-nom & iGau & Exp & Calc & $\operatorname{diff}$ & 1 & 2 \\
\hline $\mathrm{Cl}-\mathrm{CH}$ & 18 & 199.30 & 199.71 & 0.41 & [ 199.72 & 199.25 \\
\hline $\mathrm{C} 2-\mathrm{CH} 2$ & 11 & 42.40 & 46.06 & 3.66 & [ 46.09 & 45.15 \\
\hline $\mathrm{C} 3-\mathrm{CH}$ & 5 & 72.70 & 71.38 & -1.32 & 71.38 & 71.42 \\
\hline $\mathrm{C} 4-\mathrm{CH}$ & 6 & 81.60 & 78.01 & -3.59 & 78.02 & 77.81 \\
\hline $\mathrm{C} 5-\mathrm{CH} 2$ & 7 & 21.70 & 28.73 & 7.03 & 28.73 & 28.83 \\
\hline $\mathrm{C} 6-\mathrm{CH}$ & 8 & 80.90 & 82.24 & 1.34 & 82.23 & 82.63 \\
\hline $\mathrm{C} 7-\mathrm{CH}$ & 1 & 50.40 & 56.01 & 5.61 & 56.01 & 56.15 \\
\hline $\mathrm{C} 8-\mathrm{CH} 2$ & 2 & 41.70 & 44.66 & 2.96 & 44.77 & 41.07 \\
\hline $\mathrm{C} 9-\mathrm{CH}$ & 3 & 74.40 & 76.59 & 2.19 & 76.65 & 74.42 \\
\hline $\mathrm{ClO}-\mathrm{CH}$ & 12 & 64.10 & 63.65 & -0.45 & 63.70 & 61.82 \\
\hline $\mathrm{C} 11-\mathrm{CH} 2$ & 13 & 27.40 & 24.20 & -3.20 & 24.05 & 29.30 \\
\hline $\mathrm{C} 12-\mathrm{CH} 3$ & 14 & 12.80 & 12.56 & -0.24 & 12.58 & 12.00 \\
\hline
\end{tabular}

13C chem shifts: $\mathrm{RMSD}=3.35 \mathrm{ppm}(\mathrm{MAE}=2.67) \quad \mathrm{N}=12 \quad\{-3.597 .03\}$

Fractions: 0.9710 .029

\section{Conformer 1}

Energy: -5836.52648 Hartree (Rel: $0.0 \mathrm{kcal} / \mathrm{mol}$ ) XYZ coordinates for conf 1 :

$\begin{array}{lrrr}\mathrm{C} & 2.19965 & -0.12755 & -0.60869 \\ \mathrm{C} & 1.13036 & -0.97125 & 0.09183 \\ \mathrm{C} & -0.34552 & -0.50605 & -0.08939 \\ \mathrm{O} & -0.75965 & 0.29480 & 1.01101 \\ \mathrm{C} & -0.73567 & 1.71435 & 0.97488 \\ \mathrm{C} & 0.60549 & 2.35292 & 0.57629 \\ \mathrm{C} & 1.13412 & 2.23209 & -0.86873 \\ \mathrm{C} & 2.24046 & 1.35599 & -0.23694 \\ \mathrm{O} & 1.74618 & 1.62894 & 1.09584 \\ \mathrm{H} & -0.90145 & 1.97280 & 2.02861 \\ \mathrm{C} & -1.90295 & 2.30331 & 0.14492 \\ \mathrm{C} & -1.26792 & -1.73907 & -0.13014 \\ \mathrm{C} & -1.39015 & -2.49445 & 1.19163 \\ \mathrm{C} & -2.16718 & -3.81037 & 1.10335 \\ \mathrm{Br} & -3.08468 & -1.16193 & -0.76673 \\ \mathrm{H} & -0.47431 & 0.01993 & -1.04110 \\ \mathrm{Br} & 3.97835 & -0.96990 & -0.20008 \\ \mathrm{C} & -2.10855 & 3.77539 & 0.42369 \\ \mathrm{O} & -1.93635 & 4.66087 & -0.38523 \\ \mathrm{H} & 2.13517 & -0.21694 & -1.69640 \\ \mathrm{H} & 1.24224 & -1.99667 & -0.27593 \\ \mathrm{H} & 1.33578 & -0.98062 & 1.16431 \\ \mathrm{H} & 0.59861 & 3.38624 & 0.94798 \\ \mathrm{H} & 1.50407 & 3.17490 & -1.27701 \\ \mathrm{H} & 0.48775 & 1.76451 & -1.61376 \\ \mathrm{H} & 3.25363 & 1.74527 & -0.36854 \\ & & & \end{array}$

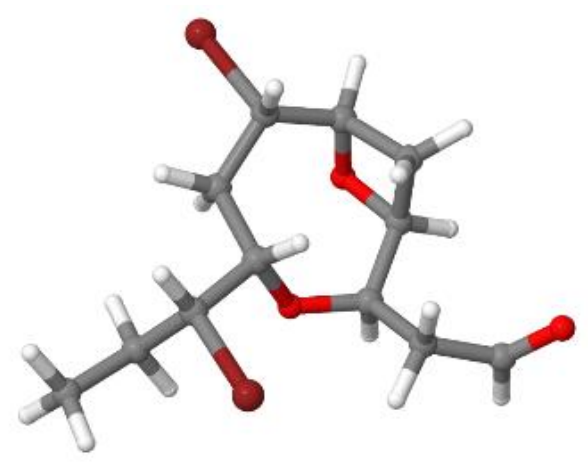




$\begin{array}{rrrr}\text { H } & -1.74635 & 2.16965 & -0.92940 \\ \mathrm{H} & -2.80841 & 1.75600 & 0.43403 \\ \mathrm{H} & -0.93522 & -2.40133 & -0.93270 \\ \mathrm{H} & -0.36696 & -2.70526 & 1.53457 \\ \mathrm{H} & -1.83344 & -1.82776 & 1.93687 \\ \mathrm{H} & -2.17009 & -4.31568 & 2.07504 \\ \mathrm{H} & -3.20595 & -3.63903 & 0.80585 \\ \mathrm{H} & -1.71500 & -4.49330 & 0.37348 \\ \mathrm{H} & -2.43276 & 4.01290 & 1.46338\end{array}$

\section{Conformer 2}

Energy: -5836.52316 Hartree (Rel: $2.1 \mathrm{kcal} / \mathrm{mol}$ ) XYZ coordinates for conf 2 :

\begin{tabular}{|c|c|c|c|}
\hline C & 0.81039 & 1.73712 & 0.58682 \\
\hline C & -0.37616 & 0.96078 & 0.00831 \\
\hline C & -0.40509 & -0.56322 & 0.28796 \\
\hline 0 & 0.15041 & -1.29130 & -0.80569 \\
\hline C & 1.49747 & -1.73209 & -0.80689 \\
\hline $\mathrm{C}$ & 2.56540 & -0.65636 & -0.54043 \\
\hline C & 2.71250 & -0.01865 & 0.85728 \\
\hline C & 2.20402 & 1.26721 & 0.16415 \\
\hline 0 & 2.22785 & 0.61914 & -1.13069 \\
\hline $\mathrm{H}$ & 1.63580 & -2.05579 & -1.84603 \\
\hline C & 1.69783 & -2.96614 & 0.11276 \\
\hline $\mathrm{C}$ & -1.82166 & -1.12152 & 0.51245 \\
\hline C & -2.49374 & -0.64921 & 1.80261 \\
\hline $\mathrm{C}$ & -3.78367 & -1.39709 & 2.15240 \\
\hline $\mathrm{Br}$ & -2.98985 & -0.72913 & -1.06272 \\
\hline $\mathrm{H}$ & 0.12604 & -0.80261 & 1.22067 \\
\hline $\mathrm{Br}$ & 0.56973 & 3.66344 & 0.06826 \\
\hline $\mathrm{C}$ & 3.04721 & -3.62144 & -0.08510 \\
\hline 0 & 3.93122 & -3.63197 & 0.74342 \\
\hline $\mathrm{H}$ & 0.77234 & 1.77768 & 1.67895 \\
\hline $\mathrm{H}$ & -1.28839 & 1.42274 & 0.39500 \\
\hline $\mathrm{H}$ & -0.39140 & 1.08081 & -1.07616 \\
\hline $\mathrm{H}$ & 3.51504 & -1.03367 & -0.94354 \\
\hline $\mathrm{H}$ & 3.74850 & 0.04860 & 1.19594 \\
\hline $\mathrm{H}$ & 2.11004 & -0.42102 & 1.67392 \\
\hline $\mathrm{H}$ & 2.90634 & 2.10438 & 0.19689 \\
\hline $\mathrm{H}$ & 1.59963 & -2.69801 & 1.16949 \\
\hline $\mathrm{H}$ & 0.91257 & -3.68777 & -0.14735 \\
\hline $\mathrm{H}$ & -1.74807 & -2.21081 & 0.49731 \\
\hline $\mathrm{H}$ & -1.76469 & -0.79280 & 2.61541 \\
\hline $\mathrm{H}$ & -2.69184 & 0.42736 & 1.74904 \\
\hline $\mathrm{H}$ & -4.18614 & -1.03848 & 3.10566 \\
\hline $\mathrm{H}$ & -4.54561 & -1.24843 & 1.38207 \\
\hline $\mathrm{H}$ & -3.60358 & -2.47464 & 2.24781 \\
\hline $\mathrm{H}$ & 3.19483 & -4.10173 & -1.07924 \\
\hline
\end{tabular}

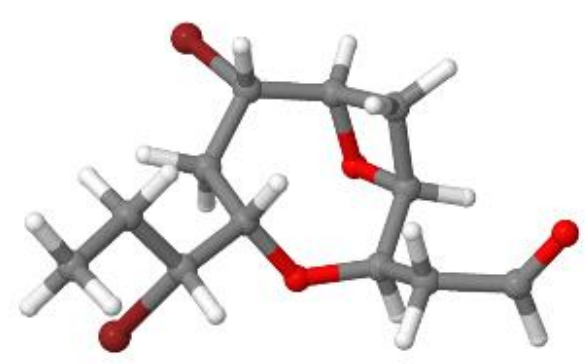


revised structure of okamuragenin, Fig.8

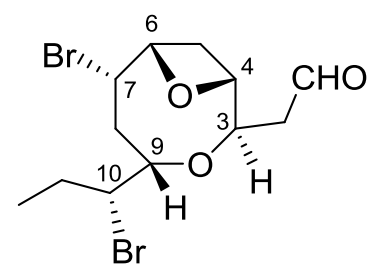

Rel energy $(\mathrm{kcal} / \mathrm{mol}): \begin{array}{cc}\text { Conf } 1 & \text { Conf } 2 \\ 0.0 & 0.9\end{array}$

\begin{tabular}{|c|c|c|c|c|c|c|c|}
\hline iGau & jGau & Jexp & Jcalc & diff & & 1 & 2 \\
\hline 23 & 25 & 5.00 & 5.12 & 0.12 & {[} & 5.13 & $5.11]$ \\
\hline 23 & 24 & 8.70 & 8.13 & -0.57 & {[} & 8.21 & 8.10 \\
\hline 20 & 21 & 5.80 & 5.84 & 0.04 & {[} & 5.61 & 5.94 \\
\hline 21 & 22 & 14.10 & 15.38 & 1.28 & {[} & -14.79 & -15.63 \\
\hline 16 & 22 & 7.40 & 7.75 & 0.35 & {[} & 8.02 & 7.64 \\
\hline \multirow[t]{2}{*}{16} & 29 & 3.50 & 2.07 & -1.43 & [ & 1.61 & 2.27 \\
\hline & & For Js: & rmsd & $=0.83 \mathrm{~Hz}$ & $\mathrm{~N}=6$ & $\{-1,4\}$ & $\{1.28\}$ \\
\hline
\end{tabular}

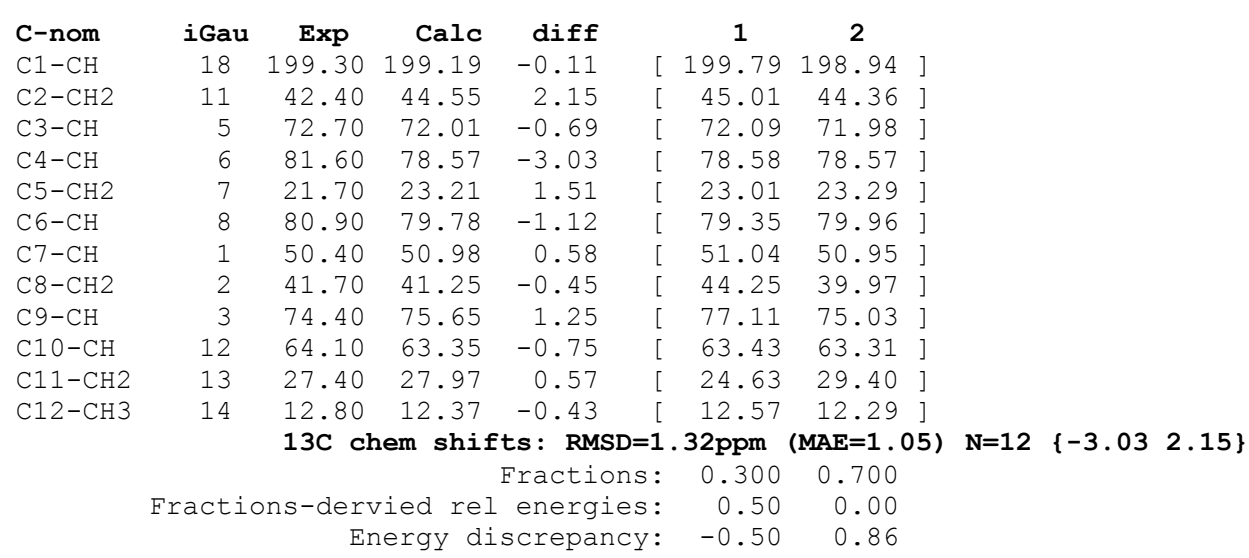

Conformer 1

Energy: -5836.53617 Hartree (Rel: $0.0 \mathrm{kcal} / \mathrm{mol}$ ) XYZ coordinates for conf 1 :

$\begin{array}{lrrr}\mathrm{C} & 2.20503 & -0.11974 & -0.64862 \\ \mathrm{C} & 1.13238 & -1.01270 & -0.01845 \\ \mathrm{C} & -0.33440 & -0.50391 & -0.13389 \\ \mathrm{O} & -0.66027 & 0.31228 & 0.99412 \\ \mathrm{C} & -0.71560 & 1.73426 & 0.89293 \\ \mathrm{C} & 0.63258 & 2.37399 & 0.56812 \\ \mathrm{C} & 1.89675 & 1.75612 & 1.20066 \\ \mathrm{C} & 2.25392 & 1.34882 & -0.24369 \\ \mathrm{O} & 1.08009 & 2.02338 & -0.76977 \\ \mathrm{H} & -0.97837 & 2.03469 & 1.91743 \\ \mathrm{C} & -1.81083 & 2.25231 & -0.06857 \\ \mathrm{C} & -1.27977 & -1.72253 & -0.12959 \\ \mathrm{C} & -1.36641 & -2.46552 & 1.20277 \\ \mathrm{C} & -2.16373 & -3.77158 & 1.15683 \\ \mathrm{Br} & -3.11695 & -1.15411 & -0.71239 \\ \mathrm{H} & -0.47138 & 0.04331 & -1.06972 \\ \mathrm{Br} & 3.99541 & -0.91844 & -0.23389 \\ \mathrm{C} & -2.27715 & 3.63462 & 0.33178 \\ \mathrm{O} & -2.17190 & 4.63010 & -0.34893 \\ \mathrm{H} & 2.14623 & -0.15926 & -1.73911 \\ \mathrm{H} & 1.21982 & -1.99470 & -0.49630 \\ \mathrm{H} & 1.34980 & -1.16300 & 1.04284 \\ \mathrm{H} & 0.54665 & 3.46383 & 0.66945 \\ \mathrm{H} & 2.58436 & 2.48115 & 1.63971 \\ \mathrm{H} & 1.72124 & 0.94253 & 1.90570\end{array}$

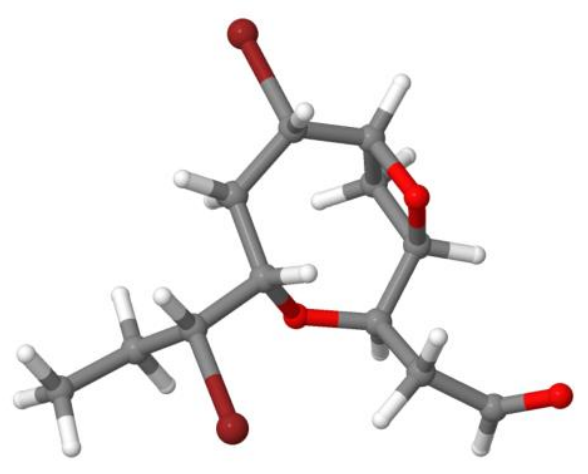




$\begin{array}{rrrr}\text { H } & 3.16920 & 1.80129 & -0.63840 \\ \mathrm{H} & -1.45843 & 2.27986 & -1.10308 \\ \mathrm{H} & -2.66521 & 1.56794 & 0.00232 \\ \mathrm{H} & -0.98213 & -2.39739 & -0.93533 \\ \mathrm{H} & -0.33532 & -2.68994 & 1.51159 \\ \mathrm{H} & -1.77338 & -1.78538 & 1.95687 \\ \mathrm{H} & -2.13329 & -4.26968 & 2.13185 \\ \mathrm{H} & -3.21141 & -3.58920 & 0.90059 \\ \mathrm{H} & -1.75047 & -4.46555 & 0.41443 \\ \mathrm{H} & -2.74391 & 3.68790 & 1.34392\end{array}$

\section{Conformer 2}

Energy: -5836.53480 Hartree (Rel: $0.9 \mathrm{kcal} / \mathrm{mol}$ ) XYZ coordinates for conf 2 :

$\begin{array}{lrrr}\mathrm{C} & -1.76033 & -0.79274 & 0.66049 \\ \mathrm{C} & -0.34823 & -1.03677 & 0.11813 \\ \mathrm{C} & 0.64403 & 0.13529 & 0.29645 \\ \mathrm{O} & 0.58576 & 0.99825 & -0.84772 \\ \mathrm{C} & -0.04382 & 2.26980 & -0.75678 \\ \mathrm{C} & -1.54637 & 2.18132 & -0.49051 \\ \mathrm{C} & -2.31408 & 1.03590 & -1.18275 \\ \mathrm{C} & -2.49569 & 0.47742 & 0.24362 \\ \mathrm{O} & -1.82871 & 1.63281 & 0.82529 \\ \mathrm{H} & 0.08055 & 2.67742 & -1.77022 \\ \mathrm{C} & 0.65252 & 3.22504 & 0.24422 \\ \mathrm{C} & 2.10633 & -0.31103 & 0.46053 \\ \mathrm{C} & 2.40630 & -1.03381 & 1.77467 \\ \mathrm{C} & 3.89804 & -1.23745 & 2.05526 \\ \mathrm{Br} & 2.68216 & -1.43487 & -1.09528 \\ \mathrm{H} & 0.40299 & 0.69766 & 1.20597 \\ \mathrm{Br} & -2.90945 & -2.34503 & 0.13309 \\ \mathrm{C} & 0.37546 & 4.67655 & -0.08864 \\ \mathrm{O} & -0.20940 & 5.45271 & 0.63228 \\ \mathrm{H} & -1.76232 & -0.82642 & 1.75306 \\ \mathrm{H} & 0.03787 & -1.93147 & 0.61590 \\ \mathrm{H} & -0.38048 & -1.27300 & -0.94817 \\ \mathrm{H} & -1.99538 & 3.17794 & -0.59533 \\ \mathrm{H} & -3.24605 & 1.33840 & -1.66351 \\ \mathrm{H} & -1.72537 & 0.42503 & -1.86827 \\ \mathrm{H} & -3.53345 & 0.42117 & 0.58753 \\ \mathrm{H} & 0.33638 & 3.03363 & 1.27317 \\ \mathrm{H} & 1.73547 & 3.06263 & 0.15457 \\ \mathrm{H} & 2.73527 & 0.57618 & 0.36274 \\ \mathrm{H} & 1.96778 & -0.42584 & 2.58088 \\ \mathrm{H} & 1.88940 & -1.99983 & 1.79684 \\ \mathrm{H} & 4.04201 & -1.71795 & 3.02873 \\ \mathrm{H} & 4.35886 & -1.86900 & 1.29039 \\ \mathrm{H} & 4.43206 & -0.27968 & 2.07120 \\ \mathrm{H} & 0.75319 & 5.00217 & -1.08606 \\ & & & \end{array}$

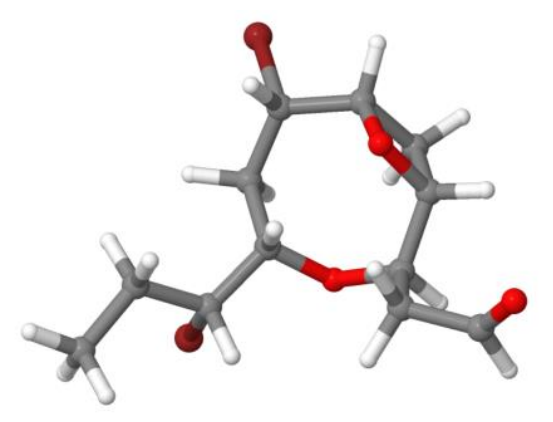


3-phenyloxete (test for the oxete structural type), Fig.9A<smiles>C1=C(c2ccccc2)CC1</smiles>

$$
\text { Rel energy (kcal/mol): } \begin{gathered}
\operatorname{Conf} 1 \\
0.0
\end{gathered}
$$

\begin{tabular}{|c|c|c|c|c|c|}
\hline C-nom & iGau & Exp & Calc & $\operatorname{diff}$ & 1 \\
\hline $\mathrm{C}$ & 8 & 149.30 & 150.14 & 0.84 & [ 150.14 \\
\hline C & 5 & 129.30 & 130.45 & 1.15 & [ 130.45 \\
\hline $\mathrm{C}$ & 1 & 128.50 & 127.68 & -0.82 & 127.68 \\
\hline $\mathrm{C}$ & 3 & 128.50 & 127.68 & -0.82 & 127.68 \\
\hline $\mathrm{C}$ & 2 & 126.10 & 124.20 & -1.90 & 124.20 \\
\hline $\mathrm{C}$ & 7 & 122.90 & 121.72 & -1.18 & [ 121.72 \\
\hline C & 4 & 122.90 & 121.65 & -1.25 & 121.65 \\
\hline $\mathrm{C}$ & 6 & 122.90 & 121.65 & -1.25 & [ 121.65 \\
\hline $\mathrm{C}$ & 10 & 80.40 & 77.48 & -2.92 & {$[\quad 77.48$} \\
\hline
\end{tabular}

13C chem shifts: RMSD=1.49ppm (MAE=1.35) $\mathrm{N}=9 \quad\left\{\begin{array}{l}-2.92 \\ 1.15\end{array}\right\}$

\section{Conformer 1}

Energy: -422.92142 Hartree (Rel: $0.0 \mathrm{kcal} / \mathrm{mol}$ ) XYZ coordinates for conf 1 :

$\begin{array}{lrrr}\mathrm{C} & 2.35420 & 1.18325 & 0.00012 \\ \mathrm{C} & 3.02284 & -0.04548 & -0.00001 \\ \mathrm{C} & 2.28185 & -1.22867 & -0.00012 \\ \mathrm{C} & 0.88857 & -1.18665 & -0.00012 \\ \mathrm{C} & 0.20245 & 0.04251 & -0.00000 \\ \mathrm{C} & 0.96375 & 1.22912 & 0.00014 \\ \mathrm{C} & -1.24449 & 0.07300 & -0.00002 \\ \mathrm{C} & -2.24882 & 0.97576 & -0.00031 \\ \mathrm{O} & -3.32756 & 0.10895 & 0.00001 \\ \mathrm{C} & -2.33359 & -0.98454 & 0.00027 \\ \mathrm{H} & 2.92134 & 2.11057 & 0.00022 \\ \mathrm{H} & 4.10874 & -0.07823 & -0.00001 \\ \mathrm{H} & 2.78977 & -2.18967 & -0.00021 \\ \mathrm{H} & 0.32226 & -2.11511 & -0.00023 \\ \mathrm{H} & 0.45549 & 2.19018 & 0.00028 \\ \mathrm{H} & -2.40399 & 2.04928 & -0.00066 \\ \mathrm{H} & -2.44683 & -1.59399 & 0.90156 \\ \mathrm{H} & -2.44689 & -1.59446 & -0.90071\end{array}$

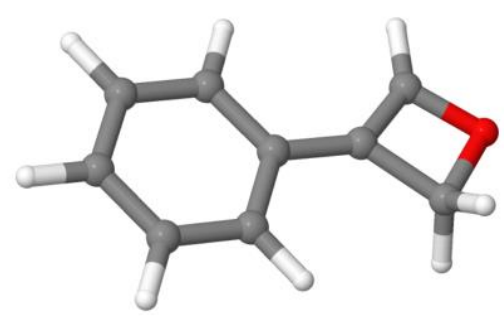


4-ethoxycarbonyl-4-trifluoromethyl-2-(p-methoxyphenyl)oxete (cmpd 4n) (Fig.9A)

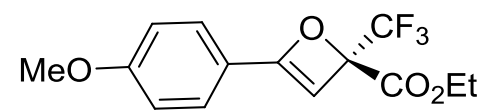

$$
\text { Rel energy (kcal/mol): } \quad 0.0
$$

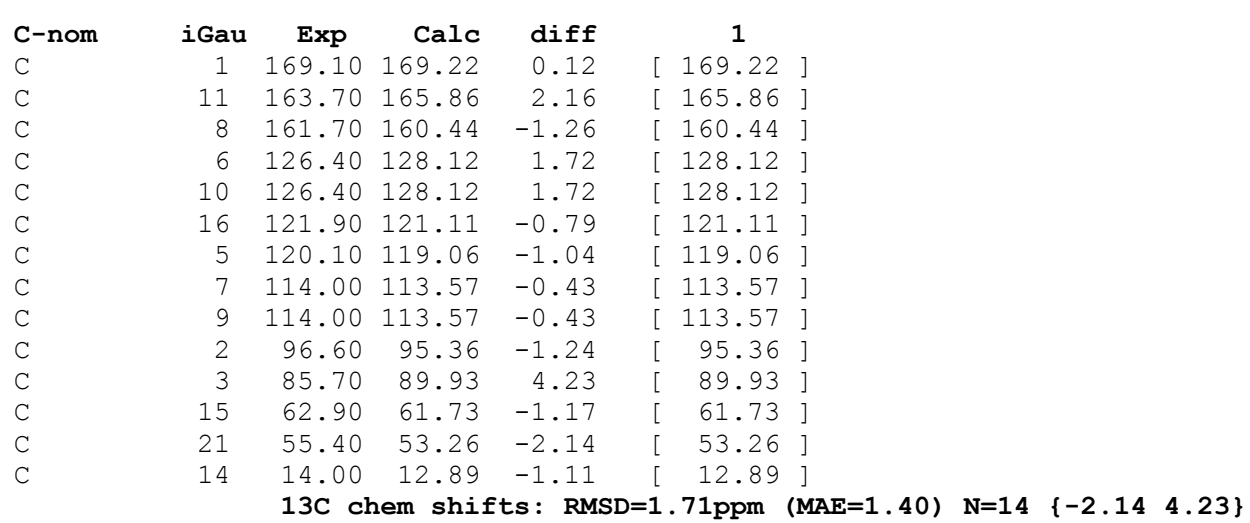

\section{Conformer 1}

Energy: -1141.66266 Hartree (Rel: $0.0 \mathrm{kcal} / \mathrm{mol}$ ) XYZ coordinates for conf 1 :

$\begin{array}{lrrr}\mathrm{C} & -0.40967 & 0.05878 & -0.16362 \\ \mathrm{C} & 0.59890 & -0.20068 & -1.02310 \\ \mathrm{C} & 1.53048 & 0.33313 & 0.04040 \\ \mathrm{O} & 0.36637 & 0.57359 & 0.88553 \\ \mathrm{C} & -1.84950 & -0.03896 & -0.04750 \\ \mathrm{C} & -2.49140 & 0.35763 & 1.14117 \\ \mathrm{C} & -3.86897 & 0.26757 & 1.26017 \\ \mathrm{C} & -4.64130 & -0.22196 & 0.19367 \\ \mathrm{C} & -4.01233 & -0.62138 & -0.99587 \\ \mathrm{C} & -2.62766 & -0.52648 & -1.10680 \\ \mathrm{C} & 2.46914 & -0.69396 & 0.68347 \\ \mathrm{O} & 2.30189 & -1.19524 & 1.76749 \\ \mathrm{O} & 3.47660 & -0.97916 & -0.15879 \\ \mathrm{C} & 5.49118 & -2.11658 & -0.76412 \\ \mathrm{C} & 4.43705 & -1.95705 & 0.31455 \\ \mathrm{C} & 2.22045 & 1.67028 & -0.25698 \\ \mathrm{~F} & 2.91102 & 2.10091 & 0.82011 \\ \mathrm{~F} & 1.33175 & 2.61864 & -0.58595 \\ \mathrm{~F} & 3.08613 & 1.54238 & -1.28057 \\ \mathrm{O} & -5.98282 & -0.26937 & 0.41061 \\ \mathrm{C} & -6.82279 & -0.75274 & -0.62732 \\ \mathrm{H} & 0.73362 & -0.60942 & -2.01088 \\ \mathrm{H} & -1.89576 & 0.73361 & 1.96653 \\ \mathrm{H} & -4.37695 & 0.56945 & 2.17042 \\ \mathrm{H} & -4.58811 & -1.00188 & -1.83141 \\ \mathrm{H} & -2.14659 & -0.83470 & -2.03122 \\ \mathrm{H} & 5.99710 & -1.16585 & -0.95935 \\ \mathrm{H} & 5.04468 & -2.46877 & -1.69953 \\ \mathrm{H} & 6.24055 & -2.84801 & -0.44247 \\ \mathrm{H} & -6.90719 & -2.89264 & 0.51922 \\ \mathrm{H} & 4.86041 & -1.59953 & 1.25818 \\ \mathrm{H} & -6.84023 & -0.69904 & -0.23649 \\ \mathrm{H} & -67679 & -0.13091 & -1.52874 \\ \mathrm{H} & -1.79372 & \end{array}$

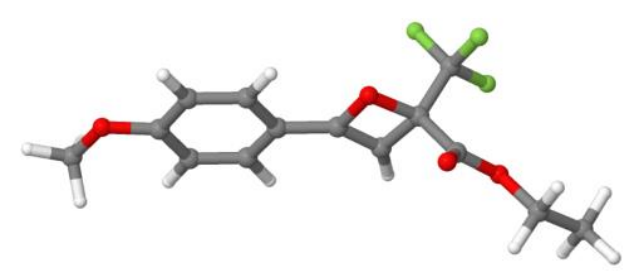


2-(o-methoxyphenyl)-3-phenyl-4-methyleneoxete - incorrect, Fig.9A<smiles>C=C1OC(c2ccccc2OC)=C1c1ccccc1</smiles>

$$
\text { Rel energy (kcal/mol): } \begin{array}{ccc}
\text { Conf } 1 & \text { Conf } 2 \\
0.0 & 2.5
\end{array}
$$

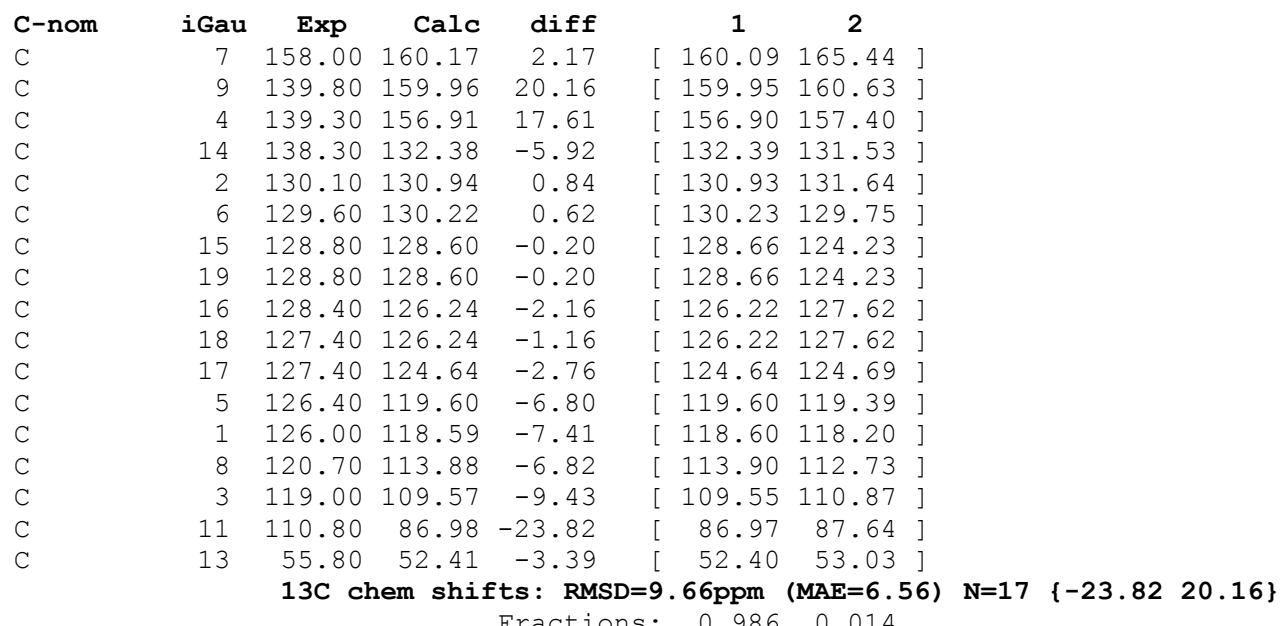

\section{Conformer 1}

Energy: -806.59026 Hartree (Rel: $0.0 \mathrm{kcal} / \mathrm{mol}$ ) XYZ coordinates for conf 1 :

$\begin{array}{lrrr}\mathrm{C} & -4.21338 & 0.52702 & 0.43317 \\ \mathrm{C} & -4.28385 & -0.84317 & 0.17882 \\ \mathrm{C} & -3.13345 & -1.56642 & -0.14512 \\ \mathrm{C} & -1.89496 & -0.92058 & -0.21122 \\ \mathrm{C} & -1.80272 & 0.46752 & 0.06596 \\ \mathrm{C} & -2.98197 & 1.17201 & 0.37113 \\ \mathrm{C} & -0.55917 & 1.19881 & 0.00723 \\ \mathrm{C} & 0.81060 & 1.10746 & -0.00938 \\ \mathrm{C} & 0.80835 & 2.57420 & -0.12001 \\ \mathrm{O} & -0.61230 & 2.61569 & -0.08992 \\ \mathrm{C} & 1.61196 & 3.62971 & -0.19582 \\ \mathrm{O} & -0.73308 & -1.53446 & -0.55034 \\ \mathrm{C} & -0.74820 & -2.91617 & -0.87760 \\ \mathrm{C} & 1.88785 & 0.13741 & 0.11501 \\ \mathrm{C} & 3.10313 & 0.36214 & -0.56083 \\ \mathrm{C} & 4.16884 & -0.52697 & -0.43441 \\ \mathrm{C} & 4.04730 & -1.66151 & 0.37094 \\ \mathrm{C} & 2.85043 & -1.89257 & 1.05487 \\ \mathrm{C} & 1.78505 & -1.00245 & 0.93539 \\ \mathrm{H} & -5.10992 & 1.08851 & 0.67760 \\ \mathrm{H} & -5.23869 & -1.35990 & 0.22380 \\ \mathrm{H} & -3.20952 & -2.62681 & -0.35549 \\ \mathrm{H} & -2.90619 & 2.23684 & 0.56264 \\ \mathrm{H} & 1.20405 & 4.63332 & -0.24786 \\ \mathrm{H} & 2.68835 & 3.50525 & -0.19706 \\ \mathrm{H} & 0.28696 & -3.17389 & -1.10499 \\ \mathrm{H} & -1.37957 & -3.11090 & -1.75378 \\ \mathrm{H} & -1.09881 & -3.52541 & -0.03460 \\ \mathrm{H} & 3.19673 & 1.23074 & -1.20669 \\ \mathrm{H} & 0.86992 & -0.33581 & -0.97099 \\ \mathrm{H} & & -2.35452 & 0.47093 \\ \mathrm{H} & -2.76335 & 1.69846 \\ \mathrm{H} & -1.17261 & 1.49143\end{array}$

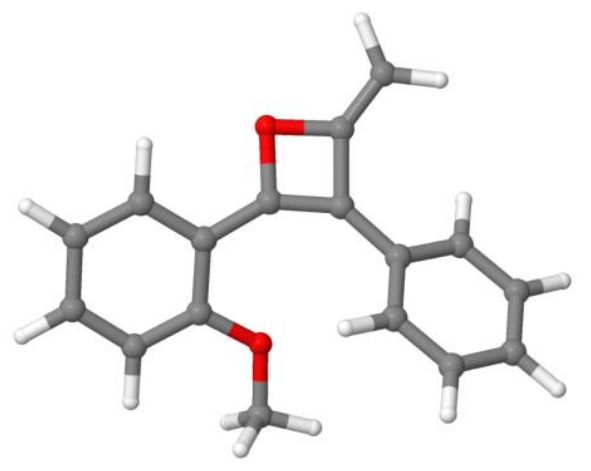




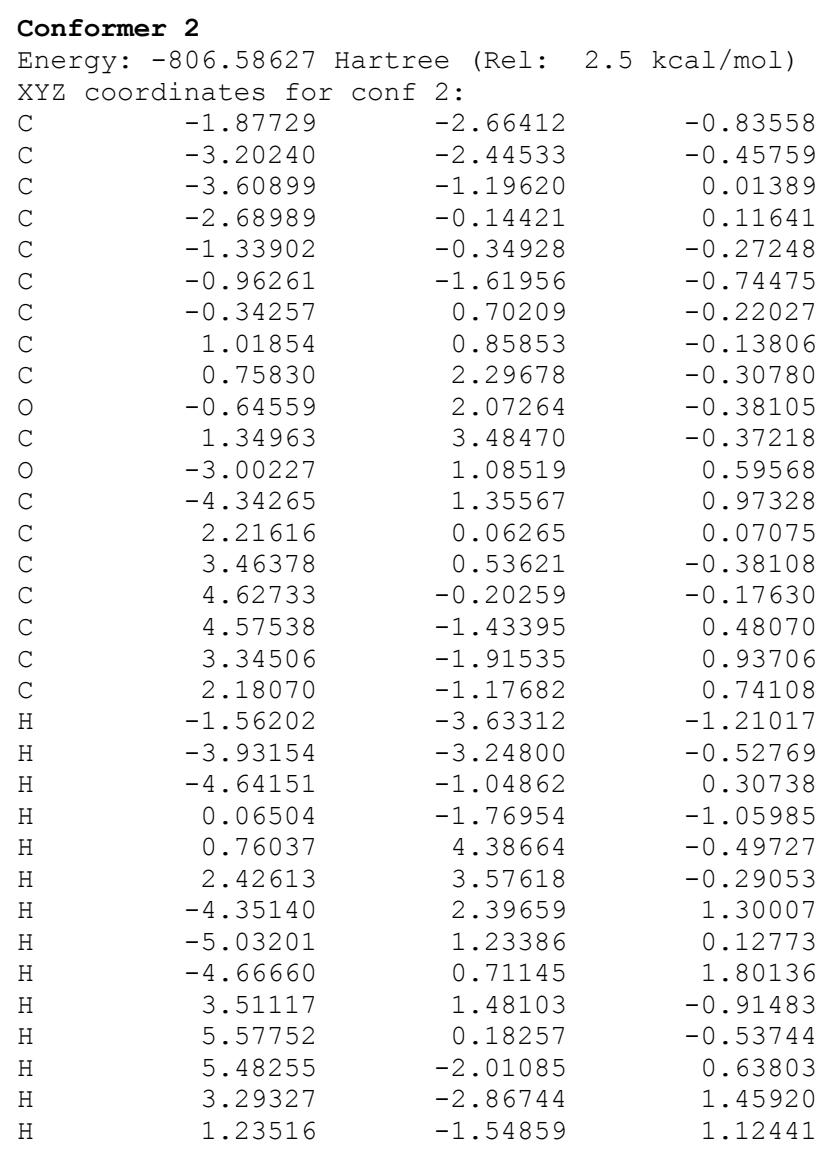

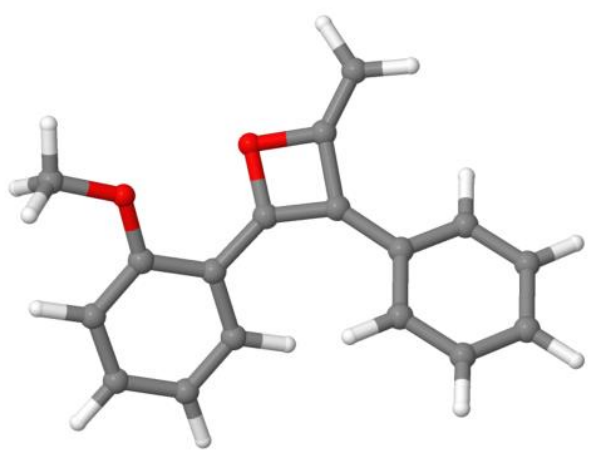


originally assigned structure of fusariumin D, Fig.9B<smiles>CCC(C)CC(C)/C=C(\C)C1=CC(=CC(=O)OC)O1</smiles>

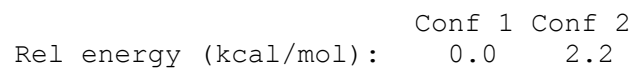

\begin{tabular}{|c|c|c|c|}
\hline C & 6.71410 & -1.98762 & 0.03217 \\
\hline 0 & 6.12913 & -0.71182 & -0.25137 \\
\hline C & 4.78011 & -0.64581 & -0.06470 \\
\hline C & 4.30126 & 0.70623 & -0.38628 \\
\hline C & 3.01866 & 1.09018 & -0.30267 \\
\hline C & 2.18874 & 2.26581 & -0.51960 \\
\hline C & 1.13944 & 1.48895 & -0.13360 \\
\hline C & -0.28503 & 1.54540 & 0.06493 \\
\hline C & -0.91153 & 0.42223 & 0.48691 \\
\hline C & -2.37491 & 0.21357 & 0.76264 \\
\hline C & -2.92641 & -0.83939 & -0.23629 \\
\hline C & -4.43363 & -1.17070 & -0.15182 \\
\hline C & -5.31569 & 0.05944 & -0.45103 \\
\hline 0 & 4.11668 & -1.59118 & 0.31255 \\
\hline 0 & 1.90462 & 0.31037 & 0.09449 \\
\hline C & -0.92609 & 2.88289 & -0.22826 \\
\hline $\mathrm{C}$ & -2.56424 & -0.21650 & 2.23270 \\
\hline C & -4.74352 & -2.34332 & -1.09813 \\
\hline C & -6.82304 & -0.17204 & -0.28356 \\
\hline $\mathrm{H}$ & 7.78114 & -1.87238 & -0.16386 \\
\hline $\mathrm{H}$ & 6.54448 & -2.26914 & 1.07560 \\
\hline $\mathrm{H}$ & 6.28770 & -2.76204 & -0.61223 \\
\hline $\mathrm{H}$ & 5.03380 & 1.43657 & -0.71118 \\
\hline $\mathrm{H}$ & 2.34765 & 3.28244 & -0.84084 \\
\hline $\mathrm{H}$ & -0.28446 & -0.45487 & 0.64780 \\
\hline $\mathrm{H}$ & -2.91249 & 1.15566 & 0.61199 \\
\hline $\mathrm{H}$ & -2.70154 & -0.49865 & -1.25725 \\
\hline $\mathrm{H}$ & -2.35643 & -1.76932 & -0.09675 \\
\hline $\mathrm{H}$ & -4.66057 & -1.50525 & 0.87141 \\
\hline $\mathrm{H}$ & -5.10851 & 0.40272 & -1.47586 \\
\hline $\mathrm{H}$ & -5.02135 & 0.88468 & 0.21030 \\
\hline $\mathrm{H}$ & -2.00551 & 2.86676 & -0.06739 \\
\hline $\mathrm{H}$ & -0.50106 & 3.66659 & 0.41097 \\
\hline $\mathrm{H}$ & -0.74485 & 3.18152 & -1.26812 \\
\hline $\mathrm{H}$ & -3.62457 & -0.33017 & 2.48133 \\
\hline
\end{tabular}

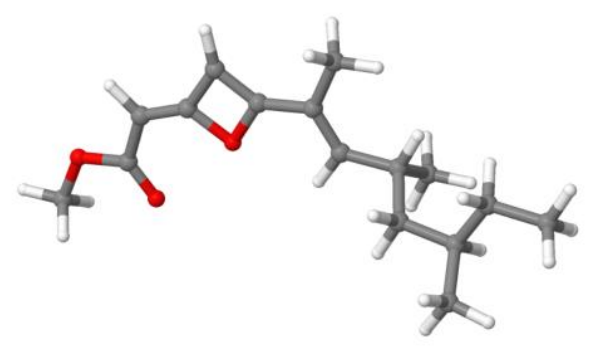




$\begin{array}{rrrr}\text { H } & -2.13686 & 0.52643 & 2.91465 \\ \mathrm{H} & -2.06739 & -1.17509 & 2.42741 \\ \mathrm{H} & -5.78332 & -2.67504 & -1.01209 \\ \mathrm{H} & -4.10355 & -3.20548 & -0.87670 \\ \mathrm{H} & -4.56796 & -2.06005 & -2.14445 \\ \mathrm{H} & -7.37861 & 0.76139 & -0.42963 \\ \mathrm{H} & -7.05692 & -0.54225 & 0.72274 \\ \mathrm{H} & -7.21162 & -0.89983 & -1.00368\end{array}$

\section{Conformer 2}

Energy: -849.73941 Hartree (Rel: $2.2 \mathrm{kcal} / \mathrm{mol}$ ) XYZ coordinates for conf 2 :

$\begin{array}{lrrr}\mathrm{C} & 7.57386 & 0.59916 & -0.47307 \\ \mathrm{O} & 6.52710 & -0.37654 & -0.42017 \\ \mathrm{C} & 5.27744 & 0.13359 & -0.22837 \\ \mathrm{C} & 4.29692 & -0.96164 & -0.19032 \\ \mathrm{C} & 2.97868 & -0.78718 & -0.01444 \\ \mathrm{C} & 1.75271 & -1.56062 & 0.08056 \\ \mathrm{C} & 1.11642 & -0.36637 & 0.24977 \\ \mathrm{C} & -0.18418 & 0.23315 & 0.45919 \\ \mathrm{C} & -1.25394 & -0.58733 & 0.56685 \\ \mathrm{C} & -2.69984 & -0.22978 & 0.77978 \\ \mathrm{C} & -3.51809 & -0.68570 & -0.45833 \\ \mathrm{C} & -5.03389 & -0.38395 & -0.45396 \\ \mathrm{C} & -5.32472 & 1.13052 & -0.40127 \\ \mathrm{O} & 5.05787 & 1.32262 & -0.11141 \\ \mathrm{O} & 2.28469 & 0.43812 & 0.16277 \\ \mathrm{C} & -0.19938 & 1.74276 & 0.53644 \\ \mathrm{C} & -3.20402 & -0.87597 & 2.08745 \\ \mathrm{C} & -5.68239 & -1.05130 & -1.67896 \\ \mathrm{C} & -6.80967 & 1.49710 & -0.28379 \\ \mathrm{H} & 8.49512 & 0.03457 & -0.62402 \\ \mathrm{H} & 7.41260 & 1.29706 & -1.29996 \\ \mathrm{H} & 7.62158 & 1.16812 & 0.46005 \\ \mathrm{H} & 4.66763 & -1.97337 & -0.31153 \\ \mathrm{H} & 1.48701 & -2.60422 & 0.03445 \\ \mathrm{H} & -1.06536 & -1.66044 & 0.48995 \\ \mathrm{H} & -2.79213 & 0.85645 & 0.88030 \\ \mathrm{H} & -3.07334 & -0.22778 & -1.35350 \\ \mathrm{H} & -3.38136 & -1.77103 & -0.57474 \\ \mathrm{H} & -5.47710 & -0.84206 & 0.44289 \\ \mathrm{H} & -4.89933 & 1.60479 & -1.29848 \\ \mathrm{H} & -4.79492 & 1.57030 & 0.45351 \\ \mathrm{H} & -1.20089 & 2.13380 & 0.72409 \\ \mathrm{H} & 0.46540 & 2.09473 & 1.33306 \\ \mathrm{H} & 0.17344 & 2.18155 & -0.39612 \\ \mathrm{H} & -4.23782 & -0.58664 & 2.30288 \\ \mathrm{H} & -2.58534 & -0.56925 & 2.93753 \\ \mathrm{H} & -3.16837 & -1.97102 & 2.02361 \\ \mathrm{H} & -6.77133 & -0.93903 & -1.67733 \\ \mathrm{H} & -5.46274 & -2.12521 & -1.70303 \\ \mathrm{H} & -5.30199 & -0.61361 & -2.61141 \\ \mathrm{H} & -6.93501 & 2.58009 & -0.17190 \\ \mathrm{H} & -7.26732 & 1.01877 & 0.59149 \\ \mathrm{H} & -7.38121 & 1.19114 & -1.16640 \\ & & & \end{array}$

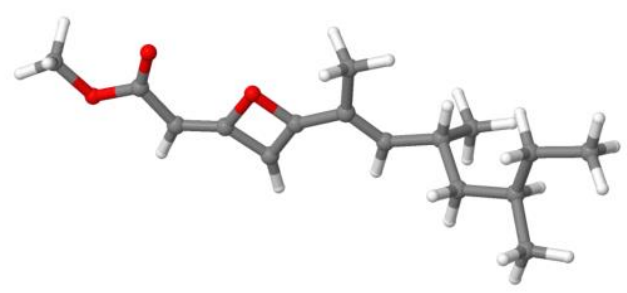


revised structure of fusariumin D, Fig.9B<smiles>CCC(C)CC(C)/C=C(\C)c1cc(=O)cc(OC)o1</smiles>

$$
\text { Rel energy (kcal/mol): } \begin{array}{cccc}
0.0 & 0.5 & 0.6 & 0.0
\end{array}
$$

\begin{tabular}{|c|c|c|c|c|c|c|c|c|}
\hline C-nom & iGau & $\operatorname{Exp}$ & Calc & $\operatorname{diff}$ & 1 & 2 & 3 & 4 \\
\hline $\mathrm{C} 1-\mathrm{C}$ & 3 & 167.90 & 168.05 & 0.15 & {$[168.03$} & 168.07 & 168.08 & $168.05]$ \\
\hline $\mathrm{C} 2-\mathrm{CH}$ & 2 & 89.30 & 90.39 & 1.09 & {$\left[\begin{array}{l}90.42 \\
0\end{array}\right.$} & 90.37 & 90.37 & 90.37 \\
\hline C3-C & 1 & 182.40 & 179.83 & -2.57 & [ 179.91 & 179.70 & 179.68 & 179.87 \\
\hline $\mathrm{C} 4-\mathrm{CH}$ & 6 & 109.20 & 110.73 & 1.53 & {$[110.71$} & 110.89 & 110.88 & 110.64 \\
\hline $\mathrm{C} 5-\mathrm{C}$ & 5 & 161.10 & 160.64 & -0.46 & {$[160.52$} & 160.88 & 160.88 & 160.56 \\
\hline $\mathrm{C} 6-\mathrm{C}$ & 9 & 124.10 & 123.13 & -0.97 & {$[123.76$} & 121.54 & 121.49 & 123.78 \\
\hline $\mathrm{C} 7-\mathrm{CH}$ & 10 & 141.90 & 143.67 & 1.77 & {$[143.62$} & 143.90 & 144.03 & 143.48 \\
\hline $\mathrm{C} 8-\mathrm{CH}$ & 11 & 30.80 & 31.45 & 0.65 & {$[\quad 31.56$} & 31.23 & 31.30 & 31.49 \\
\hline $\mathrm{C} 9-\mathrm{CH} 2$ & 15 & 44.10 & 43.81 & -0.29 & 44.87 & 42.91 & 38.62 & 44.96 \\
\hline $\mathrm{C} 10-\mathrm{CH}$ & 16 & 32.10 & 32.05 & -0.05 & 32.36 & 31.20 & 31.06 & 32.47 \\
\hline $\mathrm{C} 11-\mathrm{CH} 2$ & 18 & 29.30 & 28.10 & -1.20 & 27.11 & 31.01 & 31.03 & 26.77 \\
\hline $\mathrm{C} 12-\mathrm{CH}_{3} * *$ & 19 & 11.20 & 12.12 & 0.92 & 12.24 & 12.16 & 11.85 & 12.07 \\
\hline $\mathrm{C} 6 \mathrm{Me}-\mathrm{CH} 3$ & 12 & 12.70 & 12.19 & -0.51 & 12.30 & 12.03 & 12.05 & 12.20 \\
\hline $\mathrm{C} 8 \mathrm{Me}-\mathrm{CH} 3$ & 17 & 20.20 & 20.07 & -0.13 & 21.31 & 16.96 & 17.02 & $21.26]$ \\
\hline $\mathrm{C} 10 \mathrm{Me}-\mathrm{CH} 3 *$ & $* * 34$ & 19.40 & 18.91 & -0.49 & 19.55 & 15.09 & 19.51 & 19.73 \\
\hline $\mathrm{COMe}-\mathrm{CH} 3$ & 13 & $\begin{array}{l}56.20 \\
13 \mathrm{ch}\end{array}$ & $\begin{array}{l}54.44 \\
\text { m shift }\end{array}$ & $\begin{array}{l}-1.76 \\
\text { ts: } \text { RMSI }\end{array}$ & $\begin{array}{c}54.33 \\
1.14 \mathrm{ppm}\end{array}$ & $\begin{array}{r}54.51 \\
(\mathrm{MAE}=0.9\end{array}$ & 31) $\quad \mathrm{N}=16$ & $\begin{array}{l}54.50 \\
\{-2.57\end{array}$ \\
\hline
\end{tabular}

\section{Conformer 1}

Energy: -849.79562 Hartree (Rel: $0.0 \mathrm{kcal} / \mathrm{mol}$ ) XYZ coordinates for conf 1 :

$\begin{array}{lrrr}\text { C } & -3.55681 & -1.49585 & -0.39169 \\ \text { C } & -3.88112 & -0.08249 & -0.51915 \\ \text { C } & -2.94790 & 0.85577 & -0.21507 \\ \text { O } & -1.70328 & 0.55296 & 0.20911 \\ \text { C } & -1.32006 & -0.76144 & 0.35664 \\ \text { C } & -2.19371 & -1.75980 & 0.07433 \\ \text { O } & -3.04897 & 2.18500 & -0.26433 \\ \text { O } & -4.35927 & -2.40394 & -0.65224 \\ \text { C } & 0.06637 & -0.91928 & 0.82887 \\ \text { C } & 0.82629 & 0.17286 & 1.06159 \\ \text { C } & 2.24389 & 0.25140 & 1.56322 \\ \text { C } & 0.52884 & -2.34578 & 1.02237 \\ \text { C } & -4.30267 & 2.73718 & -0.68691 \\ \text { H } & -4.87014 & 0.19677 & -0.85671 \\ \text { C } & 3.11124 & 1.11618 & 0.61504 \\ \text { C } & 3.30242 & 0.58167 & -0.82094 \\ \text { C } & 2.25195 & 0.85622 & 2.98488 \\ \text { C } & 4.17537 & -0.69101 & -0.84558 \\ \text { C } & 4.26313 & -1.38554 & -2.21066 \\ \text { H } & -1.89724 & -2.79410 & 0.18926 \\ \text { H } & 0.36895 & 1.14585 & 0.88987 \\ \text { H } & 2.68205 & -0.74927 & 1.62855 \\ \text { H } & -0.10133 & -2.87008 & 1.75099 \\ \text { H } & 0.47654 & -2.91154 & 0.08422 \\ \text { H } & 1.55733 & -2.39126 & 1.38170 \\ \text { H } & -4.17034 & 3.81795 & -0.65178 \\ \text { H } & -4.53884 & 2.42152 & -1.70808 \\ \text { H } & -5.10571 & 2.43547 & -0.00701 \\ \text { H } & 3.98514 & 1.36884 & -2.75471 \\ \text { H } & 4.88131 & 1.99237 & -1.36008 \\ \text { H } & 3.24531 & 2.58024 & -1.70144 \\ \text { H } & 4.09903 & 1.25360 & 1.07901 \\ \text { H } & 2.65839 & 2.11729 & 0.56367 \\ \text { C } & 3.88585 & 1.69028 & -1.71261 \\ & & & \end{array}$

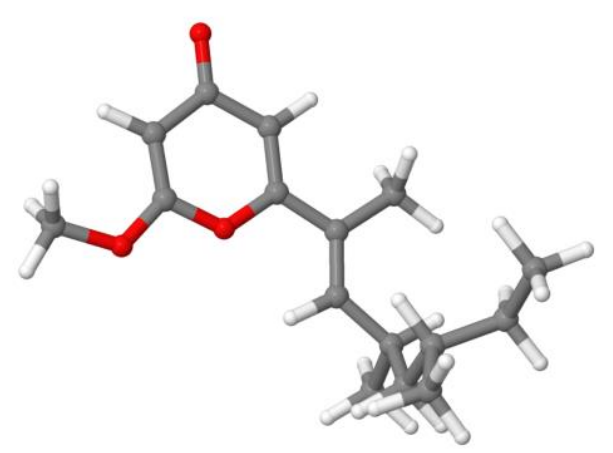




$\begin{array}{rrrr}\mathrm{H} & 2.31052 & 0.31643 & -1.21814 \\ \mathrm{H} & 3.27789 & 0.93167 & 3.36413 \\ \mathrm{H} & 1.67324 & 0.23924 & 3.68067 \\ \mathrm{H} & 1.81740 & 1.86351 & 2.98582 \\ \mathrm{H} & 3.78283 & -1.41348 & -0.11837 \\ \mathrm{H} & 5.18707 & -0.43134 & -0.49895 \\ \mathrm{H} & 4.83892 & -2.31554 & -2.13831 \\ \mathrm{H} & 4.75074 & -0.75661 & -2.96336 \\ \mathrm{H} & 3.26476 & -1.64186 & -2.58723\end{array}$

\section{Conformer 2}

Energy: -849.79483 Hartree (Rel: $0.5 \mathrm{kcal} / \mathrm{mol}$ ) XYZ coordinates for conf 2 :

\begin{tabular}{lrrr} 
C & -4.18927 & -1.11313 & -0.56595 \\
$\mathrm{C}$ & -4.33343 & 0.31995 & -0.35563 \\
$\mathrm{C}$ & -3.25507 & 1.06242 & 0.00430 \\
$\mathrm{O}$ & -2.02367 & 0.54187 & 0.18598 \\
$\mathrm{C}$ & -1.80590 & -0.80611 & 0.00681 \\
$\mathrm{C}$ & -2.83119 & -1.61782 & -0.35363 \\
$\mathrm{O}$ & -3.18187 & 2.37349 & 0.23997 \\
$\mathrm{O}$ & -5.13008 & -1.85020 & -0.89535 \\
$\mathrm{C}$ & -0.40980 & -1.21291 & 0.24286 \\
$\mathrm{C}$ & 0.50847 & -0.28959 & 0.60090 \\
$\mathrm{C}$ & 1.97590 & -0.47729 & 0.88316 \\
$\mathrm{C}$ & -0.12199 & -2.68526 & 0.05512 \\
$\mathrm{C}$ & -4.38707 & 3.13753 & 0.10377 \\
$\mathrm{H}$ & -5.30664 & 0.77171 & -0.49227 \\
$\mathrm{C}$ & 2.79254 & 0.31292 & -0.17386 \\
$\mathrm{C}$ & 4.33149 & 0.23178 & -0.07180 \\
$\mathrm{C}$ & 2.28194 & -0.01809 & 2.32428 \\
$\mathrm{C}$ & 4.96156 & 1.15169 & -1.14079 \\
$\mathrm{C}$ & 6.47713 & 1.34892 & -1.01279 \\
$\mathrm{H}$ & -2.66456 & -2.67743 & -0.49567 \\
$\mathrm{H}$ & 0.16985 & 0.73989 & 0.70286 \\
$\mathrm{H}$ & 2.23844 & -1.53657 & 0.80284 \\
$\mathrm{H}$ & -0.73451 & -3.29782 & 0.72783 \\
$\mathrm{H}$ & -0.34779 & -3.00623 & -0.96914 \\
$\mathrm{H}$ & 0.92417 & -2.92076 & 0.25304 \\
$\mathrm{H}$ & -4.10721 & 4.16467 & 0.33519 \\
$\mathrm{H}$ & -4.77014 & 3.07501 & -0.91976 \\
$\mathrm{H}$ & -5.14741 & 2.78809 & 0.80949 \\
$\mathrm{H}$ & 5.93227 & -1.26320 & -0.13107 \\
$\mathrm{H}$ & 4.53666 & -1.65472 & -1.14932 \\
$\mathrm{H}$ & 4.44578 & -1.85128 & 0.60908 \\
$\mathrm{H}$ & 2.49314 & 1.36986 & -0.11775 \\
$\mathrm{H}$ & 2.49123 & -0.03666 & -1.17191 \\
$\mathrm{C}$ & 4.83997 & -1.21399 & -0.19006 \\
$\mathrm{H}$ & 4.63603 & 0.62233 & 0.91052 \\
$\mathrm{H}$ & 3.33398 & -0.18228 & 2.57981 \\
$\mathrm{H}$ & 1.67099 & -0.57001 & 3.04689 \\
$\mathrm{H}$ & 2.06752 & 1.05083 & 2.44906 \\
$\mathrm{H}$ & 4.47441 & 2.13510 & -1.08393 \\
$\mathrm{H}$ & 4.72558 & 0.75313 & -2.13874 \\
$\mathrm{H}$ & 6.84049 & 2.06292 & -1.76097 \\
$\mathrm{H}$ & 7.02941 & 0.41412 & -1.15797 \\
$\mathrm{H}$ & 6.74161 & 1.74192 & -0.02289 \\
& & & \\
\hline & & &
\end{tabular}

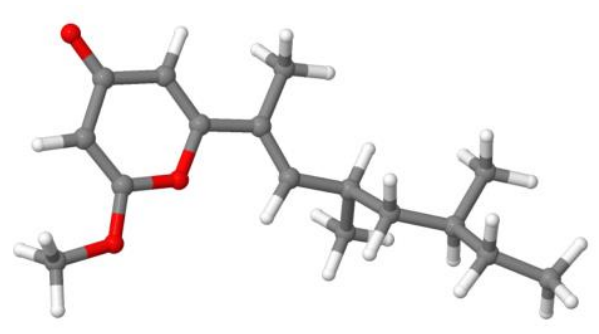

Conformer 3

Energy: -849.79462 Hartree (Rel: $0.6 \mathrm{kcal} / \mathrm{mol}$ ) XYZ coordinates for conf 3 :

$\begin{array}{llrr}C & -4.11377 & -1.07996 & -0.57427 \\ C & -4.22810 & 0.34637 & -0.30723 \\ C & -3.13282 & 1.05334 & 0.07242 \\ O & -1.91043 & 0.50223 & 0.22340 \\ C & -1.72038 & -0.84142 & -0.01064 \\ C & -2.76385 & -1.61852 & -0.39436 \\ O & -3.03202 & 2.35250 & 0.35812 \\ O & -5.07137 & -1.78496 & -0.92436 \\ C & -0.33069 & -1.28360 & 0.19848 \\ C & 0.60733 & -0.39214 & 0.58481 \\ C & 2.07228 & -0.61765 & 0.85135\end{array}$

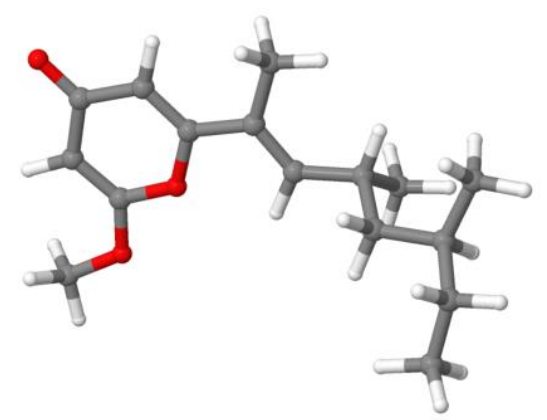




\begin{tabular}{|c|c|c|c|}
\hline C & -0.07263 & -2.75258 & -0.04882 \\
\hline C & -4.22253 & 3.14495 & 0.25723 \\
\hline $\mathrm{H}$ & -5.19343 & 0.82156 & -0.41799 \\
\hline C & 2.89834 & 0.17831 & -0.19497 \\
\hline C & 4.43475 & 0.05306 & -0.10527 \\
\hline C & 2.39787 & -0.19547 & 2.29954 \\
\hline C & 5.12900 & 0.93960 & -1.16335 \\
\hline C & 4.93203 & 2.45041 & -0.98715 \\
\hline $\mathrm{H}$ & -2.61915 & -2.67474 & -0.57962 \\
\hline $\mathrm{H}$ & 0.28892 & 0.63908 & 0.72675 \\
\hline $\mathrm{H}$ & 2.31116 & -1.68039 & 0.74746 \\
\hline $\mathrm{H}$ & -0.68800 & -3.37955 & 0.60787 \\
\hline $\mathrm{H}$ & -0.31740 & -3.02950 & -1.08150 \\
\hline $\mathrm{H}$ & 0.97152 & -3.01466 & 0.12517 \\
\hline $\mathrm{H}$ & -3.92043 & 4.15738 & 0.52289 \\
\hline $\mathrm{H}$ & -4.61285 & 3.12612 & -0.76525 \\
\hline $\mathrm{H}$ & -4.98542 & 2.78591 & 0.95533 \\
\hline $\mathrm{H}$ & 5.99752 & -1.46625 & -0.21831 \\
\hline $\mathrm{H}$ & 4.57738 & -1.82302 & -1.21523 \\
\hline $\mathrm{H}$ & 4.51222 & -2.04806 & 0.54060 \\
\hline $\mathrm{H}$ & 2.61554 & 1.23603 & -0.11057 \\
\hline $\mathrm{H}$ & 2.58338 & -0.14336 & -1.19845 \\
\hline C & 4.90341 & -1.40350 & -0.25410 \\
\hline $\mathrm{H}$ & 4.75879 & 0.41512 & 0.88174 \\
\hline $\mathrm{H}$ & 3.44740 & -0.38888 & 2.54449 \\
\hline $\mathrm{H}$ & 1.77892 & -0.74809 & 3.01480 \\
\hline $\mathrm{H}$ & 2.20888 & 0.87527 & 2.44769 \\
\hline $\mathrm{H}$ & 4.78543 & 0.63727 & -2.16389 \\
\hline $\mathrm{H}$ & 6.20578 & 0.72245 & -1.13513 \\
\hline $\mathrm{H}$ & 5.52389 & 3.00657 & -1.72335 \\
\hline $\mathrm{H}$ & 5.25253 & 2.77652 & 0.01053 \\
\hline $\mathrm{H}$ & 3.88656 & 2.75173 & -1.11528 \\
\hline
\end{tabular}

\section{Conformer 4}

Energy: -849.79560 Hartree (Rel: $0.0 \mathrm{kcal} / \mathrm{mol}$ ) XYZ coordinates for conf 4 :

\begin{tabular}{|c|c|c|c|}
\hline C & -3.58572 & -1.39334 & -0.52346 \\
\hline C & -3.86531 & 0.03512 & -0.51408 \\
\hline C & -2.90349 & 0.91020 & -0.12329 \\
\hline 0 & -1.66934 & 0.52935 & 0.26741 \\
\hline C & -1.32716 & -0.80439 & 0.28560 \\
\hline C & -2.23158 & -1.74333 & -0.08902 \\
\hline 0 & -2.96285 & 2.24057 & -0.04570 \\
\hline O & -4.41620 & -2.24652 & -0.86820 \\
\hline C & 0.05464 & -1.05011 & 0.73289 \\
\hline C & 0.84716 & -0.01119 & 1.07472 \\
\hline C & 2.26745 & -0.03187 & 1.57509 \\
\hline C & 0.47579 & -2.50146 & 0.77669 \\
\hline C & -4.19743 & 2.86906 & -0.41488 \\
\hline $\mathrm{H}$ & -4.84441 & 0.37652 & -0.82198 \\
\hline C & 3.14330 & 0.96571 & 0.77830 \\
\hline C & 3.28861 & 0.70129 & -0.73595 \\
\hline C & 2.29018 & 0.32951 & 3.07736 \\
\hline C & 4.07480 & -0.59579 & -1.02039 \\
\hline C & 4.12303 & -1.00919 & -2.49696 \\
\hline $\mathrm{H}$ & -1.96745 & -2.79267 & -0.07597 \\
\hline $\mathrm{H}$ & 0.41817 & 0.98751 & 1.01155 \\
\hline $\mathrm{H}$ & 2.69296 & -1.03505 & 1.47263 \\
\hline $\mathrm{H}$ & -0.16638 & -3.07879 & 1.45285 \\
\hline $\mathrm{H}$ & 0.40373 & -2.96765 & -0.21349 \\
\hline $\mathrm{H}$ & 1.50374 & -2.61276 & 1.12344 \\
\hline $\mathrm{H}$ & -4.03112 & 3.93714 & -0.27938 \\
\hline $\mathrm{H}$ & -4.44049 & 2.65718 & -1.46092 \\
\hline $\mathrm{H}$ & -5.01131 & 2.53095 & 0.23429 \\
\hline $\mathrm{H}$ & 3.99836 & 1.80399 & -2.49850 \\
\hline $\mathrm{H}$ & 4.95515 & 2.08175 & -1.03413 \\
\hline $\mathrm{H}$ & 3.36031 & 2.83268 & -1.21075 \\
\hline $\mathrm{H}$ & 4.14391 & 0.98765 & 1.23493 \\
\hline $\mathrm{H}$ & 2.72251 & 1.97203 & 0.92125 \\
\hline C & 3.93621 & 1.92141 & -1.41159 \\
\hline $\mathrm{H}$ & 2.27826 & 0.57934 & -1.15602 \\
\hline
\end{tabular}

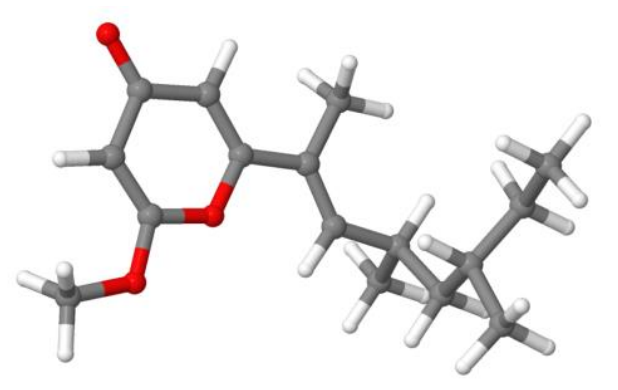




$\begin{array}{rrrr}\mathrm{H} & 3.31955 & 0.33386 & 3.45486 \\ \mathrm{H} & 1.71153 & -0.39035 & 3.66607 \\ \mathrm{H} & 1.86425 & 1.32576 & 3.24927 \\ \mathrm{H} & 3.63049 & -1.42038 & -0.44864 \\ \mathrm{H} & 5.10001 & -0.47863 & -0.63784 \\ \mathrm{H} & 4.63254 & -1.97260 & -2.61487 \\ \mathrm{H} & 4.65799 & -0.27846 & -3.11315 \\ \mathrm{H} & 3.11184 & -1.11596 & -2.90977\end{array}$


revised structure of cheliensisamine (cmpd IV), Fig.9B<smiles>CC(=O)C1=C(N)C(=O)c2cccc(O)c2C1=O</smiles>

$$
\text { Rel energy (kcal/mol): }
$$

\begin{tabular}{|c|c|c|c|c|c|}
\hline C-nom & iGau & $\operatorname{Exp}$ & Calc & $\operatorname{diff}$ & 1 \\
\hline $\mathrm{C}-\mathrm{C}$ & 14 & 200.42 & 203.33 & 2.91 & [ 205.77 \\
\hline$C-C$ & 7 & 183.51 & 181.12 & -2.39 & [ 183.13 \\
\hline$C-C$ & 10 & 179.85 & 178.94 & -0.91 & 180.91 \\
\hline$C-C$ & 3 & 160.19 & 161.60 & 1.41 & 163.23 \\
\hline$C-C$ & 8 & 153.78 & 152.88 & -0.90 & 154.34 \\
\hline$C-C$ & 5 & 134.00 & 133.44 & -0.56 & [ 134.52 \\
\hline$C-C$ & 4 & 114.58 & 114.16 & -0.42 & [ 114.87 \\
\hline$C-C$ & 9 & 107.88 & 105.75 & -2.13 & [ 106.30 \\
\hline $\mathrm{C}-\mathrm{CH}$ & 1 & 138.17 & 139.95 & 1.78 & [ 141.16 \\
\hline $\mathrm{C}-\mathrm{CH}$ & 2 & 121.62 & 121.15 & -0.47 & 122.00 \\
\hline $\mathrm{C}-\mathrm{CH}$ & 6 & 118.48 & 119.01 & 0.53 & 119.82 \\
\hline $\mathrm{C}-\mathrm{CH} 3$ & 16 & 32.77 & 35.20 & 2.43 & 34.38 \\
\hline
\end{tabular}

$13 \mathrm{C}$ chem shifts: $\mathrm{CRMSD}=1.64 \mathrm{ppm} \quad(\mathrm{CMAE}=1.40) \quad \mathrm{N}=12 \quad\left\{\begin{array}{lll}-2.39 & 2.91\end{array}\right\}$

\section{Conformer 1}

Energy: -818.36506 Hartree (Rel: $0.0 \mathrm{kcal} / \mathrm{mol}$ ) XYZ coordinates for conf 1 :

$\begin{array}{lrrr}\mathrm{C} & -2.97940 & -2.01739 & 0.00034 \\ \mathrm{C} & -3.57108 & -0.76327 & 0.00027 \\ \mathrm{C} & -2.76401 & 0.38776 & 0.00003 \\ \mathrm{C} & -1.35050 & 0.24803 & -0.00010 \\ \mathrm{C} & -0.76739 & -1.04250 & -0.00003 \\ \mathrm{C} & -1.58130 & -2.16509 & 0.00017 \\ \mathrm{C} & -0.50552 & 1.41715 & -0.00018 \\ \mathrm{C} & 0.99749 & 1.22865 & 0.00007 \\ \mathrm{C} & 1.59120 & -0.04389 & 0.00005 \\ \mathrm{C} & 0.72844 & -1.22791 & -0.00028 \\ \mathrm{O} & 1.16802 & -2.38318 & -0.00077 \\ \mathrm{O} & -0.94380 & 2.58153 & -0.00031 \\ \mathrm{H} & -1.12332 & -3.14694 & 0.00018 \\ \mathrm{C} & 3.06468 & -0.14514 & 0.00011 \\ \mathrm{~N} & 1.65608 & 2.37865 & 0.00020 \\ \mathrm{C} & 3.76761 & -1.48589 & 0.00023 \\ \mathrm{O} & 3.77221 & 0.87813 & 0.00019 \\ \mathrm{H} & -3.60949 & -2.90199 & 0.00054 \\ \mathrm{H} & -4.64927 & -0.64268 & 0.00040 \\ \mathrm{O} & -3.36557 & 1.58618 & -0.00007 \\ \mathrm{H} & -2.65276 & 2.27390 & -0.00015 \\ \mathrm{H} & 2.67423 & 2.34069 & 0.00021 \\ \mathrm{H} & 1.12084 & 3.23960 & 0.00010 \\ \mathrm{H} & 4.84390 & -1.29783 & 0.00050 \\ \mathrm{H} & 3.48774 & -2.07986 & -0.87437 \\ \mathrm{H} & 3.48741 & -2.07979 & 0.87481\end{array}$

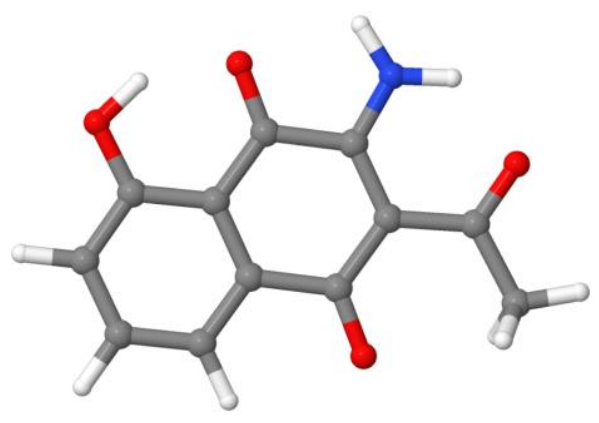


2-acetyl-2-methyl-6-methoxybenzoxete (cmpd 4d) Fig.10<smiles>COc1cccc2c1OC2(C)C(C)=O</smiles>

$$
\text { Rel energy (kcal/mol): } \begin{array}{cc}
\text { Conf } 1 & \operatorname{Conf} 2 \\
0.9 & 0.0
\end{array}
$$

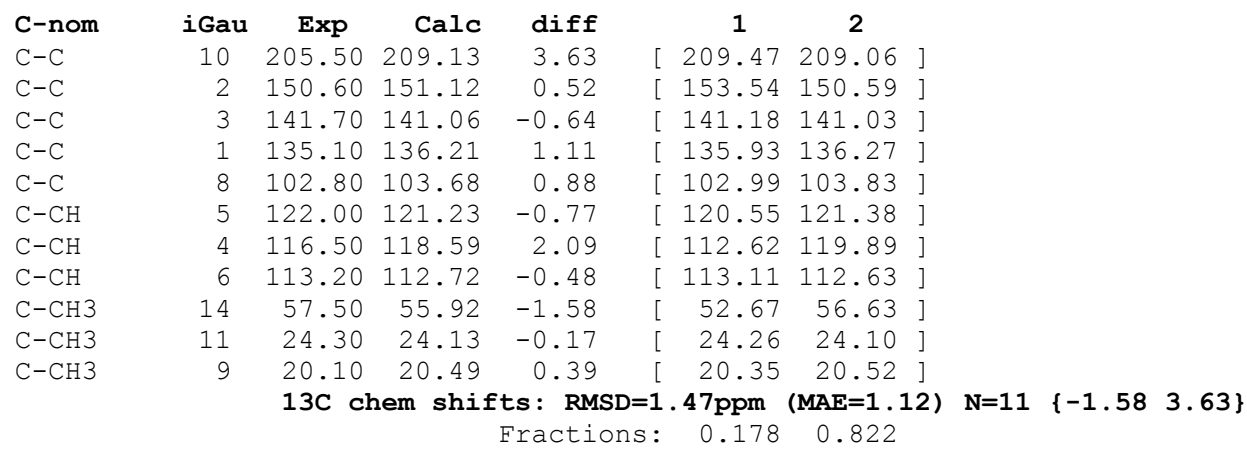

\section{Conformer 1}

Energy: -651.99068 Hartree (Rel: $0.9 \mathrm{kcal} / \mathrm{mol}$ ) XYZ coordinates for conf 1 :

\begin{tabular}{|c|c|c|c|}
\hline C & -0.39958 & 0.85562 & 0.15021 \\
\hline C & 0.46040 & -0.21377 & 0.29496 \\
\hline C & 1.83028 & -0.14874 & 0.05227 \\
\hline C & 2.26768 & 1.12776 & -0.35900 \\
\hline C & 1.39012 & 2.21622 & -0.51005 \\
\hline C & 0.01205 & 2.11223 & -0.26162 \\
\hline O & -0.46982 & -1.16334 & 0.69878 \\
\hline C & -1.51959 & -0.09351 & 0.58212 \\
\hline C & -2.20118 & 0.14600 & 1.91484 \\
\hline C & -2.51091 & -0.39439 & -0.55330 \\
\hline C & -2.15548 & -1.48148 & -1.54127 \\
\hline 0 & -3.52951 & 0.26608 & -0.62746 \\
\hline 0 & 2.59902 & -1.25860 & 0.21941 \\
\hline C & 3.98997 & -1.14334 & -0.03899 \\
\hline $\mathrm{H}$ & 3.32009 & 1.28442 & -0.57019 \\
\hline $\mathrm{H}$ & 1.80489 & 3.16755 & -0.83108 \\
\hline $\mathrm{H}$ & -0.65682 & 2.95732 & -0.38699 \\
\hline $\mathrm{H}$ & -2.93324 & 0.95031 & 1.80498 \\
\hline $\mathrm{H}$ & -2.72840 & -0.75822 & 2.23796 \\
\hline $\mathrm{H}$ & -1.46439 & 0.41934 & 2.67446 \\
\hline $\mathrm{H}$ & -2.12478 & -2.45313 & -1.03528 \\
\hline $\mathrm{H}$ & -2.90049 & -1.49819 & -2.33922 \\
\hline $\mathrm{H}$ & -1.15647 & -1.31561 & -1.95870 \\
\hline $\mathrm{H}$ & 4. 41171 & -2.13179 & 0.15100 \\
\hline $\mathrm{H}$ & 4.18365 & -0.85938 & -1.08194 \\
\hline $\mathrm{H}$ & 4.46422 & -0.41136 & 0.62815 \\
\hline
\end{tabular}

\section{Conformer 2}

Energy: -651.99213 Hartree (Rel: $0.0 \mathrm{kcal} / \mathrm{mol})$ XYZ coordinates for conf 2:

$\begin{array}{lrrr}\text { C } & -0.28685 & 0.92552 & 0.21854 \\ C & 0.63405 & -0.10920 & 0.19935 \\ C & 1.98331 & 0.07073 & -0.08956 \\ C & 2.32577 & 1.41304 & -0.36473 \\ C & 1.39385 & 2.45605 & -0.35163 \\ C & 0.03287 & 2.24020 & -0.05772 \\ \text { O } & -0.24650 & -1.14586 & 0.52555 \\ C & -1.34284 & -0.11967 & 0.57811 \\ C & -1.97407 & -0.06954 & 1.95575 \\ C & -2.37153 & -0.32942 & -0.54473\end{array}$

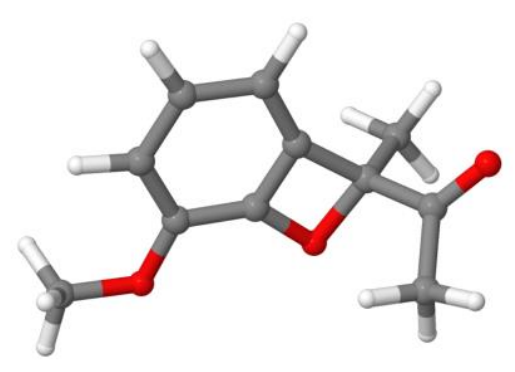




$\begin{array}{lrrr}\mathrm{C} & -2.01080 & -1.25941 & -1.68075 \\ \mathrm{O} & -3.42370 & 0.27797 & -0.49186 \\ \mathrm{O} & 2.96625 & -0.86721 & -0.13523 \\ \mathrm{C} & 2.58913 & -2.20626 & 0.17987 \\ \mathrm{H} & 3.36738 & 1.61763 & -0.59493 \\ \mathrm{H} & 1.73918 & 3.46138 & -0.57521 \\ \mathrm{H} & -0.68776 & 3.05128 & -0.05362 \\ \mathrm{H} & -2.74492 & 0.70527 & 1.96972 \\ \mathrm{H} & -2.44677 & -1.02938 & 2.19108 \\ \mathrm{H} & -1.21715 & 0.15092 & 2.71277 \\ \mathrm{H} & -1.91473 & -2.28626 & -1.30973 \\ \mathrm{H} & -2.78980 & -1.21063 & -2.44411 \\ \mathrm{H} & -1.04129 & -0.98796 & -2.11221 \\ \mathrm{H} & 3.50036 & -2.80128 & 0.09755 \\ \mathrm{H} & 2.19212 & -2.27822 & 1.19911 \\ \mathrm{H} & 1.83765 & -2.58416 & -0.52312\end{array}$


originally assigned structure of phomopsidone A, Fig.10<smiles>Cc1cc2c(c3c1C(=O)Oc1c(C)c4c(c(O)c1O3)C(=O)OC4)CO2</smiles>

Rel energy (kcal/mol):

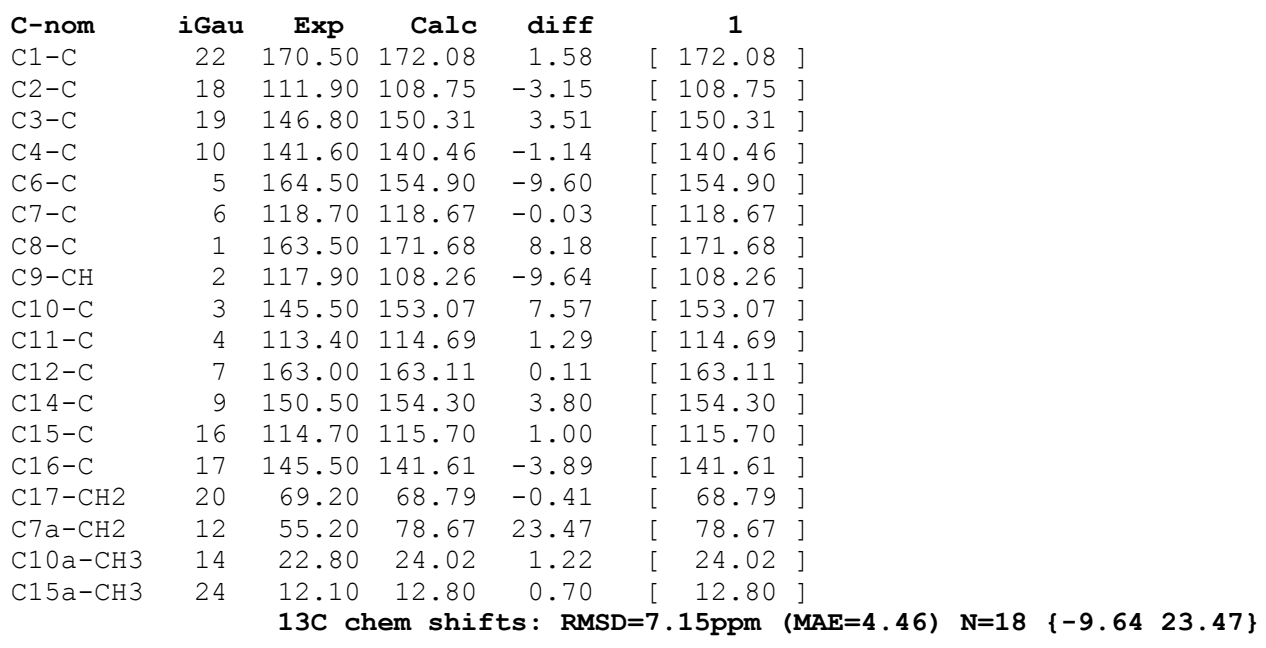

\section{Conformer 1}

Energy: -1219.64196 Hartree (Rel: $0.0 \mathrm{kcal} / \mathrm{mol}$ ) XYZ coordinates for conf 1 :

\begin{tabular}{lrrr} 
XYZ Coordinates for Conf & \multicolumn{1}{l}{ : } & \\
C & 3.51234 & -1.53282 & 0.57486 \\
C & 4.01807 & -0.35171 & 1.08285 \\
C & 3.33621 & 0.81639 & 0.68646 \\
C & 2.17604 & 0.74517 & -0.14377 \\
C & 1.69274 & -0.51270 & -0.60404 \\
C & 2.39602 & -1.63017 & -0.24105 \\
C & 1.57928 & 2.00707 & -0.65195 \\
O & 0.21021 & 2.08996 & -0.89978 \\
C & -0.75734 & 1.18098 & -0.54654 \\
C & -0.58535 & -0.17915 & -0.83044 \\
O & 0.58608 & -0.61384 & -1.42249 \\
C & 2.60648 & -3.12614 & -0.22149 \\
O & 3.79247 & -2.87899 & 0.65696 \\
C & 3.88530 & 2.13622 & 1.18405 \\
O & 2.20187 & 3.01638 & -0.87329 \\
C & -1.94217 & 1.71882 & 0.00643 \\
C & -2.94058 & 0.78804 & 0.27085 \\
C & -2.76577 & -0.56607 & 0.00742 \\
C & -1.59693 & -1.10221 & -0.53436 \\
C & -4.33035 & 0.92678 & 0.85336 \\
O & -4.86717 & -0.41769 & 0.89321 \\
C & -3.95620 & -1.30647 & 0.39799 \\
O & -4.15193 & -2.50599 & 0.31776 \\
C & -2.07222 & 3.19843 & 0.26499 \\
O & -1.44236 & -2.41947 & -0.76691 \\
H & 4.88266 & -0.30155 & 1.73487 \\
H & 2.91899 & -3.60402 & -1.15254 \\
H & 1.86289 & -3.72936 & 0.30390
\end{tabular}

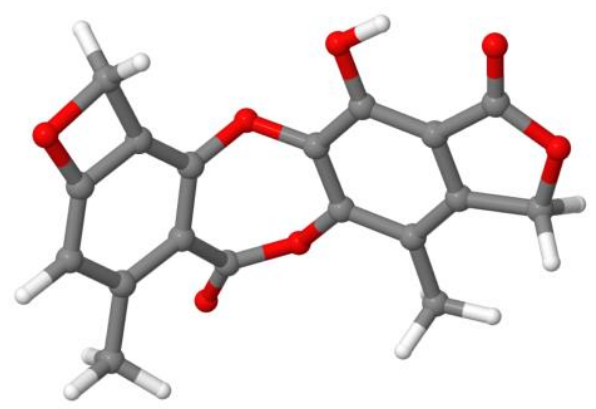




$\begin{array}{rrr}4.59285 & 1.96292 & 2.00011 \\ 4.40150 & 2.67152 & 0.38157 \\ 3.09793 & 2.80499 & 1.54269 \\ -4.33744 & 1.32134 & 1.87526 \\ -5.00066 & 1.53780 & 0.23896 \\ -1.31798 & 3.54885 & 0.97895 \\ -3.05925 & 3.44185 & 0.66968 \\ -1.92709 & 3.77690 & -0.65388 \\ -2.28119 & -2.85684 & -0.49696\end{array}$


revised phomopsidone A - excelsione (cmpd 1), Fig.10<smiles>Cc1cc(O)c(CO)c2c1C(=O)Oc1c(C)c3c(c(O)c1O2)C(=O)OC3</smiles>

Free "mixing" of the two conformers is predicated on the hypothesis that pyridine (solvent) successfully competes for H-bond, disrupting intramolecular H-bonds

$$
\text { Rel energy (kcal/mol): } \begin{array}{ccc}
\text { Conf } 1 & \text { Conf } 2 \\
0.0 & 6.1
\end{array}
$$

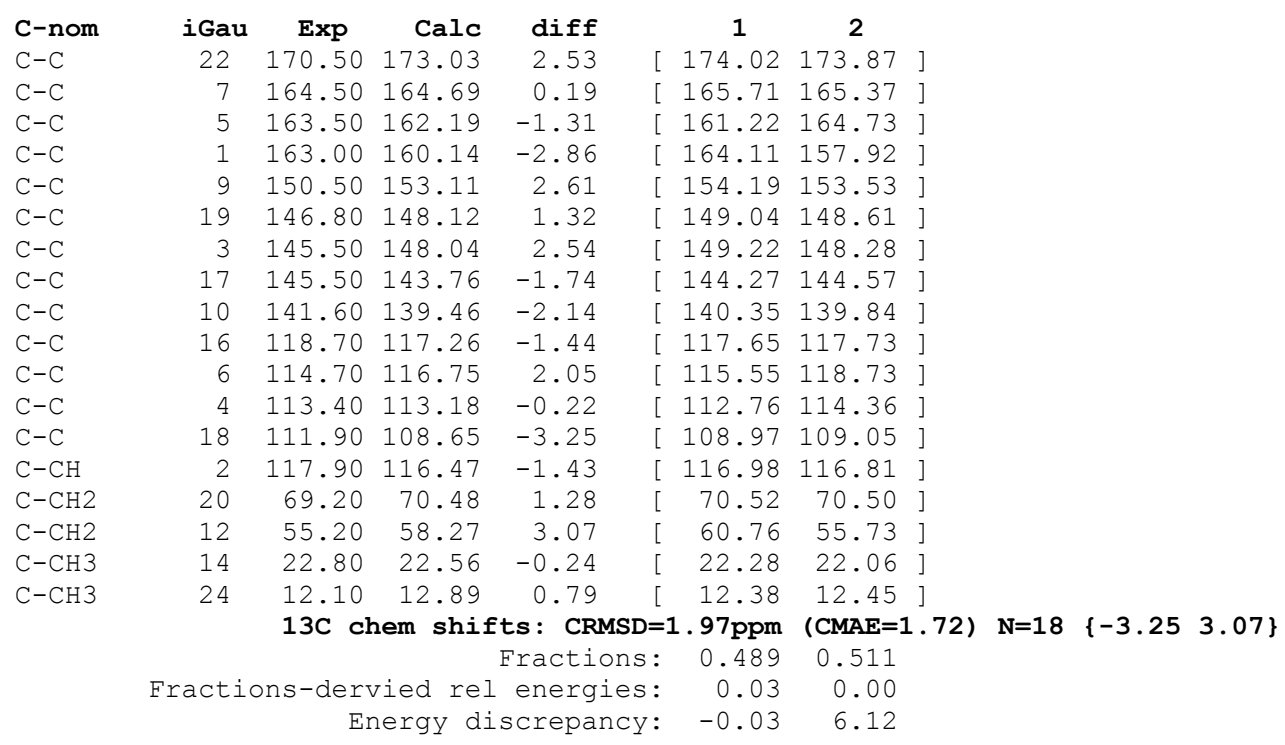

\section{Conformer 1}

Energy: -1296.13503 Hartree (Rel: $0.0 \mathrm{kcal} / \mathrm{mol}$ ) XYZ coordinates for conf 1 :

$\begin{array}{llrr}\text { C } & 3.52871 & -1.06181 & 0.56889 \\ C & 3.84481 & 0.22039 & 1.03604 \\ C & 3.09718 & 1.33674 & 0.67744 \\ C & 1.94834 & 1.15318 & -0.14049 \\ C & 1.62961 & -0.15176 & -0.56601 \\ C & 2.40306 & -1.26867 & -0.25678 \\ C & 1.23569 & 2.33674 & -0.67242 \\ O & -0.12807 & 2.29444 & -0.91716 \\ C & -0.99956 & 1.29724 & -0.54143 \\ C & -0.68411 & -0.04073 & -0.80882 \\ O & 0.52948 & -0.35426 & -1.39637 \\ C & 2.10168 & -2.63823 & -0.83248 \\ O & 4.32806 & -2.08387 & 0.94287 \\ C & 3.53029 & 2.68981 & 1.19302 \\ O & 1.76875 & 3.39484 & -0.92399 \\ C & -2.22708 & 1.72120 & 0.01233 \\ C & -3.12541 & 0.69713 & 0.29213 \\ C & -2.81844 & -0.63492 & 0.03359 \\ C & -1.60413 & -1.05389 & -0.51123 \\ C & -4.51501 & 0.70854 & 0.88559 \\ C & -4.91885 & -0.68814 & 0.92699 \\ O & -3.93182 & -1.48122 & 0.43058 \\ C & -4.01952 & -2.69794 & 0.35542 \\ O & -2.50682 & 3.18135 & 0.26040\end{array}$

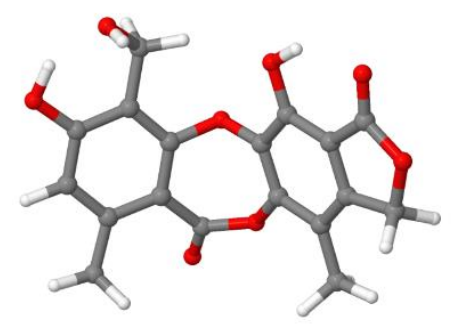




$\begin{array}{rrr}-1.32702 & -2.35287 & -0.75016 \\ 4.70881 & 0.32635 & 1.68442 \\ 2.74296 & -2.83407 & -1.69860 \\ 1.06191 & -2.70464 & -1.15528 \\ 4.25119 & 2.56694 & 2.00609 \\ 4.00098 & 3.27897 & 0.39991 \\ 2.68586 & 3.27883 & 1.56281 \\ -4.54919 & 1.09520 & 1.90858 \\ -5.24674 & 1.24869 & 0.27744 \\ -2.42865 & 3.76318 & -0.66450 \\ -1.78947 & 3.60946 & 0.96981 \\ -3.51144 & 3.32358 & 0.66730 \\ -2.11239 & -2.87498 & -0.47226 \\ 2.40979 & -3.70400 & 0.09204 \\ 3.85819 & -2.92372 & 0.71364 \\ 1.74489 & -3.68576 & 0.80151\end{array}$

\section{Conformer 2}

Energy: -1296.12527 Hartree (Rel: $6.1 \mathrm{kcal} / \mathrm{mol}$ ) XYZ coordinates for conf 2 :

$\begin{array}{lrrr}\text { C } & -3.52184 & -1.18075 & -0.84662 \\ \mathrm{C} & -3.88903 & 0.13154 & -1.17799 \\ \mathrm{C} & -3.18809 & 1.23468 & -0.70944 \\ \mathrm{C} & -2.03665 & 1.01239 & 0.09562 \\ \mathrm{C} & -1.66862 & -0.31366 & 0.37307 \\ \mathrm{C} & -2.38014 & -1.43370 & -0.06460 \\ \mathrm{C} & -1.37586 & 2.16169 & 0.75707 \\ \mathrm{O} & -0.01282 & 2.15563 & 0.99748 \\ \mathrm{C} & 0.90757 & 1.23534 & 0.54885 \\ \mathrm{C} & 0.65153 & -0.13550 & 0.67547 \\ \mathrm{O} & -0.56171 & -0.56800 & 1.18654 \\ \mathrm{C} & -1.89652 & -2.82362 & 0.31928 \\ \mathrm{O} & -4.32236 & -2.15321 & -1.35139 \\ \mathrm{C} & -3.67761 & 2.61475 & -1.08213 \\ \mathrm{O} & -1.95778 & 3.15972 & 1.11870 \\ \mathrm{C} & 2.12869 & 1.76421 & 0.07667 \\ \mathrm{C} & 3.08690 & 0.81355 & -0.25880 \\ \mathrm{C} & 2.84219 & -0.54925 & -0.12656 \\ \mathrm{C} & 1.63472 & -1.07236 & 0.33573 \\ \mathrm{C} & 4.49446 & 0.94508 & -0.79235 \\ \mathrm{O} & 4.97263 & -0.42097 & -0.93622 \\ \mathrm{C} & 4.01270 & -1.30226 & -0.54741 \\ \mathrm{O} & 4.16207 & -2.51478 & -0.56932 \\ \mathrm{C} & 2.34427 & 3.25245 & -0.02575 \\ \mathrm{O} & 1.41745 & -2.39743 & 0.46517 \\ \mathrm{H} & -4.76496 & 0.26839 & -1.80398 \\ \mathrm{H} & -0.91850 & -3.00740 & -0.14167 \\ \mathrm{H} & -2.57065 & -3.59702 & -0.06172 \\ \mathrm{H} & -4.39399 & 2.54733 & -1.90552 \\ \mathrm{H} & -4.17085 & 3.09805 & -0.23320 \\ \mathrm{H} & -2.85846 & 3.27276 & -1.38675 \\ \mathrm{H} & 4.54576 & 1.42111 & -1.77609 \\ \mathrm{H} & 5.17347 & 1.46538 & -0.11036 \\ \mathrm{H} & 2.25551 & 3.73503 & 0.95402 \\ \mathrm{H} & 1.59982 & 3.71816 & -0.68096 \\ \mathrm{H} & 3.33630 & 3.47791 & -0.42588 \\ \mathrm{H} & 2.23692 & -2.85792 & 0.17708 \\ \mathrm{O} & -1.83745 & -3.03327 & 1.72557 \\ \mathrm{H} & -4.04216 & -3.02988 & -1.04383 \\ \mathrm{H} & -1.12046 & -2.46078 & 2.04607 \\ & & & \end{array}$

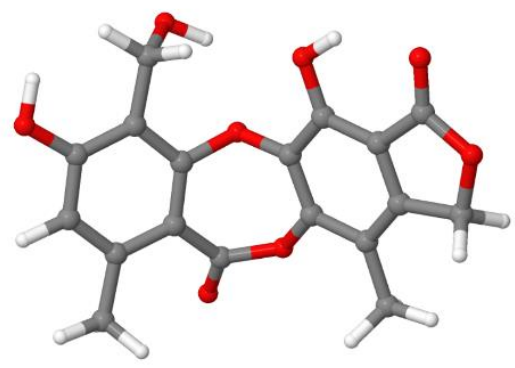


originally assigned structure of amentotaxone, Fig.10<smiles>CC1=C(O)C(=O)c2c(C)cc3c(c2C1=O)C(C)(C)O3</smiles>

\begin{tabular}{|c|c|c|c|c|c|}
\hline $\mathrm{C}-$ nom & iGau & Exp & Calc & $\operatorname{diff}$ & 1 \\
\hline $\mathrm{C} 1-\mathrm{C}$ & 10 & 178.80 & 175.53 & -3.27 & [ 175.53 \\
\hline $\mathrm{C} 2-\mathrm{C}$ & 9 & 170.80 & 153.30 & -17.50 & 153.30 \\
\hline $\mathrm{C} 3-\mathrm{C}$ & 8 & 109.00 & 116.48 & 7.48 & 116.48 \\
\hline $\mathrm{C} 4-\mathrm{C}$ & 7 & 182.70 & 185.29 & 2.59 & 185.29 \\
\hline $\mathrm{C} 4 \mathrm{a}-\mathrm{C}$ & 2 & 137.60 & 130.31 & -7.29 & 130.31 \\
\hline $\mathrm{C} 5-\mathrm{C}$ & 3 & 132.90 & 138.70 & 5.80 & 138.70 \\
\hline $\mathrm{C} 6-\mathrm{C}$ & 4 & 160.10 & 170.69 & 10.59 & 170.69 \\
\hline $\mathrm{C} 7-\mathrm{CH}$ & 5 & 121.50 & 112.70 & -8.80 & 112.70 \\
\hline $\mathrm{C} 8-\mathrm{C}$ & 6 & 148.70 & 152.03 & 3.33 & 152.03 \\
\hline $\mathrm{C} 8 \mathrm{a}-\mathrm{C}$ & 1 & 117.20 & 117.76 & 0.56 & 117.76 \\
\hline $\mathrm{C} 9-\mathrm{C}$ & 12 & 98.00 & 104.44 & 6.44 & 104.44 \\
\hline $\mathrm{C} 10-\mathrm{CH} 3$ & 13 & 25.90 & 23.69 & -2.21 & 23.69 \\
\hline $\mathrm{C} 11-\mathrm{CH} 3$ & 14 & 25.90 & 23.69 & -2.21 & 23.69 \\
\hline $\mathrm{C} 12-\mathrm{CH} 3$ & 20 & 7.70 & 9.88 & 2.18 & 9.88 \\
\hline $\mathrm{C} 13-\mathrm{CH} 3$ & 15 & 20.60 & 25.56 & 4.96 & 25.56 \\
\hline
\end{tabular}

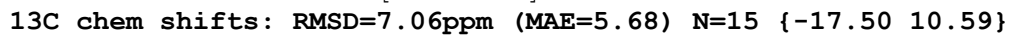

\section{Conformer 1}

Energy: -880.88744 Hartree (Rel: $0.0 \mathrm{kcal} / \mathrm{mol}$ ) XYZ coordinates for conf 1 :

\begin{tabular}{|c|c|c|c|}
\hline C & 0.65133 & 1.02917 & 0.00005 \\
\hline C & -0.11972 & -0.17809 & 0.00023 \\
\hline $\mathrm{C}$ & -1.48050 & -0.01739 & 0.00015 \\
\hline $\mathrm{C}$ & -2.07815 & 1.23681 & -0.00008 \\
\hline C & -1.37769 & 2.42960 & -0.00019 \\
\hline C & 0.02973 & 2.31369 & -0.00009 \\
\hline C & 0.48649 & -1.54789 & 0.00063 \\
\hline C & 1.94329 & -1.67317 & 0.00016 \\
\hline C & 2.67951 & -0.53073 & -0.00001 \\
\hline C & 2.10116 & 0.86371 & 0.00002 \\
\hline 0 & -3.38187 & 0.83282 & -0.00024 \\
\hline C & -2.87420 & -0.62347 & 0.00002 \\
\hline C & -3.31038 & -1.31879 & 1.27486 \\
\hline C & -3.30998 & -1.31912 & -1.27477 \\
\hline C & 0.83241 & 3.59370 & -0.00022 \\
\hline 0 & 2.93925 & 1.77639 & 0.00063 \\
\hline 0 & -0.25338 & -2.53544 & -0.00028 \\
\hline 0 & 4.01838 & -0.52787 & -0.00036 \\
\hline $\mathrm{H}$ & -1.85595 & 3.40316 & -0.00038 \\
\hline C & 2.54384 & -3.04953 & -0.00010 \\
\hline $\mathrm{H}$ & -2.80159 & -2.28577 & 1.33961 \\
\hline $\mathrm{H}$ & -4.39312 & -1.48398 & 1.27007 \\
\hline $\mathrm{H}$ & -3.04664 & -0.72457 & 2.15433 \\
\hline $\mathrm{H}$ & -3.04602 & -0.72510 & -2.15431 \\
\hline $\mathrm{H}$ & -2.80112 & -2.28609 & -1.33914 \\
\hline $\mathrm{H}$ & -4.39271 & -1.48437 & -1.27026 \\
\hline $\mathrm{H}$ & 0.16271 & 4.45890 & -0.00035 \\
\hline $\mathrm{H}$ & 1.48977 & 3.65612 & 0.87242 \\
\hline $\mathrm{H}$ & 1.48983 & 3.65589 & -0.87282 \\
\hline $\mathrm{H}$ & 4.25727 & 0.43143 & -0.00076 \\
\hline $\mathrm{H}$ & 3.63446 & -3.00315 & 0.00030 \\
\hline $\mathrm{H}$ & 2.21093 & -3.61509 & -0.87824 \\
\hline $\mathrm{H}$ & 2.21023 & -3.61568 & 0.87738 \\
\hline
\end{tabular}

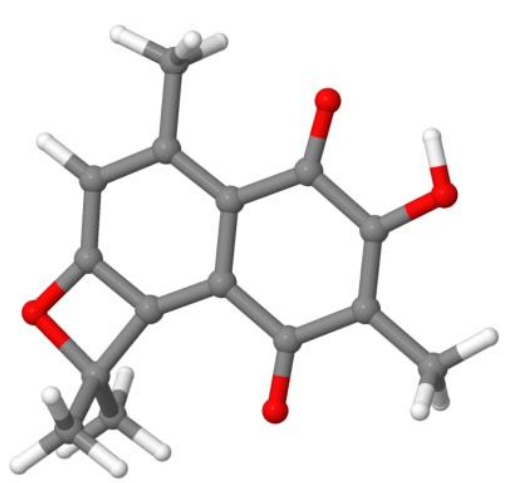


revised structure of amentotaxone (PCM methanol), Fig.10<smiles>CC1=C2OC(C)(C)c3c(O)cc(C)c(c32)C(=O)C1=O</smiles>

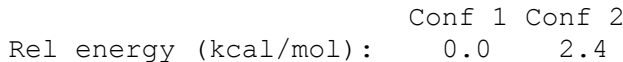

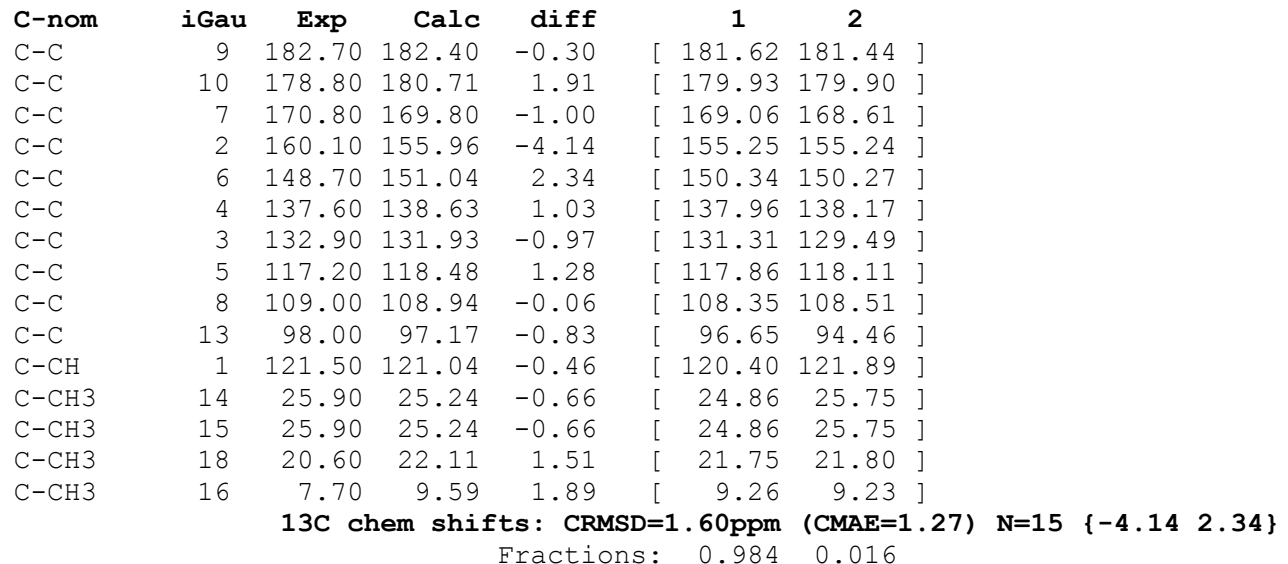

\section{Conformer 1}

Energy: -880.92704 Hartree (Rel: $0.0 \mathrm{kcal} / \mathrm{mol}$ ) XYZ coordinates for conf 1 :

\begin{tabular}{|c|c|c|c|}
\hline C & -1.24012 & 2.49423 & -0.00012 \\
\hline C & -2.06295 & 1.35332 & 0.00009 \\
\hline C & -1.45464 & 0.09086 & 0.00003 \\
\hline $\mathrm{C}$ & -0.07712 & 0.04411 & -0.00018 \\
\hline C & 0.77311 & 1.15196 & -0.00036 \\
\hline $\mathrm{C}$ & 0.16282 & 2.42928 & -0.00031 \\
\hline $\mathrm{C}$ & 0.33893 & -1.34288 & -0.00018 \\
\hline C & 1.64136 & -1.74351 & -0.00028 \\
\hline $\mathrm{C}$ & 2.63876 & -0.67907 & -0.00042 \\
\hline $\mathrm{C}$ & 2.21027 & 0.84303 & -0.00052 \\
\hline 0 & 3.10208 & 1.68167 & 0.00112 \\
\hline 0 & -0.74942 & -2.13794 & -0.00002 \\
\hline C & -1.99843 & -1.32256 & 0.00007 \\
\hline $\mathrm{C}$ & -2.76927 & -1.68070 & 1.27189 \\
\hline C & -2.76952 & -1.68072 & -1.27157 \\
\hline C & 2.09410 & -3.17659 & -0.00023 \\
\hline 0 & 3.84764 & -0.90901 & 0.00047 \\
\hline $\mathrm{C}$ & 0.96475 & 3.70518 & -0.00040 \\
\hline O & -3.41478 & 1.41790 & 0.00033 \\
\hline $\mathrm{H}$ & -1.71664 & 3.47172 & -0.00019 \\
\hline $\mathrm{H}$ & -3.03953 & -2.74120 & 1.26338 \\
\hline $\mathrm{H}$ & -3.68580 & -1.08559 & 1.32559 \\
\hline $\mathrm{H}$ & -2.16592 & -1.47449 & 2.16081 \\
\hline $\mathrm{H}$ & -2.16636 & -1.47449 & -2.16063 \\
\hline $\mathrm{H}$ & -3.03972 & -2.74124 & -1.26301 \\
\hline $\mathrm{H}$ & -3.68609 & -1.08567 & -1.32509 \\
\hline $\mathrm{H}$ & 1.23976 & -3.85827 & -0.00251 \\
\hline $\mathrm{H}$ & 2.71317 & -3.39302 & -0.87915 \\
\hline $\mathrm{H}$ & 2.70933 & -3.39435 & 0.88110 \\
\hline $\mathrm{H}$ & 1.61799 & 3.75894 & 0.87679 \\
\hline $\mathrm{H}$ & 1.62223 & 3.75629 & -0.87450 \\
\hline $\mathrm{H}$ & 0.30651 & 4.57830 & -0.00312 \\
\hline
\end{tabular}

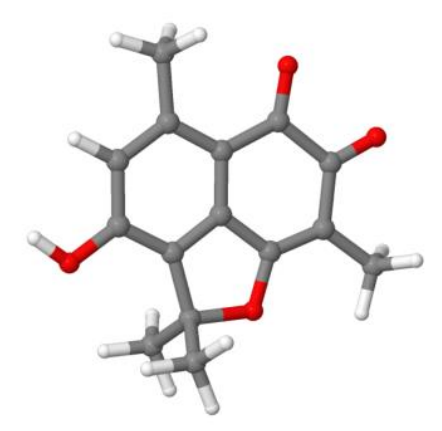


Conformer 2

Energy: -880.92314 Hartree (Rel: $2.4 \mathrm{kcal} / \mathrm{mol})$ XYZ coordinates for conf 2 :

$\begin{array}{lrrr}\mathrm{C} & 1.18748 & 2.52682 & 0.00013 \\ \mathrm{C} & 2.03760 & 1.40538 & -0.00023 \\ \mathrm{C} & 1.46157 & 0.12590 & -0.00030 \\ \mathrm{C} & 0.08083 & 0.05379 & -0.00021 \\ \mathrm{C} & -0.79240 & 1.14189 & -0.00003 \\ \mathrm{C} & -0.21035 & 2.43397 & 0.00024 \\ \mathrm{C} & -0.31299 & -1.33960 & -0.00012 \\ \mathrm{C} & -1.60574 & -1.76766 & 0.00002 \\ \mathrm{C} & -2.62437 & -0.72378 & -0.00000 \\ \mathrm{O} & -2.22373 & 0.80515 & -0.00018 \\ \mathrm{O} & -3.13071 & 1.62712 & -0.00071 \\ \mathrm{C} & 0.78849 & -2.11728 & -0.00013 \\ \mathrm{C} & 2.02124 & -1.28885 & 0.00005 \\ \mathrm{C} & 2.78982 & -1.65070 & -1.27446 \\ \mathrm{C} & 2.78914 & -1.64989 & 1.27522 \\ \mathrm{O} & -2.02771 & -3.20993 & 0.00016 \\ \mathrm{C} & -3.82811 & -0.97755 & 0.00016 \\ \mathrm{O} & -1.03985 & 3.69201 & 0.00049 \\ \mathrm{H} & 3.37026 & 1.65535 & -0.00021 \\ \mathrm{H} & 1.65843 & 3.50519 & 0.00031 \\ \mathrm{H} & 3.04710 & -2.71411 & -1.26652 \\ \mathrm{H} & 3.72092 & -1.07801 & -1.34379 \\ \mathrm{H} & 2.18769 & -1.43525 & -2.16148 \\ \mathrm{H} & 2.18598 & -1.43497 & 2.16167 \\ \mathrm{H} & 3.04755 & -2.71302 & 1.26764 \\ \mathrm{H} & 3.71949 & -1.07607 & 1.34531 \\ \mathrm{H} & -1.15939 & -3.87356 & 0.00221 \\ \mathrm{H} & -2.64182 & -3.43886 & 0.87934 \\ \mathrm{H} & -2.63849 & -3.44015 & -0.88103 \\ \mathrm{H} & -1.69427 & 3.73192 & -0.87660 \\ \mathrm{H} & -0.39959 & 3.72912 & 0.87467 \\ \mathrm{H} & 3.88193 & 4.57832 & 0.00317 \\ & & 0.83120 & -0.00247 \\ \mathrm{C} & -1.69823 & & \end{array}$

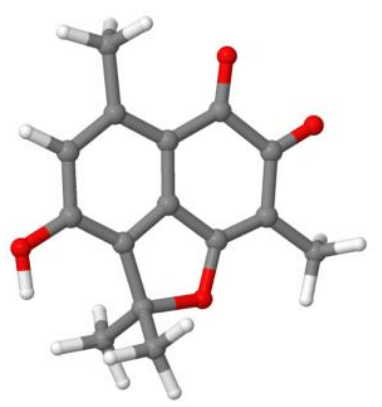


originally assigned structure of buxifoliadine (PCM DMSO), Fig.11<smiles>COc1cc2c(=O)c3c(OC)c(O)ccc3n(C)c2c2c1OCO2</smiles><smiles>COc1cc2c(=O)c3c(OC)c(O)ccc3n(C)c2c2ooc12</smiles>

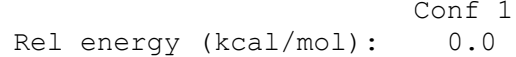

\begin{tabular}{|c|c|c|c|c|c|}
\hline C-nom & iGau & $\operatorname{Exp}$ & Calc & diff & 1 \\
\hline $\mathrm{C}-\mathrm{CH} 3-\mathrm{N}$ & 19 & 45.40 & 39.90 & -5.50 & 38.19 \\
\hline $\mathrm{C}-\mathrm{CH} 3-8$ & 18 & 56.40 & 66.35 & 9.95 & 62.82 \\
\hline $\mathrm{C}-\mathrm{CH} 3-2$ & 21 & 60.50 & 60.30 & -0.20 & 57.19 \\
\hline $\mathrm{Cl}-\mathrm{CH}$ & 14 & 107.80 & 115.53 & 7.73 & 108.61 \\
\hline $\mathrm{C} 2-\mathrm{C}$ & 13 & 157.90 & 145.16 & -12.74 & 136.19 \\
\hline C3-C & 12 & 173.90 & 156.43 & -17.47 & 146.69 \\
\hline $\mathrm{C} 4-\mathrm{C}$ & 11 & 183.00 & 146.38 & -36.62 & 137.33 \\
\hline $\mathrm{C} 4 \mathrm{a}-\mathrm{C}$ & 4 & 137.50 & 133.29 & -4.21 & 125.14 \\
\hline $\mathrm{C} 5-\mathrm{CH}$ & 10 & 116.20 & 117.63 & 1.43 & 110.56 \\
\hline $\mathrm{C} 6-\mathrm{CH}$ & 9 & 121.70 & 130.70 & 9.00 & 122.73 \\
\hline $\mathrm{C} 7-\mathrm{C}$ & 8 & 155.70 & 155.42 & -0.28 & 145.75 \\
\hline $\mathrm{C} 8-\mathrm{C}$ & 7 & 148.90 & 157.49 & 8.59 & 147.67 \\
\hline $\mathrm{C} 8 \mathrm{a}-\mathrm{C}$ & 1 & 124.30 & 127.26 & 2.96 & 119.53 \\
\hline $\mathrm{C} 9-\mathrm{C}$ & 6 & 179.10 & 190.79 & 11.69 & 178.68 \\
\hline $\mathrm{C} 9 \mathrm{a}-\mathrm{C}$ & 5 & 113.40 & 127.28 & 13.88 & 119.55 \\
\hline $\mathrm{C} 10 \mathrm{a}-\mathrm{C}$ & 2 & 139.10 & 145.60 & 6.50 & 136.60 \\
\hline
\end{tabular}

13C chem shifts: CRMSD $=12.63 \mathrm{ppm}$ (CMAE=9.30) $\mathrm{N}=16 \quad\left\{\begin{array}{lll}-36.62 & 13.88\}\end{array}\right.$

\section{Conformer 1}

Energy: -1123.44479 Hartree (Rel: $0.0 \mathrm{kcal} / \mathrm{mol}$ ) XYZ coordinates for conf 1 :

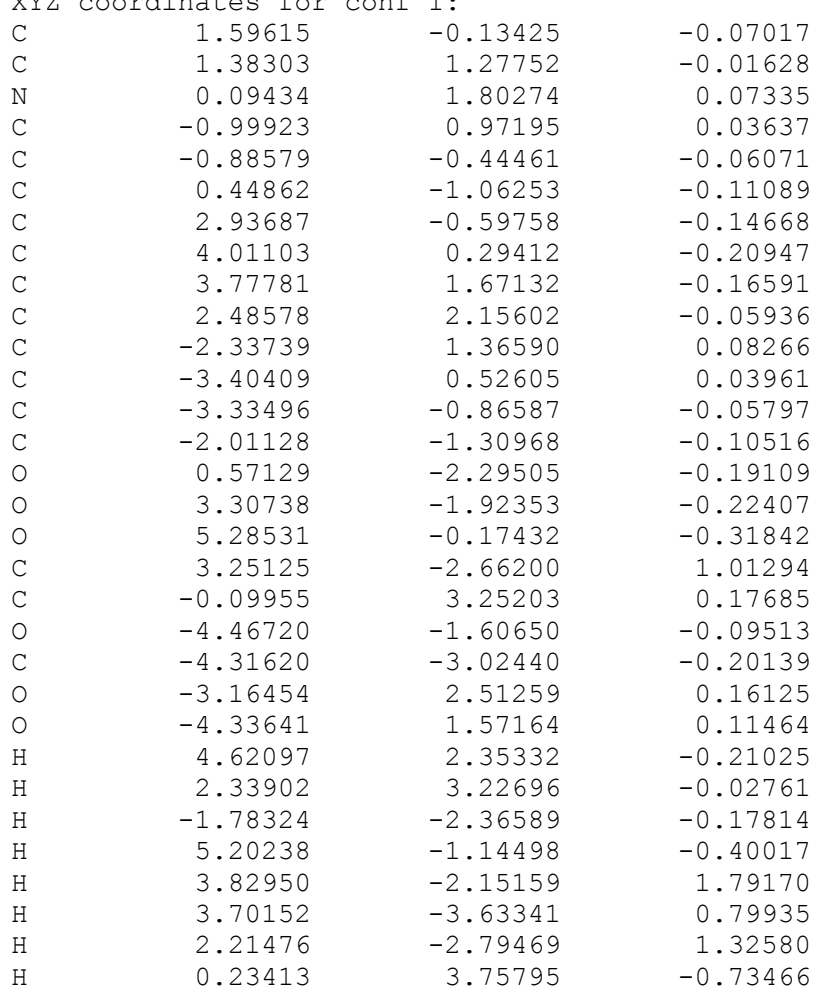

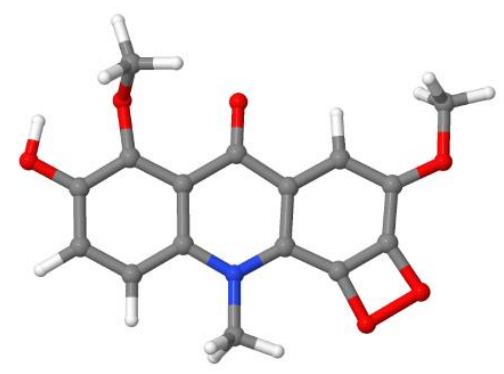




$\begin{array}{rrrr}\text { H } & -1.15498 & 3.47238 & 0.32655 \\ \mathrm{H} & 0.45097 & 3.64958 & 1.03393 \\ \mathrm{H} & -5.32850 & -3.42867 & -0.21742 \\ \mathrm{H} & -3.79489 & -3.29562 & -1.12643 \\ \mathrm{H} & -3.77092 & -3.42712 & 0.65965\end{array}$


revised buxifoliadine (PCM DMSO), Fig 11.<smiles>COC1=CC(=O)c2c(n(C)c3c(OC)c(O)ccc3c2=O)C1=O</smiles>

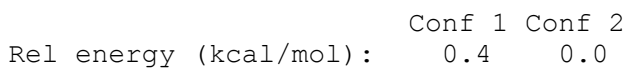

\begin{tabular}{|c|c|c|c|c|c|c|c|}
\hline C-nom & iGau & Exp & Calc & $\operatorname{diff}$ & 1 & 2 & \\
\hline $\mathrm{C}-\mathrm{C}$ & 14 & 183.00 & 181.64 & -1.36 & [ 184.15 & $184.49]$ & \\
\hline $\mathrm{C}-\mathrm{C}$ & 11 & 179.10 & 179.94 & 0.84 & {$[182.50$} & $182.39]$ & \\
\hline $\mathrm{C}-\mathrm{C}$ & 10 & 173.90 & 175.17 & 1.27 & [ 177.57 & 177.67 ] & \\
\hline $\mathrm{C}-\mathrm{C}$ & 2 & 155.70 & 155.01 & -0.69 & [ 156.91 & $157.02]$ & \\
\hline$C-C$ & 12 & 157.90 & 154.87 & -3.03 & {$[156.83$} & $156.64]$ & \\
\hline$C-C$ & 8 & 148.90 & 149.93 & 1.03 & [ 151.76 & $151.62]$ & \\
\hline$C-C$ & 4 & 139.10 & 139.67 & 0.57 & {$[140.89$} & $142.46]$ & \\
\hline $\mathrm{C}-\mathrm{C}$ & 3 & 137.50 & 136.23 & -1.27 & [ 137.53 & $138.31]$ & \\
\hline$C-C$ & 5 & 124.30 & 124.95 & 0.65 & [ 126.05 & $126.43]$ & \\
\hline$C-C$ & 9 & 113.40 & 114.85 & 1.45 & {$[115.73$} & $115.98]$ & \\
\hline $\mathrm{C}-\mathrm{CH}$ & 6 & 121.70 & 124.81 & 3.11 & {$[126.16$} & $125.31]$ & \\
\hline $\mathrm{C}-\mathrm{CH}$ & 1 & 116.20 & 114.09 & -2.11 & [ 115.09 & $114.65]$ & \\
\hline $\mathrm{C}-\mathrm{CH}$ & 13 & 107.80 & 109.19 & 1.39 & [ 109.95 & $110.10]$ & \\
\hline $\mathrm{C}-\mathrm{CH} 3$ & 20 & 60.50 & 60.03 & -0.47 & {$[\quad 59.82$} & $58.72]$ & \\
\hline $\mathrm{C}-\mathrm{CH} 3$ & 23 & 56.40 & 55.84 & -0.56 & 55.32 & $55.23]$ & \\
\hline \multirow[t]{5}{*}{$\mathrm{C}-\mathrm{CH} 3$} & 16 & 45.40 & 45.71 & 0.31 & 44.45 & $46.72]$ & \\
\hline & & $13 \mathrm{C} \mathrm{ch}$ & hem shif & ts: CRN & $=1.50 \mathrm{ppm}$ & $(\mathrm{CMAE}=1.26)$ & \multirow[t]{4}{*}{$\mathrm{N}=16 \quad\{-3.03 \quad 3.11\}$} \\
\hline & & & & Fractic & $: 0.793$ & 0.207 & \\
\hline & Fractic & ns-der & vied r & energ & 0.00 & 0.80 & \\
\hline & & & hergy & screpa & 0.37 & -0.80 & \\
\hline
\end{tabular}

\section{Conformer 1}

Energy: -1123.56524 Hartree (Rel: $0.4 \mathrm{kcal} / \mathrm{mol}$ ) XYZ coordinates for conf 1 :

\begin{tabular}{|c|c|c|c|}
\hline & & & \\
\hline C & -3.70938 & 1.71651 & -0.31167 \\
\hline C & -3.84980 & 0.32460 & -0.40349 \\
\hline C & -2.77367 & -0.53975 & -0.15024 \\
\hline C & -1.48258 & 0.00048 & 0.08176 \\
\hline C & -1.33983 & 1.40951 & 0.11883 \\
\hline C & -2.46169 & 2.23974 & -0.04233 \\
\hline $\mathrm{N}$ & -0.33936 & -0.80234 & 0.22967 \\
\hline C & 0.90132 & -0.22949 & 0.10717 \\
\hline C & 1.11785 & 1.13706 & 0.12743 \\
\hline C & -0.02343 & 2.04643 & 0.30913 \\
\hline C & 2.04869 & -1.16276 & -0.21060 \\
\hline C & 3.43371 & -0.61063 & -0.11860 \\
\hline $\mathrm{C}$ & 3.62829 & 0.72275 & -0.03780 \\
\hline C & 2.50346 & 1.67041 & 0.01049 \\
\hline O & 0.07297 & 3.25460 & 0.55139 \\
\hline C & -0.47764 & -2.16611 & 0.79833 \\
\hline O & 1.86499 & -2.30766 & -0.59048 \\
\hline O & -3.01549 & -1.89355 & -0.29365 \\
\hline O & 2.74816 & 2.87674 & -0.03260 \\
\hline C & -3.76863 & -2.51486 & 0.77418 \\
\hline O & -5.05511 & -0.17903 & -0.77590 \\
\hline O & 4.36157 & -1.57187 & -0.19957 \\
\hline C & 5.73694 & -1.16855 & -0.17971 \\
\hline $\mathrm{H}$ & -4.57269 & 2.34755 & -0.49465 \\
\hline $\mathrm{H}$ & -2.30230 & 3.31042 & 0.01753 \\
\hline $\mathrm{H}$ & 4.61270 & 1.17517 & -0.04610 \\
\hline $\mathrm{H}$ & -1.23942 & -2.12317 & 1.57505 \\
\hline $\mathrm{H}$ & -0.75477 & -2.89585 & 0.04065 \\
\hline $\mathrm{H}$ & 0.45804 & -2.45872 & 1.26409 \\
\hline $\mathrm{H}$ & -3.95819 & -3.53748 & 0.44563 \\
\hline $\mathrm{H}$ & -4.71690 & -1.99749 & 0.94633 \\
\hline
\end{tabular}

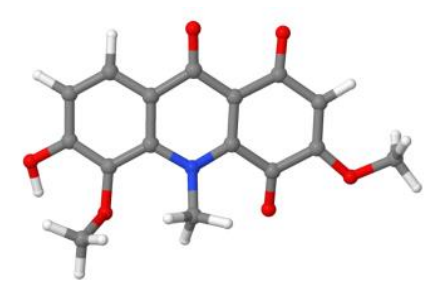




$\begin{array}{rrrr}\mathrm{H} & -3.19592 & -2.52665 & 1.70602 \\ \mathrm{H} & -4.92130 & -1.10657 & -1.04468 \\ \mathrm{H} & 6.31497 & -2.09001 & -0.24113 \\ \mathrm{H} & 5.97084 & -0.64026 & 0.75064 \\ \mathrm{H} & 5.96202 & -0.52644 & -1.03784\end{array}$

\section{Conformer 2}

Energy: -1123.56583 Hartree (Rel: $0.0 \mathrm{kcal} / \mathrm{mol}$ ) XYZ coordinates for conf 2:

$\begin{array}{lrrr}\text { C } & -3.80984 & 1.59199 & -0.05009 \\ \mathrm{C} & -3.91978 & 0.19218 & -0.00443 \\ \mathrm{C} & -2.77878 & -0.61572 & 0.11829 \\ \mathrm{C} & -1.50480 & -0.00933 & 0.23597 \\ \mathrm{C} & -1.39861 & 1.39830 & 0.14124 \\ \mathrm{C} & -2.55943 & 2.17574 & 0.00782 \\ \mathrm{~N} & -0.34382 & -0.77510 & 0.43238 \\ \mathrm{C} & 0.87933 & -0.19918 & 0.20044 \\ \mathrm{C} & 1.06184 & 1.16273 & 0.02831 \\ \mathrm{C} & -0.08813 & 2.07245 & 0.15772 \\ \mathrm{C} & 2.04214 & -1.14634 & 0.00568 \\ \mathrm{C} & 3.41298 & -0.55527 & -0.05558 \\ \mathrm{C} & 3.57283 & 0.77849 & -0.18879 \\ \mathrm{C} & 2.42576 & 1.69957 & -0.23127 \\ \mathrm{O} & -0.00303 & 3.30317 & 0.23728 \\ \mathrm{C} & -0.44238 & -2.04155 & 1.20989 \\ \mathrm{O} & 1.88098 & -2.34540 & -0.15277 \\ \mathrm{O} & -2.92901 & -1.98100 & 0.12004 \\ \mathrm{O} & 2.63655 & 2.88940 & -0.47089 \\ \mathrm{C} & -3.13858 & -2.56073 & -1.18027 \\ \mathrm{O} & -5.11251 & -0.45081 & -0.09784 \\ \mathrm{O} & 4.36507 & -1.49616 & -0.03007 \\ \mathrm{C} & 5.72729 & -1.06276 & -0.13247 \\ \mathrm{H} & -4.70599 & 2.19882 & -0.15046 \\ \mathrm{H} & -2.44055 & 3.25204 & -0.04750 \\ \mathrm{H} & 4.54281 & 1.24520 & -0.31228 \\ \mathrm{H} & -1.28555 & -1.94768 & 1.89160 \\ \mathrm{H} & -0.58696 & -2.90618 & 0.56603 \\ \mathrm{H} & 0.46730 & -2.16787 & 1.79217 \\ \mathrm{H} & -3.21280 & -3.63796 & -1.02014 \\ \mathrm{H} & -4.06511 & -2.18956 & -1.62815 \\ \mathrm{H} & -2.29249 & -2.34457 & -1.84277 \\ \mathrm{H} & -5.82054 & 0.20262 & -0.22559 \\ \mathrm{H} & 6.32981 & -1.96871 & -0.07566 \\ \mathrm{H} & 5.98175 & -0.39053 & 0.69382 \\ \mathrm{H} & 5.89947 & -0.55700 & -1.08853 \\ & & & \end{array}$

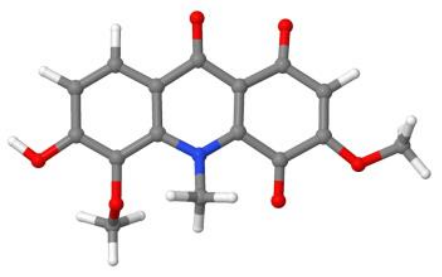


originally assigned structure of neohupehenolide B (PCM methanol), Fig.13<smiles>C[C@@]12C[C@@H]3[C@@H]4OCO[C@@H](C[C@]3(C)C1CCC2=O)OC4=O</smiles>

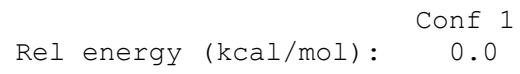

$\left.\begin{array}{lrrrrlll}\text { C-nom } & \text { iGau } & \multicolumn{1}{c}{\text { Exp }} & \text { Calc } & \text { diff } & \multicolumn{1}{c}{1} & \\ \text { C1 } & 2 & 53.30 & 51.23 & -2.07 & {[} & 50.08 & ] \\ \text { C2 } & 8 & 19.50 & 21.97 & 2.47 & {[} & 20.44 & ] \\ \text { C4 } & 10 & 221.60 & 224.40 & 2.80 & {[} & 225.50 & ] \\ \text { C5 } & 3 & 50.90 & 51.21 & 0.31 & {[} & 50.06 & ] \\ \text { C6 } & 4 & 37.20 & 29.97 & -7.23 & {[} & 28.55 & ] \\ \text { C7 } & 5 & 39.60 & 45.21 & 5.61 & {[} & 43.98 & ] \\ \text { C8 } & 6 & 81.00 & 79.55 & -1.45 & {[} & 78.77 & ] \\ \text { C9 } & 7 & 44.10 & 44.00 & -0.10 & {[} & 42.76 & ] \\ \text { C10 } & 1 & 72.20 & 72.94 & 0.74 & {[} & 72.07 & ] \\ \text { C12 } & 12 & 179.90 & 175.54 & -4.36 & {[} & 176.01 & ] \\ \text { C13 } & 22 & 73.50 & 74.71 & 1.21 & {[} & 73.87 & ] \\ \text { C14 } & 14 & 26.80 & 28.07 & 1.27 & {[} & 26.62 & ] \\ \text { C15 } & 19 & 19.70 & 20.38 & 0.68 & {[} & 18.83\end{array}\right]$

$13 \mathrm{C}$ chem shifts: $\mathrm{CRMSD}=3.13 \mathrm{ppm}(\mathrm{CMAE}=2.33) \mathrm{N}=13 \quad\{-7.235 .61\}$

\section{Conformer 1}

Energy: -1034.84458 Hartree (Rel: $0.0 \mathrm{kcal} / \mathrm{mol}$ ) XYZ coordinates for conf 1 :

\begin{tabular}{|c|c|c|c|}
\hline C & 1.80854 & -1.65811 & -0.23553 \\
\hline C & 1.95269 & -0.13573 & -0.46154 \\
\hline C & 1.39361 & 0.84975 & 0.60938 \\
\hline C & -0.14847 & 0.85930 & 0.79644 \\
\hline C & -0.93952 & 0.15890 & -0.32380 \\
\hline C & -0.77038 & -1.39275 & -0.33255 \\
\hline C & 0.43513 & -1.99354 & 0.38830 \\
\hline C & 3.35692 & 0.41416 & -0.79572 \\
\hline C & 3.13509 & 1.93746 & -0.83942 \\
\hline C & 1.90961 & 2.19182 & 0.03647 \\
\hline C & -2.45062 & 0.31774 & -0.17292 \\
\hline C & -2.89769 & -0.96827 & 0.53284 \\
\hline 0 & -1.95638 & -1.91186 & 0.36254 \\
\hline C & 2.92693 & -2.29403 & 0.60166 \\
\hline 0 & 1.86434 & -2.20005 & -1.57275 \\
\hline $\mathrm{H}$ & -0.81859 & -1.77397 & -1.35564 \\
\hline $\mathrm{H}$ & -0.66064 & 0.57614 & -1.29481 \\
\hline $\mathrm{H}$ & 1.35805 & 0.04456 & -1.36684 \\
\hline C & 2.06393 & 0.74443 & 2.00596 \\
\hline 0 & 1.41499 & 3.27961 & 0.26102 \\
\hline O & -3.93607 & -1.16094 & 1.12172 \\
\hline C & -3.16293 & 1.59604 & 0.23830 \\
\hline 0 & -3.15202 & 0.38499 & -1.46344 \\
\hline 0 & -3.98366 & 1.51090 & -0.96082 \\
\hline $\mathrm{H}$ & -0.40450 & 0.40312 & 1.75954 \\
\hline $\mathrm{H}$ & -0.45823 & 1.90811 & 0.86561 \\
\hline $\mathrm{H}$ & 0.31105 & -3.08357 & 0.38180 \\
\hline $\mathrm{H}$ & 0.41494 & -1.69016 & 1.44010 \\
\hline $\mathrm{H}$ & 3.73073 & 0.01352 & -1.74126 \\
\hline $\mathrm{H}$ & 4.08360 & 0.15599 & -0.01914 \\
\hline $\mathrm{H}$ & 3.98589 & 2.53450 & -0.49469 \\
\hline $\mathrm{H}$ & 2.89469 & 2.28503 & -1.85273 \\
\hline $\mathrm{H}$ & 2.74670 & -3.37122 & 0.70586 \\
\hline $\mathrm{H}$ & 3.89424 & -2.16016 & 0.10943 \\
\hline
\end{tabular}

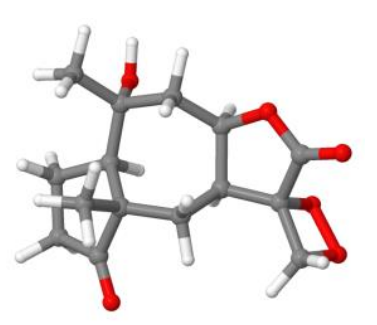




$\begin{array}{rrrr}\mathrm{H} & 2.98823 & -1.87192 & 1.60849 \\ \mathrm{H} & 1.89993 & -3.16846 & -1.49611 \\ \mathrm{H} & 1.78825 & -0.18879 & 2.50611 \\ \mathrm{H} & 3.15611 & 0.79388 & 1.95699 \\ \mathrm{H} & 1.72030 & 1.57353 & 2.63407 \\ \mathrm{H} & -3.76908 & 1.53518 & 1.14516 \\ \mathrm{H} & -2.54830 & 2.50058 & 0.23470\end{array}$


revised neohupehenolide $B$, Fig. 13<smiles>C[C@]1(O)C[C@@H]2OC(=O)[C@](C)(O)[C@H]2C[C@@]2(C)C(=O)CCC12</smiles>

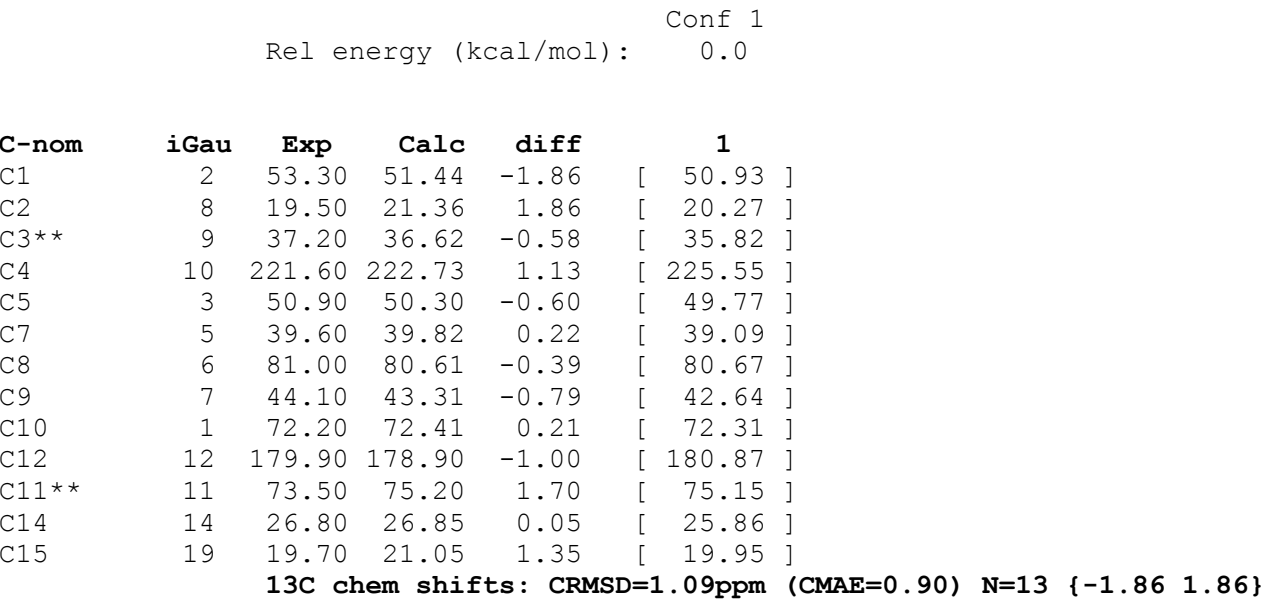

\section{Conformer 1}

Energy: -1036.16741 Hartree (Rel: $0.0 \mathrm{kcal} / \mathrm{mol}$ ) XYZ coordinates for conf 1 :

$\begin{array}{lrrr}\mathrm{C} & 1.83032 & -1.69710 & 0.00771 \\ \mathrm{C} & 2.03409 & -0.22723 & -0.42246 \\ \mathrm{C} & 1.52404 & 0.92205 & 0.49482 \\ \mathrm{C} & -0.00382 & 0.98884 & 0.73085 \\ \mathrm{C} & -0.89722 & 0.26393 & -0.29123 \\ \mathrm{C} & -0.64696 & -1.27727 & -0.43819 \\ \mathrm{C} & 0.37497 & -1.91726 & 0.49758 \\ \mathrm{C} & 3.45196 & 0.21313 & -0.84577 \\ \mathrm{C} & 3.27747 & 1.72038 & -1.11522 \\ \mathrm{C} & 2.06494 & 2.14258 & -0.28424 \\ \mathrm{C} & -2.40034 & 0.38012 & 0.05483 \\ \mathrm{C} & -2.93518 & -1.05150 & -0.09281 \\ \mathrm{O} & -1.94305 & -1.93207 & -0.24146 \\ \mathrm{C} & 2.82935 & -2.23151 & 1.04125 \\ \mathrm{O} & 2.01583 & -2.42208 & -1.22784 \\ \mathrm{H} & -0.36943 & -1.52357 & -1.46553 \\ \mathrm{H} & -0.74853 & 0.71514 & -1.27953 \\ \mathrm{H} & 1.43538 & -0.14524 & -1.34123 \\ \mathrm{C} & 2.22425 & 1.01572 & 1.87959 \\ \mathrm{O} & 1.60457 & 3.26701 & -0.23005 \\ \mathrm{O} & -4.10789 & -1.38210 & -0.05286 \\ \mathrm{C} & -3.19881 & 1.32757 & -0.85569 \\ \mathrm{O} & -2.61297 & 0.76783 & 1.40840 \\ \mathrm{O} & -4.53469 & 1.43647 & -0.37188 \\ \mathrm{H} & -0.23168 & 0.60015 & 1.72586 \\ \mathrm{H} & -0.29083 & 2.04616 & 0.75534 \\ \mathrm{H} & 0.18465 & -2.99712 & 0.52260 \\ \mathrm{H} & 0.24776 & -1.55236 & 1.52218 \\ \mathrm{H} & 3.79660 & -0.33768 & -1.72386 \\ \mathrm{H} & 4.17980 & 0.04636 & -0.04517 \\ \mathrm{H} & 4.14814 & 2.33509 & -0.86337 \\ \mathrm{H} & 3.04413 & 1.92022 & -2.16920 \\ \mathrm{H} & 2.62652 & -3.29266 & 1.23427 \\ \mathrm{H} & 3.85346 & -2.14771 & 0.66630 \\ \mathrm{H} & 2.76532 & -1.70804 & 1.99816\end{array}$

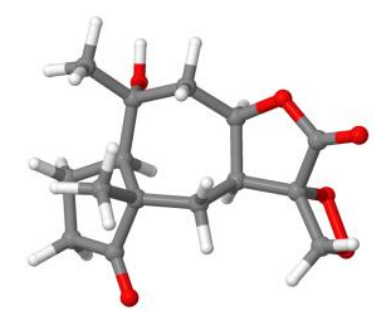




$\begin{array}{rrrr}\mathrm{H} & 2.00845 & -3.37038 & -1.01515 \\ \mathrm{H} & 1.92063 & 0.18817 & 2.52720 \\ \mathrm{H} & 3.31643 & 1.01046 & 1.80879 \\ \mathrm{H} & 1.92498 & 1.94830 & 2.37049 \\ \mathrm{H} & -2.76319 & 2.33028 & -0.80729 \\ \mathrm{H} & -3.16735 & 0.98264 & -1.89830 \\ \mathrm{H} & -3.53015 & 1.10741 & 1.42010 \\ \mathrm{H} & -4.89981 & 0.52924 & -0.38332\end{array}$


originally assigned structure of isoflavon cmpd 3, Fig.14<smiles>COc1ccc(-c2coc3c(CC=C(C)C)c4c(c(O)c3c2=O)C2OOC2C(C)(C)O4)cc1</smiles>

Rel energy (kcal/mol): Conf 1 Conf 2

\begin{tabular}{|c|c|c|c|c|c|c|}
\hline C-nom & iGau & Exp & Calc & $\operatorname{diff}$ & 1 & 2 \\
\hline $\mathrm{C} 2-\mathrm{CH}$ & 9 & 152.60 & 153.20 & 0.60 & [ 153.20 & 153.12 \\
\hline $3-C$ & 8 & 123.30 & 125.14 & 1.84 & 125.14 & 125.22 \\
\hline $4-C$ & 7 & 181.50 & 180.32 & -1.18 & 180.32 & 180.34 \\
\hline $5-C$ & 3 & 157.80 & 161.49 & 3.6 & 161.49 & 161.67 \\
\hline $\mathrm{C} 6-\mathrm{C}$ & 2 & 106.40 & 106.38 & -0.02 & 106.41 & 105.49 \\
\hline $\mathrm{C} 7-\mathrm{C}$ & 1 & 164.70 & 160. & -4 & 160.59 & 159.24 \\
\hline $8-C$ & 6 & 102.10 & 108.33 & 6.2 & 108.34 & 107.94 \\
\hline $9-C$ & 5 & 156.90 & 156.96 & 0. & 156.98 & 156.35 \\
\hline $10-C$ & 4 & 106.90 & 107.68 & & 107.68 & 107.61 \\
\hline $\mathrm{C} 1{ }^{\prime}-\mathrm{C}$ & 11 & 122.80 & 122.38 & -0.42 & 122.38 & 122.31 \\
\hline $\mathrm{C} 2{ }^{\prime}-\mathrm{CH}$ & 12 & 130.10 & 130.56 & 0 & 130.56 & 130.51 \\
\hline $3^{\prime}-\mathrm{CH}$ & 13 & 114.10 & 113. & -0 . & 113.53 & 113.57 \\
\hline $44^{\prime}-\mathrm{C}$ & 14 & 159.80 & 158.67 & -1 & 158.67 & 158.76 \\
\hline $\mathrm{C} 5^{\prime}-\mathrm{CH}$ & 15 & 114.10 & 113.53 & -0.57 & 113.53 & 113.57 \\
\hline $\mathrm{C}^{\prime}-\mathrm{CH}$ & 16 & 130.10 & 130.56 & 0 & 130.56 & 130.51 \\
\hline $\mathrm{Cl} "-\mathrm{CH}$ & 17 & 82.60 & 69.86 & -12 . & 69.78 & 72.80 \\
\hline $\mathrm{C} 2 \mathrm{~m}-\mathrm{CH}$ & 29 & 98.20 & 83. & -14 & 83.99 & 83.75 \\
\hline C3"-C & 28 & 85.60 & 77.88 & -7 & 77.79 & 81.04 \\
\hline $\mathrm{C} 4 \mathrm{~N}-\mathrm{CH} 3$ & 30 & 23.80 & 21.22 & -2 & 21.21 & 21.61 \\
\hline $\mathrm{C} 5 "-\mathrm{CH} 3$ & 31 & 18.10 & 21.25 & 3. & 21.07 & 27.72 \\
\hline $\mathrm{C} 1{ }^{\prime}+'-\mathrm{CH} 2$ & 18 & 21.20 & 23.07 & 1.87 & 23.05 & 23.69 \\
\hline $\mathrm{C} 2 \prime^{\prime} \cdot-\mathrm{CH}$ & 19 & 121.10 & 124.04 & 2.94 & 124.05 & 123.78 \\
\hline C3'' - & 25 & 132.50 & 129. & & {$[129.77$} & 129.53 \\
\hline $\mathrm{C} 4 \mathrm{I}^{\prime}-\mathrm{CH} 3$ & 26 & 25.70 & 25 & & {$\left[\begin{array}{ll}{[} & 25.18\end{array}\right.$} & 25.02 \\
\hline $\mathrm{C} 5 \mathrm{I}^{\prime}-\mathrm{CH} 3$ & 27 & 17.80 & 16. & & 16.93 & 16.88 \\
\hline COMe 4 '-CH 3 & 324 & 55.30 & 53.13 & -2.17 & 53.13 & 53.12 \\
\hline
\end{tabular}

13C chem shifts: $\mathrm{RMSD}=4.57 \mathrm{ppm}(\mathrm{MAE}=2.83) \quad \mathrm{N}=26 \quad\left\{\begin{array}{lll}-14.22 & 6.23\end{array}\right\}$

Fractions: 0.9730 .027

Conformer 1

Energy: -1532.83397 Hartree (Rel: $0.0 \mathrm{kcal} / \mathrm{mol}$ ) XYZ coordinates for conf 1 :

$\begin{array}{lrrr}\text { C } & -2.67322 & -0.26328 & -0.31690 \\ \text { C } & -2.26761 & -1.53515 & 0.13492 \\ \text { C } & -0.90228 & -1.80248 & 0.28303 \\ \text { C } & 0.05908 & -0.80263 & -0.03658 \\ \text { C } & -0.41363 & 0.43483 & -0.51480 \\ \text { C } & -1.76506 & 0.74909 & -0.66940 \\ \text { C } & 1.48332 & -1.06026 & 0.11542 \\ \text { C } & 2.37922 & 0.05283 & -0.23304 \\ \text { C } & 1.80942 & 1.18941 & -0.70961 \\ \text { O } & 0.48546 & 1.41186 & -0.85413 \\ \text { C } & 3.84856 & -0.03523 & -0.06999 \\ \text { C } & 4.58688 & 1.07665 & 0.38033 \\ \text { C } & 5.96872 & 1.02894 & 0.49621 \\ \text { C } & 6.66143 & -0.14770 & 0.17439 \\ \text { C } & 5.94437 & -1.26971 & -0.25907 \\ \text { C } & 4.55532 & -1.20758 & -0.37562 \\ \text { C } & -3.28353 & -2.61033 & 0.32517 \\ \text { C } & -2.22586 & 2.11169 & -1.15624 \\ \text { C } & -2.38680 & 3.10499 & -0.02678\end{array}$

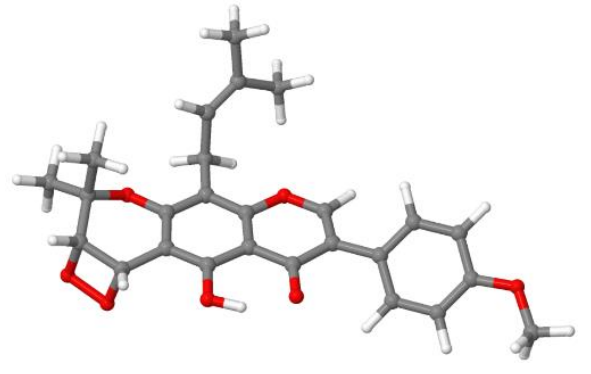




$\begin{array}{rrr}-3.99315 & 0.03856 & -0.44538 \\ -0.51765 & -3.00841 & 0.72067 \\ 1.90904 & -2.16499 & 0.52268 \\ 8.01536 & -0.09953 & 0.32560 \\ 8.76601 & -1.26984 & 0.04249 \\ -1.73978 & 4.26506 & 0.16268 \\ -2.05042 & 5.13061 & 1.36204 \\ -0.67856 & 4.82643 & -0.75275 \\ -4.95835 & -0.62725 & 0.41882 \\ -4.73398 & -2.13995 & 0.41835 \\ -6.31646 & -0.28239 & -0.18848 \\ -4.80704 & -0.07513 & 1.84339 \\ -5.02365 & -2.76699 & -0.85148 \\ -3.63369 & -3.29097 & -0.93488 \\ 2.37703 & 2.05251 & -1.03706 \\ 4.06623 & 1.98609 & 0.66901 \\ 6.53404 & 1.88483 & 0.85131 \\ 6.45215 & -2.19441 & -0.50824 \\ 4.01392 & -2.08641 & -0.70329 \\ -2.96053 & -3.34876 & 1.06115 \\ -1.52486 & 2.48068 & -1.90823 \\ -3.19201 & 1.98211 & -1.65643 \\ -3.12889 & 2.81317 & 0.71741 \\ 0.48366 & -2.98751 & 0.75213 \\ 9.80829 & -1.01150 & 0.23834 \\ 8.65940 & -1.57422 & -1.00715 \\ 8.47088 & -2.10499 & 0.69126 \\ -2.38680 & 6.13051 & 1.05303 \\ -2.82992 & 4.69117 & 1.99254 \\ -1.15593 & 5.28263 & 1.98283 \\ -1.00284 & 5.78478 & -1.18222 \\ 0.24116 & 5.03665 & -0.18897 \\ -0.41923 & 4.15832 & -1.57662 \\ -5.32459 & -2.60026 & 1.22079 \\ -6.38748 & -0.68789 & -1.20078 \\ -7.12547 & -0.70237 & 0.41876 \\ -6.44073 & 0.80398 & -0.23161 \\ -5.56955 & -0.49938 & 2.50509 \\ -3.82244 & -0.30817 & 2.26232 \\ -4.92762 & 1.01247 & 1.83208 \\ & & \end{array}$

\section{Conformer 2}

Energy: -1532.83060 Hartree (Rel: $2.1 \mathrm{kcal} / \mathrm{mol}$ ) XYZ coordinates for conf 2 :

$\begin{array}{lrrr}\text { C } & 2.65880 & -0.09622 & 0.10041 \\ C & 2.26638 & -1.36483 & -0.37117 \\ C & 0.90409 & -1.66543 & -0.46966 \\ C & -0.06950 & -0.70129 & -0.08885 \\ C & 0.38894 & 0.55004 & 0.36796 \\ C & 1.73678 & 0.89237 & 0.48769 \\ C & -1.49138 & -0.99318 & -0.19995 \\ C & -2.40286 & 0.09004 & 0.19908 \\ C & -1.84534 & 1.23865 & 0.66114 \\ C & -0.52327 & 1.50053 & 0.74712 \\ O & -3.87478 & -0.03902 & 0.09949 \\ C & -4.66443 & 1.05672 & -0.30072 \\ C & -6.04811 & 0.97016 & -0.35513 \\ C & -6.69147 & -0.23063 & -0.02028 \\ C & -5.92356 & -1.33708 & 0.36316 \\ C & -4.53303 & -1.23580 & 0.41832 \\ C & 3.29956 & -2.31263 & -0.86974 \\ C & 2.18652 & 2.25861 & 0.97277 \\ C & 2.42370 & 3.22640 & -0.16586 \\ C & 3.97199 & 0.25703 & 0.15038 \\ O & 0.54428 & -2.86349 & -0.94409 \\ O & -1.90085 & -2.10097 & -0.61380 \\ O & -8.05164 & -0.22032 & -0.10952 \\ O & -8.75449 & -1.41576 & 0.19110 \\ O & 1.79134 & 4.38174 & -0.42168 \\ C & 2.17923 & 5.21976 & -1.61811 \\ C & 0.67418 & 4.96394 & 0.41044\end{array}$

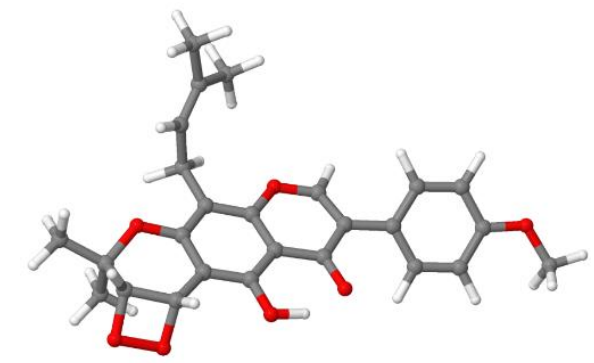




$\begin{array}{rrr}4.97822 & -0.78505 & 0.35321 \\ 4.74991 & -1.88756 & -0.71237 \\ 4.86709 & -1.31196 & 1.78709 \\ 6.30933 & -0.07405 & 0.12405 \\ 5.06385 & -3.21606 & -0.23038 \\ 3.60566 & -3.47437 & -0.01472 \\ -2.42279 & 2.08186 & 1.02153 \\ -4.18388 & 1.98493 & -0.59900 \\ -6.65318 & 1.81391 & -0.67165 \\ -6.39292 & -2.27989 & 0.61986 \\ -3.95272 & -2.10308 & 0.70794 \\ 3.05464 & -2.68290 & -1.87116 \\ 1.45184 & 2.64985 & 1.67941 \\ 3.12577 & 2.12680 & 1.52298 \\ 3.21234 & 2.91607 & -0.85170 \\ -0.45690 & -2.87624 & -0.94160 \\ -9.81166 & -1.18573 & 0.04648 \\ -8.59099 & -1.72995 & 1.23046 \\ -8.46543 & -2.23350 & -0.48205 \\ 2.49530 & 6.22694 & -1.31123 \\ 2.99750 & 4.76638 & -2.18637 \\ 1.32647 & 5.35630 & -2.29835 \\ 0.97073 & 5.93322 & 0.83554 \\ -0.20813 & 5.15873 & -0.21527 \\ 0.36193 & 4.31674 & 1.23297 \\ 5.29925 & -1.66432 & -1.63298 \\ 5.66706 & -2.02871 & 1.98673 \\ 3.91743 & -1.82403 & 1.95594 \\ 4.95587 & -0.47294 & 2.48456 \\ 7.13380 & -0.78985 & 0.21223 \\ 6.34178 & 0.37505 & -0.87424 \\ 6.45583 & 0.71816 & 0.86471\end{array}$


revised structure of isoflavon cmpd 3, Fig. 14<smiles>COc1ccc(-c2coc3c(CC=C(C)C)c4c(c(O)c3c2=O)[C@@H]2OOC[C@@H](O4)C2(C)C)cc1</smiles>

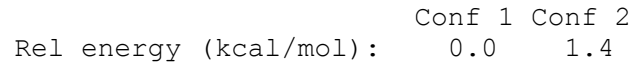

\begin{tabular}{|c|c|c|c|c|c|c|c|}
\hline C-nom & iGau & Exp & Calc & $\operatorname{diff}$ & 1 & 2 & \\
\hline $\mathrm{C} 2-\mathrm{CH}$ & 9 & 152.60 & 152.81 & 0.21 & [ 152.81 & $152.80]$ & ] \\
\hline C3-C & 8 & 123.30 & 125.20 & 1.90 & 125.19 & $125.31]$ & ] \\
\hline $\mathrm{C} 4-\mathrm{C}$ & 7 & 181.50 & 180.46 & -1.04 & 180.46 & $180.46]$ & ] \\
\hline C5-C & 3 & 157.80 & 160.46 & 2.66 & 160.45 & $160.55]$ & ] \\
\hline $\mathrm{C} 6-\mathrm{C}$ & 2 & 106.40 & 107.72 & 1.32 & [ 107.82 & $106.72]$ & ] \\
\hline C7-C & 1 & 164.70 & 165.92 & 1.22 & [ 165.82 & $166.92]$ & ] \\
\hline $\mathrm{C} 8-\mathrm{C}$ & 6 & 102.10 & 100.85 & -1.25 & {$[100.79$} & 101.49 ] & \\
\hline C9-C & 5 & 156.90 & 157.99 & 1.09 & [ 157.95 & $158.39]$ & \\
\hline $\mathrm{C} 10-\mathrm{C}$ & 4 & 106.90 & 108.93 & 2.03 & 108.95 & $108.68]$ & \\
\hline $\mathrm{C} 1^{\prime}-\mathrm{C}$ & 11 & 122.80 & 122.54 & -0.26 & [ 122.56 & $122.34]$ & \\
\hline $\mathrm{C} 2{ }^{\prime}-\mathrm{CH}$ & 12 & 130.10 & 130.61 & 0.51 & {$[130.61$} & $130.60]$ & \\
\hline $\mathrm{C}^{\prime}-\mathrm{CH}$ & 13 & 114.10 & 113.52 & -0.58 & 113.52 & $113.56]$ & \\
\hline $\mathrm{C} 4{ }^{\prime}-\mathrm{C}$ & 14 & 159.80 & 158.57 & -1.23 & {$[158.56$} & $158.67 \mathrm{]}$ & \\
\hline $\mathrm{C} 5^{\prime}-\mathrm{CH}$ & 15 & 114.10 & 113.52 & -0.58 & 113.52 & $113.56]$ & \\
\hline $\mathrm{C} 6^{\prime}-\mathrm{CH}$ & 16 & 130.10 & 130.61 & 0.51 & {$[130.61$} & $130.60]$ & \\
\hline $\mathrm{Cl} "-\mathrm{CH}$ & 17 & 82.60 & 84.61 & 2.01 & {$\left[\begin{array}{ll}{[} & 84.58\end{array}\right.$} & $84.92]$ & \\
\hline $\mathrm{C} 2 "-\mathrm{CH}$ & 29 & 98.20 & 100.01 & 1.81 & 99.68 & $103.40]$ & \\
\hline C3"-C & 28 & 85.60 & 85.73 & 0.13 & 85.62 & $86.93]$ & \\
\hline $\mathrm{C} 4=-\mathrm{CH} 3$ & 31 & 23.80 & 22.97 & -0.83 & 23.00 & $22.70]$ & \\
\hline C5"-CH3 & 30 & 18.10 & 18.58 & 0.48 & 18.39 & $20.58]$ & \\
\hline $\mathrm{C} 1 \prime^{\prime} \cdot-\mathrm{CH} 2$ & 18 & 21.20 & 23.61 & 2.41 & 23.60 & $23.66]$ & \\
\hline $\mathrm{C} 2 \mathrm{\prime}^{\prime}-\mathrm{CH}$ & 19 & 121.10 & 123.73 & 2.63 & [ 123.75 & $123.55]$ & \\
\hline C3'',-C & 25 & 132.50 & 130.03 & -2.47 & [ 130.01 & $130.22]$ & \\
\hline $\mathrm{C} 4 \mathrm{I}^{\prime}-\mathrm{CH} 3$ & 26 & 25.70 & 25.09 & -0.61 & [ 25.09 & $25.08]$ & \\
\hline $\mathrm{C} 5 \prime^{\prime} \cdot-\mathrm{CH} 3$ & 27 & 17.80 & 16.82 & -0.98 & 16.82 & $16.81]$ & \\
\hline $\mathrm{COMe} 4$ ' $^{-\mathrm{CH} 3}$ & 324 & 55.30 & 53.02 & -2.28 & 53.02 & $53.04]$ & \\
\hline
\end{tabular}

\section{Conformer 1}

Energy: -1532.86403 Hartree (Rel: $0.0 \mathrm{kcal} / \mathrm{mol}$ ) XYZ coordinates for conf 1 :

$\begin{array}{lrrr}C & 2.60567 & 0.10369 & -0.10384 \\ C & 2.28023 & -1.16652 & -0.58368 \\ C & 0.95181 & -1.56480 & -0.65941 \\ C & -0.05147 & -0.64500 & -0.21731 \\ C & 0.35478 & 0.61706 & 0.27049 \\ C & 1.68519 & 1.04712 & 0.35281 \\ C & -1.45954 & -1.00620 & -0.28225 \\ C & -2.41268 & 0.01730 & 0.17539 \\ C & -1.90162 & 1.17898 & 0.65631 \\ O & -0.59212 & 1.50373 & 0.71143 \\ C & -3.87862 & -0.18423 & 0.11326 \\ C & -4.73303 & 0.88065 & -0.23361 \\ C & -6.11181 & 0.72620 & -0.25265 \\ C & -6.68481 & -0.51414 & 0.06490 \\ C & -5.85227 & -1.59038 & 0.39560 \\ C & -4.46736 & -1.42089 & 0.41558 \\ C & 3.54075 & -1.89495 & -0.93778 \\ C & 2.08779 & 2.42288 & 0.84756\end{array}$

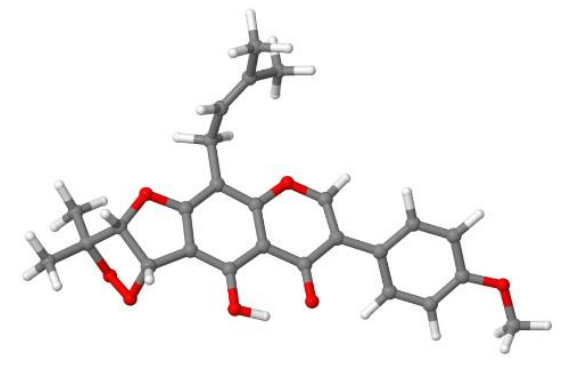




\begin{tabular}{|c|c|c|c|}
\hline C & 2.06895 & 3.46099 & -0.25302 \\
\hline 0 & 3.94420 & 0.35366 & -0.14713 \\
\hline 0 & 0.63273 & -2.76819 & -1.14285 \\
\hline 0 & -1.82968 & -2.12413 & -0.70768 \\
\hline 0 & -8.04622 & -0.57014 & 0.01256 \\
\hline C & -8.67955 & -1.80647 & 0.30109 \\
\hline C & 1.32454 & 4.57396 & -0.33658 \\
\hline C & 1.45611 & 5.49793 & -1.52527 \\
\hline C & 0.31804 & 5.02489 & 0.69410 \\
\hline C & 5.48194 & -1.51097 & 0.45875 \\
\hline C & 4.63041 & -0.82311 & -0.63541 \\
\hline C & 5.94213 & -0.59073 & 1.58585 \\
\hline C & 6.64714 & -2.28415 & -0.17175 \\
\hline O & 4.53484 & -2.42017 & 1.05422 \\
\hline 0 & 3.87035 & -3.01893 & -0.10108 \\
\hline $\mathrm{H}$ & -2.50888 & 1.98038 & 1.06036 \\
\hline $\mathrm{H}$ & -4.30768 & 1.83967 & -0.51796 \\
\hline $\mathrm{H}$ & -6.76682 & 1.54678 & -0.52811 \\
\hline $\mathrm{H}$ & -6.26666 & -2.56233 & 0.63797 \\
\hline $\mathrm{H}$ & -3.83635 & -2.26520 & 0.66385 \\
\hline $\mathrm{H}$ & 3.55527 & -2.29863 & -1.95428 \\
\hline $\mathrm{H}$ & 1.44179 & 2.71435 & 1.67928 \\
\hline $\mathrm{H}$ & 3.10699 & 2.34958 & 1.24776 \\
\hline $\mathrm{H}$ & 2.75565 & 3.24802 & -1.07311 \\
\hline $\mathrm{H}$ & -0.36536 & -2.82886 & -1.09781 \\
\hline $\mathrm{H}$ & -9.75080 & -1.62626 & 0.19389 \\
\hline $\mathrm{H}$ & -8.46886 & -2.13863 & 1.32627 \\
\hline $\mathrm{H}$ & -8.37143 & -2.59135 & -0.40213 \\
\hline $\mathrm{H}$ & 1.74775 & 6.50986 & -1.20989 \\
\hline $\mathrm{H}$ & 2.20102 & 5.13921 & -2.24258 \\
\hline $\mathrm{H}$ & 0.49744 & 5.60205 & -2.05316 \\
\hline $\mathrm{H}$ & 0.61458 & 5.98878 & 1.13125 \\
\hline $\mathrm{H}$ & -0.66285 & 5.18751 & 0.22592 \\
\hline $\mathrm{H}$ & 0.18294 & 4.31193 & 1.51018 \\
\hline $\mathrm{H}$ & 5.22344 & -0.50879 & -1.49716 \\
\hline $\mathrm{H}$ & 6.46933 & -1.17584 & 2.34587 \\
\hline $\mathrm{H}$ & 6.62857 & 0.16877 & 1.19773 \\
\hline $\mathrm{H}$ & 5.09265 & -0.08803 & 2.05089 \\
\hline $\mathrm{H}$ & 7.16225 & -2.86349 & 0.60057 \\
\hline $\mathrm{H}$ & 7.36854 & -1.59926 & -0.63188 \\
\hline $\mathrm{H}$ & 6.28839 & -2.98138 & -0.93422 \\
\hline
\end{tabular}

\section{Conformer 2}

Energy: -1532.86181 Hartree (Rel: $1.4 \mathrm{kcal} / \mathrm{mol}$ ) XYZ coordinates for conf 2 :

$\begin{array}{lrrr}\text { C } & 2.58283 & 0.08743 & -0.05230 \\ C & 2.25730 & -1.19185 & -0.52344 \\ C & 0.92900 & -1.59097 & -0.60165 \\ C & -0.07623 & -0.66444 & -0.17757 \\ C & 0.32763 & 0.60052 & 0.30342 \\ C & 1.65795 & 1.03116 & 0.39337 \\ C & -1.48457 & -1.02456 & -0.25074 \\ C & -2.44024 & 0.00598 & 0.18499 \\ C & -1.93143 & 1.17098 & 0.66009 \\ \text { O } & -0.62136 & 1.49252 & 0.72693 \\ C & -3.90585 & -0.19197 & 0.10655 \\ C & -4.75218 & 0.87226 & -0.26161 \\ C & -6.13099 & 0.72175 & -0.29756 \\ C & -6.71203 & -0.51391 & 0.02392 \\ C & -5.88730 & -1.58943 & 0.37603 \\ C & -4.50230 & -1.42393 & 0.41295 \\ C & 3.52341 & -1.92260 & -0.82690 \\ C & 2.05526 & 2.41228 & 0.87788 \\ C & 2.03187 & 3.44282 & -0.22965 \\ \text { O } & 3.92048 & 0.33921 & -0.08993 \\ O & 0.61180 & -2.80204 & -1.06503 \\ \text { O } & -1.85211 & -2.14725 & -0.66514 \\ \text { O } & -8.07250 & -0.56642 & -0.04636 \\ C & -8.71366 & -1.79876 & 0.24255 \\ C & 1.28580 & 4.55432 & -0.31796 \\ C & 1.41376 & 5.47172 & -1.51207\end{array}$

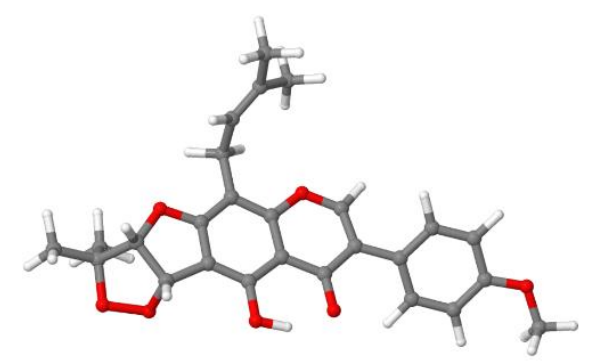




$\begin{array}{rrrr}\mathrm{C} & 0.28118 & 5.01001 & 0.71245 \\ \mathrm{C} & 5.62409 & -1.47177 & 0.32438 \\ \mathrm{C} & 4.59286 & -0.81842 & -0.64302 \\ \mathrm{C} & 5.46810 & -1.00785 & 1.77303 \\ \mathrm{C} & 7.06357 & -1.35858 & -0.17648 \\ \mathrm{O} & 5.28921 & -2.87396 & 0.20847 \\ \mathrm{O} & 3.82847 & -2.87809 & 0.21075 \\ \mathrm{H} & -2.54064 & 1.97818 & 1.04928 \\ \mathrm{H} & -4.32006 & 1.82717 & -0.54954 \\ \mathrm{H} & -6.77974 & 1.54154 & -0.58960 \\ \mathrm{H} & -6.30798 & -2.55775 & 0.62203 \\ \mathrm{H} & -3.87746 & -2.26775 & 0.67785 \\ \mathrm{H} & 3.56517 & -2.41885 & -1.80163 \\ \mathrm{H} & 1.40792 & 2.70668 & 1.70756 \\ \mathrm{H} & 3.07436 & 2.34557 & 1.27905 \\ \mathrm{H} & 2.71729 & 3.22597 & -1.04977 \\ \mathrm{H} & -0.38701 & -2.86019 & -1.02897 \\ \mathrm{H} & -9.78262 & -1.61638 & 0.11790 \\ \mathrm{H} & -8.51927 & -2.12322 & 1.27338 \\ \mathrm{H} & -8.39729 & -2.58995 & -0.44980 \\ \mathrm{H} & 1.70531 & 6.48554 & -1.20282 \\ \mathrm{H} & 2.15726 & 5.10973 & -2.22917 \\ \mathrm{H} & 0.45374 & 5.57222 & -2.03817 \\ \mathrm{H} & 0.57776 & 5.97665 & 1.14338 \\ \mathrm{H} & -0.70092 & 5.16904 & 0.24559 \\ \mathrm{H} & 0.14871 & 4.30172 & 1.53303 \\ \mathrm{H} & 5.05357 & -0.49725 & -1.58109 \\ \mathrm{H} & 6.17488 & -1.55832 & 2.40210 \\ \mathrm{H} & 4.45583 & -1.20975 & 2.13111 \\ \mathrm{H} & 5.66616 & 0.06355 & 1.87087 \\ \mathrm{H} & 7.73236 & -1.95638 & 0.45060 \\ \mathrm{H} & 7.14721 & -1.71646 & -1.20781 \\ \mathrm{H} & 7.39664 & -0.31603 & -0.13688\end{array}$


originally assigned structure of isoflavon cmpd 4, Fig. 14<smiles>COc1ccc(-c2coc3c4c(c(CC=C(C)C)c(O)c3c2=O)OC(C)(C)C2OOC42)cc1</smiles>

\begin{tabular}{|c|c|c|c|c|c|c|c|c|}
\hline \multirow[b]{2}{*}{ C-nom } & \multirow[b]{2}{*}{ iGau } & \multicolumn{2}{|c|}{ Rel energy } & \multicolumn{2}{|r|}{$\begin{array}{c}\text { Conf } 1 \\
2.0\end{array}$} & \multicolumn{2}{|c|}{1 Conf 2 Conf 3} & \\
\hline & & Exp & Calc & $\operatorname{diff}$ & 1 & 2 & 3 & \\
\hline $\mathrm{C} 2-\mathrm{CH}$ & 9 & 152.00 & 152.49 & 0.49 & [ 152.79 & 152.47 & 152.51 & ] \\
\hline C3-C & 8 & 125.10 & 125.89 & 0.79 & {$[125.96$} & 125.97 & 125.80 & ] \\
\hline $\mathrm{C} 4-\mathrm{C}$ & 7 & 180.80 & 179.94 & -0.86 & 179.80 & 179.91 & 179.97 & ] \\
\hline $\mathrm{C} 5-\mathrm{C}$ & 3 & 162.50 & 163.66 & 1.16 & {$[163.24$} & 163.48 & 163.89 & ] \\
\hline $\mathrm{C} 6-\mathrm{C}$ & 2 & 107.50 & 116.44 & 8.94 & {$[115.50$} & 116.18 & 116.77 & ] \\
\hline $\mathrm{C} 7-\mathrm{C}$ & 1 & 165.50 & 160.41 & -5.09 & [ 159.36 & 160.47 & 160.39 & ] \\
\hline $\mathrm{C} 8-\mathrm{C}$ & 6 & 100.80 & 99.16 & -1.64 & {$\left[\begin{array}{l}97.91 \\
{[}\end{array}\right.$} & 98.91 & 99.50 & ] \\
\hline $\mathrm{C} 9-\mathrm{C}$ & 5 & 152.40 & 154.28 & 1.88 & [ 154.41 & 154.43 & 154.11 & ] \\
\hline $\mathrm{C} 10-\mathrm{C}$ & 4 & 106.60 & 107.87 & 1.27 & {$[107.96$} & 107.97 & 107.76 & ] \\
\hline $\mathrm{C} 1{ }^{\prime}-\mathrm{C}$ & 11 & 122.90 & 122.39 & -0.51 & [ 122.25 & 122.52 & 122.24 & ] \\
\hline $\mathrm{C} 2{ }^{\prime}-\mathrm{CH}$ & 12 & 130.20 & 130.66 & 0.46 & {$[130.57$} & 130.70 & 130.61 & ] \\
\hline $\mathrm{C}^{\prime}-\mathrm{CH}$ & 13 & 114.10 & 113.60 & -0.50 & [ 113.62 & 113.58 & 113.62 & ] \\
\hline $\mathrm{C} 4^{\prime}-\mathrm{C}$ & 14 & 159.90 & 158.52 & -1.38 & 158.61 & 158.28 & 158.79 & ] \\
\hline $\mathrm{C} 5{ }^{\prime}-\mathrm{CH}$ & 15 & 114.10 & 113.60 & -0.50 & {$[113.62$} & 113.58 & 113.62 & ] \\
\hline $\mathrm{C} 6^{\prime}-\mathrm{CH}$ & 16 & 130.20 & 130.66 & 0.46 & {$[130.57$} & 130.70 & 130.61 & ] \\
\hline $\mathrm{C} 1 "-\mathrm{CH} 2$ & 17 & 21.60 & 23.37 & 1.77 & {$[\quad 23.28$} & 23.14 & 23.63 & ] \\
\hline $\mathrm{C} 2 "-\mathrm{CH}$ & 18 & 121.10 & 122.98 & 1.88 & [ 122.82 & 122.88 & 123.10 & ] \\
\hline C3"-C & 19 & 132.40 & 130.71 & -1.69 & [ 131.35 & 131.47 & 129.80 & ] \\
\hline $\mathrm{C} 4 "-\mathrm{CH} 3$ & 20 & 25.70 & 25.43 & -0.27 & [ 25.46 & 25.60 & 25.23 & ] \\
\hline $\mathrm{C} 5 "-\mathrm{CH} 3$ & 21 & 17.80 & 17.33 & -0.47 & 17.22 & 17.28 & 17.40 & ] \\
\hline $\mathrm{Cl} \prime^{\prime} \prime-\mathrm{CH}$ & 22 & 83.00 & 70.06 & -12.94 & 72.90 & 70.19 & 69.79 & \\
\hline $\mathrm{C} 2{ }^{\prime}, '-\mathrm{CH}$ & 23 & 98.20 & 84.17 & -14.03 & 83.68 & 83.97 & 84.42 & ] \\
\hline C3' ' & 24 & 85.80 & 77.75 & -8.05 & 80.67 & 77.62 & 77.79 & \\
\hline $\mathrm{C} 4 \mathrm{I}^{\prime} \mathrm{\prime}-\mathrm{CH} 3$ & 26 & 23.90 & 21.20 & -2.70 & 27.83 & 21.16 & 20.99 & ] \\
\hline C5' ' '-CH3 & 27 & 18.10 & 21.31 & 3.21 & 21.47 & 21.25 & 21.38 & ] \\
\hline $\mathrm{COMe} 4{ }^{\prime}-\mathrm{CH} 3$ & 31 & 55.40 & 53.05 & -2.35 & 53.05 & 53.02 & 53.08 & \\
\hline
\end{tabular}

Conformer 1

Energy: -1532.83034 Hartree (Rel: $2.0 \mathrm{kcal} / \mathrm{mol})$ XYZ coordinates for conf 1 :

\begin{tabular}{|c|c|c|c|}
\hline C & -2.53911 & -0.36697 & -0.59876 \\
\hline C & -2.14849 & 0.96270 & -0.84406 \\
\hline C & -0.78547 & 1.27086 & -0.75952 \\
\hline C & 0.18491 & 0.26771 & -0.45004 \\
\hline C & -0.27113 & -1.04049 & -0.24100 \\
\hline C & -1.61955 & -1.39248 & -0.30664 \\
\hline C & 1.60487 & 0.58240 & -0.37476 \\
\hline $\mathrm{C}$ & 2.50020 & -0.55180 & -0.09346 \\
\hline C & 1.93409 & -1.76960 & 0.10787 \\
\hline 0 & 0.61247 & -2.04568 & 0.03842 \\
\hline C & 3.97252 & -0.39783 & -0.04237 \\
\hline C & 4.81512 & -1.39658 & -0.56827 \\
\hline C & 6.19639 & -1.29479 & -0.48680 \\
\hline C & 6.78466 & -0.17398 & 0.11791 \\
\hline C & 5.96471 & 0.83866 & 0.63098 \\
\hline C & 4.57683 & 0.72248 & 0.54638 \\
\hline C & -3.16599 & 2.04358 & -1.16426 \\
\hline C & -3.77178 & 2.65824 & 0.07898 \\
\hline C & -3.63614 & 3.91290 & 0.53451 \\
\hline
\end{tabular}

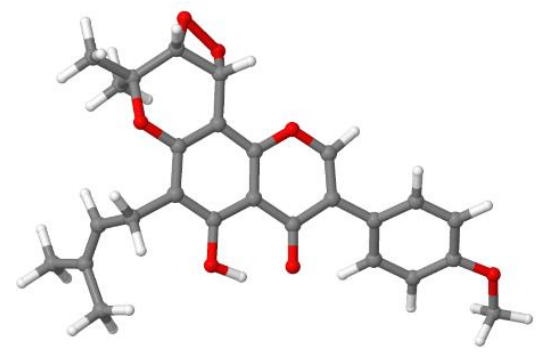




\begin{tabular}{|c|c|c|c|}
\hline C & -4.34202 & 4.34986 & 1.79748 \\
\hline C & -2.81025 & 4.99047 & -0.12571 \\
\hline C & -2.08835 & -2.79931 & -0.20322 \\
\hline C & -3.58964 & -3.01928 & -0.28109 \\
\hline C & -4.43469 & -1.75698 & 0.02750 \\
\hline 0 & -3.86745 & -0.63738 & -0.72115 \\
\hline C & -5.84987 & -1.90372 & -0.52588 \\
\hline C & -4.45438 & -1.40472 & 1.51818 \\
\hline 0 & -0.39303 & 2.53377 & -0.97728 \\
\hline 0 & 2.02895 & 1.74655 & -0.54869 \\
\hline O & 8.14729 & -0.16194 & 0.14642 \\
\hline $\mathrm{C}$ & 8.79881 & 0.95696 & 0.72698 \\
\hline 0 & -2.12550 & -3.39661 & 1.14429 \\
\hline 0 & -3.49014 & -3.93039 & 0.84141 \\
\hline $\mathrm{H}$ & 2.49569 & -2.66121 & 0.35971 \\
\hline $\mathrm{H}$ & 4.38043 & -2.25630 & -1.07159 \\
\hline $\mathrm{H}$ & 6.84296 & -2.06275 & -0.89953 \\
\hline $\mathrm{H}$ & 6.39112 & 1.71953 & 1.09700 \\
\hline $\mathrm{H}$ & 3.95662 & 1.51831 & 0.94004 \\
\hline $\mathrm{H}$ & -2.68452 & 2.80276 & -1.78319 \\
\hline $\mathrm{H}$ & -3.96865 & 1.59682 & -1.76275 \\
\hline $\mathrm{H}$ & -4.38969 & 1.97231 & 0.65939 \\
\hline $\mathrm{H}$ & -5.00873 & 5.20217 & 1.60304 \\
\hline $\mathrm{H}$ & -4.94138 & 3.54375 & 2.23292 \\
\hline $\mathrm{H}$ & -3.62159 & 4.68717 & 2.55620 \\
\hline $\mathrm{H}$ & -2.20166 & 4.62140 & -0.95269 \\
\hline $\mathrm{H}$ & -2.12906 & 5.44731 & 0.60520 \\
\hline $\mathrm{H}$ & -3.45254 & 5.80108 & -0.49915 \\
\hline $\mathrm{H}$ & -1.51657 & -3.47010 & -0.85479 \\
\hline $\mathrm{H}$ & -3.96395 & -3.53501 & -1.17133 \\
\hline $\mathrm{H}$ & -6.43560 & -1.00119 & -0.32673 \\
\hline $\mathrm{H}$ & -6.34965 & -2.75519 & -0.05135 \\
\hline $\mathrm{H}$ & -5.82740 & -2.06964 & -1.60811 \\
\hline $\mathrm{H}$ & -3.44558 & -1.27798 & 1.91671 \\
\hline $\mathrm{H}$ & -5.01686 & -0.47664 & 1.65984 \\
\hline $\mathrm{H}$ & -4.93605 & -2.20410 & 2.08609 \\
\hline $\mathrm{H}$ & 0.60171 & 2.54527 & -0.86844 \\
\hline $\mathrm{H}$ & 9.86860 & 0.75878 & 0.63927 \\
\hline $\mathrm{H}$ & 8.53729 & 1.07130 & 1.78727 \\
\hline $\mathrm{H}$ & 8.55700 & 1.88599 & 0.19415 \\
\hline
\end{tabular}

\section{Conformer 2}

Energy: -1532.83356 Hartree (Rel: $0.0 \mathrm{kcal} / \mathrm{mol}$ ) XYZ coordinates for conf 2 :

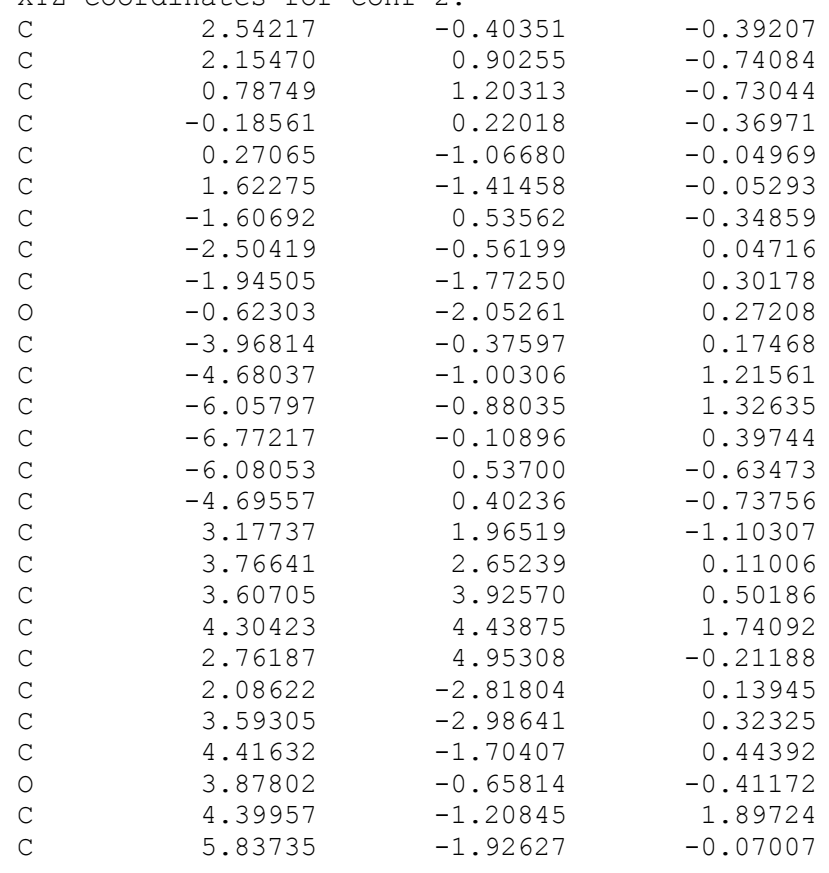

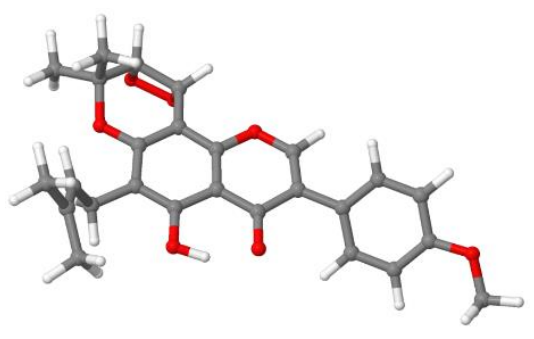




$\begin{array}{rrr}0.39523 & 2.44244 & -1.05679 \\ -2.03190 & 1.67322 & -0.65064 \\ -8.11993 & -0.04481 & 0.59080 \\ -8.89279 & 0.73396 & -0.30902 \\ 2.20387 & -3.56370 & -1.12654 \\ 3.68319 & -3.61738 & -0.97520 \\ -2.51615 & -2.65905 & 0.55049 \\ -4.14151 & -1.57650 & 1.96544 \\ -6.60311 & -1.35873 & 2.13391 \\ -6.60520 & 1.14525 & -1.36252 \\ -4.17411 & 0.91483 & -1.53642 \\ 3.98928 & 1.48625 & -1.66300 \\ 2.70496 & 2.68661 & -1.77201 \\ 4.39718 & 2.00953 & 0.72484 \\ 3.57787 & 4.80140 & 2.48214 \\ 4.91852 & 3.66689 & 2.21612 \\ 4.95585 & 5.29180 & 1.50323 \\ 2.15463 & 4.52912 & -1.01299 \\ 3.39009 & 5.75127 & -0.63297 \\ 2.07771 & 5.43929 & 0.49698 \\ 1.44832 & -3.37512 & 0.82900 \\ 3.88687 & -3.68838 & 1.11408 \\ 4.96266 & -0.27365 & 1.97401 \\ 4.86163 & -1.94511 & 2.56309 \\ 3.37842 & -1.02234 & 2.24652 \\ 5.81029 & -2.27720 & -1.10471 \\ 6.39788 & -0.98754 & -0.02862 \\ 6.35588 & -2.67039 & 0.54385 \\ -0.60318 & 2.45275 & -0.98548 \\ -9.92627 & 0.64621 & 0.03106 \\ -8.81644 & 0.35684 & -1.33743 \\ -8.59286 & 1.78996 & -0.28934\end{array}$

\section{Conformer 3}

Energy: -1532.83341 Hartree (Rel: $0.1 \mathrm{kcal} / \mathrm{mol}$ ) XYZ coordinates for conf 3 :

\begin{tabular}{|c|c|c|c|}
\hline C & -2.39551 & -0.26348 & -0.35741 \\
\hline $\mathrm{C}$ & -2.00401 & 1.03117 & -0.74234 \\
\hline $\mathrm{C}$ & -0.63589 & 1.33072 & -0.72642 \\
\hline $\mathrm{C}$ & 0.33732 & 0.35165 & -0.36072 \\
\hline C & -0.12370 & -0.91708 & 0.02013 \\
\hline C & -1.47682 & -1.25879 & 0.03122 \\
\hline $\mathrm{C}$ & 1.76028 & 0.66070 & -0.36613 \\
\hline C & 2.65810 & -0.43633 & 0.03031 \\
\hline C & 2.08961 & -1.60883 & 0.41163 \\
\hline 0 & 0.76553 & -1.88061 & 0.41393 \\
\hline C & 4.13232 & -0.29369 & 0.01601 \\
\hline C & 4.95006 & -1.36532 & -0.39310 \\
\hline C & 6.33398 & -1.26960 & -0.37070 \\
\hline C & 6.95001 & -0.08275 & 0.05341 \\
\hline C & 6.15481 & 1.00034 & 0.44777 \\
\hline C & 4.76408 & 0.88985 & 0.42515 \\
\hline C & -3.01302 & 2.09305 & -1.13963 \\
\hline C & -3.31956 & 3.04637 & -0.00444 \\
\hline C & -4.48515 & 3.23150 & 0.63320 \\
\hline C & -4.59588 & 4.24724 & 1.74692 \\
\hline C & -5.76666 & 2.49165 & 0.33531 \\
\hline $\mathrm{C}$ & -1.96787 & -2.56142 & 0.56173 \\
\hline C & -3.42681 & -2.89020 & 0.24814 \\
\hline C & -4.17104 & -1.89230 & -0.64072 \\
\hline 0 & -3.72918 & -0.53119 & -0.37326 \\
\hline C & -3.91235 & -2.21276 & -2.12006 \\
\hline $\mathrm{C}$ & -5.66164 & -1.88602 & -0.30870 \\
\hline 0 & -0.24758 & 2.56199 & -1.08705 \\
\hline O & 2.18462 & 1.79224 & -0.69034 \\
\hline 0 & 8.31293 & -0.08190 & 0.03519 \\
\hline C & 8.99015 & 1.09963 & 0.43392 \\
\hline 0 & -2.33354 & -2.50399 & 1.98788 \\
\hline 0 & -3.75657 & -2.77852 & 1.65195 \\
\hline $\mathrm{H}$ & 2.65300 & -2.46211 & 0.77001 \\
\hline $\mathrm{H}$ & 4.49261 & -2.28110 & -0.75865 \\
\hline
\end{tabular}

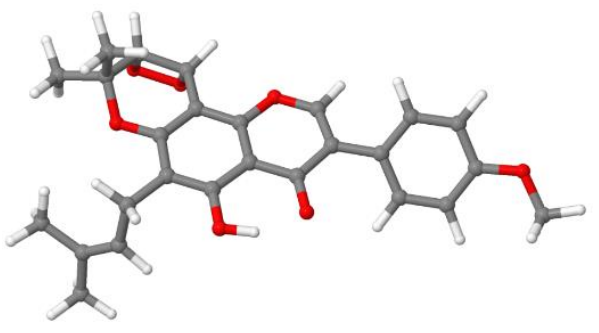




$\begin{array}{rrrr}\text { H } & 6.96097 & -2.09463 & -0.69381 \\ \mathrm{H} & 6.60312 & 1.93211 & 0.77315 \\ \mathrm{H} & 4.16317 & 1.73925 & 0.72520 \\ \mathrm{H} & -2.58762 & 2.66802 & -1.97191 \\ \mathrm{H} & -3.91767 & 1.60500 & -1.50495 \\ \mathrm{H} & -2.46416 & 3.64096 & 0.31512 \\ \mathrm{H} & -4.90459 & 3.76960 & 2.68774 \\ \mathrm{H} & -3.64774 & 4.76474 & 1.92385 \\ \mathrm{H} & -5.35927 & 5.00490 & 1.51846 \\ \mathrm{H} & -5.64789 & 1.70732 & -0.41480 \\ \mathrm{H} & -6.54577 & 3.18523 & -0.01226 \\ \mathrm{H} & -6.15590 & 2.02004 & 1.24822 \\ \mathrm{H} & -1.24979 & -3.36914 & 0.40393 \\ \mathrm{H} & -3.60942 & -3.91093 & -0.11105 \\ \mathrm{H} & -4.40779 & -1.46748 & -2.74955 \\ \mathrm{H} & -4.30910 & -3.20060 & -2.37708 \\ \mathrm{H} & -2.84241 & -2.20153 & -2.35342 \\ \mathrm{H} & -5.80833 & -1.64153 & 0.74609 \\ \mathrm{H} & -6.17784 & -1.14054 & -0.92114 \\ \mathrm{H} & -6.10323 & -2.86786 & -0.51018 \\ \mathrm{H} & 0.75125 & 2.57341 & -1.02774 \\ \mathrm{H} & 10.05518 & 0.88136 & 0.33653 \\ \mathrm{H} & 8.76747 & 1.36005 & 1.47711 \\ \mathrm{H} & 8.73310 & 1.94925 & -0.21221\end{array}$


revised structure of isoflavon cmpd 4, Fig.14<smiles>COc1ccc(-c2coc3c4c(c(CC=C(C)C)c(O)c3c2=O)O[C@@H]2[C@@H]4OOC2(C)C)cc1</smiles>

$$
\text { Rel energy (kcal/mol): } \begin{array}{ccc}
\text { Conf } 1 & \text { Conf } 2 \\
1.3 & 0.0
\end{array}
$$

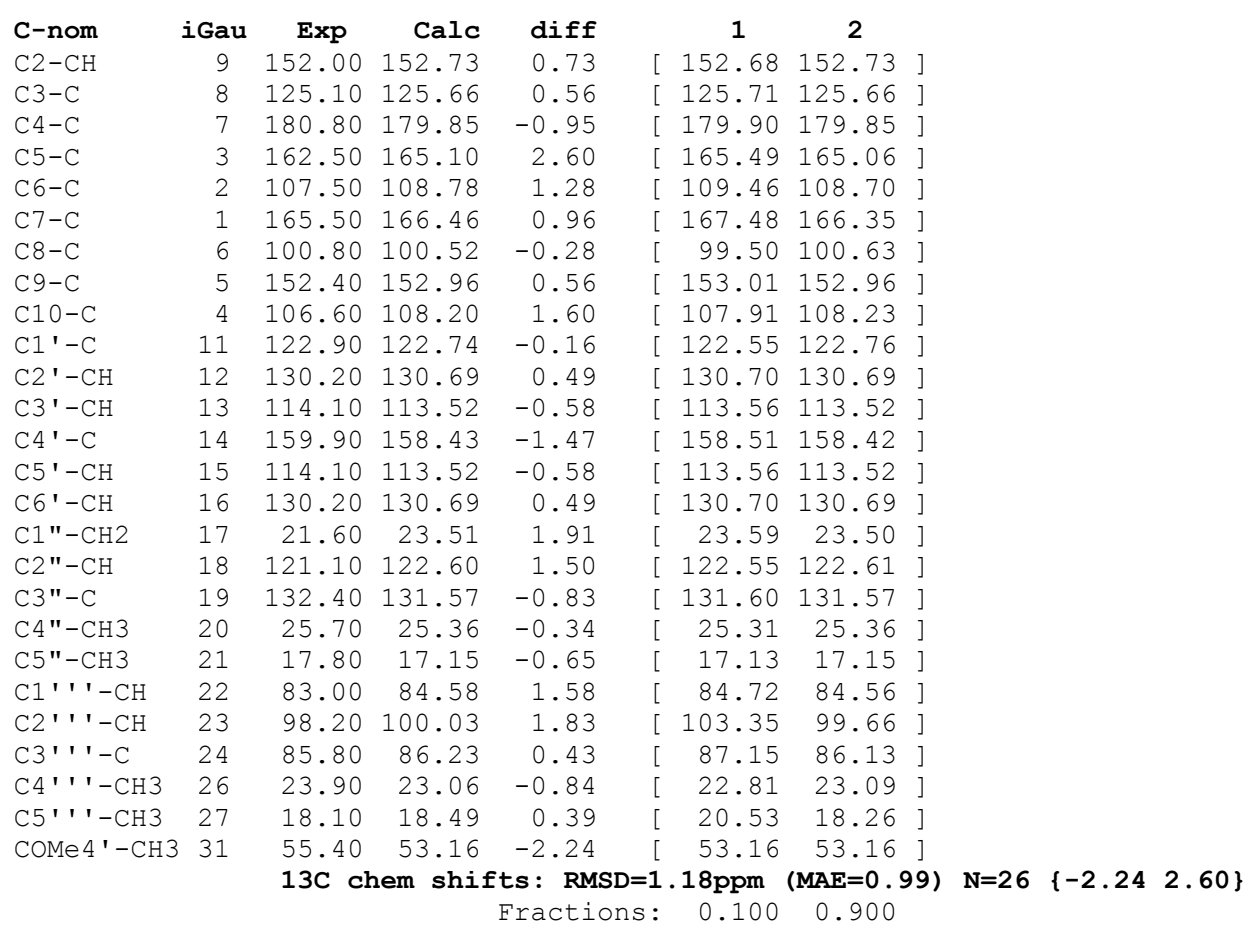

\section{Conformer 1}

Energy: -1532.86198 Hartree (Rel: $1.3 \mathrm{kcal} / \mathrm{mol}$ ) XYZ coordinates for conf 1 :

$\begin{array}{lrrr}C & -2.44345 & -0.18946 & -0.77198 \\ C & -2.03400 & 1.13478 & -0.93520 \\ C & -0.65484 & 1.36486 & -0.79860 \\ C & 0.26639 & 0.30052 & -0.52447 \\ C & -0.23565 & -1.00674 & -0.37210 \\ C & -1.59137 & -1.26509 & -0.49565 \\ C & 1.69395 & 0.55718 & -0.40444 \\ C & 2.54027 & -0.61819 & -0.13649 \\ C & 1.92791 & -1.82011 & 0.01709 \\ \text { O } & 0.60018 & -2.04986 & -0.09723 \\ C & 4.01545 & -0.51914 & -0.04244 \\ C & 4.83574 & -1.52779 & -0.58425 \\ C & 6.21720 & -1.47965 & -0.46393 \\ C & 6.82845 & -0.40440 & 0.19776 \\ C & 6.03166 & 0.61772 & 0.72822 \\ C & 4.64327 & 0.55557 & 0.60391 \\ C & -3.00865 & 2.26035 & -1.22137 \\ C & -3.59674 & 2.85309 & 0.04130\end{array}$

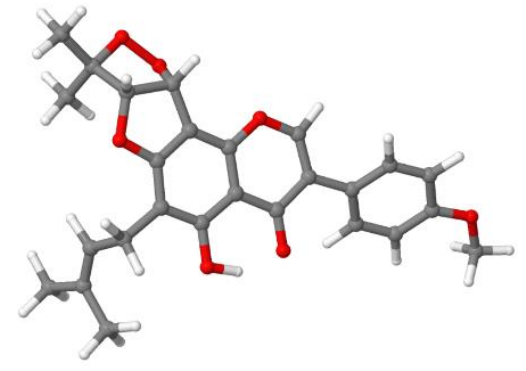




\begin{tabular}{|c|c|c|c|}
\hline C & -3.41771 & 4.08626 & 0.53833 \\
\hline C & -4.11017 & 4.50554 & 1.81448 \\
\hline C & -2.55203 & 5.15423 & -0.08548 \\
\hline C & -2.40767 & -2.50373 & -0.34375 \\
\hline C & -3.82175 & -2.00818 & -0.73954 \\
\hline C & -4.70988 & -2.46813 & 0.45492 \\
\hline 0 & -3.74535 & -0.57271 & -0.89891 \\
\hline C & -5.96802 & -3.21145 & 0.00774 \\
\hline C & -5.01313 & -1.34610 & 1.44819 \\
\hline O & -0.19971 & 2.61483 & -0.95199 \\
\hline O & 2.16932 & 1.70914 & -0.52521 \\
\hline O & 8.18966 & -0.44311 & 0.26129 \\
\hline $\mathrm{C}$ & 8.86419 & 0.62780 & 0.90266 \\
\hline O & -2.54040 & -2.87564 & 1.04407 \\
\hline 0 & -3.87322 & -3.47402 & 1.07310 \\
\hline $\mathrm{H}$ & 2.45226 & -2.73639 & 0.26052 \\
\hline $\mathrm{H}$ & 4.38396 & -2.35181 & -1.13042 \\
\hline $\mathrm{H}$ & 6.84687 & -2.25493 & -0.88898 \\
\hline $\mathrm{H}$ & 6.47670 & 1.46427 & 1.23841 \\
\hline $\mathrm{H}$ & 4.04120 & 1.35811 & 1.01189 \\
\hline $\mathrm{H}$ & -2.50238 & 3.02237 & -1.81707 \\
\hline $\mathrm{H}$ & -3.82496 & 1.85813 & -1.83531 \\
\hline $\mathrm{H}$ & -4.23787 & 2.16864 & 0.59829 \\
\hline $\mathrm{H}$ & -4.74612 & 5.38674 & 1.64779 \\
\hline $\mathrm{H}$ & -4.73814 & 3.70691 & 2.22228 \\
\hline $\mathrm{H}$ & -3.37957 & 4.79200 & 2.58423 \\
\hline $\mathrm{H}$ & -1.94792 & 4.78832 & -0.91727 \\
\hline $\mathrm{H}$ & -1.86310 & 5.56851 & 0.66315 \\
\hline $\mathrm{H}$ & -3.16454 & 5.99497 & -0.44189 \\
\hline $\mathrm{H}$ & -2.07216 & -3.37255 & -0.92004 \\
\hline $\mathrm{H}$ & -4.17246 & -2.41573 & -1.69156 \\
\hline $\mathrm{H}$ & -6.65478 & -2.52138 & -0.49363 \\
\hline $\mathrm{H}$ & -6.48390 & -3.64113 & 0.87208 \\
\hline $\mathrm{H}$ & -5.71726 & -4.02308 & -0.68297 \\
\hline $\mathrm{H}$ & -4.08650 & -0.91251 & 1.83170 \\
\hline $\mathrm{H}$ & -5.60306 & -0.55178 & 0.98153 \\
\hline $\mathrm{H}$ & -5.57746 & -1.75844 & 2.29067 \\
\hline $\mathrm{H}$ & 0.79434 & 2.57340 & -0.82981 \\
\hline $\mathrm{H}$ & 9.92830 & 0.39406 & 0.83558 \\
\hline $\mathrm{H}$ & 8.57738 & 0.71071 & 1.95936 \\
\hline $\mathrm{H}$ & 8.67071 & 1.58514 & 0.40102 \\
\hline
\end{tabular}

\section{Conformer 2}

Energy: -1532.86405 Hartree (Rel: $0.0 \mathrm{kcal} / \mathrm{mol}$ ) XYZ coordinates for conf 2 :

\begin{tabular}{|c|c|c|c|}
\hline C & -2.46151 & -0.22396 & -0.82350 \\
\hline C & -2.06986 & 1.10889 & -0.96259 \\
\hline $\mathrm{C}$ & -0.69555 & 1.35474 & -0.81031 \\
\hline $\mathrm{C}$ & 0.23672 & 0.29719 & -0.54733 \\
\hline $\mathrm{C}$ & -0.25068 & -1.01914 & -0.42715 \\
\hline $\mathrm{C}$ & -1.60193 & -1.29144 & -0.56420 \\
\hline $\mathrm{C}$ & 1.65942 & 0.56908 & -0.40921 \\
\hline C & 2.51824 & -0.60176 & -0.16123 \\
\hline $\mathrm{C}$ & 1.92034 & -1.81501 & -0.04351 \\
\hline 0 & 0.59718 & -2.05912 & -0.17482 \\
\hline C & 3.99113 & -0.48636 & -0.05090 \\
\hline C & 4.82951 & -1.47132 & -0.60828 \\
\hline $\mathrm{C}$ & 6.20910 & -1.40885 & -0.47328 \\
\hline $\mathrm{C}$ & 6.80021 & -0.34204 & 0.21953 \\
\hline $\mathrm{C}$ & 5.98541 & 0.65724 & 0.76581 \\
\hline $\mathrm{C}$ & 4.59911 & 0.58060 & 0.62652 \\
\hline C & -3.05693 & 2.22751 & -1.23389 \\
\hline $\mathrm{C}$ & -3.64287 & 2.80805 & 0.03531 \\
\hline C & -3.47054 & 4.03873 & 0.54073 \\
\hline $\mathrm{C}$ & -4.15921 & 4.44341 & 1.82362 \\
\hline $\mathrm{C}$ & -2.61584 & 5.11767 & -0.07924 \\
\hline C & -2.39252 & -2.55948 & -0.47493 \\
\hline $\mathrm{C}$ & -3.83994 & -2.04973 & -0.75210 \\
\hline C & -4.57818 & -2.42045 & 0.55827 \\
\hline 0 & -3.75766 & -0.62324 & -0.96544 \\
\hline $\mathrm{C}$ & -5.22769 & -3.80453 & 0.43540 \\
\hline
\end{tabular}

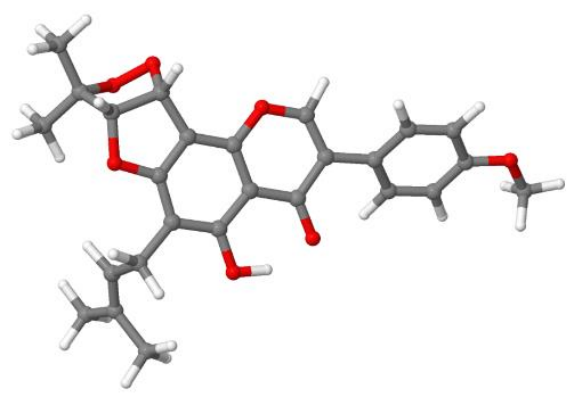




$\begin{array}{rrr}-5.55743 & -1.36438 & 1.06261 \\ -0.25548 & 2.61392 & -0.93441 \\ 2.12197 & 1.72911 & -0.50055 \\ 8.16136 & -0.36517 & 0.29572 \\ 8.81530 & 0.69867 & 0.96908 \\ -2.44696 & -3.18073 & 0.82156 \\ -3.50302 & -2.45106 & 1.51968 \\ 2.45533 & -2.73003 & 0.18101 \\ 4.39344 & -2.28790 & -1.17796 \\ 6.85262 & -2.16595 & -0.91022 \\ 6.41478 & 1.49729 & 1.29976 \\ 3.98290 & 1.36569 & 1.04714 \\ -2.56133 & 2.99847 & -1.82736 \\ -3.87339 & 1.82277 & -1.84587 \\ -4.27777 & 2.11637 & 0.59090 \\ -4.80159 & 5.32188 & 1.66738 \\ -4.77988 & 3.63753 & 2.22838 \\ -3.42643 & 4.72814 & 2.59192 \\ -2.01570 & 4.76320 & -0.91874 \\ -1.92372 & 5.52873 & 0.66823 \\ -3.23606 & 5.95808 & -0.42293 \\ -2.04898 & -3.35598 & -1.14215 \\ -4.31680 & -2.47660 & -1.63726 \\ -6.04404 & -3.78967 & -0.29553 \\ -5.63502 & -4.10545 & 1.40537 \\ -4.49429 & -4.55567 & 0.12843 \\ -5.05985 & -0.40234 & 1.19532 \\ -6.37771 & -1.23792 & 0.34882 \\ -5.97968 & -1.68189 & 2.02120 \\ 0.73723 & 2.58308 & -0.80143 \\ 9.88305 & 0.48041 & 0.90751 \\ 8.51686 & 0.75287 & 2.02445 \\ 8.61422 & 1.66511 & 0.48828\end{array}$


originally assigned structure for the sesquiterpene dimer from Xylopia aromatica, Fig.15

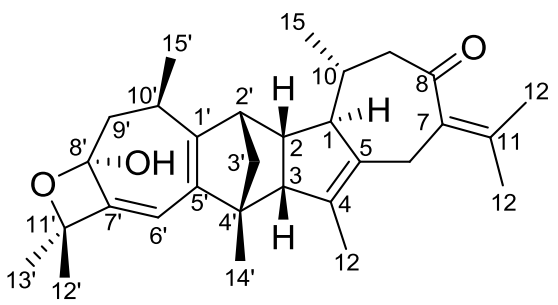

Conf 1 Conf 2

Rel energy (kcal/mol): $\quad 0.0 \quad 2.5$

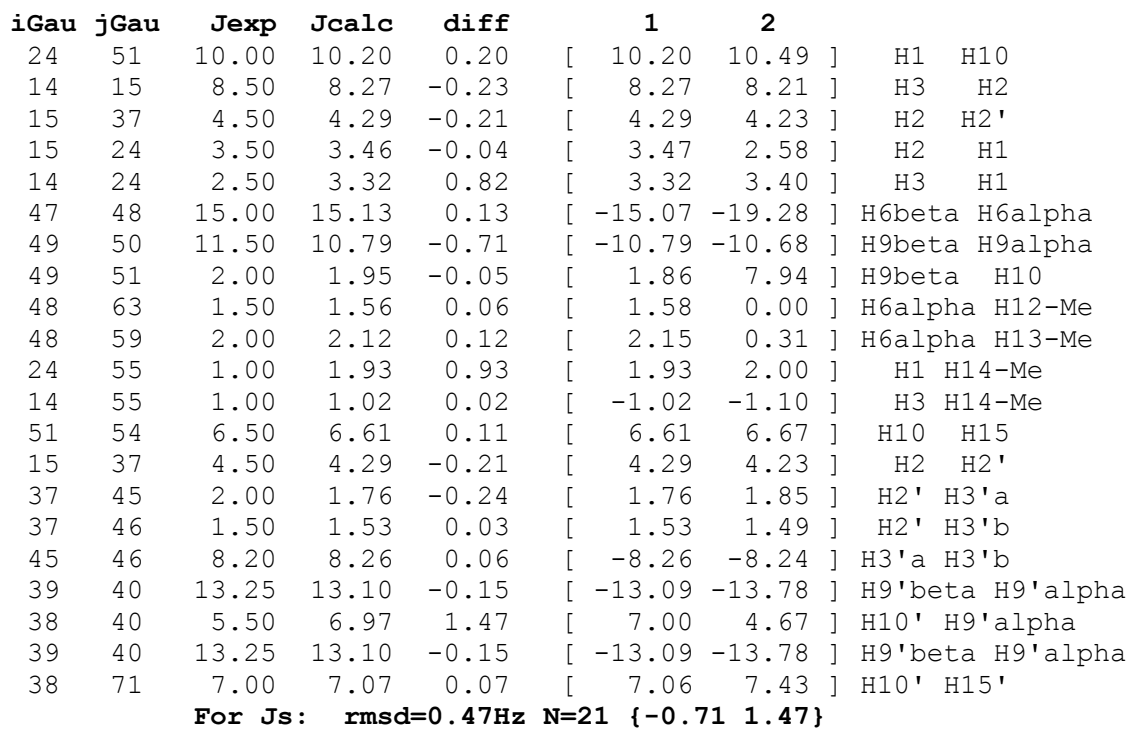

\begin{tabular}{|c|c|c|c|c|c|c|c|}
\hline H-nom & iGau & Exp & Calc & $\operatorname{diff}$ & 1 & 2 & \\
\hline $\mathrm{H} 1$ & 24 & 1.80 & 2.23 & 0.43 & 2.23 & $2.13]$ & ] \\
\hline $\mathrm{H} 2$ & 15 & 2.35 & 2.52 & 0.17 & 2.52 & $2.50 \mathrm{]}$ & 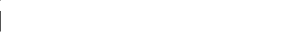 \\
\hline H3 & 14 & 2.74 & 3.00 & 0.26 & 3.00 & $3.09]$ & ] \\
\hline H6alpha & 48 & 2.60 & 2.90 & 0.30 & 2.89 & $3.46]$ & \\
\hline H6beta & 47 & 3.23 & 3.26 & 0.03 & 3.26 & $3.12]$ & \\
\hline H9alpha & 50 & 2.72 & 2.84 & 0.12 & 2.85 & $2.13]$ & ] \\
\hline H9beta & 49 & 2.19 & 2.32 & 0.13 & 2.31 & $3.08]$ & 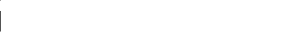 \\
\hline H1O & 51 & 1.38 & 1.74 & 0.36 & 1.74 & $1.76]$ & \\
\hline H12-Me & 63 & 1.84 & 1.94 & 0.10 & 1.94 & $2.07]$ & \\
\hline $\mathrm{H} 13-\mathrm{Me}$ & 59 & 1.96 & 2.08 & 0.12 & 2.08 & $2.38]$ & \\
\hline H1 4-Me & 55 & 1.43 & 1.60 & 0.17 & 1.60 & $1.66]$ & \\
\hline H15 & 54 & 1.07 & 1.22 & 0.15 & 1.22 & $1.29]$ & \\
\hline H2 ' & 37 & 2.84 & 2.85 & 0.01 & 2.85 & $2.90]$ & \\
\hline H3'a & 45 & 1.33 & 1.54 & 0.21 & 1.54 & 1.57 ] & \\
\hline H3'b & 46 & 1.26 & 1.42 & 0.16 & 1.42 & $1.45]$ & \\
\hline H6 ' & 41 & 5.57 & 5.82 & 0.25 & 5.82 & $5.80]$ & \\
\hline H9'alpha & 40 & 2.10 & 2.22 & 0.12 & 2.23 & $1.53 \mathrm{~J}$ & \\
\hline H9'beta & 39 & 1.69 & 1.98 & 0.29 & 1.98 & $1.94]$ & \\
\hline H10' & 38 & 2.75 & 2.94 & 0.19 & 2.94 & $2.84]$ & \\
\hline H12' & 66 & 1.44 & 1.53 & 0.09 & 1.53 & $1.53 \mathrm{~J}$ & \\
\hline H13' & 68 & 1.41 & 1.56 & 0.15 & 1.56 & $1.80 \mathrm{]}$ & \\
\hline H14' & 42 & 1.40 & 1.43 & 0.03 & 1.43 & 1.47 ] & \\
\hline \multirow[t]{2}{*}{ H15 ' } & 71 & 1.26 & 1.35 & 0.09 & {$\left[\begin{array}{ll}1.35 \\
\end{array}\right.$} & $5 \quad 1.26]$ & \\
\hline & & $\begin{array}{l}1 \mathrm{H} \text { chem } \\
\mathrm{m}=1.000\end{array}$ & $\begin{array}{r}\text { shifts: } \\
\mathrm{b}=0.00\end{array}$ & : $\mathrm{RMSD}=0$ & $.20 \mathrm{ppm}$ & $(\mathrm{MAE}=0.17)$ & $\mathrm{N}=23\left\{\begin{array}{lll}0.01 & 0.43\end{array}\right\}$ \\
\hline C-nom & iGau & $\operatorname{Exp}$ & Calc & diff & 1 & 2 & \\
\hline $\mathrm{C} 1-\mathrm{CH}$ & 18 & 57.81 & 56.94 & -0.87 & 57.01 & $52.40]$ & \\
\hline $\mathrm{C} 2-\mathrm{CH}$ & 5 & 51.82 & 53.21 & 1.39 & 53.21 & $53.32]$ & \\
\hline
\end{tabular}




\begin{tabular}{|c|c|c|c|c|c|c|c|}
\hline $\mathrm{C} 3-\mathrm{CH}$ & 4 & 61.26 & 62.53 & 1.27 & 62.53 & $62.35]$ & \\
\hline $\mathrm{C} 4-\mathrm{C}$ & 16 & 136.22 & 136.22 & -0.00 & {$[136.26$} & $133.18]$ & \\
\hline $\mathrm{C} 5-\mathrm{C}$ & 17 & 137.74 & 141.02 & 3.28 & [ 141.02 & 141.03 & \\
\hline $\mathrm{C} 6-\mathrm{CH} 2$ & 19 & 28.25 & 30.20 & 1.95 & {$[\quad 30.18$} & 31.27 & \\
\hline $\mathrm{C} 7-\mathrm{C}$ & 20 & 133.11 & 133.02 & -0.09 & {$[133.04$} & $131.50]$ & \\
\hline $\mathrm{C} 8-\mathrm{C}$ & 21 & 205.88 & 205.65 & -0.23 & {$[205.68$} & 203.89 & \\
\hline $\mathrm{C} 9-\mathrm{CH} 2$ & 22 & 50.74 & 49.87 & -0.87 & {$\left[\begin{array}{ll}49.93 \\
0\end{array}\right.$} & $45.58]$ & \\
\hline $\mathrm{Cl} 0-\mathrm{CH}$ & 23 & 41.28 & 41.02 & -0.26 & 41.03 & 40.47 & \\
\hline $\mathrm{C} 11-\mathrm{C}$ & 27 & 140.50 & 144.29 & 3.79 & {$[144.16$} & $153.40]$ & \\
\hline $\mathrm{C} 12-\mathrm{CH} 3$ & 29 & 22.56 & 22.15 & -0.41 & {$\left[\begin{array}{ll}22.13 \\
\end{array}\right.$} & $23.57]$ & \\
\hline $\mathrm{C} 13-\mathrm{CH} 3$ & 28 & 22.97 & 23.26 & 0.29 & 23.26 & $23.36]$ & \\
\hline $\mathrm{C} 14-\mathrm{CH} 3$ & 26 & 13.75 & 14.62 & 0.87 & 14.62 & $14.36]$ & \\
\hline $\mathrm{C} 15-\mathrm{CH} 3$ & 25 & 21.92 & 21.70 & -0.22 & {$\left[\begin{array}{ll}{[} & 21.75\end{array}\right.$} & 18.17 & \\
\hline $\mathrm{C} 1^{\prime}-\mathrm{C}^{\star \star}$ & 1 & 150.98 & 152.49 & 1.51 & [ 152.54 & $148.83]$ & \\
\hline $\mathrm{C} 2^{\prime}-\mathrm{CH}$ & 6 & 48.13 & 48.76 & 0.63 & {$\left[\begin{array}{l}48.76 \\
0\end{array}\right.$} & $49.11]$ & \\
\hline $\mathrm{C}^{\prime}-\mathrm{CH} 2$ & 13 & 55.89 & 55.29 & -0.60 & 55.28 & $56.21]$ & \\
\hline $\mathrm{C} 4^{\prime}-\mathrm{C}$ & 3 & 55.13 & 54.52 & -0.61 & 54.53 & $54.11]$ & \\
\hline $\mathrm{C} 5^{\prime}-\mathrm{C}^{\star *}$ & 2 & 132.31 & 135.40 & 3.09 & {$[135.37$} & $137.32]$ & \\
\hline $\mathrm{C}^{\prime}-\mathrm{CH}$ & 11 & 112.61 & 115.83 & 3.22 & {$[115.86$} & $114.02]$ & \\
\hline $\mathrm{C} 7^{\prime}-\mathrm{C}$ & 10 & 153.40 & 152.76 & -0.64 & {$[152.83$} & $147.70]$ & \\
\hline $\mathrm{C} 8^{\prime}-\mathrm{C}$ & 9 & 103.18 & 104.81 & 1.63 & {$[104.79$} & 105.85 & \\
\hline $\mathrm{C} 9^{\prime}-\mathrm{CH} 2$ & 8 & 38.93 & 45.43 & 6.50 & {$[\quad 45.46$} & $43.63]$ & \\
\hline $\mathrm{C} 10^{\prime}-\mathrm{CH}$ & 7 & 33.46 & 35.77 & 2.31 & 35.80 & $33.91]$ & \\
\hline $\mathrm{C} 11^{\prime}-\mathrm{C}$ & 32 & 85.17 & 81.71 & -3.46 & 81.65 & $86.13]$ & \\
\hline $\mathrm{C}_{12}^{\prime}-\mathrm{CH} 3$ & 33 & 24.76 & 27.51 & 2.75 & 27.52 & 27.10 & \\
\hline $\mathrm{C}^{\prime} 3^{\prime}-\mathrm{CH} 3$ & 34 & 27.50 & 29.49 & 1.99 & 29.52 & 27.24 & \\
\hline $\mathrm{C}^{4} 4^{\prime}-\mathrm{CH} 3$ & 12 & 18.31 & 18.65 & 0.34 & 18.64 & 19.05 & \\
\hline $\mathrm{C}^{\prime} 5^{\prime}-\mathrm{CH} 3$ & 36 & 19.03 & 19.29 & 0.26 & 19.28 & $19.85]$ & \\
\hline
\end{tabular}

\section{Conformer 1}

Energy: -1392.85382 Hartree (Rel: $0.0 \mathrm{kcal} / \mathrm{mol}$ ) XYZ coordinates for conf 1 :

\begin{tabular}{lrrr} 
C & 1.79677 & -1.65212 & -0.16539 \\
C & 1.96645 & -0.89728 & 0.95653 \\
C & 1.28630 & -1.62980 & 2.12728 \\
C & -0.28135 & -1.51528 & 1.89309 \\
C & -0.50374 & -2.37089 & 0.60357 \\
C & 0.94899 & -2.85533 & 0.25581 \\
C & 2.23052 & -1.46957 & -1.60945 \\
C & 3.10014 & -0.23456 & -1.93126 \\
C & 2.53452 & 1.06919 & -1.39185 \\
C & 2.74352 & 1.27128 & 0.08825 \\
C & 2.56744 & 0.42543 & 1.10780 \\
C & 1.75920 & -1.29272 & 3.53631 \\
C & 1.49307 & -3.09691 & 1.67737 \\
H & -0.77476 & -1.94938 & 2.77624 \\
H & -1.10370 & -3.25920 & 0.83252 \\
C & -0.86341 & -0.16308 & 1.55802 \\
C & -1.35660 & -0.12785 & 0.30689 \\
C & -1.25588 & -1.46501 & -0.40950 \\
C & -1.92144 & 1.08992 & -0.39889 \\
C & -3.43874 & 1.21366 & -0.29302 \\
C & -4.19028 & -0.03226 & -0.64138 \\
C & -3.52523 & -1.04362 & -1.57751 \\
C & -2.61964 & -2.06586 & -0.84312 \\
H & -0.67110 & -1.32629 & -1.33217 \\
C & -2.41239 & -3.30159 & -1.73273 \\
C & -0.85168 & 0.96105 & 2.55574 \\
C & -4.09385 & 2.34928 & 0.06441 \\
C & -5.60028 & 2.46827 & 0.16097 \\
C & -3.40541 & 3.65487 & 0.39189 \\
O & -5.30997 & -0.28235 & -0.20939 \\
O & 3.36417 & 2.23479 & -1.65562 \\
C & 3.52994 & 2.53116 & -0.21875 \\
C & 5.00585 & 2.51476 & 0.17245 \\
C & 2.86334 & 3.86164 & 0.13451 \\
O & 1.24334 & 1.21665 & -1.92511 \\
C & 2.97192 & -2.72450 & -2.12966 \\
H & 0.96483 & -3.70595 & -0.42900 \\
& & & \\
& & & \\
\hline
\end{tabular}

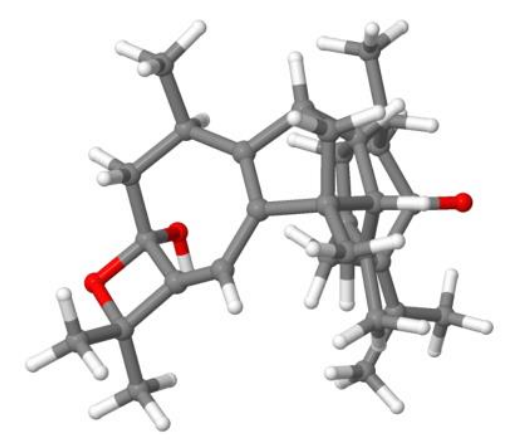




$\begin{array}{rrrr}\text { H } & 1.31024 & -1.37218 & -2.20430 \\ \mathrm{H} & 4.10729 & -0.36237 & -1.51952 \\ \mathrm{H} & 3.20343 & -0.14929 & -3.02007 \\ \mathrm{H} & 2.88772 & 0.71658 & 2.10800 \\ \mathrm{H} & 2.83733 & -1.46500 & 3.64466 \\ \mathrm{H} & 1.24681 & -1.92674 & 4.27024 \\ \mathrm{H} & 1.55809 & -0.25142 & 3.81154 \\ \mathrm{H} & 2.54699 & -3.39607 & 1.69169 \\ \mathrm{H} & 0.90170 & -3.81464 & 2.26105 \\ \mathrm{H} & -1.44243 & 1.98587 & -0.00108 \\ \mathrm{H} & -1.62235 & 1.03963 & -1.45631 \\ \mathrm{H} & -4.35036 & -1.58387 & -2.05236 \\ \mathrm{H} & -2.95025 & -0.54364 & -2.36867 \\ \mathrm{H} & -3.15710 & -2.37906 & 0.06376 \\ \mathrm{H} & -1.77580 & -4.04860 & -1.24495 \\ \mathrm{H} & -3.36863 & -3.78167 & -1.96976 \\ \mathrm{H} & -1.93259 & -3.02325 & -2.68030 \\ \mathrm{H} & -1.50137 & 1.78881 & 2.25730 \\ \mathrm{H} & -1.19502 & 0.61082 & 3.53827 \\ \mathrm{H} & 0.15989 & 1.36471 & 2.69990 \\ \mathrm{H} & -5.93826 & 2.20919 & 1.17302 \\ \mathrm{H} & -6.12937 & 1.79921 & -0.51712 \\ \mathrm{H} & -5.90708 & 3.50376 & -0.03056 \\ \mathrm{H} & -2.31841 & 3.59794 & 0.46133 \\ \mathrm{H} & -3.78515 & 4.04664 & 1.34528 \\ \mathrm{H} & -3.65330 & 4.40935 & -0.36794 \\ \mathrm{H} & 5.12032 & 2.63306 & 1.25654 \\ \mathrm{H} & 5.53714 & 3.33770 & -0.31990 \\ \mathrm{H} & 5.47462 & 1.57278 & -0.12560 \\ \mathrm{H} & 2.92561 & 4.05273 & 1.21231 \\ \mathrm{H} & 1.80585 & 3.86373 & -0.14997 \\ \mathrm{H} & 3.36098 & 4.68368 & -0.39264 \\ \mathrm{H} & 0.88193 & 2.05283 & -1.59008 \\ \mathrm{H} & 3.23629 & -2.59753 & -3.18560 \\ \mathrm{H} & 2.36278 & -3.62927 & -2.04818 \\ \mathrm{H} & 3.89849 & -2.88982 & -1.56701 \\ & & & \end{array}$

\section{Conformer 2}

Energy: -1392.84982 Hartree (Rel: $2.5 \mathrm{kcal} / \mathrm{mol}$ ) XYZ coordinates for conf 2 :

\begin{tabular}{|c|c|c|c|}
\hline C & -0.87657 & -1.38696 & 1.17705 \\
\hline $\mathrm{C}$ & -1.59455 & -1.54241 & 0.02710 \\
\hline$C$ & -1.11397 & -2.83624 & -0.65306 \\
\hline C & 0.36333 & -2.56205 & -1.17824 \\
\hline C & 1.19091 & -2.44254 & 0.14131 \\
\hline C & 0.08559 & -2.57769 & 1.24631 \\
\hline C & -0.86427 & -0.26834 & 2.20920 \\
\hline$C$ & -2.14557 & 0.59347 & 2.25908 \\
\hline C & -2.40975 & 1.35265 & 0.96716 \\
\hline C & -2.91250 & 0.54828 & -0.19192 \\
\hline C & -2.62421 & -0.69942 & -0.57287 \\
\hline C & -2.04349 & -3.49141 & -1.66777 \\
\hline $\mathrm{C}$ & -0.78038 & -3.67763 & 0.60179 \\
\hline H & 0.65900 & -3.43238 & -1.78445 \\
\hline H & 1.86868 & -3.29644 & 0.25396 \\
\hline C & 0.64411 & -1.27136 & -1.91196 \\
\hline C & 1.50340 & -0.48368 & -1.23938 \\
\hline C & 2.00921 & -1.12956 & 0.04092 \\
\hline C & 1.94224 & 0.90353 & -1.69108 \\
\hline C & 2.68026 & 1.76484 & -0.66753 \\
\hline C & 3.84728 & 1.12752 & 0.01955 \\
\hline C & 4.34333 & -0.19865 & -0.54912 \\
\hline C & 3.54190 & -1.39644 & 0.03538 \\
\hline H & 1.80523 & -0.46927 & 0.89800 \\
\hline C & 4.04427 & -1.75539 & 1.44149 \\
\hline C & -0.00125 & -0.96663 & -3.23478 \\
\hline C & 2.33407 & 3.06669 & -0.46966 \\
\hline C & 3.05985 & 4.03326 & 0.43337 \\
\hline C & 1.15420 & 3.69311 & -1.19307 \\
\hline 0 & 4.38677 & 1.57646 & 1.02808 \\
\hline 0 & -3.64659 & 2.10376 & 0.97874 \\
\hline
\end{tabular}

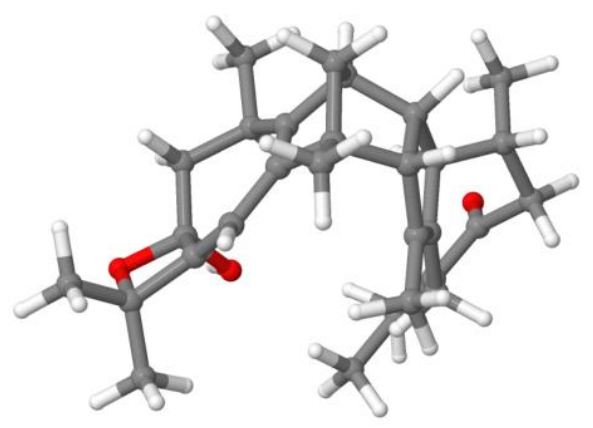




$\begin{array}{rrrr}\mathrm{C} & -4.07928 & 1.51453 & -0.31132 \\ \mathrm{C} & -5.47645 & 0.91905 & -0.18533 \\ \mathrm{C} & -3.98154 & 2.55137 & -1.42938 \\ \mathrm{O} & -1.31145 & 2.17136 & 0.60718 \\ \mathrm{C} & -0.56332 & -0.78677 & 3.63223 \\ \mathrm{H} & 0.49227 & -2.79695 & 2.23468 \\ \mathrm{H} & -0.04388 & 0.41230 & 1.93248 \\ \mathrm{H} & -3.01934 & -0.02695 & 2.48841 \\ \mathrm{H} & -2.05105 & 1.32053 & 3.07881 \\ \mathrm{H} & -3.22491 & -1.15411 & -1.35947 \\ \mathrm{H} & -3.02549 & -3.70698 & -1.22872 \\ \mathrm{H} & -1.61802 & -4.44249 & -2.01065 \\ \mathrm{H} & -2.20261 & -2.87008 & -2.55608 \\ \mathrm{H} & -1.67149 & -3.92768 & 1.18827 \\ \mathrm{H} & -0.22460 & -4.59674 & 0.37314 \\ \mathrm{H} & 2.58152 & 0.80261 & -2.58460 \\ \mathrm{H} & 1.05799 & 1.44015 & -2.03835 \\ \mathrm{H} & 4.27222 & -0.21042 & -1.64159 \\ \mathrm{H} & 5.39868 & -0.29552 & -0.27659 \\ \mathrm{H} & 3.72279 & -2.25176 & -0.63100 \\ \mathrm{H} & 3.45613 & -2.57042 & 1.87997 \\ \mathrm{H} & 5.09197 & -2.07674 & 1.41377 \\ \mathrm{H} & 3.98167 & -0.88921 & 2.10986 \\ \mathrm{H} & 0.45951 & -0.10988 & -3.73617 \\ \mathrm{H} & 0.06874 & -1.82739 & -3.91369 \\ \mathrm{H} & -1.07037 & -0.74160 & -3.11773 \\ \mathrm{H} & 2.42495 & 4.27461 & 1.29928 \\ \mathrm{H} & 4.00141 & 3.64770 & 0.81444 \\ \mathrm{H} & 3.22099 & 4.98006 & -0.09970 \\ \mathrm{H} & 1.01427 & 4.73049 & -0.87323 \\ \mathrm{H} & 0.22432 & 3.15099 & -0.98752 \\ \mathrm{H} & 1.30081 & 3.70643 & -2.28085 \\ \mathrm{H} & -5.75388 & 0.38917 & -1.10438 \\ \mathrm{H} & -6.21201 & 1.71402 & -0.01563 \\ \mathrm{H} & -5.52368 & 0.21598 & 0.65098 \\ \mathrm{H} & -4.21522 & 2.09622 & -2.39944 \\ \mathrm{H} & -2.97405 & 2.97219 & -1.47168 \\ \mathrm{H} & -4.69463 & 3.36451 & -1.24927 \\ \mathrm{H} & -1.20061 & 2.82667 & 1.31829 \\ \mathrm{H} & -0.56811 & 0.04421 & 4.34634 \\ \mathrm{H} & 0.41769 & -1.26561 & 3.69761 \\ \mathrm{H} & -1.31763 & -1.51362 & 3.95756\end{array}$


revised sesquiterpene dimer from xylopia aromatica, Fig.15

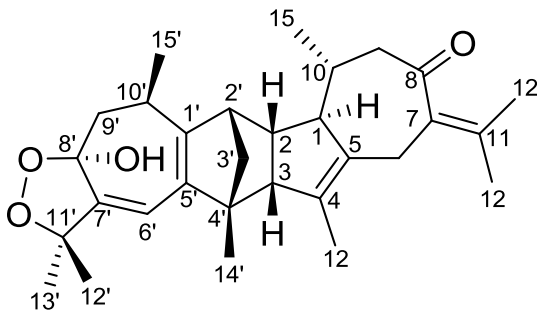

Rel energy (kcal/mol): $\begin{array}{ccc}\text { Conf } 1 \text { Conf } \\ 0.0 & 1.8\end{array}$

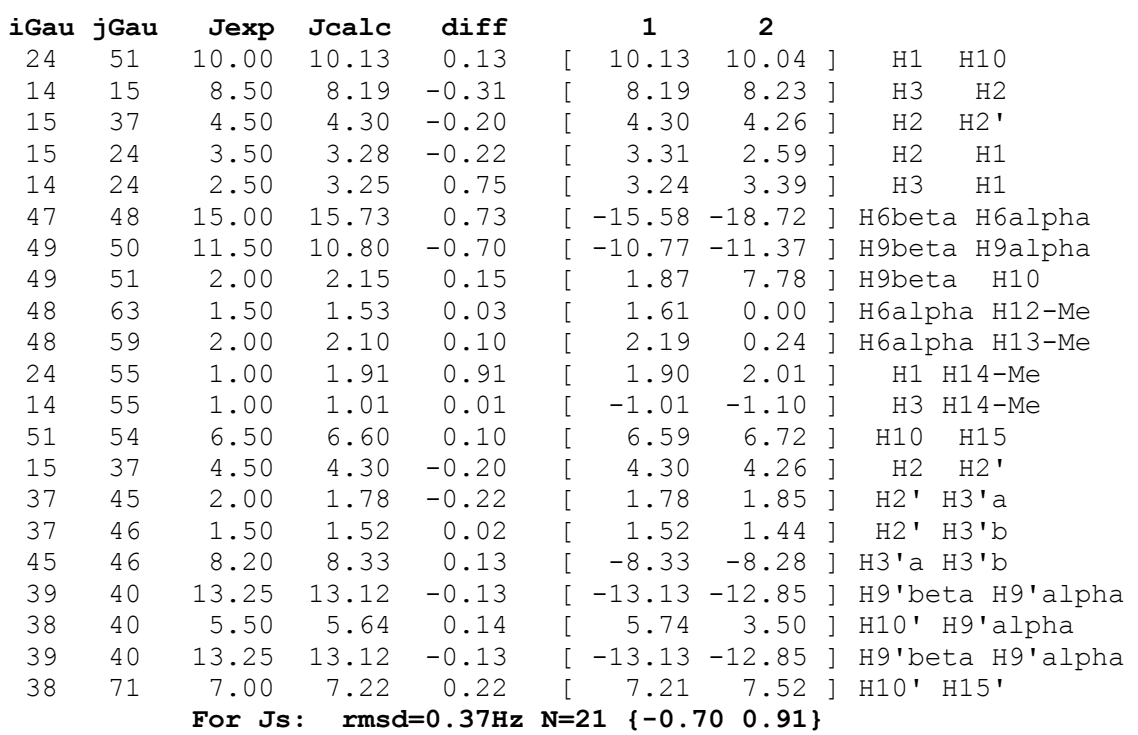

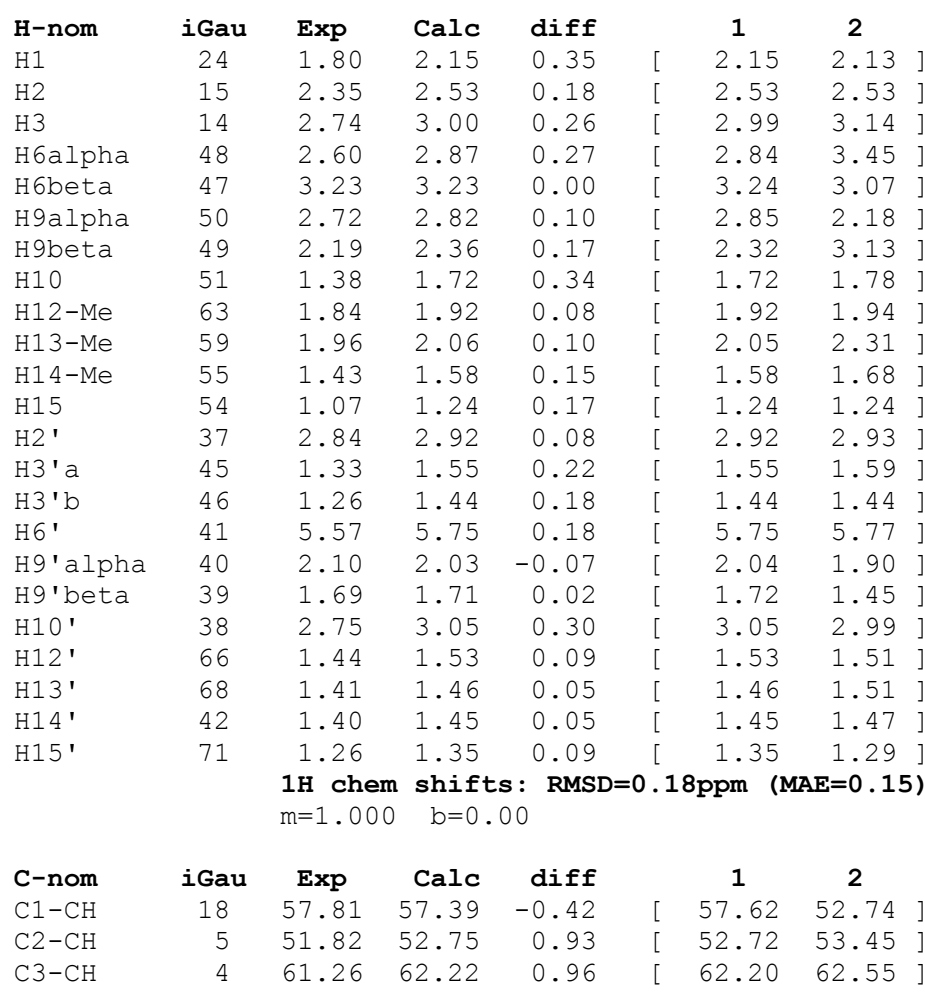




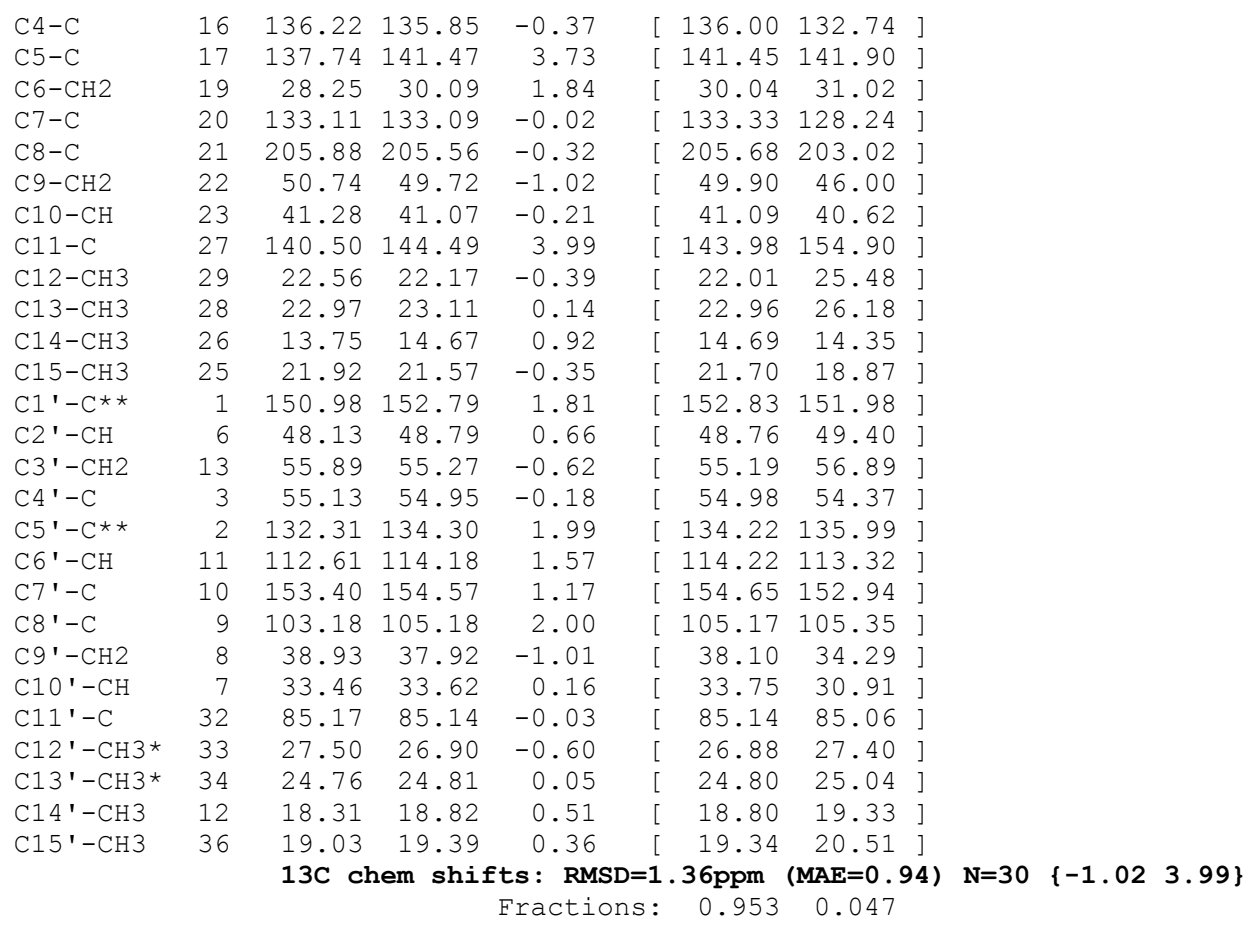

\section{Conformer 1}

Energy: -1468.01559 Hartree (Rel: $0.0 \mathrm{kcal} / \mathrm{mol}$ ) XYZ coordinates for conf 1 :

$\begin{array}{llrr}\mathrm{C} & 1.50436 & -1.89736 & -0.15984 \\ \mathrm{C} & 1.77130 & -1.11222 & 0.92120 \\ \mathrm{C} & 1.06071 & -1.73701 & 2.13822 \\ \mathrm{C} & -0.49831 & -1.50179 & 1.93158 \\ \mathrm{C} & -0.82478 & -2.39378 & 0.69022 \\ \mathrm{C} & 0.57539 & -3.00900 & 0.33116 \\ \mathrm{C} & 1.90774 & -1.76720 & -1.61264 \\ \mathrm{C} & 2.99378 & -0.71629 & -1.90272 \\ \mathrm{C} & 2.64283 & 0.69833 & -1.45569 \\ \mathrm{C} & 2.83659 & 0.97482 & 0.02050 \\ \mathrm{C} & 2.46379 & 0.16619 & 1.02429 \\ \mathrm{C} & 1.58961 & -1.38948 & 3.52497 \\ \mathrm{C} & 1.13617 & -3.23318 & 1.74847 \\ \mathrm{H} & -1.00363 & -1.85042 & 2.84510 \\ \mathrm{H} & -1.48756 & -3.21927 & 0.97378 \\ \mathrm{C} & -0.96842 & -0.12096 & 1.54165 \\ \mathrm{C} & -1.47863 & -0.09979 & 0.29710 \\ \mathrm{C} & -1.52455 & -1.47568 & -0.34816 \\ \mathrm{C} & -1.93694 & 1.12996 & -0.46188 \\ \mathrm{C} & -3.43208 & 1.40914 & -0.33754 \\ \mathrm{C} & -4.30955 & 0.22221 & -0.58347 \\ \mathrm{C} & -3.77821 & -0.90110 & -1.47620 \\ \mathrm{C} & -2.95267 & -1.96550 & -0.70846 \\ \mathrm{H} & -0.96371 & -1.44399 & -1.29555 \\ \mathrm{C} & -2.89268 & -3.26169 & -1.53200 \\ \mathrm{C} & -0.84068 & 1.04255 & 2.48425 \\ \mathrm{C} & -3.96390 & 2.62600 & -0.05074 \\ \mathrm{C} & -5.44834 & 2.90386 & 0.05616 \\ \mathrm{C} & -3.14258 & 3.87530 & 0.17511 \\ \mathrm{O} & -5.43013 & 0.10515 & -0.10051 \\ \mathrm{O} & 3.49431 & 2.78846 & -1.24005 \\ \mathrm{C} & 3.59423 & 2.28840 & 0.12093 \\ \mathrm{C} & 5.07141 & 2.08965 & 0.48956 \\ \mathrm{C} & 2.91835 & 3.34508 & 0.99707 \\ \mathrm{O} & 1.33849 & 0.99763 & -1.90877 \\ \mathrm{C} & 2.38172 & -3.11704 & -2.19939 \\ \mathrm{H} & 0.50605 & -3.88658 & -0.31449 \\ \mathrm{H} & 1.01399 & -1.46374 & -2.17772\end{array}$

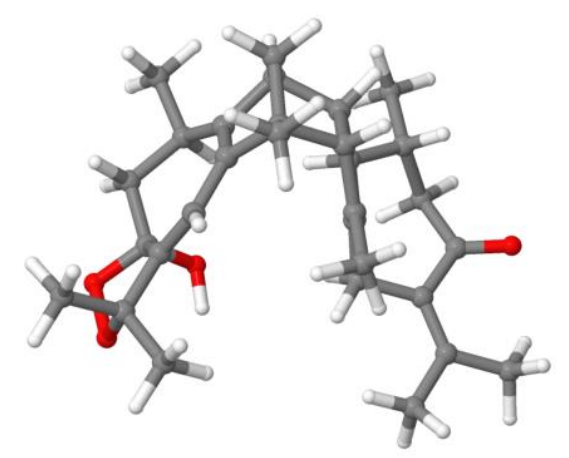




$\begin{array}{rrr}3.93947 & -1.00168 & -1.42986 \\ 3.16496 & -0.68708 & -2.98501 \\ 2.67877 & 0.49930 & 2.03927 \\ 2.65663 & -1.62816 & 3.61463 \\ 1.05499 & -1.96849 & 4.28786 \\ 1.46118 & -0.33092 & 3.77657 \\ 2.16239 & -3.61709 & 1.75146 \\ 0.50455 & -3.87498 & 2.37667 \\ -1.36175 & 1.99170 & -0.12124 \\ -1.66438 & 0.99955 & -1.51939 \\ -4.66756 & -1.38258 & -1.89509 \\ -3.18345 & -0.50643 & -2.31102 \\ -3.48749 & -2.17506 & 0.22927 \\ -2.31257 & -4.03900 & -1.02173 \\ -3.89741 & -3.66007 & -1.71325 \\ -2.42248 & -3.08153 & -2.50762 \\ -1.40347 & 1.91685 & 2.14490 \\ -1.21622 & 0.77586 & 3.48128 \\ 0.20640 & 1.34852 & 2.61323 \\ -5.77963 & 2.79928 & 1.09804 \\ -6.05932 & 2.21880 & -0.53026 \\ -5.65723 & 3.93697 & -0.24803 \\ -2.06691 & 3.71120 & 0.25060 \\ -3.47429 & 4.37616 & 1.09462 \\ -3.31524 & 4.59075 & -0.64125 \\ 5.16444 & 1.65164 & 1.48946 \\ 5.59811 & 3.05022 & 0.47512 \\ 5.54952 & 1.41892 & -0.22920 \\ 2.94453 & 3.03482 & 2.04690 \\ 1.87418 & 3.48671 & 0.70322 \\ 3.44136 & 4.30377 & 0.91279 \\ 1.19726 & 1.94220 & -1.72725 \\ 2.65486 & -2.99665 & -3.25371 \\ 1.60470 & -3.88476 & -2.14733 \\ 3.26230 & -3.49026 & -1.66282 \\ 3.60355 & 1.57140 & -2.05756\end{array}$

\section{Conformer 2}

Energy: -1468.01274 Hartree (Rel: $1.8 \mathrm{kcal} / \mathrm{mol}$ ) XYZ coordinates for conf 2 :

\begin{tabular}{lrrr} 
XYZ Coordinates for & Conf & \multicolumn{1}{l}{ L } & \\
C & -0.59656 & -1.25527 & 1.30913 \\
C & -1.35403 & -1.56860 & 0.21989 \\
C & -0.83136 & -2.90701 & -0.33533 \\
C & 0.61996 & -2.63503 & -0.93218 \\
C & 1.48048 & -2.34822 & 0.34044 \\
C & 0.41088 & -2.39305 & 1.48541 \\
C & -0.62510 & -0.01222 & 2.17551 \\
C & -2.02703 & 0.62318 & 2.29316 \\
C & -2.52729 & 1.26567 & 1.00639 \\
C & -2.98598 & 0.33019 & -0.09056 \\
C & -2.45419 & -0.85936 & -0.41653 \\
C & -1.75344 & -3.71296 & -1.24229 \\
C & -0.43017 & -3.58921 & 0.99523 \\
H & 0.93287 & -3.55283 & -1.45354 \\
H & 2.18786 & -3.16475 & 0.52522 \\
C & 0.83220 & -1.41940 & -1.80612 \\
C & 1.68568 & -0.54069 & -1.24915 \\
C & 2.25002 & -1.02929 & 0.07429 \\
C & 2.08091 & 0.80672 & -1.83926 \\
C & 2.80030 & 1.75398 & -0.88302 \\
C & 4.10875 & 1.26434 & -0.34617 \\
C & 4.54859 & -0.14375 & -0.73926 \\
C & 3.79204 & -1.24640 & 0.05252 \\
H & 2.04366 & -0.28623 & 0.86003 \\
C & 4.34625 & -1.37365 & 1.47909 \\
C & 0.14240 & -1.27785 & -3.13394 \\
C & 2.32043 & 2.98231 & -0.53825 \\
C & 3.05808 & 3.95583 & 0.35835 \\
C & 0.99435 & 3.53311 & -1.00455 \\
O & 4.81678 & 1.91719 & 0.41649 \\
O & -4.27886 & 2.24769 & -0.01741 \\
& & &
\end{tabular}

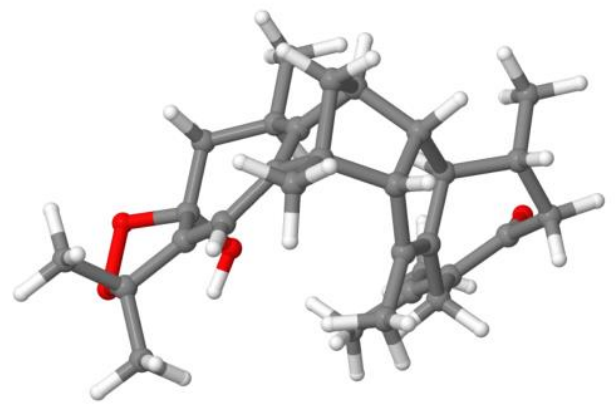




$\begin{array}{rrrr}\text { C } & -4.24594 & 0.95063 & -0.67646 \\ \mathrm{C} & -5.51014 & 0.16242 & -0.30734 \\ \mathrm{C} & -4.16923 & 1.25268 & -2.17487 \\ \mathrm{O} & -1.53972 & 2.17196 & 0.55341 \\ \mathrm{C} & -0.06957 & -0.25682 & 3.59293 \\ \mathrm{H} & 0.84871 & -2.48152 & 2.48113 \\ \mathrm{H} & 0.02047 & 0.74282 & 1.70214 \\ \mathrm{H} & -2.76080 & -0.11693 & 2.62987 \\ \mathrm{H} & -1.98615 & 1.41823 & 3.04572 \\ \mathrm{H} & -2.93277 & -1.39721 & -1.23456 \\ \mathrm{H} & -2.71833 & -3.90895 & -0.75870 \\ \mathrm{H} & -1.29721 & -4.68217 & -1.47790 \\ \mathrm{H} & -1.95225 & -3.21104 & -2.19614 \\ \mathrm{H} & -1.29428 & -3.80291 & 1.63393 \\ \mathrm{H} & 0.15440 & -4.50793 & 0.85286 \\ \mathrm{H} & 2.72993 & 0.64300 & -2.71434 \\ \mathrm{H} & 1.18961 & 1.28687 & -2.24548 \\ \mathrm{H} & 4.40425 & -0.30989 & -1.81220 \\ \mathrm{H} & 5.62025 & -0.21358 & -0.53060 \\ \mathrm{H} & 3.98011 & -2.19041 & -0.47851 \\ \mathrm{H} & 3.80011 & -2.13215 & 2.05254 \\ \mathrm{H} & 5.40408 & -1.66026 & 1.46743 \\ \mathrm{H} & 4.26847 & -0.42050 & 2.01519 \\ \mathrm{H} & 0.54177 & -0.44715 & -3.72407 \\ \mathrm{H} & 0.25110 & -2.19316 & -3.73164 \\ \mathrm{H} & -0.93638 & -1.10755 & -3.01260 \\ \mathrm{H} & 3.20499 & 3.54523 & 1.36249 \\ \mathrm{H} & 4.06278 & 4.17195 & -0.01497 \\ \mathrm{H} & 2.49718 & 4.89294 & 0.44130 \\ \mathrm{H} & 0.40564 & 3.83626 & -0.13000 \\ \mathrm{H} & 0.38660 & 2.83949 & -1.58482 \\ \mathrm{H} & 1.15899 & 4.43949 & -1.60436 \\ \mathrm{H} & -5.47912 & -0.84352 & -0.74022 \\ \mathrm{H} & -6.40179 & 0.67860 & -0.67992 \\ \mathrm{H} & -5.58751 & 0.07010 & 0.77926 \\ \mathrm{H} & -4.14338 & 0.31767 & -2.74415 \\ \mathrm{H} & -3.26906 & 1.82702 & -2.41308 \\ \mathrm{H} & -5.04710 & 1.82330 & -2.49685 \\ \mathrm{H} & -1.97114 & 2.71131 & -0.13171 \\ \mathrm{H} & -0.11571 & 0.66731 & 4.17915 \\ \mathrm{H} & 0.97603 & -0.57809 & 3.57081 \\ \mathrm{H} & -0.64793 & -1.02221 & 4.12515 \\ \mathrm{O} & -3.73277 & 1.96887 & 1.32048 \\ & & & \end{array}$


$\beta$-lactol from Caesalpinia decapetala (cmpd 1), Fig.16<smiles>O=C1c2ccc(O)c(-c3ccc4c(c3)O[C@@H]4O)c2[C@H]1O</smiles>

$$
\text { Rel energy (kcal/mol): } \begin{gathered}
\operatorname{Conf} 1 \\
0.0
\end{gathered}
$$

\begin{tabular}{|c|c|c|c|c|c|}
\hline C-nom & iGau & Exp & Calc & $\operatorname{diff}$ & 1 \\
\hline $\mathrm{C} 1-\mathrm{C}$ & 6 & 148.00 & 140.77 & -7.23 & {$[140.77$} \\
\hline $\mathrm{C} 2-\mathrm{CH}$ & 1 & 117.90 & 107.45 & -10.45 & [ 107.45 \\
\hline C3-C & 2 & 166.60 & 167.06 & 0.46 & [ 167.06 \\
\hline $\mathrm{C} 4-\mathrm{C}$ & 3 & 112.90 & 133.78 & 20.88 & [ 133.78 \\
\hline $\mathrm{C} 5-\mathrm{CH}$ & 4 & 113.00 & 122.97 & 9.97 & 122.97 \\
\hline $\mathrm{C} 6-\mathrm{CH}$ & 5 & 124.40 & 116.45 & -7.95 & [ 116.45 \\
\hline $\mathrm{C} 1{ }^{\prime}-\mathrm{C}$ & 7 & 145.50 & 121.65 & -23.85 & [ 121.65 \\
\hline$C 22^{\prime}-C$ & 8 & 145.70 & 160.69 & 14.99 & 160.69 \\
\hline C3 '-C & 9 & 123.40 & 139.68 & 16.28 & 139.68 \\
\hline $\mathrm{C} 44^{\prime}-\mathrm{CH}$ & 10 & 125.60 & 124.56 & -1.04 & 124.56 \\
\hline $\mathrm{C} 5^{\prime}-\mathrm{CH}$ & 11 & 116.00 & 119.98 & 3.98 & [ 119.98 \\
\hline $\mathrm{C} 6^{\prime}-\mathrm{C}$ & 12 & 167.40 & 158.11 & -9.29 & [ 158.11 \\
\hline $\mathrm{Cl} "-\mathrm{CH}$ & 14 & 98.30 & 106.83 & 8.53 & [ 106.83 \\
\hline $\mathrm{Cl}$ ' ' $-\mathrm{CH}$ & 18 & 111.70 & 85.45 & -26.25 & 85.45 \\
\hline C2' ' '-C & 17 & 181.00 & 183.74 & 2.74 & 183.74 \\
\hline
\end{tabular}

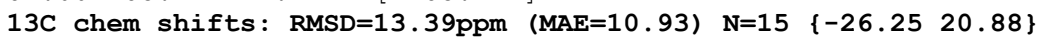

\section{Conformer 1}

Energy: -953.60168 Hartree (Rel: $0.0 \mathrm{kcal} / \mathrm{mol}$ ) XYZ coordinates for conf 1 :

$\begin{array}{lrrr}\mathrm{C} & 1.17147 & -0.29369 & -0.85853 \\ \mathrm{C} & 2.53611 & -0.39087 & -0.67559 \\ \mathrm{C} & 3.26067 & 0.35483 & 0.24681 \\ \mathrm{C} & 2.65680 & 1.25523 & 1.09637 \\ \mathrm{C} & 1.25401 & 1.35740 & 0.97250 \\ \mathrm{C} & 0.52159 & 0.61180 & 0.02137 \\ \mathrm{C} & -0.95673 & 0.75802 & -0.06041 \\ \mathrm{C} & -1.82994 & -0.32770 & -0.08790 \\ \mathrm{C} & -3.22084 & -0.19582 & -0.13353 \\ \mathrm{C} & -3.84508 & 1.05395 & -0.20250 \\ \mathrm{C} & -3.00207 & 2.15800 & -0.20735 \\ \mathrm{C} & -1.59543 & 2.02291 & -0.13529 \\ \mathrm{O} & 3.58879 & -1.08337 & -1.23615 \\ \mathrm{C} & 4.51755 & -0.29285 & -0.32610 \\ \mathrm{H} & 5.14833 & 0.33611 & -0.95605 \\ \mathrm{O} & 5.33981 & -1.07047 & 0.43743 \\ \mathrm{C} & -3.42223 & -1.66737 & -0.04211 \\ \mathrm{C} & -1.85136 & -1.86367 & -0.05057 \\ \mathrm{O} & -4.35277 & -2.42939 & 0.03698 \\ \mathrm{O} & -1.36122 & -2.51334 & 1.09697 \\ \mathrm{O} & -0.89713 & 3.18767 & -0.16516 \\ \mathrm{H} & 0.62077 & -0.84112 & -1.61537 \\ \mathrm{H} & 3.19480 & 1.84720 & 1.83112 \\ \mathrm{H} & 0.71129 & 1.99168 & 1.66796 \\ \mathrm{H} & -4.92366 & 1.17212 & -0.23926 \\ \mathrm{H} & -3.39607 & 3.16801 & -0.26354 \\ \mathrm{H} & 4.79483 & -1.58511 & 1.05705 \\ \mathrm{H} & -1.49329 & -2.34356 & -0.97404 \\ \mathrm{H} & 0.05576 & -2.70036 & 0.95932 \\ \mathrm{H} & & 2.98534 & -0.17080\end{array}$

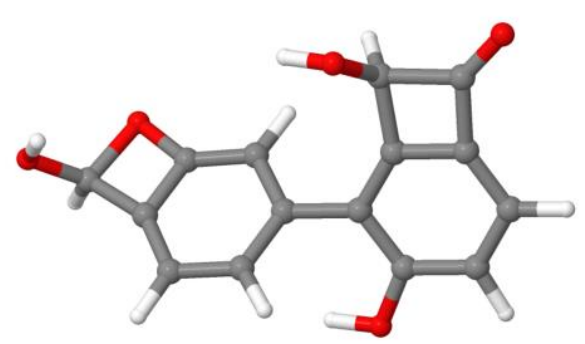


<smiles>COC1(c2ccc(C#N)cc2)OC(C)(C)C1(C)C</smiles>

Rel energy (kcal/mol): $\begin{gathered}\operatorname{Conf} 1 \\ 0.0\end{gathered}$

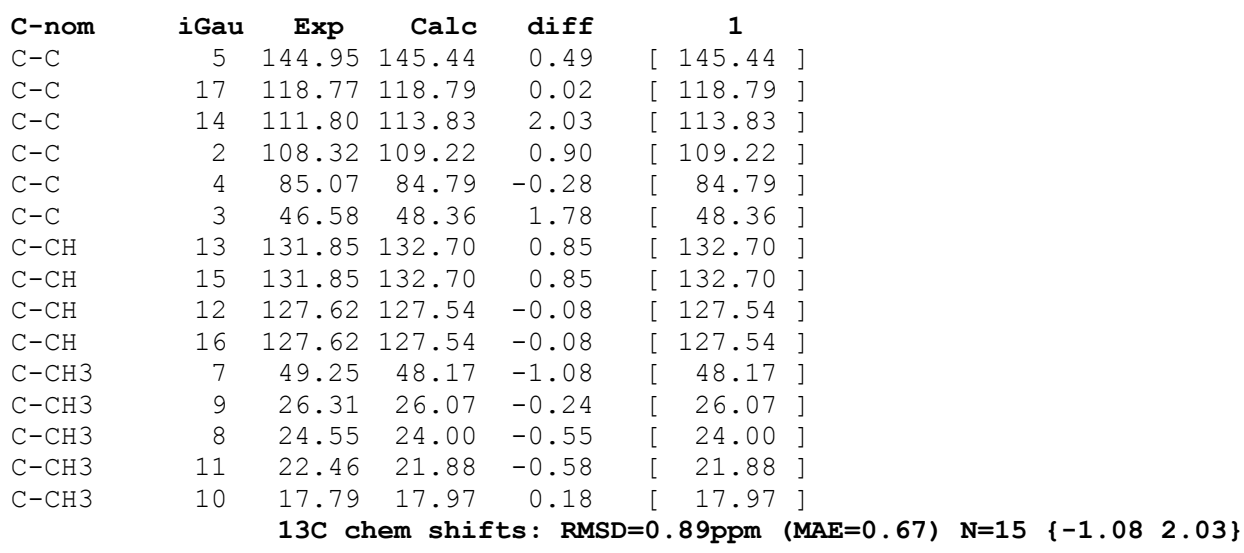

\section{Conformer 1}

Energy: -788.18927 Hartree (Rel: $0.0 \mathrm{kcal} / \mathrm{mol})$ XYZ coordinates for conf 1 :

$\begin{array}{lrrr}\mathrm{O} & -1.49812 & -0.13745 & -1.22130 \\ \mathrm{C} & -0.86133 & 0.46231 & -0.08865 \\ \mathrm{C} & -1.76603 & -0.35641 & 0.90463 \\ \mathrm{C} & -2.58053 & -0.70500 & -0.40169 \\ \mathrm{C} & 0.64309 & 0.24090 & -0.09357 \\ \mathrm{O} & -1.16014 & 1.83743 & 0.03201 \\ \mathrm{C} & -0.78666 & 2.63471 & -1.09095 \\ \mathrm{C} & -2.79893 & -2.17022 & -0.76169 \\ \mathrm{C} & -3.86492 & 0.09399 & -0.62462 \\ \mathrm{C} & -2.47814 & 0.43722 & 2.00216 \\ \mathrm{C} & -1.04713 & -1.55257 & 1.54076 \\ \mathrm{C} & 1.44428 & 0.91761 & 0.83830 \\ \mathrm{C} & 2.81894 & 0.71201 & 0.87454 \\ \mathrm{C} & 3.41792 & -0.17405 & -0.03857 \\ \mathrm{C} & 2.62218 & -0.84446 & -0.98181 \\ \mathrm{C} & 1.24511 & -0.63690 & -1.00208 \\ \mathrm{C} & 4.83556 & -0.38854 & -0.00820 \\ \mathrm{~N} & 5.98564 & -0.56270 & 0.01752 \\ \mathrm{H} & -1.15369 & 3.64217 & -0.88268 \\ \mathrm{H} & 0.30262 & 2.66702 & -1.22217 \\ \mathrm{H} & -1.24988 & 2.25537 & -2.00815 \\ \mathrm{H} & -3.17059 & -2.24187 & -1.79019 \\ \mathrm{H} & -3.54784 & -2.62480 & -0.10246 \\ \mathrm{H} & -1.87888 & -2.75455 & -0.69549 \\ \mathrm{H} & -4.20678 & -0.04767 & -1.65613 \\ \mathrm{H} & -3.70190 & 1.16143 & -0.46458 \\ \mathrm{H} & -4.66242 & -0.24615 & 0.04665 \\ \mathrm{H} & -3.24162 & -0.18907 & 2.48041 \\ \mathrm{H} & -1.76476 & 0.73252 & 2.78040 \\ \mathrm{H} & -2.95390 & 1.34427 & 1.63012 \\ & & & \end{array}$

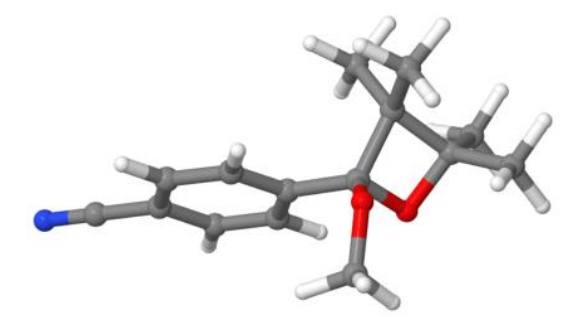




$\begin{array}{rrrr}\mathrm{H} & -1.77338 & -2.22884 & 2.00648 \\ \mathrm{H} & -0.45477 & -2.13002 & 0.82678 \\ \mathrm{H} & -0.36438 & -1.20790 & 2.32546 \\ \mathrm{H} & 0.98193 & 1.61750 & 1.52743 \\ \mathrm{H} & 3.43614 & 1.23375 & 1.59899 \\ \mathrm{H} & 3.08758 & -1.52134 & -1.69129 \\ \mathrm{H} & 0.62272 & -1.14633 & -1.72918\end{array}$


truncated bidenphytin B (hydroxydioxetane moiety), Fig.17
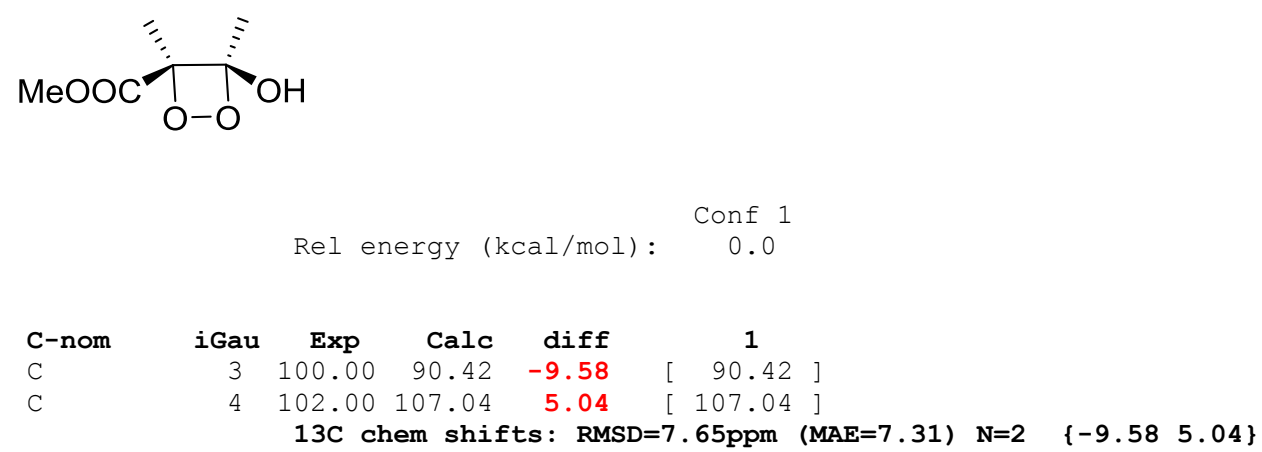

\section{Conformer 1}

Energy: -610.65674 Hartree (Rel: 0.0 kcal/mol) XYZ coordinates for conf 1 :

$\begin{array}{lrrr}\mathrm{C} & -0.18540 & 1.83975 & -0.85437 \\ \mathrm{C} & -2.80324 & 0.32596 & -0.45552 \\ \mathrm{C} & -0.22228 & 0.54789 & -0.05869 \\ \mathrm{C} & -1.49967 & -0.34860 & -0.06979 \\ \mathrm{O} & -1.46515 & -1.58631 & -0.64485 \\ \mathrm{O} & -0.44020 & 0.76462 & 1.36115 \\ \mathrm{O} & -1.37875 & -0.39367 & 1.40683 \\ \mathrm{C} & 1.08983 & -0.25081 & -0.16825 \\ \mathrm{O} & 1.17823 & -1.45742 & -0.32917 \\ \mathrm{O} & 2.15287 & 0.53913 & 0.00667 \\ \mathrm{C} & 3.43154 & -0.12562 & 0.05596 \\ \mathrm{H} & 0.66673 & 2.45014 & -0.54532 \\ \mathrm{H} & -0.08035 & 1.62288 & -1.92405 \\ \mathrm{H} & -1.10281 & 2.41029 & -0.69863 \\ \mathrm{H} & -3.62138 & -0.35043 & -0.19174 \\ \mathrm{H} & -2.84216 & 0.51299 & -1.53345 \\ \mathrm{H} & -2.93458 & 1.26379 & 0.08813 \\ \mathrm{H} & -0.56229 & -1.94711 & -0.50714 \\ \mathrm{H} & 3.45213 & -0.83961 & 0.88258 \\ \mathrm{H} & 3.62160 & -0.65263 & -0.88207 \\ \mathrm{H} & 4.16242 & 0.66745 & 0.21065\end{array}$

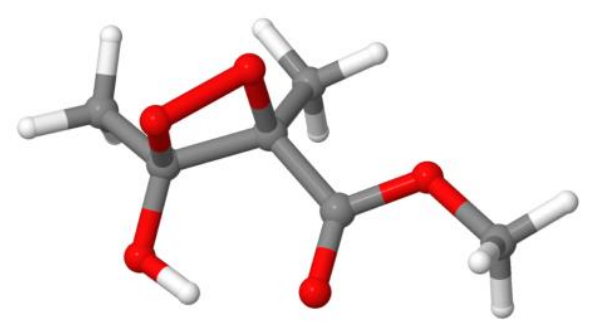


sphaerococcenol A

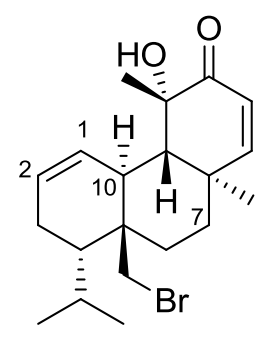

Rel energy (kcal/mol): $\begin{array}{ccc}\text { Conf } 1 & \operatorname{Conf} 2 \\ 0.0 & 0.2\end{array}$

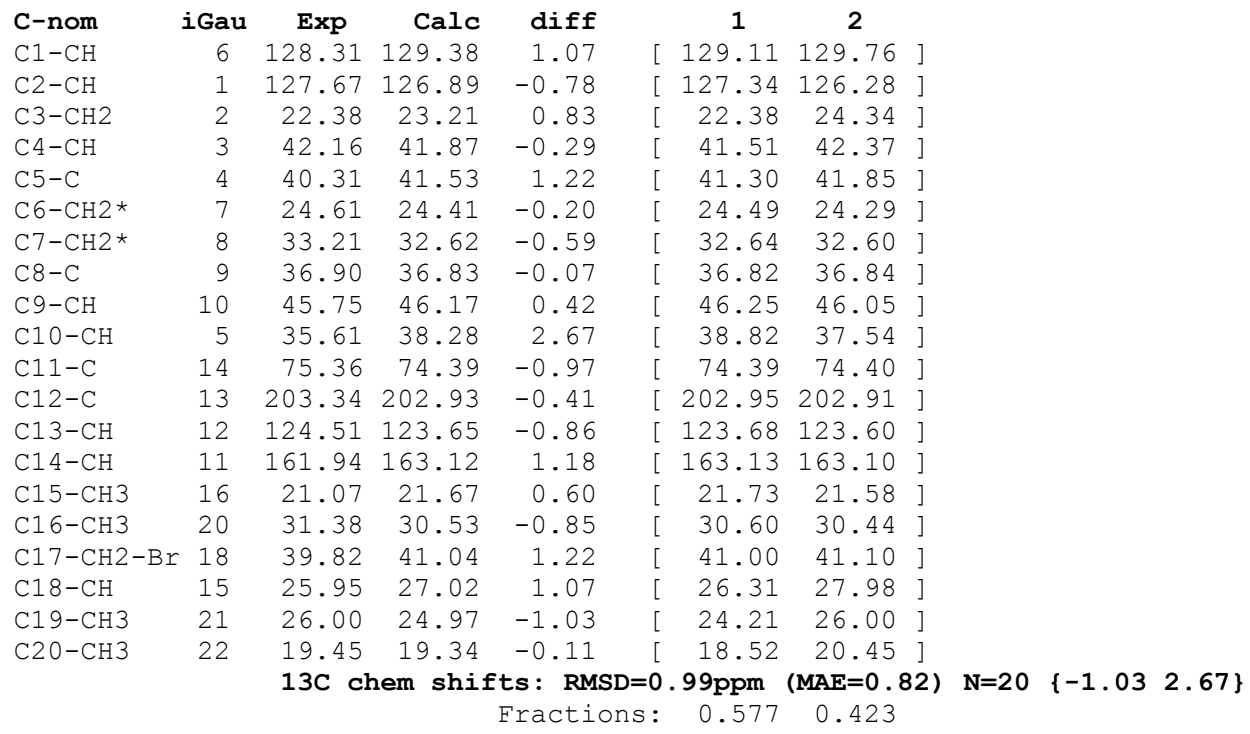

\section{Conformer 1}

Energy: -3501.38283 Hartree (Rel: $0.0 \mathrm{kcal} / \mathrm{mol}$ ) XYZ coordinates for conf 1 :

$\begin{array}{lrrr}\text { C } & 1.14595 & 1.75420 & -2.03353 \\ \text { C } & 2.43984 & 1.38904 & -1.35259 \\ \text { C } & 2.24650 & 0.91822 & 0.10833 \\ \text { C } & 1.01366 & -0.05344 & 0.19159 \\ \text { C } & -0.29180 & 0.70452 & -0.25623 \\ \text { C } & -0.06670 & 1.44184 & -1.56187 \\ \text { C } & 0.80808 & -0.62537 & 1.61440 \\ \text { C } & -0.47851 & -1.45201 & 1.76825 \\ \text { C } & -1.75329 & -0.70320 & 1.30534 \\ \text { C } & -1.54931 & -0.22155 & -0.17067 \\ \text { C } & -2.93525 & -1.65121 & 1.35058 \\ \text { C } & -4.05300 & -1.44724 & 0.63496 \\ \text { C } & -4.14378 & -0.23639 & -0.19014 \\ \text { C } & -2.85031 & 0.33841 & -0.81967 \\ \text { C } & 2.30770 & 2.10432 & 1.13196 \\ \text { C } & -2.07830 & 0.45591 & 2.28840 \\ \text { O } & -2.90943 & 1.75436 & -0.67037 \\ \text { C } & 1.23923 & -1.22714 & -0.79651 \\ \text { H } & -1.34385 & -1.13506 & -0.73978 \\ \text { C } & -2.94476 & -0.06089 & -2.31457 \\ \text { C } & 3.78213 & 2.42025 & 1.45490 \\ \text { C } & 1.58949 & 3.40968 & 0.74012 \\ \text { Br } & 2.91257 & -2.27705 & -0.50430 \\ \text { H } & 1.22086 & 2.32654 & -2.95788\end{array}$

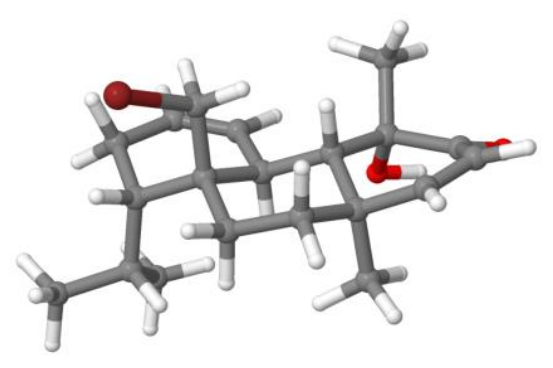




$\begin{array}{rrr}3.11922 & 2.25089 & -1.37989 \\ 2.96069 & 0.61139 & -1.93146 \\ 3.10900 & 0.29097 & 0.35935 \\ -0.94618 & 1.82247 & -2.06715 \\ 1.67164 & -1.24328 & 1.88701 \\ 0.78063 & 0.19482 & 2.33676 \\ -0.59568 & -1.74213 & 2.82150 \\ -0.39471 & -2.39369 & 1.20892 \\ -4.94645 & -2.05526 & 0.74806 \\ -2.88676 & -2.47473 & 2.06371 \\ -5.19953 & 0.34701 & -0.41058 \\ 1.85709 & 1.76376 & 2.07197 \\ -1.21404 & 1.10239 & 2.46254 \\ -2.38602 & 0.05056 & 3.25960 \\ -2.88884 & 1.08239 & 1.90705 \\ 0.46989 & -1.98944 & -0.71991 \\ 1.29974 & -0.89592 & -1.82958 \\ -3.04875 & -1.14721 & -2.42580 \\ -2.05416 & 0.25221 & -2.86365 \\ -3.82473 & 0.41235 & -2.76137 \\ 4.32827 & 2.74622 & 0.56033 \\ 3.85576 & 3.22683 & 2.19395 \\ 4.30115 & 1.54372 & 1.85980 \\ 1.69512 & 4.13922 & 1.55235 \\ 0.52161 & 3.27743 & 0.55253 \\ 2.02612 & 3.86133 & -0.15773 \\ -3.85637 & 1.97402 & -0.76651 \\ -0.46399 & 1.48541 & 0.49514\end{array}$

\section{Conformer 2}

Energy: -3501.38253 Hartree (Rel: $0.2 \mathrm{kcal} / \mathrm{mol}$ ) XYZ coordinates for conf 2 :

\begin{tabular}{|c|c|c|c|}
\hline $\mathrm{C}$ & 1.09552 & 1.73104 & -2.12369 \\
\hline C & 2.39586 & 1.36515 & -1.45791 \\
\hline C & 2.22524 & 0.95969 & 0.02448 \\
\hline C & 1.01426 & -0.03654 & 0.14465 \\
\hline C & -0.30166 & 0.71175 & -0.29809 \\
\hline C & -0.10690 & 1.43021 & -1.61950 \\
\hline C & 0.83349 & -0.56685 & 1.58717 \\
\hline C & -0.45082 & -1.38715 & 1.78959 \\
\hline C & -1.73478 & -0.65604 & 1.32401 \\
\hline C & -1.55313 & -0.21710 & -0.16804 \\
\hline C & -2.91160 & -1.60716 & 1.41379 \\
\hline C & -4.03845 & -1.43429 & 0.70430 \\
\hline C & -4.14774 & -0.25312 & -0.16021 \\
\hline C & -2.86520 & 0.31563 & -0.81709 \\
\hline C & 2.15753 & 2.16282 & 1.03979 \\
\hline C & -2.05077 & 0.52909 & 2.27829 \\
\hline 0 & -2.93550 & 1.73452 & -0.70173 \\
\hline C & 1.22789 & -1.23350 & -0.81341 \\
\hline $\mathrm{H}$ & -1.34944 & -1.14616 & -0.71240 \\
\hline C & -2.96895 & -0.12112 & -2.30064 \\
\hline C & 3.15975 & 1.94902 & 2.18987 \\
\hline C & 2.37502 & 3.56366 & 0.43923 \\
\hline $\mathrm{Br}$ & 2.93227 & -2.23691 & -0.54100 \\
\hline $\mathrm{H}$ & 1.15409 & 2.28928 & -3.05768 \\
\hline $\mathrm{H}$ & 3.10018 & 2.19920 & -1.54427 \\
\hline $\mathrm{H}$ & 2.87966 & 0.54139 & -2.00387 \\
\hline $\mathrm{H}$ & 3.11787 & 0.38243 & 0.29024 \\
\hline $\mathrm{H}$ & -0.99896 & 1.80434 & -2.10728 \\
\hline $\mathrm{H}$ & 1.69980 & -1.18113 & 1.85926 \\
\hline $\mathrm{H}$ & 0.82575 & 0.27602 & 2.28399 \\
\hline $\mathrm{H}$ & -0.54965 & -1.64434 & 2.85322 \\
\hline $\mathrm{H}$ & -0.37507 & -2.34585 & 1.25857 \\
\hline $\mathrm{H}$ & -4.92641 & -2.04388 & 0.84810 \\
\hline $\mathrm{H}$ & -2.85083 & -2.40587 & 2.15380 \\
\hline 0 & -5.21129 & 0.31159 & -0.39170 \\
\hline $\mathrm{H}$ & 1.15404 & 2.18611 & 1.48405 \\
\hline $\mathrm{H}$ & -1.18527 & 1.18079 & 2.42540 \\
\hline $\mathrm{H}$ & -2.34689 & 0.15021 & 3.26366 \\
\hline $\mathrm{H}$ & -2.86629 & 1.14362 & 1.88829 \\
\hline
\end{tabular}

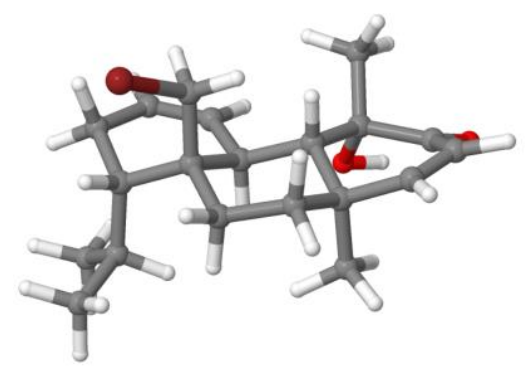




$\begin{array}{rrrr}\text { H } & 0.47747 & -2.00819 & -0.68615 \\ \mathrm{H} & 1.24665 & -0.93568 & -1.85846 \\ \mathrm{H} & -3.06578 & -1.21061 & -2.38420 \\ \mathrm{H} & -2.08478 & 0.18461 & -2.86417 \\ \mathrm{H} & -3.85600 & 0.33429 & -2.75196 \\ \mathrm{H} & 4.18965 & 1.96709 & 1.81029 \\ \mathrm{H} & 3.07050 & 2.73992 & 2.94464 \\ \mathrm{H} & 3.01295 & 0.98678 & 2.69209 \\ \mathrm{H} & 2.22515 & 4.32209 & 1.21691 \\ \mathrm{H} & 1.67442 & 3.77816 & -0.37349 \\ \mathrm{H} & 3.39539 & 3.69222 & 0.05743 \\ \mathrm{H} & -3.88465 & 1.94383 & -0.79868 \\ \mathrm{H} & -0.46597 & 1.51116 & 0.43556\end{array}$


bromosphaerol

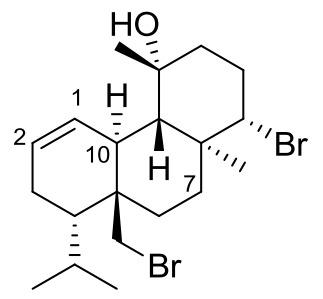

Rel energy (kcal/mol): $\begin{array}{ccc}\text { Conf } 1 & \text { Conf } 2 \\ 0.2 & 0.0\end{array}$

\begin{tabular}{|c|c|c|c|c|c|c|c|}
\hline C-nom & iGau & Exp & Calc & $\operatorname{diff}$ & 1 & 2 & \\
\hline $\mathrm{C} 1-\mathrm{CH}^{* *}$ & 6 & 126.88 & 126.63 & -0.25 & [ 126.38 & $126.81]$ & \\
\hline $\mathrm{C} 2-\mathrm{CH}^{*} *$ & 1 & 128.65 & 129.79 & 1.14 & [ 130.28 & $129.44]$ & \\
\hline $\mathrm{C} 3-\mathrm{CH} 2$ & 2 & 21.91 & 23.32 & 1.41 & 22.21 & $24.12]$ & \\
\hline $\mathrm{C} 4-\mathrm{CH}$ & 3 & 42.64 & 42.17 & -0.47 & 41.66 & $42.54]$ & \\
\hline C5-C & 4 & 40.76 & 42.22 & 1.46 & 41.84 & $42.50 \mathrm{~J}$ & \\
\hline $\mathrm{C} 6-\mathrm{CH} 2$ * & 7 & 25.04 & 24.55 & -0.49 & 24.48 & $24.60]$ & \\
\hline $\mathrm{C} 7-\mathrm{CH} 2 *$ & 8 & 36.52 & 34.16 & -2.36 & 34.17 & $34.16]$ & \\
\hline $\mathrm{C} 8-\mathrm{C}$ & 9 & 41.89 & 42.75 & 0.86 & 42.91 & $42.63]$ & \\
\hline $\mathrm{C} 9-\mathrm{CH}$ & 10 & 50.67 & 52.08 & 1.41 & 53.65 & $50.95]$ & \\
\hline $\mathrm{C} 10-\mathrm{CH}$ & 5 & 37.41 & 38.92 & 1.51 & 39.35 & $38.61]$ & \\
\hline C11-C & 14 & 72.75 & 73.21 & 0.46 & 73.29 & $73.15]$ & \\
\hline $\mathrm{C} 12-\mathrm{CH} 2$ & 13 & 46.18 & 43.29 & -2.89 & 43.98 & $42.80]$ & \\
\hline $\mathrm{C} 13-\mathrm{CH} 2$ & 12 & 30.16 & 30.72 & 0.56 & 30.96 & $30.55]$ & \\
\hline $\mathrm{C} 14-\mathrm{CH}$ & 11 & 68.80 & 67.88 & -0.92 & 67.74 & $67.98]$ & \\
\hline $\mathrm{C} 15-\mathrm{CH} 3$ & 16 & 14.07 & 12.63 & -1.44 & 12.55 & $12.69]$ & \\
\hline $\mathrm{C} 16-\mathrm{CH} 3$ & 20 & 35.02 & 34.50 & -0.52 & 36.74 & $32.89]$ & \\
\hline $\mathrm{C} 17-\mathrm{CH} 2$ & 18 & 40.36 & 40.74 & 0.38 & 40.61 & 40.83 & \\
\hline $\mathrm{C} 18-\mathrm{CH}$ & 15 & 25.88 & 27.18 & 1.30 & {$\left[\begin{array}{ll}26.34 \\
2\end{array}\right.$} & $27.78]$ & \\
\hline & & & & $\begin{array}{l}\text { ts: RM } \\
\text { Fracti }\end{array}$ & $\begin{array}{l}=1.30 \mathrm{ppm} \\
: \quad 0.419\end{array}$ & $\begin{array}{c}(\mathrm{MAE}=1.10) \\
0.581\end{array}$ & $N=18 \quad\left\{\begin{array}{lll}-2.89 & 1.51\}\end{array}\right.$ \\
\hline
\end{tabular}

Conformer 1

Energy: -5999.39321 Hartree (Rel: $0.2 \mathrm{kcal} / \mathrm{mol}$ ) XYZ coordinates for conf 1 :

$\begin{array}{lrrr}\text { C } & 2.80274 & 2.21350 & -0.74966 \\ \text { C } & 3.64870 & 1.08254 & -0.23346 \\ \text { C } & 2.86501 & 0.13031 & 0.69672 \\ \text { C } & 1.44563 & -0.16719 & 0.08706 \\ \text { C } & 0.61095 & 1.16435 & -0.05310 \\ \text { C } & 1.46636 & 2.24445 & -0.68855 \\ \text { C } & 0.65420 & -1.17281 & 0.94818 \\ \text { C } & -0.79945 & -1.36144 & 0.50304 \\ \text { C } & -1.60603 & -0.04376 & 0.37553 \\ \text { C } & -0.83180 & 0.90736 & -0.64447 \\ \text { C } & -2.97912 & -0.36555 & -0.25970 \\ \text { C } & -3.83221 & 0.86544 & -0.50025 \\ \text { C } & -3.09822 & 1.76485 & -1.49384 \\ \text { C } & -1.65652 & 2.16925 & -1.11183 \\ \text { C } & 2.94283 & 0.56988 & 2.19900 \\ \text { C } & -1.80181 & 0.60030 & 1.76986 \\ \mathrm{H} & 0.43323 & 1.50122 & 0.97665 \\ \text { C } & 1.62660 & -0.74610 & -1.33997 \\ \mathrm{H} & -0.72149 & 0.32474 & -1.56785 \\ \mathrm{C} & -1.08577 & 2.85009 & -2.38883 \\ \mathrm{C} & 4.27841 & 0.08789 & 2.80109 \\ \mathrm{C} & 2.76903 & 2.06983 & 2.50481 \\ \mathrm{Br} & 2.76900 & -2.37761 & -1.46538 \\ \mathrm{O} & -1.80421 & 3.14116 & -0.06415 \\ \mathrm{Br} & -4.05552 & -1.69717 & 0.80670 \\ \mathrm{H} & 3.32587 & 3.06183 & -1.19034 \\ \mathrm{H} & 4.52048 & 1.49253 & 0.29265 \\ \mathrm{H} & 4.07536 & 0.52050 & -1.07755 \\ \mathrm{H} & 3.37518 & -0.83864 & 0.66864\end{array}$

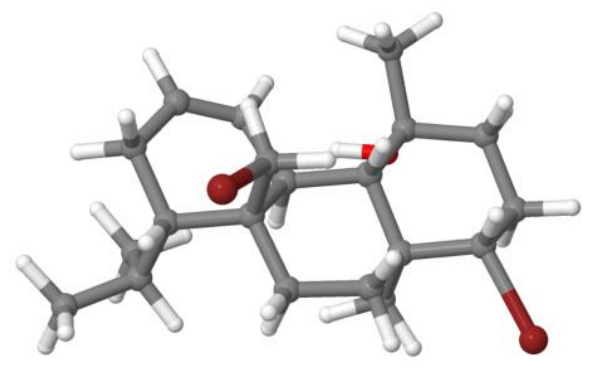




$\begin{array}{rrr}0.97589 & 3.14134 & -1.04214 \\ 1.16698 & -2.14207 & 0.93830 \\ 0.65513 & -0.84175 & 1.98935 \\ -0.83703 & -1.89405 & -0.45759 \\ -1.30613 & -2.01538 & 1.22000 \\ -2.82638 & -0.90688 & -1.19792 \\ -4.80452 & 0.57802 & -0.91294 \\ -4.02243 & 1.39138 & 0.43932 \\ -3.66015 & 2.69357 & -1.64524 \\ -3.05747 & 1.25693 & -2.46707 \\ 2.15518 & 0.04103 & 2.74777 \\ -2.19387 & 1.61583 & 1.69614 \\ -2.49561 & -0.00600 & 2.35982 \\ -0.86671 & 0.65217 & 2.33326 \\ 0.68764 & -1.08455 & -1.76741 \\ 2.08740 & -0.03670 & -2.02276 \\ -0.38126 & 3.65106 & -2.16125 \\ -1.90313 & 3.31368 & -2.95139 \\ -0.58484 & 2.13446 & -3.04974 \\ 4.34443 & 0.35093 & 3.86340 \\ 4.38628 & -0.99949 & 2.71636 \\ 5.13813 & 0.54770 & 2.29706 \\ 2.82884 & 2.22890 & 3.58817 \\ 1.80981 & 2.47141 & 2.16831 \\ 3.55868 & 2.67495 & 2.04553 \\ -0.91892 & 3.43028 & 0.20756\end{array}$

\section{Conformer 2}

Energy: -5999.39352 Hartree (Rel: $0.0 \mathrm{kcal} / \mathrm{mol})$ XYZ coordinates for conf 2 :

$\begin{array}{lrrr}\mathrm{C} & 2.77850 & 2.34859 & -0.47376 \\ \mathrm{C} & 3.62211 & 1.14922 & -0.14216 \\ \mathrm{C} & 2.85714 & 0.11690 & 0.71390 \\ \mathrm{C} & 1.45199 & -0.15327 & 0.05696 \\ \mathrm{C} & 0.60192 & 1.17747 & 0.06126 \\ \mathrm{C} & 1.44257 & 2.35684 & -0.39509 \\ \mathrm{C} & 0.66468 & -1.24008 & 0.82148 \\ \mathrm{C} & -0.79731 & -1.38941 & 0.37993 \\ \mathrm{C} & -1.60463 & -0.06532 & 0.36170 \\ \mathrm{C} & -0.82616 & 0.95412 & -0.58179 \\ \mathrm{C} & -2.98142 & -0.32134 & -0.29262 \\ \mathrm{C} & -3.82463 & 0.93546 & -0.41220 \\ \mathrm{C} & -3.10199 & 1.89958 & -1.35357 \\ \mathrm{C} & -1.63677 & 2.23756 & -0.99080 \\ \mathrm{C} & 2.78888 & 0.45983 & 2.25111 \\ \mathrm{C} & -1.79792 & 0.47378 & 1.80119 \\ \mathrm{H} & 0.39523 & 1.38550 & 1.11916 \\ \mathrm{C} & 1.63913 & -0.58855 & -1.41634 \\ \mathrm{H} & -0.69164 & 0.42934 & -1.53518 \\ \mathrm{C} & -1.07002 & 2.91696 & -2.26275 \\ \mathrm{C} & 3.22317 & -0.75671 & 3.09068 \\ \mathrm{C} & 3.59871 & 1.68866 & 2.70443 \\ \mathrm{Br} & 2.85292 & -2.14826 & -1.68971 \\ \mathrm{O} & -1.73502 & 3.18422 & 0.08733 \\ \mathrm{Br} & -4.05894 & -1.73962 & 0.65300 \\ \mathrm{H} & 3.29446 & 3.26289 & -0.76494 \\ \mathrm{H} & 4.53350 & 1.47302 & 0.37083 \\ \mathrm{H} & 3.98094 & 0.66809 & -1.06364 \\ \mathrm{H} & 3.40883 & -0.82566 & 0.63145 \\ \mathrm{H} & 0.94249 & 3.30299 & -0.57265 \\ \mathrm{H} & 1.17473 & -2.20425 & 0.70785 \\ \mathrm{H} & 0.68448 & -1.01122 & 1.88992 \\ \mathrm{H} & -0.84493 & -1.84357 & -0.62003 \\ \mathrm{H} & -1.29572 & -2.09918 & 1.04822 \\ \mathrm{H} & -2.83843 & -0.77367 & -1.27825 \\ \mathrm{H} & -4.81015 & 0.69305 & -0.82223 \\ \mathrm{H} & -3.98658 & 1.38674 & 0.57065 \\ \mathrm{H} & -3.64404 & 2.85032 & -1.40926 \\ \mathrm{H} & -3.11296 & 1.46886 & -2.36315 \\ \mathrm{H} & 1.74593 & 0.68144 & 2.51219 \\ \mathrm{H} & -2.09440 & 1.52461 & 1.80286 \\ & & & \end{array}$

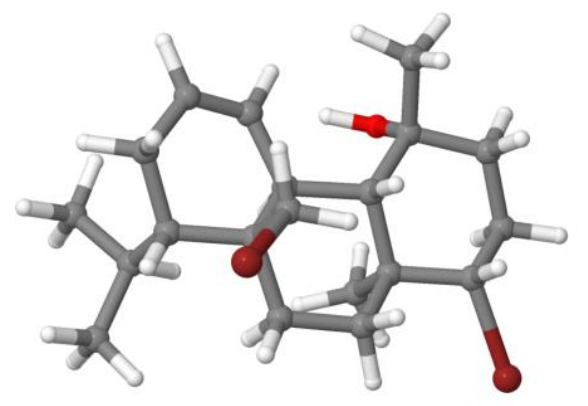




$\begin{array}{rrrr}\text { H } & -2.56764 & -0.11124 & 2.31328 \\ \mathrm{H} & -0.88695 & 0.38475 & 2.39818 \\ \mathrm{H} & 0.71252 & -0.93321 & -1.86575 \\ \mathrm{H} & 2.05763 & 0.20105 & -2.03637 \\ \mathrm{H} & -0.05769 & 3.30284 & -2.15520 \\ \mathrm{H} & -1.72105 & 3.75580 & -2.53199 \\ \mathrm{H} & -1.06055 & 2.21336 & -3.10303 \\ \mathrm{H} & 3.10558 & -0.55901 & 4.16307 \\ \mathrm{H} & 2.64542 & -1.65474 & 2.84899 \\ \mathrm{H} & 4.28083 & -0.98850 & 2.90969 \\ \mathrm{H} & 3.41424 & 1.87487 & 3.76910 \\ \mathrm{H} & 3.32092 & 2.59417 & 2.15646 \\ \mathrm{H} & 4.67850 & 1.53419 & 2.58821 \\ \mathrm{H} & -0.84036 & 3.41681 & 0.37838\end{array}$


originally assigned structure of sphaeroxetane (cmpd 3), Fig.18<smiles>CC(C)[C@H]1CC=C[C@@H]2[C@@H]3[C@](C)(CBr)CCC[C@]3(C)CC[C@]12O</smiles>

Rel energy (kcal/mol): $\begin{array}{cccc}\text { Conf 1 } & \text { Conf 2 } & \text { Conf } 3 \\ 1.2 & 0.0 & 2.7\end{array}$

\begin{tabular}{|c|c|c|c|c|c|c|c|c|}
\hline C-nom & iGau & Exp & Calc & $\operatorname{diff}$ & 1 & 2 & 3 & \\
\hline $\mathrm{C} 1-\mathrm{CH}$ & 6 & 131.03 & 135.00 & 3.97 & [ 139.11 & 134.43 & $137.26]$ & ] \\
\hline $\mathrm{C} 2-\mathrm{CH}$ & 1 & 126.95 & 128.34 & 1.39 & [ 127.21 & 128.52 & $125.79]$ & ] \\
\hline $\mathrm{C} 3-\mathrm{CH} 2$ & 2 & 22.92 & 24.16 & 1.24 & {$\left[\begin{array}{ll}{[} & 27.61\end{array}\right.$} & 23.71 & $24.22]$ & ] \\
\hline $\mathrm{C} 4-\mathrm{CH}$ & 3 & 45.25 & 42.93 & -2.32 & 46.44 & 42.43 & $46.06]$ & ] \\
\hline C5-C & 4 & 47.10 & 47.19 & 0.09 & 48.14 & 47.07 & $46.78]$ & ] \\
\hline $\mathrm{C} 6-\mathrm{CH} 2-\star$ & 7 & 23.38 & 26.88 & 3.50 & 33.24 & 25.98 & $32.79]$ & ] \\
\hline $\mathrm{C} 7-\mathrm{CH} 2-*$ & 8 & 34.35 & 34.83 & 0.48 & 36.52 & 34.60 & $35.58]$ & ] \\
\hline $\mathrm{C} 8-\mathrm{C}$ & 9 & 32.00 & 37.99 & 5.99 & 35.66 & 38.31 & $37.02]$ & ] \\
\hline $\mathrm{C} 9-\mathrm{CH}$ & 10 & 47.08 & 53.42 & 6.34 & 54.16 & 53.32 & $54.02]$ & ] \\
\hline 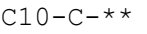 & 5 & 89.42 & 89.30 & -0.12 & 88.87 & 89.39 & $86.32]$ & ] \\
\hline $\mathrm{C} 11-\mathrm{C}-\star \star *$ & 14 & 81.40 & 84.28 & 2.88 & 86.32 & 83.99 & $86.29]$ & ] \\
\hline C12-CH2 & 13 & 41.47 & 34.54 & -6.93 & 33.89 & 34.63 & $34.59]$ & ] \\
\hline $\mathrm{C} 13-\mathrm{CH} 2$ & 12 & 29.71 & 19.25 & -10.46 & 20.05 & 19.14 & $20.08]$ & ] \\
\hline $\mathrm{C} 14-\mathrm{CH} 2$ & 11 & 34.97 & 34.13 & -0.84 & 34.09 & 34.13 & $34.31]$ & ] \\
\hline C15-CH3 & 16 & 18.25 & 33.74 & 15.49 & 33.27 & 33.80 & 34.01 ] & \\
\hline $\mathrm{C} 16-\mathrm{CH} 3$ & 20 & 27.61 & 29.20 & 1.59 & 27.75 & 29.40 & $28.71]$ & ] \\
\hline C17-CH2 & 18 & 44.41 & 46.83 & 2.42 & 54.23 & 45.78 & $54.05]$ & \\
\hline $\mathrm{C} 18-\mathrm{CH}$ & 15 & 26.21 & 26.96 & 0.75 & 30.26 & 26.52 & $27.61]$ & \\
\hline C19-CH3 & 21 & 25.85 & 25.18 & -0.67 & 25.29 & 25.15 & $26.10]$ & ] \\
\hline $\mathrm{C} 20-\mathrm{CH} 3$ & 22 & 18.75 & 17.40 & -1.35 & 19.63 & 17.09 & $19.09]$ & ] \\
\hline
\end{tabular}

\section{Conformer 1}

Energy: -3427.30811 Hartree (Rel: $1.2 \mathrm{kcal} / \mathrm{mol})$ XYZ coordinates for conf 1 :

\begin{tabular}{|c|c|c|c|}
\hline $\mathrm{C}$ & 1.44413 & 0.07804 & 2.52723 \\
\hline C & 2.15049 & -1.01619 & 1.76124 \\
\hline $\mathrm{C}$ & 2.27166 & -0.74908 & 0.21336 \\
\hline C & 1.06424 & 0.18333 & -0.27204 \\
\hline $\mathrm{C}$ & -0.15044 & -0.05094 & 0.72499 \\
\hline $\mathrm{C}$ & 0.30102 & 0.56550 & 2.03544 \\
\hline C & 0.65558 & 0.10836 & -1.77966 \\
\hline C & -0.78708 & 0.57959 & -2.10738 \\
\hline C & -1.75972 & -0.29291 & -1.30171 \\
\hline C & -1.56607 & 0.16224 & 0.19134 \\
\hline C & -3.27672 & -0.13605 & -1.51152 \\
\hline C & -4.03456 & -0.99852 & -0.43435 \\
\hline C & -3.13944 & -1.83754 & 0.53257 \\
\hline C & -2.01480 & -0.95931 & 1.12787 \\
\hline C & 2.50777 & -2.05870 & -0.60606 \\
\hline C & -1.43781 & -1.77060 & -1.74205 \\
\hline 0 & -0.60383 & -1.45672 & 1.06576 \\
\hline C & 1.68322 & 1.60376 & -0.07755 \\
\hline $\mathrm{H}$ & -2.00281 & 1.16132 & 0.30702 \\
\hline C & -2.41699 & -0.60873 & 2.56480 \\
\hline C & 3.32030 & -1.77733 & -1.88626 \\
\hline C & 3.25353 & -3.15601 & 0.18036 \\
\hline $\mathrm{Br}$ & 0.46470 & 3.19388 & -0.10312 \\
\hline $\mathrm{H}$ & 1.87050 & 0.44481 & 3.45870 \\
\hline $\mathrm{H}$ & 1.56838 & -1.93222 & 1.90409 \\
\hline $\mathrm{H}$ & 3.14811 & -1.19996 & 2.17119 \\
\hline H & 3.19181 & -0.16102 & 0.08001 \\
\hline
\end{tabular}

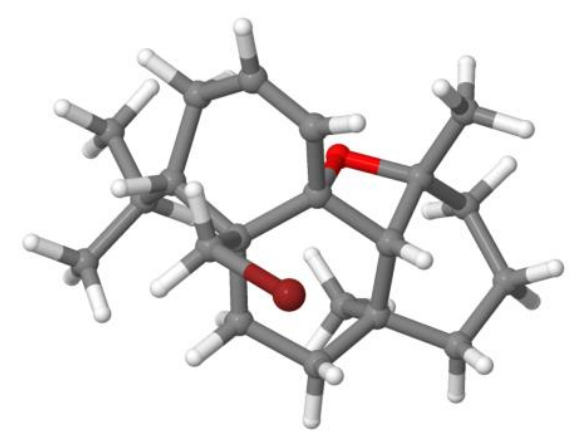




$\begin{array}{rrr}-0.23365 & 1.39332 & 2.48923 \\ 1.36129 & 0.70829 & -2.36582 \\ 0.76667 & -0.91041 & -2.14474 \\ -0.95247 & 0.45658 & -3.18648 \\ -0.91452 & 1.64146 & -1.88006 \\ -3.57482 & 0.91662 & -1.42168 \\ -3.55518 & -0.45491 & -2.52344 \\ -4.74626 & -1.67422 & -0.92204 \\ -4.64429 & -0.32781 & 0.18365 \\ -2.72600 & -2.72188 & 0.05129 \\ -3.78122 & -2.21258 & 1.33843 \\ 1.53150 & -2.47483 & -0.88480 \\ -0.72828 & -2.28459 & -1.09464 \\ -1.03339 & -1.75022 & -2.76008 \\ -2.33580 & -2.38568 & -1.80329 \\ 2.19064 & 1.71852 & 0.87254 \\ 2.38060 & 1.79914 & -0.89201 \\ -3.43405 & -0.19663 & 2.57152 \\ -1.75680 & 0.10347 & 3.05579 \\ -2.42447 & -1.52648 & 3.16469 \\ 3.39008 & -2.67810 & -2.50776 \\ 2.89729 & -0.98118 & -2.50296 \\ 4.34451 & -1.48063 & -1.62324 \\ 3.43889 & -4.01514 & -0.47506 \\ 2.69040 & -3.51966 & 1.04352 \\ 4.22948 & -2.80072 & 0.53574\end{array}$

\section{Conformer 2}

Energy: -3427.31003 Hartree (Rel: $0.0 \mathrm{kcal} / \mathrm{mol}$ ) XYZ coordinates for conf 2 :

$\begin{array}{lrrr}\mathrm{C} & 1.05907 & 1.68909 & -2.09186 \\ \mathrm{C} & 2.12071 & 1.75430 & -1.02590 \\ \mathrm{C} & 1.69674 & 1.14668 & 0.33478 \\ \mathrm{C} & 0.75100 & -0.07138 & 0.08578 \\ \mathrm{C} & -0.56152 & 0.35907 & -0.71283 \\ \mathrm{C} & -0.07548 & 0.98001 & -2.01461 \\ \mathrm{C} & 0.51407 & -0.92902 & 1.35710 \\ \mathrm{C} & -0.59016 & -1.99884 & 1.18905 \\ \mathrm{C} & -1.89392 & -1.26073 & 0.85572 \\ \mathrm{C} & -1.71201 & -0.67003 & -0.60216 \\ \mathrm{C} & -3.19683 & -2.07360 & 0.73777 \\ \mathrm{C} & -4.36307 & -1.13229 & 0.26155 \\ \mathrm{C} & -3.98192 & 0.36651 & 0.06331 \\ \mathrm{C} & -2.70014 & 0.47718 & -0.79269 \\ \mathrm{C} & 1.19813 & 2.21640 & 1.36847 \\ \mathrm{C} & -2.11706 & -0.29707 & 2.08482 \\ \mathrm{O} & -1.62173 & 1.37645 & -0.28412 \\ \mathrm{C} & 1.43757 & -1.06347 & -0.92622 \\ \mathrm{H} & -1.73457 & -1.50568 & -1.31460 \\ \mathrm{C} & -3.15679 & 0.80459 & -2.22640 \\ \mathrm{C} & 2.39192 & 2.70654 & 2.20951 \\ \mathrm{C} & 0.44742 & 3.43077 & 0.79055 \\ \mathrm{Br} & 3.18488 & -1.82885 & -0.32603 \\ \mathrm{H} & 1.27988 & 2.21018 & -3.02347 \\ \mathrm{H} & 2.46293 & 2.78824 & -0.89982 \\ \mathrm{H} & 3.00326 & 1.21466 & -1.40409 \\ \mathrm{H} & 2.59891 & 0.70931 & 0.77907 \\ \mathrm{H} & -0.72886 & 0.98089 & -2.87467 \\ \mathrm{H} & 1.46157 & -1.41039 & 1.61590 \\ \mathrm{H} & 0.26918 & -0.29095 & 2.20541 \\ \mathrm{H} & -0.69263 & -2.56516 & 2.12385 \\ \mathrm{H} & -0.32719 & -2.73170 & 0.41534 \\ \mathrm{H} & -3.07340 & -2.90291 & 0.02872 \\ \mathrm{H} & -3.44191 & -2.52667 & 1.70608 \\ \mathrm{H} & -5.20702 & -1.18975 & 0.95810 \\ \mathrm{H} & -4.74753 & -1.50586 & -0.69585 \\ \mathrm{H} & -3.86287 & 0.89215 & 1.00758 \\ \mathrm{H} & -4.81442 & 0.86410 & -0.44712 \\ \mathrm{H} & 0.50558 & 1.71759 & 2.05709 \\ \mathrm{H} & -1.75514 & 0.71808 & 1.92669 \\ \mathrm{H} & -1.60591 & -0.71715 & 2.95708 \\ & & & \end{array}$

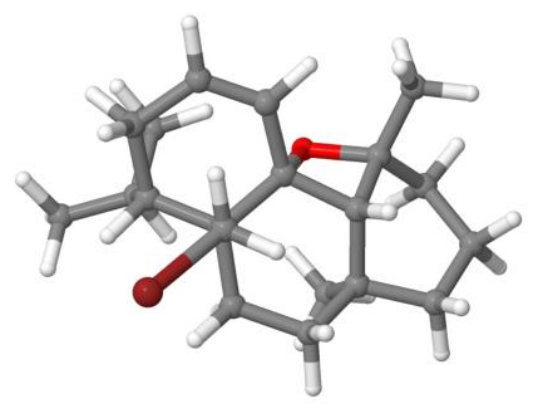




$\begin{array}{rrrr}\text { H } & -3.16719 & -0.23809 & 2.37032 \\ \mathrm{H} & 0.81962 & -1.94173 & -1.10081 \\ \mathrm{H} & 1.68654 & -0.62116 & -1.87976 \\ \mathrm{H} & -4.08111 & 0.25624 & -2.44210 \\ \mathrm{H} & -2.45107 & 0.54008 & -3.01208 \\ \mathrm{H} & -3.37853 & 1.87519 & -2.30290 \\ \mathrm{H} & 2.07291 & 3.45005 & 2.94973 \\ \mathrm{H} & 2.87602 & 1.88138 & 2.74563 \\ \mathrm{H} & 3.15263 & 3.18148 & 1.57526 \\ \mathrm{H} & 0.07546 & 4.05019 & 1.61621 \\ \mathrm{H} & -0.40695 & 3.13918 & 0.17842 \\ \mathrm{H} & 1.10835 & 4.06815 & 0.19024\end{array}$

\section{Conformer 3}

Energy: -3427.30577 Hartree (Rel: $2.7 \mathrm{kcal} / \mathrm{mol}$ ) XYZ coordinates for conf 3 :

\begin{tabular}{|c|c|c|c|}
\hline C & 1.89275 & 0.17577 & 2.20253 \\
\hline C & 2.84537 & -0.51766 & 1.25680 \\
\hline C & 2.33172 & -0.72153 & -0.21397 \\
\hline C & 1.07518 & 0.19992 & -0.48355 \\
\hline C & -0.00001 & -0.06639 & 0.64100 \\
\hline C & 0.61925 & 0.46576 & 1.91880 \\
\hline C & 0.53049 & 0.14151 & -1.94513 \\
\hline C & -0.92126 & 0.66143 & -2.11178 \\
\hline C & -1.82732 & -0.19538 & -1.21401 \\
\hline C & -1.46389 & 0.20592 & 0.27063 \\
\hline C & -3.35084 & 0.01758 & -1.26012 \\
\hline C & -4.02285 & -0.85469 & -0.13674 \\
\hline C & -3.06093 & -1.76369 & 0.69159 \\
\hline C & -1.85764 & -0.93736 & 1.20751 \\
\hline C & 2.18277 & -2.23095 & -0.64523 \\
\hline C & -1.60757 & -1.67134 & -1.72414 \\
\hline 0 & -0.47332 & -1.46657 & 0.99797 \\
\hline $\mathrm{C}$ & 1.67827 & 1.64303 & -0.34504 \\
\hline $\mathrm{H}$ & -1.85660 & 1.21116 & 0.45964 \\
\hline C & -2.13187 & -0.64839 & 2.68956 \\
\hline C & 3.36869 & -2.63576 & -1.54025 \\
\hline C & 2.01854 & -3.25857 & 0.48951 \\
\hline $\mathrm{Br}$ & 0.44275 & 3.18703 & -0.04671 \\
\hline $\mathrm{H}$ & 2.31885 & 0.50625 & 3.14954 \\
\hline $\mathrm{H}$ & 3.14484 & -1.47716 & 1.69023 \\
\hline $\mathrm{H}$ & 3.77355 & 0.07216 & 1.23912 \\
\hline $\mathrm{H}$ & 3.11729 & -0.31699 & -0.86682 \\
\hline $\mathrm{H}$ & 0.03253 & 1.06008 & 2.60841 \\
\hline $\mathrm{H}$ & 1.20114 & 0.72214 & -2.58958 \\
\hline $\mathrm{H}$ & 0.58161 & -0.87650 & -2.33080 \\
\hline $\mathrm{H}$ & -1.21413 & 0.56090 & -3.16569 \\
\hline $\mathrm{H}$ & -0.98832 & 1.72335 & -1.85878 \\
\hline $\mathrm{H}$ & -3.60152 & 1.07568 & -1.11028 \\
\hline $\mathrm{H}$ & -3.74031 & -0.25999 & -2.24718 \\
\hline $\mathrm{H}$ & -4.81425 & -1.48125 & -0.56343 \\
\hline $\mathrm{H}$ & -4.52828 & -0.18545 & 0.57070 \\
\hline $\mathrm{H}$ & -2.72601 & -2.63400 & 0.13186 \\
\hline $\mathrm{H}$ & -3.62815 & -2.16045 & 1.54154 \\
\hline $\mathrm{H}$ & 1.28007 & -2.31423 & -1.25625 \\
\hline $\mathrm{H}$ & -0.87703 & -2.23588 & -1.14663 \\
\hline $\mathrm{H}$ & -1.27937 & -1.63809 & -2.76839 \\
\hline $\mathrm{H}$ & -2.53693 & -2.24078 & -1.73536 \\
\hline $\mathrm{H}$ & 2.36009 & 1.74835 & 0.48824 \\
\hline $\mathrm{H}$ & 2.19420 & 1.90418 & -1.26813 \\
\hline $\mathrm{H}$ & -3.14116 & -0.23269 & 2.80048 \\
\hline $\mathrm{H}$ & -1.43324 & 0.03478 & 3.16464 \\
\hline $\mathrm{H}$ & -2.09985 & -1.59485 & 3.24213 \\
\hline $\mathrm{H}$ & 3.27499 & -3.67697 & -1.87153 \\
\hline $\mathrm{H}$ & 3.43646 & -2.00367 & -2.43427 \\
\hline $\mathrm{H}$ & 4.31934 & -2.54735 & -0.99711 \\
\hline $\mathrm{H}$ & 1.73297 & -4.22566 & 0.05752 \\
\hline H & 1.23862 & -2.96264 & 1.19408 \\
\hline $\mathrm{H}$ & 2.95600 & -3.42316 & 1.03545 \\
\hline
\end{tabular}

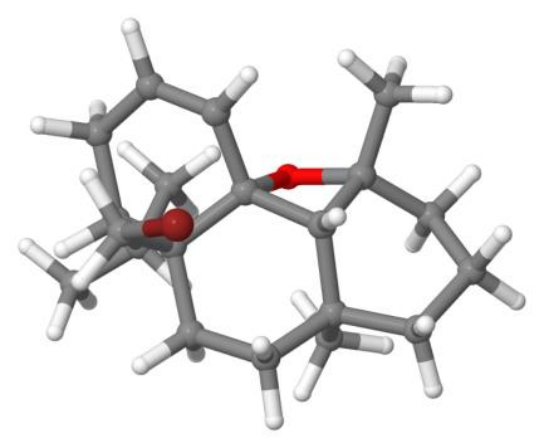


A candidate structure for sphaeroxetane (ignoring multiplicities and fitting to 13C), Fig.18

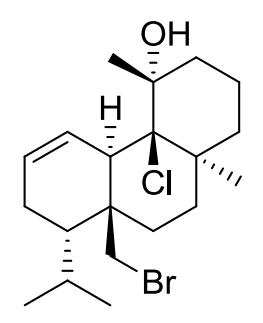

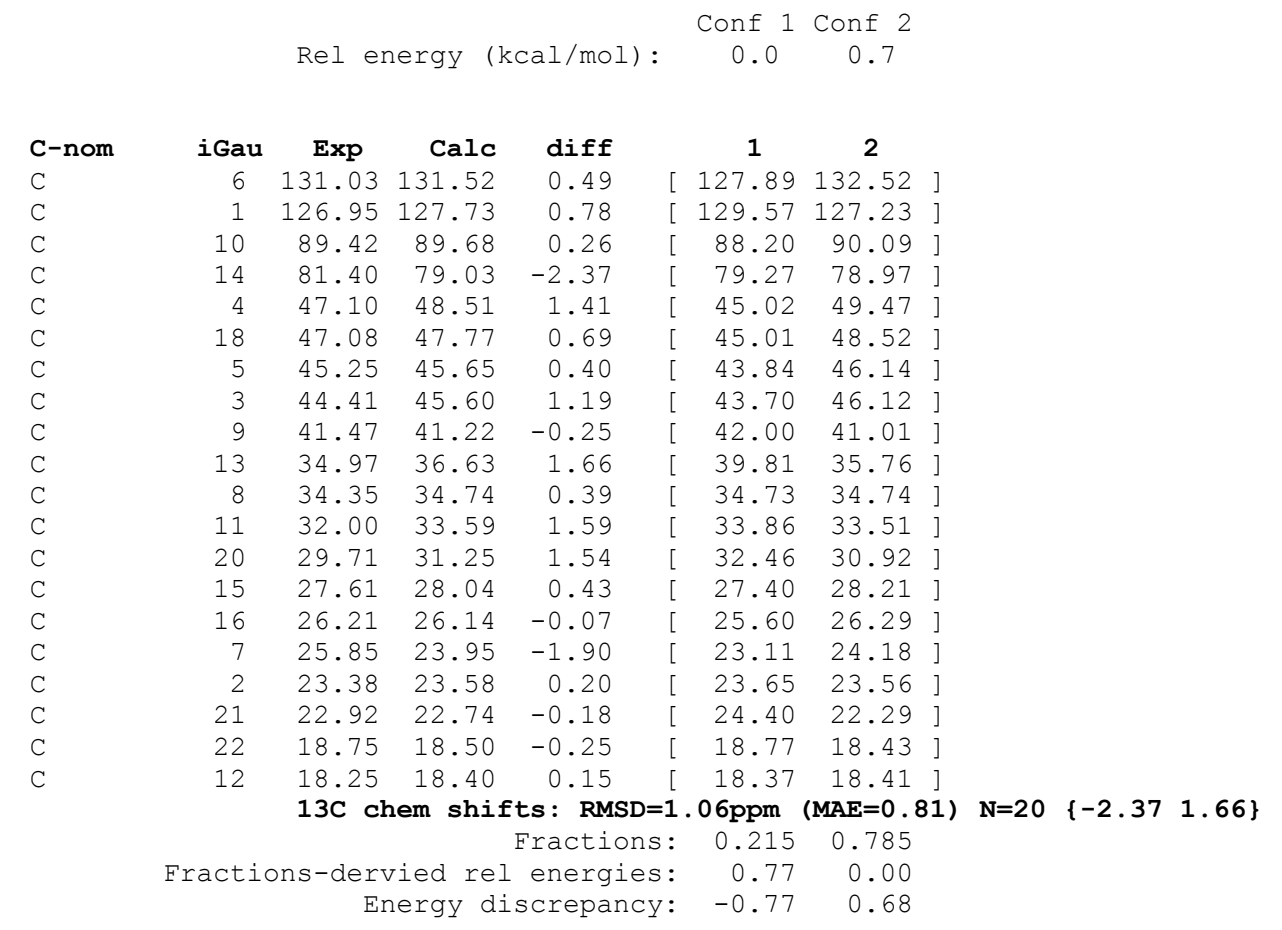

\section{Conformer 1}

Energy: -3888.17090 Hartree (Rel: $0.0 \mathrm{kcal} / \mathrm{mol}$ ) XYZ coordinates for conf 1 :

\begin{tabular}{|c|c|c|c|}
\hline $\mathrm{C}$ & 1.27197 & 1.34849 & -2.27787 \\
\hline C & 2.46349 & 1.39207 & -1.36000 \\
\hline $\mathrm{C}$ & 2.11341 & 1.10101 & 0.11849 \\
\hline C & 1.02523 & -0.03588 & 0.19765 \\
\hline C & -0.28763 & 0.56095 & -0.47730 \\
\hline $\mathrm{C}$ & 0.05477 & 0.92819 & -1.91280 \\
\hline C & 0.77246 & -0.50520 & 1.64777 \\
\hline $\mathrm{C}$ & -0.51206 & -1.33117 & 1.78052 \\
\hline C & -1.76837 & -0.55790 & 1.32013 \\
\hline C & -1.63492 & -0.18861 & -0.22187 \\
\hline $\mathrm{C}$ & -3.02396 & -1.42662 & 1.56619 \\
\hline $\mathrm{C}$ & -4.31425 & -0.74733 & 1.09722 \\
\hline C & -4.21836 & -0.28084 & -0.35787 \\
\hline C & -2.95657 & 0.54342 & -0.73740 \\
\hline $\mathrm{C}$ & 1.82413 & 2.40449 & 0.94618 \\
\hline $\mathrm{C}$ & -1.88529 & 0.69705 & 2.23850 \\
\hline 0 & -2.95440 & 1.81121 & -0.05561 \\
\hline C & 1.50866 & -1.27809 & -0.60005 \\
\hline $\mathrm{Cl}$ & -1.59552 & -1.78734 & -1.21651 \\
\hline $\mathrm{C}$ & -3.06301 & 0.83962 & -2.24861 \\
\hline $\mathrm{C}$ & 3.12205 & 2.86945 & 1.63383 \\
\hline $\mathrm{C}$ & 1.20836 & 3.59761 & 0.18604 \\
\hline $\mathrm{Br}$ & 3.32883 & -1.95034 & -0.09081 \\
\hline
\end{tabular}

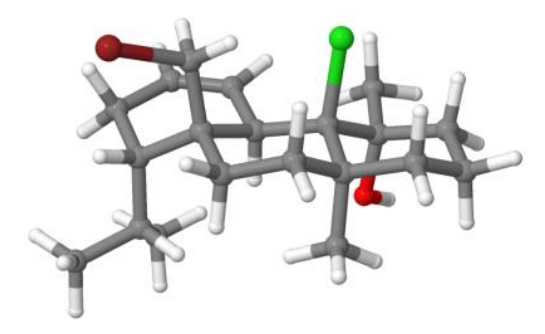




$\begin{array}{rrrr}\text { H } & 1.44828 & 1.60408 & -3.32260 \\ \mathrm{H} & 2.98456 & 2.35382 & -1.44979 \\ \mathrm{H} & 3.19260 & 0.64776 & -1.71406 \\ \mathrm{H} & 3.00631 & 0.66009 & 0.57336 \\ \mathrm{H} & -0.70331 & 0.83677 & -2.67552 \\ \mathrm{H} & 1.63285 & -1.09971 & 1.97601 \\ \mathrm{H} & 0.72144 & 0.34598 & 2.33035 \\ \mathrm{H} & -0.65351 & -1.61146 & 2.83223 \\ \mathrm{H} & -0.43224 & -2.27329 & 1.22745 \\ \mathrm{H} & -2.90699 & -2.39171 & 1.06096 \\ \mathrm{H} & -3.08583 & -1.64244 & 2.64095 \\ \mathrm{H} & -4.54984 & 0.10577 & 1.74271 \\ \mathrm{H} & -5.15760 & -1.44253 & 1.19484 \\ \mathrm{H} & -5.08881 & 0.34436 & -0.60172 \\ \mathrm{H} & -4.26974 & -1.14735 & -1.02596 \\ \mathrm{H} & 1.11993 & 2.14986 & 1.74740 \\ \mathrm{H} & -1.12292 & 1.45282 & 2.04662 \\ \mathrm{H} & -1.76341 & 0.36072 & 3.27579 \\ \mathrm{H} & -2.84392 & 1.20006 & 2.14833 \\ \mathrm{H} & 0.88090 & -2.14365 & -0.42535 \\ \mathrm{H} & 1.58130 & -1.10488 & -1.66818 \\ \mathrm{H} & -2.81172 & -0.01998 & -2.87322 \\ \mathrm{H} & -2.43819 & 1.68948 & -2.52726 \\ \mathrm{H} & -4.10340 & 1.11072 & -2.47196 \\ \mathrm{H} & 2.95257 & 3.77380 & 2.23057 \\ \mathrm{H} & 3.52273 & 2.09537 & 2.29846 \\ \mathrm{H} & 3.89768 & 3.10411 & 0.89293 \\ \mathrm{H} & 0.97677 & 4.39873 & 0.89815 \\ \mathrm{H} & 0.28521 & 3.34998 & -0.34425 \\ \mathrm{H} & 1.90709 & 4.01517 & -0.54809 \\ \mathrm{H} & -3.65992 & 2.34753 & -0.45094 \\ \mathrm{H} & -0.46949 & 1.50264 & 0.05310 \\ & & & \end{array}$

\section{Conformer 2}

Energy: -3888.16982 Hartree (Rel: XYZ coordinates for conf 2:

\begin{tabular}{|c|c|c|c|}
\hline $\mathrm{C}$ & -0.85707 & 1.53223 & 2.45329 \\
\hline C & -1.66961 & 2.16114 & 1.35479 \\
\hline C & -2.11508 & 1.09894 & 0.29918 \\
\hline C & -0.98177 & 0.01009 & -0.01941 \\
\hline C & 0.39623 & 0.55111 & 0.61667 \\
\hline C & 0.15709 & 0.73615 & 2.09956 \\
\hline C & -0.78744 & -0.30392 & -1.52135 \\
\hline $\mathrm{C}$ & 0.49693 & -1.09195 & -1.81424 \\
\hline C & 1.77148 & -0.33580 & -1.37737 \\
\hline C & 1.73219 & -0.12494 & 0.18968 \\
\hline C & 3.03317 & -1.13211 & -1.78505 \\
\hline C & 4.32976 & -0.45557 & -1.32165 \\
\hline $\mathrm{C}$ & 4.31458 & -0.13378 & 0.17758 \\
\hline $\mathrm{C}$ & 3.05902 & 0.60278 & 0.70519 \\
\hline C & -2.82689 & 1.76126 & -0.92240 \\
\hline C & 1.80029 & 1.00671 & -2.17129 \\
\hline 0 & 3.09620 & 1.92584 & 0.14791 \\
\hline $\mathrm{C}$ & -1.37943 & -1.30300 & 0.70198 \\
\hline $\mathrm{Cl}$ & 1.79831 & -1.82415 & 1.01704 \\
\hline $\mathrm{C}$ & 3.21854 & 0.74702 & 2.22978 \\
\hline $\mathrm{C}$ & -4.25412 & 2.19958 & -0.54243 \\
\hline $\mathrm{C}$ & -2.08081 & 2.94487 & -1.56648 \\
\hline $\mathrm{Br}$ & -3.14369 & -2.05432 & 0.12092 \\
\hline $\mathrm{H}$ & -1.12856 & 1.68655 & 3.49514 \\
\hline $\mathrm{H}$ & -1.07210 & 2.94494 & 0.86592 \\
\hline $\mathrm{H}$ & -2.55511 & 2.66409 & 1.75543 \\
\hline $\mathrm{H}$ & -2.90787 & 0.53120 & 0.79346 \\
\hline $\mathrm{H}$ & 0.72778 & 0.17090 & 2.82601 \\
\hline $\mathrm{H}$ & -1.65032 & -0.87479 & -1.87939 \\
\hline $\mathrm{H}$ & -0.77399 & 0.61807 & -2.10392 \\
\hline $\mathrm{H}$ & 0.56029 & -1.27269 & -2.89510 \\
\hline $\mathrm{H}$ & 0.46689 & -2.08096 & -1.34502 \\
\hline $\mathrm{H}$ & 2.97537 & -2.14678 & -1.37616 \\
\hline $\mathrm{H}$ & 3.03680 & -1.23520 & -2.87818 \\
\hline $\mathrm{H}$ & 4.50324 & 0.46316 & -1.89237 \\
\hline
\end{tabular}

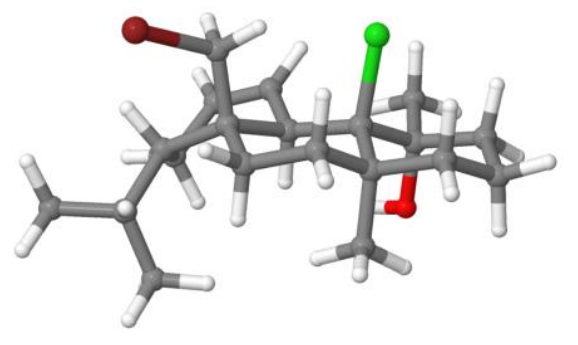




$\begin{array}{rrrr}\text { H } & 5.18429 & -1.11006 & -1.53489 \\ \mathrm{H} & 5.17344 & 0.49879 & 0.42953 \\ \mathrm{H} & 4.42144 & -1.06102 & 0.74896 \\ \mathrm{H} & -2.94263 & 0.98471 & -1.68656 \\ \mathrm{H} & 1.04113 & 1.72247 & -1.85206 \\ \mathrm{H} & 1.60614 & 0.77321 & -3.22589 \\ \mathrm{H} & 2.75547 & 1.51924 & -2.10030 \\ \mathrm{H} & -0.69198 & -2.11923 & 0.51439 \\ \mathrm{H} & -1.49660 & -1.16439 & 1.77374 \\ \mathrm{H} & 3.12472 & -0.20442 & 2.75710 \\ \mathrm{H} & 2.48949 & 1.44667 & 2.64917 \\ \mathrm{H} & 4.21708 & 1.15255 & 2.42429 \\ \mathrm{H} & -4.77927 & 2.60262 & -1.41666 \\ \mathrm{H} & -4.83863 & 1.35474 & -0.16103 \\ \mathrm{H} & -4.25087 & 2.98371 & 0.22460 \\ \mathrm{H} & -2.56873 & 3.22984 & -2.50607 \\ \mathrm{H} & -1.03418 & 2.71868 & -1.79782 \\ \mathrm{H} & -2.09083 & 3.82957 & -0.91893 \\ \mathrm{H} & 2.55416 & 2.49923 & 0.71213 \\ \mathrm{H} & 0.49001 & 1.55491 & 0.17918\end{array}$


originally assigned structure of stereumone A, Fig.18<smiles>C[C@H]1C2=C(CCO2)[C@@H]2O[C@]13C(=O)CC(C)(C)[C@H](O)[C@H]23</smiles>

$$
\text { Rel energy (kcal/mol): } \begin{gathered}
\operatorname{Conf} 1 \\
0.0
\end{gathered}
$$

\begin{tabular}{|c|c|c|c|c|c|}
\hline C-nom & iGau & Exp & Calc & diff & 1 \\
\hline $\mathrm{C} 2-\mathrm{CH} 2$ & 12 & 59.00 & 71.15 & 12.15 & 71.15 \\
\hline $\mathrm{C} 3-\mathrm{CH} 2$ & 11 & 30.50 & 30.59 & 0.09 & 30.59 \\
\hline $\mathrm{C} 3 \mathrm{a}-\mathrm{C}$ & 8 & 129.00 & 107.97 & -21.03 & 107.97 \\
\hline $\mathrm{C} 4-\mathrm{CH}$ & 7 & 90.60 & 79.35 & -11.25 & 79.35 \\
\hline $\mathrm{C} 4 \mathrm{a}-\mathrm{CH}$ & 4 & 53.90 & 51.89 & -2.01 & 51.89 \\
\hline $\mathrm{C} 5-\mathrm{CH}$ & 3 & 81.60 & 72.04 & -9.56 & 72.04 \\
\hline C6-C & 2 & 40.60 & 38.61 & -1.99 & 38.61 \\
\hline $\mathrm{C} 7-\mathrm{CH} 2$ & 1 & 41.30 & 45.08 & 3.78 & 45.08 \\
\hline $\mathrm{C} 8-\mathrm{C}$ & 6 & 195.90 & 208.17 & 12.27 & 208.17 \\
\hline $\mathrm{C} 8 \mathrm{a}-\mathrm{C}$ & 5 & 68.10 & 87.96 & 19.86 & 87.96 \\
\hline $\mathrm{C} 9-\mathrm{CH}$ & 10 & 46.20 & 36.31 & -9.89 & 36.31 \\
\hline $\mathrm{C} 9 \mathrm{a}-\mathrm{C}$ & 9 & 151.00 & 163.77 & 12.77 & 163.77 \\
\hline $\mathrm{C}-\mathrm{CH} 3-6 \mathrm{a}$ & 17 & 21.10 & 26.87 & 5.77 & 26.87 \\
\hline $\mathrm{C}-\mathrm{CH} 3-6 \mathrm{~b}$ & 18 & 27.90 & 22.81 & -5.09 & 22.81 \\
\hline $\mathrm{C}-\mathrm{CH} 3-9$ & 14 & 23.00 & 12.53 & -10.47 & 12.53 \\
\hline
\end{tabular}

13C chem shifts: RMSD $=10.97 \mathrm{ppm}(\mathrm{MAE}=9.20) \quad \mathrm{N}=15 \quad\left\{\begin{array}{l}-21.03 \\ 19.86\end{array}\right\}$

\section{Conformer 1}

Energy: -884.44341 Hartree (Rel: $0.0 \mathrm{kcal} / \mathrm{mol}$ ) XYZ coordinates for conf 1 :

$\begin{array}{lrrr}\text { C } & -2.61517 & 0.66345 & -0.78268 \\ \mathrm{C} & -2.78894 & -0.19937 & 0.49245 \\ \mathrm{C} & -1.68361 & -1.29534 & 0.48248 \\ \mathrm{C} & -0.26124 & -0.68399 & 0.56060 \\ \mathrm{C} & -0.03714 & 0.46652 & -0.46389 \\ \mathrm{C} & -1.24483 & 1.30151 & -0.91515 \\ \mathrm{C} & 0.78107 & -1.41394 & -0.32606 \\ \mathrm{C} & 2.17005 & -0.96978 & -0.00695 \\ \mathrm{C} & 2.35006 & 0.35526 & 0.02546 \\ \mathrm{C} & 1.23050 & 1.33216 & -0.19558 \\ \mathrm{C} & 3.50968 & -1.65539 & 0.11361 \\ \mathrm{C} & 4.41316 & -0.43469 & 0.45967 \\ \mathrm{O} & 3.63194 & 0.77338 & 0.21115 \\ \mathrm{C} & 1.07812 & 2.32466 & 0.97155 \\ \mathrm{O} & 0.28714 & -0.57251 & -1.46950 \\ \mathrm{O} & -1.84068 & -2.15777 & -0.64301 \\ \mathrm{C} & -4.17336 & -0.86925 & 0.46630 \\ \mathrm{C} & -2.67726 & 0.67556 & 1.75634 \\ \mathrm{O} & -1.08876 & 2.42026 & -1.36875 \\ \mathrm{H} & 0.03063 & -0.48862 & 1.59550 \\ \mathrm{H} & -3.36087 & 1.46359 & -0.83632 \\ \mathrm{H} & -2.77320 & 0.02382 & -1.66274 \\ \mathrm{H} & -1.82769 & -1.93820 & 1.36035 \\ \mathrm{H} & 0.64351 & -2.47688 & -0.52745 \\ \mathrm{H} & 1.42496 & 1.90694 & -1.11020 \\ \mathrm{H} & 3.55970 & -2.41854 & 0.90018 \\ \mathrm{H} & 3.81454 & -2.14117 & -0.82479 \\ \mathrm{H} & 5.31821 & -0.36777 & -0.14805 \\ \mathrm{H} & 4.69403 & -0.42747 & 1.51853 \\ \mathrm{H} & 0.30880 & 3.06837 & 0.74957 \\ \mathrm{H} & 2.02452 & 2.84862 & 1.13667 \\ \mathrm{H} & 0.81821 & 1.81151 & 1.90517 \\ \mathrm{H} & -1.34997 & -1.74455 & -1.37845 \\ \mathrm{H} & -4.34033 & -1.45038 & 1.38194 \\ & & & \end{array}$

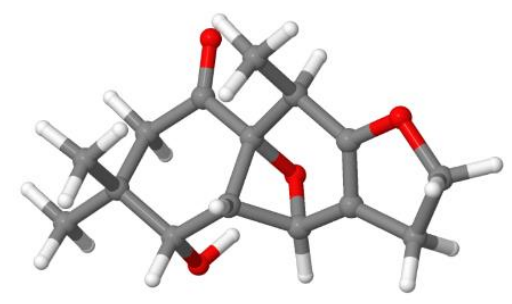




$\begin{array}{rrrr}\mathrm{H} & -4.96796 & -0.11628 & 0.39963 \\ \mathrm{H} & -4.26035 & -1.54918 & -0.38514 \\ \mathrm{H} & -1.74173 & 1.24432 & 1.80302 \\ \mathrm{H} & -3.49922 & 1.40009 & 1.79154 \\ \mathrm{H} & -2.73943 & 0.06066 & 2.66294\end{array}$

